\title{
Gauge/Gravity Duality applied to Condensed Matter Systems
}

Martin Matthias Ammon

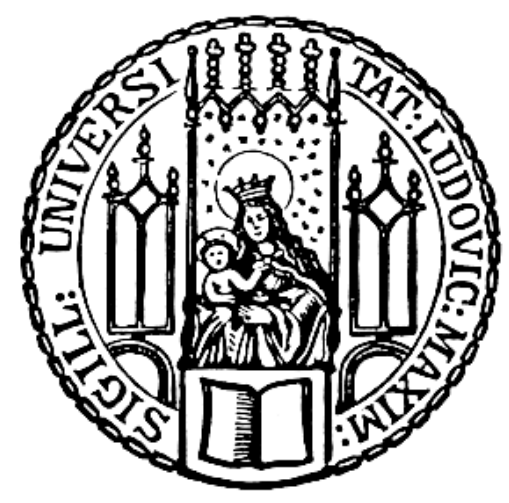

München 2010 



\title{
Gauge/Gravity Duality applied to Condensed Matter Systems
}

\author{
Martin Matthias Ammon
}

\author{
Dissertation \\ an der Fakultät für Physik \\ der Ludwig-Maximilians-Universität \\ München
}

vorgelegt von

Martin Matthias Ammon

aus Roth, Mittelfranken.

München, den 31. Mai 2010 
This thesis is based on the author's work partly published in [1-9] conducted from November 2007 until May 2010 at the Max-Planck-Institut für Physik (WernerHeisenberg-Institut), München under supervision of Priv.-Doz. Dr. Johanna Karen Erdmenger.

Erstgutachter: Priv.-Doz. Dr. Johanna Karen Erdmenger Zweitgutachter: Prof. Dr. Dieter Lüst

Tag der mündlichen Prüfung: 07. Juli 2010 


\section{Zusammenfassung}

In der hier vorliegenden Arbeit werden mit Hilfe der AdS/CFT-Korrespondenz Phänomene stark gekoppelter quantenkritischer Systeme untersucht, wie sie beispielsweise in Systemen der kondensierten Materie auftreten. Die AdS/CFT-Korrespondenz ist dabei eine aus der Stringtheorie hervorgegangene Dualität zwischen Eich- und Gravitationstheorien, die Rechnungen in starker Kopplung auf der Eichtheorieseite in perturbative Rechnungen auf der Gravitationsseite übersetzt. Dabei setzt die ursprüngliche, von Maldacena im Jahr 1997 entwickelte Vermutung vierdimensionale, $\mathcal{N}=4$ supersymmetrische YangMills-Theorie und Typ IIB-Supergravitation im fünfdimensionalen Anti-de Sitter-Raum in Beziehung. Diese Vermutung kann auf verschiedene Weise verallgemeinert werden. So können auf der Eichtheorieseite Zustände mit endlicher Temperatur und Dichte betrachtet werden oder Freiheitsgrade hinzugefügt werden, die sich in der fundamentalen Darstellung der Eichgruppe transformieren, die sogenannten Flavor-Freiheitsgrade

Diese Deformationen der Korrespondenz werden in der hier vorliegenden Arbeit verwendet, um stark gekoppelte Systeme in der Nähe von quantenkritischen Punkten besser zu verstehen. Wir approximieren hierbei die Feldtheorie am quantenkritischen Punkt durch $\mathcal{N}=4$ supersymmetrische Yang-Mills-Theorie. Die Ladungsträger des Systems werden durch supersymmetrische Flavor-Felder eingeführt. Dieses Modell ist der Ausgangspunkt von vielen Überlegungen und Untersuchungen, die im Rahmen der Dissertation durchgeführt wurden.

Beispielsweise wird in der hier vorliegenden Arbeit im Fall von zwei Flavor Feldern, die die gleiche Masse haben, ein chemisches Potential für den Isospin betrachtet und das Phasendiagramm untersucht. Das Isospin-chemische Potential bricht hierbei den nicht-Abelschen Anteil der Flavor-Symmetrie, SU(2), auf U(1). Wird ein kritischer Wert des Isospinchemischen Potentials überstiegen, so zeigt unsere Rechnung, dass das stark-gekoppelte System gegenüber Fluktuationen instabil wird. Es bildet sich ein neuer thermodynamisch favorisierter Zustand aus. Dieser Zustand bricht die restliche U(1) Flavorsymmetrie spontan und kann daher als eine Supraflüssigkeit angesehen werden. Ist nun die U(1) geeicht, erhält man auf diese Weise einen Supraleiter. Die Wechselstrom-Leitfähigkeit geht in der supraleitenden Phase für kleine Frequenzen gegen Null. Die Gleichstrom-Leitfähigkeit jedoch ist unendlich, wie das auch von einem Supraleiter erwartet wird. Da der Supraleiter stark gekoppelt ist, kann dieser nicht durch die BCS Theorie beschrieben werden. Des Weiteren berechnen wir auch die Fermifläche in der supraleitenden Phase und beobachten, dass diese auf vier ausgezeichnete Punkte kollabiert.

Außerdem wird eine holographische Methode zur Berechnung der Leitfähigkeit des Gleichstroms auf beliebige konstante elektrische und magnetische Felder weiterentwickelt. Hierbei wird die Leitfähigkeit nicht mit Hilfe von Linearer-Antwort-Theorie beschrieben, sondern ist selbst vom elektrischen Feld abhängig.

Schließlich wird auch ein zweites Modell für die Feldtheorie am quantenkritischen Punkt, eine Chern-Simons-Materie Theorie in (2+1)-Dimensionen, genauer untersucht. Die ChernSimons-Materie Theorie hat dynamische Freiheitsgrade und kann als Niederenergie-Limes 
von (2+1)-dimensionalen Membranen, sogenannter M2-Branen, aufgefasst werden. Auf verschiedene Weisen werden in der vorliegenden Arbeit auf der Eichtheorieseite Ladungsträger in Form von fundamentalen supersymmetrischen Feldern hinzugefügt und die effektive Kopplung an die Felder der Chern-Simons-Materie bestimmt. Auf der Gravitationsseite werden dabei höherdimensionale Membranen und andere nicht-perturbative Objekte, sogenannte KK-Monopole, in M-Theorie bzw. dessen Typ IIA Limes eingebettet.

Insgesamt erhoffen wir uns von diesen Untersuchungen an Modelltheorien, die sowohl Berechnungen bei starker als auch bei schwacher Kopplung zulassen, neue Impulse für die Theorie der kondensierten Materie. Dabei haben wir insbesondere quantenkritische Systeme, wie beispielsweise Hochtemperatur-Supraleiter, im Blick. 


\section{Contents}

$\begin{array}{lll}\text { Zusammenfassung } & \text { v }\end{array}$

1 Introduction and Overview 1

1.1 String Theory and strongly coupled systems . . . . . . . . . . . . . . . 3

1.2 Strongly coupled condensed matter svstems . . . . . . . . . . . . . . . 4

1.3 AdS/CFT and condensed matter systems . . . . . . . . . . . . . 8

1.4 Plan of the thesis . . . . . . . . . . . . . . . . . . . 13

\begin{tabular}{lll}
\hline Foundations of AdS/CFT & 15
\end{tabular}

2 The Anti-de Sitter/Conformal Field Theory Correspondence $\quad 17$

2.1 Review: The origin of AdS/CFT . . . . . . . . . . . . . . . 18

2.2 Review: The AdS/CFT Dictionarv . . . . . . . . . . . . . . . . . 28

2.3 Holographic renormalization for fermions . . . . . . . . . . . . 30

2.4 Review: Generalization to finite temperature . . . . . . . . . . . . . 41

2.5 Review: Adding fundamental flavor . . . . . . . . . . . . . . . . . . . . 43

2.6 Other examples of gauge/gravity dualities . . . . . . . . . . . . . . . 58

II Applications to Condensed Matter Systems 61

3 Response to external fields $\quad 63$

3.1 Review: Linear response \& retarded Green's functions . . . . . . . . . . . 63

3.2 Holographic Calculation: Linear response . . . . . . . . . . . . . . . . 65

3.3 Holographic calculation: Beyond linear response . . . . . . . . . . . . 75

4 Holographic Superconductors $\quad 87$

4.1 Introduction and summary . . . . . . . . . . . . . . . . . 88

4.2 Field theorv description . . . . . . . . . . . . . . . . . . . 90

4.3 Dual Gravity picture . . . . . . . . . . . . . . . . . . . . . . 91

4.4 String Theory Picture . . . . . . . . . . . . . . . . . . . . . . . . 99

5 Fermions in AdS/CFT 103

5.1 Introduction and Summary . . . . . . . . . . . . . . . . 103

5.2 Worldvolume Fermions of Dp-branes . . . . . . . . . . . . . 106

5.3 Emergence of the Fermi surface . . . . . . . . . . . . . . . . . 114

6 Non-relativistic systems: AC \& DC conductivities $\quad 129$

6.1 Introduction and Summarv . . . . . . . . . . . . . . . . . . . . . 129

6.2 Adding Flavor to Schrödinger Spacetime . . . . . . . . . . . . . . . 132 
6.3 DC Conductivitv of Probe Flavor . . . . . . . . . . . . . . . . . . . 139

6.4 AC Conductivity of Probe Flavor . . . . . . . . . . . . . . . . . . . . . . . 145

III Flavor in Chern-Simons-Matter Theories 151

\begin{tabular}{llr}
\hline & Adding Flavor to $A d S_{4} / C F T_{3}$ & $\mathbf{1 5 3}$
\end{tabular}

7.1 Introduction and summary . . . . . . . . . . . . . . . . 154

7.2 A M-theorv Primer . . . . . . . . . . . . . . . . . . . . 156

7.3 Review of ABJM Theorv . . . . . . . . . . . . . . . . . 159

7.4 General Analvsis of Probe Flavor . . . . . . . . . . . . . . . . . . 163

7.5 Codimension Zero $\mathcal{N}=3$ Supersvmmetric Flavor . . . . . . . . . . . . 169

7.6 Codimension One $\mathcal{N}=(0.6)$ Supersymmetric Flavor . . . . . . . . . . . 173

7.7 SU(4) Equivalence of Probe Flavor . . . . . . . . . . . . . . . . . 176

7.8 Other Flavor branes in $A d S_{4} / C F T_{3} \ldots \ldots$. . . . . . . . . . . . 179

8 Conclusions and Outlook 181

\begin{tabular}{lr}
\hline A Glossarv & 189
\end{tabular}

A.1 Bosonic String Theory . . . . . . . . . . . . . . . . . . . . . . . 189

A.2 Superstring Theory . . . . . . . . . . . . . . . . . . . . 197

A.3 D-branes . . . . . . . . . . . . . . . . . . . . . . . . . . . 205

B Appendix to Chapter 7 211

B.1 Supersvmmetrv of Type IIB Probes . . . . . . . . . . . . . . . . . . . . 211

B.2 Tvpe IIB to M-theorv . . . . . . . . . . . . . . . . . . . . . . . 214

B.3 Supersvmmetrv of M-theorv Obiects . . . . . . . . . . . . . . . . 219

B.4 $\mathcal{N}=(0,6)$ Supersymmetry Transformations . . . . . . . . . . . . . 223

\begin{tabular}{ll}
\hline Acknowledgements & 240
\end{tabular} 


\section{1 \\ Introduction and Overview}

In the twentieth century, physicists made much progress in the theoretical understanding of many phenomena in nature, related to quantum theory and gravity. The basic building blocks of the physical world are particles and their interactions. Today, four basic interactions are known: the electromagnetic force, gravity, the weak and strong nuclear forces. Except gravity, the remaining three interactions, as well as the matter fields, can be formulated in terms of a quantum field theory, known as the standard model of particle physics. Up to now, the standard model of particle physics has been very well tested at least up to energies of $100 \mathrm{GeV}$. Every high energy physics experiment has yielded results consistent with the Standard Model.

All quantized forces, which are incorporated in the standard model, can be described in terms of exchange particles. For example, the electromagnetic force is described by the exchange of massless spin- 1 particles, called photons, which we denote as $\gamma$. The quantized theory of electrodynamics, Quantum Electrodynamics (QED), is very precise. The theoretical prediction for the anomalous magnetic moment of the electron, for example, agrees with the measured value up to one part in $10^{10}$.

The weak nuclear force is described by three exchange particles, $W^{ \pm}$and $Z^{0}$. In contrast to the photon, the $W^{ \pm}$and $Z$ are massive spin-1 gauge bosons, and therefore the force is short-ranged. The weak force is responsible for nuclear beta decay, for example. Although the electromagnetic and weak forces seem to be very different, they can be formulated in a unified theory, called the electro-weak theory, in terms of a $S U(2) \times U(1)_{Y}$ gauge theory. The electro-weak symmetry breaking mechanism breaks the $S U(2) \times U(1)_{Y}$ gauge symmetry to the electromagnetic $U(1)_{\text {em }}$ group, leaving only the photon massless and giving mass to the $W^{ \pm}$and $Z$ boson. This symmetry breaking mechanism also predicts the existence of a massive scalar particle, called the Higgs boson. At colliders, such as the Large Hadron Collider (LHC) at CERN, experimentalists aim at discovering this Higgs boson.

Finally, the strong nuclear force can be described by Quantum Chromodynamics (QCD), 
which is an $S U(3)$ gauge theory. The corresponding exchange particles, gluons, are massless spin-1 gauge bosons that carry color charge. Although we know the Lagrangian of QCD, the strong nuclear force remains mysterious, since QCD is an asymptotically free theory, i.e. the coupling strength decreases if the renormalization scale increases, and vice versa. Therefore, particles, scattering with a large momentum transfer, interact more and more weakly. Such processes can be described by perturbation theory. In the opposite situation, however, i.e. for particle-scattering at small momentum transfer, the interaction strength is large and we cannot trust perturbation theory. Therefore QCD cannot be solved analytically in the infrared, i.e. for small energies. Physicists started to investigate strongly-coupled gauge theories by discretizing spacetime onto a lattice. This approach is called Lattice Gauge Theory. Though lattice gauge theories are not exactly solvable, they can be studied using simulations on a computer. The behavior of the theory defined on the continuum spacetime may be recovered by performing simulations on larger and larger lattices, while making the lattice spacing smaller.

To summarize, the Standard Model of particle physics is a gauge theory with gauge group $S U(3) \times S U(2) \times U(1)_{Y}$. It also includes matter particles of fermionic nature, which can be grouped into six quarks (up, down, strange, charm, bottom and top) and six leptons (electron, electron neutrino, muon, muon neutrino, tau and tau neutrino). Whereas quarks carry color charge and hence interact via the strong interaction, the leptons are not charged under the strong force. Quarks and the electrically charged leptons also carry weak and electric charge. Moreover, the quarks and the leptons can each be grouped into pairs since the corresponding particles exhibit similar physical behavior. Therefore we have three different copies of matter particles, called first, second and third generation.

The standard-model described above has also some shortcomings. So far, the Higgs boson and therefore also the electro-weak symmetry breaking mechanism has not been verified experimentally. It is not quite a complete description of leptons either, because it does not describe nonzero neutrino masses. Moreover, we do not understand QCD at low energies: For example, it is very difficult to find the low-energy spectrum built up of quarks. Experimentally we know that only bound states, namely baryons and mesons, exist which do not carry any color charge. But so far we have no theoretical understanding of this phenomena, referred to as color confinement.

Finally, due to the strong coupling, our understanding of many features of QCD remains incomplete. In fact, a combination of results taken from lattice QCD and analytical efforts have allowed theorists to map out parts of the phase diagram. We know that for extremely high temperature and/or density a new phase exists, the Quark-Gluon plasma. In this phase, quarks and gluons are deconfined and behave as nearly free particles. However, current experiments at Brookhaven National Laboratory's Relativistic Heavy Ion Collider (RHIC) observe a strongly coupled Quark-Gluon Plasma slightly above the confinementdeconfinement temperature, i.e. between $175 \mathrm{MeV}$ and $250 \mathrm{MeV}$. In this phase, quarks and gluons are neither confined nor free particles. The appropriate description is in terms of hydrodynamics. For the hydrodynamic description we have to determine the transport coefficients, such as bulk and shear viscosity, for QCD at strong coupling and finite density. A reliable calculation cannot be done with either lattice QCD or perturbative methods.

So far, we have not discussed the fourth fundamental interaction, namely gravity. Gravity, the most mysterious of the four fundamental interactions, is incorporated in the standard model only as a classical theory. We do not know how to quantize gravity in a consistent 
fashion since gravity is perturbatively non-renormalizable. But this is not the only problem. We have no idea why the cosmological constant $\Lambda$ is so small and what dark energy really is. Gravity is also very surprising from a different point of view. For black holes, which are an exact solutions of gravity, the entropy scales like the area of the horizon and not like the volume. This fact is the first evidence of a holographic principle in gravity: the description of space may be encoded on a boundary to that region. In a larger and more speculative sense, this holographic principle suggests that the information of the whole $(3+1)$-dimensional universe is stored on a $(2+1)$-dimensional hypersurface, for example the cosmological horizon.

String theory may resolve some of the problems mentioned above. String theory states that all particles are not point-like objects, but rather one-dimensional extended objects. The vibrations of the string correspond to different elementary particles. String theory does not only contain scalar fields and gauge fields as well as fermions, but also a spin-2 excitation, which is the graviton. The low-energy effective action, derived in string theory, includes the usual Einstein-Hilbert term of general relativity. Moreover, string theory has only one dimensionful scale, the characteristic string length. Therefore string theory is considered to be a step towards the correct fundamental description of nature. In particular, string theory is the first viable candidate for a theory of everything, describing all known forces and matter in a mathematically consistent system. Note that string theory is very difficult to test experimentally, like every other theory of quantum gravity, since the Planck length is very small. Moreover, string theorists have not succeeded in constructing the standard model within string theory, although they are very close to it. This is also due to the fact that string theory requires the existence of six extra dimensions, which are not observable, in addition to the usual four spacetime dimensions.

But let me bring you back to earth. Besides particle physics, gravity and cosmology, there are also other very interesting areas of research, e.g. condensed matter physics. Condensed matter physics deals with the macro- and microscopic physical properties of matter. It investigates the many-body interactions and collective phenomena in condensed phases of material, i.e. liquids and solids. In the last few decades, condensed matter physics has evolved and expanded to an area that encompasses frontiers in fundamental physics as well as to an interdisciplinary research field including laser cooled atoms, nanoscience, spintronics and even biophysics. Since condensed matter theory can be applied to real-world systems, you may be even more surprised that there is a connection between condensed matter physics and string theory. In this thesis we claim that we can get new insights into condensed matter physics using string theory. In particular, we use the holographic techniques mentioned above to investigate strongly correlated electron systems, such as High- $\mathrm{T}_{c}$ superconductors and Non-Fermi liquids.

\subsection{String Theory and strongly coupled systems}

In the preceding section we mentioned that string theory is one of the most promising candidates for a theory of everything. The elementary particles should be viewed as vibrations of one-dimensional extended objects, called strings. Besides strings, string theory contains also higher-dimensional objects, Dirichlet-branes, in its spectrum. The strings propagate in ten-dimensional spacetime. In fact, in ten dimensions there exists five consistent string theories. All these string theories are related by dualities. Some are strong-weak coupling 
dualities, i.e. one of the theories is strongly coupled whereas the other theory is weakly coupled.

By taking certain limits of string theory, we can establish a holographic duality between a weakly-coupled theory of gravity in asymptotically Anti-de Sitter spacetime (AdS) and a strongly-coupled conformal field theory (CFT) living on the boundary of that spacetime. This duality is called Anti-de Sitter/Conformal Field Theory correspondence (AdS/CFT) [10-12].

The most prominent example of the AdS/CFT correspondence arises from the study of D3-branes in flat space. Here the gravity theory is type IIB supergravity in the nearhorizon geometry of D3-branes, which is $A d S_{5} \times S^{5}$, and the dual strongly-coupled CFT is $(3+1)$-dimensional $\mathcal{N}=4$ supersymmetric $S U\left(N_{c}\right)$ Yang-Mills (SYM) theory in the 't Hooft limit $N_{c} \rightarrow \infty$ and additionally with large 't Hooft coupling $\lambda \equiv g_{Y M}^{2} N_{c} \rightarrow \infty$.

The AdS/CFT correspondence is a very useful tool since it relates a strongly-coupled field theory to a weakly-coupled gravity theory. Therefore we can make predictions for strongly coupled field theories by computations on the weakly-coupled gravity side. AdS/CFT is especially useful if other conventional methods are not applicable. In this thesis we apply the AdS/CFT correspondence to strongly-coupled field theories at finite density and finite temperature. In particular, we determine the phase diagram of such theories and calculate real-time transport coefficients, such as conductivities.

By construction, perturbation theory, which is essentially an expansion in small coupling, is not applicable for strongly-coupled systems. Further note that at finite density, approaches such as lattice field theory break down. Moreover it is very difficult within lattice field theory to calculate transport coefficient in the real-time formalism.

The methods developed in this thesis can be used to study a variety of systems, such as the strongly-coupled Quark-Gluon plasma and Condensed matter systems. In this thesis, we concentrate on the latter one. Let us therefore review the basic facts of strongly coupled condensed matter systems.

\subsection{Strongly coupled condensed matter systems}

The conventional approach of condensed matter theory is built on two conceptual cornerstones: Landau's symmetry breaking theory of phase transitions and the Fermi liquid theory (for a review see [13]). The Landau theory for phase transitions of matter classifies different phases of matter by their symmetries. In particular, phase transitions are associated with changes in symmetry. Fermi liquid theory treats properties of electrons in solids as small perturbations of the ground state which consists of filled single-particle energy levels.

These cornerstones of condensed matter theory are no longer valid when certain emerging phenomena in modern topics of condensed matter theory are studied. For instance, strongly correlated systems such as high temperature superconductors [14, 15], heavy fermion compounds $[16,17]$, fractional quantum hall liquids in effectively two-dimensional electron gas [18-20] and Luttinger liquids in one-dimensional conducting systems $[13,21-$ 23], all involve properties which are beyond the underlying perturbative description of 
conventional Fermi liquid theory. The Landau theory of phase transitions may be also not applicable to fractional quantum hall states and spin liquids [24-26]. In these systems, phase transitions are not associated with the breaking of symmetries. Rather, the transitions among different topological or quantum orders can occur without changing the corresponding symmetries.

Hence, new conceptional ideas are needed to describe these emerging areas of condensed matter physics. Let us discuss two examples of strongly correlated electron systems: heavy fermion compounds and high- $\mathrm{T}_{c}$ superconductors.

Let us first consider heavy fermion compounds. Heavy fermion compounds are a specific type of metallic compound in which the effective charge carrying quasiparticles have a mass of order 100 to 1,000 times of the bare electron mass. Therefore the specific heat at low temperatures is up to 1,000 times larger then the value of the expected free-electron theory. The typical phase diagram of heavy fermion compounds, depending on the pressure and the temperature, is shown in figure 1.1(a). By changing the pressure, we obtain different phases. For small pressure and small temperature, the material is antiferromagnetic. If we further increase the pressure and cool the system below $2 \mathrm{~K}$, the material is superconducting. This superconducting phase is controlled by a zero temperature phase transition [27]. Indeed, the superconductivity is magnetically mediated, not phonon-mediated as in the usual BCS theory. Since the normal-conducting phase above the superconducting dome shows non-Fermi liquid behavior, we might conclude that a perturbative quasiparticle approach is not applicable [28].

The second example are high- $\mathrm{T}_{c}$ superconductors. High- $\mathrm{T}_{c}$ superconductors are materials that have a superconducting transition temperature $T_{c}$ above $30 \mathrm{~K}$. The first high- $T_{c}$ superconductors were discovered by Karl Müller and Johannes Bednorz in 1986, for which they were awarded the Nobel price in physics in 1987. In 1986, the cuprate superconductors $\mathrm{LaBaCuO}$ and $\mathrm{La}_{2-x} \mathrm{Sr}_{x} \mathrm{CuO}_{4}$ were discovered. Until the discovery of Fe-based high- $\mathrm{T}_{c}$ superconductors in 2008, only cuprate superconductors were known to be high- $T_{c}$.

After more than twenty years of intensive research, the origin of high-temperature superconductivity is still not clear. High- $\mathrm{T}_{c}$ superconductors differ fundamentally from conventional BCS superconductors since they are doped Mott Insulators. For example, $\mathrm{La}_{2} \mathrm{CuO}_{4}$ is not superconducting but rather antiferromagnetic at low temperatures. By reducing the number of conducting electrons per $\mathrm{Cu}$ atom in the copper oxide planes, e.g. by chemical substitution to $\mathrm{La}_{2-x} \mathrm{Sr}_{x} \mathrm{CuO}_{4}$, the compound superconducts at low temperatures for sufficiently large doping $x$. For $\mathrm{LaCuO}_{4}$, the optimal doping is given by $x \approx 3 / 20$ yielding a critical temperature $T_{c} \approx 40 \mathrm{~K}$. For larger values of $x$, the compound is called over-doped, whereas for smaller values, the compound is under-doped. The schematic phase diagram is given in figure 1.1(b).

Note that in the over-doped region for temperatures $T>T_{c}$, the material behaves as a Fermi liquid. In contrast, in the under-doped region for $T>T_{c}$, we are in the still mysterious pseudogap region where the material has non-Fermi liquid properties. It is speculated that in high- $\mathrm{T}_{c}$ superconductors, there is zero temperature phase transition hidden beneath the superconducting dome. Note that also if this phase transition exists, we have to confirm that it is continuous and relevant for the dynamics in the superconducting and in the pseudogap region.

Even more mysterious is a new universal law for high- $\mathrm{T}_{c}$ superconductors. In high- $\mathrm{T}_{c}$ 


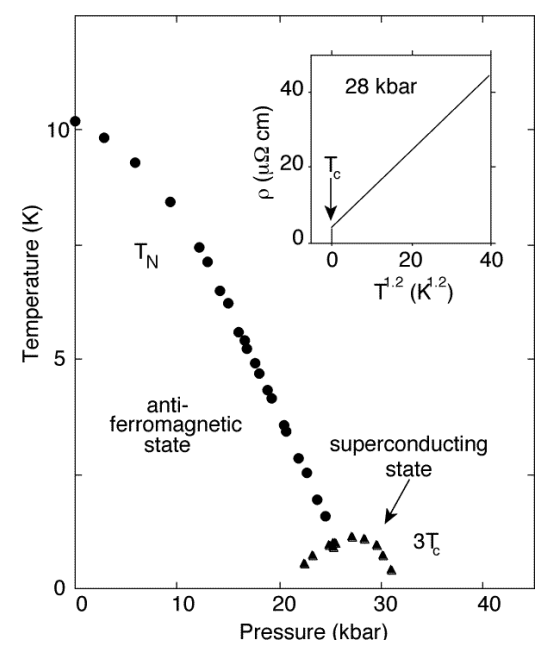

(a)

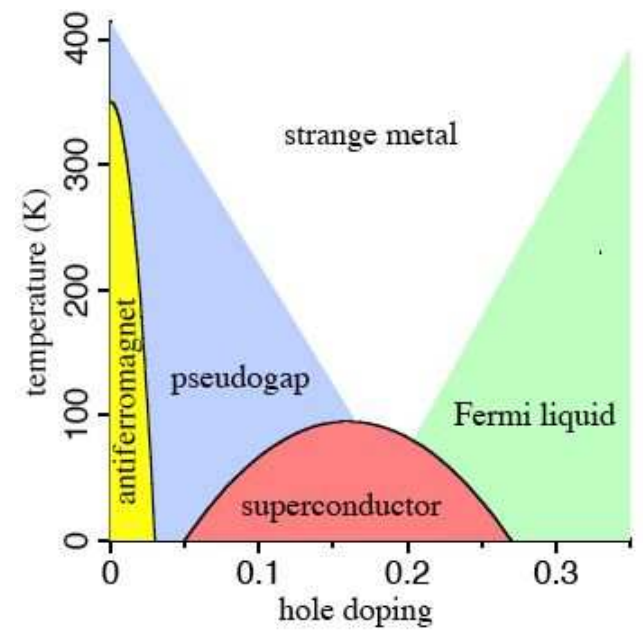

(b)

Figure 1.1: The phase diagram of two non-BCS superconductors. In both pictures, under the superconducting dome, a quantum critical point may be hidden. Figure (a) shows the phase diagram of the heavy fermion compound $\mathrm{CePd}_{2} \mathrm{Si}_{2}$ as a function of temperature and pressure. Note that the bottom left phase is antiferromagnetically ordered and the bottom middle phase is superconducting. Figure (b) shows the simplified doping-dependent phase diagram of cuprate superconductors for hole doping. The phases shown are the antiferromagnetic (AF) phase which is close to zero doping, the pseudogap, the Fermi liquid and the superconducting phase close to optimal doping. In the white area of the phase diagram, the system behaves as a strange metal. Figures taken from $[29,30]$.

superconductors we can measure the critical temperature $T_{c}$, the superconducting density close to zero temperature, $n_{s}$, and the electrical resistivity $\rho_{d c}$, measured just above the critical temperature. Surprisingly, Homes' law [31-34] states that in cuprate high- $T_{c}$ superconductors these three quantities satisfy a relation of the form

$$
n_{s} \cdot \rho_{d c} \approx 4.4 T_{c}
$$

Note that this is an experimental result. Since many novel superconductors are anisotropic, the resistivity and the superconducting density depend on the crystal direction along which these quantities are measured. Therefore both quantities have to be measured along the same direction. The constant 4.4 does neither depend on the crystal direction nor on the particular material. The expression (1.2.1) assumes that the conductivity and temperature have both been recast in units of $\mathrm{cm}^{-1}$ (or $\mathrm{s}^{-1}$ ), and that the superfluid density has units of $\mathrm{cm}^{-2}$ (or $\mathrm{s}^{-2}$ ). For BCS superconductors in the dirty limit, a similar relation holds. Only the universal constant is slightly larger, namely 8.1 [32].

There is evidence that the dynamics of both mentioned non-BCS superconductors, one of which is a high- $T_{c}$ superconductor, are controlled by quantum critical points. Let me explain why quantum critical points are relevant 11 A quantum critical point is a continuous phase transition at zero temperature. Whereas finite temperature phase transitions are driven by thermal fluctuations, zero temperature phase transitions are due to a nonanalyticity in the ground state, as we vary the coupling constant $g$. In real-world systems,

\footnotetext{
${ }^{1}$ This discussion follows closely [35].
} 


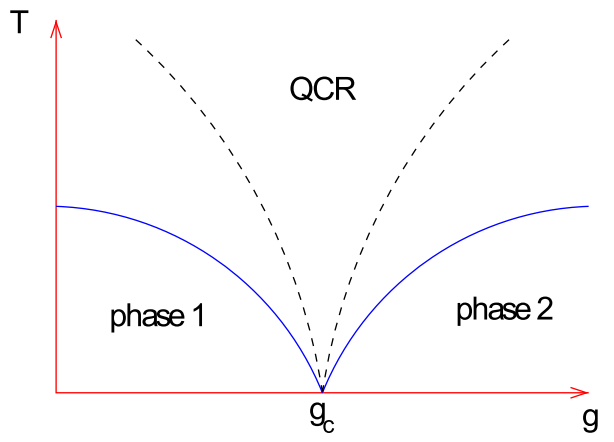

Figure 1.2: Schematic plot of a phase diagram near a continuous quantum critical point. Here, $g$ is a coupling constant of the theory, changing the dynamics of the system. Examples for $g$ include the doping parameter, a magnetic field or the pressure. On the vertical axis, the temperature $T$ is plotted. For zero temperature at $g=g_{c}$ a quantum phase transition occurs. The two blue lines are phase transitions whereas the dotted lines are cross-overs. The dotted lines define also the quantum critical region, denoted by QCR. Taken from [30].

the coupling constants are doping parameters, magnetic fields or pressure. The nonanalyticity may be a crossing of the energy levels of the first excited state and the ground state, when we vary the coupling constant, or, in the case of a system with infinite volume, it can also be traced back to an avoided crossing of the energy levels.

Typically, if we approach the continuous quantum critical point, the energy of fluctuations about the ground state, $\Delta$, vanishes 2 whereas the coherence length $3 \xi$, diverges. Let $g_{c}$ be the critical value for the coupling constant $g$, then $\xi$ and $\Delta$ scale as

$$
\xi \sim\left|g-g_{c}\right|^{-\nu}, \quad \Delta \sim\left|g-g_{c}\right|^{z \nu} .
$$

Combining both equations, we obtain

$$
\Delta \sim \xi^{-z}
$$

$z$ is called the dynamical scaling exponent. Note that energy, i.e. $\Delta$, and distance, i.e. $\xi$, need not be inversely related. The theory at the quantum critical point itself is scaleinvariant. We call this theory a quantum critical theory. For $z=1$ the quantum critical theory is relativistic, whereas for $z \neq 1$ it is non-relativistic.

Near classical critical points, the critical fluctuations are limited to a narrow region around the phase transition. However, the influence of a quantum critical point is felt over a wide range of temperatures above the quantum critical point. The region where the quantum critical point dominates is called the quantum critical region (QCR). In the quantum critical region, the deformation away of criticality, measured by $\Delta$, is less important than the scale of temperature, i.e. $\Delta \ll T$. Hence, we can describe the system in the quantum critical region by a finite temperature quantum critical theory. Note also that quantum critical theories are strongly coupled. There is no a priori reason why the phase transition should occur when $g \ll 1$.

\footnotetext{
${ }^{2}$ You might think of the energy gap as the difference of the energies between first excited state and the ground state.

${ }^{3}$ Second order phase transitions invariably have a characteristic length scale, such as the length scale determing the exponential decay of equal-time correlators.
} 


\subsection{AdS/CFT and condensed matter systems}

In the last two years, scientists have begun to merge the ideas introduced in section 1.1, the AdS/CFT correspondence, and in section 1.2, transport in quantum critical regions, in order to get new insights into phenomena of strongly correlated electron systems [30, 36-38], often called Anti-de Sitter/Condensed matter theory or AdS/CMT.

Quantum critical theories are the natural place to start investigating condensed matter systems using the AdS/CFT correspondence for several reasons. First, quantum critical theories are scale invariant and therefore allow for a holographic description by the simplest versions of AdS/CFT. Second, quantum critical theories are difficult to investigate using traditional methods due to a lack of a description in terms of weakly-coupled quasiparticles. In fact, there are no models for quantum critical theories in $(2+1)$ - and higher dimensions for which analytical results for processes like transport can be obtained. 4 The AdS/CFT correspondence, however, provides a set of exact solutions for the quantum critical transport in $2+1$ dimensions $[42,43]$.

In section 1.2 we considered quantum critical theories. Some examples of quantum critical theories can effectively be described by $\mathrm{O}(\mathrm{N})$-models (with $N=2$ or $N=3$, for more details see [35]). Here, in order to use the machinery of the AdS/CFT correspondence, we consider instead of $\mathrm{O}(\mathrm{N})$-models strongly-coupled supersymmetric large $\mathrm{N}$ field theories which are dual to some gravity theories on asymptotically AdS spacetimes. For a concrete example we have in mind $(3+1)$-dimensional $\mathcal{N}=4$ supersymmetric $S U\left(N_{c}\right)$ Yang-Mills theory in the large- $N_{c}$ limit.

Let me emphasize that we do not claim to make quantitative predictions for quantum critical theories in this thesis. Note that the models of quantum critical theories are rather artificial since such models incorporate maximal supersymmetry as well as a large $\mathrm{N}$-limit of the gauge group. Instead, the aim of the AdS/CFT correspondence is different. AdS/CFT may give us evidence for which phenomena can occur at strong coupling. By comparing the results to a weak-coupling analysis, we may get new insights into quantum critical points, which are inherently strongly coupled. We hope that the solutions provided by the AdS/CFT correspondence, lead to a significant progress in formulating a (more general) theory of quantum critical transport. Using the experience from holographic calculations of the Quark-Gluon Plasma, we also might hope to find quantitative universal behaviors in the condensed matter systems of interest. For example, it is interesting to find the explanation in the dual gravitational theory for Homes' law mentioned in section 1.2 .

Within AdS/CMT, there are two different approaches to describe condensed matter systems, the bottom-up and the top-down approach. Let us first describe the bottom-up approach. In order to describe strongly-coupled field theories at finite temperature and density the bottom-up approach studies effective gravitational systems in the background of charged black holes. In particular, the effective gravitational system is given by EinsteinHilbert gravity, where sometimes also higher-order terms are added, with other ingredients like scalar fields, vector fields and fermions added to the theory.

\footnotetext{
${ }^{4}$ There exists a theory for the quantum critical transport [39-41] of models, such as the superfluidinsulator transition in the lattice boson models and the transition between antiferromagnetic and spin gap states in Mott insulators. The theory is based on insights from various weak-coupling perturbative renormalization group analyses and is limited to a narrow range of physical parameters.
} 
In the bottom-up approach we consider an unspecified strongly-coupled field theory with a global U(1) symmetry. Moreover, we consider finite-temperature states with a finite $\mathrm{U}(1)$ chemical potential. On the gravity side, the corresponding minimal ingredients are gravity, given by an Einstein-Hilbert term, and a U(1) gauge field. The bulk geometry corresponding to a finite $\mathrm{U}(1)$ chemical potential is a Reissner-Nordström black hole. With very standard gravitational systems it is possible to describe various examples of condensed matter phenomena including superfluidity (or superconductivity) as well as (Non-)Fermi liquids. Both systems are reviewed in the following.

\section{Review: Holographic superconductors in the bottom-up approach}

The bulk theory can describe a phase transition to s-wave superfluid states, if a scalar charged under the $U(1)$ is present [44-46]. On the gravity side, the Reissner-Nordström black hole grows scalar hair at low temperature, that is, a solution with a non-trivial scalar becomes thermodynamically preferred to Reissner-Nordström. In the dual field theory, the thermodynamically-preferred state includes a nonzero expectation value for a scalar operator charged under the global $U(1)$, which we refer to as the operator condensing. The phase transition is second order with mean-field exponents $[45,46]$.

Gauge-gravity duality can also describe p-wave superfluids, that is, superfluids in which the condensing operator is a vector charged under the $U(1)$, thus breaking not only the $U(1)$ but also rotational symmetry to some subgroup [47]. On the gravity side, the minimal ingredients are gravity and non-Abelian gauge fields. The simplest case is an AdS geometry and $S U(2)$ gauge fields, $A_{M}^{a}$, with Lorentz index $M$ and $a=1,2,3$ labels the $S U(2)$ generators $\tau_{a}$. Here the $U(1)$ is a subgroup of $S U(2)$, for example the $U(1)$ in the $\tau_{3}$ direction, which we call $U(1)_{3}$. At high temperature the thermodynamically preferred geometry is Reissner-Nordström with nonzero $A_{t}^{3}$. At low temperature, the charged black hole grows vector hair: the preferred solution has non-trivial $A_{x}^{1}$. The dual field theory has three conserved currents, $J_{a}^{\mu}$, dual to the gauge fields. A chemical potential, producing a finite density $\left\langle J_{3}^{t}\right\rangle$, explicitly breaks $S U(2)$ to $U(1)_{3}$, and the transition occurs at large chemical potential, where the thermodynamically preferred state has nonzero $\left\langle J_{1}^{x}\right\rangle$.

\section{Review: Holographic fermions in the bottom-up approach}

Of central importance for potential condensed matter applications is the holographic description of a Fermi surface ${ }^{5}$ [49-52]. On the field theory side, we consider some fermionic operator, charged under the $U(1)$, in a zero temperature state. Holographic calculations of the fermionic spectral function, as a function of frequency and momentum, reveal a pole at zero frequency but finite momentum, which defines the Fermi momentum. The pole represents an excitation about a Fermi surface.

On the gravity side, we have to add some bulk Dirac fermion charged under the $U(1)$. This bulk fermion is dual to the fermionic operator. The bulk fermion is propagating in a Reissner-Nordström black hole. The spectral function of the operator is extracted from solutions of the linearized bulk equation of motion, the Dirac equation. These Fermi liquids are, generically, not Landau Fermi liquids, although the exact properties depend

\footnotetext{
${ }^{5}$ For an alternative approach, see [48].
} 
on the mass and charge of the bulk fermion.

Note that we can also consider fermions in a superconductor by using fermions and scalar fields (or non-Abelian gauge fields). Holographic calculations of fermionic spectral functions in zero-temperature s-wave superfluid states exhibit the so-called peak-dip-hump structure [53], expected to be relevant in high- $T_{c}$ superconductors [54], as well as, for suitable mass and charge of the bulk fermion, continuous bands of poles [55] and, for suitable coupling to the bulk scalar, a gap, i.e. poles in the spectral function at nonzero momentum and nonzero frequency [56].

Generally, the bulk actions used in holographic constructions of superconductors and Fermi liquids are not derived from any particular string theory construction. In other words, they are basically ad hoc models built from the minimal ingredients needed to capture the essential physics. On the one hand, simple models have one big advantage, besides simplicity, namely a kind of universality: the results may be the same for many different theories, parametrized by the masses and couplings of the fields we added by hand, regardless of the details of their dynamics.

On the other hand we cannot identify the dual field theory explicitly. This is a major drawback. For example, the holographic results obtained from a bottom-up approach may tell us that a superfluid phase transition occurs, but may not tell us why. Is a nonzero $\left\langle J_{1}^{x}\right\rangle$ the result of some pairing mechanism? If so, is the pairing mechanism the same in every dual theory? Knowing an exact dual theory may help to answer such questions, for example by providing some weak-coupling intuition. Moreover, if we have not identified the degrees of freedom and their dynamics, it is impossible to compare the effects of the strongly coupled field theory, calculated in the dual gravitational system, to the effects in the weakly coupled regime of the field theory.

Therefore it is very important to know the detailed dynamics of a specific dual theory. Finding a dual Lagrangian means embedding the bulk theory into a full string or supergravity construction. This is the goal of the top-down approach. In this thesis we aim to find embeddings of the holographic superconductor and holographic (non-)Fermi liquids into a string theory.

The theory we study here involve a in general massive sector of $\mathrm{U}(1)$ charge carriers, in a state of non-vanishing charge density $\left\langle J^{t}\right\rangle \neq 0$, interacting among themself and dissipating energy and momentum in a larger set of neutral quantum critical degrees of freedom. This situation is schematically shown in figure 1.3. In particular, in our setup the quantum critical theory with relativistic scale-invariance is given by $(3+1)$-dimensional stronglycoupled $\mathcal{N}=4$ supersymmetric Yang-Mills theory with gauge group $S U\left(N_{c}\right)$ in the large $\mathrm{N}_{c}$ 't Hooft limit. It is straightforward to add a sector of charge carriers by adding $N_{f}$ hypermultiplets or chiral multiplets to the theory, transforming in the fundamental representation of the gauge group. If $N_{f} \ll N_{c}$, the dynamics of the charge carrier does not backreact on the underlying quantum critical field theory. The new charge carriers introduce a U(1) global symmetry - the baryon or charge carrier number - under which only the new fields are charged. Transport phenomena, associated with the global U(1) charge carrier number, are dominated by the dynamics of the charge carriers which are strongly interacting with the quantum critical degrees of freedom. To be precise, the charge carriers are $S U\left(N_{c}\right)$ gauge invariant and bound states, bilinear in the fields of the hyper- or chiral multiplet. In analogy to QCD, these fields are sometimes called mesons 


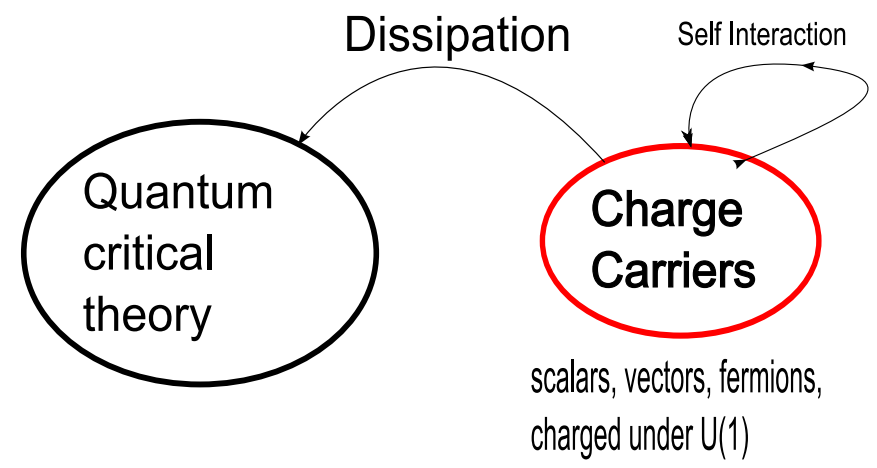

Figure 1.3: Schematic picture of our setup. The quantum critical degrees of freedom are given by $\mathcal{N}=4$ supersymmetric Yang-Mills theory in $(3+1)$-dimensions. The charge carrier sector corresponds to hypermultiplets or chiral multiplets on the field theory side.

or mesinos, depending on their bosonic or fermionic nature.

Note that the charge carriers do not necessarily propagate in $(3+1)$-dimensions. The charge carriers can also be confined to a plane, a line or even a point. Such theories are called defect theories. These defect theories are common in condensed matter applications since many real condensed matter systems are effectively $(2+1)$-dimensional degrees of freedom interacting with ambient (3+1)-dimensional degrees of freedom.

Let us now construct the embedding of the setup considered above into string theory. The gravity dual description of $\mathcal{N}=4$ supersymmetric Yang-Mills theory is given by type IIB supergravity in $A d S_{5} \times S^{5}$. The charge carriers are realized by embedding hyperplanes into string theory. These hyperplanes, known as Dp-branes, have dynamical degrees of freedom, such as scalars, vector and fermions, living on their worldvolume. These dynamical degrees of freedom play the role of the charge carriers. Since we consider $N_{f}$ hypermultiplets or chiral multiplets on the field theory, we have to embed $N_{f}$ Dp-branes. In the limit $N_{f} \ll N_{c}$, we may ignore the backreaction on the metric as well as other fields of type IIB supergravity. The global symmetry $U(1)$, under which the charge carriers are charged, corresponds to the overall $U(1)$ factor of the $U\left(N_{f}\right)$ gauge group associated with $N_{f}$ coincident Dp-branes.

After reviewing the two possible approaches to AdS/CMT, the bottom-up and the topdown approach, and discussing their advantages and disadvantages, we present the results, which I obtained using probe branes in the top-down approach.

\section{Achievements of this thesis}

Studying the probe branes introduced above, we obtain the following results:

- Calculation of the direct-current conductivity tensor

We compute the direct-current conductivity tensor associated with the transport of baryon number in arbitrary constant electric and magnetic fields to all orders in these fields.

- P-wave superconductors 
We embed holographic p-wave superconductors in string theory by considering two different kinds of mass-degenerate charge carriers, giving rise to a $S U(2)$ global symmetry. Moreover, we turn on a $S U(2)$ isospin chemical potential, breaking $S U(2)$ to $U(1)$. The two kinds of charge carriers have opposite chemical potentials. Above a critical value of the chemical potential, a non-vanishing condensate is generated breaking the remaining $\mathrm{U}(1)$ spontaneously.

- Fermions and (Non-)Fermi liquids

We embed fermions into string theory by considering the fermionic part of the probe Dp-brane action. In particular, we map the fermionic field theory operators to bulk fermions living on the worldvolume of the Dp-brane. Moreover, we study the fermionic response in the p-wave superconductor mentioned above. We find that for low temperatures a Fermi surface emerges, which collapses to points in the superconducting phase.

- Towards non-relativistic theories and their transport properties

We study the transport properties of non-relativistic field theories. In particular, we deform $\mathcal{N}=4$ super Yang-Mills theory by an irrelevant operator breaking the relativistic conformal group down to the Schrödinger group, which has non-relativistic scale invariance with dynamical exponent $z=2$. Introducing charge carriers as well as a finite number density, we compute the associated DC and AC conductivities using the dual gravitational description of probe D7-branes in an asymptotically Schrödinger spacetime.

- Adding charge carriers to (2+1)-dimensional Chern-Simons-Matter theories

We add charge carriers to $(2+1)$-dimensional conformal Chern-Simons-Matter theory, which can also be used as a model of the quantum critical theory. In particular, we calculate the effective couplings of the charge carriers to the Chern-Simons-Matter theory on the field theory side and determine the dual gravity descriptions in terms of D-branes or other non-perturbative objects.

- Holographic renormalization of fermions

As an added bonus, we also perform, to our knowledge for the first time, holographic renormalization for fermions in AdS. Holographic renormalization is a technique to cancel IR divergences on the gravity side and therefore to get finite partition functions as well as one-point functions. More precisely, we study a single free fermion in any space that asymptotically approaches Euclidean-signature AdS and determine the counterterms needed to render the on-shell action finite without spoiling the stationarity of the action. Our results rigorously justify many of the ad hoc prescriptions used in the literature, where divergences of the on-shell action were simply discarded.

- Retarded Green's function for coupled bulk fermions: An efficient way

We develop a method to compute the retarded Green's function for bulk fermions coupled to one another. Our method is actually very general, i.e. applicable to any system of coupled bulk fermions, not just to fermions on the worldvolume of probe Dp-branes, and is especially convenient for numerical analysis. 


\subsection{Plan of the thesis}

To conclude the introductory remarks, the thesis covers four main topics: the embedding of holographic superconductors and of (non-)Fermi liquids into string theory, the calculation of AC \& DC conductivities near quantum critical points with non-relativistic, $z=2$ Schrödinger symmetry and the construction of quantum critical theories with charge carriers for (2+1)-dimensional Chern-Simons theories.

The main content of the thesis is summarized below:

- The present chapter 1 gives an introduction to condensed matter systems near quantum critical points and explains the goals and the strategy of applying AdS/CFT to condensed matter systems.

- In chapter 2 we present an introduction to the AdS/CFT correspondence. In the first two sections we motivate and state the AdS/CFT correspondence. Moreover, the holographic renormalization of fermions in asymptotically AdS spacetimes is discussed. Finally, the AdS/CFT correspondence is generalized to finite temperature and fundamental matter degrees of freedom by adding probe Dp-branes.

- Chapter 3 is devoted to the response of external fields. First, we review linear response theory and the holographic calculation of the retarded Green's function. In particular, we show how to compute the conductivity tensor of alternating currents holographically as well as fermionic correlators. We also extend the analysis of fermionic correlators to the case of coupled bulk fermions and give an efficient recipe. At the end of the chapter, we calculate the direct-current conductivity tensor for arbitrary constant electric and magnetic fields to all orders in these fields.

- In chapter 4 we embed holograhic p-wave superconductors in a string theory setup. After explaining the field theory, we find a superconducting condensate on the gravity side. Finally, we give a string theory picture of the superconducting condensate.

- In chapter 5 fermions are introduced in AdS/CFT. In particular, we consider the fermions on the worldvolume of Dp-branes and determine their quantum numbers. Finally, we study the fermionic response in the p-wave superconducting state introduced in chapter 4 .

- In chapter 6 , we generalize the embedding of probe branes to non-relativistic systems with a Schrödinger symmetry. We apply this result to calculate the conductivity for alternating and direct currents.

- Chapter 7 is devoted to add probe branes to a (2+1)-dimensional conformal ChernSimons-Matter theory.

- In Chapter 8 we give a more detailed discussion of the results and we comment on open questions and further developments in the future. 

Part I

\section{Foundations of AdS/CFT}





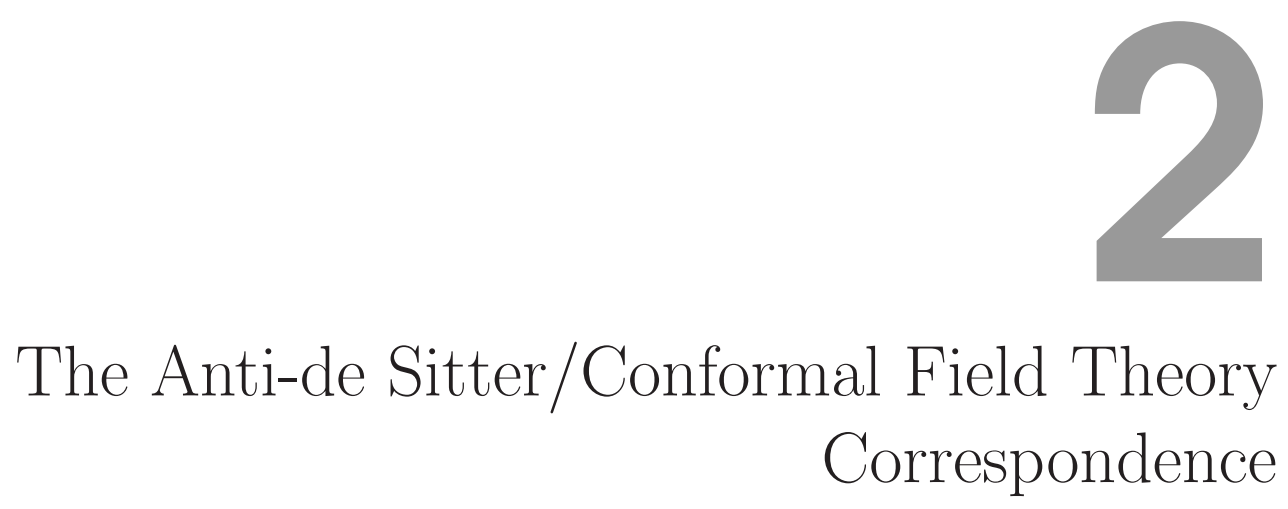

The Anti-de Sitter/Conformal Field Theory correspondence (AdS/CFT) is one of the most exciting discoveries in modern theoretical physics in the last two decades. The AdS/CFT correspondence discovered by Maldacena in 1997 [10], and more generally gauge-gravity dualities, are dualities between theories of gravity in some spacetime and field theories living on the "boundary" of that spacetime. Due to that property the AdS/CFT correspondence is a concrete realization of the holographic principle. We can map operators of the field theory side of the duality to fields of the gravity theory and relate the generating functional of the field theory to the partition function of the gravity side [11,12]. Moreover, in a particular limit, the AdS/CFT correspondence is an example of a strong/weak coupling duality: if the field theory is strongly coupled, the dual gravity theory is weakly curved. For that reason the AdS/CFT correspondence is a very promising approach to study strongly coupled field theories. Certain questions become computationally tractable on the gravity side and also conceptionally more clear.

This chapter provides a brief introduction to the AdS/CFT correspondence. In particular we emphasize facts which are highly relevant for the subsequent chapters of this thesis. For more details we refer to the standard reviews [57,58]. The outline of the chapter is as follows. In section 2.1 we first state and then motivate a particular example of the AdS/CFT correspondence. In particular we work out the precise mapping between operators on the field theory side and fields on the gravity side in section 2.2, as well as the prescription how to match the generating functional and the partition function. After explaining in section 2.3 how to renormalize holographically, i.e. how to get a finite partition function and vacuum expectation values, we generalize the AdS/CFT correspondence towards more realistic field theories. In section 2.4 we explain the implementation of finite temperature on the field theory side in the dual gravity theory. Finally, we add flavor degrees of freedom in section 2.5 which transform in the (anti-) fundamental representation of the corresponding gauge group. 


\subsection{Review: The origin of AdS/CFT}

There are many interesting examples of AdS/CFT correspondences available nowadays, relating gravity theories on asymptotically Anti-de Sitter spacetimes to Conformal Field Theories. However, for simplicity, we discuss in this chapter only the most prominent example of the AdS/CFT correspondence: between the maximally supersymmetric gauge theory in $3+1$ dimensions and a certain five-dimensional gravity theory in asymptotically Anti-de Sitter spacetime. Since a four-dimensional conformal field theory, denoted by $\mathrm{CFT}_{4}$, is mapped to a gravity theory in $\mathrm{AdS}_{5}$, we denote it by $\mathrm{AdS}_{5} / \mathrm{CFT}_{4}$ correspondence. After explicitly writing down the strongest form of the $\mathrm{AdS}_{5} / \mathrm{CFT}_{4}$ correspondence and explaining the main ingredients in section 2.1.1, we motivate the correspondence in section 2.1.2 by considering a certain limit of D-branes, which are (non-perturbative) ingredients of string theory 1 In section 2.1 .3 different limits of the $\mathrm{AdS}_{5} / \mathrm{CFT}_{4}$ correspondence are discussed which are more useful for explicit calculations.

\subsubsection{The $\mathrm{AdS}_{5} / \mathrm{CFT}_{4}$ correspondence}

The strongest form of the $\mathrm{AdS}_{5} / \mathrm{CFT}_{4}$ correspondence states that

\begin{tabular}{|}
$\mathcal{N}=4$ supersymmetric Yang-Mills (SYM) theory \\
with gauge group $S U\left(N_{c}\right)$ and Yang-Mills coupling constant $g_{Y M}$ \\
is dynamically equivalent to \\
type IIB superstring theory (with string length $l_{s}=\sqrt{\alpha^{\prime}}$ and coupling constant $g_{s}$ ) \\
on $A d S_{5} \times S^{5}$ with radius of curvature $L$ and $N_{c}$ units of $F_{(5)}$ flux on $S^{5}$. \\
The two free parameters on the field theory side, i.e. $g_{Y M}$ and $N_{c}$ are mapped to the \\
free parameters $g_{s}$ and $L / \sqrt{\alpha^{\prime}}$ of the string theory side by \\
$\qquad g_{Y M}^{2}=2 \pi g_{s}$, and $\quad 2 N_{c} g_{Y M}^{2}=L / \sqrt{\alpha^{\prime}}$. \\
\hline
\end{tabular}

Note that only the ratio $L / \sqrt{\alpha^{\prime}}$ is important and not the characteristic length scale of $A d S$, i.e. the radius of curvature $L$, and the string length $l_{s}=\sqrt{\alpha^{\prime}}$ separately. Therefore we quite often use the freedom to set the radius of curvature, $L$, to one. At the end, the dependence of $L$ can easily be restored.

What does it mean that both theories are dynamically equivalent? The correspondence states that both theories, i.e. $\mathcal{N}=4 \mathrm{SYM}$ and type IIB string theory on $A d S_{5} \times S^{5}$, are identical and therefore describe the same physics from two very different perspectives. In particular, if $\mathrm{AdS}_{5} / \mathrm{CFT}_{4}$ is true, one can map all the physics of one description onto all the physics of the other description. This is very peculiar since one can map a possible candidate for a theory of quantum gravity, i.e. type IIB string theory, to a field theory

\footnotetext{
${ }^{1}$ For those readers who are not familiar with string theory, we give a short introduction into technical terms relevant for this discussion and later chapters of the thesis. The glossary may be found in appendix A. For a more careful treatment of string theory I recommend the introductory book [59] as well as the standard textbooks [60-67].
} 
without any gravitational degrees of freedom. Moreover, the AdS/CFT correspondence is a realization of the holographic principle: the information of the five-dimensional theory, which is the Kaluza-Klein reduction of type IIB string theory on $S^{5}$, is mapped to a four-dimensional theory which lives on the "boundary" of the five-dimensional spacetime.

\subsubsection{The $\mathcal{N}=4$ supersymmetric Yang-Mills theory}

Before we motivate the correspondence in section 2.1.2, it is useful to discuss the technical terms of the conjecture in more detail. Let us start with the field theory involved, $\mathcal{N}=4$ supersymmetric Yang-Mills theory (SYM) in four spacetime dimensions.

Supersymmetry is an extension of the Poincaré symmetry including also anticommuting generators, the spinorial supercharges $\mathcal{Q}$, which relate bosonic and fermionic fields. In four dimensions, the spinorial supercharges $\mathcal{Q}$ are left-handed Weyl spinors. $\mathcal{N}$ denotes the number of those supercharges. Since a Weyl spinor in four dimensions consists of two complex components, the total number of real supercharges is $2 \times 2 \times \mathcal{N}$, i.e. 16 real supercharges in the case of $\mathcal{N}=4.2$

The supercharges act as lowering and raising operators on the helicities/spins of the fields. If $\mathcal{N}>4$, we have to include degrees of freedom with helicity/spin greater than 1 . Since we are only interested in field theories which contain only elementary fields with spin less (or equal) to one, $\mathcal{N}=4$ is the maximal number of supercharges in four spacetime dimensions 3

The supersymmetry algebras also have global symmetry rotating the supercharges into each other. This so-called $\mathcal{R}$-symmetry is given by $U(\mathcal{N})_{\mathcal{R}}$ for four-dimensional field theories.

$\mathcal{N}=4$ supersymmetry in four dimensions is very restrictive. For example, there is only one multiplet containing only degrees of freedom with helicity $\leq 1$, the $\mathcal{N}=4$ vector multiplet. This vector multiplet consists of a gauge boson $A_{\mu}$, four (left-handed) Weyl fermions $\lambda_{A}, A \in\{1, \ldots, 4\}$ as well as six real scalars $X^{k}, k \in\{1, \ldots, 6\}$, which can be grouped in three complex scalars $\Phi_{k}=X^{2 k-1}+i X^{2 k}, k \in\{1,2,3\}$. Since the gauge field transforms in the adjoint representation of the gauge groups and all other fields of the multiplet are related to the gauge field by supersymmetry, all fields have to transform in the adjoint representation of the gauge group. Under the $\mathcal{R}$-symmetry, the gauge field transforms as a $\mathbf{1}$, whereas the Weyl fermions $\lambda_{A}$ are in the $\mathbf{4}$ and the scalars $X^{i}$ are in the 6 representation of $S U(4)_{\mathcal{R}}$. The field content of $\mathcal{N}=4$ SYM is summarized in table 2.1. In this table we decomposed the $\mathcal{N}=4$ multiplet into a $\mathcal{N}=2$ vector multiplet and a $\mathcal{N}=2$ hypermultiplet. These $\mathcal{N}=2$ multiplets can be further decomposed into $\mathcal{N}=1$ multiplets: the $\mathcal{N}=2$ vector multiplet may be decomposed into a $\mathcal{N}=1$ vector multiplet $W_{\alpha}$ containing $\lambda_{4}$ and $A_{\mu}$ and into a $\mathcal{N}=1$ chiral multiplet $\Phi_{3}$ with the real scalar fields $X_{5}$ and $X_{6}$ as well as the fermion $\lambda_{3}$. The $\mathcal{N}=2$ hypermultiplet can be obtained by two $\mathcal{N}=1$ chiral multiplets each containing two real scalars and a fermion.

In order to convince ourselves that we really know the Lagrangian of the field theories

\footnotetext{
${ }^{2}$ The algebra of symmetries of the $\mathcal{N}=4$ SYM contains another set of spinorial supercharges. To distinguish both sets, $\mathcal{Q}$ are called the Poincaré supercharges.

${ }^{3}$ Therefore $\mathcal{N}=4 \mathrm{SYM}$ in four dimensions is also called maximally supersymmetric Yang-Mills theory.
} 


\begin{tabular}{|c|c|c|c|c|}
\hline $\mathcal{N}=2$ & components & spin & $S U(4)_{\mathcal{R}}$ & $\Delta$ \\
\hline$\left(W_{\alpha}, \Phi_{3}\right)$ & $A_{\mu}$ & 1 & $\mathbf{1}$ & 1 \\
vector & $\lambda_{3}, \lambda_{4}$ & $1 / 2$ & $\mathbf{4}$ & $3 / 2$ \\
& $\left(X^{5}, X^{6}\right)$ & 0 & $\mathbf{6}$ & 1 \\
\hline$\left(\Phi_{1}, \Phi_{2}\right)$ & $\lambda_{1}, \lambda_{2}$ & $1 / 2$ & $\mathbf{4}$ & $3 / 2$ \\
hyper & $X^{1}, X^{2}, X^{3}, X^{4}$ & 0 & $\mathbf{6}$ & 1 \\
\hline
\end{tabular}

Table 2.1: Fields of the $\mathcal{N}=4$ vector multiplet and their quantum numbers under the $\mathcal{R}$-symmetry group as well as their conformal dimension $\Delta$. Note that we have not assigned quantum numbers to the abelian part of $\mathcal{R}$-symmetry. Although the supersymmetry algebra for $\mathcal{N}=4 \mathrm{SYM}$ in four dimensions is invariant under $U(4)_{\mathcal{R}}$, the symmetry is broken to $S U(4)_{\mathcal{R}}$ by the action $(2.1 .2)$.

studied in this thesis, we now write down the Lagrangian of $\mathcal{N}=4 \mathrm{SYM}$ :

$$
\begin{array}{r}
S_{\mathcal{N}=4}=\int d^{4} x \operatorname{tr}[ \\
-\frac{1}{2 g_{Y M}^{2}} F_{\mu \nu} F^{\mu \nu}+\frac{\Theta}{8 \pi^{2}} F_{\mu \nu} \tilde{F}^{\mu \nu}-i \sum_{A=1}^{4} \bar{\lambda}^{A} \bar{\sigma}^{\mu} D_{\mu} \lambda_{A}-\sum_{i=1}^{6} D_{\mu} X^{i} D^{\mu} X^{i} \\
\left.+\frac{g_{Y M}^{2}}{2} \sum_{i, j}\left[X^{i}, X^{j}\right]^{2}+g_{Y M} \sum_{A, B, i}\left(C_{i}^{A B} \lambda_{A}\left[X^{i}, \lambda_{B}\right]+\text { h.c. }\right)\right] \\
=\int d^{4} x \operatorname{Im} \operatorname{tr} \tau\left[\int d^{4} \theta \bar{\Phi}_{I} e^{V} \Phi_{I} e^{-V}+\left(\int d^{2} \theta W_{\alpha} W^{\alpha}+\epsilon_{I J K} \Phi_{I} \Phi_{J} \Phi_{K}+\text { h.c. }\right)\right],
\end{array}
$$

where $\tau$ is the usual complex coupling and $W_{\alpha}$ is the chiral spinor field constructed from the vector field $V$,

$$
\tau=\frac{\Theta}{2 \pi}+i \frac{4 \pi}{g_{Y M}^{2}}, \quad W_{\alpha}=-\frac{1}{4} \bar{D}^{2}\left(e^{-V} D_{\alpha} e^{V}\right)
$$

Moreover, the constants $C_{i}^{A B}$ are related to the Clifford Dirac matrices for $S O(6)_{\mathcal{R}} \sim$ $S U(4)_{\mathcal{R}}$. In the last line of equation (2.1.2) we finally expressed the action of $\mathcal{N}=4$ super Yang-Mills theory, $S_{\mathcal{N}=4}$, in $\mathcal{N}=1$ superspace language. Besides the usual spacetime coordinates, the superspace contains also Grassmanian valued coordinates which we denote by $\theta$. The fields $\Phi_{I}, V$ and $W_{\alpha}$ are superfields and $D_{\alpha}, \bar{D}^{\dot{\alpha}}$ are superderivatives acting on these superfields. You can find more details on superspace in standard textbooks about supersymmetry, such as [68].

As you can see, the precise dynamics of $\mathcal{N}=4$ supersymmetric Yang-Mills theory is almost entirely dictated by supersymmetry and the large $\mathcal{R}$-symmetry group at the level of a renormalizable Lagrangian. Besides choosing the gauge group, we have the freedom to adjust the Yang-Mills gauge coupling $g_{Y M}$ and the $\Theta$ parameter. The $\Theta$ parameter breaks CP invariance and is set to zero from now on.

Let us list here several important facts about $\mathcal{N}=4$ supersymmetric Yang-Mills theory in four spacetime dimensions.

- Since the coupling constant is dimensionless and all fields are massless, the action of $\mathcal{N}=4 \mathrm{SYM}$ is classically scale invariant. It is quite remarkable that the theory 
is also scale invariant after quantization, 4 In fact, scale invariance is part of a larger symmetry, the conformal symmetry group $S O(4,2)$. Moreover, the Lagrangian is also invariant under $\mathcal{N}=4$ supersymmetry with $\mathcal{R}$-symmetry group $S U(4)_{\mathcal{R}}$. Combining the conformal symmetry and supersymmetry, $\mathcal{N}=4$ SYM is invariant under the superconformal symmetry given by the supergroup $P S U(2,2 \mid 4)$. Note that the bosonic subgroups of $P S U(2,2 \mid 4)$, the conformal group $S U(2,2) \sim S O(4,2)$ and the $\mathcal{R}$-symmetry group $S U(4)_{\mathcal{R}} \simeq S O(6)_{\mathcal{R}}$, are precisely the isometry groups of $\mathrm{AdS}_{5}$ and $S^{5}$, respectively.

- Using perturbative quantization, it can be shown that $\mathcal{N}=4$ SYM has no ultraviolet divergences in the correlation functions of elementary fields. Since also the corrections of instantons are finite, the theory is believed to be UV finite.

- Furthermore, Monotonen and Olive et. al. conjectured [69-71] that $\mathcal{N}=4 \mathrm{SYM}$ is invariant under the S-duality group $S L(2, \mathbb{Z})$ acting on the complex coupling constant $\tau$ as

$$
\tau \rightarrow \frac{a \tau+b}{c \tau+d}, \quad a d-b c=1, \quad a, b, c, d \in \mathbb{Z}
$$

This S-duality is quite remarkable since it implies a strong-weak duality: the coupling constant is given by $\tau=4 \pi i / g_{Y M}^{2}$. Let us now apply the S-duality transformation with $b=-c=1, a=d=0$. This transformation changes the coupling constant $g_{Y M}$ to $4 \pi / g_{Y M}$.

- The $\mathcal{N}=4 \mathrm{SYM}$ has two different classes of vacua. Since the scalar potential must vanish in the supersymmetric ground state and each interaction term $\left[X^{i}, X^{j}\right]^{2}$ is non-negative, the scalar fields have to be constant and have to satisfy $\left[X^{i}, X^{j}\right]=$ 0 for any pair of indices $i, j \in\{1, \ldots, 6\}$. This condition can be satisfied in two different ways. Either the vacuum expectation values of $X^{i}$ vanish, which is called the superconformal phase, or there exists at least one scalar $X^{i}$ for which the vacuum expectation value is non-zero. The latter case is called the Coulomb phase. In this phase, conformal invariance is broken since a length scale $\left\langle X^{i}\right\rangle$ is introduced. Moreover, the gauge symmetry is also broken down to a subgroup. For example, for gauge group $S U\left(N_{c}\right)$ the gauge symmetry may generically be broken to $U(1)^{N_{c}-1}$.

\subsubsection{The geometry of AdS spaces}

Let us also collect a few important properties of Anti-de Sitter spacetime which we need in later chapters of the thesis. $(d+1)$-dimensional Anti-de Sitter space, $A d S_{d+1}$ for short, is the unique maximally symmetric space with constant negative curvature. $A d S_{d+1}$ can be embedded into $(d+2)$-dimensional Minkowski spacetime $\left(X^{0}, X^{1}, \ldots, X^{d}, X^{d+1}\right) \in \mathbb{R}^{d, 2}$,

\footnotetext{
${ }^{4}$ Of course, every four-dimensional gauge theory with only massless flavor fields is scale invariant at the classical level. However, quantum effects dynamically generate a scale since we must specify the value of the coupling constant at some renormalization scale. If we change this energy scale, the strength of the coupling will change, i.e. the coupling runs. Therefore classical scale invariance is broken at the quantum level. This is true for pure Yang-Mills theory and also for QCD. However, $\mathcal{N}=4$ SYM theory is scale invariant also at the quantum level. This is connected to the fact that $\mathcal{N}=4 \mathrm{SYM}$ is believed to be a UV finite theory and that the $\beta$-function vanishes exactly to all orders in perturbation theory.
} 
with signature $\operatorname{diag}(-,+,+, \ldots,+,-)$, by the defining equation

$$
-\left(X^{0}\right)^{2}+\sum_{i=1}^{d}\left(X^{i}\right)^{2}-\left(X^{d+1}\right)^{2}=-L^{2},
$$

where $L$ is the radius of curvature of the Anti-de Sitter space, as we will see later. From the embedding (2.1.5) we can easily read of the isometry group $S O(d, 2)$ of $A d S_{d+1}$, which agrees with the conformal group of $d$-dimensional Minkowski spacetime. We do not review here extensively the geometrical properties of Anti-de Sitter spacetimes. For more details see [72]. Let us now introduce a particular parameterization of the hyperboloid (2.1.5). In the following we use the coordinates $t, \vec{x}=\left(x^{1}, \ldots, x^{d-1}\right) \in \mathbb{R}^{d-1}$ as well as $r>0$. The parameterization in these coordinates is given by

$$
\begin{aligned}
X^{0} & =\frac{L^{2}}{2 r}\left(1+\frac{r^{2}}{L^{4}}\left(\vec{x}^{2}-t^{2}+L^{2}\right)\right), \\
X^{i} & =\frac{r x^{i}}{L} \text { for } i \in\{1, \ldots, d-1\}, \\
X^{d} & =\frac{L^{2}}{2 r}\left(1+\frac{r^{2}}{L^{4}}\left(\vec{x}^{2}-t^{2}-L^{2}\right)\right), \\
X^{d+1} & =\frac{r t}{L} .
\end{aligned}
$$

Due to the restriction $r>0$, we cover only one half of the $A d S_{d+1}$ spacetime. These local coordinates are called Poincaré patch coordinates. In the Poincaré patch, the metric of $A d S_{d+1}$ space reads

$$
d s^{2}=\frac{L^{2}}{r^{2}} d r^{2}+\frac{r^{2}}{L^{2}}\left(-d t^{2}+d \vec{x}^{2}\right) \equiv \frac{L^{2}}{r^{2}} d r^{2}+\frac{r^{2}}{L^{2}}\left(\eta_{\mu \nu} d x^{\mu} d x^{\nu}\right),
$$

where $\mu=0, \ldots, d, \quad x^{0}=t$ and $\eta_{\mu \nu}=\operatorname{diag}(-1,+1, \ldots,+1)$. An explicit calculation of the Ricci Scalar for $A d S_{d+1}$ gives $R=-\frac{d(d+1)}{L^{2}}$, i.e. the curvature is indeed negative and constant 5 This also confirms that $L$ is the radius of curvature.

We can view $(d+1)$-dimensional Anti-de Sitter spacetime in the Poincaré patch as flat spacetime, parametrized by the coordinates $t, \vec{x}$, plus an extra warped direction, which is denoted by $r$. For a fixed value of $r$, the $d$-dimensional transverse spacetime is flat Minkowski spacetime, i.e. $\mathbb{R}^{d-1,1}$. A cartoon of Anti-de Sitter space is shown in figure 2.1. The vertical direction in the figure 2.1 displays the radial direction $r$ of Anti-de Sitter spacetime. At the bottom of the figure 2.1, $r$ is zero, whereas at the top $r$ goes to infinity. As we see now, these are two special values of the radial direction.

For $r \rightarrow 0$, i.e. at the bottom of figure 2.1, we find a horizon due to $g_{t t} \rightarrow 0$. This is the location of the Poincaré horizon, which has zero area since also $g_{x^{i} x^{i}} \rightarrow 0$ for $r \rightarrow 0$. Note that the Poincaré horizon is only a coordinate singularity: on the other side of the horizon, i.e. for $r<0$, there is another Poincaré patch, which is needed to cover the whole

${ }^{5}$ The Riemann tensor can be written in the form

$$
R_{\mu \nu \rho \sigma}=\frac{R}{d(d+1)}\left(g_{\nu \sigma} g_{\mu \rho}-g_{\nu \rho} g_{\mu \sigma}\right)=-\frac{1}{L^{2}}\left(g_{\nu \sigma} g_{\mu \rho}-g_{\nu \rho} g_{\mu \sigma}\right)
$$

Therefore we see that Anti-de Sitter spacetime is maximally symmetric. 


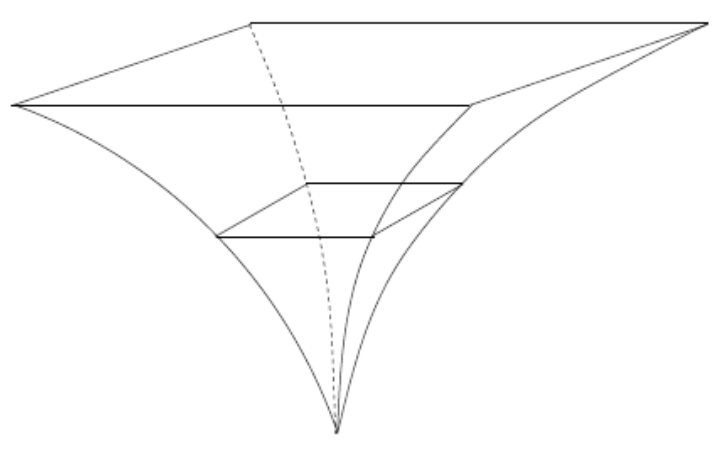

Figure 2.1: A cartoon of Anti-de Sitter spacetime, taken from [73].

AdS spacetime. It is also possible to find global coordinates which cover the whole $A d S_{d+1}$ space, but these coordinates are not important in the later chapters.

Note that the metric (2.1.10) has a "second order pole" for $r \rightarrow \infty$, i.e. $g_{x^{i} x^{i}}$ diverges quadratically for $r \rightarrow \infty$. Indeed, it is possible to show that every metric of asymptotically Anti-de Sitter spaces has always such a quadratic divergence for a particular value $r_{\star}$ of the radial direction. The slice of spacetime for fixed $r=r_{\star}$ is called the (conformal) boundary of AdS. In the coordinates (see equation (2.1.10) $)$ used above, the (conformal) boundary is located at $r \rightarrow \infty$, i.e. at the top in figure 2.1.

In order to continue the metric also on the boundary of $A d S$, we have to multiply the metric by a defining function $g(r, t, \vec{x})$ which can be constructed as follows. $g(r, t, \vec{x})$ has to be a positive smooth function of the coordinates $r, t$ and $\vec{x}$. Moreover, $g(r, t, \vec{x})$ must have a second-order zero at $r=\infty$. An example of a defining function $g(r, t, \vec{x})$ is given by $g(r, t, \vec{x})=L^{2} / r^{2} \omega(t, \vec{x})$ where $\omega$ is a smooth and positive function of $\vec{x}$ and $t$. Multiplying the metric (2.1.10) with $g$ and taking the limit $r \rightarrow \infty$, we can define a finite boundary metric given by $d s_{\partial A d S}^{2}=\omega(t, \vec{x})\left(-d t^{2}+d \vec{x}^{2}\right)$. Different choices of $\omega(t, \vec{x})$, or more generally different choices of $g(r, t, \vec{x})$, can give rise to different boundary metrics. Therefore the bulk metric determines a whole class of boundary metrics which are related by conformal transformations. Hence, the boundary of Anti-de Sitter spacetimes is called conformal.

Whereas in the defining equation (2.1.5) the isometry group $S O(d, 2)$ of $A d S_{d+1}$ is obvious, only the following subgroups of $S O(d, 2)$ are manifest for the metric in Poincaré coordinates:

- $\operatorname{ISO}(d-1,1)$, i.e. all Poincaré transformations acting on the $t, \vec{x}$ coordinates,

- $S O(1,1)$ acting on $t, \vec{x}$ and $r$ in the following way

$$
(t, \vec{x}, r) \rightarrow(\lambda t, \lambda \vec{x}, r / \lambda) .
$$

We see that we can identify the elements of $I S O(d-1,1)$ with the Poincaré transformations on the conformal boundary of AdS. But how does the other generators of the isometry group of $A d S_{d+1}$ act on the conformal boundary of $A d S$ ? One can show that the isometry group $S O(d, 2)$ acts on the boundary as the conformal group of Minkowski space. In particular the subgroup $S O(1,1)$ is identified with the dilatation $D$ of the conformal symmetry group of $\mathbb{R}^{d-1,1}$. 
Sometimes it is more convenient to invert the $r$-coordinate by defining $u=\frac{L^{2}}{r}$. As opposed to the $r$-coordinates given by $(r, t, \vec{x})$, the conformal boundary in the $u$-coordinates $(u, t, \vec{x})$ is located at $u=0$, whereas the Poincaré horizon is at $u \rightarrow \infty$. It is easy to verify that the induced metric in $u$-coordinates reads

$$
d s^{2}=\frac{L^{2}}{u^{2}}\left(d u^{2}-d t^{2}+d \vec{x}^{2}\right)=\frac{L^{2}}{u^{2}}\left(d u^{2}+\eta_{\mu \nu} d x^{\mu} d x^{\nu}\right) .
$$

To formulate the AdS/CFT correspondence, we also need to define a Euclidean-signature version of $(d+1)$-dimensional Anti-de Sitter space which we denote by $E A d S_{d+1}$. To define $E A d S_{d+1}$, we simply Wick rotate the component $X^{0}$ in the defining equation (2.1.5). Therefore the isometry group of $E A d S_{d+1}$ is given by $S O(d+1,1)$ instead of $S O(d, 2)$. Finally, for the metric in the Poincaré coordinates we have to replace $\eta_{\mu \nu}$ by $\delta_{\mu \nu}$ in equations (2.1.10) and (2.1.12). Here $\delta$ is the standard Kronecker symbol.

\subsubsection{Derivation of the $\mathrm{AdS}_{5} / \mathrm{CFT}_{4}$ correspondence}

In this section we motivate the AdS/CFT correspondence within the framework of superstring theory. Superstring theory is much more than just a theory of closed strings. Besides fundamental strings, superstring theory also contains various non-perturbative soliton-like higher-dimensional objects known as Dirichlet branes, or D-branes for short.6 As we review in section A.3 of the glossary, D-branes can be viewed from two different perspectives: the open string and the closed string perspective. Which perspective is most convenient depends on the value of the string coupling constant $g_{s} .7$

- the open string perspective (reliable for $g_{s} \ll 1$ ):

D-branes may be visualized as higher-dimensional objects where open strings can end. At low energies, the dynamics of the open strings are described by a supersymmetric gauge theory living on the worldvolume of the D-branes.

- the closed string perspective (reliable for $g_{s} N_{c} \gg 1$ ):

D-branes may be viewed as soliton-like solutions of the low-energy theory of superstring theory, i.e. of supergravity. You may consider D-branes as sources for the gravitational field which curve the surrounding spacetime. In the case of $N_{c}$ coincident D-branes, the characteristic length scale of the curved spacetime is proportional to $g_{s} N_{c}$. Note that the characteristic length scale should be large in order to trust the supergravity approximation.

These two perspectives on D-branes allow us to motivate $\mathrm{AdS}_{5} / \mathrm{CFT}_{4}$ correspondence. In the following discussion we consider $N_{c}$ coincident D3-branes in type IIB superstring theory.

\footnotetext{
${ }^{6}$ In the following, for indicating the number of spatial dimensions of the D-brane, we use the short-hand notation Dp-brane where $p$ stands for the number of spatial dimensions. A Dp-brane is also extended along a timelike direction. For example, a D-brane with three spatial and a timelike dimension is called D3-brane.

${ }^{7}$ The string coupling constant $g_{s}$ controls the interaction strength between open and closed strings. Strictly speaking, the word "constant" is wrong in this context since the string coupling $g_{s}$ is related to the expectation value of the dilaton field, as reviewed in section A.1 of the glossary, and therefore is dynamical.
} 
First, let us consider the open string perspective. As mentioned above, the $N_{c}$ coincident D3-branes may be viewed as a four-dimensional hyperplane in ten-dimensional flat spacetime. The D3-branes support open strings which can interact with closed strings. By taking a particular low-energy limit, the so-called Maldacena limit,

$$
\alpha^{\prime} \rightarrow 0 \quad \text { with } \quad u=\frac{r}{\alpha^{\prime}} \quad \text { kept fixed, }
$$

where $r$ is any distance, we can decouple open and closed strings 8 At low energies we can effectively describe the open string dynamics by a supersymmetric gauge theory living on the worldvolume of the D3-branes 9 In the case of $N_{c}$ coincident D3-branes, the effective low-energy description of open strings is given by four-dimensional maximally supersymmetric Yang-Mills theory with gauge group $U\left(N_{c}\right)$. The coupling constant $g_{Y M}$ may be expressed in terms of the string coupling constant $g_{s}$ by $g_{Y M}^{2}=2 \pi g_{s}$ (see also equation (A.3.77) of section A.3). The closed strings present in this configuration give rise to supergravity in flat ten-dimensional spacetime. Let us now take the strong coupling limit. This implies in particular that $\mathcal{N}=4 \mathrm{SYM}$ is strongly coupled.

In order to motivate the $\mathrm{AdS}_{5} / \mathrm{CFT}_{4}$ correspondence, let us now interchange the two limits, i.e. the strong coupling and the low-energy limit. Consider the $N_{c}$ D3-branes in the strongly coupled limit $g_{s} \rightarrow \infty 10$ Then, we have to take the closed string perspective. The $N_{c}$ D3-branes may be viewed as massive charged objects sourcing various fields of type IIB supergravity, and therefore also of type IIB string theory. In this background closed strings of type IIB superstring theory will propagate.

The supergravity solution of $N_{c}$ D3-branes preserving $S O(3,1) \times S O(6)$ isometries of $\mathbb{R}^{9,1}$ and half of the supercharges of type IIB supergravity, i.e. 16 out of the 32 supercharges, is given by

$$
\begin{aligned}
d s^{2} & =H_{3}(r)^{-1 / 2} \eta_{\mu \nu} d x^{\mu} d x^{\nu}+H_{3}(r)^{1 / 2} \delta_{i j} d y^{i} d y^{j} \\
\exp (2 \phi(r)) & =g_{s}^{2} \\
C_{(4)} & =\left(1-H_{3}(r)^{-1}\right) d x^{0} \wedge d x^{1} \wedge d x^{2} \wedge d x^{3}
\end{aligned}
$$

where $\mu, \nu=0, \ldots, 3$ and $i, j=4, \ldots, 9$ and $H_{3}(r)=1+\left(\frac{L_{3}}{r}\right)^{4}$ The radial coordinate $r$ is defined by $r^{2}=\sum_{i=4}^{9} y_{i}^{2}$ and $L_{3}^{4}=4 \pi g_{s} N \alpha^{\prime 2}$. From now on we just write $H(r)$ and $L$ instead of $H_{3}(r)$ and $L_{3}$. Next we introduce spherical coordinates $\left(r, \Omega_{5}\right) \in \mathbb{R}_{+} \times S^{5}$ instead of $\left(y^{4}, \ldots y^{9}\right) \in \mathbb{R}^{6}$ by

$$
\delta_{i j} d y^{i} d y^{j}=d r^{2}+r^{2} d \Omega_{5}^{2} .
$$

On the left side of the equation the summation is implicit.

The background consists of two different regions: a near-horizon region and an asymptotically flat region. Therefore we have two different types of closed strings: closed strings

\footnotetext{
${ }^{8}$ The second condition ensures that all dimensionful physical quantities, such as Higgs values, are kept constant in the Maldacena limit.

${ }^{9}$ For the precise low energy effective action for a single D-brane, as well as generalizations to coincident D-branes, see section A.3 At lowest non-trivial order in $\alpha^{\prime}$ the action indeed reduces to Yang-Mills theory.

${ }^{10}$ We can achieve this for finite $N$ by $g_{s} \rightarrow \infty$. Since a non-perturbative formulation of string theory is not known up to now, we cannot do explicit calculations in this parameter regime. But we see in section 2.1.3 that there is a weaker form of the AdS/CFT conjecture in which we consider the limit $N_{c} \rightarrow \infty$ but $g_{s} \ll 1$ such that $g_{s} N_{c} \rightarrow \infty$. In this case, the following statements are true although $g_{s}$ is small.
} 
propagating in flat ten-dimensional spacetime and closed strings propagating in the nearhorizon region. The dynamics of the closed strings in asymptotically flat spacetime are described by type IIB string theory in ten-dimensional flat spacetime. Taking the lowenergy limit (2.1.13) we can decouple both types of closed strings from each other.

Note that in this limit

$$
\frac{L^{4}}{r^{4}}=4 \pi g_{s} N_{c} \frac{\alpha^{\prime 2}}{r^{4}}=4 \pi g_{s} N_{c} \underbrace{\frac{\alpha^{\prime 4}}{r^{4}}}_{\text {const. }} \cdot \underbrace{\alpha^{\prime-2}}_{\rightarrow \infty} \rightarrow \infty,
$$

i.e. we effectively zoom into the near-horizon region. Therefore the Maldacena limit (2.1.13) is sometimes also called the near-horizon limit.

Due to equation (2.1.18), we can approximate $H(r)$ by $H(r) \simeq \frac{L^{4}}{r^{4}}$. Thus we obtain 11 for the metric and the four form potential $C_{(4)}$

$$
\begin{aligned}
d s^{2} & =\frac{r^{2}}{L^{2}} \eta_{\mu \nu} d x^{\mu} d x^{\nu}+\frac{L^{2}}{r^{2}} d r^{2}+L^{2} d \Omega_{5}^{2}, \\
C_{(4)} & =\frac{L^{4}}{r^{4}} d x^{0} \wedge d x^{1} \wedge d x^{2} \wedge d x^{3} .
\end{aligned}
$$

The metric reduces to $A d S_{5} \times S^{5}$ by taking the near-horizon limit. The radius of the sphere $S^{5}$ and of $\mathrm{AdS}_{5}$ are equal and given by

$$
L^{4}=4 \pi g_{s} N_{c} \alpha^{\prime 2}
$$

In both pictures, the open and the closed string perspective, we found two decoupled effective theories in the low-energy limits:

- closed string perspective: type IIB string Theory on $A d S_{5} \times S^{5}$ and type IIB string theory on $\mathbb{R}^{9,1}$.

- open string perspective: $\mathcal{N}=4 \mathrm{SYM}$ on flat four-dimensional spacetime and type IIB string theory on $\mathbb{R}^{9,1}$.

Both perspectives should be equivalent descriptions of the same physics, and type IIB string theory on $\mathbb{R}^{9,1}$ is present in both perspectives. Therefore Maldacena conjectured in [10] that $\mathcal{N}=4$ super Yang-Mills theory with gauge group ${ }^{12} S U\left(N_{c}\right)$ in four dimensions

\footnotetext{
${ }^{11}$ To be on the safe side, we should work only with coordinates which are kept fixed while the Maldacena limit is taken. Hence, it is useful to introduce the coordinate $w=\frac{r}{\alpha^{\prime}}$ and rewrite the metric and the four-form potential in terms of $w$. One obtains

$$
\begin{aligned}
\alpha^{\prime} d s^{2} & =\frac{w^{2}}{\tilde{L}^{2}} \eta_{\mu \nu} d x^{\mu} d x^{\nu}+\frac{\tilde{L}^{2}}{w^{2}} d w^{2}+\tilde{L}^{2} d \Omega_{5}^{2}, \\
\alpha^{\prime 2} C_{(4)} & =\frac{\tilde{L}^{4}}{w^{4}} d x^{0} \wedge d x^{1} \wedge d x^{2} \wedge d x^{3} .
\end{aligned}
$$

where $\tilde{L}=\frac{L}{\sqrt{\alpha^{\prime}}}=4 \pi g_{s} N_{c}$.

${ }^{12}$ In the discussion so far we argued that the gauge group is $U\left(N_{c}\right)$. However, it turns out that the overall $U(1) \subset U\left(N_{c}\right)$ degrees of freedom decouple from the $S U\left(N_{c}\right)$ degrees of freedom. The $U(1)$ degrees of freedom correspond to singleton fields in the gravity theory which are only located at the boundary and cannot propagate into the bulk of $\mathrm{AdS}_{5}$.
} 
is equivalent to type IIB string theory on $A d S_{5} \times S^{5}$, although the fundamental degrees of freedom on both sides are very different. A first obvious check of the conjecture are the symmetry groups on both sides which have to agree. As explained in section $2.1, \mathcal{N}=4$ super Yang-Mills theory has an $S U(4)_{\mathcal{R}} \simeq S O(6)_{\mathcal{R}} \mathcal{R}$-symmetry group and is conformal, i.e. invariant under $S O(4,2)$ transformations. The isometry groups of $\mathrm{AdS}_{5}$ and $S^{5}$ are given by $S O(4,2)$ and $S O(6)$, respectively. The matching of the symmetries will help us in section 2.2 to determine the one-to-one mapping between operators on the gauge theory side and bulk fields on the gravity side.

\subsubsection{Different versions of the $\mathrm{AdS}_{5} / \mathrm{CFT}_{4}$ correspondence}

Although the strongest form of the $\mathrm{AdS}_{5} / \mathrm{CFT}_{4}$ correspondence, discussed in section 2.1.1 and motivated in section 2.1.2, is very interesting and stimulates new ideas, it is very difficult to do explicit calculations. Moreover, it is not known how to define string theory in RR-backgrounds rigorously. Therefore it is necessary to lessen the strength, but not the importance, of the AdS/CFT correspondence by taking certain limits on both sides. We will see that we obtain more tractable forms of the $\mathrm{AdS}_{5} / \mathrm{CFT}_{4}$ correspondence.

Since we currently only understand string theory in the perturbative regime, it is useful to specialize the string theory side of the correspondence to weak coupling, i.e. to $g_{s} \ll 1$. At leading order in $g_{s}$, the AdS side reduces to classical string theory in the sense that we do not have to take into account the whole string genus expansion but only tree-level diagrams in string perturbation theory.

The question is whether we should fix $N_{c}$, which also set the characteristic length, $L / \sqrt{\alpha^{\prime}}$, while taking $g_{s} \rightarrow 0$, or if we should keep $g_{s} N_{c}$ fixed. Let us see what happens if we fix $N_{c}$ and take the limit $g_{s} \rightarrow 0$. In this case $g_{s} N_{c}$ goes to zero and therefore also the radius of curvature of $\mathrm{AdS}_{5}$ in string units, i.e. $L / \sqrt{\alpha^{\prime}} \rightarrow 0$. Although we only have to take tree-level diagrams in string perturbation theory into account, this limit is very complicated since the string is propagating on a highly curved target space.

Alternatively, we can take the limit $g_{s} \rightarrow 0$ with $N_{c} \rightarrow \infty$ such that $\lambda=g_{Y M}^{2} N_{c}=2 \pi g_{s} N_{c}$ is fixed, and thus also the radius of curvature of $\mathrm{AdS}_{5}$ is kept constant. On the field theory side this limit is known as the 't Hooft limit [74]. Hence, the field theory in the large $N_{c}$-limit, with the 't Hooft coupling $\lambda=g_{Y M}^{2} N_{c}$ fixed, corresponds to tree-level type IIB string theory on $A d S_{5} \times S^{5}$. A $1 / N_{c}$ expansion on the field theory side can be mapped to an expansion in the genus of the string worldsheet on the string theory side since $1 / N_{c}=g_{s} / \lambda$.

After taking the 't Hooft limit, there is only one free parameter on both sides: on the field theory side we can tune the 't Hooft coupling $\lambda$, whereas on the string theory side the radius of curvature $L / \sqrt{\alpha^{\prime}}$ is a free parameter. Both parameters are related by $L / \sqrt{\alpha^{\prime}}=2 \lambda$. Since we are interested in strongly-coupled field theories, we take the limit $\lambda \rightarrow \infty$ on the field theory side which corresponds to $\sqrt{\alpha^{\prime}} / L \rightarrow 0$. The string length is then very small compared to the radius of curvature. Therefore we can take a "point particle limit" of type IIB string theory which is given by type IIB supergravity on $A d S_{5} \times S^{5}$. In this limit we get an example of a strong/weak duality in the sense that we can map strongly-coupled $\mathcal{N}=4 \mathrm{SYM}$ to type IIB supergravity on weakly-curved $A d S_{5} \times S^{5}$ space. In this thesis we always use this weak form of the AdS/CFT duality. Nevertheless, we get interesting 
insights into strongly-coupled gauge theories from this weak form.

\subsection{Review: The AdS/CFT Dictionary}

In the last section, we motivated a particular example of the AdS/CFT correspondence which we called $\mathrm{AdS}_{5} / \mathrm{CFT}_{4}$. If this conjecture holds, we should be able to map every bulk field of the gravity side to some gauge invariant operator of the field theory. To establish the one-to-one mapping between operators of the conformal field theory and fields on the gravity side, various symmetries serve as a guideline. In $\mathcal{N}=4$ super YangMills theory, operators fall into multiplets of the full $\operatorname{PSU}(2,2 \mid 4)$ symmetry group. We can also group the excitations of type IIB supergravity on $A d S_{5} \times S^{5}$ into multiplets of the corresponding symmetries of $\mathrm{AdS}_{5}$ and $S^{5}$. Since these symmetries are identical on the field theory and gravity side, it may be possible to establish a one-to-one mapping between the representations of local gauge invariant operators on the field theory side and representations showing up in the Kaluza-Klein tower of type IIB supergravity on $\mathrm{AdS}_{5}$, reduced on $S^{5}$. For the concrete mapping see chapter 5.6 of [58]. On the gravity side one has to expand the ten-dimensional supergravity fields into spherical harmonics of $S^{5}$. The supergravity fields may become massive five-dimensional fields. Their mass $m$ can be mapped to the conformal dimension $\Delta$ of the dual operator. For the $A d S_{d+1} / C F T_{d}$ correspondence we obtain the following mapping:

type of field

scalars, massive spin two fields

massless spin two fields

p-form fields

spin $1 / 2$, spin $3 / 2$

$$
\begin{aligned}
& \text { relation between } m \text { and } \Delta \\
& m^{2} L^{2}=\Delta(\Delta-d) \\
& m^{2} L^{2}=0, \Delta=d \\
& m^{2} L^{2}=(\Delta-p)(\Delta+p-d) \\
& |m| L=\Delta-d / 2
\end{aligned}
$$

Note that in order to satisfy the unitarity bound $\Delta \geq \frac{d-2}{2}$ we also have to consider fields with negative $m^{2}$. In asymptotically AdS spacetimes tachyonic, i.e. negative mass squares, are allowed. Breitenlohner and Freedman showed in [75] that for scalar fields with mass given by $m^{2} L^{2} \geq-d^{2} / 4$, a quantization exists for which $\mathcal{O}$ has dimension $\Delta_{+}$, which is the larger root of the equation $m^{2} L^{2}=\Delta(\Delta-d)$. When $-d^{2} / 4<m^{2} L^{2}<-d^{2} / 4+1$, a second quantization exists for which the dual operator has conformal dimension $\Delta_{-}$(which is the smaller root). These two quantizations correspond to two different field theories, one of which (the one with $\Delta=\Delta_{-}$) is unstable against a relevant deformation by a double-trace operator of $\mathcal{O}$, and flows to the other, stable, theory. For more details see [76]. When $m>0$, the dual operator is irrelevant, i.e. $\Delta>d$. The operator is marginal for $m=0$.

Note that there is no straightforward recipe how to obtain the one-to-one mapping between operators and fields. Most of the time, however, it is enough to know the conformal dimension and the symmetries of the operator on the field theory side to determine the dual field on the gravity side.

The precise statement of the correspondence equates the partition function of type IIB string theory with the generating functional of connected CFT correlation functions. Let $\phi$ be a field propagating in the bulk and let $\phi_{0}$ be the value at the conformal boundary. For simplicity, we ignore all indices, i.e. $\phi$ can be a scalar, a vector or even the metric. 
The bulk field $\phi$ is dual to a gauge-invariant operator $\mathcal{O}$ on the field theory side. The AdS/CFT correspondence is the statement that $[11,12]$

$$
\left\langle e^{i \int d^{4} x \mathcal{O} \phi_{0}}\right\rangle_{S Y M}=\mathcal{Z}_{I I B, \text { string }}\left(\phi \rightarrow \phi_{0}\right) .
$$

In the partition function $\mathcal{Z}_{I I B, \text { string }}$ we integrate over all possible field configurations of $\phi$ as well as over all metrics that have a double pole (at the conformal boundary) and induce a given conformal structure.

Up to now it is not known how to calculate the partition function of type IIB superstring theory. Therefore let us from now on use a weaker form of the AdS/CFT correspondence (see section 2.1.3) in the rest of the thesis. If we use the weakest form of the AdS/CFT correspondence, we can argue that a saddle point to the superstring partition function $\mathcal{Z}_{I I B, \text { string }}$ is given by type IIB supergravity. Thus we can approximate the string partition function $\mathcal{Z}_{I I B, \text { string }}\left(\phi \rightarrow \phi_{0}\right)$ by

$$
\mathcal{Z}_{I I B, \text { string }}\left(\phi \rightarrow \phi_{0}\right) \approx \exp \left(i S_{I I B, \text { sugra }}\left(\bar{\phi} \rightarrow \phi_{0}\right)\right),
$$

where $\bar{\phi}$ denotes the solution of type IIB supergravity with leading asymptotic behavior $\phi_{0}$ near the conformal boundary. In the weakest form, the AdS/CFT correspondence therefore equates

$$
\left\langle e^{i \int d^{4} x \mathcal{O} \phi_{0}}\right\rangle_{S Y M}=\exp \left(i S_{I I B, \text { sugra }}\left(\bar{\phi} \rightarrow \phi_{0}\right)\right)
$$

The on-shell bulk action, $S_{I I B, \text { sugra }}$, acts as the generating functional for correlators involving the operator $\mathcal{O}$. In other words, to compute renormalized correlators of the operator $\mathcal{O}$, we take functional derivatives of $S_{I I B \text {, sugra }}$ with respect to the source $\phi_{0}$.

The precise recipe to calculate correlation functions of the operator $\mathcal{O}$ by AdS/CFT methods is as follows:

(i) Determine the bulk field $\phi$ which is dual to the operator $\mathcal{O}$.

(ii) Solve the supergravity equations of motion for $\phi$. The solution is denoted by $\bar{\phi}$.

(iii) Insert the solution $\bar{\phi}$ into the supergravity action and exponentiate the result.

(iv) Take variational derivatives with respect to the source $\phi_{0}$ which is the leading asymptotic behavior of $\bar{\phi}$.

For example the connected correlator of the operator $\mathcal{O}$, i.e. $\langle\mathcal{O}\rangle_{c}$, is given by

$$
\langle\mathcal{O}\rangle_{c}=\frac{\langle\mathcal{O}\rangle_{S Y M}}{\langle 1\rangle_{S Y M}}=i \frac{\delta}{\delta \phi_{0}} S_{I I B, \text { sugra }}
$$

Generically, both the on-shell bulk action and the CFT generating functional diverge. On the bulk side, the divergences arise from the infinite volume of $A d S$, i.e. they are long-distance or infrared (IR) divergences. In the field theory, the divergences are shortdistance ultraviolet (UV) divergences. To make the AdS/CFT correspondence meaningful we must regulate and renormalize these divergences. In the next section we discuss how

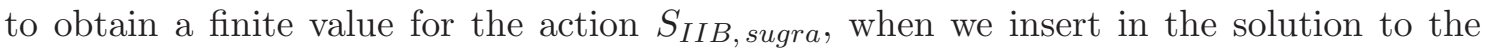
equations of motion. 


\subsection{Holographic renormalization for fermions}

In this section, we discuss holographic renormalization, a procedure which guarantees a finite partition function as well as finite one-point functions. The method presented here can not only be applied to the $\mathrm{AdS}_{5} / \mathrm{CFT}_{4}$ correspondence, but also to more general versions. In particular, we do not have to restrict the AdS/CFT correspondence to fourdimensional field theories. Hence, in this section we use the more statement that a theory of dynamical gravity 13 on $A d S_{d+1}$ is equivalent to a $d$-dimensional CFT that lives on the boundary of $A d S_{d+1}$.

The standard example for which the holographic renormalization is discussed are massive scalar fields in asymptotically AdS spacetimes (see [77,78] and references therein). The details of holographic renormalization are well-known for various other species of bulk fields, for example for the metric [77] and gauge fields [79].14 Here we study the holographic renormalization of a Dirac fermion in spaces that asymptotically approach $\mathrm{AdS}_{d+1}$. We work in Euclidean-signature $\mathrm{AdS}_{d+1}$, unless stated otherwise. We assume that the bulk metric asymptotically approaches $\mathrm{AdS}_{d+1}$, but, unlike in the previous sections, we leave the number of spatial dimensions $d$ unconstrained.

Holographic renormalization proceeds as follows. We first regulate the on-shell bulk action by introducing a cutoff on the integration in the radial direction of AdS. Using the coordinates (2.1.12), we do not integrate to $u=0$ but rather to some value $u=\epsilon$. We then add counterterms on the $u=\epsilon$ surface to cancel any terms that diverge as we remove the regulator by taking $\epsilon \rightarrow 0$. Generically, the form of the counterterms is fixed by symmetries, and the coefficients of the counterterms are adjusted to cancel the divergences. Once the counterterms are known, we can proceed to compute functional derivatives of the on-shell bulk action, always taking $\epsilon \rightarrow 0$ in the end, thus obtaining renormalized CFT correlation functions in a way that is manifestly covariant and preserves all symmetries.

The form of the counterterms is fixed by symmetries (in particular Lorentz invariance on the $u=\epsilon$ surface) and by the requirement that they cancel divergences of the on-shell action. Moreover, for fermions we have an additional constraint. The counterterms can be built only from one component of $\Psi, \Psi_{-}=1 / 2\left(1-\gamma^{u}\right) \Psi$. This component is held fixed under variations, and hence does not spoil the stationarity of the action.

Otherwise, the procedure for fermions very closely parallels that for scalars. Our main results of this section are the counterterms in equations (2.3.58) and (2.3.66), and the renormalized on-shell actions in equations (2.3.70), (2.3.71), and (2.3.72).

Let me emphasize that these are new results. The following discussion is based on work done in collaboration with Johanna Erdmenger, Matthias Kaminski and Andy O'Bannon and was published in [8].

\footnotetext{
${ }^{13}$ In the strongest form, the theory of dynamical gravity is a version of superstring theory, whereas in weaker forms it may be supergravity.

${ }^{14}$ To our knowledge, the only detailed analysis of holographic renormalization for fermions was in the (more complicated) context of non-relativistic gauge-gravity duality, in [80].
} 


\subsubsection{Review: Free Fermions}

We begin by studying a single free fermion in AdS space. In particular we review how to extract the field theory fermionic two-point function from a solution for a bulk Dirac fermion, following $[49,51,81]$.

In this section we work mainly with Euclidean-signature AdS space, with the metric of equation (2.1.12) 15 From now on, we set the characteristic radius of $A d S_{d+1}$, i.e. L, to one. The metric therefore reads

$$
d s^{2}=g_{A B} d x^{A} d x^{B}=\frac{d u^{2}}{u^{2}}+\frac{1}{u^{2}} \delta_{i j} d x^{i} d x^{j} .
$$

We study a bulk Dirac spinor $\Psi$. The Dirac action (plus boundary terms) is

$$
S=\int d^{d+1} x \sqrt{g}(\bar{\Psi} \not D \Psi-m \bar{\Psi} \Psi)+S_{b d y},
$$

where, picking one of the spatial directions to be time, with corresponding $\gamma^{t}$, we define $\bar{\Psi}=\Psi^{\dagger} \gamma^{t}$. We write the $A d S_{d+1}$ Dirac operator $\not D$ below. Here, $S_{b d y}$ includes boundary terms that do not affect the equation of motion.

Varying the above action, we obtain the bulk equation of motion, the Dirac equation,

$$
e_{A}^{M} \gamma^{A} D_{M} \Psi-m \Psi=0,
$$

where $e_{A}^{M}=u \delta_{A}^{M}$ are the inverse vielbeins associated with the metric in equation (2.1.12) 16 The curved-space covariant derivative is

$$
D_{M}=\partial_{M}+\frac{1}{4}\left(\omega_{M}\right)_{A B}\left[\gamma^{A}, \gamma^{B}\right]
$$

where $\left(\omega_{M}\right)_{A B}$ is the spin connection associated with the metric in equation (2.1.12). The only nonzero components of the spin connection are $\left(\omega_{i}\right)_{u j}=\frac{1}{u} \delta_{i j}$, so that $D_{u}=\partial_{u}$ and the other components of $D_{M}$ are

$$
D_{i}=\partial_{i}+\frac{1}{4} \frac{1}{u}\left[\gamma^{u}, \gamma^{i}\right] .
$$

We can now simplify the Dirac equation,

$$
\begin{aligned}
0 & =e_{A}^{M} \gamma^{A} D_{M} \Psi-m \Psi \\
& =u \gamma^{M} \partial_{M} \Psi+\frac{1}{4} \gamma^{i}\left[\gamma^{u}, \gamma^{i}\right] \Psi-m \Psi \\
& =\left[u \gamma^{M} \partial_{M}-\frac{d}{2} \gamma^{u}-m\right] \Psi .
\end{aligned}
$$

We work with a single Fourier mode, so we let $\Psi \rightarrow e^{i k x} \Psi$, where, without loss of generality, we have chosen the momentum to point in the $\hat{x}$ direction 17 The Dirac equation is then

$$
\left[u \gamma^{u} \partial_{u}+i k u \gamma^{x}-\frac{d}{2} \gamma^{u}-m\right] \Psi=0
$$

\footnotetext{
${ }^{15}$ Capital Latin letters $A, B, \ldots$ always denote the $A d S_{d+1}$ directions, including the radial direction $u$, while lower-case Latin letters denote field theory directions: $i, j=1, \ldots d$.

${ }^{16}$ Recall that for inverse vielbeins, the upper index is general coordinate and the lower index is local Lorentz. The $\gamma^{A}$ obey the usual algebra $\left\{\gamma^{A}, \gamma^{B}\right\}=2 \delta^{A B}$.

17 In a p-wave superfluid phase, rotational symmetry is broken. So there, to study the most general case, we must use a momentum with nonzero components in different directions, as we discuss in section 5.2
} 


\subsubsection{Solving the Equation of Motion}

In order to describe the asymptotic behavior of $\Psi$ most succinctly, we define $\Psi_{ \pm}=$ $\frac{1}{2}\left(1 \pm \gamma^{u}\right) \Psi$ so that $\gamma^{u} \Psi_{ \pm}= \pm \Psi_{ \pm}$. In terms of $\Psi_{ \pm}$, the equation of motion becomes

$$
\begin{aligned}
& \left(u \partial_{u}-\frac{d}{2}-m\right) \Psi_{+}+k u \sigma_{3} \Psi_{-}=0 \\
& \left(u \partial_{u}-\frac{d}{2}+m\right) \Psi_{-}+k u \sigma_{3} \Psi_{+}=0
\end{aligned}
$$

These first-order equations give rise to the second-order equations

$$
\left[\partial_{u}^{2}-\frac{d}{u} \partial_{u}+\frac{1}{u^{2}}\left(-m^{2} \pm m+\frac{d^{2}}{4}+\frac{d}{2}\right)-k^{2}\right] \Psi_{ \pm}=0
$$

We can solve equation (2.3.35) exactly. The form of the solution depends on the value of $m$.

First, suppose $m$ is not a half-integer. The solution of equation (2.3.35) is then

$$
\Psi_{ \pm}=u^{\frac{d+1}{2}}\left[C_{1}^{ \pm}(k) J_{m \mp \frac{1}{2}}\left(\sqrt{-k^{2}} u\right)+C_{2}^{ \pm}(k) J_{-\left(m \mp \frac{1}{2}\right)}\left(\sqrt{-k^{2}} u\right)\right]
$$

where the J's denote Bessel functions of the first kind 18 and $C_{1}^{ \pm}(k)$ and $C_{2}^{ \pm}(k)$ are spinors of the same chirality as $\Psi_{ \pm}$, which may depend on $k$, as indicated.

When $m$ is half-integer, $m \pm \frac{1}{2}$ is an integer and hence the order of the Bessel functions is an integer. In that case the two Bessel functions are not linearly independent: if the order $n$ of $J_{n}(x)$ is an integer, then $J_{n}(x)$ obeys the special identity $J_{-n}(x)=(-1)^{n} J_{n}(x)$, so in that case $J_{n}(x)$ and $J_{-n}(x)$ are obviously linearly related 19 When $m$ is half-integer, we must introduce a Bessel function of the second kind, $Y_{n}(x)$, which is linearly independent from $J_{n}(x)$. When $m$ is half-integer, the solution thus becomes

$$
\Psi_{ \pm}=u^{\frac{d+1}{2}}\left[C_{1}^{ \pm}(k) J_{m \mp \frac{1}{2}}\left(\sqrt{-k^{2}} u\right)+C_{2}^{ \pm}(k) Y_{m \mp \frac{1}{2}}\left(\sqrt{-k^{2}} u\right)\right] .
$$

Notice that if the order $n$ of $Y_{n}(x)$ is an integer, then $Y_{n}(x)$ obeys a relation similar to that for $J_{n}(x)$, namely $Y_{-n}(x)=(-1)^{n} Y_{n}(x)$.

The key difference between the Bessel functions of the first and second kinds that will be important for us is that the small- $x$ expansion of $J_{n}(x)$ involves only powers of $x$, whereas

\footnotetext{
${ }^{18}$ Notice also that since the arguments of the Bessel functions are imaginary, we could equally well write them using the modified Bessel functions $I_{n}(x)$ and $K_{n}(x)$. For our asymptotic analysis, the distinction is not important. If the space was pure $A d S_{d+1}$ (not just asymptotically $A d S_{d+1}$ ), however, equation (2.3.36) is the solution for all $u$, and regularity in the bulk would force us to discard the $I_{n}(x)$ solution.

${ }^{19}$ The Wronskian

$$
W\left(J_{n}(x), J_{-n}(x)\right)=-\frac{\sin n \pi}{\pi x},
$$
}

clearly vanishes when $n$ is integer, indicating linear dependence. The Wronskian

$$
W\left(J_{n}(x), Y_{n}(x)\right)=\frac{2}{\pi x},
$$

for any $n$. 
the expansion of $Y_{n}(x)$ involves both powers of $x$ and logarithms of $x$. Indeed, $J_{n}(x)$ has a series expression (for any $n$ ),

$$
J_{n}(x)=\left(\frac{x}{2}\right)^{n} \sum_{k=0}^{\infty} \frac{(-1)^{k}}{k ! \Gamma(k+n+1)}\left(\frac{x}{2}\right)^{2 k}
$$

from which we can immediately read the expansion for small $x . Y_{n}(x)$ has a series expression, when $n$ is a non-negative integer,

$$
\begin{aligned}
Y_{n}(x)= & \frac{2}{\pi} J_{n}(x) \log \frac{x}{2}-\frac{1}{\pi}\left(\frac{x}{2}\right)^{-n} \sum_{k=0}^{n-1} \frac{(n-k-1) !}{k !}\left(\frac{x}{2}\right)^{2 k} \\
& -\frac{1}{\pi}\left(\frac{x}{2}\right)^{n} \sum_{k=0}^{\infty} \frac{(-1)^{k}}{k !(n+k) !}[\psi(n+k+1)+\psi(k+1)]\left(\frac{x}{2}\right)^{2 k},
\end{aligned}
$$

where $\psi(x)=\frac{\Gamma^{\prime}(x)}{\Gamma(x)}$ is the digamma function. We can obtain the series form of $Y_{-n}(x)$ simply from $Y_{-n}(x)=(-1)^{n} Y_{n}(x)$. The two features to notice are that the logarithmic terms in $Y_{n}(x)$ are simply of the form $J_{n}(x) \log x$, and $Y_{n}(x)$ also includes terms with inverse powers of $x$, from the $x^{-n}$ multiplying the first sum.

Using the series expressions for the Bessel functions, we can rewrite the solutions in a form useful for our purposes (and introduce some notation to keep expressions simple and compact). First, consider $m$ not-half-integer. We write

$$
\begin{aligned}
\Psi_{ \pm} & =u^{\frac{d+1}{2}}\left[C_{1}^{ \pm}(k) J_{m \mp \frac{1}{2}}\left(\sqrt{-k^{2}} u\right)+C_{2}^{ \pm}(k) J_{-\left(m \mp \frac{1}{2}\right)}\left(\sqrt{-k^{2}} u\right)\right] \\
& =u^{\frac{d+1}{2}}\left[c_{1}^{ \pm}(k) u^{m \mp \frac{1}{2}}\left(1+s_{a}^{ \pm}(u, k)\right)+c_{2}^{ \pm}(k) u^{-\left(m \mp \frac{1}{2}\right)}\left(1+s_{b}^{ \pm}(u, k)\right)\right], \\
& =c_{1}^{ \pm}(k) u^{\frac{d}{2}+m \mp \frac{1}{2}+\frac{1}{2}}\left(1+s_{a}^{ \pm}(u, k)\right)+c_{2}^{ \pm}(k) u^{\frac{d}{2}-m \pm \frac{1}{2}+\frac{1}{2}}\left(1+s_{b}^{ \pm}(u, k)\right),
\end{aligned}
$$

where in the second line we have absorbed various factors into $C_{1}^{ \pm}(k)$ and $C_{2}^{ \pm}(k)$, which we then relabeled as $c_{1}^{ \pm}(k)$ and $c_{2}^{ \pm}(k)$, and we have defined the series

$$
s_{a}^{ \pm}(u, k) \equiv \sum_{j=1}^{\infty} a_{j}^{ \pm}(m)\left(-k^{2}\right)^{j} u^{2 j}, \quad a_{j}^{ \pm}(m) \equiv \frac{(-1)^{j}}{j ! 2^{2 j}} \frac{\Gamma\left(1+\left(m \mp \frac{1}{2}\right)\right)}{\Gamma\left(j+1+\left(m \mp \frac{1}{2}\right)\right)},
$$

and $s_{b}^{ \pm}(u, k)$ and $b_{j}^{ \pm}(m)$ are defined similarly, but with $\left(m \mp \frac{1}{2}\right) \rightarrow-\left(m \mp \frac{1}{2}\right)$. We have isolated the leading powers of $u$ near the boundary $u \rightarrow 0$ : the sums $s_{a}^{ \pm}(u, k)$ and $s_{b}^{ \pm}(u, k)$ involve sub-leading powers of $u$ (each sum starts at order $u^{2}$ ). Written separately (to facilitate counting powers of $u), \Psi_{ \pm}$are

$$
\begin{aligned}
& \Psi_{+}=c_{1}^{+}(k) u^{\frac{d}{2}+m}\left(1+s_{a}^{+}(u, k)\right)+c_{2}^{+}(k) u^{\frac{d}{2}-m+1}\left(1+s_{b}^{+}(u, k)\right) \\
& \Psi_{-}=c_{1}^{-}(k) u^{\frac{d}{2}+m+1}\left(1+s_{a}^{-}(u, k)\right)+c_{2}^{-}(k) u^{\frac{d}{2}-m}\left(1+s_{b}^{-}(u, k)\right) .
\end{aligned}
$$

The coefficients in equation (2.3.42) are actually not independent. If we return to equation (2.3.32) and insert $\Psi=\Psi_{+}+\Psi_{-}$, using our solutions for $\Psi_{ \pm}$, then we find, upon 
generalizing $k \gamma^{x} \rightarrow \not k$ and collecting powers 20 of $u$

$$
\begin{aligned}
0= & {\left[(-2 m+1) c_{2}^{+}(k)+i \not k c_{2}^{-}(k)\right] u^{\frac{d}{2}-m+1} } \\
+ & {\left[-(2 m+1) c_{1}^{-}(k)+i \not k c_{1}^{+}(k)\right] u^{\frac{d}{2}+m+1}+O\left(u^{\frac{d}{2}-m+2}\right) . }
\end{aligned}
$$

We thus conclude that

$$
c_{1}^{-}(k)=\frac{1}{2 m+1} i \not k c_{1}^{+}, \quad c_{2}^{+}(k)=\frac{1}{2 m-1} i \not k c_{2}^{-}(k) .
$$

When $m$ is half-integer, we can insert the series expressions for the Bessel functions in equations (2.3.38) and (2.3.39) into the solutions for $\Psi_{ \pm}$, and rearranging various terms, we can write

$$
\begin{aligned}
\Psi_{ \pm} & =u^{\frac{d+1}{2}}\left[C_{1}^{ \pm}(k) J_{m \mp \frac{1}{2}}\left(\sqrt{-k^{2}} u\right)+C_{2}^{ \pm}(k) Y_{m \mp \frac{1}{2}}\left(\sqrt{-k^{2}} u\right)\right], \\
& =u^{\frac{d+1}{2}}\left[c_{1}^{ \pm}(k) u^{m \mp \frac{1}{2}}(\ln u)\left(1+s_{a}^{ \pm}(u, k)\right)+c_{2}^{ \pm}(k) u^{-\left(m \mp \frac{1}{2}\right)}\left(1+s_{d}^{ \pm}(u, k)\right)\right], \\
& =c_{1}^{ \pm}(k) u^{\frac{d}{2}+m \mp \frac{1}{2}+\frac{1}{2}}(\ln u)\left(1+s_{a}^{ \pm}(u, k)\right)+c_{2}^{ \pm}(k) u^{\frac{d}{2}-m \pm \frac{1}{2}+\frac{1}{2}}\left(1+s_{d}^{ \pm}(u, k)\right),
\end{aligned}
$$

where in the second line we have absorbed various factors into $C_{1}^{ \pm}(k)$ and $C_{2}^{ \pm}(k)$, which we then relabeled as $c_{2}^{ \pm}(k)$ and $c_{1}^{ \pm}(k)$. Notice in particular that we exchanged the indices 1 and 2. Recall that we are using units in which the radius of $A d S_{d+1}$ is equal to one. The arguments of the logarithms include factors of the $A d S_{d+1}$ radius to render them dimensionless. The sums $s_{a}^{ \pm}(u, k)$ are defined in equation (2.3.41), while the sums $s_{d}^{ \pm}(u, k)$ are

$$
s_{d}^{ \pm}(u, k)=\sum_{j=1}^{\infty} d_{j}^{ \pm}(m, k)\left(-k^{2}\right)^{j} u^{2 j},
$$

where the coefficients $d_{j}^{ \pm}(m, k)$ are not particularly illuminating to see, so we will not write them down. They can be derived straightforwardly from equations (2.3.38) and (2.3.39). That derivation also shows that they can depend not only on $m$ but also on $k$, as indicated. We have defined our notation to isolate the leading powers of $u$ near the boundary $u \rightarrow 0$, and also to show that the solutions are identical in form to those for the $m$-not-half-integer case in equation (2.3.40), except for an extra logarithmic factor in the $c_{1}^{ \pm}(k)$ terms. Written separately (to facilitate counting powers of $u$ ), $\Psi_{ \pm}$are

$$
\begin{aligned}
& \Psi_{+}=c_{1}^{+}(k) u^{\frac{d}{2}+m}(\ln u)\left(1+s_{a}^{+}(u, k)\right)+c_{2}^{+}(k) u^{\frac{d}{2}-m+1}\left(1+s_{d}^{+}(u, k)\right) \\
& \Psi_{-}=c_{1}^{-}(k) u^{\frac{d}{2}+m+1}(\ln u)\left(1+s_{a}^{-}(u, k)\right)+c_{2}^{-}(k) u^{\frac{d}{2}-m}\left(1+s_{d}^{-}(u, k)\right) .
\end{aligned}
$$

Here again, the coefficients in equation (2.3.46) are not independent. Inserting the solutions for $\Psi_{ \pm}$into the Dirac equation, equation (2.3.32), and generalizing $k \gamma^{x} \rightarrow \not k$, produces

$$
\begin{aligned}
0= & {\left[-(2 m+1) c_{1}^{-}(k)+i \not k c_{1}^{+}(k)\right] u^{\frac{d}{2}+m+1} \ln u+O\left(u^{\frac{d}{2}+m+2} \ln u\right) } \\
+ & (-2 m+1) c_{2}^{+}(k) u^{\frac{d}{2}-m+1}\left(1+s_{d}^{+}(u, k)\right)+i \not k c_{2}^{-}(k) u^{\frac{d}{2}-m+1}\left(1+s_{d}^{-}(u, k)\right) \\
+ & c_{1}^{+}(k) u^{\frac{d}{2}+m}+c_{2}^{+}(k) u^{\frac{d}{2}-m+1}\left[u \partial_{u} s_{d}^{+}(u, k)\right]+O\left(u^{\frac{d}{2}-m+2}\right),
\end{aligned}
$$

\footnotetext{
${ }^{20}$ When $m$ is an integer, another term of order $u^{\frac{d}{2}+m+1}$ appears in equation (2.3.43), with a coefficient proportional to $b_{m}^{+}(m) c_{2}^{+}(k)+b_{m}^{-}(m) i \not k c_{2}^{-}(k)$. This term has opposite chirality from the term shown in equation (2.3.43), hence we set them to zero independently. Using the definition of the $b_{j}^{ \pm}(m)$, we then recover exactly the same relation between $c_{2}^{+}(k)$ and $c_{2}^{-}(k)$ as in equation (2.3.44), so we obtain no new information.
} 
where in the first line we have indicated the sub-leading order of terms involving powers and the logarithm of $u$, while in the third line we have indicated the sub-leading order of terms involving just powers of $u$. When $m \neq 1 / 2$, the vanishing of the $u^{\frac{d}{2}+m+1} \ln u$ and $u^{\frac{d}{2}-m+1}$ terms in equation (2.3.47) requires

$$
c_{1}^{-}(k)=\frac{1}{2 m+1} i \not k c_{1}^{+}, \quad c_{2}^{+}(k)=\frac{1}{2 m-1} i \not k c_{2}^{-}(k),
$$

which are identical to what we found in the $m$ non-half-integer case, equation (2.3.44). In what follows, we will also need to write $c_{1}^{+}(k)$ in terms of $c_{2}^{-}(k)$. We thus turn to higher-order terms in equation (2.3.47). When $m \neq 1 / 2$, the $u^{\frac{d}{2}+m}$ term will always have the same power of $u$ as $u^{\frac{d}{2}-m+1}$ times a particular term in the summations $s_{d}^{+}(u, k)$, $s_{d}^{-}(u, k)$ and $u \partial_{u} s_{d}^{+}(u, k)$. Recall that these summations involve powers of $u^{2}$. Some term in the summations, say the $j$ th term, will have $j=m-\frac{1}{2}$. Recalling the definition of the summations in equation (2.3.45), we find from the second and third lines in equation $(2.3 .47)$

$$
\begin{aligned}
c_{1}^{+}(k) & =-\left[c_{2}^{+}(k)(-2 m+1) d_{j}^{+}(m, k)+i \not k c_{2}^{-}(k) d_{j}^{-}(m, k)+c_{2}^{+}(k) d_{j}^{+}(m, k) 2 j\right]\left(-k^{2}\right)^{j}, \\
& =-i \not k c_{2}^{-}(k) d_{m-\frac{1}{2}}^{-}(m, k)\left(-k^{2}\right)^{m-\frac{1}{2}},
\end{aligned}
$$

where we inserted $j=m-\frac{1}{2}$ into the first line, so that the two $c_{2}^{+}(k)$ terms canceled, producing the second line. The factor of $2 j=2 m-1$ in the first line is crucial for the cancellation: it comes from the $u \partial_{u}$ acting on $s_{d}^{+}(m, k)$ in the third line of equation (2.3.47). The $m=1 / 2$ story is similar: $u^{\frac{d}{2}-m+1}$ and $u^{\frac{d}{2}+m}$ become the same power $u^{\frac{d}{2}+\frac{1}{2}}$. Thus, from the second and third lines in equation (2.3.47), we immediately find

$$
c_{1}^{+}(k)=-i \not k c_{2}^{-}(k) \text {. }
$$

In the following subsections we restrict to positive values of $m$ unless stated otherwise. We can recover results for negative $m$ as follows. For $m$ not-half-integer, if $m<0$, then we can insert $m=-|m|$ in equation (2.3.36) to obtain

$$
\begin{aligned}
\Psi_{ \pm} & =u^{\frac{d+1}{2}}\left[C_{1}^{ \pm}(k) J_{m \mp \frac{1}{2}}\left(\sqrt{-k^{2}} u\right)+C_{2}^{ \pm}(k) J_{-\left(m \mp \frac{1}{2}\right)}\left(\sqrt{-k^{2}} u\right)\right] \\
& =u^{\frac{d+1}{2}}\left[C_{1}^{ \pm}(k) J_{-\left(|m| \pm \frac{1}{2}\right)}\left(\sqrt{-k^{2}} u\right)+C_{2}^{ \pm}(k) J_{|m| \pm \frac{1}{2}}\left(\sqrt{-k^{2}} u\right)\right] .
\end{aligned}
$$

To obtain results for negative $m$, we can work with positive $m$ and then in all formulas take $m \rightarrow|m|$ and exchange $C_{1}^{ \pm}(k) \leftrightarrow C_{2}^{\mp}(k)$, which means

$$
c_{1}^{ \pm}(k) \rightarrow c_{2}^{\mp}(k), \quad c_{2}^{ \pm}(k) \rightarrow c_{1}^{\mp}(k), \quad m \text { not-half-integer. }
$$

For $m$ half-integer, if $m<0$, then we can insert $m=-|m|$ in equation (2.3.37) to obtain

$$
\begin{aligned}
\Psi_{ \pm} & =u^{\frac{d+1}{2}}\left[C_{1}^{ \pm}(k) J_{m \mp \frac{1}{2}}\left(\sqrt{-k^{2}} u\right)+C_{2}^{ \pm}(k) Y_{m \mp \frac{1}{2}}\left(\sqrt{-k^{2}} u\right)\right] \\
& =u^{\frac{d+1}{2}}\left[C_{1}^{ \pm}(k) J_{-\left(|m| \pm \frac{1}{2}\right)}\left(\sqrt{-k^{2}} u\right)+C_{2}^{ \pm}(k) Y_{-\left(|m| \pm \frac{1}{2}\right)}\left(\sqrt{-k^{2}} u\right)\right] \\
& =(-1)^{|m| \pm \frac{1}{2}} u^{\frac{d+1}{2}}\left[C_{1}^{ \pm}(k) J_{|m| \pm \frac{1}{2}}\left(\sqrt{-k^{2}} u\right)+C_{2}^{ \pm}(k) Y_{|m| \pm \frac{1}{2}}\left(\sqrt{-k^{2}} u\right)\right]
\end{aligned}
$$

where in the third equality we have used $J_{-n}(x)=(-1)^{n} J_{n}(x)$ and $Y_{-n}(x)=(-1)^{n} Y_{n}(x)$. To recover results for negative $m$, we can work with positive $m$ and then in all formulas take $m \rightarrow|m|, C_{1}^{ \pm}(k) \rightarrow(-1)^{|m| \mp \frac{1}{2}} C_{1}^{\mp}(k)$, and $C_{2}^{ \pm}(k) \rightarrow(-1)^{|m| \mp \frac{1}{2}} C_{2}^{\mp}(k)$, which means

$$
c_{1}^{ \pm}(k) \rightarrow(-1)^{|m| \mp \frac{1}{2}} c_{1}^{\mp}(k), \quad c_{2}^{ \pm}(k) \rightarrow(-1)^{|m| \mp \frac{1}{2}} c_{2}^{\mp}(k), \quad m \text { half-integer. }
$$




\subsubsection{Determining the Counterterms}

As reviewed in section 2.2, the AdS/CFT dictionary equates the exponential of (minus) the on-shell supergravity action with the generating functional of field theory correlation functions. For the action in equation (2.3.27), clearly the bulk term vanishes when evaluated on a solution. The only nonzero contribution to the on-shell action comes from the boundary terms. We split $S_{b d y}$ into two terms,

$$
S_{b d y}=S_{v a r}+S_{C T}
$$

where $S_{v a r}$ are terms required for the variational principle to be well-posed $[82,83]$.

Since $\Psi_{+}(u, k)$ and $\Psi_{-}(u, k)$ are not independent $[83,84]$, each one determines the canonical momentum associated with the other (see for example [81]). In the bulk Dirichlet problem, then, we cannot fix their asymptotic values $c_{ \pm}(k)$ simultaneously, but can fix only one, the coefficient of the dominant term, $c_{-}(k)$, and then vary the field. As shown in [83], for the action to remain stationary under such variations, we must add the following boundary term to the action,

$$
S_{v a r}=\int d^{d} x \sqrt{\gamma} \bar{\Psi}_{+} \Psi_{-},
$$

where the integration is over the $u=\epsilon$ hypersurface, $\sqrt{\gamma}=\epsilon^{-d}$ is the square root of the determinant of the induced metric at $u=\epsilon$, and $\Psi_{ \pm}$are evaluated at $u=\epsilon$.

We insert the solutions for $\Psi_{ \pm}$into $S_{v a r}$ and isolate any terms that diverge as $\epsilon \rightarrow 0$. We then introduce into $S_{C T}$ any terms necessary to cancel the divergences and hence render the on-shell action finite. The terms in $S_{C T}$ must respect the symmetries of the on-shell $S_{v a r}$ and must be built only from $\Psi_{-}$, to preserve stationarity of the action, as explained above.

For $m$ not half-integer (and positive), if we insert the solutions for $\Psi_{ \pm}$from equation (2.3.42) into equation (2.3.55), we find 21

$$
\begin{aligned}
S_{v a r}=\int d^{d} x & \frac{1}{\epsilon^{d}}\left[\bar{c}_{1}^{+} c_{2}^{-} \epsilon^{d}\left(1+f_{a^{+} b^{-}}(\epsilon, k)\right)+\bar{c}_{2}^{+} c_{1}^{-} \epsilon^{d+2}\left(1+f_{a^{-} b^{+}}(\epsilon, k)\right)\right. \\
& \left.+\bar{c}_{1}^{+} c_{1}^{-} \epsilon^{d+2 m+1}\left(1+f_{a^{+} a^{-}}(\epsilon, k)\right)+\bar{c}_{2}^{+} c_{2}^{-} \epsilon^{d-2 m+1}\left(1+f_{b^{+} b^{-}}(\epsilon, k)\right)\right]
\end{aligned}
$$

where we have introduced one more piece of notation: we have defined

$$
f_{a^{+} a^{-}}(\epsilon, k)=s_{a}^{+}(\epsilon, k)+s_{a}^{-}(\epsilon, k)+s_{a}^{+}(\epsilon, k) s_{a}^{-}(\epsilon, k),
$$

and similarly for $f_{b^{+} b^{-}}(\epsilon, k), f_{a^{+} b^{-}}(\epsilon, k)$, and $f_{a^{-} b^{+}}(\epsilon, k)$, all of which are summations in powers of $\epsilon^{2}$, starting with $\epsilon^{2}$. We now ask what happens to each of the terms in the brackets in equation (2.3.56) as $\epsilon \rightarrow 0$. The first term clearly remains finite (the $\epsilon^{d}$ 's cancel). The second and third terms vanish as $\epsilon^{2}$ and $\epsilon^{2 m+1}$, respectively. The fate of the fourth term depends on $m$. If $m<1 / 2$, then the fourth term vanishes as $\epsilon^{-2 m+1}$, and so we are done: no divergences appear and no counterterms are necessary. If $m>1 / 2$, however, then the fourth term may have one or more divergent terms, with the leading divergence going as $\epsilon^{-2 m+1}$, so we must add counterterms. Starting now, we assume $m>1 / 2$. Using

\footnotetext{
${ }^{21}$ Starting now, we drop the $k$ dependence in the coefficients: $c_{1}^{ \pm}(k) \rightarrow c_{1}^{ \pm}$and $c_{2}^{ \pm}(k) \rightarrow c_{2}^{ \pm}$.
} 
equation (2.3.44), we can write the on-shell $S_{\text {var }}$ as

$$
S_{v a r}=\int d^{d} x \frac{1}{\epsilon^{d}}\left[\bar{c}_{1}^{+} c_{2}^{-} \epsilon^{d}+\frac{1}{2 m-1} \bar{c}_{2}^{-} i \not k c_{2}^{-} \epsilon^{d-2 m+1}\left(1+f_{b^{+} b^{-}}(\epsilon, k)\right)+O\left(\epsilon^{d+2}\right)\right] .
$$

Having isolated the divergences in $S_{v a r}$, we now must write an $S_{C T}$ that obeys the following constraints: it must cancel all divergences, while respecting all symmetries of, the on-shell $S_{v a r}$, and must be built only from the boundary value of $\Psi_{-}$. The $S_{C T}$ that does the job is

$$
S_{C T}=\int d^{d} x \sqrt{\gamma} \sum_{j=0}^{\infty} \alpha_{j}(m) \bar{\Psi}_{-} \partial_{\epsilon} \square_{\epsilon}^{j} \Psi_{-}=\int d^{d} x \frac{1}{\epsilon^{d}} \sum_{j=0}^{\infty} \epsilon^{1+2 j} \alpha_{j}(m) \bar{\Psi}_{-} \not \partial \square^{j} \Psi_{-},
$$

where $\not \partial_{\epsilon}=\epsilon \not \partial$ (the power of $\epsilon$ comes from the inverse vielbein evaluated at $u=\epsilon$ ) and $\square_{\epsilon}^{j}$ is some power $j$ of the scalar Laplacian $\square_{\epsilon}$ on the $u=\epsilon$ surface (so the derivatives act only in field theory directions), which in our case is simply $\square_{\epsilon}=\epsilon^{2} \partial^{2}$. When we take $\Psi \rightarrow e^{i k x} \Psi$, the counterterms become

$$
S_{C T}=\int d^{d} x \frac{1}{\epsilon^{d}} \sum_{j=0}^{\infty} \epsilon^{1+2 j} \alpha_{j}(m) \bar{\Psi}_{-} i \not h\left(-k^{2}\right)^{j} \Psi_{-},
$$

so that, inserting in the solutions for $\Psi_{ \pm}$, we find

$$
\begin{aligned}
& S_{C T}=\int d^{d} x \frac{1}{\epsilon^{d}} \sum_{j=0}^{\infty} \epsilon^{1+2 j} \alpha_{j}(m) \\
& \times\left[\epsilon^{d+2 m+2} \bar{c}_{1}^{-} i \not k\left(-k^{2}\right)^{j} c_{1}^{-}\left(1+f_{a^{-} a^{-}}(\epsilon, k)\right)+\epsilon^{d+1} \bar{c}_{1}^{-} i \not k\left(-k^{2}\right)^{j} c_{2}^{-}\left(1+f_{a^{-} b^{-}}(\epsilon, k)\right)\right. \\
& \left.\quad+\epsilon^{d+1} \bar{c}_{2}^{-} i \not k\left(-k^{2}\right)^{j} c_{1}^{-}\left(1+f_{b^{-} a^{-}}(\epsilon, k)\right)+\epsilon^{d-2 m} \bar{c}_{2}^{-} i \not k\left(-k^{2}\right)^{j} c_{2}^{-}\left(1+f_{b^{-} b^{-}}(\epsilon, k)\right)\right] .
\end{aligned}
$$

Among the terms in the bracket, the one that is potentially divergent when $\epsilon \rightarrow 0$ goes as $\epsilon^{d-2 m}$. All of the other terms vanish when $\epsilon \rightarrow 0$. We fix the coefficients $\alpha_{j}(m)$ by demanding that the divergent terms in equations (2.3.57) and (2.3.60) cancel each other, which means that the quantity

$$
\frac{1}{2 m-1}\left(1+f_{b^{+} b^{-}}(\epsilon, k)\right)+\sum_{j=0}^{\infty} \alpha_{j}(m)\left(-\epsilon^{2} k^{2}\right)^{j}\left(1+f_{b^{-} b^{-}}(\epsilon, k)\right)
$$

must vanish order-by-order in $-\epsilon^{2} k^{2}$, up to order $\epsilon^{2 m-1}$. We immediately see that $\alpha_{0}(m)=$ $-\frac{1}{2 m-1}$, and we can write a formal recursive solution for all the other $\alpha_{j}(m)$ (here we drop the dependence on $m$ for notational clarity, so $\alpha_{j}(m) \rightarrow \alpha_{j}$, etc.),

$$
\alpha_{j}=-\frac{1}{(2 m-1)}\left[b_{j}^{+}+b_{j}^{-}+\sum_{i=1} b_{i}^{+} b_{j-i}^{-}\right]-\sum_{i<j} \alpha_{i}\left[2 b_{j-i}^{-}+\sum_{k=1} b_{k}^{-} b_{j-i-k}^{-}\right],
$$

where we define $b_{j}^{ \pm}(m) \equiv 0$ if $j \leq 0$. The first four $\alpha_{j}(m)$ are

$$
\begin{aligned}
& \alpha_{0}=-\frac{1}{2 m-1}, \quad \alpha_{1}=-\frac{b_{1}^{-}+b_{1}^{+}}{(2 m-1)}-\alpha_{0} 2 b_{1}^{-}, \\
& \alpha_{2}=-\frac{b_{2}^{-}+b_{2}^{+}+b_{1}^{-} b_{1}^{+}}{(2 m-1)}-\alpha_{1} 2 b_{1}^{-}-\alpha_{0}\left(2 b_{2}^{-}+b_{1}^{-2}\right), \\
& \alpha_{3}=-\frac{b_{3}^{+}+b_{3}^{-}+b_{2}^{+} b_{1}^{-}+b_{1}^{+} b_{2}^{-}}{(2 m-1)}-\alpha_{2} 2 b_{1}^{-}-\alpha_{1}\left(2 b_{2}^{-}+b_{1}^{-2}\right)-\alpha_{0}\left(2 b_{3}^{-}+2 b_{1}^{-} b_{2}^{-}\right) .
\end{aligned}
$$


Inserting in the explicit forms for the $b_{j}^{ \pm}(m)$ (which are just the $a_{j}^{ \pm}(m)$ from equation (2.3.41) with $\left.m \mp \frac{1}{2} \rightarrow-\left(m \mp \frac{1}{2}\right)\right)$, we can write these explicitly:

$$
\begin{aligned}
\alpha_{0}(m) & =-\frac{1}{2 m-1}, & \alpha_{2}(m) & =\frac{2}{(2 m-1)^{3}(2 m-3)(2 m-5)}, \\
\alpha_{1}(m) & =-\frac{1}{(2 m-1)^{2}(2 m-3)}, & \alpha_{3}(m) & =\frac{17-10 m}{(2 m-1)^{4}(2 m-3)^{2}(2 m-5)(2 m-7)} .
\end{aligned}
$$

We have thus determined the counterterms when $m$ is not half-integer.

We now consider half-integer $m$, in which case the solutions for $\Psi_{ \pm}$appear in equation (2.3.46). Once again, to determine the divergences, we insert the solutions for $\Psi_{ \pm}$into $S_{v a r}$, with the result

$$
\begin{aligned}
S_{v a r}= & \int d^{d} x \sqrt{\gamma} \bar{\Psi}_{+} \Psi_{-} \\
= & \int d^{d} x \frac{1}{\epsilon^{d}}\left[\bar{c}_{1}^{+} c_{2}^{-} \epsilon^{d}(\ln \epsilon)\left(1+f_{a^{+} d^{-}}(\epsilon, k)\right)+\bar{c}_{2}^{+} c_{1}^{-} \epsilon^{d+2}(\ln \epsilon)\left(1+f_{a^{-} d^{+}}(\epsilon, k)\right)\right. \\
& \left.+\bar{c}_{1}^{+} c_{1}^{-} \epsilon^{d+2 m+1}(\ln \epsilon)^{2}\left(1+f_{a^{+} a^{-}}(\epsilon, k)\right)+\bar{c}_{2}^{+} c_{2}^{-} \epsilon^{d-2 m+1}\left(1+f_{d^{+} d^{-}}(\epsilon, k)\right)\right]
\end{aligned}
$$

where $f_{a^{+} d^{-}}(\epsilon, k), f_{a^{-} d^{+}}(\epsilon, k)$ and $f_{d^{+} d^{-}}(\epsilon, k)$ are defined similarly to $f_{a^{+} a^{-}}(\epsilon, k)$. We now ask what happens to each of the terms in brackets in equation (2.3.64) when $\epsilon \rightarrow 0$. The first term diverges as $\ln \epsilon$. The second and third terms vanish as $\epsilon^{2} \ln \epsilon$ and $\epsilon^{2 m+1}(\ln \epsilon)^{2}$, respectively. The fate of the fourth term depends on $m$. If $m=1 / 2$, then the fourth term is finite. The first term still diverges, however, so in this case we need counterterms (in contrast to the $m$-not-half-integer cases). If $m>1 / 2$, then the fourth term may have one or more power-law divergent terms, with the leading divergence going as $\epsilon^{-2 m+1}$. Notice, however, that the fourth term will also always produce something finite, since the sum $f_{d^{+} d^{-}}(\epsilon, k)$ will always have a term that goes as $\epsilon^{2 m-1}$ which will cancel the $\epsilon^{-2 m+1}$. When $m \neq 1 / 2$, we can use equations (2.3.48) and (2.3.49) to rewrite the $S_{v a r}$ in equation $(2.3 .64)$ as

$$
\begin{aligned}
& S_{v a r}=\int d^{d} x \frac{1}{\epsilon^{d}}[ \bar{c}_{1}^{+} c_{2}^{-} \epsilon^{d}(\ln \epsilon)\left(1+f_{a^{+} d^{-}}(\epsilon, k)\right) \\
&\left.+\bar{c}_{2}^{+} c_{2}^{-} \epsilon^{d-2 m+1}\left(1+f_{d^{+} d^{-}}(\epsilon, k)\right)+O\left(\epsilon^{d+2} \ln \epsilon\right)\right] \\
&=\int d^{d} x \frac{1}{\epsilon^{d}} \bar{c}_{2}^{-} i \not k c_{2}^{-}\left[-d_{m-\frac{1}{2}}^{-}(m, k)\left(-k^{2}\right)^{m-\frac{1}{2}} \epsilon^{d} \ln \epsilon\left(1+f_{a^{+} d^{-}}(\epsilon, k)\right)\right. \\
&\left.\quad+\frac{1}{2 m-1} \epsilon^{d-2 m+1}\left(1+f_{d^{+} d^{-}}(\epsilon, k)\right)+O\left(\epsilon^{d+2} \ln \epsilon\right)\right] .
\end{aligned}
$$

The $m=1 / 2$ case is similar, except in the third line $d_{m-\frac{1}{2}}^{-}(m, k)\left(-k^{2}\right)^{m-\frac{1}{2}} \rightarrow 1$, and the fourth line remains identical to the first line.

We now include logarithmic terms in $S_{C T}$,

$$
S_{C T}=\int d^{d} x \sum_{j=0}^{\infty}\left(\alpha_{j}(m, k)+\beta_{j}(m, k) \ln \epsilon\right) \sqrt{\gamma} \bar{\Psi}_{-} \not_{\epsilon} \square_{\epsilon}^{j} \Psi_{-} .
$$


Inserting the solutions for $\Psi_{ \pm}$into $S_{C T}$, we find

$$
\begin{array}{rl}
S_{C T}=\int d^{d} & x \frac{1}{\epsilon^{d}} \sum_{j=0}^{\infty}\left(\alpha_{j}(m, k)+\beta_{j}(m, k) \ln \epsilon\right) \epsilon^{2 j} \epsilon^{d-2 m+1} \\
& \times\left[\bar{c}_{2}^{-} i \not \not\left(-k^{2}\right)^{j} c_{2}^{-}\left(1+f_{d^{-} d^{-}}(\epsilon, k)\right)+O\left(\epsilon^{2 m+1} \ln \epsilon\right)\right] .
\end{array}
$$

In this equation we have just isolated the potentially divergent terms. All other terms vanish for $\epsilon \rightarrow 0$. We now compare equation (2.3.65) and equation (2.3.67), and adjust the coefficients $\alpha_{j}(m, k)$ and $\beta_{j}(m, k)$ such that all divergences cancel.

We first consider the logarithmically-divergent terms. For these we must adjust the coefficients $\beta_{j}(m, k)$ such that

$$
-d_{m-\frac{1}{2}}^{-}(m, k)\left(-k^{2}\right)^{m-\frac{1}{2}}+\sum_{j=0}^{\infty} \beta_{j}(m, k)\left(-k^{2}\right)^{j} \epsilon^{2 j-2 m+1}=0 .
$$

We thus conclude that all $\beta_{j}(m, k)$ are zero except for one, $\beta_{m-\frac{1}{2}}(m, k)=d_{m-\frac{1}{2}}^{-}(m, k)$. If $m=1 / 2$, the result is that only $\beta_{0}(m, k)=1$ is nonzero.

Next we consider the power-law divergences, which appear when $m \neq 1 / 2$. For these we adjust the coefficients $\alpha_{j}(m, k)$ such that the quantity

$$
\frac{1}{2 m-1}\left(1+f_{d^{+} d^{-}}(\epsilon, k)\right)+\sum_{j=0}^{\infty} \alpha_{j}(m, k)\left(-\epsilon^{2} k^{2}\right)^{j}\left(1+f_{d^{-} d^{-}}(\epsilon, k)\right)
$$

vanishes order-by-order in $-\epsilon^{2} k^{2}$, up to order $\epsilon^{2 m-3}$, to guarantee that all divergences cancel. The $\alpha_{j}(m, k)$ are thus straightforward to obtain, indeed, they are identical in form to the $\alpha_{j}(m, k)$ in equation (2.3.62), but with $b_{j}^{ \pm}(m) \rightarrow d_{j}^{ \pm}(m, k)$. We have thus determined the counterterms when $m$ is half-integer.

Notice that when $m$ is half-integer, a finite counterterm is also possible: we may introduce a nonzero $\alpha_{m-1 / 2}$, producing a finite counterterm proportional to $\bar{c}_{2}^{-} i \not k\left(-k^{2}\right)^{m-1 / 2} c_{2}^{-}$. Something similar happens for scalars [78]. The scalar equation of motion has Besselfunction solutions, and for certain values of the scalar mass $m$ the linearly-independent solutions are Bessel functions of the first and second kind, which means the asymptotic expansion includes logarithmic terms. In those cases, a finite counterterm also appears, and is proportional to the matter conformal anomaly of the dual CFT [78] (and references therein). Here, we are simply seeing the fermionic version of the scalar story.

To summarize: for the action in equation (2.3.27), clearly the bulk term vanishes when evaluated on a solution. The only nonzero contribution to the on-shell action comes from the boundary terms, hence $S=S_{b d y}$ on-shell. Generically, $S_{b d y}$ diverges and we must add counterterms. When $m$ is not half-integer, the resulting on-shell action is 22

$$
S=\int d^{d} x \bar{c}_{1}^{+} c_{2}^{-}+O\left(\epsilon^{2}\right)
$$

\footnotetext{
${ }^{22}$ Recall once again that we restricted to positive $m$. At the end of the last subsection we explained how to recover results for negative $m$ from the results for positive $m$.
} 
while when $m$ is half-integer but $m \neq 1 / 2$ (here we take $\left.d_{j}^{ \pm}(m, k) \rightarrow d_{j}^{ \pm}\right)$,

$$
S=\int d^{d} x \bar{c}_{2}^{+} c_{2}^{-}\left(d_{m-\frac{1}{2}}^{+}+d_{m-\frac{1}{2}}^{-}+\sum_{i=1} d_{i}^{+} d_{m-\frac{1}{2}-i}^{-}\right)\left(-k^{2}\right)^{m-\frac{1}{2}}+O\left(\epsilon^{2} \ln \epsilon\right),
$$

and for $m=1 / 2$,

$$
S=\int d^{d} x \bar{c}_{2}^{+} c_{2}^{-}+O\left(\epsilon^{2} \ln \epsilon\right) .
$$

These on-shell actions remain finite as $\epsilon \rightarrow 0$, and hence, upon functional differentiation, will produce renormalized field theory correlators, as we next discuss.

\subsubsection{Computing Renormalized Correlators}

The field $\Psi$ in the bulk is dual to some fermionic operator $\mathcal{O}$. As reviewed in section 2.2 , the renormalized on-shell bulk action, $S_{r e n}=\lim _{\epsilon \rightarrow 0} S$, acts as the generating functional for correlators involving $\mathcal{O}$. In other words, to compute renormalized correlators of $\mathcal{O}$, we take functional derivatives of $S$ with respect to some source. We identify the source for $\mathcal{O}$ as the coefficient of the dominant term in $\Psi$ 's near-boundary expansion. In equations (2.3.42) and (2.3.46), the dominant term is the $u^{\frac{d}{2}-m}$ term, hence we identify $c_{2}^{-}(k)$ as the source for $\mathcal{O}$. More formally, we equate

$$
e^{-S_{r e n}\left[c_{2}^{-}, \bar{c}_{2}^{-}\right]}=\left\langle\exp \left[\int d^{d} x\left(\bar{c}_{2}^{-} \mathcal{O}+\overline{\mathcal{O}} c_{2}^{-}\right)\right]\right\rangle
$$

where the left-hand-side is the exponential of minus the on-shell bulk action in equation (2.3.27), and the right-hand-side is the generating functional of the dual field theory, with $c_{2}^{-}(k)$ acting as the source for the operator $\mathcal{O}$. Upon taking minus the logarithm of both sides, we find that the on-shell bulk action is the generator of connected correlators.

For any value of the bulk fermion's mass $m$, a quantization exists for which $\mathcal{O}$ has dimension $\Delta=\frac{d}{2}+|m|$. Like in the case of a scalar field, when $|m| \in[0,1 / 2)$, a second quantization exists for which $\Delta=\frac{d}{2}-|m|$. These two quantizations correspond to two different field theories, one of which (the one with $\Delta=\frac{d}{2}-|m|$ ) is unstable against a relevant deformation by a double-trace operator of $\mathcal{O}$, and flows to the other, stable, theory, as in the scalar case [76]. When $|m|>d / 2$, the dual operator is irrelevant: $\Delta>d$.

When $d$ is even, $\mathcal{O}$ will be an operator of definite chirality since in that case $\gamma^{u}$ is the chirality operator of the field theory and $c_{2}^{-}(k)$ has definite chirality. Notice that taking $m \rightarrow-m$ takes $c_{2}^{-}(k) \rightarrow c_{1}^{+}(k)$ when $m$ is not half-integer and $c_{2}^{-}(k) \rightarrow(-1)^{|m|+\frac{1}{2}} c_{2}^{+}(k)$ when $m$ is half-integer, and hence switches the chirality of $\mathcal{O}$.

Let us calculate the renormalized one- and two-point functions for fermions. For simplicity, we only consider the case $m$ positive and not half integer. Therefore the renormalized action reads

$$
S_{r e n}=\int d^{d} x \bar{c}_{1}^{+} c_{2}^{-} .
$$

We can now easily compute the renormalized connected correlators of $\mathcal{O}$ and $\overline{\mathcal{O}}$ by taking functional derivatives of $S_{r e n}$. For example, the renormalized one-point function of $\overline{\mathcal{O}}$ is

$$
\langle\overline{\mathcal{O}}\rangle_{\text {ren }}=-\frac{\delta S_{r e n}}{\delta c_{2}^{-}}=-\bar{c}_{1}^{+}
$$


If we use the fact that the on-shell bulk action must be Hermitian, $S=S^{\dagger}$, then we also have

$$
S_{\text {ren }}=S_{\text {ren }}^{\dagger}=\int d^{d} x\left[\bar{c}_{1}^{+} c_{2}^{-}\right]^{\dagger}=\int d^{d} x \bar{c}_{2}^{-} c_{1}^{+},
$$

hence we also find, as we should,

$$
\langle\mathcal{O}\rangle_{\text {ren }}=-\frac{\delta S_{\text {ren }}}{\delta \bar{c}_{2}^{-}}=-c_{1}^{+}
$$

We can obtain two-point functions via second functional derivatives, for example

$$
\langle\mathcal{O} \overline{\mathcal{O}}\rangle_{r e n}=-\frac{\delta^{2} S_{r e n}}{\delta c_{2}^{-} \delta \bar{c}_{2}^{-}}=-\frac{\delta c_{1}^{+}}{\delta c_{2}^{-}}
$$

Let us consider the simplest case to calculate this two-point function: pure $A d S_{d+1}$ with $m$ positive and not half-integer. The solution in equation (2.3.36) is then the solution for all $u$, not just the asymptotic solution as $u \rightarrow 0$. We need to write $c_{1}^{+}$in terms of $c_{2}^{-}$. To do so, we impose a regularity condition in the bulk of $A d S_{d+1}$. Deep in the interior of $A d S_{d+1}$, where $u \rightarrow \infty$, the solution in equation (2.3.36) diverges unless $C_{1}^{+}=(-1)^{m+1 / 2} C_{2}^{+}$. Translating that condition into a condition on $c_{1}^{+}$and $c_{2}^{+}$is trivial, once we recall the definition of $c_{1}^{+}$and $c_{2}^{+}$in equation $(2.3 .40)$. We find

$$
c_{1}^{+}=-2^{-2 m}(2 m-1) \frac{\Gamma\left(\frac{1}{2}-m\right)}{\Gamma\left(\frac{1}{2}+m\right)} k^{2 m-1} c_{2}^{+} .
$$

We then use equation (2.3.44) to write $c_{2}^{+}$in terms of $i \not k c_{2}^{-}$. We finally obtain for the two-point function,

$$
\langle\mathcal{O} \overline{\mathcal{O}}\rangle_{\text {ren }}=2^{-2 m} \frac{\Gamma\left(\frac{1}{2}-m\right)}{\Gamma\left(\frac{1}{2}+m\right)} k^{2 m-1} i \not \not,
$$

which agrees with the result in [83-85] and, up to normalization, is the correct momentumspace form for the two-point function of a quasi-primary operator of dimension $\Delta=$ $d / 2+m$. Similar arguments work when $m$ is half-integer, where again we find the correct momentum-space form for a quasi-primary operator of dimension $\Delta=d / 2+m$. In that case we can also adjust the normalization to any value we like using the finite counterterm.

\subsection{Review: Generalization to finite temperature}

The generalization of the AdS/CFT correspondence to finite temperature is very intuitive. In order to generate finite temperature and entropy, we have to consider $N_{c}$ black threebranes instead of $N_{c}$ coincident extremal D3-branes (and their supergravity solution). This leads us to an asymptotically Anti-de Sitter Schwarzschild black hole (AdS-Sch BH) background on the gravity side (instead of ordinary Anti-de Sitter spacetime). In [86] Witten showed that we can identify the Hawking temperature $T_{H}$ and the BekensteinHawking Entropy $S_{B H}$ of the Schwarzschild black hole with the temperature $T$ and the entropy $S$ on the field theory side 23

\footnotetext{
${ }^{23}$ Therefore we drop the labels $\mathrm{BH}$ and $\mathrm{H}$ from the entropy $S$ and the temperature $T$ from now on.
} 
Let us consider asymptotically AdS Schwarzschild black holes in more detail. The fivedimensional metric of the AdS Schwarzschild black hole may be written as 24

$$
d s^{2}=\frac{1}{u^{2}}\left(\frac{d u^{2}}{f(u)}-f(u) d t^{2}+d \vec{x}^{2}\right), \quad \text { with } f(u)=1-\frac{u^{4}}{u_{h}^{4}},
$$

where $u \in] 0, u_{h}\left[\right.$. Recall that the conformal boundary is at $u=0$. The $S^{5}$ part is not modified by finite temperature. The temperature is related to the location of the horizon, $u_{h}$, by

$$
u_{h}=\frac{1}{\pi T} .
$$

Note that in comparison to equation (2.1.12) we only have to add the function $f(u)$. Taking the limit $u_{h} \rightarrow \infty$, we recover the ordinary Anti-de Sitter metric.

Another useful coordinate system for AdS Schwarzschild black holes is given by

$$
d s_{A d S_{5}}^{2}=\frac{L^{2}}{u^{2}}\left(d u^{2}-\frac{\left(1-u^{4} / u_{h}^{4}\right)^{2}}{1+u^{4} / u_{h}^{4}} d t^{2}+\left(1+u^{4} / u_{h}^{4}\right) d \vec{x}^{2}\right) .
$$

The conformal boundary is still at $u=0$ and the horizon is at $u=u_{h}$ with

$$
u_{h}=\frac{\sqrt{2}}{\pi T} .
$$

If we are interested in thermodynamics, we must Wick rotate the metric to Euclidean signature by analytic continuation $\tau=i t$. Moreover, we have to compactify the time direction into a circle of circumference $T^{-1}$, as it is well-known from thermal field theory.

It is worth mentioning that if the temperature is the only scale we introduce on the field theory side, no thermal phase transition can happen. Once we have characterized the state of the theory at a particular temperature, we know the state of the system at any other temperature. Since we have only one dimensionful scale in our system, the temperature $T$, the dependence of the free energy $F$, the entropy $S$ or any other thermodynamic quantity is fixed by dimensional analysis. It is very easy to guess the entropy density $s$ for $\mathcal{N}=4$ super Yang-Mills theory with gauge group $S U\left(N_{c}\right)$ in the large- $N_{c}$ limit,

$$
s=f(\lambda) N_{c}^{2} T^{3},
$$

where $f$ is a function which depends on the 't Hooft coupling constant $\lambda$. The factors of $N_{c}^{2}$ is very intuitive since we have $N_{c}^{2}-1$ degrees of freedom and we work in the large- $N_{c}$ limit. A perturbative calculation gives

$$
\lim _{\lambda \rightarrow 0} f(\lambda)=\frac{\pi^{2}}{2}
$$

Let us now calculate the entropy for $\mathcal{N}=4 \mathrm{SYM}$ at strong coupling using the machinery of AdS/CFT. As reviewed in section 2.2 , the partition function $\mathcal{Z}$ for Euclidean signature 25 is given by

$$
\mathcal{Z}\left(\phi_{0}\right)=\exp \left(-S_{I I B, \text { sugra }}\left(\bar{\phi} \rightarrow \phi_{0}\right)\right)
$$

\footnotetext{
${ }^{24}$ Note that we set the characteristic radius of AdS space, L, to one.

${ }^{25}$ In section 2.2 the equation was written for Minkowskian signature. Note that we have to replace $i$ by -1 in the exponent for Euclidean signature.
} 
where $\phi_{0}$ are dual to sources (like finite densities, magnetic and electric fields,...) on the field theory side. Note that we have to add appropriate boundary terms to the action $S_{I I B, \text { sugra }}$ in order to regulate the partition function.

The free energy $\mathcal{F}$ is then given by

$$
\mathcal{F}\left(\phi_{0}\right)=-T \ln \mathcal{Z}\left(\phi_{0}\right)=T S_{I I B, \text { sugra }}\left(\bar{\phi} \rightarrow \phi_{0}\right) .
$$

From the free energy we can compute the entropy density $s$ for $\mathcal{N}=4$ SYM at strong coupling. Surprisingly, the supergravity result for $s$ is precisely $3 / 4$ of the free field value [87].

\subsection{Review: Adding fundamental flavor}

So far, we have considered a particular example of AdS/CFT correspondences, $\mathrm{AdS}_{5} / \mathrm{CFT}_{4}$, which arises from the study of $N_{c}$ D3-branes in flat spacetime. The dual field theory is $(3+1)$-dimensional $\mathcal{N}=4$ supersymmetric $S U\left(N_{c}\right)$ Yang-Mills (SYM) theory. The degrees of freedom of $\mathcal{N}=4 \mathrm{SYM}$ transform only in the adjoint representation of the gauge group $S U\left(N_{c}\right)$. In order to model more realistic systems, we have to extend the AdS/CFT correspondence to theories with degrees of freedom transforming in the fundamental representation of the gauge group.

To introduce fundamental flavor fields, we return to D3-branes in flat space and introduce additional open string degrees of freedom, i.e. a stack of $N_{f}$ Dp-branes, which we call flavor branes. In addition to 3-3 strings beginning and ending on the stack of D3-branes there are other types of strings present: 3-p beginning on the stack of D3 branes and ending on the stack of Dp-branes as well as p-3 and p-p strings. The latter ones begin and end on the stack of Dp-branes. We see later that the p-p strings decouple from $\mathrm{p}-3$, 3-p and 3-3 strings. But the endpoints of 3-p and p-3 strings act as pointlike excitations in the $N_{c}$ or $\bar{N}_{c}$ of $S U\left(N_{c}\right)$ on the D3-branes' worldvolume.

By now a large literature exists on the physical properties of these gauge/gravity models with flavor, beginning with [88-91] (for a review see [92]). Therefore we only review the facts relevant for the subsequent chapters of the thesis.

We review in section 2.5.1 all known candidates for flavor branes giving rise to supersymmetric flavor fields. In particular, we consider the properties of the brane intersections and deduce the field theory. Moreover, we consider two examples of flavor branes in more detail which are highly relevant for the discussion in the thesis. The standard example of flavor branes are D7-branes that intersect the D3-branes along their $(3+1)$-dimensional worldvolume [88]. We will see that the additional 3-7 and 7-3 strings can effectively be described by $\mathcal{N}=2$ hypermultiplets. In the thesis we also consider cases in which the flavor fields are confined to a lower-dimensional defect. For example, a supersymmetric D3/D5 intersection can give rise to supersymmetric flavor fields confined to propagate only in $(2+1)$-dimensions of the $(3+1)$-dimensional theory, i.e. along a codimension-one defect [93-95] 26 Since in this thesis the top-down approach is emphasized, 27 we want

\footnotetext{
${ }^{26}$ However note that the adjoint degrees of freedom, described by the open 3-3 strings, can propagate in all $3+1$ dimensions.

${ }^{27}$ Note that a major drawback of the bottom-up approach is the missing knowledge of the dual field theory.
} 
to convince the reader that we know the dual field theory. Therefore we extensively review the field theory and write down the mesonic operators in full detail for a particular example.

In section 2.5 .2 the dual gravity theory is constructed. We simplify the gravity side by neglecting the backreaction of the $N_{f}$ Dp-branes to the $A d S_{5} \times S^{5}$ background created by $N_{c}$ D3-branes. This can be justified in the large- $N_{c}$ limit if we fix the number of flavors, $N_{f}$ since then $N_{f} \ll N_{c}$. Finally, we write down the action of D-branes, and we show that gauge-invariant operators involving flavor fields, in particular mesons, can be mapped to fluctuations of the Dp-branes.

In section 2.5.3 we review a recipe how to generalize AdS/CFT to finite densities and chemical potentials. Finally, in section 2.5 .4 we give a short overview of the phase transitions present in D3/Dp systems.

\subsubsection{D3/Dp systems and the dual field theory}

Let us add a stack of $N_{f}$ Dp-branes to the stack of $N_{c}$ D3-branes which are extended along the spacetime directions $x^{0}, x^{1}, x^{2}$ and $x^{3}$. For simplicity, we refer to the $0,1,2$ and 3 direction, respectively.

Since we consider type IIB string theory, we can add Dp-branes with p odd. However, we consider only D3-, D5- and D7-branes. D(-1)-branes do not introduce flavor degrees of freedom on the D3-brane worldvolume, and D9-branes are unstable without orientifold planes, so we will not consider these cases. Although D1-branes are present in type IIB string theory we cannot use them for flavor branes since the 1-1 strings, i.e. the open strings which begin and end on the stack of D1-branes will be dynamical and therefore not decouple in the Maldacena limit. Besides the $\mathcal{N}=4$ vector multiplet and the flavor degrees of freedom (given by the 3-1 and 1-3 strings), we therefore have unwanted additional degrees of freedom, namely gauge bosons due to 1-1 strings. ${ }^{28}$

In table 2.2 we list all possible flavor Dp-branes satisfying the following conditions:

(i) the D3/Dp brane intersection is supersymmetric, i.e. the number of NeumannDirichlet directions is 0,4 or 8 .

\footnotetext{
${ }^{28}$ To judge whether the gauge theory arising from p-p strings are non-dynamical, we have to compare the 't Hooft coupling $\lambda_{D p}$ of the flavor Dp-brane to that of the color D3-branes. For the color D3-branes we obtain (see equation (A.3.76) of appendix $\mathrm{A}$ )

$$
\lambda_{D 3}=N_{c} g_{Y M, D 3}=2 \pi g_{s} N_{c},
$$

whereas for the flavor Dp-brane

$$
\lambda_{D p}=N_{f} g_{Y M, D p}=(2 \pi)^{p-2} \alpha^{\prime(p-3) / 2} g_{s} N_{f}
$$

Therefore the quotient reads

$$
\frac{\lambda_{D p}}{\lambda_{D 3}}=\frac{N_{f}}{N_{c}}(2 \pi)^{p-3} \alpha^{\prime(p-3) / 2} .
$$

For $p>3$ the quotient vanishes in the Maldacena limit $\alpha^{\prime} \rightarrow 0$, i.e. the p-p strings are non-dynamical in this limit. For $p<3$ it clearly diverges, i.e. we have to take gauge bosons into account which arise due to p-p strings. For $p=3$ the quotient is finite. However, since we work in the limit $N_{f} \ll N_{c}$, we can treat the p-p strings to be non-dynamical.
} 


\begin{tabular}{|l|cccccccccc|}
\hline & 0 & 1 & 2 & 3 & 4 & 5 & 6 & 7 & 8 & 9 \\
\hline$N_{c} D 3$ & $\bullet$ & $\bullet$ & $\bullet$ & $\bullet$ & - & - & - & - & - & - \\
$N_{f} D 7$ & $\bullet$ & $\bullet$ & $\bullet$ & $\bullet$ & $\bullet$ & $\bullet$ & $\bullet$ & $\bullet$ & - & - \\
$N_{f} D 7$ & $\bullet$ & $\bullet$ & - & - & $\bullet$ & $\bullet$ & $\bullet$ & $\bullet$ & $\bullet$ & $\bullet$ \\
$N_{f} D 5$ & $\bullet$ & $\bullet$ & $\bullet$ & - & $\bullet$ & $\bullet$ & $\bullet$ & - & - & - \\
$N_{f} D 5$ & $\bullet$ & - & - & - & $\bullet$ & $\bullet$ & $\bullet$ & $\bullet$ & $\bullet$ & - \\
$N_{f} D 3$ & $\bullet$ & $\bullet$ & - & - & $\bullet$ & $\bullet$ & - & - & - & - \\
\hline
\end{tabular}

Table 2.2: List of all possible flavor branes satisfying the conditions discussed in the main text. Directions which are wrapped by the D-brane are marked by $\bullet$. If the D-brane does not wrap the direction we use the symbol -.

\begin{tabular}{|c|c|c|c|}
\hline D3/Dp & Intersection & \# ND & Reference(s) \\
\hline D3/D7 & $(3+1)$ & 4 & {$[92]$} \\
\hline D3/D7 & $(1+1)$ & 8 & {$[96-98]$} \\
\hline D3/D5 & $(2+1)$ & 4 & {$[94,95]$} \\
\hline D3/D5 & $(0+1)$ & 8 & {$[99]$} \\
\hline D3/D3 & $(1+1)$ & 4 & {$[100]$} \\
\hline
\end{tabular}

Table 2.3: known Field Theories of D3/Dp Systems listed in table 2.2.

(ii) The flavor Dp-branes wrap the timelike direction, i.e. $x^{0}$.

(ii) The branes wrap at least one of the six extra dimensions, i.e. $y^{4}, y^{5}, \ldots, y^{8}$ or $y^{9}$. This condition is necessary since the flavor branes should wrap the radial direction of $\mathrm{AdS}_{5}, r$ defined by $r=\sqrt{\sum_{i=4}^{9}\left(y^{i}\right)^{2}}$. Otherwise the flavor degrees would be only present for one particular energy scale.

Fortunately, for many examples the D3/Dp theory is already known. The table 2.3 lists various D3/Dp systems for which the field theory has been determined explicitly. The first column indicates the D3/Dp system. The second column indicates the dimension of the intersection (the subspace of the D3-brane worldvolume in which the flavor fields propagate). In the third column the number of Neumann-Dirichlet (ND) directions is displayed. The fourth column lists references in which the D3/Dp theory is written explicitly. Note that all of the systems listed in table 2.3 preserve 8 (of the 32 ) real supercharges.

Every Dp-brane in table 2.2 is described by one of the theories above. If the D3/Dp intersection has 4 Neumann-Dirichlet (ND) directions then the corresponding flavor fields (from 3-p and p-3 strings) produce non-chiral flavor, simply because the fields are arranged in hypermultiplets [62], whereas with 8 ND directions we can obtain chiral flavor, as occurs for the 8 ND D3/D7 intersection [96-98].

Let us consider two examples of brane intersections in more detail which are highly relevant for the subsequent chapters of the thesis: the supersymmetric codimension zero D3/D7 intersection and the supersymmetric codimension one D3/D5 intersection. 


\subsubsection{The supersymmetric D3/D7 intersection}

The D3-branes are extended along 0123 whereas the D7-branes wrap 01234567. The configuration preserves $1 / 4$ of the total amount of supersymmetry in type IIB string theory (corresponding to 8 real supercharges) and has an $S O(4) \times S O(2)$ isometry in the directions transverse to the D3-branes. The $S O(4)$ rotates $x^{4}, x^{5}, x^{6}, x^{7}$, while the $S O(2)$ group acts on $x^{8}, x^{9}$. Separating the D3-branes from the D7-branes in the 89 direction by a distance $L$ explicitly breaks the $S O(2)$ group. We will confirm that these geometrical symmetries are also present in the dual field theory.

The dual field theory is $(3+1)$-dimensional $\mathcal{N}=4$ super Yang-Mills theory coupled to flavor fields preserving $\mathcal{N}=2$ supersymmetry. The Lagrangian of this field theory can conveniently be written down in $\mathcal{N}=1$ superspace formalism. As reviewed in section 2.1.1.1 the $\mathcal{N}=4$ vector multiplet decomposes into the vector multiplet $W_{\alpha}$ and the three chiral superfields $\Phi_{1}, \Phi_{2}, \Phi_{3}$ under $\mathcal{N}=1$ supersymmetry. The vector multiplet $W_{\alpha}$ and one of the three chiral superfields (e.g. $\Phi_{3}$ without loss of generality) can be grouped into a $\mathcal{N}=2$ vector multiplet. The remaining two chiral multiplets $\Phi_{1}$ and $\Phi_{2}$ are components of a $\mathcal{N}=2$ hypermultiplet. Moreover, the flavor fields are given in terms of the $\mathcal{N}=1$ chiral multiplets $Q^{r}, \tilde{Q}_{r}\left(r=1, \ldots, N_{f}\right)$. The Lagrangian is thus given by

$$
\begin{gathered}
\mathcal{L}=\operatorname{Im}\left[\tau \int d^{4} \theta\left(\operatorname{tr}\left(\bar{\Phi}_{I} e^{V} \Phi_{I} e^{-V}\right)+Q_{r}^{\dagger} e^{V} Q^{r}+\tilde{Q}_{r}^{\dagger} e^{-V} \tilde{Q}^{r}\right)\right. \\
\left.+\tau \int d^{2} \theta\left(\operatorname{tr}\left(W^{\alpha} W_{\alpha}\right)+W\right)+\text { c.c. }\right]
\end{gathered}
$$

where the superpotential $W$ is

$$
W=\operatorname{tr}\left(\varepsilon_{I J K} \Phi_{I} \Phi_{J} \Phi_{K}\right)+\tilde{Q}_{r}\left(m+\Phi_{3}\right) Q^{r}
$$

and $\tau$ is the complex gauge coupling as given in equation (2.1.3). $m$ is the mass of the hypermultiplet of flavor fields.

For massless flavor fields, i.e. for $m=0$, the Lagrangian is classically invariant under conformal transformations $S O(4,2) \sqrt{29}$ Moreover, if we assign the quantum numbers listed in table 2.4 to the components of the $\mathcal{N}=1$ superfields, the theory is invariant under the following global symmetries: the $\mathcal{R}$-symmetries $S U(2)_{\mathcal{R}}$ and $U(1)_{\mathcal{R}}$ as well as $S U(2)_{\Phi}$. The global symmetry $S U(2)_{\Phi}$ rotates the scalars in the adjoint hypermultiplet. Note that the mass term in the Lagrangian breaks the $U(1)_{\mathcal{R}}$ symmetry explicitly.

If all $N_{f}$ flavor fields have the same mass $m$, the field theory is invariant under a global $U\left(N_{f}\right)$ flavor group. The baryonic $U(1)_{B}$ symmetry is a subgroup of the $U\left(N_{f}\right)$ flavor group. The fundamental superfields $Q^{r}\left(\tilde{Q}_{r}\right)$ are charged $+1(-1)$ under $U(1)_{B}$, while the adjoint fields are inert.

These symmetries of the field theory side can be mapped to symmetries of the D3/D7 brane intersection and hence also to the dual gravity description. The $U\left(N_{f}\right)$ flavor symmetry and therefore also the baryonic $U(1)_{B}$ symmetry are realized by the gauge group of $N_{f}$

\footnotetext{
${ }^{29}$ However note that the scale-invariance is broken at the quantum level since the beta function is proportional to $N_{f} / N_{c}$ and therefore non-vanishing. In the limit $N_{c} \rightarrow \infty$ with $N_{f}$ being fixed, which we will use in later chapters, the beta function is approximately zero, i.e. we can treat the theory as being scale-invariant also at the quantum level.
} 


\begin{tabular}{|c|c|c|c|c|c|c|c|}
\hline $\mathcal{N}=2$ & components & spin & $S U(2)_{\Phi} \times S U(2)_{\mathcal{R}}$ & $U(1)_{\mathcal{R}}$ & $\Delta$ & $U\left(N_{f}\right)$ & $U(1)_{B}$ \\
\hline$\left(\Phi_{1}, \Phi_{2}\right)$ & $X^{4}, X^{5}, X^{6}, X^{7}$ & 0 & $\left(\frac{1}{2}, \frac{1}{2}\right)$ & 0 & 1 & 1 & 0 \\
hyper & $\lambda_{1}, \lambda_{2}$ & $\frac{1}{2}$ & $\left(\frac{1}{2}, 0\right)$ & -1 & $\frac{3}{2}$ & 1 & 0 \\
\hline$\left(\Phi_{3}, W_{\alpha}\right)$ & $X_{V}^{A}=\left(X^{8}, X^{9}\right)$ & 0 & $(0,0)$ & +2 & 1 & 1 & 0 \\
vector & $\lambda_{3}, \lambda_{4}$ & $\frac{1}{2}$ & $\left(0, \frac{1}{2}\right)$ & +1 & $\frac{3}{2}$ & 1 & 0 \\
& $A_{\mu}$ & 1 & $(0,0)$ & 0 & 1 & 1 & 0 \\
\hline$(Q, \tilde{Q})$ & $q^{m}=(q, \overline{\tilde{q}})$ & 0 & $\left(0, \frac{1}{2}\right)$ & 0 & 1 & $N_{f}$ & +1 \\
fund. hyper & $\psi_{i}=\left(\psi, \tilde{\psi}^{\dagger}\right)$ & $\frac{1}{2}$ & $(0,0)$ & $\mp 1$ & $\frac{3}{2}$ & $N_{f}$ & +1 \\
\hline
\end{tabular}

Table 2.4: Fields of the D3/D7 low-energy effective field theory and their quantum numbers under the global symmetries. Note that $U(1)_{B} \subset U\left(N_{f}\right)$. Taken from [92] with slight modifications.

D7-branes, $U\left(N_{f}\right)$. The $U(1)_{\mathcal{R}}$ symmetry corresponds to the $S O(2)$ symmetry of rotations in the 89-plane. An evidence for the matching of these symmetries is the fact that both symmetries are only present for massless flavor fields, i.e. if the D3- and D7-branes are not separated in the transverse 89-plane. The $S O(4)$ rotational invariance in the 4567subspace can be decomposed into two $S U(2)$ groups, denoted $S U(2)_{L}$ and $S U(2)_{R}$. The $S U(2)_{R}$ symmetry of the brane intersection is mapped to the $\mathcal{N}=2 \mathcal{R}$-symmetry $S U(2)_{\mathcal{R}}$ on the field theory side. Finally, the global $S U(2)_{L}$ symmetry of the brane intersection is identified with $S U(2)_{\Phi}$.

We do not discuss here the corresponding mesonic operators. The explicit form of these operators may be found in $[89,101]$ and are reviewed in [92].

\subsubsection{The supersymmetric D3/D5 codimension one intersection}

Now we consider the supersymmetric D3/D5 codimension one intersection. In the following we list the field content and map the symmetries of the brane intersection with the symmetries of the field theory. Then, we construct the mesonic operators in full detail. Note that I wrote down the explicit form of the mesino operators for the first time 30 For simplicity, we restrict ourselves to $N_{f}=1$. At the end of this section we will generalize the operators to the case $N_{f}>1$.

The D3-branes are extended along 0123 whereas the D5-branes wrap the subspace 012456. The brane intersection preserves 8 of the 32 real supercharges. Hence the dual field theory is $(3+1)$-dimensional $\mathcal{N}=4 \mathrm{SYM}$ coupled to defect flavor fields preserving $(2+1)$ dimensional $\mathcal{N}=4$ supersymmetry (eight real supercharges). Here, we will not write down the Lagrangian of the field theory. The couplings of the theory were determined in $[94,95]$. Coupling the defect fields to the ambient fields requires decomposing the $(3+1)$-dimensional $\mathcal{N}=4$ multiplet into two $(2+1)$-dimensional $\mathcal{N}=4$ multiplets, a vector multiplet and a hypermultiplet. The bosonic content of the $(3+1)$-dimensional $\mathcal{N}=4$ multiplet is the vector $A_{\mu}$ and six scalars $X^{4}, X^{5}, \ldots, X^{9}$. The bosonic content of the (2+1)-dimensional vector multiplet is the $(2+1)$-dimensional vector field $A_{k}$ and the three scalars $X_{V}=\left(X^{7}, X^{8}, X^{9}\right)$. The bosonic content of the $(2+1)$-dimensional

\footnotetext{
${ }^{30}$ We later use this result and compare the quantum numbers with the fluctuations of the fermionic D5-brane action. See chapter 5 for more details. The results were obtained in collaboration with Johanna Erdmenger, Matthias Kaminski and Andy O'Bannon and were published in [8].
} 


\begin{tabular}{|c|c|c|c|c|c|}
\hline Mode & Spin & $S U(2)_{H}$ & $S U(2)_{V}$ & $S U\left(N_{c}\right)$ & $\Delta$ \\
\hline$A_{k}$ & 1 & 0 & 0 & adj & 1 \\
$X_{V}^{A}$ & 0 & 0 & 1 & adj & 1 \\
$A_{3}$ & 0 & 0 & 0 & adj & 1 \\
$X_{H}^{I}$ & 0 & 1 & 0 & adj & 1 \\
$\lambda^{i m}$ & $\frac{1}{2}$ & $\frac{1}{2}$ & $\frac{1}{2}$ & adj & $\frac{3}{2}$ \\
$q^{m}$ & 0 & $\frac{1}{2}$ & 0 & $\mathbf{N}$ & $\frac{1}{2}$ \\
$\psi^{i}$ & $\frac{1}{2}$ & 0 & $\frac{1}{2}$ & $\mathbf{N}$ & 1 \\
\hline
\end{tabular}

Table 2.5: The field content of the D3/D5 theory, adapted from [94]. Here, $A_{k}, X_{V}^{A}, A_{3}, X_{H}^{I}$ and $\lambda^{i m}$ are the adjoint fields of $(3+1)$-dimensional $\mathcal{N}=4$ SYM decomposed into $(2+1)$-dimensional $\mathcal{N}=4$ multiplets. $A_{k}$ and $X_{V}^{A}$ are the bosons in a (2+1)-dimensional vector multiplet while $A_{3}$ and $X_{H}^{I}$ are the bosons in a (2+1)-dimensional hypermultiplet. $q^{m}$ and $\psi^{i}$ are the $(2+1)$-dimensional flavor fields, which are in an $\mathcal{N}=4$ hypermultiplet.

\begin{tabular}{|c|c|c|c|c|}
\hline Operator & $\Delta$ & \multicolumn{1}{|c|}{$S U(2)_{H}$} & $S U(2)_{V}$ & Operator in lowest multiplet \\
\hline $\mathcal{J}_{l}$ & $l+2$ & $l, \quad l \geq 0$ & 0 & $i \bar{q}^{m} \stackrel{D}{k}^{k} q^{m}+\bar{\psi}^{i} \rho^{k} \psi^{i}$ \\
$\mathcal{E}_{l}$ & $l+2$ & $l, \quad l \geq 0$ & 1 & $\bar{\psi}_{i} \sigma_{i j}^{A} \psi_{j}+2 \bar{q}^{m} X_{V}^{A a} T^{a} q^{m}$ \\
$\mathcal{C}_{l}$ & $l+1$ & $l+1, \quad l \geq 0$ & 0 & $\bar{q}^{m} \sigma_{m n}^{I} q^{n}$ \\
$\mathcal{D}_{l}$ & $l+3$ & $l-1, \quad l \geq 1$ & 0 & - \\
\hline
\end{tabular}

Table 2.6: The bosonic mesonic operators of the field theory corresponding to the D3/D5 system and their quantum numbers, adapted from [94].) Here $\sigma$ are Pauli matrices, $T^{a}$ are the generators of $S U(2)_{V}$, and $\rho^{k}$ are the $(2+1)$-dimensional $\Gamma$-matrices.

hypermultiplet is the scalar $A_{3}$ and the three scalars $X_{H}=\left(X_{4}, X_{5}, X_{6}\right)$. The flavor fields form a $(2+1)$-dimensional hypermultiplet with two fermions (quarks) $\psi$ and two complex scalars (squarks) $q$.

The classical Lagrangian preserves $(2+1)$-dimensional $S O(3,2)$ conformal symmetry for massless flavor degrees but breaks the $S O(6)_{\mathcal{R}} \mathcal{R}$-symmetry down to a subgroup $S U(2)_{H} \times$ $S U(2)_{V}$, under which the scalars in $X_{H}$ transform in the $(1,0)$ representation and the scalars in $X_{V}$ transform in the $(0,1)$. We use an upper index to denote these representations: $X_{V}^{A}$ and $X_{H}^{I}$. The adjoint fermions $\lambda^{i m}$ transform in the $(1 / 2,1 / 2)$ representation. Here, $i$ is the $S U(2)_{V}$ index and $m$ is the $S U(2)_{H}$ index. The quarks $\psi^{i}$ transform in the $(1 / 2,0)$ and the squarks $q^{m}$ transform in the $(0,1 / 2)$. In table 2.5 (borrowed from [94]), we summarize the field content and quantum numbers, including the conformal dimensions of the fields.

Let us now consider the mesonic operators in the field theory dual to the D3/D5 intersection which can be arranged into a $(2+1)$-dimensional massive $\mathcal{N}=4$ supersymmetric multiplet. The bosonic operators and their quantum numbers are summarized in table 2.6, whereas the fermionic operators are listed in table 2.7. In the text we write down explicitly the form of the fermionic operators.

Let us first review the meson multiplets. All operators with the same $l$ are in one multiplet. 


\begin{tabular}{|c|c|c|c|}
\hline Operator & $\Delta$ & $S U(2)_{H}$ & $S U(2)_{V}$ \\
\hline $\mathcal{F}_{l}$ & $l+3 / 2$ & $l+1 / 2, \quad l \geq 0$ & $1 / 2$ \\
$\mathcal{G}_{l}$ & $l+5 / 2$ & $l-1 / 2, \quad l \geq 1$ & $1 / 2$ \\
\hline
\end{tabular}

Table 2.7: The fermionic mesonic operators and their quantum numbers. The explicit form of the fermionic operators $\mathcal{F}_{l}$ and $\mathcal{G}_{l}$ are given in the text.

Note that we have to distinguish two cases: the $l=0$ multiplet which will be short and the $l>0$ multiplets.

The lowest multiplet, i.e. $l=0$, already appeared in [94] including the fermionic operators. According to tables 2.6 and 2.7, the operator $\mathcal{C}_{0}^{I}=q^{\dagger m} \sigma_{m n}^{I} q^{n}$, where $\sigma^{I}$ are the Pauli matrices of $S U(2)_{H}$, is the lowest chiral primary in the multiplet since all other operators dual to D5-brane fluctuations have larger conformal dimensions. $\mathcal{C}_{0}$ transforms in the $(1,0)$ representation of $S U(2)_{H} \times S U(2)_{V}$. We can thus construct all operators in the same multiplet as $\mathcal{C}_{0}$ by applying supersymmetry generators to $\mathcal{C}_{0}$. The supersymmetry generators form a $2 \times 2$ matrix of Majorana spinors $\eta^{i m}$, which transforms like $\lambda^{i m}$, i.e. in the representation $(1 / 2,1 / 2)$ of $S U(2)_{H} \times S U(2)_{V}$. Applying the supersymmetry generators to $\mathcal{C}_{0}$ we obtain the fermionic operator $\mathcal{F}_{0}^{i m}=\bar{\psi}^{i} q^{m}+q^{\dagger m} \psi^{i}$ with conformal dimension $\Delta=$ $3 / 2$ and $S U(2)_{H} \times S U(2)_{V}$ quantum numbers $(1 / 2,1 / 2)$. Applying another supersymmetry generator to $\mathcal{F}_{0}^{\text {im }}$, we obtain either $\mathcal{J}_{0}$ or $\mathcal{E}_{0}$, the forms of which appear in table 2.6. Both $\mathcal{J}_{0}$ and $\mathcal{E}_{0}$ have conformal dimension $\Delta=2$ and are singlets under $S U(2)_{H}$ but can be distinguished by their $S U(2)_{V}$ quantum number: $\mathcal{J}_{0}$ is a singlet whereas $\mathcal{E}_{0}$ is a triplet under $S U(2)_{V}$.

Now we discuss the general multiplet dual to the higher-l mesonic operators. As in the $l=0$ case, we construct the multiplet by applying supersymmetry generators to the lowest chiral primary in the multiplet, $\mathcal{C}_{l}$. According to [94], the lowest chiral primary is $\mathcal{C}_{l}^{I_{0} I_{1} \ldots I_{l}}=C_{0}^{\left(I_{0}\right.}\left(X_{H}^{l}\right)^{\left.I_{1} \ldots I_{l}\right)}$, where $\left(X_{H}^{l}\right)$ stands for the traceless symmetric product of $l$ copies of the field $X_{H}^{I} \cdot \mathcal{C}_{l}$ has conformal dimension $\Delta=l+1$ and is in the $(l+1,0)$ representation of $S U(2)_{H} \times S U(2)_{V}$. Applying a supersymmetry generator to $\mathcal{C}_{l}$ we find the fermionic operator $\mathcal{F}_{l}$ with conformal dimension $\Delta=l+3 / 2 . \mathcal{F}_{l}$ is in the $(l+1 / 2,1 / 2)$ representation of $S U(2)_{H} \times S U(2)_{V}$. Explicitly, $\mathcal{F}_{l}$ is of the form

$$
\mathcal{F}_{l}^{I_{1} \ldots I_{l} i m}=\bar{\psi}^{i}\left(X_{H}^{l}\right)^{I_{1} \ldots I_{l}} q^{m}+q^{\dagger m}\left(X_{H}^{l}\right)^{I_{1} \ldots I_{l}} \psi^{i} .
$$

Applying another supersymmetry generator to $\mathcal{F}_{l}$, we obtain $\mathcal{J}_{l}$ or $\mathcal{E}_{l}$, which have the same conformal dimension $\Delta=l+2$, but differ in the $S U(2)_{H} \times S U(2)_{V}$ representation. $\mathcal{J}_{l}$ transforms in the $(l, 0)$ representation whereas $\mathcal{E}_{l}$ has quantum numbers $(l, 1)$. To obtain the precise form of $\mathcal{J}_{l}$ or $\mathcal{E}_{l}$, we insert the operator $X_{H}^{l}$ into the operator $\mathcal{J}_{0}$ or $\mathcal{E}_{0}$, respectively.

In contrast to the $l=0$ multiplet, other operators also appear in the multiplet for $l \geq 1$, which we construct by applying three or four supersymmetry generators to $\mathcal{C}_{l}$ : a fermionic operator $\mathcal{G}_{l}$ and a bosonic operator $\mathcal{D}_{l}$. $\mathcal{G}_{l}$ has conformal dimension $\Delta=l+5 / 2$ and $S U(2)_{H} \times S U(2)_{V}$ quantum numbers $(l-1 / 2,1 / 2)$. Explicitly, $\mathcal{G}_{l}$ has the form

$$
\mathcal{G}_{l}^{I_{1} \ldots I_{l-1} i m}=\bar{\psi}^{j}\left(X_{H}^{l-1}\right)^{I_{1} \ldots I_{l-1}} \lambda^{i m} \psi_{j}+q^{\dagger n}\left(X_{H}^{l-1}\right)^{I_{1} \ldots I_{l-1}} \lambda^{i m} X_{H, I} \sigma_{n p}^{I} q^{p} .
$$

Finally, $\mathcal{D}_{l}$ has conformal dimension $\Delta=l+3$ and $S U(2)_{H} \times S U(2)_{V}$ quantum numbers $(l-1,0)$. 
We have constructed the supermultiplet for the cases $l=0$ and $l \geq 1$. For $l=0$ they multiplet consists of the bosonic operators $\mathcal{C}_{0}, \mathcal{J}_{0}$ and $\mathcal{E}_{0}$ and of the fermionic operator $\mathcal{F}_{0}$. The multiplet includes eight bosonic and eight fermionic degrees of freedom. The multiplet containing $\mathcal{C}_{l}, l \geq 1$ has $16 l+1$ fermionic and bosonic degrees of freedom:

- One real scalar $\mathcal{C}_{l}$ in the $(l+1,0)$ representation with $\Delta=l+1$,

- One spinor $\mathcal{F}_{l}$ in the $(l+1 / 2,1 / 2)$ representation with $\Delta=l+3 / 2$,

- One massive vector $\mathcal{J}_{l}$ in the $(l, 0)$ representation with $\Delta=l+2$,

- One real scalar $\mathcal{E}_{l}$ in the $(l, 1)$ representation with $\Delta=l+2$,

- One spinor $\mathcal{G}_{l}$ in the $(l-1 / 2,1 / 2)$ representation with $\Delta=l+5 / 2$,

- One real scalar $\mathcal{D}_{l}$ in the $(l-1,0)$ representation with $\Delta=l+3$.

Moreover, we mapped the operators in the supermultiplet to the fluctuations of the probe brane summarized in tables 2.6 and 2.7 .

Finally, we consider $N_{f}>1$ coincident D5-branes. The dual field theory then has $N_{f}$ flavors with the same mass giving rise to a global $U\left(N_{f}\right)$ flavor symmetry. The overall $U(1)$ we identify as baryon (more accurately quark) number, while the $S U\left(N_{f}\right)$ subgroup we identify as isospin. The mesino operators $\mathcal{F}_{l}$ and $\mathcal{G}_{l}$ of course have zero baryon number charge and are valued in the adjoint of $S U\left(N_{f}\right)$. For example, in our case with $N_{f}=2$ the mesinos acquire an $S U(2)$ isospin index, $\mathcal{F}_{l}^{a}$ and $\mathcal{G}_{l}^{a}$.

\subsubsection{Probe Dp-branes in $A d S_{5} \times S^{5}$}

In order to construct the gravity dual, we have to determine the supergravity solution for the various D3/Dp systems. This is very difficult. For simplicity, we therefore keep the number $N_{f}$ of Dp-branes fixed as $N_{c} \rightarrow \infty$, so that $N_{f} \ll N_{c}$. In this limit we may neglect the Dp-branes' contribution to the stress-energy tensor, and hence we may ignore their effect on the metric 31 This limit is called the probe limit because in this limit the Dp-brane cleanly probes the geometry without deforming it.

The limit is clearest on the field theory side: since the geometry encodes the gauge configuration in which the quarks move, we are simply neglecting diagrams with quark (and/or squark) loops in the language of perturbation theory. This limit is well-known in field theory and is sometimes called the quenched approximation. In this approximation we may ignore quantum effects due to the flavor, such as the running of the coupling, because these effects are suppressed by $N_{f} / N_{c}$. Therefore the $N_{f}$ flavor degrees of freedom appear in the supergravity description as a number $N_{f}$ of Dp-branes [88] embedded in the $A d S_{5} \times S^{5}$ background generated by $N_{c}$ D3-branes.

\subsubsection{Embedding in $\mathbf{A d S}_{5}$}

Let us first study the embedding of the probe Dp-branes into $A d S_{5} \times S^{5}$. As we will see, the probe Dp-branes are extended along $A d S_{P} \times S^{Q}$ with $2 \leq P \leq 5$ and $1 \leq Q \leq 5$.

\footnotetext{
${ }^{31}$ Analogous statements apply for the other fields of type IIB supergravity.
} 


\begin{tabular}{|c|c|c|}
\hline D3/Dp system & Intersection & worldvolume geometry \\
\hline D3/D7 & $3+1$ & $A d S_{5} \times S^{3}$ \\
D3/D7 & $1+1$ & $A d S_{3} \times S^{5}$ \\
D3/D5 & $2+1$ & $A d S_{4} \times S^{2}$ \\
D3/D5 & $0+1$ & $A d S_{2} \times S^{4}$ \\
D3/D5 & $1+1$ & $A d S_{3} \times S^{1}$ \\
\hline
\end{tabular}

Table 2.8: Worldvolume geometry of probe Dp-branes. The first column lists the various D3/Dp systems considered in table 2.3. In the second column the dimension of the intersection on the field theory side is displayed. Finally, the induced worldvolume geometry on the probe Dp-brane is indicated in the last column.

Moreover, in order to preserve supersymmetry we have to require $|P-Q|=2[102]$.

Let us determine all possible pairs $P$ and $Q$ for a Dp-brane. The induced worldvolume geometry can be guessed in the following way 32 The worldvolume coordinates giving rise to the induced $A d S_{P}$ factor are the coordinates of the intersection on the field theory side as well as the radial direction of AdS spacetime. The dimension of the sphere wrapped by the probe $D p$-brane can easily figured out since the number of dimensions of the Dp-brane has to agree with the dimension of the induced geometry. Therefore, $Q=p+1-P$. The result is summarized in table 2.8 .

Let us specify now the precise embedding. The supergravity solution includes a tendimensional metric with a (4+1)-dimensional AdS-Schwarzschild factor and an $S^{5}$ factor. We use an AdS-Schwarzschild metric

$$
d s_{A d S_{5}}^{2}=g_{u u} d u^{2}+g_{t t} d t^{2}+g_{x x} d \vec{x}^{2},
$$

where $u$ is the AdS radial coordinate. When we need an explicit metric, we use the metric components (2.4.80) and (2.4.82) in the case of finite temperature 33 The Dp-brane wrap the radial coordinate $u$ of $\mathrm{AdS}_{5}$ and the time $t$ as well as $P-2$ of the three spatial coordinates $\vec{x}$.

For the sphere $S^{5}$ we use a metric of the form

$$
d s_{S^{5}}^{2}=d \theta^{2}+\sin ^{2} \theta d s_{S^{4-Q}}^{2}+\cos ^{2} \theta d s_{S^{Q}}^{2},
$$

where $\theta$ is an angle between zero and $\pi / 2$ and $d s_{S^{4-Q}}^{2}$ and $d s_{S Q}^{2}$ are metrics for the $(4-Q)$ dimensional and the $Q$-dimensional sphere, respectively. The probe $D p$-brane wrap the $Q$-dimensional sphere $S^{Q}$. The supergravity solution also includes $N_{c}$ units of five-form flux through the $S^{5}$ given by equation (2.1.20).

The embedding of a hyperplane (in our case of the probe Dp-brane) is determined by the transverse coordinates which are in general functions of all worldvolume coordinates. But using the symmetries present in the dual D3/Dp system, only the transverse coordinate $\theta$ can be a non-trivial function of the radial coordinate $u$, i.e. $\theta=\theta(u)$. In this case the induced metric on the worldvolume of the probe Dp-brane reads

$$
d s_{D p}^{2} \equiv\left(G_{u u} d u^{2}+G_{t t} d t^{2}+G_{x x} d \vec{x}^{2}\right)+\cos ^{2} \theta(u) d s_{S^{Q}}^{2},
$$

where $G_{u u}=g_{u u}+\theta^{\prime}(u)^{2}$ and $G_{t t}=g_{t t}, G_{x x}=g_{x x}$.

\footnotetext{
${ }^{32}$ However, note that we use a particular embedding into $A d S_{5} \times S^{5}$ which is described in the text below.

${ }^{33}$ Here we are using units in which the radius of AdS is equal to one. In these units, we convert from string theory to SYM theory quantities using $\alpha^{\prime-2}=2 \lambda$.
} 


\subsubsection{Effective D-brane action}

In this section we discuss how to determine the embedding function $\theta(u)$. For this we need the effective action for a Dp-brane 34 specialized to the supergravity background discussed above. In the thesis only the Dirac-Born-Infeld part of the D-brane action is important 35

$$
S_{D p}=-N_{f} \tau_{p} \int_{D p} d^{p+1} \zeta e^{-\Phi} \sqrt{-\operatorname{det}\left(\mathcal{P}[\mathrm{g}+\mathrm{B}]_{\mathrm{ab}}+\left(2 \pi \alpha^{\prime}\right)^{2} \mathrm{~F}_{\mathrm{ab}}\right)},
$$

where $\mathcal{P}[g+B]_{a b}$ are the pullback of $g_{\mu \nu}$ and $B_{\mu \nu} \cdot \zeta^{a}$ are the worldvolume coordinates of the Dp-brane. In the supergravity background $\Phi$ and $B_{\mu \nu}$ are zero. $F_{a b}$ is the U(1) field strength tensor

$$
F_{a b}=\partial_{a} A_{b}-\partial_{b} A_{a}
$$

of the gauge field $A$ living on the Dp-brane.

Since the gauge fields and the scalars should only depend on the radial coordinate of AdS spacetime (and not on the coordinates of the spacetime and of the sphere), we can integrate over these coordinates. Starting now we divide by the volume of the field theory, i.e. of $\mathbb{R}^{P-2,1}$, such that $S_{D p}$ actually denotes an action density,

$$
S_{D p}=-\mathcal{N} \int d u \sqrt{-\operatorname{det}\left(\mathcal{P}[\mathrm{g}]_{\mathrm{ab}}+\left(2 \pi \alpha^{\prime}\right)^{2} \mathrm{~F}_{\mathrm{ab}}\right)},
$$

where we have to integrate from $u=0$ to $u=u_{h}$. The constant $\mathcal{N}$ is given by

$$
\mathcal{N}=N_{f} \tau_{p} \operatorname{Vol}\left(S^{Q}\right)=4 N_{f} N_{c}(2 \lambda)^{\frac{p-3}{4}}(2 \pi)^{-p+1} \frac{\pi^{\frac{Q+1}{2}}}{\Gamma\left(\frac{Q+1}{2}\right)} .
$$

In the last step, we used $\alpha^{-2}=2 \lambda$ and $g_{s}=2 \pi g_{Y M}^{2}=2 \pi \lambda / N_{c}$ to rewrite $\mathcal{N}$ in terms of field theory quantities. Note that $S_{D p}$ is only of order $N_{c}$ compared to the type IIB supergravity action which is of order $N_{c}^{2}$. In particular, for the D3/D7 codimension zero flavor brane, we obtain

$$
\mathcal{N}=\frac{2 N_{f} N_{c} \lambda}{(2 \pi)^{4}}
$$

We set the field strength tensor $F_{a b}$ to zero in this section and consider a non-vanishing worldvolume scalar field $\theta=\theta(u)$. In order to determine the embedding we have to solve the equations of motion for $\theta(u)$. The action in the metric (2.4.80) reads

$$
S_{D p}=-\mathcal{N} \int d u u^{-P}(\cos \theta(u))^{Q} \sqrt{1+\theta^{\prime}(u)^{2} u^{2} f(u)},
$$

Note that the action of the probe brane, $S_{D p}$, is divergent due to integration all the way to the $\mathrm{AdS}_{5}$ boundary at $u=0$, and thus requires renormalization. The general recipe for holographic renormalization of the Dp-brane action appears in [103-106].

\footnotetext{
${ }^{34}$ For more details see section A.3.

${ }^{35}$ We can slightly generalize the configuration by considering $N_{f}$ coincident Dp-branes. The massless effective degrees of freedom of $N_{f}$ coincident Dp-branes are a $U\left(N_{f}\right)$ valued gauge field living on the brane as well as $U\left(N_{f}\right)$ valued scalars transversal to the Dp-brane. If we are only interested in the dynamics of the overall $U(1)$ subgroup of $U\left(N_{f}\right)$, then we can use the action (2.5.95). Therefore we included a factor of $N_{f}$ infront of the action (2.5.95).
} 
The field $\theta(u)$ is holographically dual to an operator that is given by taking $\frac{\partial}{\partial m}$ of the SYM Lagrangian. We denote the operator by $\mathcal{O}_{m}$. The operator $\mathcal{O}_{m}$ is a supersymmetric version of the quark bilinear, and it takes the schematic form

$$
\mathcal{O}_{m}=\bar{\psi} \psi+q^{\dagger} \Phi_{3} q+m q^{\dagger} q
$$

where $\Phi_{3}$ is one of the adjoint scalars and $q, \psi$ are the flavor fields. $m$ is the mass of the flavor fields.

We do not explicitly write down the equations of motion for $\theta(u)$. We can read off the boundary asymptotics of $\theta(u)$ by expanding the Lagrangian (2.5.100) since $\theta(u)$ is very small at the boundary. Hence, we obtain the Lagrangian of a canonically normalized scalar with mass $M^{2}=-Q$. Therefore the boundary asymptotics of $\theta(u)$ reads

$$
\theta(u)=\theta_{1} u^{P-1-\Delta_{+}}+\theta_{2} u^{\Delta_{+}}+\ldots,
$$

where dots indicate higher order terms in $u$. The exponent $\Delta_{+}$is the larger root of the equation $M^{2}=\Delta(\Delta-P+1)$. Recall that the field theory is effectively $(P-1)$-dimensional. $\Delta_{+}$is also the conformal dimension of the dual operator. The parameters $\theta_{1}$ and $\theta_{2}$ can be mapped to the mass $m$ and the expectation value $\left\langle\mathcal{O}_{m}\right\rangle$. The precise mapping depend on the dimension of the flavor brane and the precise embedding. Therefore we will now discuss only the case of a codimension zero D3/D7 brane. As shown in [103, 104], we identify the mass as $m \propto \theta_{1}$ and the expectation value as $\left\langle\mathcal{O}_{m}\right\rangle \propto-2 \theta_{1}+\frac{1}{3} \theta_{2}^{3}$. From the point of view of field theory the expectation value $\left\langle\mathcal{O}_{m}\right\rangle$ is fixed, if $m$ is fixed. But how can we relate these two parameters on the gravity side?

We cannot derive such a relation by a near-boundary analysis. But $\theta_{1}$ and $\theta_{2}$ are related due to a condition we have to impose at or near the horizon. In fact there are two topologically distinct ways to embed the Dp-brane in the AdS-Schwarzschild background.

The first type of embedding is a Minkowski embedding, in which the worldvolume $S^{Q}$ shrinks as we move along the Dp-brane away from $u=0$ and eventually collapses to a point at some $u=u^{\prime}$ outside the horizon, $u^{\prime}<u_{h}$. We then have the boundary conditions $\theta\left(u^{\prime}\right)=\frac{\pi}{2}$, such that $\cos \theta\left(u^{\prime}\right)=0$ and the $S^{Q}$ has zero volume, and $\theta^{\prime}\left(u^{\prime}\right)=\infty$, so that the Dp-brane does not develop a conical singularity when the $S^{Q}$ collapses to zero volume [104]. The Dp-brane then does not extend past $u^{\prime}$, but rather appears to end abruptly at $u^{\prime}$.

The second type of embedding is a black hole embedding, in which the $S^{Q}$ shrinks but does not collapse, and the Dp-brane intersects the horizon. We can then choose the value of $\theta(u)$ at the horizon, $\theta\left(u_{h}\right) \in\left[0, \frac{\pi}{2}\right)$, while for the derivative we must have $\theta^{\prime}\left(u_{h}\right)=0$ for the embedding to be static.

A discontinuous (first order) transition between the two types of embeddings occurs as a function of $m / T$. The transition has been studied in great detail [91, 104, 107-112]. Roughly speaking, large values of $m / T$ (above a critical value) correspond to Minkowski embeddings while small values of $m / T$ correspond to black hole embeddings.

Finally, let us consider $\theta(u)$ in two limits. The first limit is $m=0$, which corresponds to the trivial solution $\theta(u)=0$ and hence has $\theta\left(u_{h}\right)=0$. The second limit is $m \rightarrow \infty$, where $\theta\left(u_{h}\right) \rightarrow \frac{\pi}{2}$. 


\begin{tabular}{|c|c|c|}
\hline Operator & $l$ & Fluctuation \\
\hline $\mathcal{J}_{l}$ & $l \geq 0$ & $b_{l}^{k}$ \\
$\mathcal{E}_{l}$ & $l \geq 0$ & $\phi_{l}$ \\
$\mathcal{C}_{l}$ & $l \geq 0$ & $(b+z)_{l+1}^{(-)}$ \\
$\mathcal{D}_{l}$ & $l \geq 1$ & $(b+z)_{l-1}^{(+)}$ \\
\hline $\mathcal{F}_{l}$ & $l \geq 0$ & $\Psi_{l}^{-}$ \\
$\mathcal{G}_{l}$ & $l \geq 1$ & $\Psi_{l-1}^{+}$ \\
\hline
\end{tabular}

Table 2.9: Mapping of operators in the field theory of D3/D5 codimension one intersection to fluctuations of the D5-brane. The fluctuations are explained in the text.

\subsubsection{Mapping between fluctuations and mesonic operators}

In this section we show how to map fluctuations of a probe Dp-brane to mesonic operators on the field theory side. We will not do this in generality but rather study an example: the D3/D5 codimension one intersection. In particular we map the mesino operators, i.e. fermionic mesonic operators, to the fluctuations of the fermionic part of the D5-brane action which we investigate in section 5.2.1. Let me emphasize that this result is new and was obtained in collaboration with Johanna Erdmenger, Matthias Kaminski and Andy O'Bannon 36

First, consider the bosonic fluctuations of the D5-brane, as studied in $[94,113,114] .37$ The bosonic fluctuations consists of three real scalars, which in the notation of [94] are $\phi_{l},(b+z)_{l}^{(-)}$and $(b+z)_{l}^{(+)}$, as well as a vector $b_{l}^{k}$. Here $\phi_{l}$ corresponds to fluctuations of the embedding in the $S^{5}$ directions (transverse to the $\left.S^{2}\right),(b+z)_{l}^{(-)}$and $(b+z)_{l}^{(+)}$are linear combinations of the fluctuations of the $S^{2}$ components of the worldvolume gauge field and of the fluctuation of the embedding in $\mathrm{AdS}_{5}$ transverse to $A d S_{4}$. Note that $(b+z)_{l}^{(+)}$is not present in the lowest multiplet with $l=0$. Finally, $b_{l}^{k}(k=0,1,2)$ corresponds to fluctuations of the worldvolume gauge field in the $A d S_{4}$ directions.

Let us borrow here a result from chapter 5. The fermionic fluctuations of the D5-brane consists of two real spinors $\Psi_{l}^{ \pm}$with two components. Their quantum numbers are determined in section 5.2.1. The fluctuations $\Psi_{l}^{-}$with $l \geq 0$ transform in the $(l+1 / 2,1 / 2)$ representation of $S U(2)_{H} \times S U(2)_{V}$ and are dual to operators with conformal dimension $\Delta=l+3 / 2$. The fluctuations $\Psi_{l-1}^{+}$with $l \geq 1$ are in the $(l-1 / 2,1 / 2)$ representation of $S U(2)_{H} \times S U(2)_{V}$ and correspond to operators with conformal dimension $\Delta=l+5 / 2$.

Since we already mapped the symmetries of the gravity side to the field theory side, we can work out the mapping between the fluctuations and the mesonic operators discussed in the previous section. The results are summarized in table 2.9 .

\footnotetext{
${ }^{36}$ The result is published in [8].

${ }^{37}$ The notation is borrowed from [94] but slightly modified. Notice that our definition of $l$ differs from that in [94]. In our notation, fluctuations with the same $l$ have the same mass.
} 


\subsubsection{Finite baryon number density}

In this section we discuss how to generalize gauge/gravity dualities derived from D3/Dp intersections to describe plasmas with finite density/chemical potential for the $U(1)$ baryon number.

Recall that the $U\left(N_{f}\right)$ gauge invariance of the coincident Dp-branes is dual to the $U\left(N_{f}\right)$ symmetry of the mass-degenerate flavor fields in the SYM theory. We identify the $U(1) \subset$ $U\left(N_{f}\right)$ subgroup as baryon number, $U(1)_{B}$. The D7-brane worldvolume Abelian gauge field $A_{\mu}$ is dual to the SYM $U(1)_{B}$ current $J^{\mu}$. Hence to introduce a finite $U(1)_{B}$ density in the SYM theory, we must introduce a non-zero worldvolume gauge field $A_{t}$.

As in the case of the worldvolume scalar $\theta(u)$, we use symmetry arguments to constrain the dependence on the worldvolume fields. Preserving rotational and time-translation invariance, $A_{t}$ should not depend on the field theory coordinates. Moreover, it should also not depend on coordinates of the sphere $S^{Q}$. Therefore the remaining option for $A_{t}$ is to depend on the radial direction of $A d S$ spacetime, i.e. $A_{t}=A_{t}(u)$. Since we work in a gauge with $A_{u}=0$, the only non-vanishing component of the field strength tensor is $F_{u t}=\partial_{u} A_{t}(u)$. This may be interpreted as an electric field on the Dp-brane pointing in the radial direction of AdS spacetime.

As in the previous section 2.5.2, we now have to solve the equations of motions for the action (2.5.97). For simplicity, we consider a massless embedding of the flavor branes corresponding to $\theta(u)=0$.

The action (2.5.97) in the coordinates (2.4.80) with trivial embedding $\theta(u)=0$ reads

$$
S_{D p}=-\mathcal{N} \int d u \frac{1}{u^{P}} \sqrt{1+\left(2 \pi \alpha^{\prime}\right)^{2} u^{4} A_{t}^{\prime}(u)^{2}} .
$$

Since the action does not explicitly depend on $A_{t}(u)$, the equations of motion can be written in the form

$$
\frac{\partial \mathcal{L}}{\partial A_{t}^{\prime}(u)}=-\mathcal{N} u^{4-P}\left(2 \pi \alpha^{\prime}\right)^{2} \frac{A_{t}^{\prime}(u)}{\sqrt{1+\left(2 \pi \alpha^{\prime}\right)^{2} u^{4} A_{t}^{\prime}(u)^{2}}}=\text { const. . }
$$

This constant of motion can be identified with the baryon number density $\left\langle J^{t}\right\rangle$ on the field theory side by 38

$$
\left\langle J^{\mu}\right\rangle=-\frac{\partial \mathcal{L}}{\partial A_{\mu}^{\prime}(u)} .
$$

Let us now expand the equation (2.5.104) near the boundary $u \rightarrow 0$. We obtain $A_{t}^{\prime}(u) u^{4-P}=$ const. and therefore

$$
A_{t}(u)=\mu-d_{t} u^{P-3}+\ldots,
$$

where ... represent terms that decay faster than $u^{P-3}$ as $u \rightarrow 0$. Here, the constant $\mu$ is the chemical potentia 39 of the field theory side and $d_{t}$ is a constant which is related to

\footnotetext{
${ }^{38}$ Since we have to renormalize (due to the infinite volume of AdS space), the precise expression for $\left\langle J^{\mu}\right\rangle$ is

$$
\left\langle J^{\mu}\right\rangle=\lim _{\epsilon \rightarrow 0}\left(\frac{1}{\epsilon^{4}} \frac{1}{\sqrt{-\gamma}} \frac{\delta S_{r e g}}{\delta A_{\mu}(\epsilon)}\right),
$$

where $\gamma$ is the determinant of the induced metric on the $u=\epsilon$ hypersurface and $S_{\text {reg }}$ denotes the regulated action $S_{r e g}=S_{D p}+S_{C T}$.

${ }^{39}$ To be precise, $\mu$ is the chemical potential (associated with the baryon number) of the quarks. The chemical potential of the baryons, denoted by $\mu_{b}$ is given by $\mu_{b}=N_{c} \mu$.
} 
$\left\langle J_{t}\right\rangle$ by

$$
\left\langle J^{t}\right\rangle=\mathcal{N}\left(2 \pi \alpha^{\prime}\right)^{2} d_{t},
$$

where $\mathcal{N}$ is the constant (2.5.98).

In order to relate $d_{t}$ and $\mu$ or, equivalently, density $\left\langle J^{t}\right\rangle$ and the chemical potential $\mu$, we have to impose a condition for $A_{t}(u)$ on the horizon which were discussed in [115] (see also appendix A of [116]): since the Killing vector corresponding to time translations becomes degenerate at the horizon, we have to impose

$$
A_{t}\left(u_{h}\right)=0
$$

such that the gauge field remains well-defined as a one-form at the horizon.

Let us discuss now what happens if we allow for massive flavor degrees of freedom at finite density. In this case we have to solve the equations of motion for $\theta(u)$ and for $A_{t}(u)$. But as we will see in the next paragraph, not every solution is also meaningful.

As argued in [115], for finite density, i.e. when $A_{t}(u)$ is a non-trivial function of $u$, only black hole embeddings are allowed, for a simple physical reason. With nonzero $A_{t}(u)$, the Dp-brane has a worldvolume electric field pointing in the $u$ direction, $F_{t u}$. What source produces the electric field? The simplest possible source is a density $\left\langle J^{t}\right\rangle$ of strings ending on the Dp-brane. Such a picture is nicely consistent with the field theory picture of a $U(1)_{B}$ density $\left\langle J^{t}\right\rangle$. A straightforward analysis then shows that the force the strings exert on the Dp-brane is greater than the tension of the Dp-brane [115]. We thus expect the strings to pull the Dp-brane into the black hole, producing a Dp-brane black hole embedding with electric field lines in the $u$ direction.

\subsubsection{Phase Transitions at finite baryon density}

Next, we consider phase transitions in the D3/Dp systems which arise when we consider a finite baryon number density. We specialize to the case of the D3/D7 system at finite temperature. The flavor fields have mass $m$. Moreover, we also consider a finite $U(1)_{B}$ baryon density. Therefore we have three independent parameters in our system: the temperature $T$, the chemical potential $\mu$ and the mass of the flavor fields $m$. Since our system is conformal, the phase diagram depends only on two ratios, e.g. $m / T$ and $\mu / m$.

In order to determine the phase diagram (and possibly phase transitions), we need a thermodynamical potential. Since we work at fixed chemical potential $\mu$, i.e. in the grand canonical ensemble 40 we calculate the grand potential $\Omega$. The grand potential is proportional to the Euclidean renormalized on-shell action of the probe Dp-branes, ${ }^{41}$

$$
\Omega_{f l .}(T, m, \mu)=-T \ln \mathcal{Z}_{f l .}=T S_{D p, r e n+o n s h e l l}^{E} .
$$

\footnotetext{
${ }^{40}$ We can also consider the canonical potential in which the baryon number density $\left\langle J_{t}\right\rangle$ is kept fixed. The canonical potential is given by the Legendre transformed on-shell action of probe Dp-branes.

${ }^{41}$ To be precise, we should use the action of the whole system, i.e. of type IIB supergravity and of the probe Dp-brane. Note that the type IIB supergravity action is not sensitive to the parameters since we neglect the backreaction of the gauge fields. Therefore we can calculate the contribution of the flavor fields to the thermodynamic potentials. For example the grand potential for the whole system is
}

$$
\Omega(T, m, \mu)=\Omega_{\mathcal{N}=4 S Y M}(T)+\Omega_{f l .}(T, m, \mu) .
$$




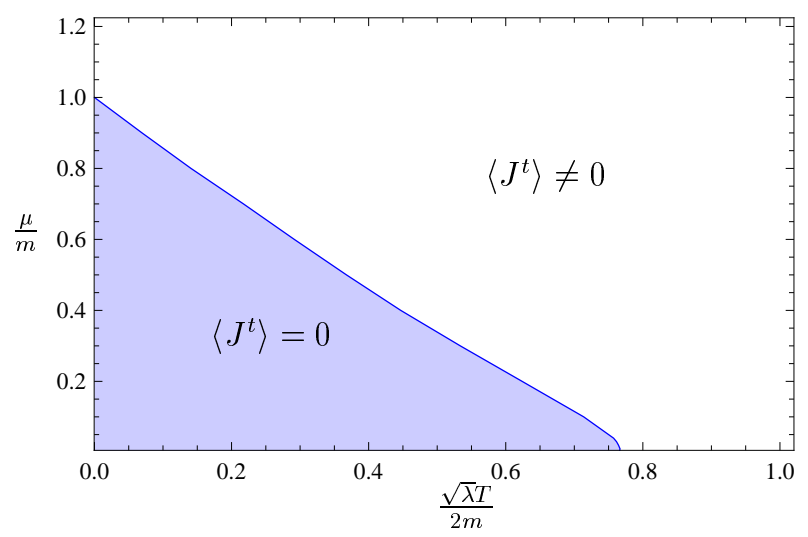

Figure 2.2: The phase diagram for the D3/D7 system in the $\mu / m-T / m$ plane. The blue line is a phase transition from a zero density phase $\left(\left\langle J^{t}\right\rangle=0\right)$ to a finite density state $\left(\left\langle J^{t}\right\rangle \neq 0\right)$.

The phase diagram of the system is shown in figure 2.2. In the blue shadowed region of the phase diagram, the baryon number density $\left\langle J^{t}\right\rangle$ is zero, whereas in the white area, the baryon number density is non-zero.

There is a phase transition between the areas with zero and finite baryon number density $\left\langle J^{t}\right\rangle$. For $\left\langle J^{t}\right\rangle \neq 0$, we only have black hole embeddings, whereas for $\left\langle J^{t}\right\rangle=0$ the Minkowski embeddings are preferred. Note that at the bottom of the separation line between the phases with $\left\langle J^{t}\right\rangle=0$ and $\left\langle J^{t}\right\rangle \neq 0$ there exists a multi-valued region. This multi-valued region is not resolved here. For more details, see the original paper [117].

An interesting question is the order of the phase transition. For small values of the chemical potential (and in particular for vanishing chemical potential) the phase transition is first order $[104,107-112]$. However, above a critical value of the chemical potential, the phase transition is third-order [118]. In [118], it is argued that the third order phase transition is driven by the condensation of worldsheet instantons. The third order and first order phase transitions are separated by a tricritical point. When the chemical potential equals the mass of the quarks, i.e. for $\mu / m=1$, the phase transition is second order [119].

It is also possible to study the phases in the presence of non-vanishing electric and magnetic fields. For more details see [120-123]. In particular something interesting happens, if a finite baryon density of fundamental matter is exposed to an external magnetic field $[124,125]$. There exists an isolated critical point in the phase diagram of the D3/D7 model at zero temperature depending on the magnetic field $B$, the bare mass $m$ of the quarks and the finite density $\left\langle J^{t}\right\rangle$. The quantum phase transition is second order with mean field exponents.

As shown in figure 2.3, the quantum phase transition occurs for zero mass of the flavor fields. The phase transition arises due to a competition between finite density and magnetic fields. The order parameter of the transition is $\left\langle\mathcal{O}_{m}\right\rangle$, i.e. the chiral condensate. It is wellknown from the field theory point of view that a magnetic field at zero density triggers chiral symmetry breaking, i.e. $\left\langle\mathcal{O}_{m}\right\rangle \neq 0$. In contrast, at finite density but no magnetic field, the vacuum is chiral symmetric, $\left\langle\mathcal{O}_{m}\right\rangle=0$.

We can generalize these results in various ways. For example, we can consider other D3/Dp systems. For condensed matter applications also codimension one and two sys- 


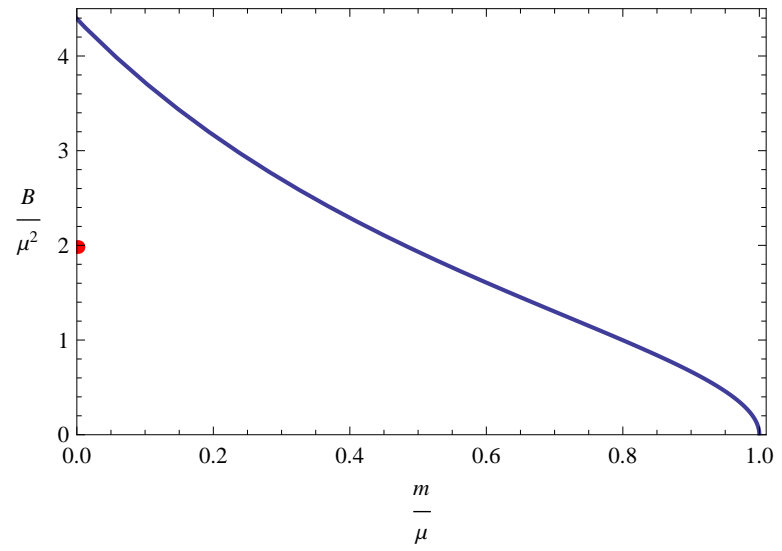

Figure 2.3: The phase diagram for the D3/D7 system in the $m / \mu-B / \mu^{2}$ plane at zero temperature, taken from [124]. The red dot indicates the critical point mentioned in the text. The blue line indicates the phase transition to a zero density phase, $\left\langle J^{t}\right\rangle=0$, located which is located in the upper right hand side of the diagram. The numerics of [124] suggest that the phase transition is at least second order (or higher) away from $B=0$.

tems are relevant. One example is the D3/D5 system which is studied in this thesis. The thermodynamics and hydrodynamics were studied in [126-132]. It is interesting to investigate this system at nonzero density and magnetic field [133,134]. Also in this configuration a quantum critical point exists which is the first holographic example of a quantum Berenzinskii-Kosterlitz-Thouless (BKT) phase transition in two spatial dimensions.

Another possibility is to consider a finite isospin chemical potential instead of a baryon chemical potential. Although a part of the phase diagram is similar to figure 2.2, we will see in chapter 4 that there is an unstable phase. This instability leads to condensation of mesons which breaks the remaining $U(1)$ flavor symmetry spontaneously.

\subsection{Other examples of gauge/gravity dualities}

So far, we only considered one example of the AdS/CFT correspondence which can be derived from the near-horizon limit of coincident D3-branes in flat Minkowski spacetime. The dual field theory is given by four-dimensional $\mathcal{N}=4$ supersymmetric Yang-Mills theory with gauge group $S U\left(N_{c}\right)$. Similar gauge/gravity dualities may also be derived [135] by taking the corresponding limit for Dp-branes with $p<6.42$ The dual field theory is maximally supersymmetric Yang-Mills in a $(\mathrm{p}+1)$-dimensional Minkowski spacetime. However, note that only in the case $p=3$, i.e. for D3-branes, the dual field theory is conformal. For $p \neq 3$ the coupling constant $g_{Y M}$ of the dual gauge theory is dimensionful. Moreover, the effective coupling constant for $N_{c}$ Dp-branes satisfies a power-law running

$$
g_{\text {eff }}^{2}=g_{Y M}^{2} N_{c}\left(r / \alpha^{\prime}\right)^{p-3},
$$

where $r$ is the radial coordinate transverse to the $D p$-brane which can be interpreted as the energy scale on the field theory side. In the bulk, the absence of conformality on the field

\footnotetext{
${ }^{42}$ For Dp-branes, with $p \geq 6$, there exists no decoupling limit between the field theory and the bulk as shown in [135].
} 
theory side is manifest in the variation of the string coupling constant $g_{s}$ (or equivalently the dilaton field) and the spacetime curvature in the radial direction. In particular the spacetime is not asymptotically AdS. We can also add fundamental degrees of freedom to the super Yang-Mills theory by considering probe branes in the background generated by $D p$-branes. The mesons in those backgrounds were studied in [113].

An very important example for a top-down approach towards a dual description of QCD was discussed by Sakai and Sugimoto in [136]. The background is generated by D4branes wrapped on a circle $S^{1}$. The boundary conditions implemented on the circle break supersymmetry. Moreover, Sakai and Sugimoto added D8- and anti-D8-branes giving rise to left- and right-handed quarks. Many interesting features of QCD can also be found in this holographic model.

In this thesis, however, we are interested in scale-invariant field theories since we aim to describe condensed matter field theories near quantum critical points. Therefore the SakaiSugimoto model and the near-horizon limit of Dp-branes (with $p \neq 3$ ) play no role in this thesis. Besides D3-branes also solitonic solutions of M-theory are interesting. In particular the near-horizon limit of multiple M2-branes and M5-branes provide holographic duals for $2+1$ and 5+1-dimensional supersymmetric conformal field theories.

The low-energy description of multiple M2-branes at a $\mathbb{C}^{4} / \mathbb{Z}_{k}$ singularity is a $(2+1)$ dimensional $\mathcal{N}=6$ supersymmetric $U\left(N_{c}\right) \times U\left(N_{c}\right)$ Chern-Simons matter theory, the ABJM theory [137]. In the large- $N_{c}$ limit, its holographic dual is supergravity in $A d S_{4} \times$ $S^{7} / \mathbb{Z}_{k}$. In chapter 7 we study various ways to add fields that transform in the fundamental representation of the gauge groups, i.e. flavor fields, to the ABJM theory. We work in a probe limit and perform analyses in both the supergravity and field theory descriptions. In the supergravity description we find a large class of supersymmetric embeddings of probe flavor branes. In the field theory description, we present a general method to determine the couplings of the flavor fields to the fields of the ABJM theory. We then study two different examples 43 in detail: codimension-zero $\mathcal{N}=3$ supersymmetric flavor, described in supergravity by Kaluza-Klein monopoles or D6-branes and codimension-one $\mathcal{N}=(0,6)$ supersymmetric chiral flavor, described by D8-branes. Finally, we discuss special physical equivalences between brane embeddings in M-theory, and their interpretation in the field theory description.

Finally, we can also generalize the AdS/CFT correspondence to scale-invariant but nonrelativistic field theories. These theories are relevant for the description of a quantum critical point with a dynamical scaling exponent $z \neq 1$. In chapter 6 we explain how to obtain the dual gravity description for such field theories. There we discuss how to embed D7-branes into a background with Schrödinger symmetry and how to calculate conductivities for flavor fields.

\footnotetext{
${ }^{43}$ In the paper [6] you will also find two other examples in full detail: codimension-one $\mathcal{N}=(3,3)$ supersymmetric non-chiral flavor, described by M5/D4-branes and codimension-two $\mathcal{N}=4$ supersymmetric flavor, described by M2/D2-branes.
} 



\section{Part II}

\section{Applications to Condensed Matter Systems}





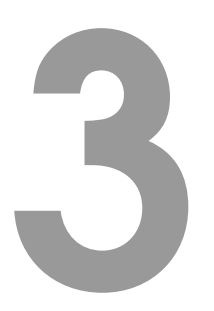

\section{Response to external fields}

In the preceding chapter we reviewed the AdS/CFT correspondence and its generalizations to finite temperature and density. In particular in section 2.5.4 we reviewed the thermodynamics of probe branes, i.e. equilibrium properties of the dual field theory.

Let us consider now small space- and time-dependent perturbations about equilibrium. This is the domain where we can apply linear response theory. Within the linear response approximation, it is possible to investigate interesting physical processes, such as transport and spectroscopy, which are accessible in experiments.

We first review linear response theory in section 3.1. In particular we determine conductivities of alternating currents. In section 3.2 the holographic calculation of the retarded Green's function is explained. In particular we consider the conductivity tensor for alternating currents (section 3.2.2) and the linear response of fermions (section 3.2.3). Moreover, we present simple prescription in section 3.2 .4 how to compute the matrix-valued retarded two-point function from bulk solutions for coupled fermions.

In section 3.3 we calculate the direct current conductivity tensor for flavor fields in arbitrary constant electric and magnetic fields. By using a peculiar property of the DiracBorn-Infeld action, we are able to calculate the conductivity tensor to all orders in the electric and magnetic fields.

\subsection{Review: Linear response \& retarded Green's functions}

In this section we consider the linear response of the system at equilibrium to weak spaceand time-dependent perturbations. In the following we denote the weak external fields by $\phi^{I}(x)$ where $x$ is a short-hand notation for the time $t$ and the space $\vec{x}$. The corresponding operators to which the external fields couple are called $\mathcal{O}_{I}(x)$. The interaction between the external fields $\phi^{I}(x)$ and the corresponding operators $\mathcal{O}_{I}(x)$ are taken into account by 
adding the term 1

$$
\delta \hat{H}=-\int d^{d} \vec{x} \phi^{I}(t, \vec{x}) \mathcal{O}_{I}(t, \vec{x})
$$

to the Hamiltonian $\hat{H}$ of the system. Using standard methods of time-dependent perturbation theory, one can show that a weak external perturbation causes a change in the vacuum expectation value of $\mathcal{O}_{I}(x)$ of the form

$$
\delta\left\langle\mathcal{O}_{I}(x)\right\rangle=\int d^{d+1} x^{\prime} \mathcal{G}_{I J}^{R}\left(x, x^{\prime}\right) \phi^{J}\left(x^{\prime}\right)+\ldots,
$$

where the dots indicate that we have omitted terms which are higher order in $\phi_{J}$. Here, $\mathcal{G}_{I J}^{R}\left(x, x^{\prime}\right)$ is the retarded Green's function given by

$$
\mathcal{G}_{I J}^{R}\left(x, x^{\prime}\right)=i \theta\left(t-t^{\prime}\right)\left\langle\left\{\hat{\mathcal{O}}_{I}(x), \hat{\mathcal{O}}_{J}\left(x^{\prime}\right)\right\}_{ \pm}\right\rangle
$$

The bracket $\{\cdot, \cdot\}_{ \pm}$denotes a commutator in the case of bosonic fields and a anticommutator in the case of fermionic fields.

If the system is invariant under spacetime translations, the Green's function $\mathcal{G}_{I J}^{R}\left(x, x^{\prime}\right)$ depends only on the difference $x-x^{\prime}$, and not separately on $x$ and $x^{\prime}$. Moreover, in translational invariant systems it can be convenient to decompose the external fields in Fourier components. Using the Fourier transformed retarded Green's function

$$
\tilde{\mathcal{G}}_{I J}^{R}(k)=\int d^{d+1} x \mathcal{G}_{I J}^{R}(x, 0) e^{-i k \cdot x},
$$

we can rewrite equation $(\overline{3.1 .3})$ in the form

$$
\delta\left\langle\mathcal{O}_{I}(k)\right\rangle=\tilde{\mathcal{G}}_{I J}^{R}(k) \phi^{J}(k)+\ldots
$$

In equation (3.1.3) we see explicitly that the retarded Green's function is causal since $\delta\left\langle\mathcal{O}_{I}(x)\right\rangle$ is only influenced by sources $\phi^{I}\left(t^{\prime}, \vec{x}\right)$ with $t^{\prime}<t$. Causality implies that the momentum-space Green's function $\tilde{\mathcal{G}}_{I J}^{R}(\omega, \vec{k})$ is analytic in $\omega$ for $\operatorname{Im} \omega>0$. Assume there is a pole at $\omega_{\star}$ in the upper half plane of complex frequencies. This leads to an exponentially growing mode

$$
\mathcal{G}_{I J}^{R}(t, \vec{k}) \sim e^{-i w_{\star} t} \sim e^{\left|\operatorname{Im} \omega_{\star}\right| t},
$$

which indicates that the vacuum (in which the Green's function has been computed) is instable against perturbations.

In the retarded Green's function important information is encoded. For example, the spectral function $\mathcal{R}_{I J}$ is defined as the anti-Hermitian part of the retarded Green's function,

$$
\mathcal{R}_{I J}(\omega, \vec{q}) \equiv i\left(\tilde{\mathcal{G}}_{I J}^{R}(\omega, \vec{q})-\tilde{\mathcal{G}}_{I J}^{R \dagger}(\omega, \vec{q})\right) .
$$

The external sources $\phi^{I}(t, \vec{x})$ do work on the system. The time-averaged rate of change of the total energy, $\frac{\overline{d W}}{d t}$, in leading order of the (time-varying) external sources is measured by the spectral function $\mathcal{R}_{I J}$. To be precise, the dissipation of the system is captured by the spectral function times $i \omega$ where $\omega$ is the frequency of the external source.

\footnotetext{
${ }^{1}$ Note that $\delta \hat{H}$ is not the variation of the Hamilton operator $\hat{H}$.
} 
Stability requires the eigenvalues of $\mathcal{R}_{I J}$, and hence both the diagonal elements of $\mathcal{R}_{I J}$ and the spectral measure, i.e. the sum of the eigenvalues, to be strictly non-negative. Otherwise, if we perturb the medium with one of the operators appearing in the spectral function, the resulting excitation would experience negative energy dissipation into the medium, i.e. the excitation would extract energy from the medium, signaling an instability. The eigenvalues of the spectral function are a direct measure of the states of the theory that have an overlap with the relevant operators. The off-diagonal elements of $\mathcal{R}_{I J}$, however, need not obey any positivity requirement.

Note that in the case of only one source (or more sources which do not couple to each other), the spectral function $\mathcal{R}_{I J}$ can be written in the simple form

$$
\mathcal{R}(\omega, \vec{q})=-2 \operatorname{Im} \tilde{\mathcal{G}}^{R}(\omega, \vec{q}) .
$$

\subsubsection{AC-conductivities}

Let us rephrase Ohm's Law in this language. Ohm's Law states that for a spatially constant electric field $\vec{E}(\omega)$, which may oscillate in time with frequency $\omega$, the spatial part of the charge current response, $J^{i}(\omega)$, is determined by

$$
\left\langle J_{i}(\omega)\right\rangle=\sigma_{i j}(\omega) E^{j}(\omega) .
$$

$\sigma^{i j}(\omega)$ is the conductivity tensor for alternating currents. In the language of the previous paragraphs, we consider an external vector potential $A^{\mu}(x)$ (playing the role of $\phi^{J}(x)$ ) and a conserved current $J_{\mu}(x)$ which corresponds to the operator $\mathcal{O}_{J}$. In the gauge $A_{t}=0$, the electric field $E_{k}$ is given by $E_{k}=-\partial_{t} A_{k}$. Fourier decomposing $A_{k} \sim e^{-i w t}$, we obtain for the electric field $E_{k}=i \omega A_{k}$. Comparing Ohm's Law (3.1.9) to the definition of the retarded Green's function (3.1.5), we obtain a simple expression for the conductivity tensor of alternating currents,

$$
\sigma_{i j}(\omega)=\frac{\tilde{\mathcal{G}}_{i j}^{R}(\omega, \overrightarrow{0})}{i \omega} .
$$

Here, $\tilde{\mathcal{G}}_{i j}^{R}$ are the components of the retarded correlator of currents in Fourier space. Note that the spatial momentum $\vec{q}$ is set to zero.

\subsection{Holographic Calculation: Linear response}

In the last section we saw that retarded Green's functions play an important role in linear response theory. In this section we first give a general recipe how to calculate the retarded Green's function. We apply this recipe to two examples: correlators of currents, which are important for the calculation of the conductivity tensor for alternating currents, and fermionic correlators.

\subsubsection{The retarded Greens function: A general recipe}

In principle some real-time Green's functions can be deduced from the Euclidean Green's function by analytical continuation. This is the case for the advanced and retarded realtime Green's functions. However, it is very difficult to calculate the quantity of interest. 
In particular we are interested in the hydrodynamic limit (small $\omega$ and small $\vec{q}$ of the retarded Green's function $\mathcal{G}^{R}(\omega, \vec{q})$. This limit of real-time correlators is very difficult to extract from Euclidean correlators since we need to perform an analytical continuation from a discrete set of points in Euclidean frequencies, the so-called Matsubara frequencies $\omega=2 \pi i n T, n \in \mathbb{N}$. The smallest frequency is therefore $|\omega|=2 \pi T$.

Therefore we need a real-time AdS/CFT prescription which allows us to directly compute the real-time retarded Green's function of the operator $\mathcal{O}$. $\phi$ is the bulk field dual to the operator $\mathcal{O}$. Let us briefly review the recipe presented in [138]:

(i) Find the part of the action which is quadratic in $\phi$.

(ii) Linearize the equations of motion for $\phi$ and solve them in Fourier space $(\omega, \vec{q})$. It is convenient to split the solution $\phi(u, \omega, \vec{q})$ into a function $\phi_{0}(\omega, \vec{q})$, depending on the frequency $\omega$ and the momentum $\vec{q}$, and a function $f_{(\omega, \vec{q})}(u)$,

$$
\phi(u, \omega, \vec{q})=\phi_{0}(\omega, \vec{q}) \cdot f_{(\omega, \vec{q})}(u) .
$$

At the boundary, horizon, $f_{(\omega, \vec{q})}$ is one. At the horizon $f_{(\omega, \vec{q})}$ has to satisfy in-falling wave boundary condition, i.e. $f_{(\omega, \vec{q})}(u) \sim\left(u_{h}-u\right)^{-i \omega \alpha}$, where $\alpha$ is a real positive number.

(iii) The action, evaluated for the solution of the form (3.2.11), reduces to a surface integral

$$
S=\left.\int \frac{d \omega d \vec{q}}{(2 \pi)^{4}} \phi_{0}(-\omega,-\vec{q}) \mathcal{F}(\omega, \vec{q}, u) \phi_{0}(\omega, \vec{q})\right|_{u=0} ^{u=u_{h}} .
$$

Remember that the conformal boundary in the $\mathrm{u}$-coordinates is located at $u_{b}=0$.

(iv) The retarded Green's function is given by

$$
\tilde{\mathcal{G}}^{R}(\omega, \vec{q})=-2 \mathcal{F}(\omega, \vec{q}, u=0) .
$$

A more advanced analysis is performed in [139], using the Schwinger-Keldysh formalism which reduces to the same result.

Let us illustrate this recipe by three examples. First we consider AC conductivities which can be calculated using current-current correlators. In the second example the recipe is extended to fermionic correlators. Finally, we generalize the calculation of the retarded Green's function to operators which mix in the renormalization group flow. In this case, the retarded Green's function is promoted to a matrix with entries $\tilde{\mathcal{G}}_{I J}^{R}(\omega, \vec{q})$.

\subsubsection{Example I: AC conductivities}

In this section we review the recipe how to determine the $\mathrm{AC}$ conductivity associated with charge carriers. Therefore we have to apply the recipe provided in the previous section to retarded Green's functions of current-current correlators. In the dual gravity picture, the conserved current $J^{\mu}$ couples to gauge field $A_{\mu}$.

In particular we consider $N_{f}$ probe Dp-branes in $A d S_{5} \times S^{5}$. The induced metric on the world-volume of the probe Dp-brane is given by equation (2.5.94). For simplicity, we 
set the world-volume scalar $\theta(u)$ to zero, i.e. we only study massless flavor degrees. We derive the Green's function for the conserved $U(1)$ baryon current $J^{\mu}$, which is dual to the diagonal $U(1)$ gauge field on the world-volume of $N_{f}$ Dp-branes. We may choose a gauge in which $A_{u}=0$. Moreover, the gauge field components on the sphere vanish. Therefore only the Dirac-Born-Infeld part of the Dp-brane action is relevant for our discussion, which is given by

$$
S_{D p}=-\mathcal{N} \int d u d t d \vec{x} \sqrt{-\operatorname{det}\left(\mathcal{P}[\mathrm{g}]_{\mathrm{ab}}+(2 \pi \alpha)^{\prime 2} \mathrm{~F}_{\mathrm{ab}}\right)}
$$

Note that we have not integrated over the spacetime coordinates $t$ and $\vec{x}$. In comparison to equation (2.5.97), which is an action density, equation (3.2.14) describes an action (since we have not divided by the spacetime volume).

In order to introduce a finite density of charge carriers, we allow for a non-trivial gauge field $A_{t}(u)$ as discussed in section 2.5.3 and solve the equations of motion. To determine the retarded Green's function $\mathcal{G}^{R}$, we now investigate small fluctuations of the background field configuration, given by $A_{t}(r)$. Therefore we add small fluctuations $\tilde{A}$ to the gauge field of the background, $\bar{A}$. Since only $\bar{A}_{t}$ is non-vanishing, we can write

$$
A_{\mu}(t, \vec{x}, u)=\delta_{\mu}^{t} \bar{A}_{t}(u)+\tilde{A}_{\mu}(t, \vec{x}, u) .
$$

The field strength tensor of the fluctuations and the background add up, i.e. $F=\bar{F}+$ $\tilde{F}$, where $\bar{F}$ is the field strength of the background and $\tilde{F}$ is the field strength of the fluctuations. In the next step we derive the linearized equations of motion for $\tilde{A}$ in the background of $\bar{A}$. Transforming the fluctuations to momentum space,

$$
\tilde{A}_{\mu}(t, \vec{x}, u)=\int \frac{d \omega d \vec{q}}{(2 \pi)^{4}} e^{-i \omega t+i \vec{q} \cdot \vec{x}} \tilde{A}_{\mu}(\omega, \vec{q}, u)
$$

the solution of the linearized equations of motion can be written in the form

$$
\tilde{A}_{\mu}(\omega, \vec{q}, u)=f_{(\omega, \vec{q})}(u) \tilde{A}_{\mu}^{(0)}(\omega, \vec{q}) .
$$

Note that $f_{\omega, \vec{q}}$ is only a function of $u$, the radial coordinate of AdS. The index indicates that the precise form of the function $f$ depends on the parameters $\omega$ and $\vec{q} . \tilde{A}_{\mu}^{(0)}(\omega, \vec{q})$ does not depend on the radial direction of AdS space. In fact, later we will vary two times with respect to $\tilde{A}_{\mu}^{(0)}(\omega, \vec{q})$, in order to obtain the retarded Green's function $\mathcal{G}^{R}(\omega, \vec{q})$. Since we are interested in the $\mathrm{AC}$ conductivity, which is related to the retarded correlator of the current for zero spatial momentum, we can set $\vec{q}$ to zero.

The function $f_{(\omega, \vec{q})}$ asymptotes $f_{(\omega, \vec{q})} \sim\left(u_{h}-u\right)^{ \pm i \omega \alpha}$ near the horizon $u \rightarrow u_{h}$. Here, $\alpha$ is a positive real number. These two solutions correspond to infalling and outgoing waves at the horizon. In order to determine the retarded Green's function we have to use the infalling wave-boundary conditions at the horizon, as reviewed in section 3.2.1. Choosing outgoingwave boundary conditions corresponds to computing the advanced Green's function.

After imposing the correct boundary conditions and solving the linearized equations of motion, we insert the solution of the fluctuations $\tilde{A}_{\mu}$ into the action (3.2.14). If we rewrite the action in the form (3.2.12), we can easily read of the retarded Green's function given by (3.2.13). Finally, the AC-conductivity is then given by (3.1.10). 


\subsubsection{Example II: Fermionic response}

In this section we discuss how to obtain the Green's function for massive fermions. The general case for arbitrary values of the mass $m$ is complicated. For simplicity, we therefore restrict our discussion to values of $m$ that are positive and not half-integer. Our arguments are easy to generalize to any other value of $m$.

In this section we will also restrict to four- and five-dimensional AdS spaces, which we will collectively denote as $A d S_{d+1}$ with $d=3,4$, primarily for pedagogical reasons: in these cases the bulk Dirac spinor has four complex components, and we can write explicit $4 \times 4$ bulk Dirac $\Gamma$-matrices. Additionally, we note that $A d S_{d+1}$ spaces with $d \leq 4$ are the cases most relevant for condensed matter applications (as opposed to, say, $A d S_{7}$ ). The generalization to other dimensions is straightforward.

We use an explicit basis for the $\Gamma$-matrices. It is convenient to choose a basis in which all the $\Gamma$-matrices are Hermitian,

$$
\gamma^{u}=\left(\begin{array}{cc}
-\sigma_{3} & 0 \\
0 & -\sigma_{3}
\end{array}\right), \quad \gamma^{t}=\left(\begin{array}{cc}
\sigma_{1} & 0 \\
0 & \sigma_{1}
\end{array}\right), \quad \gamma^{x}=\left(\begin{array}{cc}
-\sigma_{2} & 0 \\
0 & \sigma_{2}
\end{array}\right)
$$

where $\sigma_{1}, \sigma_{2}$ and $\sigma_{3}$ are the usual Pauli matrices,

$$
\sigma_{1}=\left(\begin{array}{cc}
0 & 1 \\
1 & 0
\end{array}\right), \quad \sigma_{2}=\left(\begin{array}{cc}
0 & -i \\
i & 0
\end{array}\right), \quad \sigma_{3}=\left(\begin{array}{cc}
1 & 0 \\
0 & -1
\end{array}\right) .
$$

Next we define two sets of projectors. The first set is

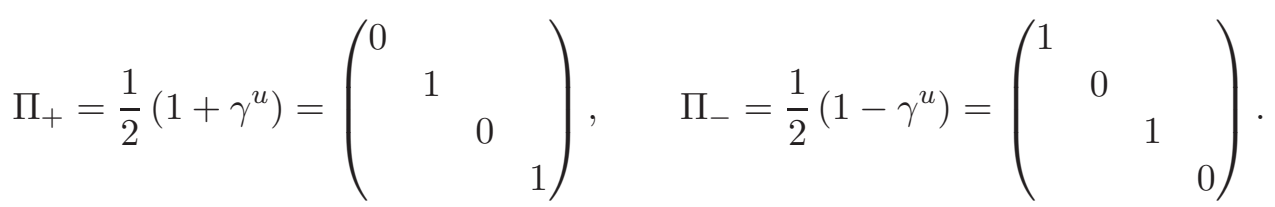

We use these to define $\Psi_{ \pm}=\frac{1}{2}\left(1 \pm \gamma^{u}\right) \Psi$ so that $\gamma^{u} \Psi_{ \pm}= \pm \Psi_{ \pm}$. This set has been already used in section 2.3 . The second set of projectors is very useful for the numerical calculation presented in chapter 5 ,

$$
\Pi_{1}=\frac{1}{2}\left(1+i \gamma^{u} \gamma^{t} \gamma^{x}\right)=\left(\begin{array}{cccc}
0 & & & \\
& 0 & & \\
& & 1 & \\
& & & 1
\end{array}\right), \quad \Pi_{2}=\frac{1}{2}\left(1-i \gamma^{u} \gamma^{t} \gamma^{x}\right)=\left(\begin{array}{cccc}
1 & & \\
& 1 & & \\
& & 0 & \\
& & 0
\end{array}\right)
$$

To make converting between $\Psi_{ \pm}$and $\Psi_{1,2}$ easy, we explicitly write $\Psi$ first as $\Psi_{+}+\Psi_{-}$and then as $\Psi_{1}+\Psi_{2}$,

$$
\Psi=\left(\begin{array}{c}
0 \\
\Psi_{+u} \\
0 \\
\Psi_{+d}
\end{array}\right)+\left(\begin{array}{c}
\Psi_{-u} \\
0 \\
\Psi_{-d} \\
0
\end{array}\right)=\left(\begin{array}{c}
0 \\
0 \\
\Psi_{1 u} \\
\Psi_{1 d}
\end{array}\right)+\left(\begin{array}{c}
\Psi_{2 u} \\
\Psi_{2 d} \\
0 \\
0
\end{array}\right)
$$

where the subscripts $u$ and $d$ indicate the up and down components of the effectively two-component $\Psi_{ \pm}$and $\Psi_{1,2}$. Identifications such as $\Psi_{+u}=\Psi_{2 d}$ are then obvious. 
We have a choice of whether to use $\Psi_{ \pm}$or $\Psi_{1,2}$, although of course, we can easily translate between the two options using equation (3.2.22). We choose whatever is most convenient for a given question.

For example, the projectors $\Pi_{1,2}$ commute with the operator in equation (2.3.32), which tells us that, for a free fermion, the equations for $\Psi_{1,2}$ decouple. That makes $\Psi_{1,2}$ especially attractive for numerical analysis, hence we employ them in sections 5.2 and 5.3 . Explicitly, the equations for $\Psi_{1,2}$ are

$$
\begin{aligned}
& {\left[u \partial_{u}-\frac{d}{2}+m \sigma_{3}-k u\right] \Psi_{1}=0} \\
& {\left[u \partial_{u}-\frac{d}{2}+m \sigma_{3}+k u\right] \Psi_{2}=0 .}
\end{aligned}
$$

On the other hand, the asymptotic behavior of $\Psi$ is most succinctly described using $\Psi_{ \pm}$. Therefore we used these projectors in section 2.3. Recall that the leading asymptotic behaviors of $\Psi_{ \pm}$are

$$
\Psi_{ \pm}=c_{ \pm}(k) u^{\frac{d}{2} \pm m}+O\left(u^{\frac{d}{2}+1 \pm m}\right)
$$

where $c_{ \pm}(k)$ are spinors that obey $\Pi_{ \pm} c_{ \pm}(k)= \pm c_{ \pm}(k)$, and which may depend on $k$, as indicated.

As reviewed in section 2.3.4, to compute renormalized correlators of the dual operator $\mathcal{O}$, we take functional derivatives of $S$ with respect to some source. We identify the source for $\mathcal{O}$ as the coefficient of the dominant term in $\Psi$ 's near-boundary expansion, i.e. as the term that grows most quickly as $u \rightarrow 0$. From equation (3.2.25), we see that the dominant term is the $u^{\frac{d}{2}-m}$ term, hence we identify $c_{-}(k)$ as the source for $\mathcal{O}$.

More formally in equation (2.3.73), we have equated the generating functional of the dual field theory with the saddle point of the partition function given by the exponential of minus the action in equation (2.3.27), evaluated on a solution and properly renormalized.

The connected one- and two-point correlators are given by equations (2.3.75) and (2.3.77). In order to calculate the renormalized two-point function, we need a relation between $c_{+}$ and $c_{-}$.

This relation can be obtained from the equations of motion plus some regularity condition in the interior of the spacetime. The equation is linear, hence the relation will be linear: $c_{+}=-\mathcal{G}(k) \gamma^{t} c_{-}$, for some matrix $\mathcal{G}(k)$ which will turn out to be the Euclidean Green's function. We include a factor of $\gamma^{t}$ because, as discussed in [49,51], the Euclidean Green's function is actually $\left\langle\mathcal{O} \mathcal{O}^{\dagger}\right\rangle_{r e n}$, which differs from $\langle\mathcal{O} \overline{\mathcal{O}}\rangle_{r e n}$ by a factor of $\gamma^{t}$. We indeed find

$$
\langle\mathcal{O} \overline{\mathcal{O}}\rangle_{\text {ren }}=\mathcal{G}(k) \gamma^{t}, \quad\left\langle\mathcal{O O}^{\dagger}\right\rangle_{\text {ren }}=\mathcal{G}(k)
$$

In general, we must extract $\mathcal{G}(k) \gamma^{t}$ from a solution by imposing some regularity condition in the bulk of the spacetime (in our coordinates, the $u \rightarrow \infty$ region), which fixes $c_{+}$ in terms of $c_{-}$. We have already calculated the Euclidean Green's function in equation (2.3.79).

Let us switch to $\Psi_{1,2}$ which we will need in chapter 5. We reproduce the formulas used in $[49,51]$. In that case, the equations for $\Psi_{1}$ and $\Psi_{2}$ decouple for a free fermion, hence 
in the Green's function the $\Pi_{1}$ and $\Pi_{2}$ subspaces will not mix. Writing $c_{+}=-\mathcal{G}(k) \gamma^{t} c_{-}$ explicitly, we will have (suppressing the $k$ dependence of $c_{ \pm}(k)$ )

$$
\begin{aligned}
& \left(\begin{array}{c}
0 \\
c_{+u} \\
0 \\
c_{+d}
\end{array}\right)=-\left(\begin{array}{ccc}
\mathcal{G}_{22}(k) \mathbf{1}_{2} & \\
& \mathcal{G}_{11}(k) \mathbf{1}_{2}
\end{array}\right)\left(\begin{array}{cccc}
0 & 1 & & \\
1 & 0 & & \\
& & 0 & 1 \\
& & 1 & 0
\end{array}\right)\left(\begin{array}{c}
c_{-u} \\
0 \\
c_{-d} \\
0
\end{array}\right) \\
& =-\left(\begin{array}{cc}
\mathcal{G}_{22}(k) \mathbf{1}_{2} & \\
& \mathcal{G}_{11}(k) \mathbf{1}_{2}
\end{array}\right)\left(\begin{array}{c}
0 \\
c_{-u} \\
0 \\
c_{-d}
\end{array}\right)
\end{aligned}
$$

where blank entries represent zero, $\mathbf{1}_{2}$ is the $2 \times 2$ identity matrix, and $\mathcal{G}_{11}$ and $\mathcal{G}_{22}$ represent the components of the Green's function in the $\Pi_{1}$ and $\Pi_{2}$ subspaces, respectively. Given a bulk solution for $\Psi$, we obtain the Green's functions simply by reading off the asymptotic values of $c_{+}(k)$ and $c_{-}(k)$ and then constructing

$$
\mathcal{G}_{22}(k)=-\frac{c_{+u}}{c_{-u}}, \quad \mathcal{G}_{11}(k)=-\frac{c_{+d}}{c_{-d}} .
$$

Finally, we review the prescription of [81] to compute the retarded two-point function in the finite-temperature, Lorentzian-signature case. Here the geometry is AdS-Schwarzschild, with a horizon at some position $u_{h}$. To obtain the retarded two-point function, we require that, near the horizon, the bulk solution for $\Psi$ has the form of wave traveling into the horizon (out of the spacetime), i.e. an in-going wave. The asymptotic form for $\Psi$ near the boundary is the same as in equation (3.2.25) (for positive, non-half-integer $m$ ). Following [81], in the regime of linear response, we have

$$
c_{+}(\omega, k)=-i \mathcal{G}^{R}(\omega, k) \gamma^{t} c_{-}(\omega, k),
$$

where $\mathcal{G}^{R}(\omega, k)$ is the retarded Green's function. Notice that here we distinguish the frequency $\omega$ from the momentum $k$, and $\gamma^{t}$ is now anti-Hermitian,

$$
\gamma^{t}=\left(\begin{array}{cc}
i \sigma_{1} & 0 \\
0 & i \sigma_{1}
\end{array}\right)
$$

Equation (3.2.30) is essentially just an analytic continuation from the Euclidean case: $\gamma^{t} \rightarrow i \gamma^{t}$. For a free fermion, we obtain (see also equation (A17) of [51])

$$
\mathcal{G}_{22}^{R}(\omega, k)=\frac{c_{+u}}{c_{-u}}, \quad \mathcal{G}_{11}^{R}(\omega, k)=\frac{c_{+d}}{c_{-d}} .
$$

\subsubsection{Holographic Operator mixing: Coupled Fermions}

We now consider multiple bulk fermions, say $N$ of them, $\Psi_{a}$ with $a=1, \ldots, N$, coupled to one another. The fact that the linearized fluctuation of the $\Psi_{a}$ couple in the bulk is dual to the statement that the fermionic operators in the field theory mix with one another under renormalization group flow.

Here, we will develop a method how to obtain the retarded Green's function in a simple way. Let me emphasize that this section reviews my own work done in collaboration with 
Andy O'Bannon, Johanna Erdmenger and Matthias Kaminski. It is originally published in $[8]$.

In particular, we will work in Lorentzian signature, and finite temperature, so that the bulk geometry is AdS-Schwazrschild, with a horizon at some position $u_{h}$. We consider fermions with quadratic couplings of the form (with implicit summation over $a, b$ )

$$
S=i \int d^{d+1} x \sqrt{g}\left(\bar{\Psi}_{a} \not D \Psi_{a}-\bar{\Psi}_{a} \Lambda_{a b} \Psi_{b}\right)+S_{b d y},
$$

for some matrix $\Lambda_{a b}$ that need not be diagonal in either the $a, b$ indices or the spinor indices ${ }^{2}$

For the following arguments, we do not need to know any details about the equations of motion. We will only exploit one important feature. Using the $\Pi_{ \pm}$projectors, we will always obtain equations similar to equations (2.3.33) and (3.2.24). We will then always be able to write these equations in the form

$$
\nabla_{a b \pm} \Psi_{b \pm}=M_{a c \pm} \Psi_{c \mp}
$$

where $\nabla_{a b \pm}$ is some differential operator, involving in particular $\partial_{u}$, and $M_{a c \pm}$ is a matrix representing the couplings among not only the $\Psi_{a}$, which come from $\Lambda_{a b}$, but also the terms from $\not D \Psi_{a}$ that produce couplings between $\Psi_{a+}$ and $\Psi_{a-}$, for example the terms proportional to the momentum $k$ in equations (2.3.33) and (3.2.24). The key feature is that only the $\Psi_{a \pm}$ are on the left-hand-side, while only the $\Psi_{a \mp}$ are on the right-hand-side.

In practical terms, the total number of complex functions for which we must solve is $4 \times N$, since each $\Psi_{a}$ has four complex components. In other words, we need to decompose the $\Psi_{a}$ not only into $\Psi_{a+}$ and $\Psi_{a-}$, but also into the up and down components, $\Psi_{a+u}, \Psi_{a+d}, \Psi_{a-u}$, and $\Psi_{a-d}$. When convenient, we may sometimes think of equation (3.2.34) as equations describing these $4 \times N$ coupled functions, which we may sometimes refer to as fields.

Clearly, if we solve for all the $\Psi_{a}$, insert the solutions into the bulk action, and take functional derivatives, we obtain field theory retarded Green's functions that are matrices, $\mathcal{G}_{a b}^{R}(\omega, k)$. In principle, we may be able to diagonalize the equations of motion and obtain decoupled equations, in which case the Green's function is diagonal. Given the bulk solutions for the $\Psi_{a}$, we then extract the elements of $\mathcal{G}_{a b}^{R}(\omega, k)$ using equation (3.2.32). In some cases, however, diagonalizing the equations of motion may be prohibitively difficult, i.e. practically impossible. We can always resort to numerics to find solutions, but we will then be forced to compute elements of the un-diagonalized $\mathcal{G}_{a b}^{R}(\omega, k)$. We thus need to know what combinations of the asymptotic values $c_{a+}$ and $c_{a-}$ give an arbitrary element $\mathcal{G}_{a b}^{R}(\omega, k)$.

We will describe a prescription to obtain the matrix $\mathcal{G}_{a b}^{R}(\omega, k)$, assuming we have bulk solutions for the $\Psi_{a}$. The method is a hybrid of the methods in [140,141] and [50,82]. $[140,141]$ described a general method to construct a retarded Green's function for coupled bulk scalar and gauge fields, while $[50,82]$ described general methods for computing Green's functions from fermions in the bulk.

\footnotetext{
${ }^{2}$ As a concrete example, in chapter $\left[5\right.$ we will introduce a bulk $S U(2)$ gauge field $A_{M}$ and a bulk fermion valued in the adjoint of $S U(2)$. The indices $a, b$ are then $S U(2)$ indices, hence we will have three bulk fermions (for $\tau_{1}, \tau_{2}$, and $\tau_{3}$ ) with a coupling, coming from the gauge-covariant derivative, of the form $\epsilon_{a b c} \bar{\Psi}_{a} e_{A}^{M} \gamma^{A}\left(A_{M}\right)_{b} \Psi_{c}$, which is obviously not diagonal in either $S U(2)$ indices or in spinor indices (because of the $\gamma^{A}$ ).
} 
The first observation is that we can construct second-order equations for the bulk fields, the $\Psi_{a \pm}$, that is similar to equation (2.3.35). We actually don't care about the exact form of these equations. We only need to know that such equations exist. We thus have a system of $2 N$ second-order linear equations, for which we expect $2 \times 2 N$ linearlyindependent solutions. We must therefore fix two boundary conditions for each field to specify a solution for the entire system. Following [141], we fix these boundary conditions near the horizon $u_{h}$. For example, the $\Psi_{a+u}$ have the near-horizon form

$$
\Psi_{a+u}=n_{a+u}\left(u-u_{h}\right)^{i \alpha}+\ldots,
$$

where $n_{a+u}$ and $\alpha$ are constants, independent of $u$, and $\ldots$ represents terms that decay faster, as $u \rightarrow u_{h}$, than the terms shown. The two constants $n_{a+u}$ and $\alpha$ are the two degrees of freedom we have to specify the solution. Generically, the equation of motion will only be satisfied for two values of $\alpha$, one describing an in-going wave and the other describing an out-going wave. As reviewed in section 3.2.1, to obtain the retarded Green's function, we must use an in-going wave. We still need to choose the normalization $n_{a+u}$. As shown in $[49,51]$, for fermions, once we choose an in-going wave solution, if we use the projectors $\Pi_{1,2}$, then when we fix the normalization of the up component $\Psi_{a 1 u}$ to be $n_{a 1 u}$, the equation of motion fixes the down component $\Psi_{a 1 d}$ to have normalization $i$ times $n_{a 1 u}$. The same applies to the up and down components of $\Psi_{a 2}$. Switching to the $\Pi_{ \pm}$projectors (recall equation (3.2.22) ), the statement is that once we fix the normalization of $\Psi_{a-d}$ to be $n_{a-d}$, then $\Psi_{a+d}$ must have normalization $i$ times $n_{a-d}$. The same statement applies to $\Psi_{a-u}$ and $\Psi_{a+u}$.

We thus need only fix $2 N$ normalizations, for the up and down components of the $\Psi_{a-}$. Let us arrange these normalizations into a row vector $\vec{n}$

$$
\vec{n}=\left(n_{1-u}, n_{1-d}, n_{2-u}, n_{2-d}, \ldots, n_{N-u}, n_{N-d}\right) .
$$

Following [141], we use these horizon normalizations to construct a basis of solutions as follows. We solve the equations of motion $2 N$ times, each time with a different choice of $\vec{n}$. The first time we use $\vec{n}=(+1,+1,+1, \ldots,+1,+1)$, the second time we use $\vec{n}=$ $(+1,-1,+1, \ldots,+1,+1)$, the third time we use $\vec{n}=(+1,+1,-1, \ldots,+1,+1)$, and so on. We label these choices $\vec{n}^{(i)}$, with $i=1, \ldots, 2 N$. For each choice of normalizations, we obtain solutions $\Psi_{a \pm}^{(i)}$. We now have a basis of solutions, so we can write any particular solution as a linear combination of these. To do so, we construct matrices that we call $\tilde{P}_{a j}^{ \pm}(u, \omega, k)$ from the basis solutions, where each row corresponds to a field and each column corresponds to a choice of normalization, the $i$ index. For example, suppressing the $\Psi_{a-}$ 's dependence on all variables,

$$
\left[\tilde{P}_{a j}^{-}(u, \omega, k)\right]=\left(\begin{array}{cccc}
\Psi_{1-}^{(1)} & \Psi_{1-}^{(2)} & \ldots & \Psi_{1-}^{(2 N)} \\
\Psi_{2-}^{(1)} & \Psi_{2-}^{(2)} & \ldots & \Psi_{2-}^{(2 N)} \\
\ldots & \ldots & & \ldots \\
\Psi_{N-}^{(1)} & \Psi_{N-}^{(2)} & \ldots & \Psi_{N-}^{(2 N)}
\end{array}\right)
$$

with $\tilde{P}_{a j}^{+}(u, \omega, k)$ defined similarly. The $\tilde{P}_{a j}^{ \pm}(u, \omega, k)$ are $2 N \times 2 N$ matrices. For later convenience, we factor out the leading asymptotic behavior of the solutions, defining new matrices $P_{a j}^{ \pm}(u, \omega, k)$,

$$
\tilde{P}_{a j}^{ \pm}(u, \omega, k) \equiv u^{\frac{d}{2} \pm m} P_{a j}^{ \pm}(u, \omega, k)
$$


We can now write any solution as a linear combination of the basis solutions:

$$
\begin{aligned}
& \Psi_{a+}(u, \omega, k)=u^{\frac{d}{2}+m} P_{a j}^{+}(u, \omega, k)\left(P^{+}(\epsilon, \omega, k)^{-1}\right)_{j b} c_{b+}(\omega, k), \\
& \Psi_{a-}(u, \omega, k)=u^{\frac{d}{2}-m} P_{a j}^{-}(u, \omega, k)\left(P^{-}(\epsilon, \omega, k)^{-1}\right)_{j b} c_{b-}(\omega, k),
\end{aligned}
$$

with a summation over the $j$ index. Notice that we take the solutions $\Psi_{a \pm}$ to be linear in the sources, $c_{a \pm}$. As emphasized in [141], equation (3.2.39) is simply saying that the sources $c_{a \pm}$ will source various linear combinations of fields in the bulk, and that we can write those linear combinations as linear combinations of our basis solutions. Notice that when we evaluate the solutions at $u=\epsilon$, we reproduce the leading asymptotic form, $\Psi_{a \pm} \sim c_{a \pm} u^{\frac{d}{2} \pm m}$.

Now we arrive at the main difference between bulk fermions and bulk bosons: $c_{a+}$ and $c_{a-}$ are not independent. The equation of motion relates them [83,84]. Indeed, we saw above that only the $c_{a-}$ are sources, while the $c_{a+}$ give one-point functions. To relate them, we follow $[50,82]$. We return to the equation of motion as written in equation (3.2.34). We focus only on the equation with $\Psi_{a+}$ on the left-hand-side, and simply insert solutions as written in equation (3.2.39) (suppressing all $\omega$ and $k$ dependence)

$$
\nabla_{a b+} u^{\frac{d}{2}+m} P_{b j}^{+}(u)\left(\left(P^{+}(\epsilon)^{-1}\right)_{j d} c_{d+}\right)=M_{a e+} u^{\frac{d}{2}-m} P_{e j}^{-}(u)\left(\left(P^{-}(\epsilon)^{-1}\right)_{j f} c_{f-}\right),
$$

where the parentheses separate $u$-dependent factors from $u$-independent factors. We now observe that the matrices $P_{a j}^{ \pm}$also solve the equation of motion, by construction, since they are built from solutions. We thus have

$$
\nabla_{a b+} u^{\frac{d}{2}+m} P_{b j}^{+}(u)=M_{a c+} u^{\frac{d}{2}-m} P_{c j}^{-}(u) .
$$

Here we have a free $j$ index, so we actually have $2 N$ such equations. Recall that the index $j$ labels the choice of normalization vector $\vec{n}$. The above equation is just the statement that one column of the $P_{a j}^{ \pm}$matrices solves the equation of motion. We are free to act on the right with the vector $\left(P^{+}(\epsilon)^{-1}\right)_{j d} c_{d+}$, so that we obtain

$$
\nabla_{a b+} u^{\frac{d}{2}+m} P_{b j}^{+}(u)\left(\left(P^{+}(\epsilon)^{-1}\right)_{j d} c_{d+}\right)=M_{a c+} u^{\frac{d}{2}-m} P_{c j}^{-}(u)\left(\left(P^{+}(\epsilon)^{-1}\right)_{j d} c_{d+}\right) .
$$

We now simply compare equations (3.2.40) and (3.2.42). The left-hand sides are identical, so we may equate the right-hand sides. Acting on the left with some inverse matrices, we obtain the desired relation between the $c_{a+}$ and $c_{a-}$,

$$
c_{a+}=P^{+}(\epsilon)_{a j}\left(P^{-}(\epsilon)^{-1}\right)_{j b} c_{b-} .
$$

Invoking equation (3.2.30), we now just need to perform two operations to extract the retarded two-point function $\mathcal{G}_{a b}^{R}(\omega, k)$ from $P^{+}(\epsilon)_{a j}\left(P^{-}(\epsilon)^{-1}\right)_{j b}$ : we take $\epsilon \rightarrow 0$ and then act on the right with $-i \gamma^{t}$.

The effect of taking $\epsilon \rightarrow 0$ is easy to understand. From the definition of the $\tilde{P}_{a j}^{ \pm}(u)$ in equation (3.2.37) and the definition of the $P_{a j}^{ \pm}(u)$ in equation (3.2.38), we can identify the $\epsilon \rightarrow 0$ limit of the $P_{a j}^{ \pm}(u)$ as

$$
\lim _{\epsilon \rightarrow 0}\left[P_{a j}^{-}(\epsilon)\right]=\left(\begin{array}{cccc}
c_{1-}^{(1)} & c_{1-}^{(2)} & \ldots & c_{1-}^{(2 N)} \\
c_{2-}^{(1)} & c_{2-}^{(2)} & \ldots & c_{2-}^{(2 N)} \\
\ldots & \ldots & & \ldots \\
c_{N-}^{(1)} & c_{N-}^{(2)} & \ldots & c_{N-}^{(2 N)}
\end{array}\right)
$$


and similarly for $\lim _{\epsilon \rightarrow 0} P_{a j}^{+}(\epsilon)$. In short, the matrices $P_{a j}^{ \pm}$, when evaluated at the boundary, are simply matrices of the $c_{a+}$ and $c_{a-}$.

Notice that the $P^{-}(\epsilon)^{-1}$ matrix introduce a factor of $\operatorname{det} P^{-}(\epsilon)$ in the denominator of the Green's function. Generically, then, if $\operatorname{det} P^{-}(\epsilon)$ has a zero, the Green's function has a pole, which means a quasi-normal mode appears in the bulk spectrum, as in the bosonic cases of [141]. Given the identification in equation (3.2.44), then, to identify quasi-normal modes we need only identify the zeroes of the matrix of $c_{-}$'s.

Understanding how $\gamma^{t}$ acts on $P^{+}(\epsilon)_{a j}\left(P^{-}(\epsilon)^{-1}\right)_{j b}$ is a little tricky. Luckily, the way we have written $P^{+}(\epsilon)_{a j}\left(P^{-}(\epsilon)^{-1}\right)_{j b}$ means that $-i \gamma^{t}$ acts trivially. To see that, notice that equation (3.2.43) is written in a two-component form: here $c_{a \pm}$ are two component spinors. To restore them to four-component form, we take a direct product,

$$
c_{a+}=\left(\begin{array}{c}
c_{a+u} \\
c_{a+d}
\end{array}\right) \rightarrow c_{a+} \otimes\left(\begin{array}{l}
0 \\
1
\end{array}\right)=\left(\begin{array}{c}
0 \\
c_{a+u} \\
0 \\
c_{a+d}
\end{array}\right), \quad c_{a-}=\left(\begin{array}{c}
c_{a-u} \\
c_{a-d}
\end{array}\right) \rightarrow c_{a-} \otimes\left(\begin{array}{l}
1 \\
0
\end{array}\right)=\left(\begin{array}{c}
c_{a-u} \\
0 \\
c_{a-d} \\
0
\end{array}\right) .
$$

To restore the $P_{a j}^{ \pm}$matrices to the same four-component form, we recall equation (3.2.37), which shows that we should perform exactly the same direct products (suppressing the dependence on all variables):

$$
P_{a j}^{+} \rightarrow P_{a j}^{+} \otimes\left(\begin{array}{l}
0 \\
1
\end{array}\right), \quad P_{a j}^{-} \rightarrow P_{a j}^{-} \otimes\left(\begin{array}{l}
1 \\
0
\end{array}\right)
$$

which implies $\left(P^{-}\right)_{j a}^{-1} \rightarrow\left(P^{-}\right)_{j a}^{-1} \otimes\left(\begin{array}{ll}1 & 0\end{array}\right)$. Equation $(3.2 .43)$ thus becomes

$$
c_{a+} \otimes\left(\begin{array}{l}
0 \\
1
\end{array}\right)=\left\{\left[P^{+}(\epsilon)_{a j}\left(P^{-}(\epsilon)^{-1}\right)_{j b}\right] \otimes\left(\begin{array}{ll}
1 & 0 \\
0 & 0
\end{array}\right)\right\}\left[c_{b-} \otimes\left(\begin{array}{l}
1 \\
0
\end{array}\right)\right] .
$$

We now simply observe that, in such a representation, $-i \gamma^{t}=\mathbf{1}_{N} \otimes \sigma_{1}$. In the $N \times N$ subspace, $-i \gamma^{t}$ merely acts as the identity.

In summary, the retarded Green's function for coupled bulk fermions is

$$
\mathcal{G}_{a b}^{R}(\omega, k)=\lim _{\epsilon \rightarrow 0}\left(P^{+}(\epsilon)_{a j} P^{-}(\epsilon)_{j b}^{-1}\right),
$$

with the matrices $P_{a j}^{ \pm}$defined in equation (3.2.38).

Finally, as an important check, let us use our prescription to reproduce the result for free fermions, equation (3.2.32). For illustration, we consider $N=2$, so we have two bulk fermions, which we call $\Psi_{a}$ and $\Psi_{b}$. We return to the equation of motion as written in equation (3.2.34), and assume the equations for $\Psi_{a}$ and $\Psi_{b}$ decouple, so that $\nabla_{a b \pm}$ and $M_{a b \pm}$ become diagonal in the $a$ and $b$ indices. We can further decouple the equations of motion by using the projectors $\Pi_{1,2}$. Acting with these, we obtain equations similar to equation (3.2.23). We thus find four decoupled equations, for $\Psi_{a 1}, \Psi_{a 2}, \Psi_{b 1}$ and $\Psi_{b 2}$.

We now solve the equations $2 N=4$ times, each time with a different normalization vector $\vec{n}$ for the $\Psi_{a-}$ and $\Psi_{b-}$ fields. In the first solution, all four fields have normalizations $\vec{n}=\left(n_{a-u}, n_{a-d}, n_{b-u}, n_{b-d}\right)=(+1,+1,+1,+1)$. In the second solution, we use $\vec{n}=$ $(+1,-1,+1,+1)$. The key observation is that the field $\Psi_{a-d}$ whose normalization we 
change is $\Psi_{a-d}=\Psi_{a 1 u}$ (recall equation (3.2.22)), and hence couples only to $\Psi_{a 1 d}=\Psi_{a+d}$. The change in normalization thus leaves the other three fields, $\Psi_{a-u}, \Psi_{b-u}$, and $\Psi_{b-d}$ unchanged. The solutions for these fields thus is identical to what they were using the original +1 normalizations. The $P_{a j}^{-}$matrix thus takes the form (here we must write the up and down components explicitly)

$$
\left[\tilde{P}_{a j}^{-}(u, \omega, k)\right]=\left(\begin{array}{llll}
\Psi_{1-u}^{(1)} & \Psi_{1-u}^{(1)} & \Psi_{1-u}^{(1)} & \Psi_{1-u}^{(1)} \\
\Psi_{1-d}^{(1)} & \Psi_{1-d}^{(2)} & \Psi_{1-d}^{(1)} & \Psi_{1-d}^{(1)} \\
\Psi_{2-u}^{(1)} & \Psi_{2-u}^{(1)} & \Psi_{2-u}^{(3)} & \Psi_{2-u}^{(1)} \\
\Psi_{2-d}^{(1)} & \Psi_{2-d}^{(1)} & \Psi_{2-d}^{(1)} & \Psi_{2-d}^{(4)}
\end{array}\right)
$$

with $\tilde{P}_{a j}^{+}(u, \omega, k)$ being identical except all - subscripts become + . The main feature here is that all the superscripts are the same, except on the diagonal. A straightforward exercise (especially simple for $2 \times 2$ matrices) then shows that taking the inverse $\left(P^{-}\right)_{j a}^{-1}$ and then contracting with $P_{a j}^{+}$, and taking $\epsilon \rightarrow 0$, reproduces exactly the purely diagonal $c_{+u} / c_{-u}$ and $c_{+d} / c_{-d}$ form of equation (3.2.32).

In summary: by combining the methods of $[140,141]$ and $[50,82]$, we have provided a relatively simple prescription to compute the matrix-valued retarded two-point function from bulk solutions for coupled fermions. We simply solve the equations of motion (typically numerically) $2 N$ times, using a different normalization vector $\vec{n}$ each time, use those solutions to construct the matrices $P_{a j}^{ \pm}(\epsilon)$, and then take $\lim _{\epsilon \rightarrow 0} P^{+}(\epsilon)_{a j}\left(P^{-}(\epsilon)^{-1}\right)_{j b}$.

Using this method we calculate the fermionic response in a p-wave superconductor in chapter 5 .

\subsection{Holographic calculation: Beyond linear response}

The first sections of the chapter dealt with linear response theory. For example we gave a recipe how to calculate the conductivity tensor for probe brane in linear response. In this section we demonstrate that it is possible to do explicit calculations beyond linear response. The method presented here uses peculiar properties of the effective action of probe branes, in particular of the Dirac-Born-Infeld action, to calculate the DC conductivity to all orders in the electric field $\vec{E}$ and in the magnetic field $\vec{B}$.

This section is based on my own work done in collaboration with Hai Ngo and Andy O'Bannon and was published in [5].

We consider flavor fields propagating through an $\mathcal{N}=4 \mathrm{SYM}$ plasma with finite $U(1)_{B}$ density. In particular we calculate the DC conductivity for the flavor fields to completely arbitrary (constant) electric and magnetic fields generalizing the results of $[105,106]$. Though we focus on probe D7-branes, i.e. on $\mathcal{N}=2$ supersymmetric hypermultiplets which may propagate in all $(3+1)$ spacetime dimensions, our analysis easily extends to other probe Dp-brane systems.

For an arbitrary configuration of constant electric and magnetic fields, we may sum all the electric fields into a single vector, and similarly for the magnetic fields. The most general configuration is thus an electric field $\vec{E}$ pointing in some direction, which we take to be $\hat{x}$, 
and a magnetic field $\vec{B}$ that may be decomposed into two components, one along $\hat{x}$, which we call $B_{x}$, and one perpendicular to it, along the $\hat{z}$ direction, which we call $B_{z} \cdot 3$

Let us briefly summarize the main results of the section: we calculate all components of the direct current conductivity tensor of flavor fields at finite temperature. The conductivity tensor $\sigma_{i j}$ measures the electrical response of a conducting medium to externally applied fields. It is defined by

$$
\left\langle J_{i}\right\rangle=\sigma_{i j} E_{j},
$$

where $E_{j}$ are the components of externally applied electric fields and $\left\langle J_{i}\right\rangle$ are the components of electrical currents induced in the medium. Using the most general configuration introduced above the components $\sigma_{x x}, \sigma_{x y}$ and $\sigma_{x z}$ of the conductivity tensor are given by equations (3.3.71). In particular we notice that $\sigma_{x z} \propto \sigma_{x x}$. Moreover, $\sigma_{x x}$ and therefore also $\sigma_{x z}$ consist of two terms adding in quadrature. One of the term is proportional to the finite density $\rho$. This contribution to the conductivity tensor can be traced back to the finite density of charge carriers. The other contribution to $\sigma_{x x}$ depends on the mass of the flavor fields. We give several arguments indicating that this term arises due to pair production. Note that in linear response theory such a term cannot be present since the electric field has to be a small perturbation.

Introducing a nonzero $\vec{E} \cdot \vec{B}$ is worthwhile for a number of reasons:

- With perpendicular electric and magnetic fields $E$ and $B_{z}$, we expect a current $\left\langle J^{x}\right\rangle$ parallel to the electric field (because it pushes the charges) and a Hall current $\left\langle J^{y}\right\rangle$ orthogonal to both the electric and magnetic field. With nonzero $B_{x}$, we expect a current $\left\langle J^{z}\right\rangle$, and hence we can compute a new transport coefficient, $\sigma_{x z}$. More generally, we can compute the entire conductivity tensor and determine its dependence on $B_{x}$. We find that, generically, $B_{x}$ enhances the contribution from the pair-produced charges.

- Additionally, in a Lorentz-invariant system, we can build two Lorentz-invariant quantities from $\vec{E}$ and $\vec{B}$, namely $|\vec{E}|^{2}-|\vec{B}|^{2}$ and $\vec{E} \cdot \vec{B}$. When $\vec{E} \cdot \vec{B}=0$, and $|\vec{B}|>|\vec{E}|$, we can boost to a frame where the electric field is zero, which immediately tells us that all the physics must be equilibrium. For example, as reviewed in [142], the form of the Hall conductivity is fixed by Lorentz invariance to be $\left\langle J^{t}\right\rangle / B_{z}$. When $\vec{E} \cdot \vec{B}$ is non-zero, we can no longer boost to a frame in which the electric field is zero, hence the physics cannot be purely equilibrium.

- Many (though not all) previous gauge-gravity calculations of conductivities were in $(3+1)$-dimensional AdS space, so that the boundary CFT was $(2+1)$-dimensional [42, $43,142,143]$, which precludes the existence of $\vec{E} \cdot \vec{B}$. Another drawback of the systems studied in $[42,43,142,143]$ was translation invariance, which implies momentum conservation. The system thus has no way to dissipate momentum, so the DC transport behavior was singular. For example, the DC conductivity at finite density is infinite because the charge carriers, in the presence of an external electric field but without frictional forces, accelerate forever.

Let us also emphasize that the probe limit allows our system to mimic a dissipative system. As explained in more detail in $[73,105,106,116]$, and as we review below,

\footnotetext{
${ }^{3}$ Stated simply, then, we generalize the results of $[105,106,116]$ to include a magnetic field with nonzero $\hat{x}$ component, or equivalently a nonzero $\vec{E} \cdot \vec{B} \sim F \wedge F$.
} 
the charge carriers do indeed transfer energy and momentum to the $\mathcal{N}=4$ plasma, but the rates at which they do so are of order $N_{c}$. That means that only at times of order $N_{c}$ the charge carriers have transferred order $N_{c}^{2}$ amounts of energy and momentum to the plasma, and hence the motion of the $\mathcal{N}=4$ SYM plasma is no longer negligible. For earlier times, we may treat the $\mathcal{N}=4$ SYM plasma as a motionless reservoir into which the charge carriers may dump their energy and momentum, thus providing the charge carriers with an (apparent) mechanism for dissipation.

\subsubsection{Holographic setup}

In this section we present the holographic setup of massive hypermultiplets propagating through an $\mathcal{N}=4$ SYM plasma with finite $U(1)_{B}$ density and in the presence of external electric and magnetic fields. In particular we introduce $N_{f}$ coincident probe D7-branes in a Schwarzschild AdS black hole as described in section 2.5.2. The induced metric on the worldvolume of the D7-branes reads

$$
d s_{D 7}^{2}=G_{u u} d u^{2}+G_{t t} d t^{2}+G_{x x} d \vec{x}^{2}+\cos ^{2} \theta(u) d s_{S^{3}}^{2} .
$$

The embedding profile is specified by the function $\theta=\theta(u)$. The most convenient metric for the computation of the direct current conductivity is given by equation 2.4 .82 which we use in the subsequent discussion (if needed). Therefore $G_{u u}, G_{t t}$ and $G_{x x}$ are given by

$$
G_{u u}=\frac{1}{u^{2}}+\theta^{\prime}(u)^{2}, \quad G_{t t}=-\frac{1}{u^{2}} \frac{\left(1-u^{4} / u_{h}^{4}\right)^{2}}{1+u^{4} / u_{h}^{4}}, \quad G_{x x}=\frac{1}{u^{2}}\left(1+u^{4} / u_{h}^{4}\right)
$$

where prime denotes differentiation with respect to $u$.

As we are interested only in the $U(1)$ part of the $U\left(N_{f}\right)$ worldvolume gauge field, the relevant part of their action is the usual Abelian Dirac-Born-Infeld term as discussed in section 2.5.2.

In order to describe a plasma at finite density $\left\langle J^{t}\right\rangle$, we have to consider a non-trivial gauge field component $A_{t}(u)$. Electric and magnetic fields, and the resulting currents $\left\langle J^{x}\right\rangle,\left\langle J^{y}\right\rangle$, and $\left\langle J^{z}\right\rangle$ are introduced by the following ansatz for the gauge field components

$$
A_{x}(t, u)=-E t+f_{x}(u), \quad A_{y}(x, u)=B_{z} x+f_{y}(u), \quad A_{z}(y, u)=B_{x} y+f_{z}(u) .
$$

In each case, the leading term is a non-normalizable mode that introduces an external field into the SYM theory. Choosing a gauge in which $A_{u}=0$, we can write the nonzero elements of $F_{a b}$

$$
\begin{gathered}
F_{t x}=-E, \quad F_{x y}=B_{z}, \quad F_{y z}=B_{x}, \\
F_{u t}=A_{t}^{\prime}, \quad F_{u x}=A_{x}^{\prime}, \quad F_{u y}=A_{y}^{\prime}, \quad F_{u z}=A_{z}^{\prime} .
\end{gathered}
$$

As explained in section 2.5.2, since the fields in our ansatz depend only on $u$, we can immediately perform in the Abelian D7-brane action the integration over the SYM theory directions $(t, x, y, z)$ and over the $S^{3}$ directions. 
We plug our ansatz into the Abelian DBI action density (2.5.97),

$$
S_{D 7}=-\mathcal{N} \int d u \cos ^{3} \theta \sqrt{G_{u u} G_{t t} G_{x x}^{3}-G_{x x} A_{2}-A_{4}},
$$

where $\mathcal{N}$ is the constant given in equation (2.5.99). $A_{2}$ and $A_{4}$ contain terms with two or four factors of $\tilde{F}_{a b}$, respectively 4

$$
\begin{aligned}
A_{2}= & G_{u u} G_{x x} \tilde{E}^{2}+G_{t t} G_{u u}\left(\tilde{B}_{x}^{2}+\tilde{B}_{z}^{2}\right)+G_{x x}^{2} \tilde{A}_{t}^{\prime 2}+G_{t t} G_{x x}\left(\tilde{A}_{x}^{\prime 2}+\tilde{A}_{y}^{\prime 2}+\tilde{A}_{z}^{\prime 2}\right) \\
A_{4}= & G_{x x} \tilde{E}^{2}\left(\tilde{A}_{y}^{\prime 2}+\tilde{A}_{z}^{\prime 2}\right)+G_{x x} \tilde{A}_{t}^{\prime 2}\left(\tilde{B}_{x}^{2}+\tilde{B}_{z}^{2}\right)+G_{u u} \tilde{E}^{2} \tilde{B}_{x}^{2}+G_{t t} \tilde{B}_{z}^{2} \tilde{A}_{z}^{\prime 2}+G_{t t} \tilde{B}_{x}^{2} \tilde{A}_{x}^{\prime 2} \\
& +2 G_{t t} \tilde{B}_{x} \tilde{B}_{z} \tilde{A}_{x}^{\prime} \tilde{A}_{z}^{\prime}-2 G_{x x} \tilde{E} \tilde{B}_{z} \tilde{A}_{t}^{\prime} \tilde{A}_{y}^{\prime} .
\end{aligned}
$$

The action only depends on the $u$ derivatives of $A_{t}, A_{x}, A_{y}$, and $A_{z}$, so the system has four constants of motion. As shown in section 2.5.3, we can identify these as the components of the $U(1)_{B}$ current density in the SYM theory 5

$$
\left\langle J^{\mu}\right\rangle=-\frac{\delta L}{\delta A_{\mu}^{\prime}},
$$

where $L$ denotes the Lagrangian density, $S_{D 7} \equiv \int d u L$. Our ansatz thus allows for a nonzero $U(1)_{B}$ density $\left\langle J^{t}\right\rangle$ as well as $U(1)_{B}$ currents $\left\langle J^{x}\right\rangle,\left\langle J^{y}\right\rangle$, and $\left\langle J^{z}\right\rangle$. Given these constants of motion, we can solve algebraically for the derivatives of the gauge field, the field strength components:

$$
\begin{aligned}
& A_{t}^{\prime}(u)=-\frac{\sqrt{G_{u u}\left|G_{t t}\right|}}{G_{x x}^{2}+\tilde{B}_{x}^{2}} \frac{\left\langle J^{t}\right\rangle \xi-B_{z} a_{1}}{\sqrt{\xi \chi-\frac{a_{1}^{2}}{G_{x x}^{2}+\tilde{B}_{x}^{2}}+\frac{a_{2}^{2}}{G_{t t} G_{x x}-\tilde{E}^{2}}}}, \\
& A_{x}^{\prime}(u)=\sqrt{\frac{G_{u u}}{\left|G_{t t}\right|}} \frac{1}{G_{x x}} \frac{\left\langle J^{x}\right\rangle \xi-B_{x} a_{2}}{\sqrt{\xi \chi-\frac{a_{1}^{2}}{G_{x x}^{2}+\tilde{B}_{x}^{2}}+\frac{a_{2}^{2}}{G_{t t} G_{x x}-\tilde{E}^{2}}}}, \\
& A_{y}^{\prime}(u)=\sqrt{\frac{G_{u u}}{\left|G_{t t}\right|}} \frac{1}{G_{x x}} \frac{\left\langle J^{y}\right\rangle \xi+E a_{1}}{\sqrt{\xi \chi-\frac{a_{1}^{2}}{G_{x x}^{2}+\tilde{B}_{x}^{2}}+\frac{a_{2}^{2}}{G_{t t} G_{x x}-\tilde{E}^{2}}}}, \\
& A_{z}^{\prime}(u)=\frac{\sqrt{G_{u u}\left|G_{t t}\right|}}{G_{t t} G_{x x}-\tilde{E}^{2}} \frac{\left\langle J^{z}\right\rangle \xi-B_{z} a_{2}}{\sqrt{\xi \chi-\frac{a_{1}^{2}}{G_{x x}^{2}+\tilde{B}_{x}^{2}}+\frac{a_{2}^{2}}{G_{t t} G_{x x}-\tilde{E}^{2}}}},
\end{aligned}
$$

where we have defined

$$
\xi=\left|G_{t t}\right| g_{x x}^{3}-G_{x x}^{2} \tilde{E}^{2}+G_{t t} G_{x x}\left(\tilde{B}_{x}^{2}+\tilde{B}_{z}^{2}\right)-\tilde{E}^{2} \tilde{B}_{x}^{2},
$$

\footnotetext{
${ }^{4}$ A tilde over a quantity denotes a factor of $\left(2 \pi \alpha^{\prime}\right)$, for example, $\tilde{F}_{a b} \equiv\left(2 \pi \alpha^{\prime}\right) F_{a b}$.

${ }^{5}$ Strictly speaking the action is not finite due to the infinite volume of $A d S_{5}$. Therefore holographic renormalization (see section 2.3) is required, i.e. we have to add counterterms $S_{C T}$ to the action. In the $B_{x}=0$ case, the counterterms appearing in $S_{C T}$ were computed in [106]. A straightforward analysis reveals that no new counterterms are necessary with non-zero $B_{x}$ and that, as in [106], the counterterms do not contribute to $\left\langle J^{\mu}\right\rangle$. Equation (3.3.58) then follows for on-shell $A_{\mu}$. For more details, see the appendix of $[106]$.
} 


$$
\begin{gathered}
\chi=G_{t t} G_{x x}^{2} \mathcal{N}^{2}\left(2 \pi \alpha^{\prime}\right)^{4} \cos ^{6} \theta-\left(2 \pi \alpha^{\prime}\right)^{2}\left(\left\langle J^{x}\right\rangle^{2}+\left\langle J^{y}\right\rangle^{2}\right) \\
+\left(2 \pi \alpha^{\prime}\right)^{2}\left(\frac{G_{t t} G_{x x}}{G_{x x}^{2}+\tilde{B}_{x}^{2}}\left\langle J^{t}\right\rangle^{2}-\frac{G_{t t} G_{x x}}{G_{t t} G_{x x}-\tilde{E}^{2}}\left\langle J^{z}\right\rangle^{2}\right), \\
a_{1}=\left(2 \pi \alpha^{\prime}\right)^{2}\left(G_{t t} G_{x x} B_{z}\left\langle J^{t}\right\rangle+\left(G_{x x}^{2}+\tilde{B}_{x}^{2}\right) E\left\langle J^{y}\right\rangle\right), \\
a_{2}=\left(2 \pi \alpha^{\prime}\right)^{2}\left(\left(G_{t t} G_{x x}-\tilde{E}^{2}\right) B_{x}\left\langle J^{x}\right\rangle+G_{t t} G_{x x} B_{z}\left\langle J^{z}\right\rangle\right) .
\end{gathered}
$$

Notice that $\xi$ is the value of $-\operatorname{det}\left(G_{a b}+\left(2 \pi \alpha^{\prime}\right) F_{a b}\right)$ in the $(t, x, y, z)$ subspace.

We can obtain $\theta(u)$ 's equation of motion in two ways. We can find its Euler-Lagrange equation of motion from the original D7-brane action, equation (3.3.55), and then plug into that equation of motion the solutions for the field strengths in equation (3.3.59). Equivalently, we can plug the solutions for the field strengths into the D7-brane action, equation (3.3.55), to obtain an effective action for $\theta(u)$, perform a Legendre transform, and then find the Euler-Lagrange equation of motion. Plugging the solutions in equation (3.3.59) into $S_{D 7}$, we find

$$
S_{D 7}=-\mathcal{N}^{2}\left(2 \pi \alpha^{\prime}\right)^{2} \int d u \cos ^{6} \theta G_{x x} \sqrt{G_{u u} G_{t t}} \frac{\xi}{\sqrt{\xi \chi-\frac{a_{1}^{2}}{G_{x x}^{2}+\tilde{B}_{x}^{2}}+\frac{a_{2}^{2}}{G_{t t} G_{x x}-\tilde{E}^{2}}}} .
$$

The Legendre-transformed on-shell action, $\hat{S}_{D 7}$, is then

$$
\begin{aligned}
\hat{S}_{D 7} & =S_{D 7}-\int d u\left(A_{t}^{\prime} \frac{\delta S_{D 7}}{\delta A_{t}^{\prime}}+A_{x}^{\prime} \frac{\delta S_{D 7}}{\delta A_{x}^{\prime}}+A_{y}^{\prime} \frac{\delta S_{D 7}}{\delta A_{y}^{\prime}}+A_{z}^{\prime} \frac{\delta S_{D 7}}{\delta A_{z}^{\prime}}\right) \\
& =-\frac{1}{\left(2 \pi \alpha^{\prime}\right)^{2}} \int d u G_{x x}^{-1} \sqrt{\frac{G_{u u}}{\left|G_{t t}\right|}} \sqrt{\xi \chi-\frac{a_{1}^{2}}{G_{x x}^{2}+\tilde{B}_{x}^{2}}+\frac{a_{2}^{2}}{G_{t t} G_{x x}-\tilde{E}^{2}}} .
\end{aligned}
$$

To complete our solution, we must specify boundary conditions for the worldvolume fields, namely $\theta(u)$ and the gauge fields.

The boundary condition for the time-like component of the gauge field, $A_{t}(u)$, is discussed in section 2.5.3: we must impose $A_{t}\left(u_{h}\right)=0$. What about the other components of the gauge field? The key point is that the calculation of the next section implicitly fixes the values of these components at the horizon. In the next section we demand that the on-shell Lagrangian remains real for all $u$. For given values of $E, B_{x}, B_{z}$ and $\left\langle J^{t}\right\rangle$, that only happens for particular values of $\left\langle J^{x}\right\rangle,\left\langle J^{y}\right\rangle$ and $\left\langle J^{z}\right\rangle$. For those values of $\left\langle J^{x}\right\rangle,\left\langle J^{y}\right\rangle$, and $\left\langle J^{z}\right\rangle$, the solutions for $A_{x}, A_{y}$ and $A_{z}$ are fixed by our solutions above, and hence we can then (working backwards) infer their values at the horizon. In other words, we implicitly choose the values of $A_{x}, A_{y}$, and $A_{z}$ at the horizon to produce exactly the values of $\left\langle J^{x}\right\rangle,\left\langle J^{y}\right\rangle$ and $\left\langle J^{z}\right\rangle$ such that the Lagrangian remains real for all $u$. Unfortunately, our solution for $A_{x}(t, u)$ diverges at the horizon. The conductivity tensor does not depend on the values of the gauge fields at the horizon, so it is safe from the divergence, 6

Let us now turn to the boundary conditions on $\theta(u)$. The boundary conditions for $\theta(u)$ are discussed in section 2.5.2. Please note that our calculation of the conductivity rely

\footnotetext{
${ }^{6}$ The stress-energy tensor does depend on the values at the horizon, but as explained in [116], these divergences (suitably regulated) have a sensible interpretation in the field theory as rates of energy and momentum loss. For more details see section 4 of my paper [5].
} 
on the fact that the D7-brane intersects the horizon, so our results should be applicable in any region of the phase diagram whose description in supergravity is a D7-brane black hole embedding (in the language of section 2.5.2).

But the phase diagram of our system has not been explored for all values of $T, m,\left\langle J^{t}\right\rangle, E$, $B_{z}$, and $B_{x}$. To date, only certain regions, with some subset of the parameters nonzero, have been explored [91,104,107-112,115,117-123,129,144-148].

Nevertheless we expect that for finite density black hole embeddings are favored, i.e. we can apply our method to any value of $T, m,\left\langle J^{t}\right\rangle, E, B_{z}$, and $B_{x}$. Crucially, however, as shown in [120-123], for the case with $B_{z}$ nonzero but $\left\langle J^{t}\right\rangle, E$ and $B_{x}$ zero, an infinite number of solutions describing $m=0$ exist, and all but one are unstable. The stable solution is not the trivial solution $\theta(u)=0$. On general grounds, we expect that the same should be true for our solution, which has nonzero $\left\langle J^{t}\right\rangle, E$ and $B_{x}$. Nevertheless, whenever we consider the zero mass limit, we use the trivial solution as a simple example.

\subsubsection{The Conductivity tensor}

In order to compute the conductivity tensor, we have to fix the horizon conditions for the gauge fields $A_{x}, A_{y}$ and $A_{z}$. We do not specify explicitly the conditions. Rather, we demand that the Lagrangian of the on-shell action (3.3.61) is real for all values of the radial coordinate $u$. This will implicitly fix the gauge fields $A_{x}, A_{y}$ and $A_{z}$ at the horizon. Let us justify this method in the next few paragraphs.

For simplicity, consider first $(9+1)$-dimensional Minkowski space. Now introduce $N_{f}$ coincident probe D7-branes, 7 and consider a solution in which the only nontrivial worldvolume field is a constant $U(1)$ electric field $F_{t x}=-E$.

Our ansatz for the gauge field involves only the $(u, t, x)$ directions,${ }^{8}$ hence we may write $S_{D 7}$ as a $(3+1)$-dimensional DBI action times some extra factors, with the $(3+1)$-dimensional part being the $(u, t, x, y)$ subspace:

$$
\begin{aligned}
S_{D 7} & =-N_{f} T_{D 7} \int d^{8} x \sqrt{1-\frac{1}{2} \tilde{F}^{2}-\frac{1}{4}(\tilde{F} \wedge \tilde{F})^{2}} \\
& =-N_{f} T_{D 7} \int d^{8} x \sqrt{1-\left(2 \pi \alpha^{\prime}\right)^{2} E^{2}} .
\end{aligned}
$$

Clearly when the electric field is greater than the string tension, $E>1 /\left(2 \pi \alpha^{\prime}\right)$, the DBI action becomes imaginary. That signals the well-known tachyonic instability of open strings in an electric field [149-151]. The electric field pulls the endpoints of an open string in opposite directions. When the electric field is large enough to overcome the tension of the string, it rips the string apart. Another way to say the same thing is that the electric field reduces the effective tension of open strings. The instability appears when that effective tension becomes negative. Notice that if we additionally introduce a

\footnotetext{
${ }^{7}$ Strictly speaking, to avoid constraints on the number of D7-branes we should set the string coupling to be precisely zero. Many of the arguments that follow rely only on the form of the DBI action, rather than any properties unique to D7-branes, however.

${ }^{8}$ Later we also turn on a constant magnetic field $F_{x y}=B_{z}$. This magnetic field can be obtained from the gauge field configuration $A_{y}(x)=B_{z} x$. The analysis presented here can also be extended to the most general configuration of constant electric and magnetic fields considered above.
} 
magnetic field $F_{x y}=B_{z}$ orthogonal to the electric field, then the DBI action becomes

$$
S_{D 7}=-N_{f} T_{D 7} \int d^{8} x \sqrt{1-\left(2 \pi \alpha^{\prime}\right)^{2}\left(E^{2}-B_{z}^{2}\right)}
$$

If $E>B_{z}$ we can boost to a frame in which the magnetic field vanishes, and the arguments above still apply. If $E \leq B_{z}$ then the instability never appears. Indeed, in that case we can boost to a frame where the electric field is zero.

Now instead of flat space consider $A d S_{5}$-Schwarzschild times $S^{5}$, as in equation (3.3.51). Here the effective tension of strings already decreases as a function of $u$, going to zero at the horizon. Probe D7-branes with a constant worldvolume electric field will reduce the effective tension by the same amount at every value of $r$. We thus expect that for any nonzero $E$ the effective tension will go to zero at some radial position $u_{*}$ outside the horizon, and to be negative between $u_{*}$ and the horizon. With an asymptotically AdS space, however, we have a dual field theory, so we can use our field theory intuition to guess the endpoint of the instability. The endpoint of a string looks like a quark. The electric field ripping a string apart should look like a Schwinger pair-production process. We should thus see a current.

The current stabilizes the system. Note that the instability in equation (3.3.63) occurs if the Lagrangian becomes imaginary, i.e. the term under the square root changes sign. Therefore we have to adjust the currents in such a way that the term under the square root (in equation (3.3.61)) is always positive.

We now calculate the conductivity tensor by demanding that the Lagrangian of the onshell action (3.3.61) is real for all values of the radial coordinate $u$. But let us make two important comments beforehand.

First, note that from equation (3.3.60a), we see that $\xi$ is negative at the horizon but positive at the boundary, thus $\xi$ must change sign at some value of $u$, which we call $u_{*}$. We can straightforwardly calculate $u_{*}$ from the equation $\xi\left(u_{*}\right)=0$,

$$
\frac{u_{*}^{4}}{u_{h}^{4}}=G-\sqrt{G^{2}-1},
$$

with

$$
G \equiv e^{2}-b_{z}^{2}-b_{x}^{2}+\sqrt{\left(e^{2}-b_{z}^{2}\right)^{2}+\left(b_{x}^{2}+1\right)\left(b_{x}^{2}+1+2\left(e^{2}+b_{z}^{2}\right)\right)},
$$

where we have introduced the dimensionless quantities

$$
e \equiv \pi \alpha^{\prime} u_{h}^{2} E=\frac{E}{\frac{\pi}{2} \sqrt{2 \lambda} T^{2}}, \quad b_{z} \equiv \pi \alpha^{\prime} u_{h}^{2} B_{z}=\frac{B_{z}}{\frac{\pi}{2} \sqrt{2 \lambda} T^{2}}, \quad b_{x} \equiv \pi \alpha^{\prime} u_{h}^{2} B_{x}=\frac{B_{x}}{\frac{\pi}{2} \sqrt{2 \lambda} T^{2}} .
$$

Moreover, we need later $G_{x x}^{2}$ evaluated at $u_{*}$ in order to translate our result for the conductivity tensor into SYM theory quantities. Using equation (3.3.65), we find

$$
\left.G_{x x}^{2}(u)\right|_{u=u_{*}}=\frac{\pi^{4} T^{4}}{2}(1+G) \equiv \pi^{4} T^{4} \mathcal{F}\left(e, b_{x}, b_{z}\right),
$$

\footnotetext{
${ }^{9}$ We actually find four solutions for $u_{*}^{4} / u_{h}^{4}$. The one we present is the only one for which $u_{*}^{4} / u_{h}^{4}$ takes physical values, between 0 and 1 .
} 
where in the last step we removed a factor of $\pi^{4} T^{4}$ and defined the rest to be $\mathcal{F}\left(e, b_{x}, b_{z}\right)$, which will appear in our result for the conductivity tensor. A useful limit is $e=0$, where $G=1$ and hence $\mathcal{F}=1$.

Following $[105,106]$, we now focus on the on-shell action, equation (3.3.61), and in particular we focus on the square root in the denominator of equation (3.3.61), which we reproduce here for convenience,

$$
\sqrt{\xi \chi-\frac{a_{1}^{2}}{G_{x x}^{2}+\tilde{B}_{x}^{2}}+\frac{a_{2}^{2}}{G_{t t} G_{x x}-\tilde{E}^{2}}}
$$

and which also appears in the solutions for the field strengths $A_{\mu}^{\prime}(u)$ for $\mu=t, x, y, z$, equation (3.3.59), as well as the Legendre-transform of the on-shell action, equation (3.3.62). We will argue that the four functions $\xi, \chi, a_{1}$ and $a_{2}$, must all vanish at $u_{*}$ in order for the above square root, and hence the on-shell action, to remain real for all $u$.

When $\xi=0$ the $a_{2}^{2}$ term is negative, because the equation $\xi\left(u_{*}\right)=0$ itself tells us that $\left(G_{t t} G_{x x}-\tilde{E}^{2}\right)=-\frac{G_{t t} G_{x x} \tilde{B}_{z}^{2}}{\left(G_{x x}^{2}+\tilde{B}_{x}^{2}\right)}<0$ at $u_{*}$. To avoid an imaginary action at $u_{*}$ we must have $a_{1}\left(u_{*}\right)=a_{2}\left(u_{*}\right)=0$.

Arguing why $\chi$ has to vanish at $u_{*}$ is more subtle. $\chi$ has the same behavior as $\xi$ : it is positive at the boundary and negative at the horizon, so it must have a zero at some $u$ value, which we will call $u_{\chi}$. If $u_{*}$ and $u_{\chi}$ are not the same, so that $\xi$ and $\chi$ have distinct zeroes, then the product $\xi \chi$ will be negative on the interval between $u_{*}$ and $u_{\chi}$. The crucial question then is whether the $a_{2}^{2}$ term is positive or negative on that interval. If it is positive (and sufficiently large) it could keep the action real. The sign of the $a_{2}^{2}$-term is determined by $\left(G_{t t} G_{x x}-\tilde{E}^{2}\right)$, which (like $\xi$ and $\chi$ ) is positive at the boundary and negative at the horizon, and hence must have have a zero at some value of $u$ that we will call $u_{E^{2}}$. We showed above that $\left(G_{t t} G_{x x}-\tilde{E}^{2}\right)$ is negative at $u_{*}$, so the zero must obey $u_{E^{2}}<u_{*}$ (it is closer to the boundary than $u_{*}$ ). Now suppose $\chi$ changes sign at $u_{\chi}>u_{*}$. As we just showed, the $a_{2}^{2}$ term is negative there, so the on-shell action would be imaginary on the interval $\left(u_{*}, u_{\chi}\right)$, hence we demand $u_{\chi} \leq u_{*}$. We exclude the possibility that $u_{\chi}<u_{*}$. We know that $u_{E^{2}}$ is also less than $u_{*}$, so we must compare $u_{\chi}$ and $u_{E^{2}}$. If $u_{\chi}<u_{E^{2}}$, then the on-shell action is imaginary on the interval $\left(u_{E^{2}}, u_{*}\right)$, and if $u_{\chi}>u_{E^{2}}$, the action is imaginary on the interval $\left(u_{\chi}, u_{*}\right)$. In order for the on-shell action to remain real for all $u$, then, we demand that $u_{\chi}=u_{*}$.

The upshot is that we obtain four equations, $\xi\left(u_{*}\right)=\chi\left(u_{*}\right)=a_{1}\left(u_{*}\right)=a_{2}\left(u_{*}\right)=0$, for four unknowns, $u_{*},\left\langle J^{x}\right\rangle,\left\langle J^{y}\right\rangle$, and $\left\langle J^{z}\right\rangle$. The equation $\xi\left(u_{*}\right)=0$ gives us $u_{*}$, as we explained above. We will now solve for the currents $\left\langle J^{x}\right\rangle,\left\langle J^{y}\right\rangle$, and $\left\langle J^{z}\right\rangle$.

The equation $a_{1}\left(u_{*}\right)=0$ gives us $\left\langle J^{y}\right\rangle$, while the equation $a_{2}\left(u_{*}\right)=0$ gives us $\left\langle J^{z}\right\rangle$. We then plug the results for $\left\langle J^{y}\right\rangle$ and $\left\langle J^{z}\right\rangle$ into $\chi\left(u_{*}\right)=0$ to find $\left\langle J^{x}\right\rangle$. The result for the current in each case includes an overall factor of $E$, so invoking Ohm's law $\left\langle J^{i}\right\rangle=\sigma_{i x} E$, we identify the components of the conductivity tensor

$\sigma_{x x}=\frac{G_{x x}^{2}+\tilde{B}_{x}^{2}}{G_{x x}\left(G_{x x}^{2}+\tilde{B}_{x}^{2}+\tilde{B}_{z}^{2}\right)} \sqrt{\mathcal{N}^{2}\left(2 \pi \alpha^{\prime}\right)^{4} G_{x x}\left(G_{x x}^{2}+\tilde{B}_{x}^{2}+\tilde{B}_{z}^{2}\right) \cos ^{6} \theta\left(u_{*}\right)+\left(2 \pi \alpha^{\prime}\right)^{2}\left\langle J^{t}\right\rangle^{2}}$, 


$$
\begin{gathered}
\sigma_{x y}=\frac{\left(2 \pi \alpha^{\prime}\right) \tilde{B}_{z}\left\langle J^{t}\right\rangle}{G_{x x}^{2}+\tilde{B}_{x}^{2}+\tilde{B}_{z}^{2}}, \\
\sigma_{x z}=\frac{\tilde{B}_{x} \tilde{B}_{z}}{G_{x x}^{2}+\tilde{B}_{x}^{2}} \sigma_{x x},
\end{gathered}
$$

where all functions of $u$ are evaluated at $u_{*}$. In analogy with equation (3.3.67), we define

$$
\rho \equiv \pi \alpha^{\prime} u_{h}^{2}\left\langle J^{t}\right\rangle=\frac{\left\langle J^{t}\right\rangle}{\frac{\pi}{2} \sqrt{\lambda} T^{2}} .
$$

We then use the result for $G_{x x}^{2}\left(u_{*}\right)$ in equation (3.3.68) to write the components of the conductivity tensor in terms of SYM theory quantities

$$
\begin{gathered}
\sigma_{x x}=\sqrt{\frac{N_{f}^{2} N_{c}^{2} T^{2}}{16 \pi^{2}} \frac{\left(\mathcal{F}+b_{x}^{2}\right)^{2}}{\sqrt{\mathcal{F}}\left(\mathcal{F}+b_{x}^{2}+b_{z}^{2}\right)} \cos ^{6} \theta\left(u_{*}\right)+\frac{\rho^{2}\left(\mathcal{F}+b_{x}^{2}\right)^{2}}{\mathcal{F}\left(\mathcal{F}+b_{x}^{2}+b_{z}^{2}\right)^{2}}}, \\
\sigma_{x y}=\frac{\rho b_{z}}{\mathcal{F}+b_{x}^{2}+b_{z}^{2}}, \\
\sigma_{x z}=\frac{b_{x} b_{z}}{\mathcal{F}+b_{x}^{2}} \sigma_{x x} .
\end{gathered}
$$

As in $[105,106]$, the result for $\sigma_{x x}$ includes two terms adding in quadrature. As discussed in $[105,106,116]$, these two terms have different physical interpretations. The system has two types of charge carriers. First we have the density of charge carriers we introduced explicitly in $\left\langle J^{t}\right\rangle$, whose contribution appears as the second term under the square root in $\sigma_{x x}$. Even when $\left\langle J^{t}\right\rangle=0$ we find a nonzero $\sigma_{x x}$ and hence a nonzero current, however, so the system must have some other source of charge carriers.

The other type of charge carriers come from pair production in the electric field. Their contribution appears as the term in $\sigma_{x x}$ with the $\cos ^{6} \theta\left(u_{*}\right)$ factor. We have two pieces of evidence that suggests the $\cos ^{6} \theta\left(u_{*}\right)$ term represents pair production. First is the behavior of the pair-production term as a function of the mass $m$. When $m \rightarrow \infty$, so that the pair production should be suppressed, we indeed have $\cos ^{6} \theta\left(u_{*}\right) \rightarrow 0$, while when $m \rightarrow 0$, so that the pair production should be maximal, we have $\cos ^{6} \theta\left(u_{*}\right) \rightarrow 1$. Second, as shown in [116] for the case with $B_{x}=0$, when the density $\left\langle J^{t}\right\rangle=0$ the flavor fields have zero momentum in the $\hat{x}$ direction, which is consistent with pair production: the oppositelycharged particles in each pair move in opposite directions, producing a finite $\left\langle J^{x}\right\rangle$ but zero net momentum. For our case, with $B_{x} \neq 0$, we see that $\sigma_{x z} \propto \sigma_{x x}$, so both types of charge carriers contribute to $\left\langle J^{z}\right\rangle$, too. Using the stress-energy tensor as calculated in [5], in particular for $\left\langle T_{x}^{t}\right\rangle$ and $\left\langle T_{z}^{t}\right\rangle$, we can show that when $\left\langle J^{t}\right\rangle=0$, the flavor fields have zero momentum in the $\hat{x}$ and $\hat{z}$ directions, so we again find a nicely consistent picture.

\subsubsection{Interesting limits of the conductivity tensor}

We will check now our result in the classical quasi-particle limit. More specifically, we take $m$ to be much larger than any other scale in the problem, which includes not only $T$ but also the scale of thermal corrections to the energy of a heavy quark, $\frac{1}{2} \sqrt{\lambda} T$ [152]. We 
will call this the " $m \rightarrow \infty$ " limit. As explained in section 2.5 .2 .2 in that limit, $\theta(u) \rightarrow \frac{\pi}{2}$ and hence $\cos ^{6} \theta\left(u_{*}\right) \rightarrow 0$.

In this limit, we expect the charge carriers to behave as classical quasi-particles experiencing a drag force due to the $\mathcal{N}=4 \mathrm{SYM}$ plasma and a Lorentz force due to the external electric and magnetic fields. Our answer for the conductivity should then reduce to the Drude form. Let us briefly review what the Drude result is. Consider a density $\left\langle J^{t}\right\rangle$ of massive quasi-particles propagating through an isotropic, homogeneous, dissipative neutral medium. In the rest frame of the medium we introduce an electric field $\vec{E}$ in the $\hat{x}$ direction, and a magnetic field $\vec{B}$ with a component $B_{z}$ in the $\hat{z}$ direction and a component $B_{x}$ in the $\hat{x}$ direction. The force on a quasi-particle is then

$$
\frac{d \vec{p}}{d t}=\vec{E}+\vec{v} \times \vec{B}-\mu \vec{p}
$$

where our quasi-particle has charge +1 and $\mu$ is a drag coefficient. We replace the momentum with the velocity using $\vec{p}=M \vec{v}$ for quasi-particle mass $M$. We then replace the velocity with the induced current using $\vec{v}=\langle\vec{J}\rangle /\left\langle J^{t}\right\rangle$. Imposing the steady-state condition $\frac{d \vec{p}}{d t}=0$ and solving for $\langle\vec{J}\rangle$ yields

$$
\sigma_{x x}=\sigma_{0} \frac{\left(B_{x} / \mu M\right)^{2}+1}{|\vec{B}|^{2} /(\mu M)^{2}+1}, \quad \sigma_{x y}=\sigma_{0} \frac{\left(B_{z} / \mu M\right)}{|\vec{B}|^{2} /(\mu M)^{2}+1}, \quad \sigma_{x z}=\sigma_{0} \frac{\left(B_{x} / \mu M\right)\left(B_{z} / \mu M\right)}{|\vec{B}|^{2} /(\mu M)^{2}+1}
$$

where $\sigma_{0}=\left\langle J^{t}\right\rangle / \mu M$ is the conductivity when $\vec{B}=0$.

To show that our answer reduces to the Drude result, equation (3.3.73), when $m \rightarrow \infty$, we need to know what $\mu M$ is for our charge carriers, that is, we must compute the drag force on the charge carriers, following $[105,106]$. We begin by rewriting the force law equation (3.3.72), in the steady state, as

$$
\begin{aligned}
\mu|\vec{p}| & =\sqrt{E^{2}+|\vec{v} \times \vec{B}|^{2}+2 \vec{E} \cdot(\vec{v} \times \vec{B})} \\
& =\sqrt{E^{2}+v_{y}^{2}\left(B_{x}^{2}+B_{z}^{2}\right)+\left(v_{z} B_{x}-v_{x} B_{z}\right)^{2}+2 E_{x} v_{y} B_{z}} .
\end{aligned}
$$

As $m \rightarrow \infty$, pair creation will be suppressed and only the charge carriers in $\left\langle J^{t}\right\rangle$ will contribute to $\langle\vec{J}\rangle$, hence we may write $\langle\vec{J}\rangle=\left\langle J^{t}\right\rangle \vec{v}$, where we drop the $\cos \theta\left(u_{*}\right)$ terms in $\left\langle J^{x}\right\rangle$ and $\left\langle J^{z}\right\rangle$, as these vanish in our $m \rightarrow \infty$ limit. Notice that all components of the conductivity tensor are then proportional to $\left\langle J^{t}\right\rangle$, so from our answer for the conductivity tensor we find the components of $\vec{v}=\langle\vec{J}\rangle /\left\langle J^{t}\right\rangle$ as functions of $E, B_{x}$ and $B_{z}$. What is more instructive, however, is to use the original equations $\xi\left(u_{*}\right)=\chi\left(u_{*}\right)=a_{1}\left(u_{*}\right)=a_{2}\left(u_{*}\right)=0$ to write $\vec{v}$ in terms of $G_{x x}\left(u_{*}\right)$ and $G_{t t}\left(u_{*}\right)$. For example, the speed of the heavy charge carriers is

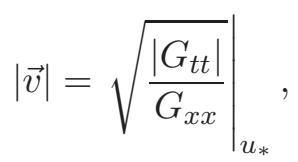

which is the local speed of light at $u_{*}$. The drag force is

$$
\mu|\vec{p}|=\frac{1}{2 \pi \alpha^{\prime}} \sqrt{\left|G_{t t}\left(u_{*}\right)\right| G_{x x}\left(u_{*}\right)}
$$

which is simply the Nambu-Goto Lagrangian (density) for a string extended in the $\hat{x}$ direction, sitting at fixed radial position $u_{*}$. Following $[105,106,152,153]$, if we employ 
the relativistic relation $|\vec{p}|=\gamma M v$ with $\gamma=\frac{1}{\sqrt{1-v^{2}}}$ and $M$ the quasi-particle mass, then we find

$$
\mu M=\frac{1}{2 \pi \alpha^{\prime}} \sqrt{G_{x x}\left(u_{*}\right)^{2}-\left|G_{t t}\left(u_{*}\right)\right| G_{x x}\left(u_{*}\right)}=\frac{\pi}{2} \sqrt{\lambda} T^{2},
$$

which is identical to the zero-density result of $[152,153]$ and the finite density results of $[105,106]$, but now with nonzero $B_{x}$. That we recover the same answer is not surprising in the probe limit $N_{f} \ll N_{c}$. In the probe limit, the flavor excitations are too dilute to experience a significant number of collisions with one another. Most of their energy loss comes from their interactions with the $\mathcal{N}=4 \mathrm{SYM}$ plasma, rather than with other flavor excitations, hence the drag force is independent of $\left\langle J^{t}\right\rangle$. See $[73,106]$ for more detailed explanations.

We can now compare to the Drude form (3.3.73). We take $m \rightarrow \infty$, so that $\cos ^{6} \theta\left(u_{*}\right) \rightarrow 0$ in the conductivity tensor. We also linearize in the electric field, that is, we consider the regime of linear response, where the currents are linear in $E$ and hence the conductivity is constant in $E$. (Recall that the Drude form relies on Maxwell's equations, which are linear.) In practical terms, that means setting $E=0$ in our result for the conductivity. That means we take $\mathcal{F}\left(e=0, b_{x}, b_{z}\right)=1$ as explained above. Lastly, using our identification of $\mu M$ in equation (3.3.77), we can write

$$
\rho=\frac{\left\langle J^{t}\right\rangle}{\frac{\pi}{2} \sqrt{\lambda} T^{2}}=\frac{\left\langle J^{t}\right\rangle}{\mu M},
$$

and similarly for $b_{x}$ and $b_{z}$ (recall equation (3.3.67)). We immediately find that our result for the conductivity tensor is identical to the Drude form, equation (3.3.73).

Finally, given that the novelty of our result is the presence of $B_{x}$, we can take limits that highlight the effects of $B_{x}$. For example, we can show that, generically, $B_{x}$ enhances the process of pair production. We first linearize in the electric field again, so $\mathcal{F}=1$, and then isolate the pair production term by taking zero density $\left(\left\langle J^{t}\right\rangle=0\right.$, hence $\left.\rho=0\right)$. The result for $\sigma_{x x}$ is then

$$
\sigma_{x x}=\frac{N_{f} N_{c} T}{4 \pi} \frac{1+b_{x}^{2}}{\sqrt{1+b_{x}^{2}+b_{z}^{2}}} \cos ^{3} \theta\left(u_{*}\right) .
$$

If we further consider $b_{x} \gg b_{z}$, then we see that $\sigma_{x x}$ has a $\sqrt{1+b_{x}^{2}}$ factor. Clearly, increasing $B_{x}$ increases the contribution to $\left\langle J^{x}\right\rangle$ from pair production. Conversely, if we suppress the pair production by taking $m \rightarrow \infty$, so that $\cos ^{6} \theta\left(u_{*}\right) \rightarrow 0$, while keeping $\left\langle J^{t}\right\rangle$ finite, then $\sigma_{x x}$ reduces to

$$
\sigma_{x x}=\rho \frac{1+b_{x}^{2}}{1+b_{x}^{2}+b_{z}^{2}}
$$

(which is of course the Drude result from equation (3.3.73)) so that now taking $b_{x} \gg b_{z}$ we find that $\sigma_{x x} \rightarrow \rho$. Increasing $B_{x}$ does not enhance the contribution to $\left\langle J^{x}\right\rangle$ coming from the net density $\left\langle J^{t}\right\rangle$ of charge carriers. By contrast, the limit $b_{z} \gg b_{x}$ clearly suppresses both contributions to the current.

\subsubsection{The Stress-Energy tensor}

In addition to the results presented here we also used our holographic setup to compute the contribution that the flavor fields make to the expectation value of the stress-energy tensor 
of the field theory. We will call this contribution $\left\langle T_{\nu}^{\mu}\right\rangle$. We identified certain divergences in the stress-energy tensor which are related to the rates of energy and momentum loss of the charge carriers, the flavor fields. Moreover, we were able to identify two special quantities that are free from these IR divergences, 10

The detailed calculations and results may be found in the paper [5] written in collaboration with Hai Ngo and Andy O'Bannon.

${ }^{10}$ These results are a direct extension of the work published in [116] to include nonzero $B_{x}$. 


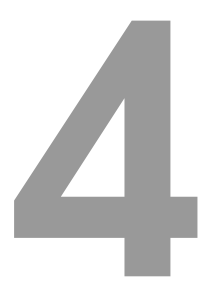

\section{Holographic Superconductors}

So far we have considered only one species of flavor fields and a finite $U(1)$ baryon chemical potential for the flavor fields. In this chapter we generalize the approach to $N_{f}=2$ species of flavor fields, with equal masses, which we call $u$ and $d$. Moreover, we turn on a nonvanishing isospin chemical potential $\mu_{I}$, which breaks the $S U(2)$ flavor symmetry to $U(1)_{3}$.

We find a phase transition at a critical value of the isospin chemical potential $\mu_{I}$. For larger values of the chemical potential we observe a condensation of a gauge-invariant bilinear of the flavor fields, i.e. a meson. This condensation breaks the remaining $U(1)_{3}$ flavor symmetry spontaneously. The phase transition is second order with mean field values. Since the order parameter of the phase transition is a vector and the signatures are similar to those of superconductors, we call the new phase a holographic p-wave superfluid/superconductor.

Note that the setup presented in this chapter may be viewed as the first embedding of holographic superconductors in string theory [2]. As reviewed in section 1.3, physicists investigated superconductors using phenomenological models in the bottom-up approach. Here, we investigate superconductors in the top-down approach. In particular, we investigate a consistent embedding of such models in string theory using probe Dp-branes. This enables us to identify explicitly the degrees of freedom which become unstable for sufficiently large isospin chemical potential. The gravity setup also allows for a dual string theory picture of the instability and the resulting condensation process. Finally, since the configuration is rather generic, we identified a whole class of string theory embeddings with superconducting phases.

This chapter is based on my own work done in collaboration with Johanna Erdmenger, Viviane Grass, Matthias Kaminski, Patrick Kerner and Andy O'Bannon. The work is published in $[2,3,7,8]$. 


\subsection{Introduction and summary}

As discussed in section 1.3 of the introduction, the holographic realization of superconductors were first realized using phenomenological models in the bottom-up approach. The starting point is Einstein-Hilbert gravity with negavite cosmological constant coupled to a U(1) gauge field. Superconductors can be built by adding new fields to this holographic setup, which may condense for low temperatures. In particular, to model s- or p-wave superconductors, we add a scalar field to the theory or promote the $\mathrm{U}(1)$ gauge field to a non-abelian $\mathrm{SU}(2)$ gauge field.

As a new result, we propose in this chapter a model which represents a stringy realization of superfluidity/superconductivity in a relativistic framework ${ }^{1}$ A string theory realization of holographic superconductors has two advantages compared to the bottom-up approaches discussed in the introduction of the thesis: First, we embed holographic superconductors in a consistent string background. Second, we know the dual field theory side explicitly and we are able to write down the corresponding Lagrangian. Therefore it is possible to identify the condensate in terms of elementary fields and to compare the results found in the holographic description at strong coupling with the corresponding results at weak coupling.

For the stringy realization, we use $N_{f}=2$ coincident probe Dp-branes in the supergravity background of $N_{c}$ D3-branes which correspond to two different species of fundamental matter having the same mass. Moreover, we consider a finite isospin chemical potential $\mu_{I}$ for the two species of fundamental matter. The dual field theory is described in section 4.2. In particular we argue that mesons have to condense above a critical value of the isospin chemical potential (or, equivalently, below a critical temperature $T_{c}$ ).

The precise holographic setup is described in section 4.3.1. In order to determine the dynamics of the flavor degrees of freedom, we have to use a non-Abelian version of the effective action for Dp-branes. However, note that such an effective action is not known beyond $F^{4}$. Therefore we have to modify the effective action. We use three different approximations: (1) truncate the action to the lowest non-trivial order in the field strength tensor, i.e. to $F^{2}$, (2) truncate the action to the largest order in the field strength tensor (which we trust), i.e. to $F^{4}$ and (3) use an effective action which captures all orders in the field strength tensor. Although we used these three different approximations for the effective Dp-brane action, it is amazing that the results agree qualitatively.

In all three approaches, we find a second order phase transition with mean field values between a phase with no meson condensate, i.e. $\left\langle J_{1}^{x}\right\rangle=0$, and phase in which the meson condenses, $\left\langle J_{1}^{x}\right\rangle \neq 0$. Note that the order parameter of the phase transition is a vector (here we choose the vector to be in $\mathrm{x}$-direction). Hence, the phase transition breaks the rotational invariance and is called $p$-wave. Furthermore, the vacuum expectation value $\left\langle J_{1}^{x}\right\rangle$ breaks the remaining $U(1)_{3}$ flavor symmetry spontaneously. We can draw the following analog: the $U(1)_{3}$ flavor symmetry can be related to the gauge group $U(1)_{e m}$ of electromagnetism and $J_{1}^{x}$ can be identified with the superconducting condensate. Therefore this phase has properties well-known from superfluids and superconductors. In section 4.3.4 we also calculate the frequency dependent ac conductivity $\sigma(\omega)$ and find a gap in the superconducting phase for small frequencies $\omega$. Moreover, the dc conductivity is infinite.

\footnotetext{
${ }^{1}$ Other String (and M-) theory embeddings of holographic s-wave superfluids appear in [154-156].
} 


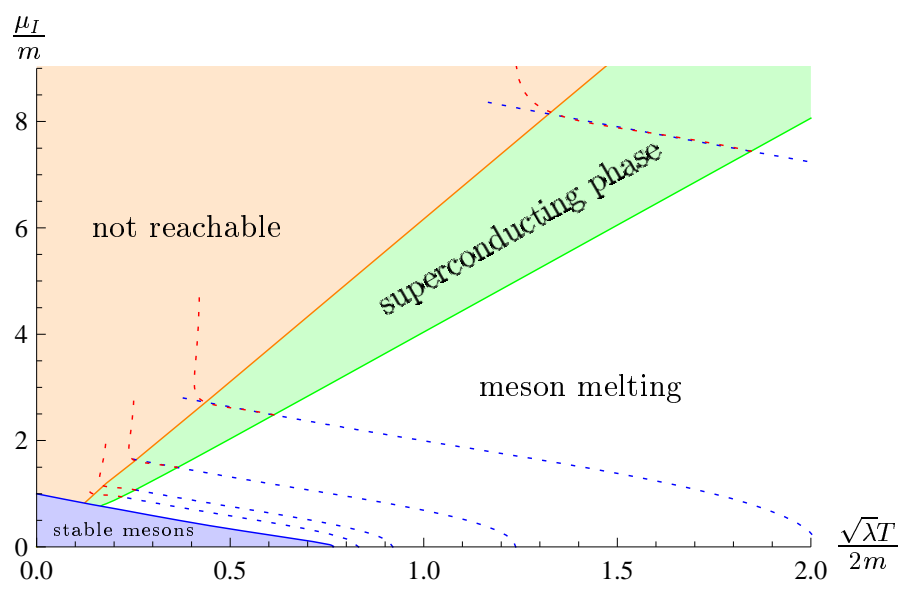

Figure 4.1: Phase diagram for two $\mathcal{N}=2$ supersymmetric hypermultiplets with mass $m$ coupled to a $\mathcal{N}=4$ supersymmetric vector multiplet at finite temperature $T$ and finite isospin chemical potential $\mu_{I}$. This field theory is dual to two probe D7 branes in $A d S_{5} \times S^{5}$. The phase diagram was obtained with the adapted symmetrized trace prescription: In the blue phase, in which the isospin density is zero, the mesons are stable, i.e. the decay width is zero. In the other phases, the mesons have a finite decay width. We refer to this as meson melting. The new superfluid phase is green. In the yellow phase we cannot trust our numerics since we ignore the backreaction. The dotted curves correspond to lines at finite mass. These curves are parametrized by the density $\tilde{d}_{t}^{3}$. Along the blue curves the field $A_{x}^{1}$ is zero while along the red ones the field $A_{x}^{1}$ is non-zero. The endpoints of the red curves determine the second order phase transition to the superconducting phase. The dotted, red curves diverge inside the superconducting phase since the backreaction of the condensate on the background is not considered. This divergence determines the boundary of the orange region which is not reachable without backreaction. Figure made by Patrick Kerner.

Although we refer to the new phase as a holographic p-wave superconductor, the spontaneous breaking of a global $U(1)$ corresponds, strictly speaking, to a superfluid. To view our system as a superconductor, it would be necessary to gauge the global $U(1)_{3}$ flavor symmetry which is broken spontaneously in our model. However, many features of superconductivity do not depend on whether the $U(1)_{3}$ is gauged. One exception to this is the Meissner-Ochsenfeld effect. To generate the currents expelling the magnetic field, the $U(1)_{3}$ symmetry has to be gauged.

Indeed we find a remnant of the Meissner-Ochsenfeld effect [3]. Since the spontaneously broken $U(1)_{3}$ flavor symmetry is global, magnetic fields cannot be expelled by the system. We note that a constant magnetic field reduces the critical temperature [3]. We can argue as follows: if we weakly gauge the global $U(1)_{3}$ symmetry, i.e. if we promote the global symmetry to a local symmetry and introduce the corresponding $U(1)$ gauge bosons, the superconducting current $\left\langle J_{1}^{x}\right\rangle$ generates a magnetic field opposite to the constant external field. Thus the phase observed in [3] is a necessary condition, in the case of a global symmetry, to find the Meissner-Ochsenfeld effect, if the symmetry is gauged. Hence, after gauging the local $U(1)_{3}$ symmetry, the phase is superconducting.

Furthermore, in section 4.4 we present a string-theoretical picture, why the system is instable above a critical value of the isospin chemical potential. Moreover, we identify the condensate $\left\langle J_{1}^{x}\right\rangle$ as condensing D7-D7 strings in this picture and therefore have a geometrical understanding of the superconducting condensate.2

\footnotetext{
${ }^{2}$ This phenomena can also be viewed as the dynamical generation of meson masses.
} 
The main result of this chapter can be summarized in figure 4.1. This figure shows the phase diagram for two $\mathcal{N}=2$ supersymmetric hypermultiplets with mass $m$. The hypermultiplets are coupled to the $\mathcal{N}=4$ super Yang-Mills theory as described in section 2.5.1.1 and can propagate in 3+1-dimensions. The dual gravity theory consists of two coincident D7-branes wrapping asymptotically $A d S_{5} \times S^{3}$. We consider this field theory at finite temperature $T$ and finite isospin chemical potential $\mu_{I}$.

We can compare figure 4.1 to the phase diagram for baryon chemical potential (see figure 2.2). Besides the green and yellow areas, both figures agree. In both figures, the charge density is zero in the blue phase: $\left\langle J^{t}\right\rangle=0$ for the baryon case and $\left\langle J_{3}^{t}\right\rangle=0$ for the isospin case. In both blue shadowed phases, the mesons are stable, i.e. the decay width is zero. In the rest of the phase diagram, there exists a finite charge density and the mesons have a finite lifetime. We refer to those phases as meson melting phases. Let us discuss the new phase: in the yellow and green areas mesons condense. For sufficiently large, but not too large, values of the isospin chemical potential, only vector mesons condense, i.e. $\left\langle J_{1}^{x}\right\rangle \neq 0$. If we further increase the isospin chemical potentials, other mesons may also condense. The yellow and green shadowed phases are superconducting since the remaining $U(1)_{3}$ flavor symmetry is spontaneously broken due to a finite meson condensate $\left\langle J_{1}^{x}\right\rangle$.

\subsection{Field theory description}

Before we discuss the holographic setup, we investigate the field theory side in more detail in the this section. We consider a strongly-coupled $\mathcal{N}=4$ supersymmetric Yang-Mills theory with gauge group $S U\left(N_{c}\right)$ in the large- $N_{c}$ limit coupled to $N_{f}=2$ species of flavor fields (called $\mathrm{u}$ and $\mathrm{d}$ ) with the same mass $m$, which may be confined to a defect. We study thermal equilibrium states with temperature $T$, and introduce an isospin chemical potential $\mu_{I}$ as a source of the operator $J_{3}^{t}$. The hypermultiplets $\mathrm{u}(\mathrm{d})$ have a positive (negative) chemical potential, respectively. Note that the $U(2)$ flavor symmetry of the theory is broken to $U(1)_{B} \times U(1)_{3}$ by considering a non-vanishing chemical potential. Here, $U(1)_{B}$ is the usual baryon symmetry, acting schematically as $u \rightarrow e^{i \alpha} u, d \rightarrow e^{i \alpha} d$. In contrast, the symmetry $U(1)_{3}$ acts as $u \rightarrow e^{i \alpha} u, d \rightarrow e^{-i \alpha} d$. The global symmetry $U(1)_{3}$ plays a crucial role in this chapter.

For sufficiently large $\mu_{I}$, the system develops a nonzero $\left\langle J_{1}^{x}\right\rangle$. The operator $J_{1}^{x}$ is a gaugeinvariant bilinear in the flavor fields, valued in the adjoint of $U\left(N_{f}\right)$. The precise form of the operator $J_{1}^{x}$ depends on the details of the flavor fields. For example, for two $\mathcal{N}=2$ supersymmetric hypermultiplets $\sqrt[3]{3}$ called $u=\left(q_{u}, \psi_{u}\right)$ and $d=\left(q_{d}, \psi_{d}\right)$, the operators $J_{3}^{t}$ and $J_{1}^{x}$ read

$$
\begin{aligned}
J_{3}^{t} & =\bar{\psi} \sigma^{3} \gamma^{t} \psi+q \sigma^{3} \partial_{t} \bar{q}=\bar{\psi}_{u} \gamma^{t} \psi_{u}-\bar{\psi}_{d} \gamma^{t} \psi_{d}+\text { bosons }=n_{u}-n_{d} \\
J_{1}^{x} & =\bar{\psi} \sigma^{1} \gamma^{x} \psi+q \sigma^{1} \partial_{x} \bar{q}=\bar{\psi}_{u} \gamma^{x} \psi_{d}+\bar{\psi}_{d} \gamma^{x} \psi_{u}+\text { bosons }
\end{aligned}
$$

Here, $n_{u}$ and $n_{d}$ are the charge density of the isospin fields, $q=\left(q_{u}, q_{d}\right)$ and $\psi=\left(\psi_{u}, \psi_{d}\right)$. $\sigma^{i}$ are the usual Pauli matrices.

The operator $J_{1}^{x}$ is precisely what we call a vector meson, and the phase transition appears to be vector meson condensation. To be precise, the spectrum of the D3/Dp theory includes gauge-invariant bound states of flavor fields. We refer to such bosonic or

\footnotetext{
${ }^{3}$ For more details see section 2.5.1.1.
} 
fermionic bound states as mesons or mesinos, respectively. For massive flavor fields, these mesons/mesinos are typically the lightest flavor degrees of freedom in the theory $[89,113]$. We may thus imagine writing an effective theory for these degrees of freedom, analogous to the chiral Lagrangian of QCD. An isospin chemical potential $\mu_{I}$ acts as a negative masssquared for any mesons/mesinos charged under $U(1)_{3}$. If we make $\mu_{I}$ sufficiently large, then we expect Bose-Einstein condensation of mesons. In QCD, we expect the lightest charged mesons, the pions, to condense first producing a scalar condensate (and hence an s-wave superfluid), while the heavier vector mesons may condense at higher $\mu_{I}[157,158]$. Which mesons condense first in D3/Dp systems depends on the details of the system. 4 The general lesson from these D3/Dp systems is that the p-wave superfluid phase transition appears to be vector meson condensation, which is in line with our weak-coupling intuition.

Moreover, thinking of the p-wave states as a Bose-Einstein condensate makes many potentially confusing features of the p-wave state transparent. For example, the p-wave transition appears to involve the spontaneous generation of a persistent current $\left\langle J_{1}^{x}\right\rangle$, that is, at high density charges begin moving without experiencing dissipation 5 While not impossible, such a scenario naturally raises some questions. Why do charges start moving? How does that lower the free energy? Vector meson condensation neatly accounts for all of the physics: we merely see Bose-Einstein condensation, i.e. bosons populating a zero-momentum state, the main novelty being that the bosons are vectors, not scalars.

\subsection{Dual Gravity picture}

\subsubsection{Holographic setup}

We study $\mathcal{N}=4$ super Yang-Mills coupled to $N_{f}=2$ species of massless flavor degrees of freedom at finite temperature. The flavor degrees of freedom may be constrained to a defect. Moreover, we turn on a non-vanishing isospin chemical potential.

In the dual holographic setup the $\mathcal{N}=4 \mathrm{SYM}$ degrees of freedom are given by the background supergravity solution including a metric and Ramond-Ramond five-form. In order to describe a state with finite temperature, the spacetime is (4+1)-dimensional AdSSchwarzschild times $S^{5}$ with metric $(2.4 .80) \cdot 6$

The flavor fields are introduced by two coincident probe Dp-branes, extended along $A d S_{P} \times$ $S^{Q}$. In this section we consider only the trivial embedding of the Dp-branes, i.e. we consider solutions in which all the Dp-branes' worldvolume scalars, including scalars in

\footnotetext{
${ }^{4}$ In other holographic models which are more realistic for describing QCD, such as the Sakai-Sugimoto model [136], holographic calculations suggest that indeed the pions condense first and the vector mesons second, as we increase $\mu_{I}[159,160]$.

${ }^{5}$ Crucially, however, no net momentum is flowing. In holographic calculations, in both the probe and fully back-reacted cases [7,161,162], the Yang-Mills stress-energy tensor and the metric are diagonal, and indeed the bulk spacetime is static, which indicates that the expectation value of the field theory stressenergy tensor is strictly diagonal. The system thus has zero net momentum. If charges are moving, they must be doing so in pairs that move in opposite directions. A static bulk spacetime also indicates that the energy density of the field theory is not changing in time: the system is not heating up, consistent with the fact that the moving charges experience no dissipation.

${ }^{6}$ In our units, where the AdS radius is one, we can convert between string theory and field theory quantities using $\alpha^{\prime-2}=4 \pi g_{s} N_{c}=2 g_{Y M} N_{c}=2 \lambda$.
} 
$A d S_{5}$ directions, are zero. The dual flavor fields are then massless. The induced metric on the Dp-branes reads

$$
d s_{D p}^{2}=\frac{1}{u^{2}}\left(\frac{d u^{2}}{f(u)}-f(u) d t^{2}+d \vec{x}^{2}\right)+d s_{S^{Q}}^{2},
$$

where now $d \vec{x}^{2}$ represents the $(P-2)$-dimensional Euclidean metric.

The dynamics of the flavor degrees of freedom is encoded in the non-Abelian effective action for Dp-branes. The precise form is not known as reviewed in section A.3.1. The action

$$
S_{\mathrm{DBI}}=-\tau_{p} \operatorname{Str} \int_{D p} \mathrm{~d}^{p+1} \xi \sqrt{\operatorname{det} \mathrm{Q}}\left[\operatorname{det}\left(\mathcal{P}_{\mathrm{ab}}\left[\mathrm{E}_{\mu \nu}+\mathrm{E}_{\mu \mathrm{i}}\left(\mathrm{Q}^{-1}-\delta\right)^{\mathrm{ij}} \mathrm{E}_{\mathrm{j} \nu}\right]+2 \pi \alpha^{\prime} \mathrm{F}_{\mathrm{ab}}\right)\right]^{\frac{1}{2}}
$$

is only correct up to order $F^{4}$ in the field strength 7

\subsubsection{Truncation to $\alpha^{2}$}

Therefore we have to use an approximation. In this section we truncate the action to leading non-trivial order in $\alpha^{\prime}$, i.e. to order $\alpha^{\prime 2}$. The action (4.3.3) reduces then to a $U\left(N_{f}\right)$ non-Abelian Yang-Mills theory in $A d S_{P} \times S^{Q}$

$$
S_{D p}=-\tau_{p} N_{f} \int d^{p+1} \xi \sqrt{-G_{D p}}\left[1+\left(2 \pi \alpha^{\prime}\right)^{2} \frac{1}{2} \operatorname{Tr}\left(F_{\mu \nu} F^{\mu \nu}\right)\right] .
$$

Here, the integral is over the worldvolume coordinates $\xi^{\mu}, N_{f}=2, G_{D p}$ is the determinant of the induced metric, and the trace is taken over gauge indices. We use $S U(2)$ generators $\tau_{a}=\frac{1}{2} \sigma_{a}$ such that, with $\epsilon_{123}=+1$,

$$
\left[\tau_{a}, \tau_{b}\right]=i \epsilon_{a b c} \tau_{c}
$$

The field strength $F_{\mu \nu}=F_{\mu \nu}^{a} \tau_{a}$ is given by

$$
F_{\mu \nu}^{a}=\partial_{\mu} A_{\nu}^{a}-\partial_{\nu} A_{\mu}^{a}+\epsilon^{a b c} A_{\mu}^{b} A_{\nu}^{c} .
$$

Since we turn on a non-vanishing isospin chemical potential, we consider the following ansatz for the gauge fields

$$
A=A_{x}^{1}(u) \tau_{1} d x+A_{t}^{3}(u) \tau_{3} d t,
$$

where the chemical potential is the leading order of $A_{t}^{3}(u)$ in a near-boundary expansion. We also have included a gauge field component $A_{x}^{1}(u)$ which describes the non-zero meson condensate. Inserting the ansatz (4.3.6) into the action (4.3.4) and using the explicit form of the metric components (2.4.80), the action density reads

$$
S_{D p}=-\frac{\left(2 \pi \alpha^{\prime}\right)^{2} \mathcal{N}}{4} \int \frac{d u}{u^{P}}\left(1-u^{4}\left(A_{t}^{3 \prime}(u)\right)^{2}+u^{4} f(u)\left(A_{x}^{1 \prime}(u)\right)^{2}-\frac{u^{4}}{f(u)}\left(A_{x}^{1}(u) A_{t}^{3}(u)\right)^{2}\right),
$$

\footnotetext{
${ }^{7}$ For more details, in particular for the precise definition of $Q$ and $E$, please consult section A.3.1 of the appendix A
} 
where $\mathcal{N}$ is given by equation (2.5.98).

For numerics, it is better to use the dimensionless quantities $\chi=\frac{u}{u_{h}}$ and $\tilde{A}_{t}^{3}=u_{h} A_{t}^{3}$, $\tilde{A}_{x}^{1}=u_{h} A_{x}^{1}$. The action density in these quantities is given by

$$
S_{D p}=-\frac{\left(2 \pi \alpha^{\prime}\right)^{2} \mathcal{N}}{4 u_{h}^{P-1}} \int_{0}^{1} \frac{d \chi}{\chi^{P}}\left(1-\chi^{4}\left(\partial_{\chi} A_{t}^{3}\right)^{2}+\chi^{4} f(\chi)\left(\partial_{\chi} A_{x}^{1}\right)^{2}-\frac{\chi^{4}}{f(\chi)}\left(A_{x}^{1} A_{t}^{3}\right)^{2}\right),
$$

where $f(\chi)=1-\chi^{4}$. The equations of motion for the gauge fields $\tilde{A}_{t}^{3}(\chi)$ and $\tilde{A}_{x}^{1}(\chi)$ are given by

$$
\begin{gathered}
\partial_{\chi}^{2} \tilde{A}_{t}^{3}(\chi)+\frac{4-P}{\chi} \partial_{\chi} \tilde{A}_{t}^{3}(\chi)-\frac{1}{f(\chi)} \tilde{A}_{t}^{3}(\chi)\left(\tilde{A}_{x}^{1}(\chi)\right)^{2}=0, \\
\partial_{\chi}^{2} \tilde{A}_{x}^{1}(\chi)+\left(\frac{4-P}{\chi}+\frac{\partial_{\chi} f(\chi)}{f(\chi)}\right) \partial_{\chi} \tilde{A}_{x}^{1}(\chi)+\frac{1}{f(\chi)^{2}}\left(\tilde{A}_{t}^{3}(\chi)\right)^{2} \tilde{A}_{x}^{1}(\chi)=0 .
\end{gathered}
$$

The equations of motion near the boundary $\chi \rightarrow 0$ determine the asymptotic forms of the solutions,

$$
\tilde{A}_{t}^{3}(\chi)=\tilde{c}_{t}^{3}-\tilde{d}_{t}^{3} \chi^{P-3}+\ldots, \quad \tilde{A}_{x}^{1}(\chi)=\tilde{d}_{x}^{1} \chi^{P-3}+\ldots,
$$

where ... represent terms that decay faster than $\chi^{P-3}$ as $\chi \rightarrow 0$. Note that there is no constant term in $\tilde{A}_{x}^{1}$. In principle, such a term would be allowed. However, we do not source the operator $\left\langle J_{1}^{x}\right\rangle$ on the field theory side. Therefore we set the parameter $\tilde{c}_{x}^{1}$ to zero.

The constants $\tilde{c}_{t}^{3}, \tilde{d}_{t}^{3}$ and $\tilde{d}_{x}^{1}$ are related to the chemical potential $\mu_{I}$, to the finite isospin density $\left\langle J_{3}^{t}\right\rangle$ and to the meson condensate $\left\langle J_{1}^{x}\right\rangle$, respectively. The precise mapping is given by

$$
\begin{aligned}
\tilde{c}_{t}^{3} & =\mu_{I} u_{h}=\frac{\mu_{I}}{\pi T}, \\
\tilde{d}_{t}^{3} & =u_{h}^{P-2} \mathcal{N}^{-1}\left(2 \pi \alpha^{\prime}\right)^{-2}\left\langle J_{3}^{t}\right\rangle \\
& =\frac{\left\langle J_{3}^{t}\right\rangle \Gamma((Q+1) / 2)}{4 N_{f} N_{c}(2 \lambda)^{(P+Q-8) / 4}(2 \pi)^{-P-Q+4} \pi^{(Q+1) / 2}(\pi T)^{P-2}}=\frac{\left\langle J_{3}^{t}\right\rangle}{\tilde{\mathcal{N}}_{P, Q} T^{P-2}},
\end{aligned}
$$

where in the second equality we converted to field theory quantities. Similarly,

$$
\left\langle J_{1}^{x}\right\rangle=\tilde{\mathcal{N}}_{P, Q} T^{P-2} \tilde{d}_{t}^{3} \sim N_{f} N_{c} T^{P-2} \tilde{d}_{x}^{1} .
$$

$\mathcal{N}_{P, Q}$ is given by

$$
\tilde{\mathcal{N}}_{P, Q}=\frac{4 N_{f} N_{c}(2 \lambda)^{(P+Q-8) / 4}(2 \pi)^{-P-Q+4} \pi^{(Q+1) / 2} \pi^{P-2}}{\Gamma\left(\frac{Q+1}{2}\right)} .
$$

Notice that $\tilde{\mathcal{N}}_{P, Q}$ and therefore also $\left\langle J_{3}^{t}\right\rangle$ and $\left\langle J_{1}^{x}\right\rangle$ are proportional to $N_{f} N_{c}$.

One solution of equations (4.3.9) has $\tilde{A}_{x}^{1}(\chi)=0$ and $\tilde{A}_{t}^{3}(\chi)=\tilde{c}_{t}^{3}\left(1-\chi^{P-3}\right)$, i.e. $A_{t}^{3}(u)$ reads

$$
A_{t}^{3}(u)=\mu_{I}\left(1-\frac{u^{P-3}}{u_{h}^{P-3}}\right) .
$$


Such a solution corresponds in the field theory to the normal phase, in which the chemical potential $\mu_{I}$ explicitly breaks the $S U(2)$ isospin symmetry down to the $U(1)_{3}$ flavor symmetry, but no spontaneous symmetry breaking occurs. These solutions exist for all values of $\mu_{I}$.

For sufficiently low temperatures $T$ or equivalently 8 for sufficiently large isospin chemical potential $\mu_{I}$, other solutions of equation (4.3.9) exists in which $\tilde{A}_{x}^{1}(\chi)$ is nonzero. These solutions correspond in the field theory to superfluid states, with nonzero $\left\langle J_{x}^{1}\right\rangle$, so $U(1)_{3}$ is spontaneously broken. For $P \geq 4$, the field theory's spatial rotational symmetry ${ }^{9}$ is also broken from $S O(P-2)$ down to $S O(P-3)$.

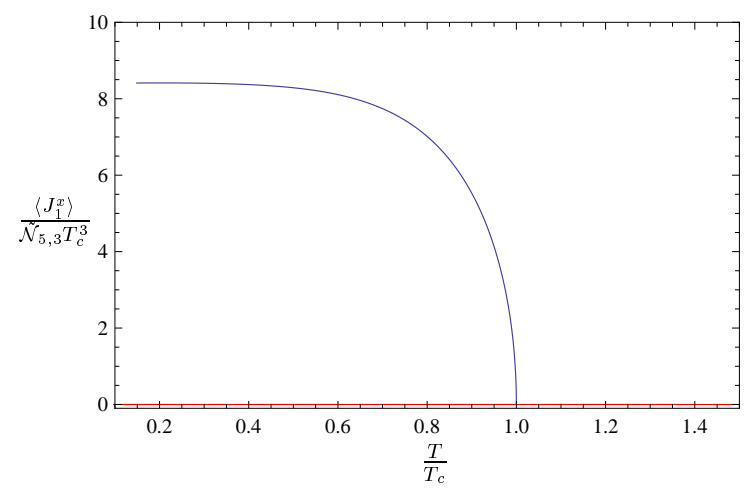

(a)

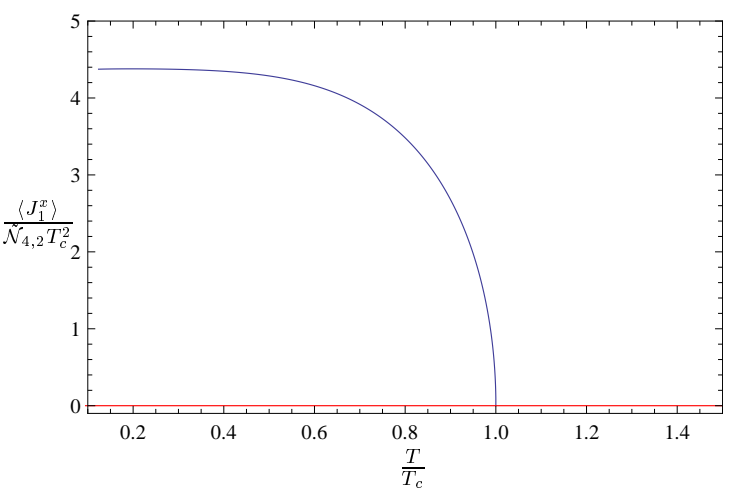

(b)

Figure 4.2: The superconducting condensate $\left\langle J_{1}^{x}\right\rangle$ as a function of the temperature $T / T_{c}$ for (a) D7-branes wrapping $A d S_{5} \times S^{3}$ and for (b) D5-branes wrapping $A d S_{4} \times S^{2} . T_{c}$ is the critical temperature where the phase transition happens. Note that for $T \rightarrow 0$ we obtain a finite value for $\left\langle J_{1}^{x}\right\rangle$. Near $T_{c}$ the superconducting condensate $\left\langle J_{1}^{x}\right\rangle$ behaves as $\left\langle J_{1}^{x}\right\rangle \propto\left(1-T / T_{c}\right)^{1 / 2}$. Therefore the critical exponent of the phase transition is $1 / 2$. Figures made by Patrick Kerner.

For temperatures below a critical temperature $T_{c}$, we have two solutions, so we need to determine which is thermodynamically preferred. Therefore we Wick-rotate our setup to Euclidean signature. In order to calculate the grand potential, we have to compute the Euclidean on-shell action of probe Dp-branes,

$$
\Omega_{f l .}\left(T, \mu_{I}\right)=-T \ln \mathcal{Z}_{f l .}=T S_{\star}^{E} \operatorname{Vol}\left(\mathbb{R}^{1, P-2}\right) .
$$

Here, $S_{\star}^{E}$ denotes the on-shell value of the renormalized action density in Euclidean signature. In order to get the action, and not the action density, we have to include a factor of $\operatorname{Vol}\left(\mathbb{R}^{1, P-2}\right)$. Recall that the time is compactified on a circle with circumference $T^{-1}$. This factor cancels the explicit temperature dependence in equation (4.3.16).

\footnotetext{
${ }^{8}$ Notice that these D3/Dp theories in the probe limit are scale-invariant since in the probe limit we neglect the quantum effects that would cause the $\mathcal{N}=4 \mathrm{SYM}$ coupling to run, and we are working with massless flavor fields. The theory is thus in a limit where no intrinsic scale appears. Therefore the only meaningful physical quantity is $\mu_{I} / T$, so fixing $T$ and increasing $\mu_{I}$ is equivalent to fixing $\mu_{I}$ and reducing $T$. We think in terms of the latter. Any transition must occur at a temperature $T_{c}$ set by the chemical potential, $T_{c} \propto \mu_{I}$

${ }^{9}$ When $P=3$ the flavor fields are confined to a $(1+1)$-dimensional defect and hence have no spatial rotational symmetry. Notice that in such cases the large $N_{c}$ limit is what permits spontaneous symmetry breaking to occur, by suppressing the fluctuations that would destroy long-range order.
} 
Using the explicit form of the action density (4.3.8), we obtain

$$
\Omega_{f l .}\left(T, \mu_{I}\right)=\frac{\tilde{\mathcal{N}}_{P, Q}}{4 \pi} T^{P-1} \int_{0}^{1} \frac{d \chi}{\chi^{P-4}}\left(-\left(\partial_{\chi} \bar{A}_{t}^{3}\right)^{2}+f(\chi)\left(\partial_{\chi} \bar{A}_{x}^{1}\right)^{2}-\frac{1}{f(\chi)}\left(\bar{A}_{x}^{1} \bar{A}_{t}^{3}\right)^{2}\right)
$$

Here, $\bar{A}_{t}^{3}(\chi)$ and $\bar{A}_{x}^{1}(\chi)$ are solutions of the equations of motion (4.3.9) with boundary asymptotics (4.3.10) corresponding to an isospin chemical potential $\mu_{I}$. This expression for the grand potential can be simplified by integration by parts and by using the equations of motion. We obtain

$$
\Omega_{f l .}\left(T, \mu_{I}\right)=\frac{\tilde{\mathcal{N}}_{P, Q}}{4 \pi} T^{P-1}\left((P-3) \tilde{c}_{t}^{3} \tilde{d}_{t}^{3}-\int_{0}^{1} \frac{d \chi}{\chi^{P-4}} f(\chi)\left(\partial_{\chi} \bar{A}_{x}^{1}\right)^{2}\right) .
$$

Let us discuss now two cases in more detail: two coincident D7-branes with $P=5$ and $Q=3$ and two coincident D5-branes with $P=4$ and $Q=2$. We will see that the results are qualitatively the same although the field theories are different. In the case of the D5branes, the hypermultiplets can only propagate in $2+1$ dimensions, whereas in the case of D7-branes there is no defect. The results are summarized in figures 4.2 and 4.3 .

Let us discuss the case of two coincident D7-branes with $P=5, Q=3$ first. In figure 4.2 (a) we plot $\left\langle J_{1}^{x}\right\rangle$ versus the rescaled temperature $T / T_{c}$. Note that $T_{c}$ is defined being the highest temperature below which a non-zero condensate $\left\langle J_{1}^{x}\right\rangle$ exists. Therefore the blue curve, corresponding to the superconducting phase with $\left\langle J_{1}^{x}\right\rangle \neq 0$, exists only for $T / T_{c} \leq 1$.

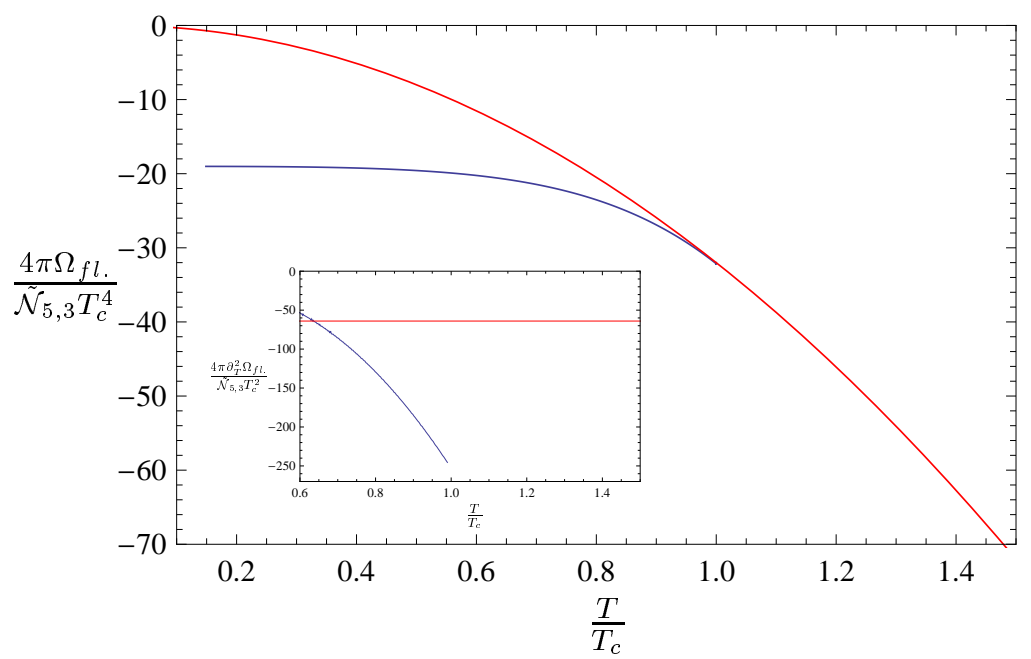

Figure 4.3: The rescaled grand potential $\Omega_{f l}$. for the D7-brane (with $P=5$ and $Q=3$ ) as a function of the temperature $T / T_{c}$. We see that the grand potential for the superfluid phase (blue curve) is smaller than for the normal phase (red curve). Therefore the superfluid phase is thermodynamically preferred. Note that the grand potential is differentiable for $T=T_{c}$. Moreover, we also plot the second derivative of the grand potential which is discontinuous. Figure made by Patrick Kerner.

To determine which is the thermodynamically preferred phase, we plot the grand potential $\Omega_{f l}$. as a function of the temperature $T / T_{c}$ in figure 4.3. We see that the grand potential of the superconducting phase, given by the red curve, is smaller than of the normal phase. 
Therefore the superconducting phase is thermodynamically preferred. We can also read off the order of the phase transition. The second derivative is discontinuous but not the first derivative.

From figure 4.2 (a) we can determine the critical exponent by fitting near the critical temperature. It turns out that the critical exponent is $1 / 2$, i.e.

$$
\left\langle J_{1}^{x}\right\rangle \propto\left(1-T / T_{c}\right)^{1 / 2} .
$$

In summary, the phase transition is second order with a mean field exponent of $1 / 2$.

Finally, the story for the case of two coincident D5-branes with $P=4$ and $Q=2$ is qualitatively the same. For $\mu_{I} \geq 3.81 \times(\pi T)$ the state with nonzero $\left\langle J_{x}^{1}\right\rangle$ has lower free energy. In other words, $T_{c}=\mu_{I} /(3.81 \times \pi)$. In figure 4.2 (b) we plot $\left\langle J_{1}^{x}\right\rangle /\left(T_{c}^{2} \tilde{\mathcal{N}}_{4,2}\right)$ versus the rescaled temperature $T / T_{c}$. Near the transition, $\left\langle J_{1}^{x}\right\rangle$ appears to have a mean-field exponent of $1 / 2$ as in the D7-brane case.

\subsubsection{Beyond the probe limit: backreaction}

Notice that these D3/Dp systems give rise to probe gauge fields, as defined in section 2.5.2, rather than gauge fields coming from the supergravity sector. The probe limit is sufficient to study many properties of the p-wave phase transition, however, the probe limit is known to fail at low temperatures. The solutions with nonzero $A_{x}^{1}(u)$ have a field strength that increases as we cool the system. Hence, we can no longer neglect the backreaction. To reach zero temperature, we must solve the fully coupled equations of motion.

In [7] we have included the backreaction of the gauge fields for a toy-model. We considered the action (4.3.7) (with $P=5, Q=3$ ) and coupled it to the usual Einstein-Hilbert term with negative cosmological constant. Note that this is only a toy-model for the case of coincident D7-branes since D7-branes would also source other fields, such as RR-potentials and the dilaton, in type IIB supergravity.

The only free parameter in [7] is the ratio $\alpha$ of the gravitational constant, $\kappa_{5}$ and the Yang-Mills coupling constant, $\hat{g}$,

$$
\alpha=\frac{\kappa_{5}}{\hat{g}} .
$$

Since $1 / \hat{g}^{2} \propto N_{f} N_{c}$ and $1 / \kappa_{5}^{2} \propto N_{c}^{2}, \alpha^{2}$ is proportional to $N_{f} / N_{c}$. Therefore the probe limit corresponds to $\alpha \rightarrow 0$.

In [7] we construct the backreacted solution for different values of $\alpha$ and confirm that the superconducting phase is thermodynamically preferred below a critical temperature. For small $\alpha$, including the probe limit, the phase transition is second order. Above the critical value $\alpha_{c}=0.365 \pm 0.001$, the phase transition is first order.

Moreover, we find 10 that the zero-temperature limits of the bulk hairy black hole solutions involves a domain wall interpolating between two regions, one near the boundary and one deep in the interior of the spacetime. The geometry interpolates between a near-boundary AdS space and an interior AdS space with a different radius of curvature and speed of light $[154,162-165]$. In field theory language, the interior AdS space represents an emergent

\footnotetext{
${ }^{10}$ We have not yet published this calculation. Similar results for $\mathrm{AdS}_{4}$ may be found in [161].
} 
conformal symmetry at low temperature and finite charge density. In other words, the emergent AdS represents a quantum critical point.

\subsubsection{Beyond $F^{2}$ and massive embeddings}

So far we have only discussed the truncation to order $\alpha^{\prime 2}$ and considered massless flavor fields. In [3] we extended our analysis for two coincident D7-branes with $P=5, Q=3$ to massive flavor fields and by including higher order terms of the field strength tensor. Since the non-Abelian version of the Dirac-Born-Infeld action is known only up to order $F^{4}$, we use the two following prescriptions:

(i) we truncate the non-Abelian Dirac-Born-Infeld action (4.3.3) to order $F^{4}$,

(ii) we incorporate all higher order terms in the field strength tensor $F$. For simplicity, we change the prescription of the symmetrized trace which is denoted by Str: we omit all commutators of the generators $\sigma^{i}$ and use $\left(\sigma^{i}\right)^{2}=\mathbb{1}$. We call this approximation the adapted symmetrized trace prescription.

Although these two prescriptions for the non-Abelian Dirac-Born-Infeld action (4.3.3) are not related in an obvious way, the results obtained in both prescriptions agree qualitatively. In both prescriptions, the superconducting phase exists below a critical temperature $T_{c}$, and is thermodynamically preferred. Moreover, the phase transition is second order with mean field values for the critical exponent.

The main results of [3] are the phase diagram in figure 4.1, which we already discussed in the introductory section 4.1, and the retarded Green's function for a particular component of the currents. As explained in section 3.1.1, we can calculate the ac conductivity from the retarded Green's function of the currents

$$
\sigma(\omega)=\frac{\tilde{\mathcal{G}}^{R}(\omega, \overrightarrow{0})}{i \omega} .
$$

Here we consider the component $J_{3}^{y}$ of the current. Note that $J_{3}^{y}$ is charged under the $U(1)_{3}$ symmetry and therefore is influenced by the spontaneous symmetry breaking. Moreover, the current is transverse to the superconducting condensate in spacetime.

The calculation of the frequency-dependent conductivity for alternating currents in the gravity setup is reviewed in section 3.2.2. Here we only list the results obtained in [3]. The real part of the ac conductivity, $\operatorname{Re} \sigma(\omega)$, is presented in figure 4.4 for the symmetrized trace prescription. It shows the appearance and growth of a gap in the frequency as we decrease the temperature below the critical temperature $T_{c}$. The conductivity gap originates in a pseudo gap already present right above $T_{c}$, as can be seen from the red curve in figure 4.4. By a pseudo gap we mean a well-defined gap in the ac conductivity at low frequency $\omega$ in which the conductivity is not identically zero [166]. If the mass of the hypermultiplet is zero we also find prominent peaks in the ac conductivity. If we increase the mass $m$ of the hypermultiplets from zero to a finite value, as shown in figures 4.4 and 4.5 , these prominent peaks become sharper which we may interpret as mesonic excitations. Note that the prominent peaks in figure 4.5 are inside the gap.

Since we obtained the results numerically, we cannot resolve the value of the conductivity at $\omega \rightarrow 0$. However, for small frequencies $\omega$, the imaginary part of the conductivity behaves 


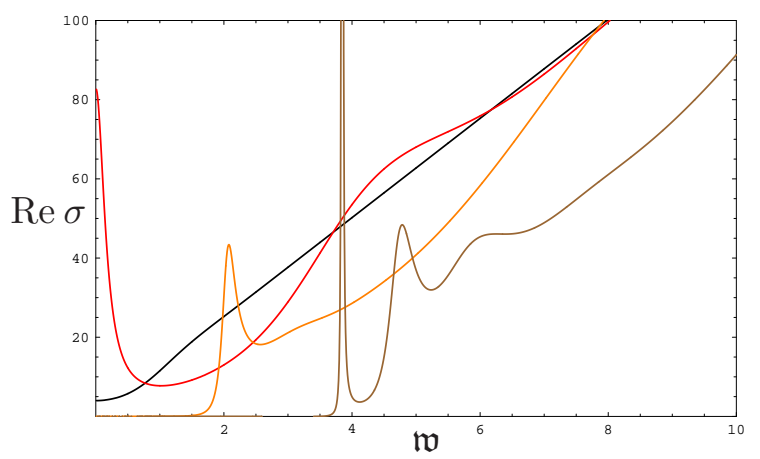

Figure 4.4: Real part of conductivity, Re $\sigma$, in units of $N_{f} N_{c} T /(16 \pi)$ versus the dimensionless frequency $\mathfrak{w}=\omega /(2 \pi T)$ for massless quarks computed from the adapted symmetrized trace prescription. Distinct curves correspond to $T / T_{c}=\infty$ (black), 1 (red), 0.5 (orange) and 0.28 (brown). By decreasing the temperature below the critical one, a gap where the conductivity is approximately zero appears which is a characteristic feature of a superconductor. In addition prominent peaks arise. Figure made by Matthias Kaminski and Patrick Kerner.

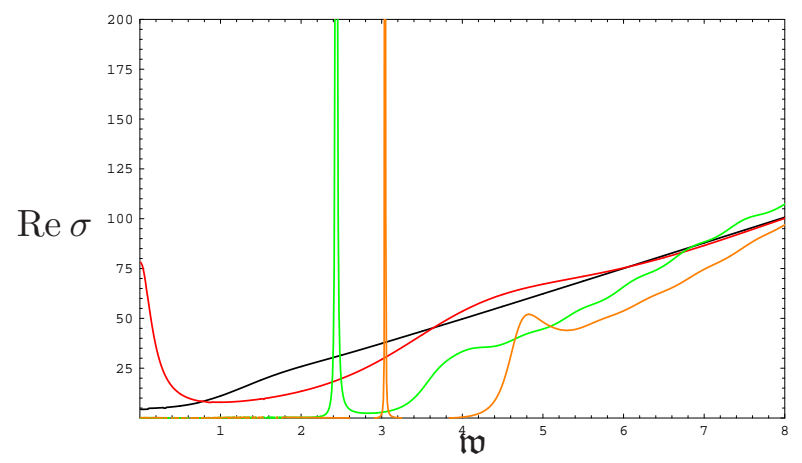

Figure 4.5: Real part of conductivity, Re $\sigma$, in units of $N_{f} N_{c} T /(16 \pi)$ versus the dimensionless frequency $\mathfrak{w}=\omega /(2 \pi T)$ for massive quarks at fixed $\mu / M_{q}=3$ computed from the adapted symmetrized trace prescription. Distinct curves correspond to $T / T_{c}=10$ (black), 1(red), 0.6 (green) and 0.5 (orange). As in figure 4.4 a gap appears. Moreover, we observe a prominent peak inside the gap where the conductivity is approximately zero in the green and orange curves. Figure made by Matthias Kaminski and Patrick Kerner.

as

$$
\operatorname{Im} \sigma(\omega) \sim \frac{n_{s}}{\omega} \quad \text { for } \omega \rightarrow 0
$$

Here, $n_{s}$ is a real parameter. Using the Kramers-Kronig relations, which connects the real and imaginary part of the conductivity, we therefore find a delta peak at $\omega=0$ in the real part of the conductivity,

$$
\operatorname{Re} \sigma(\omega) \sim \pi n_{s} \delta(\omega) .
$$

Therefore $n_{s}$ is the density of the superconducting condensate. The corresponding behavior of the imaginary part of the conductivity (multiplied by $\omega$ ) is visualized in figure 4.6. Taking the limit $\omega \rightarrow 0$, we can read off the superconducting density $n_{s}$ from figure 4.6 .

As expected from Ginzburg-Landau theory, our numerics show that the superconducting density $n_{s}$ vanishes linearly at the critical temperature, $n_{s} \propto\left(1-T / T_{c}\right)$ for $T \approx T_{c}$. As expected, for decreasing temperature $T$, the superconducting density $n_{s}$ increases. 


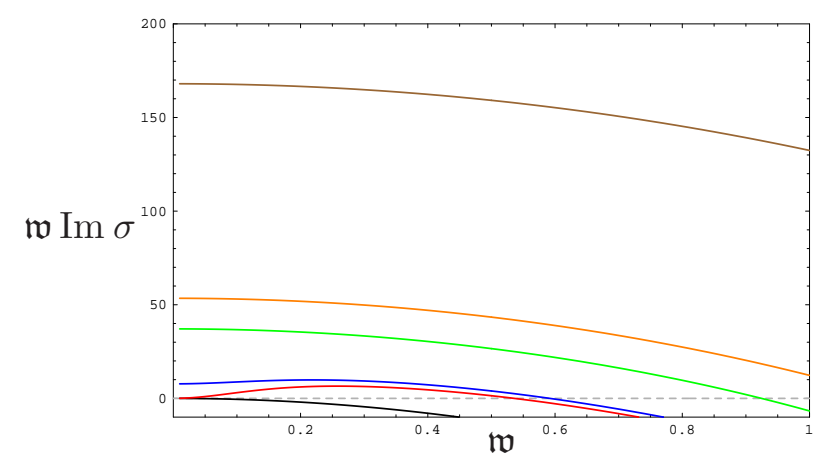

Figure 4.6: Scaled imaginary part of conductivity $\mathfrak{w} \operatorname{Im} \sigma$ in units of $N_{f} N_{c} T /(16 \pi)$ versus the dimensionless frequency $\mathfrak{w}=\omega /(2 \pi T)$ for massless quarks computed from the adapted symmetrized trace prescription. Distinct curves correspond to $T / T_{c}=\infty$ (black), 1 (red), 0.9 (blue), 0.6 (green), 0.5 (orange) and 0.28 (brown). This figure has been scaled to asymptote to a constant at $\mathfrak{w}=0$. This constant determines the superconducting density $n_{s}$. Figure made by Matthias Kaminski and Patrick Kerner.

Both calculational prescriptions - the adapted symmetrized trace prescription and the expansion of the DBI action to fourth order in the field strength - yield qualitatively very similar results. Therefore we have only present the results obtained from the symmetrized trace prescription.

The only difference are the prominent peaks as seen in figures 4.4 and 4.5 . From the Dirac-Born-Infeld action expanded to fourth order we obtain less prominent peaks. These peaks do not appear until we approach small temperatures. For the truncation to $\alpha^{\prime 2}$, i.e. for the Yang-Mills approximation of the Dirac-Born-Infeld action we do not see any prominent peaks in the ac conductivity. Therefore we conclude that the terms higher order in the field strength dominate the generation of the prominent peaks.

\subsection{String Theory Picture}

In this section we discuss why a non-zero gauge field $A_{x}^{1}$ stabilizes the system for large chemical potentials. For simplicity, we use the D3/D7 model. However, the arguments, presented in this section, can be generalized to other D3/Dp systems.

In the string context, the non-zero fields $A_{t}^{3}$ and $A_{x}^{1}$ induce two non-zero flavorelectric $S U(2)$ fields $E_{x}^{2}=F_{t x}^{2}=A_{t}^{3} A_{x}^{1}$ and $E_{u}^{3}=F_{t u}^{3}=-\partial_{u} A_{t}^{3}$ as well as a non-zero flavormagnetic field $B_{x u}^{1}=F_{x u}^{1}=-\partial_{u} A_{x}^{1}$ on the D7-branes where $u$ is the radial AdS direction. These fields are generated by D7-D7 strings stretched between the two probe branes and by strings stretched from the D7-branes to the horizon. In order to balance the flavorelectric and gravitational forces, the D7-D7 strings, which are responsible for the symmetrybreaking condensate, move from the horizon into the bulk and thus distribute the isospin charge. This stabilizes the system.

Let us discuss this stabilization mechanism in more detail. First we describe the unstable configuration in absence of the field $A_{x}^{1}$. As known from $[115,119,147]$, the non-zero field $A_{t}^{3}$ is induced by fundamental strings which are stretched from the D7-brane to the horizon of the black hole. Since the tension of these strings would increase as they move to 


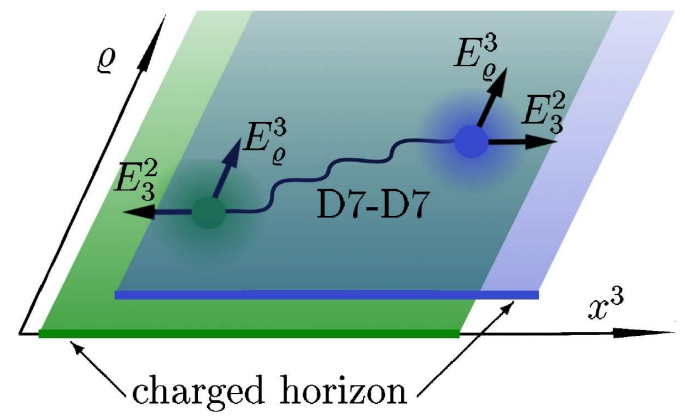

Figure 4.7: Sketch of our string setup: Strings spanned from the horizon of the AdS black hole to the D7-branes (green and blue plane) induce a charge at the horizon $[115,119,147]$. In the setup considered here, there are also D7-D7 strings present as shown in the figure. These D7-D7 strings are distributed along the AdS radial coordinate $u$, since they have to balance the flavorelectric and gravitational, i.e. tension forces. Thus these D7-D7 strings distribute the charges along the AdS radial coordinate, leading to a stable configuration of reduced energy. This corresponds to a superconducting condensate given by the Cooper pairs. Figure made by Patrick Kerner.

the boundary, they are localized at the horizon, i.e. the horizon is effectively charged. By increasing their density, the charge on the D7-brane at the horizon and therefore the energy of the system grows. In [147], the critical density was found beyond which this setup becomes unstable. In this case, the strings would prefer to move towards the boundary due to the repulsive force on their charged endpoints generated by the field $E_{u}^{3}$.

The setup is now stabilized by the new non-zero field $A_{x}^{1}$. This field is induced by D7-D7 strings moving in the $x$ direction. This may be interpreted as a current in $x$ direction which induces the magnetic field $B_{x u}^{1}=-\partial_{u} A_{x}^{1}$.

Let us now explain the mechanism by which the D7-D7 strings may propagate into the bulk. Due to the non-Abelian structure, the field $E_{u}^{3}$ and the magnetic field $B_{x u}^{1}$ induce the field $E_{x}^{2}$, corresponding to an interaction between the two string types. This field $E_{x}^{2}$ stretches the D7-D7 strings in the $x$ direction. The position of the string in the $u$ coordinate is fixed such that the gravitational force induced by the change in tension balances the force induced by the field $E_{u}^{3}$. This means that the energy of the setup is minimized. Our numerical calculations show that this is the case.

The double importance of the D7-D7 strings is given by the fact that they are both responsible for stabilizing the new phase by lowering the charge density, as well as being the dual of the Cooper pairs since they break the remaining $U(1)_{3}$ flavor symmetry.

In summary, in this chapter we have embedded a p-wave superconductor into string theory using probe Dp-branes. We have determined the holographic setup on the gravity side and have solved the equations of motions numerically. We find that below a critical temperature two solutions exit in the gravity theory, one with a non-vanishing gauge field component $A_{x}^{1}$. We confirm that the solution with non-zero $A_{x}^{1}$ is thermodynamically preferred by calculating the grand potential. Since $A_{x}^{1}$ breaks the remaining $U(1)_{3}$ symmetry spontaneously, we can view the system as a superconductor or, more precisely, as a superfluid. The phase transition separating the normal phase and the superconducting phase is second order with a mean field critical exponent of $1 / 2$. The non-vanishing gauge field $A_{x}^{1}$ can be identified as a meson condensate on the field theory side. 
Moreover, we have also determined the response to electric fields. In particular, we are interested in the conductivity of alternating currents in the superconducting phase. We find a gap in the AC conductivity for small frequencies and, by using Kramers-Kronig relations, a delta peak in the direct-current conductivity. In the next chapter, we study the response of fermionic operators in the background constructed here. 



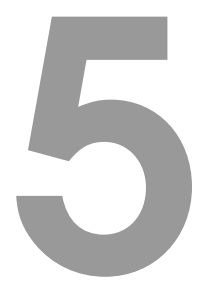

\section{Fermions in AdS/CFT}

Of central importance for potential condensed matter applications is the holographic description of fermions, in particular of Fermi liquids. Since a proper theoretical understanding on Non-Fermi liquids is lacking, we investigate a new class of (non-)Fermi liquids using techniques of the AdS/CFT correspondence.

In this chapter we compute spectral functions of fermionic operators in a strongly-coupled (defect) field theory. In particular we consider the defect field theory discussed in chapter 4. We map the fluctuations of the fermionic part of the D-brane action to the dual field theory operators corresponding to the superpartners of the mesons, i.e. to mesinos and compute the spectral function for certain mesinos in the p-wave state.

We identify an emergent Fermi surface in the superfluid phase. As we cool down the system through the superfluid phase transition, we find strong evidence of Fermi surfaces: gapless fermionic excitations for discrete values of the momentum. Due to the p-wave symmetry of the superconducting condensate, the rotational symmetry in the spectral functions is broken. For sufficiently low temperature, the Fermi surface is comprised of isolated points. This signature can also be observed in p-wave superfluids in real-world systems, like in ruthenate compounds. Moreover, the properties of low-energy excitations about the Fermi surface, in particular the scaling behavior, differs significantly from Landau liquid theory.

This chapter of the thesis is based on work done in collaboration with Johanna Erdmenger, Matthias Kaminski and Andy O'Bannon and was published in [8].

\subsection{Introduction and Summary}

In this chapter we embed the fermions into string theory by considering supersymmetric probe Dp-branes in the supergravity background of $N_{c}$ coincident D3-branes. The probe Dp-branes are extended along $A d S_{P} \times S^{Q}$, where supersymmetry requires $|P-Q|=2$ [102], as reviewed in section 2.5.2. For simplicity, we study only trivial embeddings of such 
Dp-branes, that is, we study only solutions in which all Dp-brane worldvolume scalars are trivial. This also implies that the flavor degrees of freedom are massless. Our bulk fermions are the Dp-branes' worldvolume fermions. These fermions are in a supermultiplet with the worldvolume scalars and gauge field, hence they are in the adjoint of the worldvolume $U\left(N_{f}\right)$, and couple to the gauge field via the gauge-covariant derivative 11 In other words, supersymmetry determines the charges of the fermions. For example, we use $N_{f}=2$, where we find three fermions with charges $+1,-1$ and 0 under $U(1)_{3}$.

To compute fermionic spectral functions we need the linearized equations of motion, the Dirac equation, for these fermions. Fortunately, the fermionic part of the D-brane action, for a D-brane in arbitrary backgrounds (including RR fields) is known to quadratic order in the fermionic fields [167-169]. The form of the action is determined by supersymmetry and T-duality [169], as we review in section 5.2. For our Dp-branes extended along $A d S_{P} \times S^{Q}$, we perform a reduction on the $S^{Q}$ to obtain a Dirac equation in $A d S_{P}$, following [101] very closely. The spectrum of $A d S_{P}$ fermion masses are fixed by $P, Q$ and the coupling to the background RR five-form.

We emphasize a major difference between our systems and the models of of the bottom-up approach described in the introduction: in our embedding of the Dirac equation into string theory, the mass and charge of the fermions are fixed by supersymmetry and T-duality. Therefore we are not free to dial the values of the mass and charge, unlike in the models of the bottom-up approach.

Much of our analysis is valid for any supersymmetric Dp-brane extended along $A d S_{P} \times S^{Q}$, with $P \geq 3.2$ but one particular Dp-brane is attractive for a number of reasons, namely the D5-brane extended along $A d S_{4} \times S^{2}(P=4$ and $Q=2)$. From the bulk point of view, this D5-brane is the only Dp-brane with a massless worldvolume fermion, as we show in section 5.2.1. That makes both our numerical analysis, and comparison to [49,51] (in which the fermions were massless), much easier. The dual field theory and in particular the explicit form of the fermionic operators dual to the D5-branes' worldvolume fermions was discussed in section 2.5.1. These fermionic operators are mesinos, the supersymmetric partners of mesons.

On a technical level, we consider more than one $D p$-brane giving rise to the flavor degrees of freedom. The dynamics of coincident $D p$-branes is described by a non-Abelian Dirac-BornInfeld action (as well as a non-Abelian version of the Chern Simons action which vanishes in the background considered). However note that the precise form of the non-Abelian DiracBorn-Infeld action is only known up to $F^{4}$. Therefore we truncated the Dirac-Born-Infeld action to $\alpha^{\prime 2}$ in section 4.3.1. In section 4.3.1 we studied two isospin charged flavor degrees at finite isospin chemical potential $\mu_{I}$. Above a critical value of the chemical potential (or equivalently, for low temperatures) we identified a second order phase transition. The order parameter is a rho-meson condensate which breaks the remaining $U(1)$ spontaneously. The gravity dual of the symmetry broken phase is given by a non-vanishing component $A_{x}^{1}$.

Here, we use this gravity background considered in section 4.3 .1 and study the fermionic fluctuations therein. In order to be consistent we also truncate the fermionic part of the Dp-brane action to $\alpha^{\prime 2}$. Hence, the equations of motions are given by a Dirac equation for

\footnotetext{
${ }^{1}$ Like all worldvolume fields, they are not charged under the diagonal $U(1) \subset U\left(N_{f}\right)$. However, note that in the fermionic D-brane action proposed in [167-169] there are also dipole-like couplings present.

${ }^{2}$ We focus on $P \geq 3$, since only in those cases is a vector condensate $\left\langle J_{1}^{x}\right\rangle$ possible. One exception is a D5-brane along $A d S_{2} \times S^{4}$, which we study in section 5.2 .1 (but not in p-wave states).
} 
a fermion in the adjoint of $S U(2)$ confined to an $A d S_{P}$ submanifold of (4+1)-dimensional AdS-Schwarzschild. For any Dp-brane, the three worldvolume fermions decouple in the normal (non-superfluid) phase, where $A_{x}^{1}$ is zero, but couple to one another in the superfluid phase, where $A_{x}^{1}$ is nonzero. These couplings indicate that, in the field theory, the dual fermionic operators experience operator mixing under renormalization group flow $[140,141]$. In the field theory, the retarded Green's function, and hence the spectral function, becomes a matrix with off-diagonal entries.

For the $P=4, Q=2$ D5-brane, using our method for coupled bulk fermions (see section 3.2.4), we numerically compute spectral functions for mesinos as we cool the system through the p-wave superfluid phase transition. Due to the operator mixing, or equivalently the coupling of the fermions in the bulk, we see that the spectral function of even a neutral fermion develops a nontrivial feature, a peak, as the system enters the p-wave phase.

Furthermore, as we lower the temperature 3 the zero-frequency spectral measure, which is defined as the trace of the spectral function, is clearly no longer rotationally invariant. In fact at the lowest temperatures we can reliably access in the probe limit, the main features of the spectral measure are five largely isolated peaks in the $\left(k_{x}, k_{y}\right)$ plane, two on the $k_{x}$ axis, two on the $k_{y}$ axis, and one at the origin 4

We cannot resist drawing an analogy between our system and certain experimentallyrealized p-wave superconductors (see also [170]) 5 In that context, a reduction of the Fermi surface to certain points in momentum space has been proposed for the ruthenate compound $\mathrm{Sr}_{2} \mathrm{RuO}_{4}$ [171]: the p-wave state is supported by ferromagnetic fluctuations that increase the propensity for electrons to form spin triplet Cooper pairs, with an odd (p-wave) Cooper pair wave function 6 Scattering channels with momentum transfer $Q=$ $(0,0)$, as is the case in a ferromagnet, should be enhanced in the system, as opposed to scattering channels of $Q=(\pi, \pi)$, which is the case in an anti-ferromagnet. Small momentum transfer is best accomplished by a strongly-peaked density of states at the Fermi level, as occurs for example with van Hove singularities, where the density of states diverges. This lies at the heart of the strong suspicion that a Fermi surface localized to certain points with a high density of states may account for a suitable setup to support p-wave pairing.

The chapter of the thesis is organized as follows. In section 5.2 we write the fermionic part of the Dp-brane action, perform the reduction of the worldvolume Dirac equation to $A d S_{P}$, and, for the $P=4, Q=2$ D5-brane, we match bulk fermions to the dual field theory operators discussed in section 2.5.1.2. In section 5.3 we present our numerical

\footnotetext{
${ }^{3}$ As always in the probe limit, note that we cannot access the zero temperature ground state with finite chemical potential.

${ }^{4}$ These results are very similar to the $T=0$ results of [161], where the bulk theory was gravity and $S U(2)$ gauge fields in (3+1)-dimensions, in the $T=0$ vector-hairy black hole geometry. In that case, for a fermion in the fundamental representation of $S U(2)$, the spectral measure consisted of two points on the $k_{x}$ axis, located symmetrically about the origin. The prediction of [161] for fermions in the adjoint representation would be three points on the $k_{x}$ axis, one at the origin and two at finite $k_{x}$, positioned symmetrically about the origin. At finite temperature we see five points emerging, but we strongly suspect that, if we could access the $T=0$ limit, we would indeed see only three points, as we discuss in section 5.3

${ }^{5}$ I want to thank Ronny Thomale for many useful conversations about real p-wave superconductors.

${ }^{6}$ This is rather particular, bearing in mind that a large number of generic spin interactions, for example induced by superexchange processes, favor antiferromagnetic fluctuations.
} 
results for the fermionic retarded Green's functions using the $P=4, Q=2$ D5-brane.

\subsection{Worldvolume Fermions of Dp-branes}

We study fluctuations of fermionic operators of the D3/D5 theory with finite temperature and isospin chemical potential, in the two phases described in section 4.3.1, i.e. the normal (non-superfluid) phase and the superfluid phase. On the field theory side, we study mesino operators which are valued in the adjoint representation of the $S U(2)$ isospin symmetry. As we will see below, we thus have three mesinos, two with equal and opposite charges under the $U(1)_{3}$, and one that is neutral. The precise form of the mesino operator for the field theory dual to the supersymmetric D3/D5 intersection is discussed in section 2.5.1.

To be specific, we compute holographically the retarded two-point function of mesinos as a function of frequency and momentum. On the gravity side, that means studying fermionic fluctuations of the Dp-branes, and in particular solving their linearized equation of motion in the background we found in section 4.3.2. The geometry is $(4+1)$-dimensional AdS-Schwarzschild and the D5-branes have non-trivial worldvolume gauge fields. As for all supersymmetric Dp-branes, the worldvolume fermions are in a supermultiplet with the worldvolume gauge field and scalars, and hence are in the adjoint of the worldvolume $S U(2)$ gauge group, which is dual to the statement that the mesinos are in the adjoint of the isospin symmetry 7

As we will see, in the normal phase the three bulk fermions decouple, which is dual to the statement that the retarded Green's function is diagonal. In the superfluid phase, however, where $A_{x}^{1}(u)$ is nonzero, the three fermions couple, indicating that the dual operators mix under renormalization group flow in the field theory. The Green's function is then a $6 \times 6$ matrix, where the 6 is the number of fermions times the two components of the fermions (two, using the $\Pi_{1,2}$ projectors which are defined in equation 3.2.21). We thus have a perfect testing ground for the method we developed in section 3.2.4 for computing Green's functions from coupled bulk fermions.

The fermionic part of general Dp-brane actions, to quadratic order in the fermionic fields and in backgrounds with non-trivial RR forms, was determined in [167-169]. The general couplings were derived by starting with the action for a supermembrane in M-theory, written in a superspace formalism, expanding the action to second order in the Grassmann variables, reducing to type IIA supergravity, and then performing a T-duality to type IIB. The form of the quadratic fermionic action on the Dp-brane worldvolume is thus determined completely by supersymmetry and T-duality.

Using the worldvolume fermion actions of [167-169], the spectra of mesinos in the D3/D7 theory (for the D7-brane with $P=5$ and $Q=3$ ) and in the Sakai-Sugimoto model were determined in $[101,172]$. We will very closely follow the D7-brane analysis of [101], which in turn was the fermionic generalization of the analysis of [89] for mesons. For a Dp-brane extended along $A d S_{P} \times S^{Q}$, we consider a worldvolume spinor that is a spherical harmonic on the $S^{Q}$. We reduce the worldvolume spinor on the $S^{Q}$, obtaining an effective Dirac action in $A d S_{P}$. This procedure fixes the masses of the bulk fermionic excitations, which

\footnotetext{
${ }^{7}$ Obviously, the mesinos carry no baryon number, which is dual to the statement that the worldvolume fermions, like all of the worldvolume fields, do not couple via a gauge-covariant derivative to the diagonal $U(1)$ part of the worldvolume gauge field.
} 
allows us to identify the dimensions of the dual mesinos, and more generally to map bulk fluctuations to mesino operators. As emphasized in [101], the coupling to the RR five-form is crucial to obtain the correct bulk masses 8

\subsubsection{Equation of Motion I: Reduction to AdS}

In this section we now reduce the fermionic action of a Dp-brane extended along $A d S_{P} \times S^{Q}$ to an effective Dirac action in $A d S_{P}$, with emphasis on D5-branes with $P=4$ and $Q=2.9$

The quadratic action for fermionic fluctuations of the Dp-branes truncated to second order in $\alpha^{\prime}$ is (for more details see $[101,169]$ )

$S_{D p}=N_{f} T_{D p} \int d^{p+1} \xi \sqrt{-g_{D p}} \frac{1}{2} \operatorname{Tr}\left[\hat{\bar{\Psi}} P_{-} \Gamma^{\hat{A}}\left(D_{\hat{A}}+\frac{1}{8} \frac{i}{2 * 5 !} F_{\hat{N} \hat{P} \hat{Q} \hat{R} \hat{S}} \Gamma^{\hat{N} \hat{P} \hat{Q} \hat{R} \hat{S}} \Gamma_{\hat{A}}\right) \hat{\Psi}\right]$.

Here, $\hat{\Psi}$ is a ten-dimensional positive-chirality Majorana-Weyl spinor of type IIB supergravity, the $\Gamma_{\hat{A}}$ are the pullback of the ten-dimensional $\Gamma$-matrices to the Dp-brane worldvolume, $\Gamma_{\hat{A}}=\Gamma_{\hat{M}} \partial_{\hat{A}} x^{\hat{M}}$ (we use a trivial embedding, so the pullback is trivial), $P_{-}$is a $\kappa$-symmetry projector that ensures $\kappa$-symmetry invariance of the action, $D_{A}$ is a (gauge and curved-space) covariant derivative, and $F_{\hat{N} \hat{P} \hat{Q} \hat{R} \hat{S}}$ is the five-form of the background. Notice that here $F_{\hat{N} \hat{P} \hat{Q} \hat{R} \hat{S}}$ is not the pullback of the five-form to the Dp-brane worldvolume, rather, it is the five-form evaluated on the submanifold spanned by the Dp-brane. Indeed,

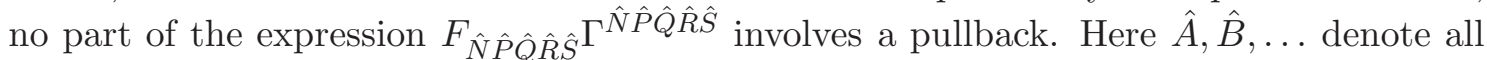
worldvolume indices, while below $A, B, \ldots$ denote AdS-Schwarzschild coordinates which are wrapped by the probe brane. Moreover, the indices of the coordinates on the sphere $S^{Q}$ are labelled by $a, b, \ldots$ Notice that the fermion $\hat{\Psi}$ is in the adjoint representation of $S U(2)$.

The equation of motion for the fermion is

$$
\left[\Gamma^{\hat{A}} D_{\hat{A}}+\frac{1}{8} \frac{i}{2 * 5 !} \Gamma^{\hat{A}} F_{\hat{N} \hat{P} \hat{Q} \hat{R} \hat{S}} \Gamma^{\hat{N} \hat{P} \hat{Q} \hat{R} \hat{S}} \Gamma_{\hat{A}}\right] \hat{\Psi}=0,
$$

where we have suppressed gauge indices. We reduce the equation of motion for the fermion to a Dirac equation in $A d S_{P}$, following [101] very closely. First we decompose every tendimensional spinor and $\Gamma$-matrix into parts associated with $A d S_{5}$ and $S^{5}$. In a local Lorentz frame, the $\Gamma$-matrices decompose as

$$
\Gamma^{M}=\sigma_{2} \otimes \mathbb{1}_{4} \otimes \gamma^{M}, \quad \Gamma^{m}=\sigma_{1} \otimes \gamma^{m} \otimes \mathbb{1}_{4},
$$

where $\mathbf{1}_{4}$ is the $4 \times 4$ identity matrix, the index $M$ runs over $A d S_{5}$ directions, which we generically call 01234 , and the index $m$ runs over $S^{5}$ directions, which we call 56789 . The $\gamma$-matrices are five-dimensional, obeying the usual relations

$$
\left\{\gamma^{M}, \gamma^{N}\right\}=2 \eta^{M N}, \quad\left\{\gamma^{m}, \gamma^{n}\right\}=2 \delta^{m n}
$$

\footnotetext{
${ }^{8}$ The authors of [173] appear to omit the coupling to the five-form when they study fermionic fluctuations of the probe D3-brane extended along $A d S_{3} \times S^{1}$.

${ }^{9}$ In section 4.3 .1 we were interested in $\mathrm{p}$-wave states and hence required $P \geq 3$. In this subsection we relax that constraint. Our results thus also apply for D5-branes extended along $A d S_{2} \times S^{4}$, which were used in [174] to construct a holographic model of fermions at lattice sites (that can pair to form dimers).
} 
Given the above decompositions, we then have

$$
\begin{gathered}
\Gamma^{01234}=i \sigma_{2} \otimes \mathbb{1}_{4} \otimes \mathbb{1}_{4}, \quad \Gamma^{56789}=\sigma_{1} \otimes \mathbb{1}_{4} \otimes \mathbb{1}_{4}, \\
\Gamma^{11}=\Gamma^{0123456789}=\sigma_{3} \otimes \mathbb{1}_{4} \otimes \mathbb{1}_{4} .
\end{gathered}
$$

The ten-dimensional spinor $\hat{\Psi}$ has positive chirality, $\Gamma^{11} \hat{\Psi}=\hat{\Psi}$, and decomposes as

$$
\hat{\Psi}=\uparrow \otimes \chi \otimes \Psi,
$$

where $\uparrow=\left(\begin{array}{l}1 \\ 0\end{array}\right)$, and $\chi$ and $\Psi$ are four-component spinors of $S O(5)$ and $S O(4,1)$, which act on the tangent spaces of $S^{5}$ and $A d S_{5}$, respectively. The spinor $\chi$ further decomposes as $\chi=\chi_{\|} \otimes \chi_{\perp}$, where $\chi_{\|}$is a spinor associated with the $S^{Q}$ that the Dp-brane wraps and $\chi_{\perp}$ is associated with the $S^{5}$ directions transverse to the $S^{Q}$.

We parameterize the five-form in terms of the volume forms of $A d S_{5}$ and $S^{5}$, which we denote as $\Omega_{A d S_{5}}$ and $\Omega_{S^{5}}$,

$$
F_{N P Q R S}=4\left(\Omega_{A d S_{5}}\right)_{N P Q R S}, \quad F_{n p q r s}=4\left(\Omega_{S^{5}}\right)_{n p q r s} .
$$

Using the decomposition of the type IIB spinor in equation (5.2.6) and of the $\gamma$-matrices in equation (5.2.2) we obtain

$$
\begin{aligned}
\Gamma^{a}\left(\Gamma^{01234}+\Gamma^{56789}\right) \Gamma_{a}(\uparrow \otimes \chi \otimes \Psi) & =2 \downarrow \otimes \chi \otimes \Psi, \\
\Gamma^{A}\left(\Gamma^{01234}+\Gamma^{56789}\right) \Gamma_{A}(\uparrow \otimes \chi \otimes \Psi) & =-2 \downarrow \otimes \chi \otimes \Psi,
\end{aligned}
$$

In equation (5.2.7) we have not summed over $a$ or $A$. Using this result we can simplify the coupling of the spinor to the five-form,

$$
\begin{aligned}
\frac{1}{8} \frac{i}{2 * 5 !} \Gamma^{\hat{A}} F_{\hat{N} \hat{P} \hat{Q} \hat{R} \hat{S}} \Gamma^{\hat{N} \hat{P} \hat{Q} \hat{R} \hat{S}} \Gamma_{\hat{A}} \hat{\Psi} & =\frac{i}{4} \Gamma^{\hat{A}}\left(\left(\sigma_{1}+i \sigma_{2}\right) \otimes \mathbf{1}_{4} \otimes \mathbf{1}_{4}\right) \Gamma_{\hat{A}} \hat{\Psi} \\
& =-\frac{i}{2}(P-Q)(\downarrow \otimes \chi \otimes \Psi)
\end{aligned}
$$

where here we do sum over $\hat{A}$. We can also extract the $S^{Q}$ and the $A d S_{P}$ part of the derivative terms as

$$
\begin{aligned}
\Gamma^{\hat{A}} D_{\hat{A}} \hat{\Psi} & =\Gamma^{A} D_{A} \hat{\Psi}+\Gamma^{a} D_{a} \hat{\Psi} \\
& =\left(\left(\sigma_{2} \otimes \mathbf{1}_{4} \otimes \gamma^{A} D_{A}\right)+\left(\sigma_{1} \otimes \gamma^{a} D_{a} \otimes \mathbf{1}_{4}\right)\right)(\uparrow \otimes \chi \otimes \Psi) \\
& =\left(i\left(\mathbf{1}_{2} \otimes \mathbf{1}_{4} \otimes \gamma^{A} D_{A}\right)+\left(\mathbf{1}_{2} \otimes \gamma^{a} D_{a} \otimes \mathbf{1}_{4}\right)\right)(\downarrow \otimes \chi \otimes \Psi) \\
& \equiv\left(i \Delta_{A d S_{P}}+\Delta_{S^{Q}}\right)(\downarrow \otimes \chi \otimes \Psi),
\end{aligned}
$$

where $\Delta_{A d S_{P}}$ and $\Delta_{S}$ are the Dirac operators of $A d S_{P}$ and $S^{Q}$, respectively. The Dirac operator on a sphere $S^{Q}$ has spinor spherical harmonics $\chi_{\ell}^{ \pm}$that obey

$$
\Delta_{S Q} \chi_{\ell}^{ \pm}=\mp \frac{i}{R_{Q}}\left(\ell+\frac{Q}{2}\right) \chi_{\ell}^{ \pm},
$$

where $\ell \geq 0$ and $R_{Q}$ is the radius of the $S^{Q}$. In our units, $R_{Q}=1$. For $Q=3$, relevant for the D7-brane along $A d S_{5} \times S^{3}$, the spinors $\chi_{\ell}^{+}$are in the $\left(\frac{\ell+1}{2}, \frac{\ell}{2}\right)$ representation of 
the $S O(4)$ that acts on $S^{3}$, while the spinors $\chi_{\ell}^{-}$are in the $\left(\frac{\ell}{2}, \frac{\ell+1}{2}\right)$ representation. For $Q=2$, relevant for the D5-brane along $A d S_{4} \times S^{2}$, the spinors $\chi_{\ell}^{ \pm}$are in the $\left(\ell+\frac{1}{2}\right)$ of the $S U(2) \simeq S O(3)$ that acts on $S^{2}$.

Inserting everything into equation (5.2.1), we find

$$
\left(\Delta_{A d S_{P}} \mp\left(\ell+\frac{Q}{2}\right)-\frac{1}{2}(P-Q)\right) \Psi_{\ell}^{ \pm}=\left\{\begin{array}{c}
\left(\Delta_{A d S_{P}}-\left(\ell+\frac{1}{2} P\right)\right) \Psi_{\ell}^{+} \\
\left(\Delta_{A d S_{P}}+\left(\ell-\frac{1}{2} P+Q\right)\right) \Psi_{\ell}^{-}
\end{array}\right\}=0 .
$$

The fermions $10 \Psi^{ \pm}$thus have masses

$$
m_{\ell}^{+}=\ell+\frac{P}{2}, \quad m_{\ell}^{-}=-\left(\ell+Q-\frac{1}{2} P\right) .
$$

Note that we use units, where the radius of AdS is one. We collect the values of $m_{\ell}^{ \pm}$for our Dp-branes of interest in the table 5.1 .

\begin{tabular}{|c|c|c|c|c|c|c|c|c|}
\hline Dp & $P$ & $Q$ & {$[\psi]$} & {$[q]$} & $m_{\ell}^{+}=\ell+P / 2$ & $\Delta_{\ell}^{+}$ & $\left|m_{\ell}^{-}\right|=\ell+Q-P / 2$ & $\Delta_{\ell}^{-}$ \\
\hline \hline D7 & 5 & 3 & $3 / 2$ & 1 & $\ell+5 / 2$ & $\ell+9 / 2$ & $\ell+1 / 2$ & $\ell+5 / 2$ \\
\hline D7 & 3 & 5 & $1 / 2$ & - & $\ell+3 / 2$ & $\ell+5 / 2$ & $\ell+7 / 2$ & $\ell+9 / 2$ \\
\hline D5 & 4 & 2 & 1 & $1 / 2$ & $\ell+2$ & $\ell+7 / 2$ & $\ell$ & $\ell+3 / 2$ \\
\hline D5 & 2 & 4 & 0 & - & $\ell+1$ & $\ell+3 / 2$ & $\ell+3$ & $\ell+7 / 2$ \\
\hline D3 & 3 & 1 & $1 / 2$ & 0 & $\ell+3 / 2$ & $\ell+5 / 2$ & $\ell-1 / 2$ & $\ell+1 / 2$ \\
\hline
\end{tabular}

Table 5.1: Masses of fermionic excitations on the worldvolume of a Dp-brane extended along $A d S_{P} \times S^{Q}$ inside $A d S_{5} \times S^{5}$. We list Dp-branes that are known to preserve eight real supercharges (at zero temperature and density), in which case $|P-Q|=2$. Here, $\psi$ denotes a generic quark field and $q$ denotes a generic squark field. $\Delta_{\ell}^{ \pm}$denotes the dimension of the operator dual to the bulk fermion with mass $m_{\ell}^{ \pm}$, with $\Delta_{\ell}^{ \pm}=\frac{P-1}{2}+\left|m_{\ell}^{ \pm}\right|$. For the D3-brane, the values of $m_{\ell}^{-}$and $\Delta_{\ell}^{-}$ shown are for $\ell \geq 1$ only, whereas for $\ell=0,\left|m_{0}^{-}\right|=1 / 2$ and $\Delta_{0}^{-}=3 / 2$.

Notice that since $P$ and $Q$ are integers, the $m_{\ell}^{ \pm}$are always integer or half-integer. As we reviewed in section 2.3.1, a bulk fermion with an integer or half-integer mass $m$ is dual to a fermionic operator of dimension $\Delta=\frac{P-1}{2}+|m|$. We include the values of $\Delta_{\ell}^{ \pm}=\frac{P-1}{2}+\left|m_{\ell}^{ \pm}\right|$in the table.

To get a rough idea of which operators correspond to which bulk fermion, we can do some dimension counting. Let us denote a generic quark as $\psi$, a generic squark as $q$, a generic adjoint Majorana fermion as $\lambda$, and a generic adjoint real scalar as $X$. The dimensions of the fields are $[\psi]=\frac{P-2}{2},[q]=\frac{P-3}{2},[\lambda]=3 / 2,[X]=1$.

For the D7-brane extended along $A d S_{5} \times S^{3}$, the D5-brane along $A d S_{4} \times S^{2}$, and the D3-brane along $A d S_{3} \times S^{1}$, all of which have $P-Q=2$, the dual flavor fields comprise a supermultiplet with both quarks $\psi$ and squarks $q$. In these cases, we can build a gaugeinvariant mesino in two ways [101]. One way is to construct an operator of the form $\bar{\psi} \lambda \psi+q^{\dagger} X \lambda q$, with dimension $\Delta=P-1 / 2$. We can additionally include some number $\ell$ of adjoint scalars ${ }^{11}$ as $\bar{\psi} \lambda X^{\ell} \psi+q^{\dagger} X \lambda X^{\ell} q$, so that the dimension is $\Delta=\ell+P-1 / 2$.

\footnotetext{
${ }^{10}$ The \pm superscript here refers to the sign of the eigenvalue of the Dirac operator in equation (5.2.10), not to the projectors $\Pi_{ \pm}$defined in section 2.3.1.

${ }^{11}$ Notice these are not necessarily all the same scalar, i.e. $X^{\ell}$ could represent $\ell$ distinct scalars. At the moment we are just counting dimensions, ignoring this subtlety.
} 
Inspecting the table, these are precisely the $\Delta_{\ell}^{+}$, so apparently these kinds of mesinos are dual to the bulk fermions with masses $m_{\ell}^{+}$. The other way to build a mesino is to construct $\bar{\psi} X^{\ell} q$ (plus the Hermitian conjugate), with dimension $\Delta=\ell+P-5 / 2$. For Dp-branes with $P-Q=2$, these dimensions are precisely the $\Delta_{\ell}^{-}$, so apparently mesinos of this type are dual to the fermions with masses $m_{\ell}^{-}$.

The mesino operators for the D5-brane along $A d S_{4} \times S^{2}$ were already studied in section 2.5.1. The explicit form of the fermionic operators $\mathcal{F}_{l}$ and $\mathcal{G}_{l}$ are given by equation (2.5.90) and equation (2.5.91), respectively. Furthermore, in section 2.5.2 we used the quantum numbers of the fluctuations $\Psi_{\ell}^{ \pm}$of the fermionic D5-brane action to establish a mapping between the fluctuations of the D5-brane and the operators $\mathcal{F}_{l}$ and $\mathcal{G}_{l}$. The matching is summarized in table $2.9^{12}$

For the D7-brane extended along $A d S_{3} \times S^{5}$ and D5-brane along $A d S_{2} \times S^{4}$, which have $P-Q=-2$, the dual flavors fields are quarks alone, with no squarks [96-99]. The mesinos with dimensions $\Delta_{\ell}^{+}$are of the same form, $\bar{\psi} \lambda X^{\ell} \psi$, but the mesinos with dimensions $\Delta_{\ell}^{-}$ must obviously have a different form. We leave a detailed study of these mesinos for the future.

Looking at the table, we immediately notice that the D5-branes are special: for these, the masses of the worldvolume fermions are integers. The reason is that the D5-branes wrap even-dimensional spheres, so the eigenvalue in equation (5.2.10) is $\pm i$ times an integer.

In the next subsection we focus on two coincident D5-branes extended along $A d S_{4} \times S^{2}$. We calculate the spectral function for the mesino operators with lowest conformal dimension, i.e. for $\mathcal{F}_{0}$. In the dual gravitational description the mesino $\mathcal{F}_{0}$ corresponds to worldvolume fermions with mass $m_{0}^{-}=0$ (since $\Delta=3 / 2$, see section 2.5.1.2). Since the fermion's mass is zero the numerical analysis is much simpler and a massless fermion requires no counterterms (as we showed in section 2.3). Moreover, with a massless bulk fermion we can directly compare to the results of $[49,51]$, where most of the analysis focused on massless bulk fermions.

\subsubsection{Equation of Motion II: Gauge Couplings}

In this section we return to the equation of motion for the worldvolume fermions, equation (5.2.11), and specialize to our case of interest, namely two coincident Dp-branes in (4+1)dimensional AdS-Schwarzschild with trivial worldvolume scalars but non-trivial worldvolume gauge fields $A_{t}^{3}(u)$ and $A_{x}^{1}(u)$. More specifically, we explicitly unpack the gaugeand curved-space covariant Dirac operator $\Delta_{A d S_{P}}$ for the $A d S_{P}$ submanifold of (4+1)dimensional AdS-Schwarzschld and see how, when $A_{x}^{1}(u)$ is nonzero, the three worldvolume fermions couple to one another. In this subsection we assume $P \geq 3$.

The linearized equation of motion for the worldvolume fermions in equation (5.2.11) is 13

$$
\left(\Delta_{A d S_{P}}-m_{l}^{ \pm}\right) \Psi_{l}^{ \pm}=0
$$

where the masses $m_{l}^{ \pm}$appear in equation (5.2.12). To simplify the notation we write $m$

\footnotetext{
${ }^{12}$ In this chapter we use the subscript $\ell$, while in the sections 2.5 .1 and 2.5 .2 we used a subscript $l$. For $\Psi_{\ell}^{-}$the two are identical, i.e. $\ell=l$ whereas for $\Psi_{\ell}^{+}$we take $\ell=l-1$.

${ }^{13}$ Here the \pm does not refer to the projectors $\Pi_{ \pm}$of section 2.3 .1 , but rather to the \pm sign labeling the eigenvalues of the Dirac operator of $S^{Q}$ in equation (5.2.10).
} 
instead of $m_{l}^{ \pm}$as well as $\Psi$ instead of $\Psi_{l}^{ \pm} . \Delta_{A d S_{P}}=e_{A}^{M} \gamma^{A} D_{M}$ is the gauge and curvedspace covariant Dirac operator. The index $A$ runs over the worldvolume directions inside $A d S_{5}$. Notice that here $e_{A}^{M}$ are the inverse vielbeins. The $\gamma^{A}$ are the $\Gamma$-matrices of $(4+1)$ dimensional Minkowski space, which obey $\left\{\gamma^{A}, \gamma^{B}\right\}=2 \eta^{A B}$.

The nonzero vielbeins of (4+1)-dimensional AdS-Schwarzschild (2.4.80) are (recall that upper index is local Lorentz, and the lower index is general coordinate),

$$
e^{u}{ }_{u}=\frac{1}{u \sqrt{f}}, \quad e_{t}^{t}=\frac{\sqrt{f}}{u}, \quad e_{j}^{i}=\frac{1}{u} \delta^{i}{ }_{j} .
$$

The spin connection $\omega$ of $(4+1)$-dimensional AdS-Schwarzschild then has the nonzero components

$$
\omega_{t u}=\left(\frac{f(u)}{u}-\frac{f^{\prime}(u)}{2}\right) d t, \quad \omega_{\vec{x} u}=-\frac{\sqrt{f(u)}}{u} d \vec{x}
$$

where $\omega_{\vec{x} u}$ indicates the three components $\omega_{x u}, \omega_{y u}$, and $\omega_{z u}$.

For the Dirac operator, we have (here $a$ is a gauge index)

$$
\begin{aligned}
{\left[\left(\Delta_{A d S_{P}}-m\right) \Psi\right]_{a} } & =u\left(\sqrt{f} \gamma^{u} \partial_{u}+\frac{1}{\sqrt{f}} \gamma^{t} \partial_{t}+\gamma^{i} \partial_{i}+\left[-\frac{P-1}{2 u} \sqrt{f}+\frac{1}{4} \frac{f^{\prime}}{\sqrt{f}}\right] \gamma^{u}\right) \Psi_{a} \\
& +e_{A}^{M} i \gamma^{A}\left[A_{M}, \Psi\right]_{a}-m \Psi_{a}
\end{aligned}
$$

where $f^{\prime}=\partial_{u} f$. When $T=0$ and hence $f(u)=1$, the operator in parentheses on the right-hand side in the first line is the Dirac operator of $A d S_{P}$. At finite temperature, where $f(u)=1-u^{4} / u_{b}^{4}$, the operator is that of an $A d S_{P}$ submanifold of (4+1)-dimensional AdSSchwarzschild 14 The coupling to the gauge field in the second line is of course fixed by gauge invariance.

We need an ansatz for $\Psi_{a}$. For the coordinate dependence, our ansatz is similar to the one in [49-51],

$$
\Psi=\Psi_{a} \sigma_{a}=u^{(P-1) / 2} f^{-\frac{1}{4}} e^{i k_{\mu} x^{\mu}} \psi_{a}(u) \tau_{a},
$$

where $\mu$ runs over field theory directions, the $\psi_{a}(u)$ are three spinor functions for which we must solve, and we extract a factor of $u^{(P-1) / 2} f^{-\frac{1}{4}}$ to make the Dirac equation look nice later.

Using our ansatz for the fermion in equation (5.2.17) and the ansatz for the gauge field in equation (4.3.6), we find three Dirac equations,

$$
\begin{aligned}
0 & =\left(\sqrt{f} \gamma^{u} \partial_{u}-\frac{i \omega}{\sqrt{f}} \gamma^{t}+i k_{i} \gamma^{i}-\frac{1}{u} m\right) \psi_{1}+\frac{A_{t}^{3}(u)}{\sqrt{f}} \gamma^{t} \psi_{2} \\
0 & =\left(\sqrt{f} \gamma^{u} \partial_{u}-\frac{i \omega}{\sqrt{f}} \gamma^{t}+i k_{i} \gamma^{i}-\frac{1}{u} m\right) \psi_{2}-\frac{A_{t}^{3}(u)}{\sqrt{f}} \gamma^{t} \psi_{1}+A_{x}^{1}(u) \gamma^{x} \psi_{3} \\
0 & =\left(\sqrt{f} \gamma^{u} \partial_{u}-\frac{i \omega}{\sqrt{f}} \gamma^{t}+i k_{i} \gamma^{i}-\frac{1}{u} m\right) \psi_{3}-A_{x}^{1}(u) \gamma^{x} \psi_{2}
\end{aligned}
$$

In what follows we use the Lorentzian-signature $\gamma^{A}$ from section 2.3.1, in which all the $\gamma^{A}$

\footnotetext{
${ }^{14}$ For a general $(\mathrm{d}+1)$-dimensional AdS-Schwarzschild space, $f(u)=1-u^{d} / u_{h}^{d}$.
} 
are Hermitian except for $\gamma^{t}$, which are anti-Hermitian, 15

$$
\begin{aligned}
\gamma^{u} & =\left(\begin{array}{cc}
-\sigma_{3} & 0 \\
0 & -\sigma_{3}
\end{array}\right), & \gamma^{t} & =\left(\begin{array}{cc}
i \sigma_{1} & 0 \\
0 & i \sigma_{1}
\end{array}\right), \\
\gamma^{x} & =\left(\begin{array}{cc}
-\sigma_{2} & 0 \\
0 & \sigma_{2}
\end{array}\right), & \gamma^{y} & =\left(\begin{array}{cc}
0 & \sigma_{2} \\
\sigma_{2} & 0
\end{array}\right) .
\end{aligned}
$$

We also use the projectors $\Pi_{1,2}$, which in Lorentzian signature are defined as

$$
\Pi_{\alpha} \equiv \frac{1}{2}\left(1-(-1)^{\alpha} \gamma^{u} \gamma^{t} \gamma^{x}\right)
$$

with $\alpha=1,2$.

\subsubsection{Normal Phase}

First consider the normal phase, where $A_{x}^{1}(u)=0$. In that case, $\psi_{3}$ decouples from $\psi_{1}$ and $\psi_{2}$, and its equation of motion becomes that of a free neutral fermion, as expected. We can then simplify the remaining two equations by taking linear combinations of them. Defining $\psi_{ \pm} \equiv \psi_{2} \pm i \psi_{1}$, we find three decoupled equations,

$$
\begin{aligned}
0 & =\left(\sqrt{f} \gamma^{u} \partial_{u}-\frac{i \omega}{\sqrt{f}} \gamma^{t}+i k_{i} \gamma^{i}-\frac{1}{u} m\right) \psi_{+}+i \frac{A_{t}^{3}(u)}{\sqrt{f}} \gamma^{t} \psi_{+}, \\
0 & =\left(\sqrt{f} \gamma^{u} \partial_{u}-\frac{i \omega}{\sqrt{f}} \gamma^{t}+i k_{i} \gamma^{i}-\frac{1}{u} m\right) \psi_{-}-i \frac{A_{t}^{3}(u)}{\sqrt{f}} \gamma^{t} \psi_{-}, \\
0 & =\left(\sqrt{f} \gamma^{u} \partial_{u}-\frac{i \omega}{\sqrt{f}} \gamma^{t}+i k_{i} \gamma^{i}-\frac{1}{u} m\right) \psi_{3}
\end{aligned}
$$

which are precisely the equations of motion for fermions (in the fundamental representation of the unbroken $\left.U(1)_{3} \subset S U(2)\right)$ with charges $q=\mp 1,0$. As in section 5.2, we emphasize that, because we consider a particular embedding of the Dirac equation into string theory, the allowed values of the mass and charge of the fermions are constrained by supersymmetry and T-duality.

We now follow appendix A of [51] to simplify the equation of motion further. First we rewrite the equation as 17

$$
\begin{gathered}
\left(\sqrt{f} \gamma^{u} \partial_{u}-\frac{1}{u} m\right) \psi+i K_{\mu}(u) \gamma^{\mu} \psi=0, \\
K_{\mu}(u)=\left(-v(u), k_{i}\right), \quad v(u)=\frac{1}{\sqrt{f}}\left(\omega+q A_{t}(u)\right),
\end{gathered}
$$

where the index $i$ runs over spatial directions, $q=\mp 1$ for $\psi_{ \pm}$and $q=0$ for $\psi_{3}$. Notice that near the boundary, $v(u) \rightarrow \omega+q \mu$, so the frequency $\omega$ is measured relative to $(q$ times) the chemical potential.

\footnotetext{
${ }^{15}$ We only need $\gamma^{y}$ when $P \geq 4$.

${ }^{16}$ Here the \pm index refers to linear combinations of the worldvolume fermions that diagonalize the equations of motion when $A_{x}^{1}(u)=0$. Nowhere in this subsection or the next do we use a \pm index to refer to the projectors $\Pi_{ \pm}$of section 2.3.1 or to the eigenvalues of the Dirac operator on $S^{Q}$ of equation (5.2.10).

${ }^{17}$ Starting now, we use the notation $\psi$ to refer to any of our three fermions, when we are making general statements.
} 
The system is rotationally invariant, so without loss of generality we can take only $k_{x}$ to be nonzero. Obviously, this is not the case in the superfluid phase, where rotational symmetry is broken. The fermion's equation of motion then depends only on $\gamma^{u}, \gamma^{t}$ and $\gamma^{x}$, so the projectors $\Pi_{\alpha}$ commute with the operator acting on the $\psi$ in equation (5.2.27), hence the equations for $\phi_{\alpha} \equiv \Pi_{\alpha} \psi$ decouple from one another. In terms of the $\phi_{\alpha}$, the equation of motion becomes

$$
\left(\partial_{u}+\frac{1}{u \sqrt{f}} m \sigma_{3}\right) \phi_{\alpha}+\frac{1}{\sqrt{f}}\left(-i v(u) \sigma_{2}-(-1)^{\alpha} k_{x} \sigma_{1}\right) \phi_{\alpha}=0 .
$$

We thus obtain six decoupled equations, four for the $\phi_{ \pm \alpha}$ and two for the $\phi_{3 \alpha}$.

Equation (5.2.29) is almost identical to equation (A14) of [51]. The biggest difference is the function $f(u)$, which for us is the $f(u)$ of $(4+1)$-dimensional AdS-Schwarzschild and in [51] was the $f(u)$ of $(3+1)$-dimensional AdS-Schwarzschild. Given that we solve nearly identical equations of motion, we obtain qualitatively similar finite-temperature results. As mentioned above, however, we cannot reach $T=0$ within the probe approximation, so we are not able to reproduce the $T=0$ results of $[49,51]$, including in particular the influence of an emergent $A d S_{2}$.

\subsubsection{Superfluid Phase}

In the solution corresponding to the superfluid phase, where $A_{x}^{1}(u)$ is nonzero, we cannot write linear combinations of $\psi_{1}, \psi_{2}$ and $\psi_{3}$ to diagonalize the system and produce three decoupled equations. To make comparison to the normal phase easier, we again work with $\psi_{ \pm}=\psi_{2} \pm i \psi_{1}$, so that equations (5.2.18)-(5.2.20) become

$$
\begin{aligned}
0 & =\left(\sqrt{f} \gamma^{u} \partial_{u}-\frac{i \omega}{\sqrt{f}} \gamma^{t}+i k_{i} \gamma^{i}-\frac{1}{u} m\right) \psi_{+}+i \frac{A_{t}^{3}(u)}{\sqrt{f}} \gamma^{t} \psi_{+}+A_{x}^{1}(u) \gamma^{x} \psi_{3}, \\
0 & =\left(\sqrt{f} \gamma^{u} \partial_{u}-\frac{i \omega}{\sqrt{f}} \gamma^{t}+i k_{i} \gamma^{i}-\frac{1}{u} m\right) \psi_{-}-i \frac{A_{t}^{3}(u)}{\sqrt{f}} \gamma^{t} \psi_{-}+A_{x}^{1}(u) \gamma^{x} \psi_{3}, \\
0 & =\left(\sqrt{f} \gamma^{u} \partial_{u}-\frac{i \omega}{\sqrt{f}} \gamma^{t}+i k_{i} \gamma^{i}-\frac{1}{u} m\right) \psi_{3}-\frac{1}{2} A_{x}^{1}(u) \gamma^{x}\left(\psi_{+}+\psi_{-}\right)
\end{aligned}
$$

Clearly the three fermions $\psi_{ \pm}$and $\psi_{3}$ couple to one another via a nonzero $A_{x}^{1}(u)$. Here we have a concrete example of the couplings described in section 3.2 .4 (especially around equation (3.2.34) $)$. We simplify the $\psi_{+}$and $\psi_{-}$equations again by writing them as

$$
\begin{aligned}
0 & =\left(\sqrt{f} \gamma^{u} \partial_{u}-\frac{1}{u} m\right) \psi_{+}+i K_{\mu}(u) \gamma^{\mu} \psi_{+}+A_{x}^{1}(u) \gamma^{x} \psi_{3}, \\
0 & =\left(\sqrt{f} \gamma^{u} \partial_{u}-\frac{1}{u} m\right) \psi_{-}+i K_{\mu}(u) \gamma^{\mu} \psi_{-}+A_{x}^{1}(u) \gamma^{x} \psi_{3}, \\
0 & =\left(\sqrt{f} \gamma^{u} \partial_{u}-\frac{1}{u} m\right) \psi_{3}+i K_{\mu}(u) \gamma^{\mu} \psi_{3}-\frac{1}{2} A_{x}^{1}(u) \gamma^{x}\left(\psi_{+}+\psi_{-}\right),
\end{aligned}
$$

where $K_{\mu}$ is defined the same way as in equation (5.2.28). Recall that $\psi_{+}$has charge $q=-1, \psi_{-}$has charge $q=+1$ and $\psi_{3}$ has charge $q=0$.

Now we come to a big difference from the solution corresponding to the normal phase, at least for $D p$-branes wrapping $A d S_{P}$ with $P \geq 4$. In the solution corresponding to the 
superfluid phase, rotational symmetry is broken from $S O(P-2)$ to $S O(P-3)$. Using the $S O(P-3)$ rotational symmetry, the most general momentum we can pick has nonzero $k_{x}$ and nonzero $k_{y}$. The equations for $\psi_{+}$and $\psi_{-}$then depend on $\gamma^{u}, \gamma^{t}, \gamma^{x}$ and now also $\gamma^{y}$, hence the $\Pi_{\alpha}$ projectors no longer commute with the operators acting on the fermions in the equations of motion, and the equations for the $\phi_{\alpha} \equiv \Pi_{\alpha} \psi$ do not decouple from each other. Upon acting with the projectors $\Pi_{\alpha}$, the equations of motion become

$$
\begin{gathered}
0=\left[\partial_{u}+\frac{m}{u \sqrt{f}} \sigma_{3}+\frac{1}{\sqrt{f}}\left(-i v(u) \sigma_{2}-(-1)^{\alpha} k_{x} \sigma_{1}\right)\right] \phi_{+\alpha} \\
\quad-\frac{k_{y}}{\sqrt{f}} \sigma_{1}(-1)^{\alpha+1} \epsilon^{\alpha \beta} \phi_{+\beta}-\frac{A_{x}^{1}}{\sqrt{f}}(-1)^{\alpha} i \sigma_{1} \phi_{3 \alpha}, \\
0=\left[\partial_{u}+\frac{m}{u \sqrt{f}} \sigma_{3}+\frac{1}{\sqrt{f}}\left(-i v(u) \sigma_{2}-(-1)^{\alpha} k_{x} \sigma_{1}\right)\right] \phi_{-\alpha} \\
\quad-\frac{k_{y}}{\sqrt{f}} \sigma_{1}(-1)^{\alpha+1} \epsilon^{\alpha \beta} \phi_{-\beta}-\frac{A_{x}^{1}}{\sqrt{f}}(-1)^{\alpha} i \sigma_{1} \phi_{3 \alpha}, \\
0=\left[\partial_{u}+\frac{m}{u \sqrt{f}} \sigma_{3}+\frac{1}{\sqrt{f}}\left(-i v(u) \sigma_{2}-(-1)^{\alpha} k_{x} \sigma_{1}\right)\right] \phi_{3 \alpha} \\
-\frac{k_{y}}{\sqrt{f}} \sigma_{1}(-1)^{\alpha+1} \epsilon^{\alpha \beta} \phi_{3 \beta}+\frac{1}{2} \frac{A_{x}^{1}}{\sqrt{f}}(-1)^{\alpha} i \sigma_{1}\left(\phi_{+\alpha}+\phi_{-\alpha}\right) .
\end{gathered}
$$

Here $\epsilon^{\alpha \beta}$ is antisymmetric with $\epsilon^{12}=+1$. Notice that when $k_{y}$ is nonzero, the $\phi_{1}$ and $\phi_{2}$ couple.

Equation (5.2.36) is the result for any Dp-brane extended along $A d S_{P} \times S^{Q}$. What will change from one Dp-brane to another are the allowed values of $m$ and the solutions for $A_{t}^{3}(u)$ and $A_{x}^{1}(u)$. In the next section we specialize to the D5-brane extended along $A d S_{4} \times S^{2}(P=4$ and $Q=2)$, and to the massless worldvolume fermion.

\subsection{Emergence of the Fermi surface}

\subsubsection{Properties of the Spectral Function}

For the probe D5-brane worldvolume fermions of the last section, we solved the linearized equations of motion, equations (5.2.36), numerically, and used these solutions to extract the fermionic spectral functions. In this section we present a selection of our numerical results.

We work with two D5-branes extended along (when $T=0$ ) $A d S_{4} \times S^{2}$ inside $A d S_{5} \times S^{5}$. As shown in section 5.2.1, we have many worldvolume fermions to choose from, with many different masses. In our numerical analysis we work exclusively with the massless worldvolume fermion. The dual operator is then the $l=0$ case of the mesino operator $\mathcal{F}_{l}$ written explicitly in equation (2.5.90). These mesinos are valued in the adjoint of the $S U(2)$ isospin symmetry, so we actually have three mesinos, $\mathcal{F}_{0}^{+}, \mathcal{F}_{0}^{-}$, and $\mathcal{F}_{0}^{0}$, where the superscript denotes the charge under $U(1)_{3}$. These are dual to the three fermions $\psi^{ \pm}$and $\psi^{0}$ in subsection 5.2.2.2. 
In more detail, our procedure is as follows. We first choose the values of $T$ and $\mu$ that we want, and solve for the background $S U(2)$ gauge field functions $A_{t}^{3}(u)$ and $A_{x}^{1}(u)$, as in section 4.3.1. Plugging the gauge field solution into equations (5.2.36), we then solve for the bulk fermions. Near the horizon the fermions have the form of an in-going wave, equation (3.2.35), with the $\alpha$ in that equation being $\alpha=\frac{\omega}{4 \pi T}$ for our system. When $A_{x}^{1}(u)$ is nonzero, the fermions couple to one another, hence we employ the technique of section 3.2.4 to compute the retarded Green's function, which then gives us the spectral function, as we explain below.

The normalization of our Green's functions is fixed by the normalization of the fermionic part of the D5-brane action, equation (5.2.1). The normalization includes various numerical factors, and in particular depends on the normalization of the $S^{5}$ spinor $\chi$ defined in equation (5.2.6). We omit the details, but we mention that the normalization includes a factor of $N_{f} T_{D 5} \propto \sqrt{\lambda} N_{f} N_{c}$. In what follows we rescale our Green's functions by the overall normalization. In other words, we divide the action by the normalization factor, so that we obtain an effective $A d S_{4}$ Dirac action with a Lagrangian of the form $i \bar{\Psi} \Delta \Psi$.

As explained in section 3.2.4, with three bulk fermions, the field theory retarded Green's function is a $6 \times 6$ matrix. In the normal phase where the three bulk fermions decouple, the Green's function is diagonal in isospin indices and in the subspaces defined by the $\Pi_{1,2}$ projectors defined in equation (3.2.21) (see also equation (3.2.22)). Explicitly, the retarded Green's function has the form

$$
\left[G_{A B}^{R}\left(\omega, k_{x}, k_{y}\right)\right]=\operatorname{diag}\left(G_{-2}^{R}, G_{-1}^{R}, G_{+2}^{R}, G_{+1}^{R}, G_{02}^{R}, G_{01}^{R}\right)
$$

with $A, B=1, \ldots, 6$, so that $A=1$ corresponds to the components of the $\mathcal{F}_{0}^{-}$mesino in the $\Pi_{2}$ subspace, $A=2$ corresponds to $\mathcal{F}_{0}^{-}$in the $\Pi_{1}$ subspace, $A=3$ corresponds to the components of the $\mathcal{F}_{0}^{+}$mesino in the $\Pi_{2}$ subspace, and so on. In the superfluid phase, due to the bulk couplings, for generic momenta all the off-diagonal elements become nonzero.

In section 3.1 we defined the spectral function $\mathcal{R}_{a b}$ as the anti-Hermitian part of the retarded Green's function,

$$
\mathcal{R}_{A B}\left(\omega, k_{x}, k_{y}\right) \equiv i\left(G_{A B}^{R}\left(\omega, k_{x}, k_{y}\right)-G_{A B}^{R \dagger}\left(\omega, k_{x}, k_{y}\right)\right)
$$

As review in section 3.1, stability requires that the eigenvalues of $\mathcal{R}_{A B}$ are positive and note an arbitrary component of the matrix $\mathcal{R}$. In particular the off-diagonal elements $\mathcal{R}_{A B}$ with $A \neq B$ can be negative. But the diagonal elements of the matrix $\mathcal{R}$ have to be positive and therefore also the spectral measure $\mathcal{R}\left(\omega, k_{x}, k_{y}\right)$ which is defined as the trace over $\mathcal{R}_{A B}\left(\omega, k_{x}, k_{y}\right)$ (a trace over both flavor and spinor indices),

$$
\mathcal{R}\left(\omega, k_{x}, k_{y}\right) \equiv \operatorname{tr} \mathcal{R}_{A B}\left(\omega, k_{x}, k_{y}\right)
$$

We have confirmed that our numerical result for the spectral function obeys the following symmetries in both the normal and superfluid phases (in each case, any argument not 
shown is invariant):

$$
\begin{aligned}
\mathcal{R}_{11}(-\omega)=\mathcal{R}_{44}(\omega), & \mathcal{R}_{22}(-\omega)=\mathcal{R}_{33}(\omega), \\
\mathcal{R}_{55}(-\omega)=\mathcal{R}_{66}(\omega), & \\
\mathcal{R}_{11}\left(-k_{x}\right)=\mathcal{R}_{22}\left(k_{x}\right), & \mathcal{R}_{33}\left(-k_{x}\right)=\mathcal{R}_{44}\left(k_{x}\right), \\
\mathcal{R}_{55}\left(-k_{x}\right)=\mathcal{R}_{66}\left(k_{x}\right), & \\
\mathcal{R}_{11}\left(-\omega,-k_{y}\right)=\mathcal{R}_{44}\left(\omega, k_{y}\right), & \mathcal{R}_{22}\left(-\omega,-k_{y}\right)=\mathcal{R}_{33}\left(\omega, k_{y}\right), \\
\mathcal{R}_{55}\left(-\omega,-k_{y}\right)=\mathcal{R}_{66}\left(\omega, k_{y}\right) & .
\end{aligned}
$$

An example for frequency and momentum symmetries in the off-diagonal elements is

$$
\mathcal{R}_{A B}\left(-k_{y}\right)=(-1)^{A+B} \mathcal{R}_{B A}{ }^{*}\left(k_{y}\right),
$$

which is also true for all diagonal elements, stating their invariance under $k_{y} \rightarrow-k_{y}$. We have also confirmed that our numerical results obey

$$
\mathcal{R}_{A B}=\mathcal{R}_{B A^{*}}
$$

which follows directly from the definition of the spectral function in equation (5.3.38).

Lastly, notice that because we study a fermionic operator of dimension $3 / 2$, the retarded Green's function, and hence the spectral function and measure, are dimensionless.

\subsubsection{Numerical Results}

First we compute the spectral function for temperatures below $T_{c}$ but in the normal (nonsuperfluid) phase, which we know is thermodynamically disfavored. We do so for two reasons: first, to compare later to the superfluid phase, and second, to reproduce some of the finite-temperature results of [49], as a check of our methods. In practical terms, we use the solution for the gauge field with $A_{t}^{3}(u)$ (from equation (4.3.15) with $P=4$ ) and zero $A_{x}^{1}(u)$. Figure 5.1 shows two diagonal spectral function components, $\mathcal{R}_{A A}\left(\omega, k_{x}, k_{y}\right)$ with $A=1,5$, as functions of $k_{x} / \pi T$, with $\omega=k_{y}=0$, for $T \leq T_{c}$ in the normal phase.

Figure 5.1 (a) shows the component $\mathcal{R}_{11}$, which corresponds to the components of the mesino $\mathcal{F}_{0}^{-}$in the $\Pi_{2}$ subspace, at temperature $T=0.61 T_{c}$. For the moment, our main point is that figure 5.1 (a) is qualitatively similar to figure 4 of [49]. As we lower the temperature, the peaks in the figure move to larger momenta and additional peaks appear near zero momentum. In fact, in figure 5.1 (a), $\mathcal{R}_{11}$ along negative momenta (dashed blue curve) already displays a small bump near $k_{x} / \pi T=0$, which grows into a peak as we cool the system. Similar effects were observed in [49], and were interpreted as the emergence of multiple Fermi surfaces at different momenta. Additionally, the spectral functions for our other charged fermions in the low-temperature normal phase are similar to those in $[49,51]$, so we do not present them.

Figure 5.1 (b) shows the component $\mathcal{R}_{55}$, which corresponds to the neutral operator $\mathcal{F}_{0}^{0}$, in the $\Pi_{2}$ subspace, at temperatures $T=T_{c}, 0.75 T_{c}$, and $0.61 T_{c} . \mathcal{R}_{55}$ is featureless here, but will not be so in the p-wave phase. Notice that $\mathcal{R}_{55}$ also does not change as the temperature decreases, or equivalently as the chemical potential increases, since when $q=0$ the chemical potential does not enter the relevant bulk fermion's equation of motion: 


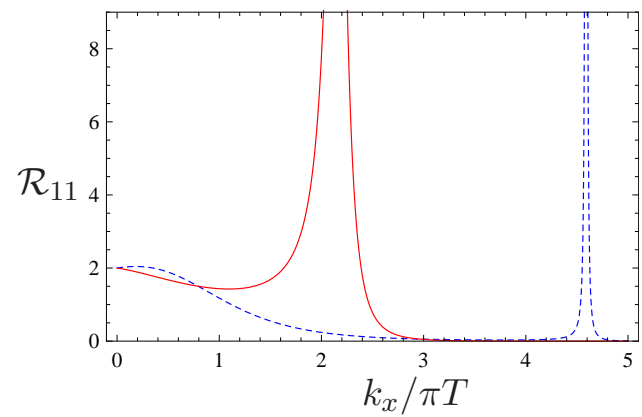

(a)

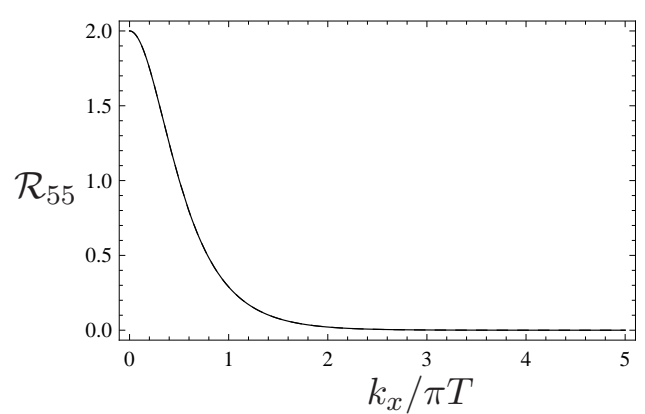

(b)

Figure 5.1: Two spectral function components $\mathcal{R}_{A A}\left(\omega, k_{x}, k_{y}\right)$, for $A=1,5$, plotted versus the rescaled momentum $k_{x} / \pi T$, with $\omega=k_{y}=0$, at temperatures $T \leq T_{c}$ but in the normal (nonsuperfluid) phase, i.e. in the thermodynamically disfavored phase. (a) $\mathcal{R}_{11}$, corresponding to the fermionic operator with charge $q=-1$, at $T=0.61 T_{c}$. The two curves are for positive momentum $k_{x}$ (solid red curve) and negative momentum $-k_{x}$ (dashed blue curve), the latter case being equal to $\mathcal{R}_{22}\left(\omega, k_{x}, k_{y}\right)$ at positive momentum due to the symmetries of the spectral function. Multiple peaks are visible, just as in [49]. (b) $\mathcal{R}_{55}$, corresponding to a fermionic operator with charge $q=0$, at temperatures $T=T_{c}$ (solid curve), $T=0.75 T_{c}$, (grey dotted curve), $T=0.61 T_{c}$ (dashed curve). The curves remain coincident since changing the chemical potential does not affect the uncharged operator.

see equation (5.2.28). Next we plot the essentially the same thing as in figure 5.1, but now in the thermodynamically favored superfluid phase. More precisely, figure 5.2 shows two diagonal spectral function components, $\mathcal{R}_{A A}\left(\omega, k_{x}, k_{y}\right)$ with $A=1,5$, as a function of the rescaled momentum $k_{x} / \pi T$, with $\omega=k_{y}=0$, for $T \leq T_{c}$ in the superfluid phase, i.e. now with nonzero $A_{x}^{1}(u)$. Figure 5.2 (a) shows the same component of the spectral function as in figure 5.1 (a), $\mathcal{R}_{11}$, again with $T=0.61 T_{c}$. Figure 5.2 (b) shows the same component of the spectral function as in figure 5.1 (b), $\mathcal{R}_{55}$, at the same temperatures $T=T_{c}, 0.75 T_{c}$, and $0.61 T_{c}$.

The operator mixing is obvious in figure 5.2: the spectral function for a neutral fermion, $\mathcal{R}_{55}$, develops a bump that grows into a small peak located at the same momentum as the peak in $\mathcal{R}_{11}, k_{x} / \pi T=3.87$. In bulk terms, the coupling between $\phi_{3}$ and $\phi_{ \pm}$in equation (5.2.36) is allowing the peak in the charged fermions' spectral functions to "leak" into the spectral functions of the neutral fermions. That coupling is proportional to $A_{x}^{1}(u)$, hence the peak should grow as the temperature decreases and $A_{x}^{1}(u)$ grows, which is indeed what we see in figure 5.2 (b). The method we developed in section 3.2 .4 for computing retarded Green's functions for coupled bulk fermions seems to work very well. As another comparison between the normal and broken phases when $T \leq T_{c}$, we focus on the pole in the retarded Green's function $G_{11}^{R}\left(\omega, k_{x}, k_{y}\right)$, for the mesino with charge $q=-1$ in the $\Pi_{2}$ subspace, that is closest to the origin of the complex frequency plane, $\omega=0$, and follow the movement of the pole in the frequency plane as we change the momentum. 18

Figure 5.3 (a) shows the movement of the pole in the normal phase when $T=0.91 T_{c}$ for values of $k_{x} / \pi T \in[2.579,12.580]$, which in the figure corresponds to starting at the

\footnotetext{
${ }^{18}$ Poles in the retarded Green's function are holographically equivalent to the bulk fermion's quasinormal modes $[138,175,176]$. As explained in section 3.2.4 we can detect these quasi-normal modes from the vanishing of $\operatorname{det} P^{-}(\epsilon)$, where the matrix $P^{-}$is defined in equations (3.2.37) and (3.2.38).
} 


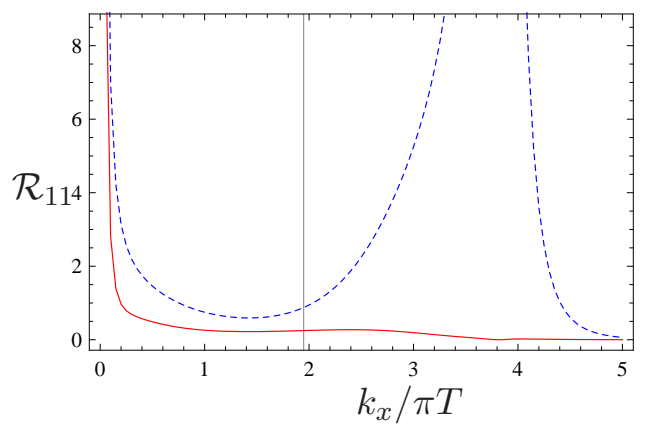

(a)

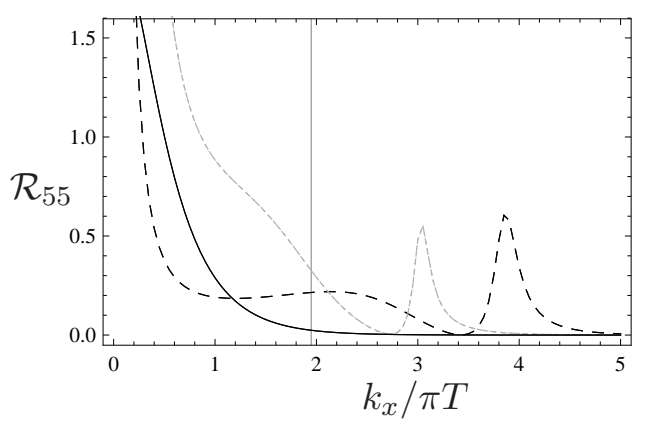

(b)

Figure 5.2: Two spectral function components $\mathcal{R}_{A A}\left(\omega, k_{x}, k_{y}\right)$ plotted versus the rescaled momentum $k_{x} / \pi T$ with $\omega=k_{y}=0$ in the superfluid phase. (a) Exactly the same spectral function as in figure 5.1 (a), $\mathcal{R}_{11}$, at the same temperature $T=0.61 T_{c}$, but now in the superfluid phase. The two curves correspond to positive momentum $k_{x}$ (solid red curve) or negative momentum $-k_{x}$ (dashed blue curve). (b) Exactly the same spectral function component as in 5.1 (b), $\mathcal{R}_{55}$, at the same temperatures $T_{c}$ (solid curve), $T=0.75 T_{c}$ (grey dotted curve), $T=0.61 T_{c}$ (dashed curve). Here we see operator mixing: a feature develops in the neutral fermion's spectral function in the p-wave phase. A bump grows into a peak at the same momentum $k_{x} / \pi T \approx 3.87$ as the peak in $\mathcal{R}_{11}$.

point nearest $\operatorname{Re}[\omega / \pi T]=0$ and moving toward the upper left. The pole asymptotically approaches the real frequency axis $\operatorname{Im} \omega / \pi T=0$, as $k_{x}$ increases.

At a temperature $T^{*} \approx 0.6 T_{c}$, however, the distance to the real axis develops a local minimum. Figure 5.3 (b) shows the movement of the same pole as figure 5.3 (a) at a temperature $T=0.48 T_{c}<T^{*}$, still in the normal phase. Here we see that the distance to the real frequency axis has a local minimum at $(\operatorname{Re} \omega / \pi T, \operatorname{Im} \omega / \pi T)=(-1.79,-7.21 \times$ $10^{-6}$ ) when $k_{x} / \pi T=8.15$. Such behavior persists to lower temperatures, and indeed, the distance to the real frequency axis decreases. The lowest temperature we studied was $T=$ $0.19 T_{c}$, where the local minimum occurred at $(\operatorname{Re} \omega / \pi T, \operatorname{Im} \omega / \pi T)=\left(5.32,2.64 \times 10^{-18}\right)$ when $k_{x} / \pi T=23.22$.

We seem to be seeing the emergence of a Fermi surface, which, as in [49], would occur at $T=0$ when the pole would reach the origin of the complex frequency plane at some finite momentum $k_{F}$, the Fermi momentum. Let us consider low temperature, and define $k^{*}$ as the value of momentum where the closest approach to the real frequency axis occurs. When $T=0, k^{*}$ would be the Fermi momentum, $k^{*}=k_{F}$. At our lowest temperature, $T=0.19 T_{c}, k_{x}^{*} / \pi T \equiv 23.22$, and the closest approach to the real frequency axis occurs at an $\omega^{*}$ given by $\left(\operatorname{Re} \omega^{*}, \operatorname{Im} \omega^{*}\right)=\left(5.32,2.64 \times 10^{-18}\right) \pi T$. Letting $k^{*}$ play the role of $k_{F}$, we see behavior similar to the results of [49]: for small but nonzero temperature, as we change the momentum the frequency of the pole behaves as

$$
\omega-\omega^{*} \sim\left(k-k^{*}\right)^{z},
$$

where our numerical results indicate that the exponent $z=1.00 \pm 0.01$, and the spectral function behaves as

$$
\mathcal{R}_{11} \sim\left(k-k^{*}\right)^{-\alpha},
$$

where our numerical results indicate that the exponent $\alpha=2.0 \pm 0.1$. In fact, these results are rather robust: we find the same $z$ and $\alpha$ for many values of $T<T^{*}$, and for several 


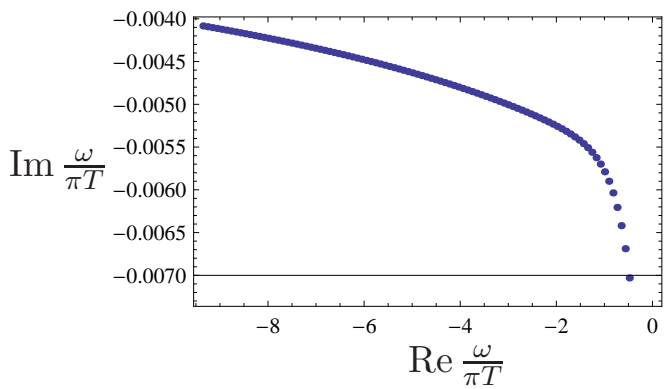

(a)

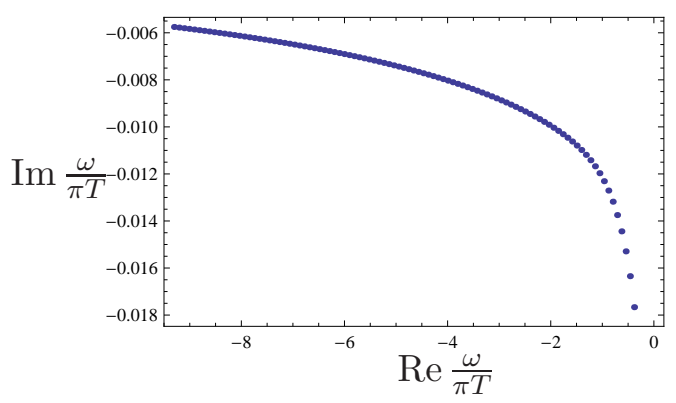

(c)

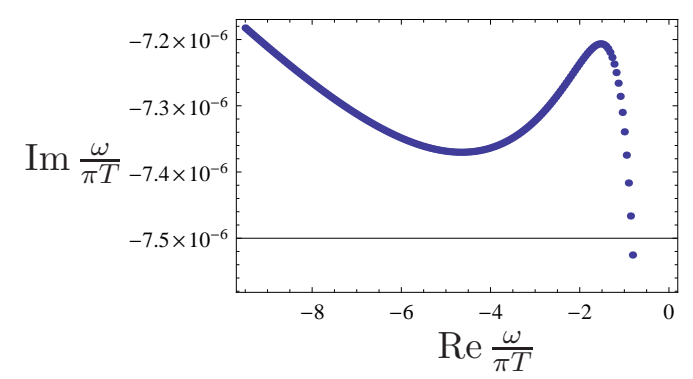

(b)

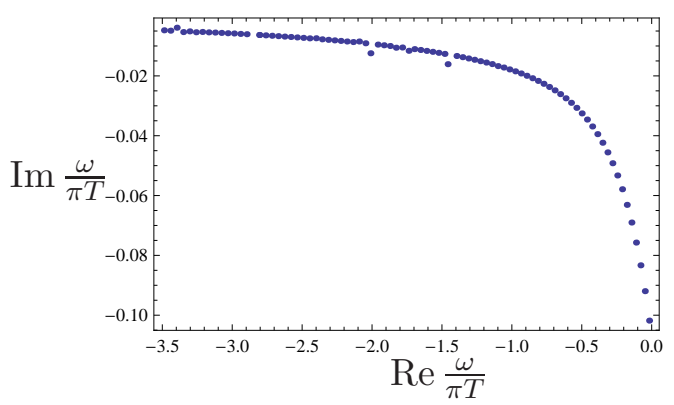

(d)

Figure 5.3: The movement of the pole in the retarded Green's function $G_{11}^{R}\left(\omega, k_{x}, k_{y}\right)$, for a mesino of charge $q=-1$, that is closest to $\omega=0$, as a function of momentum, for $T<T_{c}$, in both the normal, thermodynamically disfavored, phase and superfluid, thermodynamically favored, phase. (a) The movement of the pole in the normal phase at $T=0.91 T_{c}$, for $k_{x} / \pi T \in[2.58,12.58]$. As the momentum increases, the pole moves from the lower right, near $\operatorname{Re} \omega / \pi T=0$, toward the upper left. The same applies for the following three figures. The pole asymptotically approaches the real frequency axis, $\operatorname{Im} \omega / \pi T=0$, as $k_{x}$ increases. (b) The movement of the pole in the normal phase at $T=0.48 T_{c}$, for $k_{x} / \pi T \in[6.15,16.15]$. Here we see that the distance to the real axis does not decrease monotonically, but rather a local minimum develops at $(\operatorname{Re} \omega / \pi T, \operatorname{Im} \omega / \pi T)=$ $\left(-1.79,-7.21 \times 10^{-6}\right)$ when $k_{x} / \pi T=8.15$. (c) The movement of the pole in the superfluid phase at $T=0.91 T_{c}$, for $k_{x} / \pi T \in[2.49,12.29]$ and $k_{y}=0$. The movement is qualitatively similar to (a). (d) The movement of the pole in the superfluid phase at $T=0.48 T_{c}$, for $k_{x} / \pi T \in[4.83,8.98]$ and $k_{y}=0$. The movement is again qualitatively similar to (a), in particular, the distance to the real frequency axis does not develop a local minimum, in contrast to the normal phase result in (b). We see qualitatively similar behavior when we set $k_{x}=0$ and increase $k_{y}$. 
other poles. These results suggest that the low-temperature normal phase may not be a Landau Fermi liquid, which would have $z=\alpha=1$.

Figures 5.3 (c) and (d) show the movement of the same pole, at the same temperatures, but in the superfluid phase 19 Figure 5.3 (c) shows the movement of the pole at $T=0.91 T_{c}$ for $k_{x} / \pi T \in[2.49,12.29]$ and $k_{y}=0$. Figure 5.3 (d) shows the movement of the pole at $T=0.48 T_{c}$ for $k_{x} / \pi T \in[4.83,8.98]$ and $k_{y}=0$. Unlike the normal phase result, here the distance to the real frequency axis does not develop a local minimum. In other words, here we do not see a Fermi surface emerge in the same fashion as in the normal phase.

To see the emergence of the p-wave superfluid Fermi surface, we study the spectral measure, which as mentioned above, provides a direct measure of the density of states that have overlap with our fermionic operators.

Our main results concern the evolution of the spectral measure $\mathcal{R}\left(\omega, k_{x}, k_{y}\right)$ as we cool the system through the superfluid phase transition. In the spectral measure we see the breaking of rotational symmetry as we take $T$ below $T_{c}$, and we see the emergence of the Fermi surface as we approach $T=0$, although in the probe limit we do not reach $T=0$. Our results agree qualitatively with the $T=0$ results of [161], in which the Fermi surface in the p-wave phase consists of isolated points.

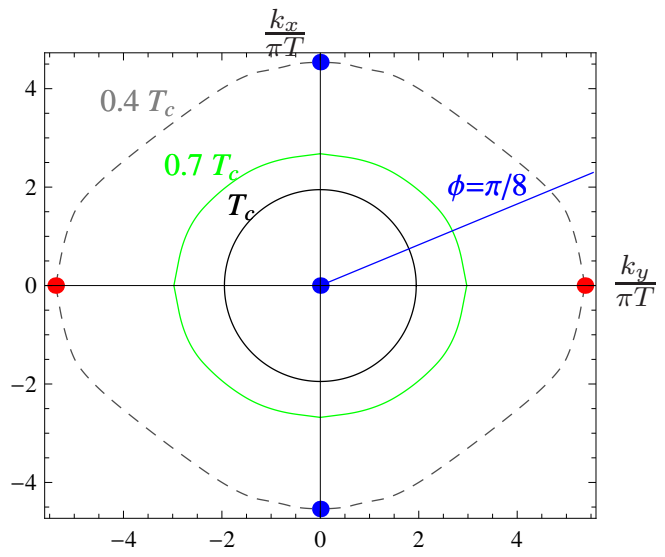

(a)

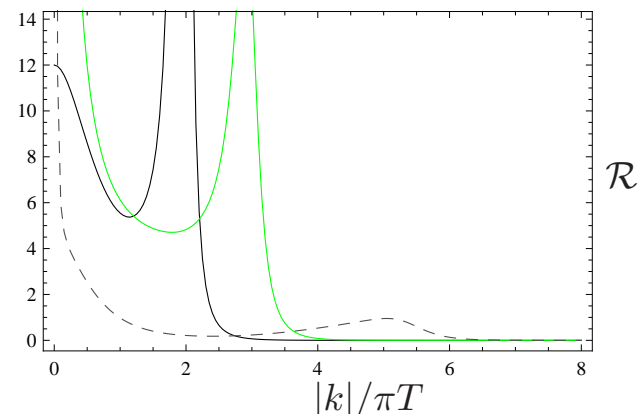

(b)

Figure 5.4: The evolution of the spectral measure $\mathcal{R}\left(\omega, k_{x}, k_{y}\right)=\operatorname{tr} \mathcal{R}_{A B}\left(\omega, k_{x}, k_{y}\right)$ in the $\left(k_{x} / \pi T, k_{y} / \pi T\right)$ plane at $\omega=0$, as we lower the temperature. (a.) The position of peaks in the spectral measure $\mathcal{R}$ are indicated by the curves as we lower the temperature from $T_{c}$ (black curve) through $0.7 T_{c}$ (green curve) to $0.43 T_{c}$ (dashed grey curve). The $T=0.43 T_{c}$ case exhibits a small bump rather than a sharp peak, except for points on the $k_{x}$ and $k_{y}$ axes. We have indicated the bump with the grey dashed curve and the peaks with red and blue dots, including the blue dot at the origin. (b.) The spectral measure $\mathcal{R}$ plotted for a representative slice of the $\left(k_{x} / \pi T, k_{y} / \pi T\right)$ plane (still with $\omega=0$ ), namely along the line given by the polar angle $\phi=\pi / 8$ drawn in (a.). We plot $\mathcal{R}$ versus the magnitude of the momentum $|k|=\sqrt{k_{x}^{2}+k_{y}^{2}}$ divided by $\pi T$, at $T=T_{c}$ (black curve), $T=0.7 T_{c}$ (green curve), and $T=0.43 T_{c}$ (dashed grey curve).

Figure 5.4 provides a road map for the evolution of the spectral measure as we lower the

\footnotetext{
${ }^{19}$ Our independent calculations in the normal and superfluid phases yield the same position for the pole at $T=T_{c}$ to within $0.1 \%$.
} 
temperature. Here we set $\omega=0$, so we are studying fluctuations with zero energy above the chemical potential. Figure 5.4 (a) indicates the locations of peaks in the spectral measure, in the $\left(k_{x} / \pi T, k_{y} / \pi T\right)$ plane, with solid lines and the locations of small bumps as the dashed grey line. At $T=T_{c}$ we see rotational symmetry: the black solid line indicates peaks for any values of $\left(k_{x} / \pi T, k_{y} / \pi T\right)$ on the black circle. At $T=0.7 T_{c}$ the rotational symmetry is mildly broken: the green line is not a perfect circle. At $T=0.43 T_{c}$, sharp peaks only appear at isolated points on the axes, denoted by the red and blue dots (and also at the blue dot at the origin), while the dashed line indicates a small bump, rather than a sharp peak. Here we are clearly seeing the emergence of the Fermi surface at isolated points.

To illustrate what the peaks and bumps look like, we choose a representative slice of the $\left(k_{x} / \pi T, k_{y} / \pi T\right)$ plane, namely the line given by the polar angle $\phi=\pi / 8$, which is drawn in figure 5.4 (a), and plot the $(\omega=0)$ spectral measure versus the magnitude of the momentum $|k|=\sqrt{k_{x}^{2}+k_{y}^{2}}$ divided by $\pi T$. In figure 5.4 (b) a distinct peak is visible for both $T=T_{c}$ (black curve) and $T=0.7 T_{c}$ (green curve), while for this generic (off-axis) value of $\phi$ the only feature of the spectral measure at $T=0.43 T_{c}$ (dashed grey curve) is a small bump. On the axes $(\phi=0, \pi / 2)$, the picture is similar, except the bump becomes a sharp peak, corresponding to the red or blue dots in figure 5.4 (a).

To illustrate the evolution of the spectral measure in more detail, we present threedimensional plots of $\mathcal{R}\left(\omega, k_{x}, k_{y}\right)$, for $\omega=0$, over the $\left(k_{x} / \pi T, k_{y} / \pi T\right)$ plane, for temperatures $T=T_{c}$ (figure 5.5 (a)), $T=0.91 T_{c}$ (figure 5.5 (b)), $T=0.69 T_{c}$ (figure 5.5 (c)) and $T=0.4 T_{c}$ (figure $5.5(\mathrm{~d})$ ). In figure 5.5 (a) we see the peaks corresponding to the black circle in figure 5.4 (a). Clearly here the spectral measure is rotationally symmetric. When we cool the system to $T=0.69 T_{c}$ (figure 5.5 (c)), we clearly see the emergence of five distinct peaks, two on the $k_{x} / \pi T$ axis, two on the $k_{y} / \pi T$ axis, and one at the origin. The circle of peaks corresponds to the green circle in figure 5.4 (a). When we further cool the system to $T=0.4 T_{c}$, the five peaks are still present, although the resolution of our three-dimensional plot is insufficient to resolve the two on the $k_{y} / \pi T$ axis away from the origin.

Although these peaks have a much smaller footprint in the $\left(k_{x} / \pi T, k_{y} / \pi T\right)$ plane than the peaks on the $k_{x} / \pi T$ axis, they are much taller. The spectral measure $\mathcal{R}$ is of order $5 \times 10^{5}$ at the peaks on the $k_{y} / \pi T$ axis, but only order $10^{2}$ at the peaks on the $k_{x} / \pi T$ axis, and order $5 \times 10^{4}$ at the peak at the origin. Apparently a large number of states are piling up at two precise locations on the $k_{y} / \pi T$ axis.

For a bulk theory with $S U(2)$ gauge fields and fermions in the fundamental representation, a combination of analytic and numerical results reveal that the $\omega=0$ spectral measure at $T=0$ consists of two isolated points on the $k_{x}$ axis, located symmetrically about the origin [161]. The analytic arguments of [161] indicate that, for the same bulk theory but for fermions in the adjoint representation, the $\omega=0$ spectral measure at $T=0$ should consist of three isolated points, one at the origin and two on the $k_{x}$ axis, located symmetrically about the origin.

What we see is at least consistent with the results of [161]. The main obstacle to a direct comparison is the probe limit, which restricts us to finite temperatures: we do not know which peaks in our spectral measure persist to $T=0$. Nevertheless, given that we see the three peaks on the $k_{x} / \pi T$ that we generically expect, and that the two peaks on the 


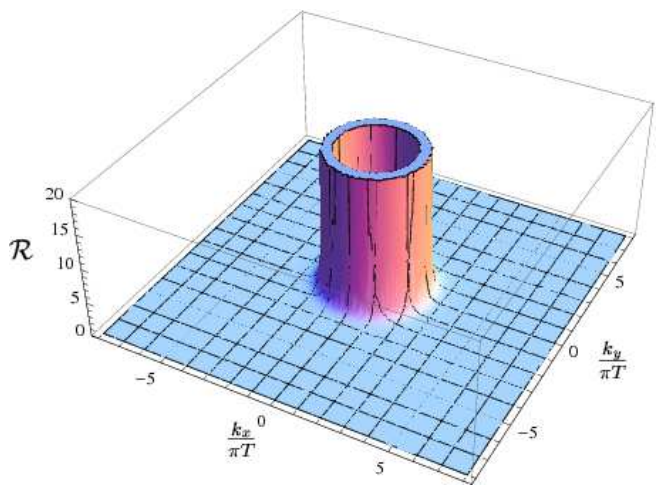

(a) $T=T_{c}, \omega=0$

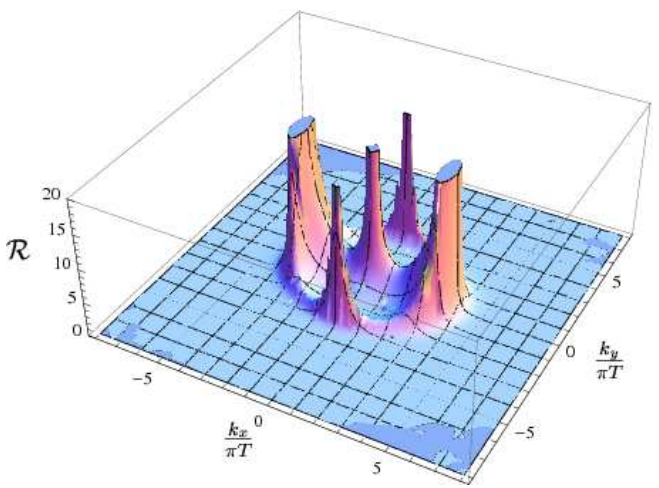

(c) $T=0.69 T_{c}, \omega=0$

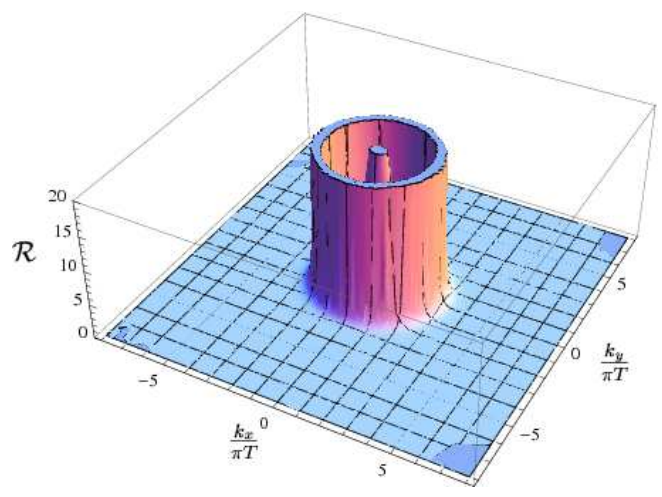

(b) $T=0.91 T_{c}, \omega=0$

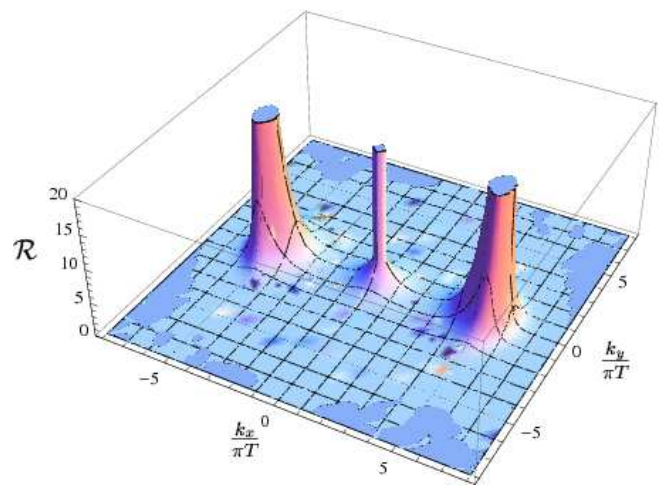

(d) $T=0.43 T_{c}, \omega=0$

Figure 5.5: Three-dimensional plots of the spectral measure $\mathcal{R}\left(\omega, k_{x}, k_{y}\right)$ in the superfluid phase over the $\left(k_{x} / \pi T, k_{y} / \pi T\right)$ plane at zero frequency, $\omega=0$, and for distinct temperatures $T \leq T_{c}$. (a) The $T=T_{c}$ case, which is clearly rotationally invariant. The peaks correspond to the black circle in figure 5.4 (a). (b) The $T=0.91 T_{c}$ case, where the spectral measure does not yet display any dramatic breaking of rotational symmetry. Notice the peak a the origin of the $\left(k_{x} / \pi T, k_{y} / \pi T\right)$ plane. (c) The $T=0.69 T_{c}$ case, where the breaking of rotational symmetry is obvious. We see that the "cylinder" of (a) breaks into five distinct peaks on the $k_{x} / \pi T$ and $k_{y} / \pi T$ axes. The peaks correspond to the green circle in figure 5.4 (a). (d) The $T=0.4 T_{c}$ case, which still has five peaks, labeled by the red and blue dots in figure 5.4 (a). The peaks along the $k_{y} / \pi T$ axis are too narrow to appear in the three-dimensional plot with the resolution we use. 
$k_{y} / \pi T$ axis have a shrinking footprint as we cool the system, we have good reason to believe that the results of [161] may apply to our system. To answer the question fully requires computing the back-reaction of the D5-branes in the bulk. Whether that produces a domain-wall geometry of the kind used in [161] is not guaranteed.

We begin to explore the $\omega$ dependence of the spectral measure $\mathcal{R}\left(\omega, k_{x}, k_{y}\right)$ in figure 5.6. Figure 5.6 (a) simply reproduces the $T=0.4 T_{c}$ part of figure 5.4 (a), where $\omega=0$. Figure 5.6 (b) shows the spectral function along three lines in the $\left(k_{x} / \pi T, k_{y} / \pi T\right)$ plane: along the positive $k_{x} / \pi T$ axis (red curve), along the positive $k_{y} / \pi T$ axis (blue curve), and along the line given by the polar angle $\phi=\pi / 8$ in figure 5.4 (a). Here we see explicitly the difference in widths of the peaks on the $k_{x} / \pi T$ and $k_{y} / \pi T$ axes (the red and blue peaks).

To explore the $\omega$ dependence, we choose a few representative points in the $\left(k_{x} / \pi T, k_{y} / \pi T\right)$ plane and, for each point, plot the spectral measure versus $\omega$. For these points, we consider not only $T=0.4 T_{c}$, as in figure 5.6 (a), but also the slightly higher temperature $T=0.55 T_{c}$, in order to study the behavior as we cool the system.

Our points are similar to those in figure 8 of [161], where the same quantities were plotted (for the slightly different system of [161]): the spectral measure versus $\omega$ for fixed $k_{x}$ and $k_{y}$. We thus compare our results to those of figure 8 of [161] along the way.

In figure 5.6 (1) we plot the $\omega$ dependence of $\mathcal{R}\left(\omega, k_{x}, k_{y}\right)$ for the point (1) labeled in figure 5.6 (a). The dotted line is for $T=0.55 T_{c}$ and the solid line is for $T=0.40 T_{c}$. Here we see that as we cool the system, a small gap (a depletion of states) opens near $\omega=0$, while a sharp peak emerges near $\omega / \pi T \approx 1.7$. Such behavior at least appears to be approaching that of figure 8 (1) in [161], where a genuine gap (zero states) appeared at $\omega=0$.

In figure 5.6 (2) we plot the $\omega$ dependence of $\mathcal{R}\left(\omega, k_{x}, k_{y}\right)$ for the point (2) labeled in figure 5.6 (a). The dotted line is for $T=0.55 T_{c}$ and the solid line is for $T=0.40 T_{c}$. Here we see that a sharp peak near $\omega / \pi T \approx 1.5$ when $T=0.55 T_{c}$ shrinks and begins moving toward $\omega=0$ as we lower the temperature to $T=0.4 T_{c}$. Moreover, the small peak near $\omega / \pi T \approx 3.5$ when $T=0.55 T_{c}$ grows much sharper at $T=0.4 T_{c}$. As in [161], here we seem to see the emergence of the well-known "peak-dip-hump" shape, with the peak being at $\omega / \pi T \approx 3.5$, the dip being at $\omega / \pi T \approx 5.2$, and the hump being at $\omega / \pi T \approx 6.5$.

In figure 8 (2) of [161], a gap was present in the spectral measure for small frequencies, except for a single genuine delta-function peak at finite frequency, and at larger frequency a continuum of states appears (the "hump"). As argued in [161], at finite temperature the delta-function peak acquires a finite width and merge with the hump, producing the peak-dip-hump. Our spectral measure appears to be approaching the form of the spectral measure in figure 8 (2) of [161] (with the usual caveat that we cannot actually reach $T=0)$.

In figure $5.6(3)$ we plot the $\omega$ dependence of $\mathcal{R}\left(\omega, k_{x}, k_{y}\right)$ for the point (3) labeled in figure 5.6 (a), which is sitting right on top of the peak on the positive $k_{x} / \pi T$ axis. The dotted line is for $T=0.55 T_{c}$ and the solid line is for $T=0.40 T_{c}$. At the higher temperature (the dotted line), the primary feature is the peak near $\omega / \pi T \approx 1.2$, which moves toward $\omega=0$ and also shrinks (the peak is lower) as we lower the temperature, becoming the peak in the solid line. Assuming such a trend continues, our results would be consistent with figure 8 (3) of [161], where, sitting right on top of the peak on the $k_{x}$ axis, the spectral measure went to a finite constant at $\omega=0$. 


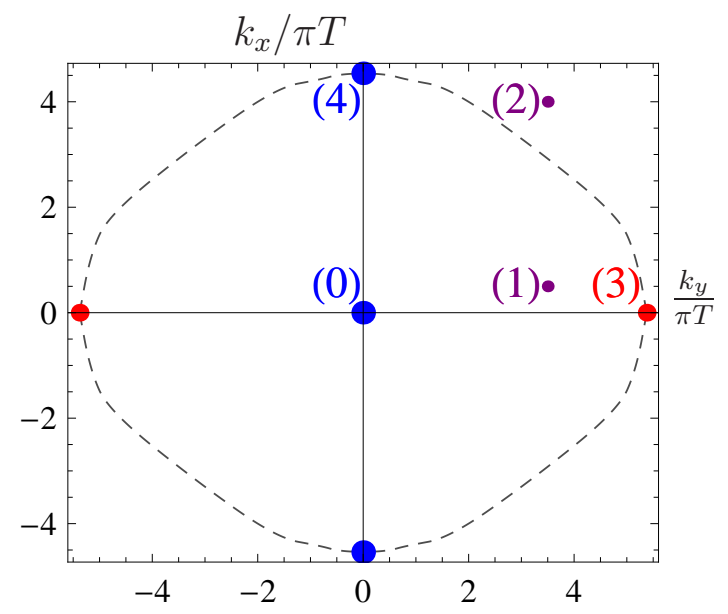

(a)

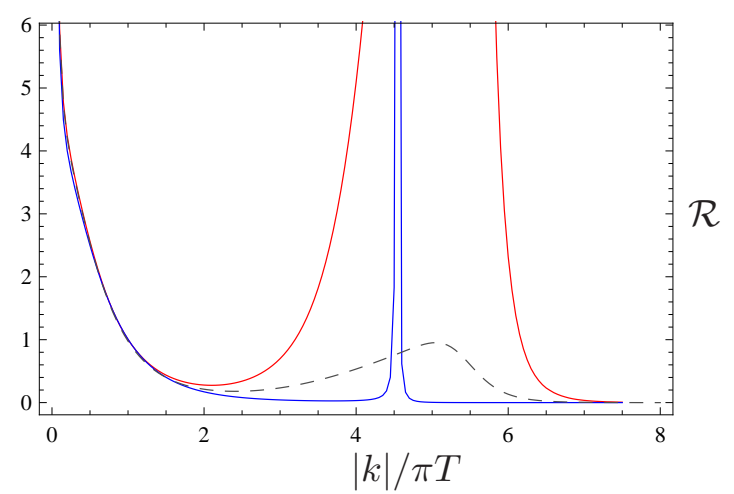

(b)

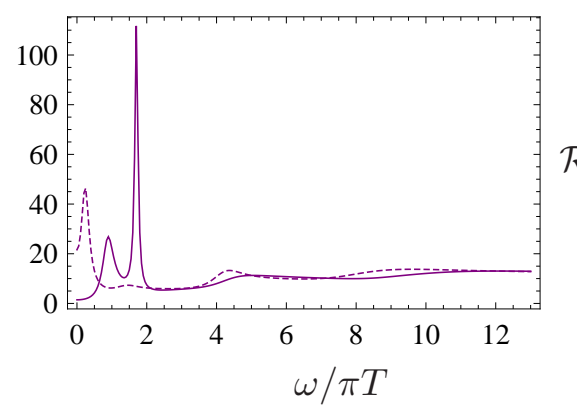

(1)

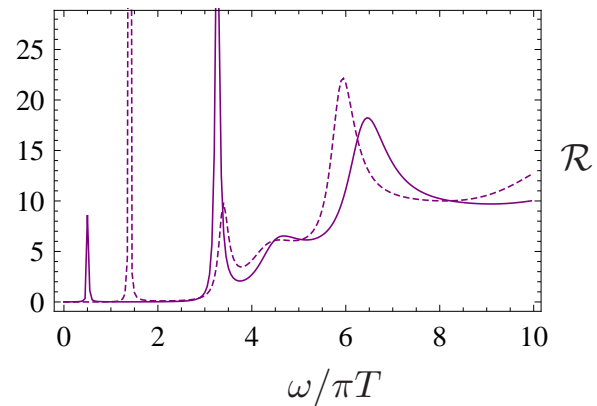

(2)

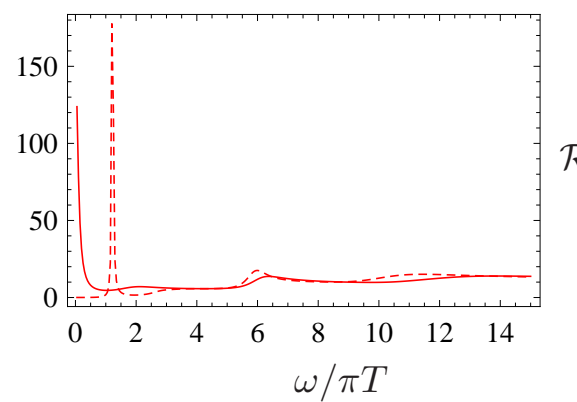

(3)

Figure 5.6: (a) Peaks in the $\omega=0$ spectral measure in the $\left(\frac{k_{x}}{\pi T}, \frac{k_{y}}{\pi T}\right)$ plane at $T=0.4 T_{c}$. The labeling is the same as figure 5.4 (a). (b) The $\omega=0$ spectral measure along three lines in (a): along the $\frac{k_{x}}{\pi T}$ axis (red curve) and the $\frac{k_{y}}{\pi T}$ axis (blue curve), so the peaks at nonzero momenta correspond to (3) and (4) in (a), respectively, and along the polar angle $\phi=\pi / 8$ (dashed grey curve), drawn in figure 5.4 (a), so the bump corresponds to where $\phi=\pi / 8$ intersects the dashed grey line in (a). In each case the peak at zero momentum corresponds to (0) in (a). (1) $\mathcal{R}$ as a function of $\omega / \pi T$ for the point labeled (1) in (a). The dotted and solid lines are for $T=0.55 T_{c}$ and $T=0.4 T_{c}$, respectively. (2) and (3) show the same thing for the corresponding points $(2)$ and (3) in (a). 


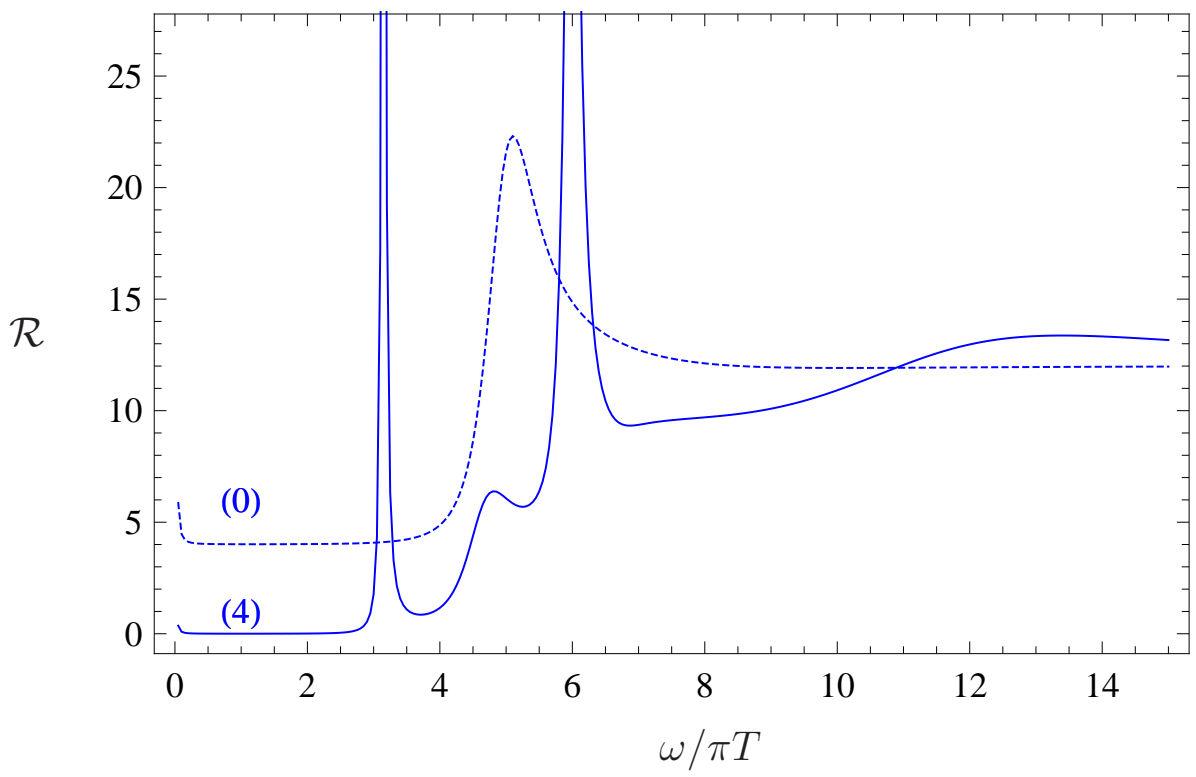

(a)

Figure 5.7: The spectral measure $\mathcal{R}\left(\omega, k_{x}, k_{y}\right)$ as a function of $\omega / \pi T$ for $\left(k_{x} / \pi T, k_{y} / \pi T\right)$ values corresponding to points (0) (dotted blue curve) and (4) (solid blue curve) in figure 5.6 (a). These points sit right on top of the peaks at the origin and on the positive $k_{y} / \pi T$ axis, respectively. Here $T=0.4 T_{c}$.

Figure 5.7 shows $\mathcal{R}\left(\omega, k_{x}, k_{y}\right)$ versus $\omega / \pi T$ for the points (0) and (4) in figure 5.6 (a), sitting right on top of the peaks at the origin and on the positive $k_{y} / \pi T$ axis, respectively. Here we use only $T=0.4 T_{c}$. We clearly see a gap developing at low frequency in both cases. Such behavior is similar to the gap that develops in the spectral function of vector fluctuations (see section 4.3.4), which is immediately related (via a Kubo formula) to a gap in the conductivity. Whether these two gaps are related is unclear, but deserves further study.

Finally, to explore further the $\omega$ dependence of $\mathcal{R}\left(\omega, k_{x}, k_{y}\right)$, we do not restrict to points in the $\left(k_{x} / \pi T, k_{y} / \pi T\right)$ plane, but rather restrict to a single nonzero value of frequency, $\omega / \pi T=0.25$, and plot the spectral measure over the entire $\left(k_{x} / \pi T, k_{y} / \pi T\right)$ plane. The result appears in figure 5.8, where (a) is for $T=T_{c}$, (b) is for $T=0.91 T_{c}$, (c) is for $T=0.54 T_{c}$ and $(\mathrm{d})$ is for $T=0.4 T_{c}$. We see a number of differences from the $\omega=0$ case of figure 5.5. At the transition, $T=T_{c}$, the spectral measure is again rotationally symmetric, but now with two concentric "cylinders." By the time we cool the system to $T=0.54 T_{c}$, we see a number of peaks clumped near the momentum axes. If we compare the spectral measure at $T=0.4 T_{c}$ at $\omega=0$ and $\omega / \pi T=0.25$, figures 5.5 (d) and 5.8 (d), respectively, then we see that the $\omega=0$ peaks on the $k_{y} / \pi T$ axis each split into a number of peaks at $\omega / \pi T=0.25$ which then move apart along the $k_{x} / \pi T$ axis.

To summarize, in the superfluid phase we observe isolated peaks in the spectral measure, whose locations appear to be consistent with the p-wave nature of the condensate, as well as with the fact that our fermions are in the adjoint representation of $S U(2)$. Such a structure suggests nodes in the energy gap on the normal-phase Fermi surface. We plan to investigate this further in the near future, for instance by identifying the appropriate 


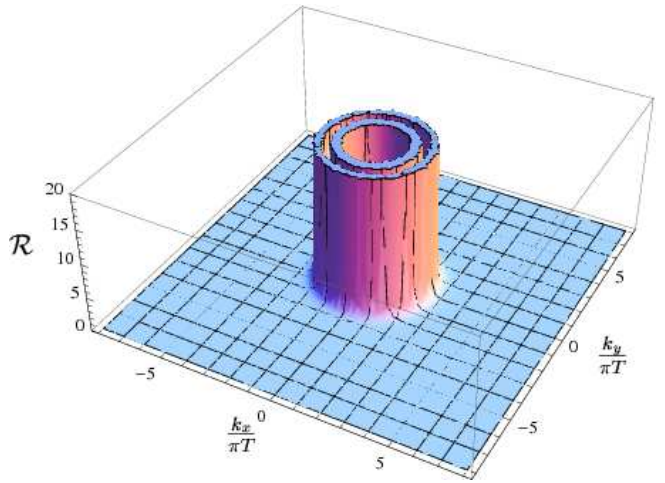

(a) $T=T_{c}, \omega / \pi T=0.25$

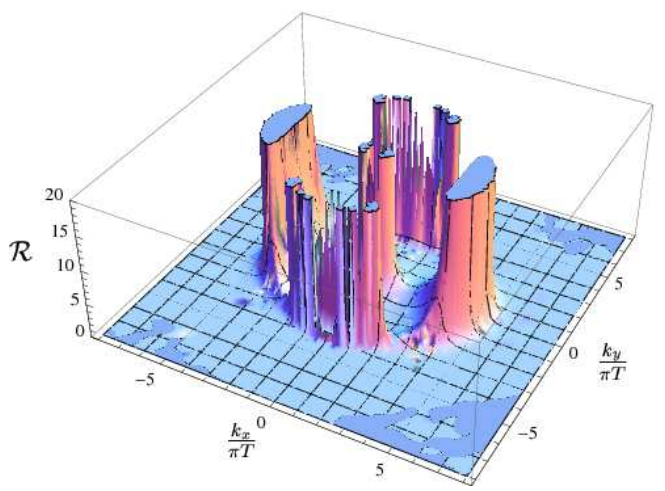

(c) $T=0.54 T_{c}, \omega / \pi T=0.25$

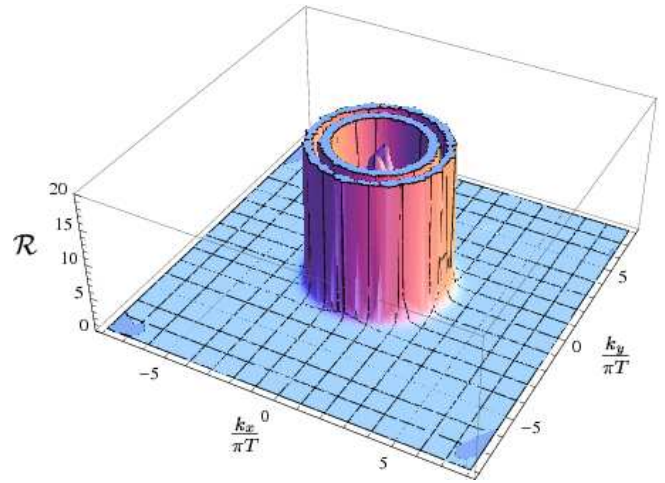

(b) $T=0.91 T_{c}, \omega / \pi T=0.25$

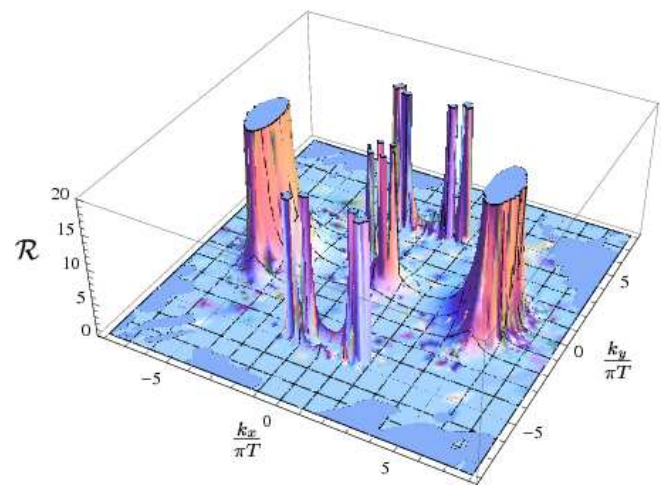

(d) $T=0.43 T_{c}, \omega / \pi T=0.25$

Figure 5.8: Three-dimensional plots of the spectral measure $\mathcal{R}\left(\omega, k_{x}, k_{y}\right)$ in the superfluid phase over the $\left(k_{x} / \pi T, k_{y} / \pi T\right)$ plane at $\omega / \pi T=0.25$, and for distinct temperatures $T \leq T_{c}$. The four plots are arranged in a similar fashion as in figure 5.5 . (a) is the $T=T_{c}$ case, (b) is the $T=0.91 T_{c}$ case, (c) is the $T=0.54 T_{c}$ case, and (d) is the $T=0.4 T_{c}$ case. The main differences from figure 5.5 are that the "cylinder" we saw in figure 5.5 (a) is now two concentric cylinders, and the peaks along the $k_{y} / \pi T$ axis each split into multiple peaks which then move away from one another along the $k_{x} / \pi T$ axis as we cool the system. 
Dirac cones and by studying the spectral measure for fixed values of $\omega$, as well as for further values of fixed $k_{x}, k_{y}$.

This concludes the chapter about fermions and (Non-)Fermi liquid behavior in the AdS/CFT correspondence. Therefore let me recall briefly what we have achieved. First, we have embedded fermions in the top-down approach of AdS/CMT using probe branes and the fermionic part of their effective action. In particular, we have calculated the masses of the bulk fields and determined the conformal dimension of the dual operator. Finally, we studied the fermionic response in the superconducting state, which we found in section 4.3.2. 



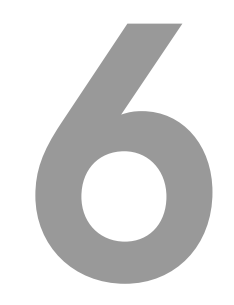

\section{Non-relativistic systems: AC \& DC conductivities}

So far we have considered only relativistic systems. As reviewed in section 1.2 , the dynamical scaling exponent $z$ of quantum critical theories does not have to be one. In this chapter we consider the gravity dual description of non-relativistic quantum critical theories with $z \neq 1$. In particular we compute conductivities associated with a finite density of charge carriers in a strongly-coupled theory with non-relativistic symmetry. The theory is $\mathcal{N}=4$ SYM theory deformed by a dimension-five operator that breaks the relativistic conformal group down to the Schrödinger group, with dynamical scaling exponent $z=2$, and the charge carriers are comprised of a finite baryon density of massive $\mathcal{N}=2$ supersymmetric hypermultiplets.

In this chapter, we calculate the conductivity of alternating currents in linear response theory. Moreover, we determine the conductivity tensor of direct currents to all orders in the electric field using the holographic methods presented in section 3.3 . We find that, generally speaking, both the DC and AC conductivities exhibited relativistic scaling, with temperature or frequency, in the IR and non-relativistic scaling, with $z=2$, in the UV. These results are in accord with our expectations, given the origin of the non-relativistic symmetry via an irrelevant deformation of the theory.

This chapter of the thesis is based on work done in collaboration with Carlos Hoyos, Andy O'Bannon and Jackson Wu and was published in [9].

\subsection{Introduction and Summary}

In the chapters so far we studied theories with relativistic scaling to get novel insights into low-temperature systems controlled by quantum critical points. As reviewed in section 1.2, quantum critical theories are invariant under scale transformations of the form

$$
t \rightarrow \lambda^{z} t, \quad \vec{x} \rightarrow \lambda \vec{x}
$$


where $\lambda$ is some real, positive scaling parameter, $z$ is the dynamical scaling exponent, and we have assumed spatial isotropy. If rotations, space translations, and time translations are also symmetries, then the theory is invariant under the so-called Lifshitz symmetry algebra.

This Lifshitz symmetry algebra can be extended for two particular values of $z$. When $z=1$ the Lifshitz algebra may be enhanced to the usual relativistic conformal algebra. When $z=2$ the Lifshitz algebra may be generalized to the Schrödinger symmetry. The Schrödinger symmetry includes time translations, spatial translations, spatial rotations, and Galilean boosts, as well as scale transformations with $z=2$, a special conformal transformation, and a number symmetry with generator $N$, which is a central element of the Schrödinger algebra. An example for a theory invariant under the Schrödinger symmetry $[177,178]$ are fermions at unitarity, which can be realized experimentally using cold atoms.

In this chapter we study transport coefficients in a particular strongly-coupled field theory with Schrödiner symmetry. In particular we are interested in AC and DC conductivities. Our methods presented in this chapter can be used to engineer a holographic model for fermions at unitarity. Since we are able to calculate transport coefficients at strong coupling we might get interesting new insights into the theory of cold atoms. To construct such a holographic model, we first of all need a gravitational dual description for theories with Schrödinger symmetry.

First, note that the Schrödinger algebra is in fact easy to obtain from the relativistic conformal algebra in one higher spatial dimension. If we use the extra spatial dimension to form light-cone coordinates, $x^{ \pm}$, and then retain only those generators that commute with the translation generator in the $x^{-}$direction, $P_{-}$, the resulting algebra is precisely the Schrödinger algebra, if we make some identifications, including identifying the relativistic generator $P_{+}$with the non-relativistic Hamiltonian (generator of time translations) and $P_{-}$with the number operator $N$. Notice that if the spectrum of eigenvalues of $N$ should be discrete, which is the case for a non-relativistic theory, then we must compactify $x^{-}$. In other words, if we begin with a relativistic conformal theory, break the symmetry group down to the subgroup that commutes with $P_{-}$(via some deformation), and then perform a Discrete Light-Cone Quantization (DLCQ), then we obtain a non-relativistic theory with Schrödinger symmetry, in one lower spatial dimension.

Some gravitational duals for theories with Schrödinger symmetry were discovered. Via the gauge-gravity dictionary, the Schrödinger symmetry group translates into the isometry group of the metric. We thus call such spacetimes Schrödinger spacetimes [179,180]. A direct method to obtain Schrödinger spacetimes is to apply a solution-generating technique of type II supergravity, the Null Melvin Twist (NMT), to known solutions [181-183]. We review the NMT in section 6.2.2. We apply the Null Melvin Twist to our basic example, type IIB supergravity on $A d S_{5} \times S^{5}$, where $A d S_{5}$ is (4+1)-dimensional anti-de Sitter space and $S^{5}$ is a five-sphere. We obtain $S c h_{5} \times S^{5}$, where $S c h_{5}$ is $(4+1)$-dimensional Schrödinger spacetime. The solution also includes a non-trivial Neveu-Schwarz (NS) twoform, B. The dual theory is then $\mathcal{N}=4 \mathrm{SYM}$ deformed by a particular dimension-five operator that breaks the relativistic conformal group down to the Schrödinger group. We may then additionally compactify $x^{-\sqrt[1]{1}}$ The generalization to thermal equilibrium states

\footnotetext{
${ }^{1}$ Note that doing so makes the supergravity approximation to string theory unreliable since $x^{-}$is a null circle [182].
} 
with temperature $T$ is straightforward [181-183].

In this background we introduce charge carriers by considering probe branes. For simplicity, we restrict ourself to D7-branes. Moreover, we introduce a finite baryon number density, $\left\langle J^{t}\right\rangle$. We then compute (holographically) both the DC and AC conductivities associated with baryon number transport. In the relativistic case these computations were reviewed in sections 3.3 and 3.2.2, respectively. As holographic models of fermions at unitarity, these systems have various advantages and disadvantages, some of which we review below. We mention here two of the biggest disadvantages, however. First, the NMT does not produce a genuinely non-relativistic theory, but rather a deformation of a relativistic theory which then has Schrödinger symmetry. Second, as emphasized in [184], the $U(1)$ number symmetry generated by $N$ is not spontaneously broken in any of the known supergravity solutions, whereas real systems are typically superfluids.

Our study is complementary to that of [185], where the DC and AC conductivities of probe flavor were computed holographically using probe branes in Lifshitz spacetimes, that is, spacetimes whose isometry group is the Lifshitz group, with general $z$. Two of the main results of [185] were that at temperatures low compared to the density and mass the DC conductivity $\sigma$ scales as $\left\langle J^{t}\right\rangle T^{-2 / z}$ (for all $z$ ) and the AC conductivity $\sigma(\omega)$ scales as

$$
\sigma(\omega) \propto \begin{cases}\left\langle J^{t}\right\rangle z / 2 \omega^{-1} & \text { for } z<2, \\ \left\langle J^{t}\right\rangle(\omega \log \omega \Lambda)^{-1} & \text { for } z=2 \\ \left\langle J^{t}\right\rangle \omega^{-2 / z} & \text { for } z>2\end{cases}
$$

Here $\Lambda$ is a dimensionful scale that renders the argument of the logarithm dimensionless. The authors of [185] then suggested that, by introducing a scalar field, such as a dilaton, with nontrivial dependence on the holographic radial coordinate, the powers of $T$ and $\omega$ in the $\mathrm{DC}$ and $\mathrm{AC}$ conductivities, respectively, can be engineered to take essentially any value we like. In such a fashion we can produce holographic systems with scalings that match any number of real strongly-coupled electron systems. Moreover, with varying scalars we can even engineer flows in which the scalings change between the ultraviolet (UV) and infrared (IR). An example of such a flow, with an exponent $z=2$ in the UV and $z=1$ in the IR, produced by a marginally relevant deformation of Lifshitz spacetime, appears in [186].

Using Schrödinger instead of Lifshitz spacetime, we find that, in appropriate limits, for example low-temperature and large mass, the scalings with temperature or frequency in the IR are relativistic, meaning $z=1$, while in the UV the scalings are non-relativistic, meaning $z=2$. That is precisely what we expect in the dual (relativistic) field theory, in the presence of an irrelevant operator that, roughly speaking, produces $z=2$ in the UV. Schrödinger spacetime is thus a good example of a flow from non-relativistic scaling in the UV to relativistic scaling in the IR.

Our results suggest that the NMT may be a useful tool for the kind of model-building proposed in [185]. We can imagine starting with a relativistic bulk system, introducing a scalar and engineering whatever exponents we like, and then performing a NMT. We generically obtain a theory with Schrödinger symmetry, and exponents that flow from UV values, presumably with $z=2$, to the IR values we gave them in the original relativistic setting. Such an approach of engineering scaling exponents directly in a relativistic system may be technically easier than engineering them in a non-relativistic system. 2

\footnotetext{
${ }^{2} \mathrm{AdS}$ space is a solution of Einstein gravity plus a negative cosmological constant. Gravity alone cannot
} 
The chapter is organized as follows. In section 6.2 we review how to add probe D7-branes to $A d S_{5} \times S^{5}$, review the NMT and $S c h_{5}$ solution, and then discuss how the NMT affects the D7-branes' action, the Dirac-Born-Infeld (DBI) action. In section 6.3 we compute the $\mathrm{DC}$ conductivity and in section 6.4 we compute the $\mathrm{AC}$ conductivity.

\subsection{Adding Flavor to Schrödinger Spacetime}

In this section we review how to obtain Schrödinger spacetime from a NMT of $A d S_{5} \times S^{5}$, and review the field theory dual to supergravity on Schrödinger spacetime. Our new ingredient are probe D7-branes. We discuss in general terms what effect the NMT has on the D7-branes' worldvolume action.

\subsubsection{Review: D7-Branes in AdS}

In type IIB supergravity, the solution describing the near-horizon geometry of non-extremal D3-branes is given by $A d S_{5}$-Schwarzschild times $S^{5}$. We use the metric in the coordinates 3 $(2.4 .80)$

$$
\begin{aligned}
d s^{2} & =g_{r r} d r^{2}+g_{t t} d t^{2}+g_{y y} d y^{2}+g_{x x} d \vec{x}^{2}+d s_{S^{5}}^{2} \\
& =\frac{1}{r^{2}}\left(\frac{d r^{2}}{f(r)}-f(r) d t^{2}+d y^{2}+d \vec{x}^{2}\right)+(d \chi+\mathcal{A})^{2}+d s_{\mathbb{C P}^{3}}^{2}
\end{aligned}
$$

where we have singled out one field theory spatial direction, $y$, for use in the NMT below. Moreover, we have written the $S^{5}$ metric as a Hopf fibration over $\mathbb{C P}^{3}$, with $\chi$ the Hopf fiber direction. $\mathcal{A}$ gives the Kähler form $J$ of $\mathbb{C P}^{3}$ via $d \mathcal{A}=2 J$. To write the metric of $\mathbb{C P}^{3}$ and $\mathcal{A}$ explicitly, we introduce $\mathbb{C P}^{3}$ coordinates $\alpha_{1}, \alpha_{2}, \alpha_{3}$, and $\theta$ and define the $S U(2)$ left-invariant forms

$$
\begin{aligned}
\sigma_{1} & =\frac{1}{2}\left(\cos \alpha_{2} d \alpha_{1}+\sin \alpha_{1} \sin \alpha_{2} d \alpha_{3}\right), \\
\sigma_{2} & =\frac{1}{2}\left(\sin \alpha_{2} d \alpha_{1}-\sin \alpha_{1} \cos \alpha_{2} d \alpha_{3}\right), \\
\sigma_{3} & =\frac{1}{2}\left(d \alpha_{2}+\cos \alpha_{1} d \alpha_{3}\right),
\end{aligned}
$$

so that the metric of $\mathbb{C P}^{3}$ is

$$
d s_{\mathbb{C P}^{3}}^{2}=d \theta^{2}+\cos ^{2} \theta\left(\sigma_{1}^{2}+\sigma_{2}^{2}+\sin ^{2} \theta \sigma_{3}^{2}\right),
$$

and $\mathcal{A}=\cos ^{2} \theta \sigma_{3}$. The full solution also includes a nontrivial five-form, but as shown in [181-183] that is unaffected by the NMT, so we ignore it.

produce Lifshitz or Schrödinger spacetime, however. These require matter fields. In the Schrödinger case, the NMT takes AdS and generates the needed matter fields, in particular the NS two-form B. Introducing scalars in a theory of gravity alone and then doing the NMT may be easier than introducing scalars in a theory with gravity and other matter fields.

${ }^{3}$ In equation (2.4.80) we denoted the radial coordinate of AdS by $u$. Here, we switch our notation and use $r$ instead. Moreover, in this chapter we also denote the induced metric by $g_{a b}$ instead of $G_{a b}$. Note that only $G_{r r}$, the component of the induced metric in r-direction, differs from $g_{r r}$, the metric of the background. In order to avoid confusion, we call $G_{r r}$ in this chapter $g_{r r}^{D 7}$. 
As discussed in section 2.5, we introduce a number $N_{f}$ of probe D7-branes into the above geometry. The D7-branes are extended along $A d S_{5}$-Schwarzschild times an $S^{3} \subset S^{5}$. More specifically, the D7-branes are extended along the three angular directions $\alpha_{1}, \alpha_{2}$ and $\alpha_{3}$. The two worldvolume scalars are then $\theta$ and $\chi$.

To study transport, we need to introduce a finite charge density following section 2.5.3. Therefore we consider a non-trivial gauge field component $A_{t}(r)$. An electric field in $x$ direction can be implemented by a non-vanishing field strength tensor $F_{t x}$. The resulting current $\left\langle J^{x}\right\rangle$ is given by a non-trivial gauge field $A_{x}(r)$.

The D7-brane action describing the dynamics of the worldvolume fields is the DBI action plus Wess-Zumino (WZ) terms. For our ansatz with $\theta(r)$ and only the Abelian worldvolume gauge field, we only need the Abelian D7-brane action. Additionally, for our ansatz the WZ terms vanish because the relevant form fields do not saturate the D7-branes' indices.

Since our ansatz for the gauge field involves only the $(r, t, x)$ directions, hence we may write $S_{D 7}$, given by equation (2.5.97), as a $(3+1)$-dimensional DBI action times some extra factors, with the $(3+1)$-dimensional part being the $(r, t, x, y)$ subspace:

$$
S_{D 7}=-\mathcal{N} \int d r \cos ^{3} \theta(r) g_{x x}^{1 / 2} \sqrt{-g-\frac{1}{2} g \tilde{F}^{2}-\frac{1}{4}(\tilde{F} \wedge \tilde{F})^{2}}
$$

The square root factor is the characteristic form of a $(3+1)$-dimensional DBI action, in our case in the $(r, t, x, y)$ subspace, so that $g$ is the determinant of the induced metric (2.5.94) in that subspace. Starting now, primes denote $\frac{\partial}{\partial r}$ and tildes denote factors of $\left(2 \pi \alpha^{\prime}\right)$, for example $\tilde{F}_{a b}=\left(2 \pi \alpha^{\prime}\right) F_{a b}$. We also performed the trivial integration over the field theory directions and divided both sides by this (infinite) volume, so now $S_{D 7}$ is actually an action density. We use that convention in what follows. More explicitly, for our ansatz the DBI action is

$$
S_{D 7}=-\mathcal{N} \int d r \cos ^{3} \theta(r) g_{x x} \sqrt{\left|g_{t t}\right| g_{x x} g_{r r}^{D 7}-\left(2 \pi \alpha^{\prime}\right)^{2}\left(g_{x x} A_{t}^{\prime 2}+g_{r r}^{D 7} \dot{A}_{x}^{2}-\left|g_{t t}\right| A_{x}^{\prime 2}\right)},
$$

where dots denote $\frac{\partial}{\partial t}$. We define a Lagrangian $L$ via $S_{D 7}=\int d r L$.

\subsubsection{The Null Melvin Twist}

Following [181-183], we now apply the NMT to the supergravity solution in equation (6.2.3). The NMT is a species of TsT (T-duality, shift, T-duality) transformation that produces new supergravity solutions from old ones. The input is some solution with two commuting $U(1)$ isometries. The output is a new solution with different asymptotics. In our case, we begin with $A d S_{5}$-Schwarzschild times $S^{5}$, using the Hopf fiber direction $\chi$ and the field theory spatial direction $y$ as isometry directions, and find a new solution for which the metric is asymptotically Schrödinger. The steps of the NMT are:

(1) Boost by an amount $\gamma$ in $y$,

(2) T-dualize in $y$,

(3) Shift in the $\chi$ direction $d \chi \rightarrow d \chi+\alpha d y$, 
(4) T-dualize in $y$,

(5) Boost by $-\gamma$ in $y$,

(6) Take a limit: $\gamma \rightarrow \infty$ and $\alpha \rightarrow 0$ keeping $\beta=\frac{1}{2} \alpha e^{\gamma}$ fixed.

Steps (2), (3), and (4), are the TsT part of the NMT. We do not write the explicit result of each step (for that, see [183]), but we make some generic comments about each step. The first step changes $g_{t t}$ and $g_{y y}$ and generates a $d y d t$ term in the metric. The second step produces a nontrivial NS B-field, with $d y \wedge d t$ component, and dilaton, and also changes $g_{t t}$ and $g_{y y}$. The third step changes $g_{y y}$ and also introduces a $d \chi+\mathcal{A}$ and $d y$ cross-term in metric, but leaves the B-field and dilaton unchanged. The fourth step changes $g_{t t}, g_{y y}$, $g_{\text {ty }}$, and the $(d \chi+\mathcal{A})^{2}$ term in the metric, and generates a $(d \chi+\mathcal{A}) \wedge d y$ term in B. In the end, the metric that results from the NMT is asymptotically $S_{c h}$. Explicitly, the final result for the metric is

$$
\begin{gathered}
d s^{2}=\frac{1}{r^{2}}\left(\frac{d r^{2}}{f(r)}-f(r) \frac{1+\beta^{2} r^{-2}}{K(r)} d t^{2}+\frac{1-\beta^{2} r^{-2} f(r)}{K(r)} d y^{2}-\frac{2 \beta^{2} r^{-2} f(r)}{K(r)} d t d y+d \vec{x}^{2}\right) \\
+\frac{1}{K(r)}(d \chi+\mathcal{A})^{2}+d s_{\mathbb{C P}^{3}}^{2} \\
=\frac{1}{r^{2}}\left(\frac{d r^{2}}{f(r)}-\frac{f(r)}{r^{2} K(r)} d x^{+2}+\frac{2}{K(r)} d x^{+} d x^{-}+\frac{1-f(r)}{2 K(r)}\left(\frac{d x^{+}}{\sqrt{2} \beta}-\sqrt{2} \beta d x^{-}\right)^{2}+d \vec{x}^{2}\right) \\
+\frac{1}{K(r)}(d \chi+\mathcal{A})^{2}+d s_{\mathbb{C P}^{3}}^{2},
\end{gathered}
$$

where

$$
f(r)=1-\frac{r^{4}}{r_{H}^{4}}, \quad K(r)=1+\frac{\beta^{2} r^{2}}{r_{H}^{4}}
$$

and in the second equality of equation (6.2.9) we have introduced light-cone coordinates $X^{ \pm}$

$$
X^{+}=t+y, \quad X^{-}=\frac{1}{2}(-t+y),
$$

which we then rescaled by factors of $\beta$ to produce the light-cone coordinates $x^{ \pm}$,

$$
x^{+}=\beta(t+y), \quad x^{-}=\frac{1}{2 \beta}(-t+y) .
$$

We discuss the utility of this rescaling at the end of this subsection. The solution also includes the NS two-form field

$$
\begin{aligned}
B & =-\frac{\beta}{r^{2} K(r)}(d \chi+\mathcal{A}) \wedge(f(r) d t+d y) \\
& =-\frac{1}{2 r^{2} K(r)}(d \chi+\mathcal{A}) \wedge\left((1+f(r)) d x^{+}+(1-f(r)) 2 \beta^{2} d x^{-}\right)
\end{aligned}
$$

and a dilaton

$$
\Phi=-\frac{1}{2} \log K(r)
$$

Notice that if we take $\beta \rightarrow 0$ then we recover the pre-NMT solution.

The metric in equation (6.2.9) has a horizon at $r=r_{H}$, with some associated Hawking temperature $T$. We obtain $T=0$ by sending $r_{H} \rightarrow \infty$, which sends $f(r) \rightarrow 1$ and 
$K(r) \rightarrow 1$. The resulting metric is then the metric of $S c h_{5}$ (not just asymptotically $S c h_{5}$ ), which is essentially the metric of $A d S_{5} \times S^{5}$ with an extra term $-d x^{+2} / r^{4}$,

$$
\begin{aligned}
d s^{2} & =\frac{1}{r^{2}}\left(d r^{2}-\frac{\beta^{2}}{r^{2}} d X^{+2}+2 d X^{+} d X^{-}+d \vec{x}^{2}\right)+d s_{S^{5}}^{2} \\
& =\frac{1}{r^{2}}\left(d r^{2}-\frac{1}{r^{2}} d x^{+2}+2 d x^{+} d x^{-}+d \vec{x}^{2}\right)+d s_{S^{5}}^{2}
\end{aligned}
$$

Notice that the $d x^{+2}$ term diverges faster as $r \rightarrow 0$ than the metric of $A d S_{5}$. When $T=0$ the geometry includes an $S^{5}$, however, the NS B-field breaks the $S O(6)$ isometry of the $S^{5}$ down to $S U(3) \times U(1)$, which is the isometry group of $\mathbb{C P}^{3}$. The $T=0$ solution also breaks all supersymmetry ${ }^{4}[182]$ and has a singularity at $r=\infty$ [183]. When $T$ is finite the geometry is only asymptotically $S c h_{5}$, the $S^{5}$ is deformed, the singularity is hidden behind a horizon, and the dilaton becomes non-trivial.

What is the field theory dual to type IIB supergravity on $S c h_{5}$ ? Equivalently, we can ask what field theory operation is dual to the NMT? Put briefly, the NMT is dual to adding an irrelevant operator to the $\mathcal{N}=4$ SYM theory Lagrangian. We can easily see this as follows. Given the solution above, if we perform a Kaluza-Klein reduction 5 on the $S^{5}$ the NS B-field gives rise to a massive vector in $S c h_{5}$ whose dual operator is a vector of dimension five, in the antisymmetric tensor representation of the $S U(4) \mathrm{R}$-symmetry. The dual operator, which we denote $\mathcal{O}_{\mu}$, is a linear combination of operators of the form [181]

$$
\mathcal{O}_{\mu}^{I J}=\operatorname{Tr}\left(F_{\mu}{ }^{\nu} \Phi^{[I} D_{\nu} \Phi^{J]}+\sum_{K} D_{\mu} \Phi^{K} \Phi^{[K} \Phi^{I I} \Phi^{J]}\right)+\text { fermion terms }
$$

where $\Phi^{I}$ are the adjoint scalars of $\mathcal{N}=4$ SYM transforming in the 6 of $S U(4), F_{\mu \nu}$ is the field strength, and $D_{\mu}$ is the covariant derivative. To be precise, recall that the $\mathbf{1 5}$ of $S U(4)$ decomposes into representations of $S U(3)$ as $\mathbf{1 5}=\mathbf{8}+\mathbf{3}+\overline{\mathbf{3}}+\mathbf{1}$, so that we can write $\mathcal{O}_{\mu}=M_{I J} \mathcal{O}_{\mu}^{I J}$ where $M_{I J}$ is an $S U(4)$ matrix that, after the decomposition, is in the $\mathbf{1}$ of $S U(3)$. In short, the NMT generates an NS B-field whose presence indicates an irrelevant deformation of $\mathcal{N}=4 \mathrm{SYM}$ : we have added $\mathcal{O}_{+}$to the $\mathcal{N}=4$ SYM Lagrangian. Indeed, adding $\mathcal{O}_{+}$breaks the relativistic conformal group down to the algebra of generators that commute with $P^{+} \propto P_{-}$, producing the Schrödinger group, and breaks the $S U(4)$ R-symmetry down to $S U(3) \times U(1)$. As an irrelevant deformation, we also expect the geometry to be deformed near the boundary, which is indeed the case: we see explicitly in equation (6.2.15) that the effect of the deformation (the $\beta^{2}$ term) grows near the boundary $r \rightarrow 0$.

The number generator $N$ of the Schrödinger algebra is dual to the isometry of the $x^{-}$ direction. If the eigenvalues of $N$ should be discrete, we must compactify $x^{-}$, that is, we must perform a DLCQ. When $T=0$ a DLCQ of the above geometry produces a null circle. Any closed string that wrap the null circle is massless, hence when $T=0$ and we compactify $x^{-}$the supergravity approximation becomes unreliable [182]. As emphasized in [182], if the spacetime has momentum in the $x^{-}$direction then the $x^{-}$circle is no longer

\footnotetext{
${ }^{4}$ Supersymmetric $S c h_{5}$ solutions do exist $[187,188]$, obtained by using different directions of the $S^{5}$ (besides the Hopf fiber) in the TsT transformation, which are very similar in form to the solution above. We leave a thorough analysis of probe branes in those backgrounds for the future.

${ }^{5}$ For details of the reduction, which is in fact consistent, see [182].
} 
null, and supergravity is reliable in most of the spacetime, although $x^{-}$becomes null again near the boundary $r \rightarrow 0$.

The finite- $T$ solution in fact has $x^{-}$momentum, since the NMT involves boosts, so with finite $T$ the $x^{-}$direction is no longer null (as is obvious from equation (6.2.9)). The dual field theory is in a state with a finite number density $N$, or equivalently a finite chemical potential [181,183]. As shown in [181,183], the field theory temperature $T$ and chemical potentia] $[$ are

$$
T=\frac{1}{\pi r_{H}} \frac{1}{\beta}, \quad \mu=-\frac{1}{2 \beta^{2}} .
$$

The dual field theory has the correct equation of state for a scale-invariant, non-relativistic theory with $z=2$ in two spatial dimensions (here we are performing a DLCQ), $\epsilon=P$, with $\epsilon$ the energy density and $P$ the pressure. The NMT does not change the area of horizons [189], so the metrics in equations (6.2.3) and (6.2.9) have the same horizon area, although the conversion to field theory quantities and interpretation differs in the two cases. Of central importance is the fact that the entropy, and other thermodynamic quantities, such as the free energy density, scale with negative powers of $\mu / T$, and hence diverge in the limit $\mu / T \rightarrow 0$. Such odd singular behavior appears to be a direct consequence of the DLCQ: exactly the same scalings occur in a gas of non-interacting, non-relativistic Kaluza-Klein particles [190]. Type IIB supergravity in Schrödinger spacetime is apparently not dual to a theory of fermions at unitarity. $]^{7}$

Notice that the bulk theory is relativistic, so that under a scale transformation the coordinates transform as $r \rightarrow \lambda r, t \rightarrow \lambda t, \vec{x} \rightarrow \lambda \vec{x}$ for some real positive number $\lambda$. The parameter $\beta$ has units of length and hence scales as $\beta \rightarrow \lambda \beta$. Rescaling the $X^{ \pm}$in equation (6.2.11) by powers of $\beta$ produces the light-cone coordinates $x^{ \pm}$in equation (6.2.12), such that under scalings $x^{+} \rightarrow \lambda^{2} x^{+}$while $x^{-}$is invariant. Once we perform the DLCQ and interpret $x^{+}$as the time coordinate, the resulting theory indeed exhibits the scaling of equation (6.1.1) with $z=2$. The fact that $x^{-}$is invariant indicates that the conjugate momentum $P_{-}$is also invariant, which makes sense: after DLCQ we identify $P_{-}$with the number operator $N$, which is a central element of the algebra, and in particular must commute with the dilation generator.

For a theory with $d$ spatial dimensions, if we assign momentum to have scaling dimension one, then for a given value of $z$ we have the following scaling dimensions for a density $\left\langle J^{t}\right\rangle$, current $\left\langle J^{x}\right\rangle$, electric field $E$, magnetic field $B$, chemical potential $\mu$ and temperature $T$ :

$$
\left[\left\langle J^{t}\right\rangle\right]=d, \quad\left[\left\langle J^{x}\right\rangle\right]=d+z-1, \quad[E]=z+1, \quad[B]=2, \quad[\mu]=[T]=z .
$$

From Ohm's law, $\left\langle J^{x}\right\rangle=\sigma E$, we find that the conductivity has dimension $[\sigma]=d-2$. Our system has $z=2$ and, after DLCQ, $d=2$. From equation (6.2.17) we see that the factors of $\beta$ are essential to produce a $T$ and $\mu$ with scaling dimension two.

\footnotetext{
${ }^{6}$ For us $\mu$ always denote the chemical potential associated with the $U(1)$ along the compact $x^{-}$, not the chemical potential associated with the $U(1)$ baryon number of the flavor fields.

${ }^{7}$ Curiously, however, at $T=0$ and $\mu=0$, the three-point functions computed holographically from $\mathrm{Sch}_{5}$ agree exactly, up to normalization, with the three-point functions of fermions at unitarity [191,192]. As in a relativistic conformal theory, the Schrödinger symmetry fixes the form of two-point functions but the three-point functions are only partially fixed, and so contain dynamical information. The fact that the $S_{c h}$ result agrees with fermions at unitarity is thus a non-trivial statement about the dynamics of the dual theory.
} 
In the field theory we also have background gauge fields, dual to the gauge fields on the D7-branes, such as the (relativistic) electric field $F_{t x}=\dot{A}_{x}$ in equation (6.2.8). Here again, appropriate factors of $\beta$ produce gauge fields with the correct scaling dimensions. From equation (6.2.17) we have $\beta \propto(-\mu)^{-1 / 2}$, so we may interpret all rescalings by powers of $\beta$ as rescalings by appropriate powers of the chemical potential $\mu$. Recalling that the gauge field is a one-form, we have

$$
A_{t} d t+A_{y} d y=\frac{1}{2 \beta}\left(A_{t}+A_{y}\right) d x^{+}+\beta\left(-A_{t}+A_{y}\right) d x^{-} \equiv A_{+} d x^{+}+A_{-} d x^{-},
$$

so that $A_{+}$has scaling dimension two while, after DLCQ, $A_{-}$is a dimensionless scalar. An electric field $F_{+x}=\partial_{+} A_{x}-\partial_{x} A_{+}$then indeed have scaling dimension $z+1=3$. Recalling the relativistic coupling $A_{\mu} J^{\mu}$, from the coupling $A_{+} J^{+}$we see that $J^{+}$has scaling dimension two, so that $A_{+}$and $J^{+}$have the correct scaling dimensions of a chemical potential and charge density, respectively, for $z=2$ and $d=2$. The coupling $A_{-} J^{-}$ indicates that after DLCQ $J^{-}$is a scalar with scaling dimension four.

\subsubsection{Twisting with Probe D7-branes}

We now ask what happens to our probe flavor when we perform the NMT. The field theory side is easy, so we start there: we simply write the Lagrangian of $\mathcal{N}=4 \mathrm{SYM}$ theory coupled to massive $\mathcal{N}=2$ supersymmetric hypermultiplets in the fundamental representation of the gauge group, and then add the operator $\mathcal{O}_{+}$. The flavors break the $S U(3) \times U(1)$ symmetry to the same $S O(4) \times U(1)$ as in the relativistic case. In the probe limit, massless flavors preserve the Schrödinger symmetry, while a finite mass explicitly break scale invariance.

Now we ask what happens on the gravity side, that is, we ask what happens to probe D7-branes when we perform the NMT. The effect of the boost in steps 1 and 5 is straightforward. In the T-dualities of steps 2 and 4, the D7-branes are converted into D6-branes and then back to D7-branes. The component $A_{y}$ of the worldvolume gauge field is converted into a scalar, $\Phi_{y}$, which is then converted back into $A_{y}$. Crucially, however, the DBI action is consistent with T-duality [193]. That means that when we T-dualize, the metric, NS B-field, and dilaton may change, and we replace $A_{y} \rightarrow \Phi_{y}$, but the quantity

$$
\mathcal{L} \equiv e^{-\Phi} \sqrt{\operatorname{det}\left(P[G+B]_{a b}+\left(2 \pi \alpha^{\prime}\right) F_{a b}\right)}
$$

evaluates to the same function of $r$, though now with $\Phi_{y}$ replacing $A_{y}$. If $A_{y}$ is non-trivial, so that after T-duality $\Phi_{y}$ is non-trivial, then the shift in step 3 may change the pullback of the metric to the D6-branes, and hence potentially change $\mathcal{L}$. If $A_{y}$ is trivial, however, then the TsT part of the NMT transformation leaves $\mathcal{L}$ unchanged.

The most general statement we can make is: if $\mathcal{L}$ is initially invariant under boosts in the $y$ direction, then the entire NMT has no effect on $\mathcal{L}$. In such cases the boosts in steps 1 and 5 and the TsT transformation each individually leave $\mathcal{L}$ unchanged. For boosts to be a symmetry requires $T=0$, and all worldvolume fields must be invariant under boosts in $y .8$ For example, we may introduce the worldvolume scalar $\theta(r)$, as well as

\footnotetext{
${ }^{8}$ In the Sakai-Sugimoto model [136], which is a system of intersecting D4-branes and D8-branes, the NMT seems to have no effect on the probe D8-branes' action, even in the black hole background and with nonzero $A_{t}(r)$ [194].
} 
the gauge field $A_{x}(r)$, both of which are clearly invariant under boosts in $y$. In the field theory we have flavor fields with a finite mass and some current in the $x$ direction, $\left\langle J^{x}\right\rangle .9$ Using the asymptotically Schrödinger background and these worldvolume fields, we find that the action $S_{D 7}$ is identical to the asymptotically AdS case in equation (6.2.8), with $A_{t}^{\prime}=\dot{A}_{x}=0$. We may also add field strengths describing electric and magnetic fields pointing in the $y$ direction, such as $F_{t y}$, which are invariant under boosts 10 The NMT leaves $\mathcal{L}$ invariant in all such cases.

A number of conclusions follow from the invariance of $S_{D 7}$ for $y$-boost-invariant configurations. For example, suppose that, at $T=0$, we introduce only the worldvolume scalar $\theta(r)$. After the NMT we find exactly the AdS result, equation (6.2.8), with all gauge fields set to zero. The equation of motion for $\theta(r)$ is then identical to the AdS case, hence the solution is also identical: $\theta(r)=\arcsin (c r)$, where $c$ is a constant that determines the mass $m$ of the flavor fields via $m=c /\left(2 \pi \alpha^{\prime}\right)$ [88] ${ }^{11}$ The counterterms written in [103], needed to render the on-shell action finite, are then also identical to the relativistic case. Furthermore, in the $T=0$ AdS case, solutions with nonzero $\theta(r)$ and $A_{x}(r)$ were found in [119]. These solutions will also be identical for D7-branes in $S_{c h}$.

Given that the embedding of the D7-branes, $\theta(r)$, is identical in the $T=0$ AdS and Schrödinger cases, a natural question is whether the spectra of linearized fluctuations of worldvolume fields are also the same. These spectra are dual to the spectra of mesons in the field theory. In general, the spectrum of mesons are not the same. The simplest way to see that is to consider the fermionic mesons, dual to fermionic fluctuations of the D7-branes [101]. The linearized equation of motion for these fermionic fluctuations is simply the Dirac equation. The Dirac operator is different in AdS and Schrödinger spacetimes [80]. More generally, the differential operators appearing in the fluctuations' equations of motions, for example the scalar Laplacian, differ from their AdS counterparts, so the spectrum generically is different. Some subsector of the meson spectrum may be unchanged, for example the sector with zero momentum in $x^{-}$and zero charge under the R-symmetry that corresponds to the Hopf fiber isometry. We leave a detailed investigation of the meson spectrum for the future.

When $T$ is nonzero the NMT changes $\mathcal{L}$, even when all the worldvolume fields are trivial. Introducing non-trivial worldvolume fields will then obviously not restore $\mathcal{L}$ to its AdS form. In what follows, we are interested in finite $T$ solutions with worldvolume fields that are not invariant under boosts in $y$, such as electric fields $F_{t x}$, so we will not be able to exploit the $T=0$ invariance of $\mathcal{L}$.

In what follows we study transport. We should, however, first study thermodynamics, to determine the ground state of the system for all values of the parameters (mass, density, etc.). In the relativistic case, a variety of phase transitions do indeed occur as the parameters change. The thermodynamics has been reviewed in section 2.5.4. For more details

\footnotetext{
${ }^{9}$ As mentioned in [119] in the field theory at $T=0$ such a current is not dissipate. We may introduce it simply as an external parameter.

${ }^{10}$ If we introduce an electric field $F_{t y}$ in the field theory, then we expect a resulting current in the $y$ direction, $\left\langle J^{y}\right\rangle$, which breaks the boost invariance. A bulk solution with $F_{t y}$ and no $\left\langle J^{y}\right\rangle$ would probably be pathological, exhibiting an instability of the kind that we discuss in section 6.3.1, for example.

${ }^{11}$ In AdS the $\theta(r)=\arcsin (c r)$ solution is supersymmetric, but here the background breaks all supersymmetry already, so we need not bother checking the supersymmetry of the D7-branes' embedding. A good question, though, is whether supersymmetric embeddings could be found for the supersymmetric Schrödinger solutions of $[187,188]$.
} 
see $[91,92,109,115,123,145,147,195]$ and references therein. We leave a detailed analysis of the non-relativistic case for the future. In the following, in the field theory we always assume that the D7-branes intersect the horizon, hence our results for the conductivity is only be valid when such D7-branes describe the ground state of the field theory.

\subsection{Conductivity of Probe Flavor}

In this section we compute holographically the DC conductivity associated with transport of baryon number charge. We use the method presented in section 3.3, which captures effects beyond those of linear response. Our background spacetime is asymptotically $\mathrm{Sch}_{5}$ rather than asymptotically $A d S_{5}$. One of the major differences between these is that in $\mathrm{Sch}_{5}$ we use light-cone coordinates $x^{ \pm}$, compactify $x^{-}$, and in the dual non-relativistic theory interpret $x^{+}$as the time coordinate.

\subsubsection{In the DLCQ of AdS}

Suppose we perform a DLCQ both in the bulk and in the field theory. To do so, we first write the AdS part of the metric in equation $(6.2 .3)$ in the light-cone coordinates $X^{ \pm}$of equation (6.2.11),

$$
\begin{aligned}
d s^{2} & =g_{r r} d r^{2}+g_{++} d X^{+2}+g_{--} d X^{-2}+2 g_{+-} d X^{+} d X^{-}+g_{x x} d \vec{x}^{2} \\
& =\frac{1}{r^{2}}\left(\frac{d r^{2}}{f(r)}+\frac{1}{4}(1-f(r)) d X^{+2}+(1-f(r)) d X^{-2}+(1+f(r)) d X^{+} d X^{-}+d \vec{x}^{2}\right),
\end{aligned}
$$

Notice that when $T=0$ and $f(r)=1$, the metric, and its inverse, in the light-cone directions is strictly off-diagonal, $g_{++}=g_{--}=0$ and $g^{++}=g^{--}=0$.

After the DLCQ, we interpret $X^{+}$as the new time coordinate. The boundary value of the D7-brane worldvolume field $A_{+}$acts as a source for the field theory operator $J^{+}$. In the DLCQ we assume all physical quantities are independent of $X^{-}$, that is, that $\partial_{-}$acting on any quantity gives zero. After the DLCQ, the relativistic equation for conservation of the current, $\partial_{\mu}\left\langle J^{\mu}\right\rangle=0$, reduces to $\partial_{+}\left\langle J^{+}\right\rangle+\partial_{i}\left\langle J^{i}\right\rangle=0$, with $i$ the index for the spatial directions. We thus interpret $\left\langle J^{+}\right\rangle$as the charge density after DLCQ.

To study states in the field theory with finite $\left\langle J^{+}\right\rangle$our ansatz for the worldvolume fields always includes $A_{+}(r)$, or equivalently $F_{+r}(r)=-A_{+}^{\prime}(r)$. Suppose for the moment we also introduce $A_{-}(r)$. The DBI action will then involve terms of the form

$$
\frac{1}{2} g \tilde{F}^{2} \supset g_{D 7}^{r r} g^{++} A_{+}^{\prime}(r)^{2}+g_{D 7}^{r r} g^{--} A_{-}^{\prime}(r)^{2}+2 g_{D 7}^{r r} g^{+-} A_{+}^{\prime}(r) A_{-}^{\prime}(r) .
$$

Taking variational derivatives and using equation (2.5.105), we see that a nontrivial $A_{+}^{\prime}(r)$ not only produces a finite $\left\langle J^{+}\right\rangle$in the field theory but also a finite $\left\langle J^{-}\right\rangle$. In other words, in the field theory, if we introduce $\left\langle J^{+}\right\rangle$, we must introduce $\left\langle J^{-}\right\rangle$. We discuss the meaning of this, from the field theory point of view, shortly. Furthermore, when $T=0$ and $g^{++}=0$, a nontrivial $A_{+}^{\prime}(r)$ produces only a nonzero $\left\langle J^{-}\right\rangle$. To obtain a nonzero $\left\langle J^{+}\right\rangle$at $T=0$, we thus also introduce $A_{-}(r)$. Notice that if we return to the original coordinates, with just $A_{t}(r)$, then we obtain both $A_{+}(r)$ and $A_{-}(r)$. 
We also need a constant electric field, which after DLCQ should be $F_{+x}=-E$. Introducing $F_{+x}$ alone doing does not produce an instability of the DBI action, however. When $T=0$, for example, the inverse metric component $g^{++}=0$, hence if we introduce only $F_{+x}$ then the DBI action does not depend on the electric field at all, since $g \tilde{F}^{2} \propto g^{x x} g^{++} F_{+x}^{2}=0$. Converting back to the original coordinates reveals what is happening: $F_{+x}$ describes perpendicular electric and magnetic fields $F_{t x}$ and $F_{y x}$ of equal magnitude, such that $g \tilde{F}^{2} \propto E^{2}-B^{2}=0$. We thus also introduce $F_{-x}$, in which case the DBI action depends on both $F_{+x}$ and $F_{-x}$, and exhibits the expected instability. Introducing both $F_{+x}$ and $F_{-x}$ is the same as introducing $F_{t x}$ and $F_{x y}$. Similarly to the story with $A_{+}(r)$ and $A_{-}(r)$, in what follows we begin with $F_{t x}$ and then switch to light-cone coordinates.

In summary, our ansatz for the worldvolume gauge field is identical to the relativistic case, with $F_{r t}=A_{t}^{\prime}(r), F_{r x}=A_{x}^{\prime}(r)$ and constant $F_{t x}$, but converted to light-cone coordinates.

The bulk field $A_{-}$is dual to the field theory operator $J^{-}$. Given that we are working with a nontrivial $A_{-}(r)$ in the bulk and states with nonzero $\left\langle J^{-}\right\rangle$in the field theory, a natural question is, from the field theory point of view, what is $J^{-}$?

After the DLCQ, $A_{-}$is a bulk scalar and $J^{-}$is a scalar operator. To gain some intuition for the physical meaning of $A_{-}$and $J_{-}$after DLCQ, consider a complex scalar field in the relativistic theory that carries the $U(1)$ charge associated with the current $J^{\mu}$, which in our case means the scalars in the $\mathcal{N}=2$ hypermultiplet. Consider in particular the kinetic terms, written in light-cone coordinates and with a covariant derivative $D_{\mu}=\partial_{\mu}-i q A_{\mu}$ involving the background gauge field $A_{\mu}$, with $q$ the charge of the scalar under the $U(1)$. Explicitly, we have (here $\partial_{ \pm}$are derivatives with respect to $X^{ \pm}$)

$$
\begin{aligned}
g^{\mu \nu}\left|D_{\mu} \Phi\right|^{\dagger} D_{\nu} \Phi & =g^{+-}\left|D_{+} \Phi\right|^{\dagger} D_{-} \Phi+g^{-+}\left|D_{-} \Phi\right|^{\dagger} D_{+} \Phi+\ldots \\
& =\partial_{+} \Phi^{\dagger}\left(\partial_{-}-i q A_{-}\right) \Phi+\left(\partial_{-}+i q A_{-}\right) \Phi^{\dagger} \partial_{+} \Phi+\ldots
\end{aligned}
$$

If we work with fixed $X^{-}$momentum $N$,

$$
\Phi\left(X^{-}, X^{+}, \vec{x}\right)=e^{-i N X^{-}} \phi\left(X^{+}, \vec{x}\right),
$$

then we obtain

$$
g^{\mu \nu}\left|D_{\mu} \Phi\right|^{\dagger} D_{\nu} \Phi=\left(N+q A_{-}\right) i\left[\phi^{\dagger} \partial_{+} \phi-\left(\partial_{+} \phi^{\dagger}\right) \phi\right]+\ldots
$$

Recalling that after DLCQ we interpret $N$ as the particle number quantum number 12 we see that a nonzero $A_{-}$looks like a shift in the particle number $N$. Indeed, that is true for any $U(1)$ : if we introduce a nonzero $A_{-}$, any fields charged under the $U(1)$ appear to have a shifted $N$. That makes sense since, after DLCQ, a nonzero $A_{-}$produce a Wilson loop in the $x^{-}$direction, effectively shifting the momentum $P_{-}$, and hence shifting $N$. Both $N$ and $q A_{-}$couple to the operator representing $x^{+}$momentum,

$$
P_{+}=i\left[\phi^{\dagger} \partial_{+} \phi-\left(\partial_{+} \phi^{\dagger}\right) \phi\right] .
$$

As mentioned in the introduction (and [179]), after the DLCQ, $P_{+}$plays the role of the Hamiltonian in the Schrödinger algebra. We thus see that $A_{-}$couples to the operator $J^{-}=q P_{+}$, or $q$ times the Hamiltonian. The statement above that a nonzero $\left\langle J^{+}\right\rangle$must be accompanied by a nonzero $\left\langle J^{-}\right\rangle$is thus easy to understand: from the perspective of the non-relativistic theory, a finite density of particles must be accompanied by some energy.

\footnotetext{
${ }^{12}$ Here $N$ denotes the eigenvalue of the number operator, which we also called $N$ above.
} 


\subsubsection{In Schrödinger Spacetime}

Having explained the method for computing the DC conductivity, and some of the subtleties of working in light-cone coordinates, we proceed to the case where the background spacetime is the asymptotically $S c h_{5}$ metric of equation (6.2.9).

First, we must be careful with factors of $\beta$ (see the end of section 6.2.2). As explained above, our ansatz is the same as in the relativistic case of section 6.2.1, with $A_{t}(r)$, $F_{t x}=-E$, and $A_{x}(r)$, but converted to the (rescaled) light-cone coordinates $x^{ \pm}$of equation (6.2.12). We also rescale the gauge field components as in equation (6.2.19), so that our $A_{ \pm}$obey non-relativistic scaling. Explicitly, our ansatz for the worldvolume gauge field is

$$
A_{+}(x, r)=E_{\beta} x+h_{+}(r), \quad A_{-}(x, r)=-2 \beta^{2} E_{\beta} x+h_{-}(r), \quad A_{x}=A_{x}\left(x^{+}, r\right),
$$

where we have redefined the electric field to be $E_{\beta}=E /(2 \beta)$ such that $E_{\beta}$ scales nonrelativistically, i.e. $\left[E_{\beta}\right]=3$, and $h_{ \pm}(r)$ are functions for which we most solve. We also recall the other scaling dimensions of equation (6.2.18), with $d=z=2$,

$$
\left[A_{+}\right]=2, \quad\left[J^{+}\right]=2, \quad\left[A_{-}\right]=0, \quad\left[J^{-}\right]=4, \quad\left[A_{x}\right]=1, \quad\left[J^{x}\right]=3,
$$

and the conductivity is dimensionless, $[\sigma]=d-2=0$.

We now insert our ansatz for the worldvolume fields into the DBI action, equation (2.5.95), using the background metric, B-field, and dilaton of equations (6.2.9), (6.2.13), and (6.2.14), respectively. To write the action succinctly, let us introduce some notation. We define

$$
\tilde{G}_{i_{1} \ldots i_{n}} \equiv \frac{1}{\sin ^{2} \alpha_{1}} \operatorname{det}\left(P[g+B]_{a b}\right), \quad a, b=i_{1}, \ldots, i_{n},
$$

i.e. $\tilde{G}_{i_{1} \ldots i_{n}}$ is the determinant of the $n \times n$ submatrix of $P[g+B]_{a b}$ containing only rows and columns indexed by $i_{1}, \ldots, i_{n}$. All such sub-determinants are functions of $r$ times a factor of $\sin ^{2} \alpha_{1}$, hence we divide by $\sin ^{2} \alpha_{1}$ to make $\tilde{G}_{i_{1} \ldots i_{n}}$ a function of $r$ only. Similarly we define the $3 \times 3$ submatrix determinant (divided by $\sin ^{2} \alpha_{1}$ ):

$$
\tilde{G}_{B} \equiv \frac{1}{\sin ^{2} \alpha_{1}} \operatorname{det}\left(\begin{array}{ccc}
g_{+-} & B_{+\alpha_{2}} & B_{+\alpha_{3}} \\
-B_{-\alpha_{2}} & g_{\alpha_{2} \alpha_{2}} & g_{\alpha_{3} \alpha_{2}} \\
-B_{-\alpha_{3}} & g_{\alpha_{2} \alpha_{3}} & g_{\alpha_{3} \alpha_{3}}
\end{array}\right) \text {. }
$$

Explicitly, the submatrix determinants we need are

$$
\begin{gathered}
\tilde{G}_{\alpha_{2} \alpha_{3}}=\frac{\cos ^{2} \theta(r)+K(r) \sin ^{2} \theta(r)}{16 K(r)} \cos ^{4} \theta(r) \\
\tilde{G}_{+\alpha_{2} \alpha_{3}}=\frac{r^{6}-4 r_{H}^{4} \beta^{2} f(r) \sin ^{2} \theta(r)}{64 r^{4} r_{H}^{4} \beta^{2} K(r)} \cos ^{4} \theta(r), \quad \tilde{G}_{-\alpha_{2} \alpha_{3}}=\frac{K(r)-1}{16 K(r)} \cos ^{4} \theta(r), \\
\tilde{G}_{B}=\frac{1+f(r)}{32 r^{2} K(r)} \cos ^{4} \theta(r), \quad \tilde{G}_{+-\alpha_{2} \alpha_{3}}=-\frac{f(r) \cos ^{4} \theta(r)}{16 r^{4} K(r)} .
\end{gathered}
$$

We note for later use that $\tilde{G}_{+-\alpha_{2} \alpha_{3}}$ vanishes at the horizon $r=r_{h}$ and otherwise is strictly negative, while $\tilde{G}_{\alpha_{2} \alpha_{3}}$ is strictly positive. We also define the shorthand notation

$$
\tilde{G}_{3} \equiv \tilde{G}_{-\alpha_{2} \alpha_{3}}+4 \beta^{4} \tilde{G}_{+\alpha_{2} \alpha_{3}}+4 \beta^{2} \tilde{G}_{B}=\frac{\beta^{2}\left[r^{2}-\beta^{2} f(r) \sin ^{2} \theta(r)\right]}{4 r^{4} K(r)} \cos ^{4} \theta(r) .
$$


Plugging our gauge field ansatz equation (6.3.25) into the D7-brane action, we obtain

$$
\begin{aligned}
S_{D 7} & =-N_{f} T_{D 7} \int d r d^{3} \alpha e^{-\Phi} \sqrt{-\operatorname{det}\left(P[g+B]_{a b}+\left(2 \pi \alpha^{\prime}\right) F_{a b}\right)} \\
& =-\mathcal{N} \int d r \sqrt{-K(r) \operatorname{det} M_{a b}},
\end{aligned}
$$

where the matrix $M_{a b}$ has determinant

$$
\begin{aligned}
\operatorname{det} M_{a b} \equiv g_{x x} g_{\alpha_{1} \alpha_{1}} & \left\{g_{r r}\left[E_{\beta}^{2} \tilde{G}_{3}+g_{x x} \tilde{G}_{+-\alpha_{2} \alpha_{3}}\right]+\tilde{G}_{+-\alpha_{2} \alpha_{3}}\left(A_{x}^{\prime}\right)^{2}+E_{\beta}^{2} \tilde{G}_{\alpha_{2} \alpha_{3}}\left(2 \beta^{2} A_{+}^{\prime}+A_{-}^{\prime}\right)^{2}\right. \\
& \left.+g_{x x}\left[\tilde{G}_{-\alpha_{2} \alpha_{3}}\left(A_{+}^{\prime}\right)^{2}+\tilde{G}_{+\alpha_{2} \alpha_{3}}\left(A_{-}^{\prime}\right)^{2}-2 \tilde{G}_{B} A_{+}^{\prime} A_{-}^{\prime}\right]\right\}
\end{aligned}
$$

Using equation (2.5.105) we obtain the current components

$$
\begin{aligned}
\left\langle J^{+}\right\rangle & =\frac{\mathcal{N} K g_{x x} g_{\alpha_{1} \alpha_{1}}}{\sqrt{-K \operatorname{det} M_{a b}}}\left[\left(2 \beta^{2} E_{\beta}^{2} \tilde{G}_{\alpha_{2} \alpha_{3}}-g_{x x} \tilde{G}_{B}\right) A_{-}^{\prime},+\left(4 \beta^{4} E_{\beta}^{2} \tilde{G}_{\alpha_{2} \alpha_{3}}+g_{x x} \tilde{G}_{-\alpha_{2} \alpha_{3}}\right) A_{+}^{\prime}\right] \\
\left\langle J^{-}\right\rangle & =\frac{\mathcal{N} K g_{x x} g_{\alpha_{1} \alpha_{1}}}{\sqrt{-K \operatorname{det} M_{a b}}}\left[\left(2 \beta^{2} E_{\beta}^{2} \tilde{G}_{\alpha_{2} \alpha_{3}}-g_{x x} \tilde{G}_{B}\right) A_{+}^{\prime}+\left(E_{\beta}^{2} \tilde{G}_{\alpha_{2} \alpha_{3}}+g_{x x} \tilde{G}_{+\alpha_{2} \alpha_{3}}\right) A_{-}^{\prime}\right] \\
\left\langle J^{x}\right\rangle & =\frac{\mathcal{N} K g_{x x} g_{\alpha_{1} \alpha_{1}}}{\sqrt{-K \operatorname{det} M_{a b}}} \tilde{G}_{+-\alpha_{2} \alpha_{3}} A_{x}^{\prime} .
\end{aligned}
$$

Solving equations (6.3.33) for $A_{ \pm}^{\prime}$ and $A_{x}^{\prime}$ and plugging the solutions back into the action, we obtain the on-shell action

$$
S_{D 7}=-\mathcal{N}^{2} \int d r K(r) g_{x x} g_{r r}^{1 / 2} g_{\alpha_{1} \alpha_{1}} \sqrt{\frac{g_{x x}\left|\tilde{G}_{+-\alpha_{2} \alpha_{3}}\right|-E_{\beta}^{2} \tilde{G}_{3}}{U(r)-V(r)}}
$$

where

$$
\begin{aligned}
U(r) & =\frac{\left\langle J^{x}\right\rangle^{2}}{\tilde{G}_{+-\alpha_{2} \alpha_{3}}}+\mathcal{N}^{2} K(r) g_{x x} g_{\alpha_{1} \alpha_{1}}, \\
V(r) & =\frac{E_{\beta}^{2} \tilde{G}_{\alpha_{2} \alpha_{3}}\left(\left\langle J^{+}\right\rangle-2 \beta^{2}\left\langle J^{-}\right\rangle\right)^{2}+g_{x x}\left(\tilde{G}_{+\alpha_{2} \alpha_{3}}\left\langle J^{+}\right\rangle^{2}+\tilde{G}_{-\alpha_{2} \alpha_{3}}\left\langle J^{-}\right\rangle^{2}+2 \tilde{G}_{B}\left\langle J^{+}\right\rangle\left\langle J^{-}\right\rangle\right)}{g_{x x} \tilde{G}_{\alpha_{2} \alpha_{3}}\left(g_{x x}\left|\tilde{G}_{+-\alpha_{2} \alpha_{3}}\right|-E_{\beta}^{2} \tilde{G}_{3}\right)} .
\end{aligned}
$$

We now focus on the square root factor in the on-shell action in equation (6.3.34), and demand that the action remain real for all $r$, as in the relativistic case of section 6.3.1. First, notice that as a function of $r$, the factor in the numerator, $g_{x x}\left|\tilde{G}_{+-\alpha_{2} \alpha_{3}}\right|-E_{\beta}^{2} \tilde{G}_{3}$, is negative at the horizon, $r=r_{H}$, and positive near the boundary $r \rightarrow 0$, and hence must have a zero at some $r=r_{*}$,

$$
\left[g_{x x}\left|\tilde{G}_{+-\alpha_{2} \alpha_{3}}\right|-E_{\beta}^{2} \tilde{G}_{3}\right]_{r_{*}}=0 \Longrightarrow \frac{1}{E_{\beta}^{2}}=\frac{4 r_{*}^{2} \beta^{2}}{f\left(r_{*}\right)}\left[r_{*}^{2}-\beta^{2} f\left(r_{*}\right) \sin ^{2} \theta\left(r_{*}\right)\right] .
$$

Similarly, the denominator $U(r)-V(r)$ is negative at the horizon and positive at the boundary, and so must have a zero also at some value of $r$. As in the relativistic $A d S$ case 
reviewed above, the two zeros must coincide to avoid an imaginary action, so we require $U\left(r_{*}\right)-V\left(r_{*}\right)=0$. Now consider $V(r)$, which has a factor of $g_{x x}\left|\tilde{G}_{+-\alpha_{2} \alpha_{3}}\right|-E_{\beta}^{2} \tilde{G}_{3}$ in its denominator. If the numerator of $V(r)$ is finite at $r_{*}$, then $V(r)$ diverge at $r_{*}$. Notice, however, that $U(r)$ is not divergent at $r_{*}$. The only way to achieve $U\left(r_{*}\right)-V\left(r_{*}\right)=$ 0 is thus to demand that the numerator of $V(r)$ vanish at $r_{*}$ (at least as quickly as $g_{x x}\left|\tilde{G}_{+-\alpha_{2} \alpha_{3}}\right|-E_{\beta}^{2} \tilde{G}_{3}$ ). Setting the numerator of $V(r)$ to zero at $r_{*}$, we obtain (after some algebra)

$$
\left\langle J^{-}\right\rangle=-\left.\frac{\tilde{G}_{B}+2 \beta^{2} \tilde{G}_{+\alpha_{2} \alpha_{3}}}{2 \beta^{2} \tilde{G}_{B}+\tilde{G}_{-\alpha_{2} \alpha_{3}}}\right|_{r_{*}}\left\langle J^{+}\right\rangle .
$$

Notice that equation (6.3.37) has no explicit dependence on the current $\left\langle J^{x}\right\rangle$, and depends on the electric field only implicitly through $r_{*}$. In the absence of the electric field, equation (6.3.37) becomes $\left\langle J^{-}\right\rangle=-\left\langle J^{+}\right\rangle /\left(2 \beta^{2}\right)$, which is independent of the temperature. Recalling the statements at the end of the last subsection, here we see that, indeed, we cannot introduce $\left\langle J^{+}\right\rangle$without also introducing $\left\langle J^{-}\right\rangle$.

Now the condition $U\left(r_{*}\right)=V\left(r_{*}\right)$, combined with equation (6.3.37), fixes the current to be

$$
\begin{aligned}
\left\langle J^{x}\right\rangle^{2} & =E_{\beta}^{2}\left[\mathcal{N}^{2} K g_{\alpha_{1} \alpha_{1}} \tilde{G}_{3}+\frac{\tilde{G}_{3}^{2}\left\langle J^{+}\right\rangle^{2}}{g_{x x}^{2}\left(\tilde{G}_{-\alpha_{2} \alpha_{3}}+2 \beta^{2} \tilde{G}_{B}\right)^{2}}\right]_{r_{*}} \\
& =E_{\beta}^{2} \frac{f\left(r_{*}\right)}{64 E_{\beta}^{2} r_{*}^{6}}\left[\mathcal{N}^{2} \cos ^{6} \theta\left(r_{*}\right)+\frac{16\left\langle J^{+}\right\rangle^{2} r_{*}^{2} f\left(r_{*}\right)}{\beta^{4} E_{\beta}^{2}}\right],
\end{aligned}
$$

which gives the DC conductivity, $\sigma$, through Ohm's law $\left\langle J^{x}\right\rangle=\sigma E_{\beta}$,

$$
\sigma=\sqrt{\mathcal{N}^{2} \frac{f\left(r_{*}\right)}{64 E_{\beta}^{2} r_{*}^{6}} \cos ^{6} \theta\left(r_{*}\right)+\frac{f\left(r_{*}\right)^{2}}{4 \beta^{4} r_{*}^{4} E_{\beta}^{4}}\left\langle J^{+}\right\rangle^{2}} .
$$

Note that $\sigma$ is dimensionless, as it should be. Notice that once we fix $T, E_{\beta}$ and $\left\langle J^{+}\right\rangle$, reality of the action determines $\left\langle J^{-}\right\rangle$and $\left\langle J^{x}\right\rangle$.

As in the relativistic case, the result for $\sigma$ consists of two terms adding in quadrature. Once again, the second term, proportional to $\left\langle J^{+}\right\rangle^{2}$, describes the contribution to the current from the charge carriers we introduced explicitly via the net density $\left\langle J^{+}\right\rangle$. The first term, again proportional to $\cos ^{6} \theta\left(r_{*}\right)$, appears to describe the contribution from charge-neutral pairs. We suspect that, as in the relativistic case, these pairs come from Schwinger and/or thermal pair production. From the field theory point of view, such pair production at first seems counter-intuitive, since in a non-relativistic theory, the number of particles should not change. Recall, however, that we are actually studying a relativistic theory which we deform in two ways, first by introducing an irrelevant operator and then by performing a DLCQ. From that perspective, nothing is wrong with pair production. Notice also that what plays the role of the number of particles is the eigenvalue of $N \sim P_{-}$, the momentum in the $x^{-}$direction, which is indeed fixed.

We now take two different limits to explore the scaling of the conductivity. The two limits depend on the relative strengths of $E_{\beta}$ and $T$. In the limit of a very weak electric field, $E_{\beta}^{2} \beta^{2} r_{H}^{4} \ll 1$, or equivalently $E_{\beta} \ll \beta T^{2}$, we have from equation (6.3.36) $r_{*} \rightarrow r_{H}$, and the conductivity takes the form

$$
\sigma \approx \sqrt{\frac{\pi^{2} \mathcal{N}^{2} \cos ^{2} \theta\left(r_{*}\right)}{16} T^{2} \beta^{4}+\frac{4\left\langle J^{+}\right\rangle^{2}}{\pi^{4} \beta^{4} T^{4}}}=\sqrt{\frac{\pi^{2} \mathcal{N}^{2} \cos ^{2} \theta\left(r_{*}\right)}{64} \frac{T^{2}}{\mu^{2}}+\frac{16}{\pi^{4}} \frac{\left\langle J^{+}\right\rangle^{2}}{T^{2}} \frac{\mu^{2}}{T^{2}}},
$$


where in the second equality we have replaced $\beta$ with $\mu$ using equation (6.2.17). At low temperatures or large masses, i.e. $T / \mu \rightarrow 0$ or $\cos \theta\left(r_{*}\right) \rightarrow 0$, the pair-production term is suppressed, in which case the conductivity approaches

$$
\sigma \approx \frac{4}{\pi^{2}} \frac{\left\langle J^{+}\right\rangle}{T} \frac{(-\mu)}{T}
$$

In a scale-invariant theory with dynamical exponent $z$, the conductivity should behave as $\sigma \sim\left\langle J^{+}\right\rangle T^{-2 / z}$. Here we see that, if we fix the chemical potential $\mu$, then we obtain the dynamical exponent of a relativistic theory, $z=1$. On the other hand, if we hold fixed the ratio $\mu / T$ as we vary the temperature, then we find non-relativistic scaling, with $z=2$.

The opposite limit is strong electric field, $E_{\beta}^{2} \beta^{2} r_{H}^{4} \gg 1$, or equivalently $E_{\beta} \gg \beta T^{2}$. If the mass is small, so that $\theta\left(r_{*}\right) \approx 0$ and hence $\sin \theta\left(r_{*}\right) \approx 0$, then equation (6.3.36) implies that $r_{*} \sim\left(2 E_{\beta} \beta\right)^{-1 / 2}$, and we find

$$
\sigma \approx \sqrt{\frac{\mathcal{N}^{2} \cos ^{6} \theta\left(r_{*}\right)}{8} E_{\beta} \beta^{3}+\frac{\left\langle J^{+}\right\rangle^{2}}{E_{\beta}^{2} \beta^{2}}}=\sqrt{\frac{\mathcal{N}^{2} \cos ^{6} \theta\left(r_{*}\right)}{8^{3 / 2}} \frac{E_{\beta}}{(-\mu)^{3 / 2}}+2 \frac{\left\langle J^{+}\right\rangle^{2}(-\mu)}{E_{\beta}^{2}}} .
$$

If we set the mass and density to zero, so $\cos \theta\left(r_{*}\right)=1$ and $\left\langle J^{+}\right\rangle=0$, then we obtain a finite conductivity,

$$
\sigma \approx \frac{\mathcal{N}}{8^{3 / 4}} \sqrt{\frac{E_{\beta}}{(-\mu)^{3 / 2}}}
$$

We indeed find a nonzero current, which must come from Schwinger pair production. The bulk mechanism is exactly the same as in the relativistic case: the worldvolume electric field is ripping strings apart. If we fix the value of $\mu$, then $\sigma \propto \sqrt{E_{\beta}}$, which is the same scaling with the electric field as in the $T=0$ relativistic case. If we fix the ratio $E_{\beta} /(-\mu)^{3 / 2}$ as we vary $E_{\beta}$, however, then the conductivity is a constant, which we expect for a $(2+1)$-dimensional theory with non-relativistic scale invariance, if the only scale is the electric field.

If the electric field is small, then the second term under the square root in equation (6.3.43) dominates. We then find

$$
\sigma \approx \frac{\left\langle J^{+}\right\rangle(-2 \mu)^{1 / 2}}{E_{\beta}}
$$

Switching back to relativistic coordinates,

$$
\left\langle J^{+}\right\rangle=\beta\left(\left\langle J^{t}\right\rangle+\left\langle J^{y}\right\rangle\right)=\frac{1}{(-2 \mu)^{1 / 2}}\left(\left\langle J^{t}\right\rangle+\left\langle J^{y}\right\rangle\right),
$$

we find that, for a fixed $\mu$, the equation for the current becomes

$$
\left\langle J^{x}\right\rangle=\left\langle J^{t}\right\rangle+\left\langle J^{y}\right\rangle,
$$

which is similar to the AdS result at zero temperature (see Appendix A of [116]). The physics here is simply that at zero temperature the charge carriers are accelerated to the speed of light, so the system is not really stationary. If we fix the ratio $E_{\beta} /(-\mu)^{3 / 2}$, so that we can use $(-\mu)^{1 / 2} \sim E_{\beta}^{1 / 3}$, then

$$
\sigma \propto \frac{\left\langle J^{+}\right\rangle}{E_{\beta}^{2 / 3}}
$$


which is the appropriate dependence on the electric field for a two-dimensional nonrelativistic conformal theory with a finite density.

To summarize: of the two scales $E_{\beta}$ and $T$, we can take one to be large relative to the other. If we hold the larger scale fixed relative to the scale set by $\mu$, then we obtain non-relativistic scaling. If on the other hand we hold $\mu$ fixed and vary the larger scale, we obtain relativistic scaling.

We can understand our results in terms of the geometry as follows. Consider for example the case in which we can neglect the electric field, so the temperature is the larger scale. The conductivity is then evaluated at $r_{*} \simeq r_{H}$. Fixing $\mu / T$ is the same as fixing $r_{H} / \beta$. We are thus probing the geometry on the scale of the deformation $\beta$, so intuitively we expect to obtain non-relativistic behavior. Indeed, in this case the conductivity in (6.3.42) behaves in a non-relativistic way. If instead we fix $\mu$ as we take $T / \mu \rightarrow 0$ (see the text above equation (6.3.42)), then the ratio $r_{H} / \beta \rightarrow \infty$ and the horizon enters the region where the geometry is similar to AdS-Schwarzschild. In that case (6.3.42) indeed exhibits relativistic scaling.

\subsection{AC Conductivity of Probe Flavor}

We now proceed to compute the frequency-dependent conductivity in the linear response approximation. In the field theory we consider thermal equilibrium states with temperature $T$ and massive flavor fields with a finite density $\left\langle J^{+}\right\rangle$and finite $\left\langle J^{-}\right\rangle$, but now, unlike the last section, no constant electric field.

In the holographic dual the system at equilibrium is described by probe D7-branes in the asymptotically Schrödinger black hole geometry of equation (6.2.9), with nontrivial worldvolume fields $A_{+}(r)$ and $A_{-}(r)$. We can obtain the solution for these in exactly the same way as the last section: each has an associated constant of motion, $\left\langle J^{+}\right\rangle$and $\left\langle J^{-}\right\rangle$ from equation (2.5.105), so we obtain equations similar to those in equations (6.3.33), which we then algebraically invert to find $A_{+}(r)$ and $A_{-}(r)$ in terms of $\left\langle J^{+}\right\rangle$and $\left\langle J^{-}\right\rangle$. We do not present these solutions explicitly, but we record that, in the absence of a constant worldvolume electric field, we have from the last section that $r_{*}=r_{H}$ and $\left\langle J^{-}\right\rangle=-\left\langle J^{+}\right\rangle /\left(2 \beta^{2}\right)$ (see equations (6.3.36) and (6.3.37)). Notice also that, as always with a nontrivial $A_{+}(r)$ (or $A_{t}(r)$ ), the D7-branes must extend all the way to the black hole horizon [115]. We also consider a nontrivial embedding $\theta(r)$, whose form we discuss in detail below.

To obtain the conductivity in the regime of linear response, we consider a small, frequencydependent perturbation of the worldvolume electric field about the background solution (which has $A_{+}(r), A_{-}(r)$, and $\theta(r)$ ),

$$
A_{x}\left(x^{+}, x^{-}, r\right)=\operatorname{Re}\left[e^{-i \omega\left(x^{+}+2 \beta^{2} x^{-}\right)} a_{x}(r, \omega)\right] .
$$

For simplicity we work with zero spatial momentum. Here we note that, in fact, removing all $x^{-}$dependence does not qualitatively change our results for the behavior of the conductivity with frequency.

To quadratic order, the Lagrangian density for the perturbation is

$$
\mathcal{L}_{2}=\alpha_{++} f_{+x}^{2}+\alpha_{--} f_{-x}^{2}+2 \alpha_{+-} f_{+x} f_{-x}-\alpha_{r r} f_{x r}^{2},
$$


where $f_{\alpha \beta}=\partial_{\alpha} A_{\beta}-\partial_{\beta} A_{\alpha}$ is the field strength associated with the fluctuation in equation (6.4.49). The equation of motion for the fluctuation is then

$$
a_{x}^{\prime \prime}+\frac{\alpha_{r r}^{\prime}}{\alpha_{r r}} a_{x}^{\prime}+\omega^{2} \frac{\alpha_{++}+4 \beta^{4} \alpha_{--}+4 \beta^{2} \alpha_{+-}}{\alpha_{r r}} a_{x}=0 .
$$

Here the $\alpha$ are $r$-dependent coefficients that depend on the background solutions for $A_{+}(r)$ and $A_{-}(r)$ about which we are perturbing. To write these succinctly, let us introduce some notation. We define

$$
\begin{gathered}
k_{s}(r) \equiv 1+\frac{\sin ^{2} \theta(r) \beta^{2} r^{2}}{r_{H}^{4}}, \quad \rho(r) \equiv 1+r^{2} f(r) \theta^{\prime 2}, \\
Q^{2} \equiv \frac{64\left\langle J^{+}\right\rangle^{2}}{\beta^{2}}, \quad \gamma^{2}(r) \equiv \cos ^{6} \theta(r) k_{s}(r)+Q^{2} r^{4}\left(r^{2}+\beta^{2} \sin ^{2} \theta(r)\right) .
\end{gathered}
$$

The coefficients in the quadratic Lagrangian density are then

$$
\begin{aligned}
\alpha_{r r}(r) & =\frac{\mathcal{N}}{16 r \sqrt{k_{s}(r) \rho(r)}} f(r) \gamma(r) \\
\alpha_{++}(r) & =\frac{\mathcal{N}}{16 r_{H}^{4} f(r) \gamma(r)} \beta^{2} r^{3}\left(Q^{2} r^{2} r_{H}^{4}+\cos ^{6} \theta(r)\right) \sqrt{k_{s}(r) \rho(r)} \\
\alpha_{--}(r) & =\frac{\mathcal{N}}{64 \beta^{2} r^{3} f(r) \gamma(r)}\left[Q^{2} r^{8}+\cos ^{6} \theta(r)\left(\frac{r^{6}}{r_{H}^{4}}-4 \sin ^{2} \theta(r) \beta^{2} f(r)\right)\right] \sqrt{k_{s}(r) \rho(r)} \\
\alpha_{+-}(r) & =\frac{\mathcal{N}}{32 r f(r) \gamma(r)}\left[Q^{2} r^{6}-\cos ^{6} \theta(r)\left(\frac{r^{4}}{r_{H}^{4}}-2\right)\right] \sqrt{k_{s}(r) \rho(r)}
\end{aligned}
$$

We follow the now-standard procedure to compute transport coefficients, in the regime of linear response, holographically (for a review see $[30,36,37]$ ). We must first solve the linearized equation of motion for the fluctuation $a_{x}(r, \omega)$ with the boundary condition that near the horizon the solution has the form of a traveling wave moving into the black hole, i.e. an in-going wave. We then insert that solution into the action, which then acts as a generating functional for field theory correlators. Taking two functional derivatives of the on-shell action gives us the retarded Green's function. We then extract the conductivity from the retarded Green's function via a Kubo formula. Ultimately, we find

$$
\sigma(\omega) \propto \lim _{r \rightarrow 0}\left[\frac{\alpha_{r r} a_{x}^{\prime}(r, \omega)}{\omega a_{x}(r, \omega)}\right],
$$

where, because we are primarily interested in $\sigma(\omega)$ 's scaling behavior with $\omega$, we omit the overall prefactor.

Crucially, notice that the result depend on the background solution $\theta(r)$ describing the embedding of the D7-brane. In general, with finite temperature $T$ and density $\left\langle J^{+}\right\rangle$, we can only solve for $\theta(r)$ numerically. We leave a complete numerical solution for future work. Here we focus on regimes of physical interest, applying some approximations to obtain analytic results for $\sigma(\omega)$ 's scaling with $\omega$.

We are interested in a regime dominated by the physics of a zero-temperature critical point. Remember that the Schrödinger geometry interpolates between a UV critical point with $z=2$ and an IR critical point with $z=1$. The scale that separates the two regimes 
is the chemical potential ( $\beta$ in the metric). In order to eliminate thermal effects, we work in a limit where the temperature is much smaller than any other scale, which in particular means $\omega \gg T$. We then expect that we can explore both regions by taking $\mu \gg \omega$ for the IR regime and $\mu \ll \omega$ for the $\mathrm{UV}$.

In the probe approximation the critical behavior is not spoiled by a nonzero mass or charge density for the flavors. The sector described by the probe D7-branes is sensitive to these quantities, however, so we expect deviations from scale invariance whenever $\omega \sim\left\langle J^{+}\right\rangle^{z / d}$ or $\omega \sim m^{2}$. In order to avoid such deviations we only explore frequencies below these scales, which means $\left\langle J^{+}\right\rangle^{z / d} \gg \mu$ and $m^{2} \gg \mu$.

Instead of computing the exact current-current correlator in the holographic description, we use the radial coordinate as an approximation to the frequency scale, following [185]. We call $r_{0}$ the reference scale around which we give an estimate of the conductivity. More precisely, we define a local conductivity $\sigma\left(\omega, r_{0}\right)$ to be the quantity in brackets in equation (6.4.55), evaluated at $r=r_{0}$,

$$
\sigma\left(\omega, r_{0}\right) \equiv\left[\frac{\alpha_{r r} a_{x}^{\prime}(r, \omega)}{\omega a_{x}(r, \omega)}\right]_{r_{0}}
$$

If we think about the charge carriers as strings attached to the brane, the length of the string from $r_{0}$ to the horizon 13 times the string tension, is, roughly speaking, the energy of the charge carriers we are exciting when we apply an oscillating electric field, which is on the order of $1 / r_{0}^{2}$. The holographic conductivity evaluated at $r_{0}$ should thus give us a rough idea of the response of the system to an external field with a fixed frequency of the order $1 / r_{0}^{2}$. Notice that in such a picture we would expect to produce pairs if the energy is of the order of, or larger than, the mass. That is another good reason why we only explore scales much below the mass. Notice also that in the Lifshitz case of [185], both $\sigma\left(\omega, r_{0}\right)$ and $\sigma(\omega)$ had the same scaling with frequency in the limit that $\omega r^{z} \ll 1$.

In terms of the quantities in our formulas, for frequencies small relative to the chemical potential (the IR) we should set $r_{0} \gg \beta$ while for large frequencies (the UV) we should set $r_{0} \ll \beta$. In the low temperature limit we should send $r_{H} \rightarrow \infty$ relative to any other scale, and by large mass and density we mean $r_{\Lambda} \ll r_{0}$ and $\left\langle J^{+}\right\rangle \gg 1 / r_{0}^{2}$.

We show in the following subsection that, with some assumptions, the D7-branes have a very simple embedding that we can compute analytically in our regimes of interest. Using our analytic results for $\theta(r)$, we analytically compute the $\omega$-dependence of $\sigma(\omega)$ in the subsequent subsections and compare the $\omega$ scaling with the results from Lifshitz backgrounds, equation (6.1.2).

\subsubsection{D7-brane Embeddings}

To determine analytic forms for $\theta(r)$, we return to the DBI action of equation (2.5.95) and insert our ansatz for the worldvolume fields: $A_{+}(r), A_{-}(r)$, and $\theta(r)$, using the background metric, NS B-field and dilaton of equations (6.2.9), (6.2.13), and (6.2.14), respectively. The result is precisely equation (6.3.31), if in equation (6.3.31) we take $E_{\beta}=A_{x}^{\prime}(r)=0$.

\footnotetext{
${ }^{13}$ We are using black hole embeddings only, in which case the endpoint of a string at $r_{0}$ would be free to move along the D7-brane and into the horizon. Our statements about the physical meaning of $r_{0}$ are meant only to provide intuition.
} 
Writing $S_{D 7}=-\int d r L$ (recall the text below equation (6.2.8)), we find

$$
-L=\frac{\mathcal{N}}{8 r^{5}} \cos ^{3} \theta(r) \sqrt{a_{0}-b_{0} \cos ^{2} \theta(r)+r^{2} f(r) \theta^{\prime 2}} \equiv \frac{\mathcal{N}}{8 r^{5}} \cos ^{3} \theta(r) \hat{L},
$$

where for later convenience we defined a reduced Lagrangian $\hat{L}$. Here $a_{0}(r)$ and $b_{0}(r)$ are functions of the $U(1)$ field strength, not yet evaluated on any solution for the field strength.14

$$
\begin{aligned}
& a_{0}(r)=1+[1+f(r)] r^{4} A_{+}^{\prime}(r) A_{-}^{\prime}(r)+r^{2} A_{-}^{\prime}(r)^{2}\left(f(r)-\frac{r^{6}}{4 \beta^{2} r_{H}^{4}}\right)-\frac{r^{8} \beta^{2}}{r_{H}^{4}} A_{+}^{\prime}(r)^{2} \\
& b_{0}(r)=r^{2} f(r) A_{-}^{\prime}(r)^{2}
\end{aligned}
$$

The equation of motion for $\theta(r)$ is

$$
\left(\frac{\cos ^{3} \theta(r) r^{3} f(r) \theta^{\prime}}{\hat{L}}\right)^{\prime}-\frac{\sin \theta(r)}{r^{5}}\left(3 \cos ^{2} \theta(r) \hat{L}-\frac{b_{0} \cos ^{4} \theta(r)}{\hat{L}}\right)=0 .
$$

Solving for the gauge fields by inverting equation (6.3.33), with the charge density fixed, and plugging the solutions into $a_{0}(r)$ and $b_{0}(r)$, we find the on-shell values of $a_{0}(r)$ and $b_{0}(r)$,

$$
\begin{gathered}
a_{0}(r)=\frac{\cos ^{6} \theta(r) k_{s}(r)^{2}}{\gamma^{2}(r) k_{s}(r)}+\frac{Q^{2} r^{4} f(r)}{\gamma^{2}(r) k_{s}(r)}\left[\beta^{2} \cos ^{2} \theta(r)\right. \\
\left.-r^{2} \theta^{\prime 2}\left(\beta^{2} \frac{r^{4}}{r_{H}^{4}}-\beta^{2} \cos (2 \theta(r))+r^{2}\left(1+\frac{\beta^{4} \sin ^{4} \theta(r)}{r_{H}^{4}}\right)\right)\right], \\
b_{0}(r)=Q^{2} \frac{\beta^{2} r^{4} f(r)}{\gamma^{2}(r)} \frac{\rho(r)}{k_{s}(r)}
\end{gathered}
$$

and the action evaluated on the solution is proportional to $\hat{L}=\cos ^{3} \theta(r) \sqrt{k_{s}(r) \rho(r)} / \gamma(r)$.

As we reviewed in section 6.2.1, when $A_{t}(r)$, or $A_{+}(r)$, is zero, the D7-brane can end at some value of the radial coordinate $r_{\Lambda}$, but when $A_{t}(r)$ or $A_{+}(r)$ is nontrivial, the D7brane must extend all the way to the horizon, and has a spike when $r_{\Lambda} \ll r_{H}$. Along the spike and far from the horizon, $r_{\Lambda}<r \ll r_{H}, \theta(r)$ is approximately constant. Such a region in $r$ corresponds to scales below the mass gap of the charge carriers, where scale invariance is approximately restored.

Let us consider the limits $r \ll r_{H}$ and $Q r^{3} \gg 1$, corresponding to low temperature and large density, as explained in the last subsection. If we take the IR limit $\beta \ll r$, we find

$\gamma^{2}(r)=\cos ^{6} \theta(r)\left(1+\frac{\beta^{2} \sin ^{2} \theta(r) r^{2}}{r_{H}^{4}}\right)+Q^{2} r^{4}\left(r^{2}+\beta^{2} \sin ^{2} \theta(r)\right) \approx \cos ^{6} \theta(r)+Q^{2} r^{6} \approx Q^{2} r^{6}$.

In the UV limit $\beta \gg r$ we find

$$
\gamma^{2}(r) \approx \cos ^{6} \theta(r)+Q^{2} r^{4} \beta^{2} \sin ^{2} \theta(r) \simeq Q^{2} \beta^{2} \sin ^{2} \theta(r) r^{4} .
$$

\footnotetext{
${ }^{14}$ Alternatively, we can derive $\theta(r)$ 's equation of motion by first solving for the gauge fields, plugging the results into $L$, performing a Legendre transform with respect to the gauge fields, and then finding the Euler-Lagrange equations of motion [115].
} 
The IR and UV limits of all the quantities that appear in the equation of motion equation (6.4.59) are similarly straightforward to determine. In addition, we can assume that $r^{2} \theta^{\prime 2} \ll 1$ as long as $r_{\Lambda} \ll r$, so we take $1+r^{2} f \theta^{\prime 2} \approx 1, \sin \theta(r) \approx \sin \theta_{0}$ and $\cos \theta(r) \approx \cos \theta_{0}$ with $\theta_{0}$ a constant.

We then find that in the IR limit, to leading order, the equation of motion for $\theta(r)$ equation (6.4.59) becomes

$$
\theta^{\prime \prime}+\frac{\beta^{2} \sin \theta_{0} \cos \theta_{0}}{r^{4}}=0
$$

while in the UV limit, the equation of motion becomes

$$
\left(\frac{\theta^{\prime}}{r}\right)^{\prime}+\frac{\cot \theta_{0}}{r^{3}}=0
$$

The solutions in the IR and UV limits are

$$
\begin{gathered}
\text { IR } \theta(r)=\theta_{0}-\frac{\beta^{2}}{6 r^{2}} \sin \theta_{0} \cos \theta_{0}, \\
\text { UV } \theta(r)=\theta_{0}+\frac{1}{2} \cot \theta_{0} \log \frac{r}{r_{0}},
\end{gathered}
$$

where, following [185], and as discussed above, we have introduced the reference scale $r_{0}$ obeying $r_{\Lambda}<r_{0} \ll \beta$, which makes the argument of the logarithm in the UV solution dimensionless. Notice that in order to satisfy the condition $r^{2} \theta^{\prime 2} \ll 1$ in the UV solution, we need $\left|\cot \theta_{0}\right| \ll 1$, meaning a narrow spike and probably a large mass gap $r_{\Lambda} \ll r_{0}$. The expansion is also limited to a region around the reference scale $r_{0}$, such that $\left|\log \left(r / r_{0}\right)\right| \ll$ 1. Otherwise these results are consistent with all the approximations we have made.

\subsubsection{AC conductivity in the IR}

In the IR limit the equation of motion for the gauge field fluctuation, equation (6.4.51), becomes simply

$$
a_{x}^{\prime \prime}+\frac{2}{r} a_{x}^{\prime}+4 \beta^{2} \omega^{2} a_{x}=0
$$

with solutions

$$
a_{x}(r, \omega)=a_{x}^{0} \frac{e^{ \pm 2 i \beta \omega r}}{r},
$$

with $a_{x}^{0}$ a constant. The in-going solution corresponds to the positive sign in the exponential.

Next we follow the procedure described in [185] for probe branes in Lifshitz geometries. As mentioned above, we do not directly apply the formula equation (6.4.55) in our case because the $r \rightarrow 0$ limit takes us out of the regime of our approximations. Instead we compute the local conductivity $\sigma\left(\omega, r_{0}\right)$ at a reference scale $r_{0}$ such that $\beta \omega r_{0} \ll 1$. Plugging the solution into equation (6.4.56) and expanding the result with $\beta \omega r_{0} \ll 1$, we find that at leading order the local conductivity is

$$
\sigma\left(\omega, r_{0}\right) \propto \frac{\mathcal{N}\left\langle J^{+}\right\rangle r_{0}}{2 \beta} \omega^{-1}
$$

The $\omega^{-1}$ scaling is consistent with the result of [185], our equation (6.1.2), for the relativistic case with dynamical exponent $z=1$. 


\subsubsection{AC conductivity in the UV}

In the UV the equation of motion for the gauge field fluctuation equation (6.4.51) also takes a simple form. Using $\sin \theta_{0} \simeq 1$, we find

$$
a_{x}^{\prime \prime}+\frac{1}{r} a_{x}^{\prime}+4 r^{2} \omega^{2} a_{x}=0 .
$$

The general solutions are Bessel functions. The solution describing an in-going traveling wave at the horizon is a Hankel function,

$$
a_{x}(r, \omega)=a_{x}^{0} H_{0}^{(1)}\left(\omega r^{2}\right),
$$

where again $a_{x}^{0}$ is a constant. Notice that equations (6.4.71) and (6.4.72) coincide with the equations in [185] for a fluctuation of a probe brane's worldvolume gauge field in Lifshitz spacetime with dynamical exponent $z=2$. As before, we choose a cutoff $r_{0}$ such that $\omega r_{0}^{2} \ll 1$. Plugging the solution into equation (6.4.55) and expanding the result in powers of $\omega r_{0}^{2}$, we obtain

$$
\sigma\left(\omega, r_{0}\right) \propto \frac{\mathcal{N}\left\langle J^{+}\right\rangle}{16}\left(\omega \log \left(\omega r_{0}^{2}\right)\right)^{-1},
$$

which is indeed the same scaling with $\omega$ as obtained from a probe brane in a Lifshitz spacetime with $z=2$, see equation (6.1.2).

In summary: taking the temperature scale to be very low and the mass and density scales to be very high, we find that in the IR, meaning $\omega \ll \mu$, the $\mathrm{AC}$ conductivity exhibits relativistic scaling with frequency, while in the UV, meaning $\omega \gg \mu$, we find that the AC conductivity exhibits non-relativistic scaling with dynamical exponent $z=2$. These results clearly confirm our intuition on the bulk side, where the space is similar to AdS deep in the interior but not asymptotically, and in the field theory, where we have introduced an irrelevant deformation to $\mathcal{N}=4 \mathrm{SYM}$ that breaks the relativistic conformal group down to the Schrödinger group. 


\section{Part III}

\section{Flavor in Chern-Simons-Matter Theories}





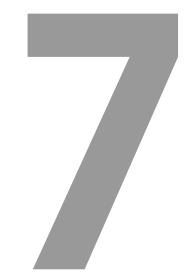

\section{Adding Flavor to $A d S_{4} / C F T_{3}$}

In this chapter we study another explicit realization of the Anti-de Sitter / Conformal Field Theory correspondence relating a $(2+1)$-dimensional superconformal field theory (denoted by $C F T_{3}$ ) to a gravity theory on $A d S_{4}$, which is therefore called $\mathrm{AdS}_{4} / \mathrm{CFT}_{3}$ correspondence. $\mathrm{AdS}_{4} / \mathrm{CFT}_{3}$ may be derived from the near-horizon limit of M2-branes. As we review in section 7.3, the low energy theory of $N_{c}$ M2-branes is given by maximally supersymmetric Chern-Simons matter theory with gauge group $U\left(N_{c}\right) \times U\left(N_{c}\right)$ in $(2+1)$ dimensions which is to eleven-dimensional supergravity on $A d S_{4} \times S^{7} / \mathbb{Z}_{k}$.

The $\mathrm{AdS}_{4} / \mathrm{CFT}_{3}$ correspondence is interesting from two different point of views. We can use this correspondence to study purely $(2+1)$-dimensional quantum critical theories, described by Chern-Simons theories. Note that Chern-Simons theories are very common in condensed matter physics. The $\mathrm{AdS}_{4} / \mathrm{CFT}_{3}$ correspondence sheds also some light on M2-branes and their interactions, and therefore is important for the further development of M-theory.

After an introduction to M-theory and its basic objects in section 7.2 , we review shortly $\mathrm{AdS}_{4} / \mathrm{CFT}_{3}$ in section 7.3. In the remaining sections we add flavor degrees to that correspondence which are confined to $2+1$ and $1+1$ dimensions. In particular we derive the Lagrangian and the symmetries of the field theory side and determine the embedding of the flavor branes in the dual gravity theory. Note that this chapter contains technical studies, parts of which may be found in appendix $B$. The field theories discussed in this chapter and their dual gravitational description are important for further studies of condensed matter theories using the AdS/CFT correspondence.

This chapter of the thesis is mainly based on work done in collaboration with Johanna Erdmenger, Réne Meyer, Andy O'Bannon and Timm Wrase and was published in [6]. 


\subsection{Introduction and summary}

So far we studied only one particular example of the AdS/CFT correspondence. In section 2.1 we reviewed that the near-horizon limit of $N_{c}$ D3-branes gives rise to a one-to-one mapping between $\mathcal{N}=4$ supersymmetric Yang-Mills theory in four-dimensional Minkowski spacetime and type IIB string theory on $A d S_{5} \times S^{5}$. Later we added flavor degrees of freedom transforming in the fundamental representation of the gauge group. The flavor degrees were allowed to propagate in four dimensions (in the case of flavor D7-branes) or can be confined to a lower-dimensional defect. Such a defect theory is realized by a supersymmetric D3/D5 intersection. The probe D5-brane is aligned along $A d S_{4} \times S^{2}$ inside $A d S_{5} \times S^{5}$ which describes supersymmetric flavor fields confined to propagate only in $(2+1)$-dimensions of the $(3+1)$-dimensional theory, i.e. along a codimension-one defect. Therefore from the point of view of the flavor degrees of freedom the theory is effectively $(2+1)$-dimensional.

Note that defect theories are very common in condensed matter systems as it was emphasized in the introduction, particularly in section 1.3. However, sometimes it is also useful to understand strongly coupled field theories which are purely $(2+1)$-dimensional theories. A natural candidate to derive a gauge/gravity duality involving a superconformal 2+1-dimensional field theory is to look at the near-horizon limit of M2-branes, which are one of the basic objects of M-theory.

Given $N_{c}$ M2-branes at a $\mathbb{C}^{4} / \mathbb{Z}_{k}$ singularity, when we take $N_{c} \rightarrow \infty$ we can replace the M2-branes with their near-horizon geometry, which is (3+1)-dimensional AdS space times a $\mathbb{Z}_{k}$ orbifold of a seven-sphere, $A d S_{4} \times S^{7} / \mathbb{Z}_{k}$. The natural conjecture was that the low-energy theory of M2-branes, in the large- $N_{c}$ limit, is dual to eleven-dimensional supergravity on $A d S_{4} \times S^{7} / \mathbb{Z}_{k}$. The exact low-energy theory of multiple M2-branes was unknown before [137], however.

In $[137]$ it was proposed that $N_{c}$ coincident M2-branes probing a $\mathbb{C}^{4} / \mathbb{Z}_{k}$ singularity have a low-energy description as a particular $\mathcal{N}=6$ supersymmetric Chern-Simons-matter theory. We refer to the theory of [137] as the ABJM theory.

Let us summarize here the main content of the chapter.

First, a short introduction to M-theory and its basic objects is given in section 7.2 for readers not familiar with the subject. In particular the properties of M2- and M5-branes are discussed. We work out the near-horizon geometry of M2- and M5-branes, whose metric is given by $A d S_{4} \times S^{7}$ (in the case of M2-branes) and $A d S_{7} \times S^{4}$ (for M5-branes), respectively.

In section 7.3 we follow [137] and derive the $\mathcal{N}=6$ supersymmetric Chern-Simons-matter theory, and its relation to M2-branes, from a particular brane construction in type IIB string theory. The initial configuration includes two stacks of $N_{c}$ D3-branes, an NS5brane, and a $(1, k) 5$-brane. At this stage we can identify the low-energy theory of the D3-branes as a $(2+1)$-dimensional $\mathcal{N}=3$ supersymmetric Chern-Simons-matter theory with product gauge group $U\left(N_{c}\right) \times U\left(N_{c}\right)$ and with equal but opposite Chern-Simons levels $k$ and $-k$ for the two gauge groups, which we denote as $U\left(N_{c}\right)_{k} \times U\left(N_{c}\right)_{-k}$. Performing

\footnotetext{
${ }^{1}$ Following the work of [196-202].
} 
a T-duality, an uplift to M-theory, and a certain kind of "near-horizon" limit 2 we obtain M2-branes probing $\mathbb{C}^{4} / \mathbb{Z}_{k}$. A supersymmetry enhancement occurs in the "near-horizon" limit, from $\mathcal{N}=3$ to $\mathcal{N}=6$ supersymmetry. The low-energy theory of the M2-branes (the ABJM theory) is thus an $\mathcal{N}=6$ supersymmetric $U\left(N_{c}\right)_{k} \times U\left(N_{c}\right)_{-k}$ theory with adjoint and bifundamental fields. Upon taking $N_{c} \rightarrow \infty$ and replacing the M2-branes with their near-horizon geometry, the appropriate description is eleven-dimensional supergravity on $A d S_{4} \times S^{7} / \mathbb{Z}_{k}$. We may then take $k \rightarrow \infty$, where the appropriate description becomes type IIA supergravity on $A d S_{4} \times \mathbb{C P}^{3}$.

The aim of the chapter is to deform the ABJM theory by introducing fields in the fundamental representation of the gauge groups, i.e flavor fields. We study flavor fields both in the brane construction and also in the field theory. In particular, for a given brane construction, we present a general recipe to determine the couplings of the flavor fields to the fields of the ABJM theory. We then apply our general recipe to two examples ${ }^{3}$ for which we write down explicitly the field theory Lagrangians. Moreover, we compare the symmetries of the string/gravity description and the field theory.

To add supersymmetric flavor to the ABJM theory, we add supersymmetric flavor branes in the type IIB brane construction of the ABJM theory in section 7.4.1 (see also appendix B.1). We begin by listing all supersymmetric Dp-branes that are extended along the coordinate axes in the type IIB construction (see table 7.1 in section 7.4.1). We then perform a T-duality, a lift to M-theory, and the "near-horizon" limit to determine where these branes end up in M-theory on $\mathbb{C}^{4} / \mathbb{Z}_{k}$, and we compute the amount of supersymmetry the object (brane or Kaluza-Klein (KK) monopole) preserves (table B.2 in appendix B.3.1 lists a few examples). Lastly we take $N_{c} \rightarrow \infty$ and determine where the objects end up in M-theory on $A d S_{4} \times S^{7} / \mathbb{Z}_{k}$, and for a few examples we compute the amount of supersymmetry the objects preserve (see appendix B.3.2).

All of the above analysis occurs on the gravity side of the AdS/CFT correspondence. How do we determine the dual field theory, including the flavor fields? As a systematic approach to construct the field theory, we start a few steps before ABJM's type IIB construction. We begin in type IIB with just a D3/Dp intersection, where the Dp-brane is the flavor brane. The actions for many D3/Dp systems are known (see for example [88,94-100]). We then follow what happens to the action, step-by-step, during ABJM's type IIB construction. Two steps are crucially important in this procedure. The first is the addition of the NS5-branes, which impose boundary conditions that set to zero some of the degrees of freedom, as explained in $[203,204]$. The second is when we take the "near-horizon" limit (after T-dualizing in $x^{6}$ and lifting to M-theory). In the field theory, this corresponds to taking a low-energy limit and writing an effective theory valid on scales below the ChernSimons mass scale $g_{Y M}^{2} k /(4 \pi)$ (with $g_{Y M}$ the Yang-Mills coupling of the (2+1)-dimensional theory). Roughly speaking, the action will be the known D3/Dp action after 1.) imposing the NS5-brane boundary conditions and 2.) taking the low-energy limit. The resulting action is the answer for the field theory, and should have the correct symmetries. As we will see, however, this procedure is not always easy to implement in concrete examples.

\footnotetext{
${ }^{2}$ Throughout this paper we use quotation marks to distinguish this "near-horizon" limit, which we explain in detail below, and the usual near-horizon limit of a stack of branes, for example the near-horizon limit of M2-branes, which produces $A d S_{4}$ (see section 7.2). We define the "near-horizon" limit precisely in section 7.3 .3 , and perform the limit explicitly in appendix B.2.

${ }^{3}$ Another two interesting examples as well as more examples of $S U(4)$ equivalence are studied in [6].
} 
We apply our general procedure to two examples. We will add two different flavor branes in type IIB, which become two objects (branes or KK monopoles) in M-theory on $\mathbb{C}^{4} / \mathbb{Z}_{k}$. The two branes, and the type of flavor fields they describe, are as follows:

- A D5-brane that becomes a D6-brane in type IIA and a KK monopole in M-theory, and which introduces codimension-zero $\mathcal{N}=3$ supersymmetric flavor fields.

- A D7-brane that becomes a D8-brane in type IIA and an M9-brane 4 in M-theory, and which introduces codimension-one, chiral, $\mathcal{N}=(0,6)$ supersymmetric flavor fields.

In particular for both cases we describe the location of the object in M-theory on $\mathbb{C}^{4} / \mathbb{Z}_{k}$, calculate the number of supercharges it preserves, and identify the isometries of the background that it preserves. Moreover we write the field theory Lagrangian describing the coupling of the flavor fields to the fields of the ABJM theory and match the symmetries between the field theory and supergravity descriptions.

In our analysis of section 7.7 we find that many different Dp-branes in type IIB, for example Dp-branes with different embeddings or even Dp-branes of different dimensionality, become the same object in M-theory. Furthermore, the embeddings of many such M-theory objects may be mapped into one another via an $S U(4)$ isometry transformation (as first noted in [206]). When that occurs, we call the two objects $S U(4)$-equivalent. A natural question is what $S U(4)$ equivalence means in the field theory. In simple terms, $S U(4)$ equivalence occurs when two different theories flow to the same low-energy fixed point (corresponding to two type IIB Dp-branes becoming the same object after the "near-horizon" limit). We will discuss $S U(4)$ equivalence in more detail, and provide an explicit example, below.

Throughout this paper we work in the probe limit whenever applicable. In other words, whenever we have $N_{f}$ flavor branes and we take $N_{c} \rightarrow \infty$, we will keep $N_{f}$ fixed, such that $N_{f} \ll N_{c}$. We will always consider $N_{f}$ coincident flavor branes; we will never separate the flavor branes from one another. We will also consider only massless flavor fields.

\subsection{A M-theory Primer}

So far we considered only type IIB string theory (see also the glossary in appendix A.2.3). Besides type IIB string theory there are well known four other consistent string theories in ten spacetime dimensions, called type IIA string theory, type I string theory, heterotic $\mathrm{SO}(32)$ and heterotic $E_{8} \times E_{8}$ string theory. All five consistent string theories are connected by a web of dualities. Examples of dualities are T-duality and S-duality. Whereas S-duality is a duality between a strongly coupled string theory and a (not necessarily different) weakly coupled string theory, T-duality (or target space duality) denotes the equivalence between two superstring theories compactified on different background spacetimes. In this chapter we will exploit the fact that type IIA superstring theory on a circle with radius $R$ and type IIB superstring theory on a circle with radius $\alpha^{\prime} / R$ are equivalent. In fact, S-duality and T-duality can be embedded into a larger duality group, U-duality [207].

\footnotetext{
${ }^{4}$ Here, and throughout the paper, M9-brane will refer to the still-mysterious M-theory description of D8-branes. The only part of the M-theory description that we really use is the name M9-brane, however. In most cases, thinking of this object as a D8-brane in type IIA suffices. For more on the conjectured M9-brane, see [205] and references therein.
} 
By using this U-duality, it was realized in 1995 that the strong coupling regime of all five consistent string theories in ten spacetime dimensions is mapped to some weakly coupled limit of another theory ( [208], for a review see [209]). For this picture to be self-contained, one has to include also eleven-dimensional supergravity. In fact, it is believed that all five consistent string theories can be unified into an eleven-dimensional parent theory, called M-theory. In other words, the five different versions of string theory are just M-theory expanded around different vacua. Although up to now a precise definition of M-theory is not available, we know that the low-energy effective action of this theory is given by the unique eleven-dimensional supergravity.

The bosonic field content of eleven-dimensional supergravity is very: a graviton $\sqrt[5]{5} G_{M N}$ and a three-form potential $A_{(3)}$ (with components $A_{M N P}$ ). There is a deep connection between M-theory and type IIA superstring theory: the low-energy limit of type IIA superstring theory, which is given by type IIA supergravity, can be obtained by a KaluzaKlein reduction of eleven-dimensional supergravity on a circle $S^{1}$ as reviewed in section 7.2.2,

\subsubsection{Membranes in M-theory}

Since eleven-dimensional supergravity has only an antisymmetric tensor field $A_{(3)}$ of rank three, the possible branes are very restricted: a 2-brane, called M2 and the magnetic dual, a M5-brane. Here we will discuss the supergravity solution of a M2-brane.

A stack of $N$ coincident M2-branes sources the following fields

$$
\begin{aligned}
d s^{2} & =h(r)^{-2 / 3}\left(-d t^{2}+d x_{1}^{2}+d x_{2}^{2}\right)+h(r)^{1 / 3}\left(d r^{2}+r^{2} d \Omega_{7}^{2}\right) \\
G_{(4)} & =d t \wedge d x_{1} \wedge d x_{2} \wedge d h(r)^{-1}
\end{aligned}
$$

where $G_{(4)}=d A_{(3)} \cdot h(r)$ and $L$ are given by

$$
h(r)=1+\frac{L^{6}}{r^{6}}, \quad L^{6}=32 \pi^{2} N l_{p}^{6} .
$$

$l_{p}$ is the Planck length of the eleven-dimensional theory. Taking the near-horizon limit, i.e. $r / L \rightarrow 0$, the metric reduces to

$$
d s^{2}=L^{2}\left(\frac{1}{4} d s_{A d S_{4}}^{2}+d s_{S^{7}}^{2}\right)
$$

$d s_{A d S_{4}}^{2}$ and $d s_{S^{7}}^{2}$ are the metrics of $A d S_{4}$ and $S^{7}$ with radius one. Note that due to the different prefactors in equation 7.2.3, the radii of $S^{7}$ and of $A d S_{4}$ are not equal.

For the strongly coupled theory of $N$ M2-branes, the AdS/CFT correspondence predicts an interesting feature: the number of degrees of freedom scales like $N^{3 / 2}$. This feature has not been completely understood yet. In contrast for $N$ D3-branes the number of degrees of freedom scales like $N^{2}$ as expected from a gauge theory. The peculiar scaling $N^{3 / 2}$ can be understood in terms of a gauge theory where not all degrees of freedom are dynamical. Note that $N$ coincident M5-branes are even mysterious since the degrees of freedom scale like $N^{3}$.

\footnotetext{
${ }^{5}$ The Latin indices $M, N$ and $P$ label the eleven dimensions. Later we will perform a Kaluza-Klein reduction to ten dimensions. In ten dimensions we use greek indices $\mu, \nu, \ldots$.
} 


\subsubsection{Relation to type IIA supergravity}

A Kaluza-Klein reduction to $D=10$ is performed by putting the eleventh coordinate $X^{10}$ on a circle of radius $R_{10}$. Decomposing the fields with respect to the ten-dimensional Lorentz group, we can identify the field content of type IIA supergravity. The metric $G_{M N}$ and three-form potential $A_{(3)}$ of eleven-dimensional supergravity is related to the dilaton $\phi$, to the metric $g_{\mu \nu}$ and to the R-R-potentials $C_{(1)}$ and $C_{(3)}$ in the following schematic way, symbolized by $\sim$ :

$$
\begin{aligned}
G_{M N} \rightarrow & \phi \sim G_{1010}, \\
& C_{\mu} \sim G_{\mu 10}, \\
& g_{\mu \nu} \sim G_{\mu \nu}, \\
A_{M N P} \rightarrow & B_{\mu \nu} \sim A_{\mu \nu 10}, \\
& C_{\mu \nu \rho} \sim A_{\mu \nu \rho},
\end{aligned}
$$

where $\mu, \nu, \rho=0, \ldots, 9$. Moreover, the ten-dimensional fields are independent of the eleventh, internal coordinate $X_{10}$. The Planck length $l_{p}$ of M-theory is related to the string length $l_{s}=\sqrt{\alpha^{\prime}}$ and the string coupling constant $g_{s}$ by

$$
l_{p}=g_{s}^{1 / 3} l_{s} .
$$

Finally, the radius $R_{10}$ of the compactified direction reads

$$
R_{10}=g_{s}^{2 / 3} l_{p}=g_{s} l_{s} .
$$

For small string coupling constant $g_{s}$ the radius $R_{10}$ of the compactified direction in units of the string length is small and the spacetime is effectively ten-dimensional. For large string coupling constants the spacetime is eleven-dimensional.

However, the relation between type IIA supergravity and $M$ theory is much deeper. Let us match non-perturbative objects on both sides. In the non-perturbative part of its spectrum eleven-dimensional supergravity has M2- and M5-branes, which are are charged under the four-form field strength $G_{(4)}=d A_{(3)}$. The non-perturbative spectrum of type IIA supergravity contain stable Dp-branes with $p$ even as well as NS5-branes.

Let us consider some examples to see how D-branes and M-branes are related. The fundamental string and D2-branes can be related to M2-branes by dimensional reduction along the circle $X^{10}$ : In the case of the fundamental string the M2-brane is wrapped on the compactified coordinate $X^{10}$. Performing a dimensional reduction we obtain a fundamental string. If the M2-brane is not wrapped along the compactified dimension we get a D2-brane. In addition D4- and NS5-branes can be mapped to M5-branes of M theory. D0-branes correspond to Kaluza Klein particles. For further details see [210].

In recent years there has been a lot of progress in the understanding of the interactions of coincident M2-branes despite not having a fundamental perturbative description. In particular, we know the low-energy effective action for M2-branes, which is called the ABJM theory. In section 7.3 we will review the derivation of the low-energy effective action following [137]. 


\subsection{Review of ABJM Theory}

In this section we review the ABJM theory [137]. In particular, we discuss its field content, Lagrangian, and symmetries. We also review the type IIB (and type IIA) brane construction of the theory, and its large- $N$ supergravity dual.

\subsubsection{The Gauge Theory}

Let us begin by writing the Lagrangian and reviewing the symmetries of the ABJM theory. The theory is a $U\left(N_{c}\right) \times U\left(N_{c}\right)$ gauge theory with a Chern-Simons term for each gauge group factor. The two Chern-Simons terms have equal but opposite levels, $k$ and $-k$, which we denote by $U\left(N_{c}\right)_{k} \times U\left(N_{c}\right)_{-k}$.

The nicest way to write the Lagrangian is in $\mathcal{N}=2$ superspace. Our conventions are those of [211]. We use a mostly-plus Minkowski metric. The $(2+1)$-dimensional $\mathcal{N}=2$ supersymmetry algebra includes two Majorana spinors, which we will combine into a single complex spinor $\theta^{\alpha}$, and its complex conjugate $\bar{\theta}^{\alpha}$, with $\alpha=1,2$ the spinor index. The superspace covariant derivatives are then

$$
D_{\alpha}=\frac{\partial}{\partial \theta^{\alpha}}+i\left(\gamma^{\mu} \bar{\theta}\right)_{\alpha} \frac{\partial}{\partial x^{\mu}}, \quad \bar{D}_{\alpha}=-\frac{\partial}{\partial \bar{\theta}^{\alpha}}-i\left(\theta \gamma^{\mu}\right)_{\alpha} \frac{\partial}{\partial x^{\mu}},
$$

where $\gamma^{0}=i \sigma_{2}, \gamma^{1}=\sigma_{1}$, and $\gamma^{2}=\sigma_{3}$, with $\sigma_{1}, \sigma_{2}$, and $\sigma_{3}$ the usual Pauli matrices. A chiral superfield $\phi$ obeys $\bar{D}_{\alpha} \phi=0$.

The ABJM theory includes the following fields:

(i) Two $\mathcal{N}=2$ vector superfields $V_{i}$, one for each gauge group, hence $i=1,2$ labels the $U\left(N_{c}\right)$ factor. An $\mathcal{N}=2$ vector superfield includes a vector potential $A_{\mu}$, a real scalar field $\sigma$, two real (Majorana) gauginos, and an auxiliary real scalar field $D$, all in the adjoint representation of the gauge group.

(ii) Two $\mathcal{N}=2$ chiral superfields $\Phi_{i}$, each of which is in the adjoint representation. An $\mathcal{N}=2$ chiral superfield includes two real (Majorana) fermions, two real scalars, and a complex auxiliary scalar $F$.

(iii) Four $\mathcal{N}=2$ chiral superfields, $A_{1}, A_{2}, B_{1}$ and $B_{2}$, where $A_{1}$ and $A_{2}$ are in the bifundamental $\left(N_{c}, \overline{N_{c}}\right)$ representation and the $B_{1}$ and $B_{2}$ are in the anti-bifundamental $\left(\overline{N_{c}}, N_{c}\right)$ representation.

We will divide the action into three pieces,

$$
S_{\mathrm{ABJM}}=S_{\mathrm{CS}}+S_{\mathrm{bifund}}+S_{\mathrm{pot}},
$$

\footnotetext{
${ }^{6}$ We can obtain the $(2+1)$-dimensional $\mathcal{N}=2$ supersymmetry algebra via dimensional reduction of the (3+1)-dimensional $\mathcal{N}=1$ supersymmetry algebra. $\theta^{\alpha}$ is precisely the single complex spinor of the $(3+1)$-dimensional $\mathcal{N}=1$ supersymmetry algebra.
} 
where, in $\mathcal{N}=2$ superspace,

$$
\begin{aligned}
S_{\mathrm{CS}} & =-i \frac{k}{4 \pi} \int d^{3} x d^{4} \theta \int_{0}^{1} d t \operatorname{Tr}\left(V_{1} \bar{D}^{\alpha}\left(e^{t V_{1}} D_{\alpha} e^{-t V_{1}}\right)\right. \\
\left.-V_{2} \bar{D}^{\alpha}\left(e^{t V_{2}} D_{\alpha} e^{-t V_{2}}\right)\right), & \\
S_{\text {bifund }} & =-\int d^{3} x d^{4} \theta \operatorname{Tr}\left(\bar{A}_{a} e^{-V_{1}} A_{a} e^{V_{2}}+\bar{B}_{a} e^{-V_{2}} B_{a} e^{V_{1}}\right), \\
S_{\text {pot }} & =\int d^{3} x d^{2} \theta W+\text { c.c. },
\end{aligned}
$$

with the superpotential

$$
W=-\frac{k}{8 \pi} \operatorname{Tr}\left(\Phi_{1}^{2}-\Phi_{2}^{2}\right)+\operatorname{Tr}\left(B_{a} \Phi_{1} A_{a}\right)+\operatorname{Tr}\left(A_{a} \Phi_{2} B_{a}\right) .
$$

In $S_{\text {bifund }}$ and the superpotential, summation over $a=1,2$ is implicit. All traces are taken in the fundamental representation. Without the superpotential the action has $\mathcal{N}=2 \mathrm{su}-$ persymmetry. The chiral superfields $\Phi_{i}$ combine with the corresponding $V_{i}$ to form $\mathcal{N}=4$ vector multiplets, although the Chern-Simons terms only preserve $\mathcal{N}=3$ supersymmetry. The form of the superpotential is completely fixed by $\mathcal{N}=3$ supersymmetry (see for example [212]).

The fields $\Phi_{i}$ have no kinetic terms, hence at low energy they can be integrated out, yielding the superpotential

$$
W_{\mathrm{ABJM}}=\frac{2 \pi}{k} \varepsilon^{a b} \varepsilon^{\dot{a} \dot{b}} \operatorname{Tr}\left(A_{a} B_{\dot{a}} A_{b} B_{\dot{b}}\right),
$$

which clearly exhibits an $S U(2)$ symmetry acting on $A_{a}$ and a separate $S U(2)$ symmetry acting on $B_{\dot{a}}$. We denote this symmetry as $S U(2)_{A} \times S U(2)_{B}$. The $\mathcal{R}$-symmetry of the theory, $S O(3)_{R} \equiv S U(2)_{R}$, does not commute with the $S U(2)_{A} \times S U(2)_{B}$ : under the $S U(2)_{R}$ symmetry, $\left(A_{1}, B_{1}^{*}\right)$ and $\left(A_{2}, B_{2}^{*}\right)$ are each a doublet. We thus conclude that the full symmetry is $S U(4)$, under which $\left(A_{1}, A_{2}, B_{1}^{*}, B_{2}^{*}\right)$ transforms as a 4 . As argued in [137], the supercharges also transform under the $S U(4)$, hence the full $\mathcal{R}$-symmetry is $S U(4)_{R} \equiv S O(6)_{R}$, and hence the theory is in fact $\mathcal{N}=6$ supersymmetric.

We emphasize that at low energy the supersymmetry is enhanced, where by low energy we mean energies lower than the mass, $g_{Y M}^{2} k /(4 \pi)$ (here we use a normalization for the kinetic terms of the vector multiplet with a $1 / g_{Y M}^{2}$ in front), of the fields in the $\mathcal{N}=4$ vector multiplet. ${ }^{7}$ We will see this supersymmetry enhancement again shortly, in the brane construction of the theory.

The theory additionally has a $U(1)_{b}$ baryon number symmetry under which $A_{i} \rightarrow e^{i \alpha} A_{i}$ and $B_{i} \rightarrow e^{-i \alpha} B_{i}$. Remarkably, the theory also has a parity symmetry, which involves inverting one spatial coordinate (say $x^{1} \rightarrow-x^{1}$ ), exchanging the two gauge groups, and performing charge conjugation on all of the fields.

Finally, as shown in [137], the moduli space of the theory is $\mathbb{C}^{4} / \mathbb{Z}_{k}$, where the $\mathbb{Z}_{k}$ acts as $\left(A_{1}, A_{2}, B_{1}^{*}, B_{2}^{*}\right) \rightarrow e^{2 \pi i / k}\left(A_{1}, A_{2}, B_{1}^{*}, B_{2}^{*}\right)$, where here $A_{a}$ and $B_{a}$ denote only the scalar component of the corresponding superfields.

\footnotetext{
${ }^{7}$ When we integrate out the fermions in the vector multiplets, we may worry that the Chern-Simons level will change: the adjoint fermions have the same mass with the same sign within the $U\left(N_{c}\right)$ multiplet, but with the opposite sign of fermions in the other $U\left(N_{c}\right)$, so the Chern-Simons level should be shifted by $\pm N_{c}$. The massive gauge fields cancel that shift, however [213].
} 


\subsubsection{Type IIB Construction}

In this section we review the type IIB brane construction (of [137]) leading to the $\mathcal{N}=$ 6 Chern-Simons-matter theory with gauge group $U\left(N_{c}\right)_{k} \times U\left(N_{c}\right)_{-k}$ described above. Consider the following brane setup in type IIB string theory

\begin{tabular}{|c|cccccccccc|}
\hline & 0 & 1 & 2 & 3 & 4 & 5 & 6 & 7 & 8 & 9 \\
\hline NS5 & $\bullet$ & $\bullet$ & $\bullet$ & $\bullet$ & $\bullet$ & $\bullet$ & - & - & - & - \\
NS5 $^{\prime}$ & $\bullet$ & $\bullet$ & $\bullet$ & $\bullet$ & $\bullet$ & $\bullet$ & - & - & - & - \\
$N_{c}$ D3 & $\bullet$ & $\bullet$ & $\bullet$ & - & - & - & $\bullet$ & - & - & - \\
$k$ D5 & $\bullet$ & $\bullet$ & $\bullet$ & $\bullet$ & $\bullet$ & - & - & - & - & $\bullet$ \\
\hline
\end{tabular}

where the $x^{6}$ direction is a circle. The NS5- and NS5'-branes are separated in the $x^{6}$ direction. The $N_{c}$ D3-branes, which are extended in the $x^{6}$ direction, break on the NS5branes. The $k$ D5-branes and the NS5'-brane are coincident in $x^{6}$.

The D3-branes, together with the NS5- and NS5'-branes, give rise to an $\mathcal{N}=4$ supersymmetric (2+1)-dimensional $U\left(N_{c}\right) \times U\left(N_{c}\right)$ Yang-Mills theory [203]. The bosonic part of the $\mathcal{N}=4$ vector multiplet in each $U\left(N_{c}\right)$ gauge group consists of the $(2+1)$-dimensional components of the D3-brane worldvolume gauge field together with the three real scalars describing each D3-brane's position in the $\left(x^{3}, x^{4}, x^{5}\right) \equiv(345)$ directions. Recall from the last subsection that each $\mathcal{N}=4$ vector multiplet consists of an $\mathcal{N}=2$ vector multiplet $V_{i}$ and an $\mathcal{N}=2$ chiral multiplet $\Phi_{i}$. The real scalars are the two real scalars in $\Phi_{i}$ plus the real scalar $\sigma_{i}$ in $V_{i}$, which thus form a vector representation of $S O(3)_{R}$. Similarly, the auxiliary fields $D$ and $F$ form a vector of the $\mathcal{R}$-symmetry.

The theory also has (anti-)bifundamental $\mathcal{N}=2$ chiral multiplets, coming from strings stretched between the two stacks of D3-branes. These are the fields $A_{a}$ and $B_{a}$ of the last subsection, with $a=1,2$.

The $k$ D5-branes coincident with the NS5'-branes introduce massless D3/D5 strings, and break the supersymmetry to $\mathcal{N}=2$. The field theory thus has $k$ massless $\mathcal{N}=2$ chiral multiplets in the fundamental and $k$ massless $\mathcal{N}=2$ chiral multiplets in the anti-fundamental of each $U\left(N_{c}\right)$ factor.

What does any of this have to do with Chern-Simons theory? If we can give the fundamental and anti-fundamental fields the same mass, then via the parity anomaly these fields will produce Chern-Simons terms at low energy. More precisely, we need real masses of equal sign. As argued in [214] (see also [215]), the deformation that produces such masses is to bind the $k$ D5-branes to the NS5'-brane, producing a $(1, k) 5$-brane. To preserve $\mathcal{N}=2$ supersymmetry, the $(1, k) 5$-brane must be tilted at an angle $\theta$ in the (59) plane, which we denote by $[5,9]_{\theta}$. The angle $\theta$ depends on the complex axion-dilaton $\tau=\frac{i}{g_{s}}+\chi$ as $\theta=\arg (\tau)-\arg (k+\tau)$. In what follows, we will always set $\tau=i$. Such a deformation actually gives the fundamental and anti-fundamental fields infinite mass. Integrating out these fields then produces Chern-Simons terms with levels $k$ and $-k$ for the two $U\left(N_{c}\right)$ gauge groups. Moreover, we can enhance the supersymmetry to $\mathcal{N}=3$ if we additionally rotate the $(1, k) 5$-brane by the same angle $\theta$ in the (37) and (48) planes. We thus arrive at the brane construction 


\begin{tabular}{|c|cccccccccc|}
\hline & 0 & 1 & 2 & 3 & 4 & 5 & 6 & 7 & 8 & 9 \\
\hline $\mathrm{NS} 5$ & $\bullet$ & $\bullet$ & $\bullet$ & $\bullet$ & $\bullet$ & $\bullet$ & - & - & - & - \\
$(1, k) 5$ & $\bullet$ & $\bullet$ & $\bullet$ & {$[3,7]_{\theta}$} & {$[4,8]_{\theta}$} & {$[5,9]_{\theta}$} & - & - & - & - \\
$N_{c}$ D 3 & $\bullet$ & $\bullet$ & $\bullet$ & - & - & - & $\bullet$ & - & - & - \\
\hline
\end{tabular}

We will henceforth refer to the final brane configuration above as the type IIB setup of the ABJM theory. The field theory associated with this setup is an $\mathcal{N}=3 U\left(N_{c}\right)_{k} \times$ $U\left(N_{c}\right)_{-k}$ Yang-Mills theory with Chern-Simons terms and four massless bifundamental matter multiplets $\left(A_{a}, B_{b}\right)$. We saw above that this theory flows in the infrared (meaning energies below $\left.g_{Y M}^{2} k /(4 \pi)\right)$ to the $\mathcal{N}=6$ superconformal $U\left(N_{c}\right)_{k} \times U\left(N_{c}\right)_{-k}$ Chern-Simons theory with the same bifundamental matter content. The easiest way to see that happen in the brane setup is to T-dualize and then lift to M-theory.

\subsubsection{Type IIA and M-theory Descriptions}

If we perform a T-duality along $x^{6}$ then the type IIB brane setup above turns into the following type IIA configuration: the $N_{c}$ D3-branes become $N_{c}$ D2-branes in the (012) directions. The NS5-brane along (012345) becomes a KK monopole associated with the $x^{6}$ circle. The $(1, k) 5$-brane becomes a KK monopole in the $(0123)[3,7]_{\theta}[4,8]_{\theta}[5,9]_{\theta}$ directions associated with the $x^{6}$ circle. Normally $k$ D5-branes would appear as $k$ D6-branes in type IIA string theory. Here the $k$ D5-branes bound into the $(1, k) 5$-brane appear as D6-brane flux on the KK monopole. The configuration in type IIA string theory is thus

\begin{tabular}{|c|cccccccccc|}
\hline & 0 & 1 & 2 & 3 & 4 & 5 & 6 & 7 & 8 & 9 \\
\hline$N_{c}$ D2 & $\bullet$ & $\bullet$ & $\bullet$ & - & - & - & - & - & - & - \\
KK monopole & $\bullet$ & $\bullet$ & $\bullet$ & $\bullet$ & $\bullet$ & $\bullet$ & - & - & - & - \\
KK monopole with D6-brane flux & $\bullet$ & $\bullet$ & $\bullet$ & {$[3,7]_{\theta}$} & {$[4,8]_{\theta}$} & {$[5,9]_{\theta}$} & - & - & - & - \\
\hline
\end{tabular}

We can now lift the configuration to M-theory, introducing a second circle direction, which we will denote $x^{\sharp}$. The D2-branes become M2-branes, whereas the KK monopole associated with the $x^{6}$ circle remains unchanged. Normally a D6-brane would lift to a KK monopole associated with the $x^{\sharp}$ circle, hence the KK monopole with D6-brane flux becomes a KK monopole associated with a circle on the $(6, \sharp)$ torus. Notice that the two 5 -branes in the type IIB picture, i.e. the NS5-brane and the $(1, k) 5$-brane, lift to pure geometry in M-theory.

The spacetime is now $\mathbb{R}^{1,2} \times X_{8}$, where the M2-branes are extended along $\mathbb{R}^{1,2}$ and $X_{8}$ is the spacetime generated by the KK monopoles. The space $X_{8}$ preserves $3 / 16$ of the 32 supersymmetries of M-theory. (Adding the M2-branes (with the right orientation) does not break any additional supersymmetries.) We thus expect the M2-branes' worldvolume theory to have $\mathcal{N}=3$ supersymmetry.

The enhancement of supersymmetry that we saw in the field theory occurs when we take a "near-horizon" limit, which we define as follows. At the intersection point of the two KK monopoles, the singularity of the space $X_{8}$ is locally $\mathbb{C}^{4} / \mathbb{Z}_{k}$. Denoting the complex coordinates of $\mathbb{C}^{4}$ by $z^{i}$, the action of the $\mathbb{Z}_{k}$ is $z^{j} \rightarrow e^{2 \pi i / k} z^{j}$. The "near-horizon" limit means retaining only the $\mathbb{C}^{4} / \mathbb{Z}_{k}$ singularity of the full $X_{8}$ space. We will often refer to this as zooming in on the singularity. $\mathbb{C}^{4} / \mathbb{Z}_{k}$ preserves 12 supersymmetries, or $3 / 8$ of the 
32 supersymmetries of M-theory. We write the metric of $X_{8}$, and take the "near-horizon" limit, explicitly in appendix B.2.

Note that 12 real supercharges is of course the correct amount for a $(2+1)$-dimensional $\mathcal{N}=6$ supersymmetric theory. Recall also that the moduli space of the $\mathcal{N}=6$ ChernSimons-matter theory is precisely $\mathbb{C}^{4} / \mathbb{Z}_{k}$. Furthermore, $\mathbb{C}^{4} \cong \mathbb{R}^{8}$ has an $S O(8)$ isometry, of which only $S U(4) \times U(1)$ remains after the $\mathbb{Z}_{k}$ orbifold. These symmetries match the $S U(4)_{R} \times U(1)_{b}$ symmetry of the $\mathcal{N}=6$ Chern-Simons theory. The central conclusion of [137] was therefore that the $\mathcal{N}=6$ superconformal $U\left(N_{c}\right)_{k} \times U\left(N_{c}\right)_{-k}$ Chern-Simons matter theory of section 7.3 .1 describes the low-energy dynamics of $N_{c}$ coincident M2branes at the $\mathbb{C}^{4} / \mathbb{Z}_{k}$ singularity.

Recalling that, in the field theory, the $\mathbb{Z}_{k}$ acts on the bifundamentals as

$$
\left(A_{1}, A_{2}, B_{1}^{*}, B_{2}^{*}\right) \rightarrow e^{2 \pi i / k}\left(A_{1}, A_{2}, B_{1}^{*}, B_{2}^{*}\right),
$$

and also that they transform as a 4 of $S U(4)_{R}$, we can (roughly) identify $\left(z^{1}, z^{2}, z^{3}, z^{4}\right)$ with $\left(A_{1}, A_{2}, B_{1}^{*}, B_{2}^{*}\right)$, where here $A_{a}$ and $B_{a}$ represent the bosonic components of the corresponding superfields. The $U(1)_{b}$ symmetry of the field theory thus appears as a phase shift $z^{i} \rightarrow e^{i \alpha} z^{i}$ (which is equivalent to shifts in the $x^{\sharp}$ circle, as we show in appendix B.2).

\subsubsection{The Dual Gravity Theory}

Consider $N_{c}$ M2-branes at the $\mathbb{C}^{4} / \mathbb{Z}_{k}$ singularity. If we take $N_{c} \rightarrow \infty$, we can replace the M2-branes with their near-horizon geometry, $A d S_{4} \times S^{7} / \mathbb{Z}_{k}$. The natural conjecture then is that eleven-dimensional supergravity on $A d S_{4} \times S^{7} / \mathbb{Z}_{k}$ is holographically dual to the $\mathcal{N}=6$ supersymmetric $U\left(N_{c}\right)_{k} \times U\left(N_{c}\right)_{-k}$ Chern-Simons-matter theory at large $N_{c}$. The $A d S_{4}$ radius of curvature $L$ is related to the 't Hooft coupling $\lambda=N_{c} / k$ and the Chern-Simons level $k$ as (with $\ell_{p}$ the eleven-dimensional Planck length),

$$
\frac{L^{3}}{\ell_{p}^{3}}=4 \pi \sqrt{2 k N_{c}}=4 \pi k \sqrt{2 \lambda}
$$

We can thus trust the M-theory description in the strong 't Hooft coupling limit $\lambda \rightarrow \infty$. If we write the $S^{7}$ as a circle fibration over $\mathbb{C P}^{3}$, then the $\mathbb{Z}_{k}$ orbifold acts on the fiber direction. The radius of the circle in Planck units is on the order of $L / k \ell_{p} \propto\left(N_{c} k\right)^{1 / 6} / k$, so we can only trust the solution when $N_{c} \gg k^{5}$. In short, when $N_{c} \rightarrow \infty$ such that $N_{c} \gg k^{5}$ (which implies $\lambda=N_{c} / k \rightarrow \infty$ ), the $\mathcal{N}=6$ supersymmetric $U\left(N_{c}\right)_{k} \times U\left(N_{c}\right)_{-k}$ Chern-Simons-matter theory is dual holographically to eleven-dimensional supergravity on $A d S_{4} \times S^{7} / \mathbb{Z}_{k}$.

When $k^{5} \gg N_{c} \gg k$, where again $\lambda \rightarrow \infty$, the circle becomes small and the appropriate description is in terms of type IIA supergravity on the spacetime $A d S_{4} \times \mathbb{C P}^{3}$.

\subsection{General Analysis of Probe Flavor}

In this section we discuss how to add flavor to the ABJM theory in general terms. We first discuss the gravity analysis and then the field theory analysis. More specifically, we 
explain in this section exactly what we compute on the gravity side and what we want to compute on the field theory side.

Our general approach is to add flavor branes in the type IIB setup and follow what happens to them, in both the gravity and field theory descriptions, in the construction of the ABJM theory (T-duality, lift to M-theory, etc.). Why start with type IIB? The main reason is that the brane description in type IIB provides an easy starting point for constructing the field theory.

\subsubsection{Gravity Analysis}

On the gravity side, we introduce flavor branes in the type IIB setup. To limit our search for supersymmetric probe branes, we impose four constraints. First, we consider only D1-, D3-, D5- and D7-branes. D(-1)-branes do not introduce flavor degrees of freedom on the D3-brane worldvolume, and D9-branes are unstable without orientifold planes, so we will not consider these cases. Second, we do not separate any probes from the D3-branes in overall transverse directions. Third, when we consider multiple probes, i.e. $N_{f}>1$, we do not separate them from each other, so that they retain a $U\left(N_{f}\right)$ symmetry. Fourth, we consider only probes aligned along the coordinate axes. More generally the probe brane could be at an angle with respect to these axes. We studied a few special cases of probes at angles (see appendix B.1) and found that all such probes appeared to preserve as much as, or less, supersymmetry, as the probes listed below, i.e. they never exhibit enhanced supersymmetry.

The counting of supercharges left unbroken by our probes in this background is a straightforward exercise, the details of which appear in appendix B.1. The main result of appendix B.1 is table 7.1, which appears below. Table 7.1 lists the flavor Dp-branes we study, exactly where they are located in the type IIB setup, and the number of real supercharges each Dp-brane preserves. Although for specific calculations we focused on the Dp-branes listed in table 7.1, most of our comments in this section will be applicable more generally.

A very important fact (mentioned in appendix B.1) is that when $k=0$, such that the type IIB setup includes just NS5-branes and no $(1, k) 5$-brane, all of our flavor branes preserve 4 real supercharges, except for two cases that preserve 8 supercharges. The first case preserving 8 supercharges is D3-branes along (0126), which are of course coincident with the D3-branes whose low-energy dynamics we are interested in. The second case preserving 8 supercharges is D5-branes along (012789), which were first studied in [203]. For all cases, table 7.1 indicates the number of supercharges that remain unbroken after forming the $(1, k) 5$-brane.

The first column of table 7.1 lists the type of brane in the type IIB construction while the second column lists the resulting type IIA description, obtained by T-dualizing in $x^{6}$, and the third column lists the M-theory description, obtained by lifting to eleven dimensions. A type IIB D-brane that becomes a D6-brane in type IIA will lift to a KK monopole associated with the M-theory circle, which we have indicated with $K K$. The fourth column lists the codimension of the defect to which the flavor fields will be confined in the (2+1)-dimensional Chern-Simons-matter theory. The fifth column indicates the directions in which the probe brane is extended in the IIB construction. The $S O(3)$ symmetry that acts simultaneously on the directions (345) and (789) gives rise to other 
supersymmetric branes, related to the ones in the table by $S O(3)$ transformations. We have indicated this by (). For example, the first brane could extend along (07), (08) or (09). The last two columns of the table indicate the number of real supercharges preserved by the probe brane or anti-brane. Recall that for codimension-zero branes the number of preserved supercharges must be even, but for higher codimension the brane may preserve an odd number of real supercharges.

\begin{tabular}{|c|c|c|c|c|c|c|}
\hline Type IIB & Type IIA & M theory & codim & wrapping & SUSY & SUSY (anti) \\
\hline \hline D1 & D2 & M2 & 2 & $0(7)$ & 2 & 2 \\
\hline D3 & D2 & M2 & 0 & 0126 & 6 & 0 \\
\hline D3 & D4 & M5 & 1 & $01(37)$ & 3 & 3 \\
\hline D3 & D4 & M5 & 1 & $01(38)$ & 2 & 2 \\
\hline D3 & D2 & M2 & 2 & $0(34) 6$ & 2 & 2 \\
\hline D3 & D2 & M2 & 2 & $06(78)$ & 2 & 2 \\
\hline D5 & D6 & KK & 0 & $012(347)$ & 2 & 2 \\
\hline D5 & D6 & KK & 0 & $012(349)$ & 4 & 2 \\
\hline D5 & D6 & KK & 0 & 012789 & 6 & 0 \\
\hline D5 & D4 & M5 & 1 & 013456 & 3 & 3 \\
\hline D5 & D4 & M5 & 1 & $01(378) 6$ & 2 & 2 \\
\hline D5 & D4 & M5 & 1 & $01(389) 6$ & 3 & 3 \\
\hline D5 & D6 & KK & 2 & $0(34) 789$ & 2 & 2 \\
\hline D7 & D6 & KK & 0 & $0126(3478)$ & 2 & 4 \\
\hline D7 & D6 & KK & 0 & $0126(3479)$ & 2 & 2 \\
\hline D7 & D8 & M9 & 1 & 01345789 & 3 & 3 \\
\hline
\end{tabular}

Table 7.1: List of D-branes (extended along the coordinate axes) that we can add to the type IIB construction while still preserving some supersymmetry.

As reviewed in section 7.3.2, to go from the type IIB setup to M2-branes on $\mathbb{C}^{4} / \mathbb{Z}_{k}$, we T-dualize in $x^{6}$, lift to M-theory, and take the "near-horizon" limit. We can easily determine what type of object the flavor Dp-branes become in M-theory: we obtain M2-, M5-, and M9-branes or KK monopoles. More difficult to determine is the exact position of the object on $\mathbb{C}^{4} / \mathbb{Z}_{k}$. To find that, we take the straightforward approach. We compute explicitly the coordinate transformations from the type IIB coordinates to the coordinates $\left(z^{1}, z^{2}, z^{3}, z^{4}\right)$ of $\mathbb{C}^{4} / \mathbb{Z}_{k}$. We present the details of the computation in appendix B.2. Given the embedding of a Dp-brane in type IIB, we can then immediately write the embedding of the corresponding object in M-theory on $\mathbb{C}^{4} / \mathbb{Z}_{k}$.

Once we know the location of the M-brane or KK monopole in $\mathbb{C}^{4} / \mathbb{Z}_{k}$, we can compute the amount of supersymmetry and the isometries that the object preserves. The details of those calculations, for a subset of our examples, appear in appendix B.3.1. Our results are summarized in table B.2 in appendix B.3.1. The locations of our objects on $\mathbb{C}^{4} / \mathbb{Z}_{k}$ are more complicated to explain, however, so we will not reproduce table B.2 here. We also studied a few examples of M-branes or KK monopoles in the near-horizon geometry of very many M2-branes, $A d S_{4} \times S^{7} / \mathbb{Z}_{k}$. The details of those calculations appear in appendix B.3.2. Knowing what symmetries the object preserves is, of course, extremely helpful when constructing the dual field theory.

In our analysis of objects on $\mathbb{C}^{4} / \mathbb{Z}_{k}$, we make use of a helpful tool, originally used in [206], 
which we call $S U(4)$-equivalence. The basic idea is that two different Dp-branes in type IIB can become the same object in M-theory on $\mathbb{C}^{4} / \mathbb{Z}_{k}$. More specifically, two Dp-branes of different dimensionality and/or located in different places in the type IIB setup (and hence possibly preserving different symmetries) can actually become the same object in M-theory. At work here is the "near-horizon" limit, which erases many of the details of the type IIB embedding 8 To be still more precise, the two Dp-branes can become the same type of object, two M5-branes for example, but located in two different places, i.e. with two different embeddings into $\mathbb{C}^{4} / \mathbb{Z}_{k}$. If we can rotate one object into the other via an $S U(4)$ isometry, however, then the two objects are physically equivalent. We may thus work with either one, and any physical results will be valid for both. On a technical level, some things may be easier to calculate for one embedding than for the other, for example the calculation of the number of preserved supercharges. We will present an explicit example of $S U(4)$ equivalence, and discuss its field theory meaning, in section 7.7 .

\subsubsection{Field Theory Analysis}

Eleven-dimensional supergravity on $A d S_{4} \times S^{7} / \mathbb{Z}_{k}$ is dual to the ABJM theory with ChernSimons level $k$, and $N_{c}$ large (such that $N_{c} \gg k^{5}$ ). What is the dual field theory when we add one of our flavor M-branes or KK monopoles, however? If the object preserves a large amount of symmetry, then that symmetry may be enough to determine the form of the field theory action. That will not always be the case, of course, so we want a more general method to determine the field theory. We will now describe a general recipe, one that is actually very straightforward and, in principle at least, is guaranteed to give the correct field theory for any flavor Dp-brane in the type IIB setup. Our recipe actually begins a few steps before the type IIB setup. We begin with D3-branes alone (so no NS5or $(1, k) 5$-branes) and flavor Dp-branes, which we considered in section 2.5. The recipe then consists of four steps, as follows.

\section{Step 1: Construct the D3/Dp Theory}

In type IIB consider D3-branes alone in flat space, so let $x^{6}$ be non-compact and remove the NS5- and $(1, k) 5$-branes. We then add supersymmetric flavor Dp-branes. In general, we next need to determine the low-energy theory living on the D3-branes, including the couplings to the (defect) flavor fields. We will generically call that theory the $D 3 / D p$ theory. Fortunately, for many examples the D3/Dp theory is already known. In fact we discussed the field theory for various D3/Dp systems in section 2.5.1. Moreover we presented in table 2.3 a complete list of D3/Dp systems for which the field theories have been written explicitly in the published literature.

Every Dp-brane in table 7.1 is described by one of the theories mentioned in table 2.2 , except for the D3/D1 system. Recall that if the D3/Dp intersection has 4 NeumannDirichlet (ND) directions then the corresponding flavor fields (from 3-p and p-3 strings) will produce non-chiral flavor, simply because the fields are arranged in hypermultiplets [62], whereas with 8 ND directions we can obtain chiral flavor, as occurs for the 8 ND D3/D7 intersection [96-98].

\footnotetext{
${ }^{8}$ That of course was an essential feature in the brane construction of the ABJM theory: the erasure produced the (super)symmetry enhancement.
} 


\section{Step 2: Add the NS5-branes}

Now we ask what happens when we construct the ABJM theory from the D3-branes. First we introduce the NS5- and NS5'-branes along (012345) and separated in $x^{6}$ (which for now is still non-compact), and let the D3-branes end on them in $x^{6}$. From a field theory point of view, adding the NS5-branes has two effects. The first effect is that on the D3-brane worldvolume the $x^{6}$ direction is now finite in extent, so the low-energy effective theory on the D3-brane worldvolume will be a $(2+1)$-dimensional field theory. In other words, we must perform a dimensional reduction in the $x^{6}$ direction. The $(3+1)$-dimensional $\mathcal{N}=4$ multiplet decomposes into two $(2+1)$-dimensional $\mathcal{N}=4$ multiplets, a vector multiplet and a hypermultiplet. The second effect of the NS5-branes is to impose boundary conditions that kill (i.e. set to zero) the adjoint $(2+1)$-dimensional $\mathcal{N}=4$ hypermultiplet $[203,204]$. We will call these the NS5-brane boundary conditions. We must thus take the D3/Dp action we wrote in Step 1 and perform a dimensional reduction in $x^{6}$ and then determine what couplings remain after we impose the NS5-brane boundary conditions.

For this procedure, a crucial distinction is whether the flavor Dp-brane is extended in $x^{6}$ or not. If not, then we need only dimensionally reduce and impose boundary conditions on the fields of the $(3+1)$-dimensional $\mathcal{N}=4$ supersymmetric Yang-Mills theory. If the flavor Dp-brane is extended in $x^{6}$, then we must also perform a dimensional reduction and impose boundary conditions on the flavor fields. In this paper we study examples in which we can avoid doing these operations explicitly.

We will also mention an alternative, but entirely equivalent, way to perform Step 2, namely to perform two T-dualities, one along $x^{6}$ and another along one of the directions 3,4 and 5 (along the NS5-branes but transverse to the D3-branes). Strictly speaking, here we must assume that $x^{6}$ is compact, and that we have two stacks of D3-branes, giving rise to two $U\left(N_{c}\right)$ gauge groups, as in the type IIB construction of the ABJM theory. The NS5-branes ultimately become the orbifold space $\mathbb{C}^{2} / \mathbb{Z}_{2} \times \mathbb{C}$, the D3-branes become D3branes located at the orbifold singularity, and the flavor branes become some Dq-branes (with $q=p, p+2$, or $p-2$ ), which may be wrapping some part of $\mathbb{C}^{2} / \mathbb{Z}_{2} \times \mathbb{C}[216-218]$. We can then use well-known machinery for studying D-branes on orbifolds (see [216-219] and references therein) to determine the field theory.

\section{Step 3: Compactify $x^{6}$, form the $(1, k) 5$-brane, and lift to M-theory}

Now we compactify $x^{6}$ and add another stack of D3-branes, so that the gauge group of the D3-branes' worldvolumes is $U\left(N_{c}\right) \times U\left(N_{c}\right)$. If the flavor Dp-brane is localized in $x^{6}$, then in this paper we will always introduce two stacks of flavor Dp-branes, each with $N_{f}$ Dp-branes, located at opposite sides of the $x^{6}$ circle, away from the NS5-branes. We will thus obtain open strings stretched from each stack of Dp-branes to the corresponding stack of D3-branes, and hence we obtain massless fields in the fundamental representation of each gauge group factor. We then introduce the $k$ D5-branes along (012349), bind them to the NS5'-brane to form a $(1, k) 5$-brane, and then rotate the $(1, k) 5$-brane. None of these operations affect the form of the action in our flavor sector: they correspond to adding additional flavor fields, which then acquire mass terms and are integrated out, producing the Chern-Simons terms. The action in our flavor sector, i.e. the coupling to adjoint fields, coming from $3-\mathrm{p}$ and p-3 strings, is unchanged 9 We then T-dualize to type IIA and lift to M-theory. The action in the flavor sector is unchanged in those two steps. In

\footnotetext{
${ }^{9}$ We can make a more direct argument for why these operations do not affect our flavor action, for flavor
} 
particular, notice that the symmetries will be unchanged. We thus arrive in M-theory on the manifold $X_{8}$ mentioned in section 7.3 .3 .

\section{Step 4: Take the low-energy limit}

In the supergravity description, the last step is to zoom in on the $\mathbb{C}^{4} / \mathbb{Z}_{k}$ singularity of $X_{8}$, which appears in the field theory description as a low-energy limit. More precisely, we are doing effective field theory: we want to write a theory valid on scales below the mass of the $\mathcal{N}=4$ vector multiplet, $g_{Y M}^{2} k /(4 \pi)$. Following the rules of effective field theory, in the low-energy action we must write all terms consistent with the symmetries, which in particular means supersymmetry and $\mathcal{R}$-symmetry. If we can determine the coefficients of these terms (using for example supersymmetry), then the action we obtain is the correct action of the theory. Furthermore, as in the ABJM theory, to determine whether a (super)symmetry enhancement occurred, a helpful step is to integrate out the fields $\Phi_{i}$. We emphasize that integrating out the $\Phi_{i}$ does not change the theory, however. The equations of motion for the $\Phi_{i}$ 's are simply algebraic constraints: the theory already has whatever symmetry it has before we formally integrate out the $\Phi_{i}$ 's.

Our recipe has advantages and disadvantages. Let us first consider the advantages. One advantage is the fact that, in principle at least, our recipe is guaranteed to produce the correct field theory. Another advantage is the fact that the input for our recipe is a known D3/Dp theory, that is, our recipe is a kind of machine that takes a known D3/Dp theory and outputs the field theory for flavor fields coupled to the ABJM theory. Notice also that the action we obtain in the flavor sector will generally be valid for all values of $N_{c}$ and $k$, although we will primarily be interested in the limits where gauge-gravity duality is under best control (such as $N_{c} \gg k^{5}$ ).

Now let us consider some disadvantages. Although in principle our recipe is guaranteed to work, in practice some of the steps can be difficult. Indeed, having studied many of the Dp-branes listed in table 7.1, we can say from experience that Steps 2 and 4 often present technical challenges, especially in cases where the flavor fields are confined to a defect.

In Step 2 for example, for defect flavor fields, naïvely imposing the NS5-brane boundary conditions often leaves us with field content that does not easily fit into simple representations of the defect's supersymmetry group. (We know what supersymmetry the system should have from the gravity analysis.) In such cases, a more rigorous analysis of supersymmetry-preserving boundary conditions, along the lines of [204], may be required. Such an example is presented in section 6 of [6].

As for Step 4, several special issues arise. Step 4 often requires careful analysis of supersymmetric non-renormalization theorems. In the ABJM construction (without flavor), we begin with an $\mathcal{N}=3$ supersymmetric Yang-Mills-Chern-Simons-matter theory. An

\footnotetext{
Dp-branes not extended along $x^{6}$. We can start with the D3/Dp intersection and immediately add an NS5brane and the $(1, k) 5$-brane. Once again, we first do a dimensional reduction to $(2+1)$ dimensions. We then impose a boundary condition for the NS5-brane and a separate boundary condition for the $(1, k) 5$-brane. Together these set to zero the $\mathcal{N}=4$ hypermultiplet and introduce a Chern-Simons term [203,204,215]. In these operations, the only changes in the flavor sector are the same that occur with just NS5-branes: some couplings are eliminated when the boundary conditions set adjoint fields to zero. Otherwise the action in the flavor sector does not change. For flavor Dp-branes extended along $x^{6}$, more work may be required to determine the effect of the $(1, k) 5$-brane boundary condition on the flavor fields, along the lines of [204].
} 
important feature of $\mathcal{N}=3$ supersymmetry is that the action is fully determined by the symmetry [212]. That means that the low-energy limit consists only of discarding the kinetic terms for the $\mathcal{N}=4$ vector multiplet (while leaving the Chern-Simons terms). The action cannot change otherwise, for example the superpotential cannot acquire new terms, and the Kähler potential cannot be renormalized. When we add defect flavor, however, the Lorentz symmetry of the ABJM theory is broken to the subgroup that leaves the defect invariant, and the amount of supersymmetry is also reduced. In such cases a prerequisite for Step 4 is to re-examine non-renormalization theorems for defect theories. For the defect field theories corresponding to the D3/D5 and D3/D3 intersections ((3+1)-dimensional $\mathcal{N}=4 \mathrm{SYM}$ with defect flavor), proofs of non-renormalization appear in [95, 100]. Our examples in sections 7.5 and 7.6 have enough symmetry to avoid this issue.

Step 4 also involves integrating out the fields $\Phi_{i}$. For codimension-zero flavor fields that is usually straightforward. If our flavor fields are codimension one or two, however, this procedure is more difficult. In particular, we would need to decompose the $(2+1)$-dimensional fields of the ABJM theory into lower-dimensional multiplets, and then integrate out the lower-dimensional fields corresponding to the $\Phi_{i}$.

A useful strategy for Step 4 is to work backwards, that is, to use symmetries of the gravity description to guess the final result. In other words, given the symmetries on the gravity side, we can write all possible terms consistent with those symmetries in the field theory. In cases where a symmetry enhancement occurs, we must demonstrate that these are all the terms allowed by the original symmetry, so that we retroactively justify the result.

Lastly, let us explain the field theory meaning of $S U(4)$ equivalence. On the gravity side, $S U(4)$ equivalence was the statement that two Dp-branes in the type IIB setup, which may be located in different places or even have different dimensionality, but which must have the same codimension in $(2+1)$ dimensions, become the same type of object in Mtheory on $\mathbb{C}^{4} / \mathbb{Z}_{k}$, where the embeddings of the the two objects are related by an $S U(4)$ isometry transformation. In the field theory what is happening is simply that two different theories, with different symmetries for example, including possibly different amounts of supersymmetry, flow to the same low-energy fixed point in Step 4. We will discuss that further, and present an explicit example, in section 7.7.

To illustrate various features of our recipe, we now turn to several examples.

\subsection{Codimension Zero $\mathcal{N}=3$ Supersymmetric Flavor}

In this section we study codimension-zero $\mathcal{N}=3$ supersymmetric flavor fields, which have already been studied in $[206,220,221]$. For us this section serves as a particularly nice illustration of our general recipe. Compared to other examples, however, this example lacks many interesting features. For example, no supersymmetry enhancement occurs in the "near-horizon" limit, as we will review.

\subsubsection{Supergravity with KK-monopoles/D6-branes}

To obtain codimension-zero $\mathcal{N}=3$ supersymmetric flavor, we follow [206,220,221], and add D5-branes extended along (012789) in the type IIB setup. We are free to choose their 
position on the $x^{6}$ circle. We will add two stacks of D5-branes, each with $N_{f}$ coincident D5-branes, on opposite sides of the circle, away from the NS5-brane and $(1, k) 5$-brane. The strings from the D5-branes to the two stacks of D3-branes thus introduce massless flavor in both gauge groups. As shown in table 7.1 in section 7.4.1, these D5-branes preserve 6 real supercharges in the type IIB setup.

After T-duality in $x^{6}$ the D5-branes become D6-branes. The $2 N_{f}$ D6-branes are coincident, and have a $U\left(2 N_{f}\right)$ symmetry broken to $U\left(N_{f}\right) \times U\left(N_{f}\right)$ by a $\mathbb{Z}_{2}$-valued Wilson line, as explained in [206]. (The Wilson line simply tells us where the D5-branes were in type IIB.) After uplift to M-theory and the "near-horizon" limit, the D6-branes become KK monopoles associated with the $x^{\sharp}$ circle in M-theory on $\mathbb{C}^{4} / \mathbb{Z}_{k}$. The authors of [206] argue that the embedding of the KK monopole is described by the equations $z^{1}=\bar{z}^{3}, z^{2}=\bar{z}^{4}$ in $\mathbb{C}^{4} / \mathbb{Z}_{k}$. The authors of [206] then showed that, by using the $S U(4)$ symmetry of $\mathbb{C}^{4} / \mathbb{Z}_{k}$, we can map this embedding to $\operatorname{Im}\left(z^{i}\right)=0$ for $i \in\{1,2,3,4\}$. In other words, the two embeddings are $S U(4)$ equivalent. The symmetries preserved by the KK monopoles are easier to see in the latter embedding, however. In the latter embedding, the KK monopoles are extended along (012) and along $\operatorname{Re}\left(z^{i}\right)$ for $i \in\{1,2,3,4\}$.

The circle direction associated with the KK monopoles corresponds to the $U(1)_{b}$ symmetry of the background, so the KK monopoles preserve this symmetry. The KK monopoles break the $S U(4)$ symmetry to an $S O(4)$ under which $\left(z^{1}, z^{2}, z^{3}, z^{4}\right)$ transforms as a 4. The total symmetry group that the KK monopoles preserve is $S O(4) \times U(1)_{b}=S U(2) \times$ $S U(2) \times U(1)_{b}[206,220,221]$. In appendix B.3.1 we find that the KK monopoles preserve 6 real supercharges.

If we take $N_{c} \rightarrow \infty$, we can replace the M2-branes by their near-horizon geometry, which is $A d S_{4} \times S^{7} / \mathbb{Z}_{k}$. The KK monopoles are extended along $A d S_{4}$ and wrap a three cycle in $S^{7} / \mathbb{Z}_{k}$. In appendix B.3.2 we analyze the $\kappa$-symmetry condition for these monopoles and find that after the near-horizon limit the number of preserved supercharges has doubled to 12 .

For large $k$, such that $k^{5} \gg N_{c}$ and the appropriate description is type IIA on $A d S_{4} \times \mathbb{C P}^{3}$, the monopoles become D6-branes wrapping $A d S_{4} \times \mathbb{R P}^{3}[206,220,221]$.

To summarize: as explained in $[206,220,221]$, in the type IIB setup we can add D5branes that produce fundamental matter for both gauge group factors. These become KK monopoles in M-theory on $\mathbb{C}^{4} / \mathbb{Z}_{k}$. These $\mathrm{KK}$ monopoles preserve 6 real supercharges, so we expect a dual field theory with $\mathcal{N}=3$ superconformal symmetry. The corresponding $\mathcal{R}$-symmetry group has to be $S O(3)$, which fits into the symmetry found above. We now proceed to review the dual field theories constructed in [206, 220,221], and check that it has the right symmetries and amount of supersymmetry.

\subsubsection{The Field Theory}

We will first review the theory described in [206,220,221], and then re-derive it using our recipe.

In the type IIB setup we introduce two stacks of $N_{f}$ coincident D5-branes along (012789) on opposite sides of the $x^{6}$ circle. These D5-branes preserve the $\mathcal{N}=3$ supersymmetry of the type IIB setup (see appendix B.1). The strings stretched between each stack of 
D3-branes and each stack of D5-branes will produce $\mathcal{N}=2$ chiral superfields transforming in the $U\left(N_{f}\right)$ and $U\left(N_{c}\right)$ representations $\left(\bar{N}_{f}, N_{c}\right)$ and $\left(N_{f}, \bar{N}_{c}\right)$ of each $U\left(N_{c}\right)$. We will denote these as $Q_{i}$ and $\tilde{Q}_{i}$, respectively, where again $i$ labels the gauge group, $i=1,2$. The field $Q_{1}$, for example, transforms in the $\bar{N}_{f}$ representation of $U\left(N_{f}\right)$ and the $N_{c}$ representation of the first $(i=1) U\left(N_{c}\right)$ gauge group, while $\tilde{Q}_{1}$ transforms in the conjugate representations, $N_{f}$ and $\bar{N}_{c}$. For notational simplicity, we will suppress flavor indices.

$\mathcal{N}=3$ supersymmetry completely determines the action [212]. The kinetic terms of the flavor fields are

$$
S_{\text {fund }}=-\int d^{3} x d^{4} \theta\left(\bar{Q}_{i} e^{-V_{i}} Q_{i}+\tilde{Q}_{i} e^{V_{i}} \overline{\tilde{Q}}_{i}\right) .
$$

Here we have left summation over $i$ implicit. The superpotential now has extra terms,

$$
W=-\frac{k}{8 \pi} \operatorname{Tr}\left(\Phi_{1}^{2}-\Phi_{2}^{2}\right)+\operatorname{Tr}\left(B_{a} \Phi_{1} A_{a}\right)+\operatorname{Tr}\left(A_{a} \Phi_{2} B_{a}\right)+\tilde{Q}_{1} \Phi_{1} Q_{1}-\tilde{Q}_{2} \Phi_{2} Q_{2} .
$$

At low energy we again integrate out $\Phi_{1}$ and $\Phi_{2}$, which gives

$$
W=\frac{2 \pi}{k} \operatorname{Tr}\left[\left(A_{a} B_{a}+Q_{1} \tilde{Q}_{1}\right)^{2}-\left(B_{a} A_{a}-Q_{2} \tilde{Q}_{2}\right)^{2}\right] .
$$

Now let us derive the action above using our recipe.

\section{Step 1: Construct the D3/D5 Theory}

We return to type IIB and consider D3-branes alone in flat space, so for now let $x^{6}$ be non-compact and remove the NS5- and $(1, k) 5$-brane. We then add $N_{f}$ flavor D5-branes, which intersect the D3-branes in $(2+1)$ dimensions. This D3/D5 intersection has 4 ND directions and preserves 8 real supercharges.

The D3/D5 theory was constructed in $[94,95]$ and discussed in section 2.5.1. Let us review the field theory shortly. In the flavor sector we have two $\mathcal{N}=2$ chiral superfields (which comprise an $\mathcal{N}=4$ hypermultiplet), which of course propagate only in $(2+1)$ dimensions. In the adjoint sector, we start with the theory on the D3-branes, $(3+1)$ dimensional $\mathcal{N}=4$ SYM theory. The $(3+1)$-dimensional $\mathcal{N}=4$ multiplet decomposes into two $(2+1)$-dimensional $\mathcal{N}=4$ multiplets, a vector multiplet and a hypermultiplet. The $(2+1)$-dimensional $\mathcal{N}=4$ vector multiplet then further decomposes into an $\mathcal{N}=2$ vector multiplet and an $\mathcal{N}=2$ chiral multiplet. The kinetic term for the flavor fields is then precisely the one above, i.e. the flavor fields have the standard coupling to the $\mathcal{N}=2$ vector superfield. The superpotential is also precisely the one above (at least, the terms involving the flavor fields are the same), i.e. a coupling to the adjoint $\mathcal{N}=2$ chiral superfield from the $\mathcal{N}=4$ vector multiplet. (See for example equation (4.7) in [95].) The entire action preserves $\mathcal{N}=4$ supersymmetry, that is, 8 real supercharges. We emphasize that the flavor fields do not couple to the $(2+1)$-dimensional $\mathcal{N}=4$ hypermultiplet at all.

\section{Step 2: Add the NS5-branes}

We add the NS5-brane and NS5'-brane along (012345) and separated in $x^{6}$ (which for now is still non-compact), and let the D3-branes end on them in $x^{6}$. We first perform a dimensional reduction in $x^{6}$, which does not affect the flavor action in this case (since it is 
already (2+1)-dimensional). The NS5-brane boundary conditions set to zero the $(2+1)$ dimensional $\mathcal{N}=4$ hypermultiplet. As we mentioned, however, the flavor fields do not couple to the $\mathcal{N}=4$ hypermultiplet, so this step actually has no effect on the action in the flavor sector. The theory retains $\mathcal{N}=4$ supersymmetry.

\section{Step 3: Compactify $x^{6}$, form the $(1, k) 5$-brane, and lift to M-theory}

Now we compactify $x^{6}$ and add another stack of D3-branes, so that the gauge group of the D3-branes' worldvolumes is $U\left(N_{c}\right) \times U\left(N_{c}\right)$, and another stack of $N_{f}$ D5-branes. We thus obtain two sets of flavor fields, the $Q_{i}$ and $\tilde{Q}_{i}$, with $i=1,2$, mentioned above, and correspondingly, two copies of the defect action. As we argued in section 7.4, forming the $(1, k) 5$-brane and lifting to M-theory does not change the defect action. The action acquires Chern-Simons terms, however, which break the supersymmetry to $\mathcal{N}=3$.

\section{Step 4: Take the low-energy limit}

Lastly, we must take the low-energy limit, which means writing all terms consistent with the symmetries of the field theory. Our theory has $\mathcal{N}=3$ supersymmetry. As mentioned above, $\mathcal{N}=3$ supersymmetry completely determines the form of the action [212]. The flavor action thus remains the same, and hence we arrive at equations (7.5.16) and (7.5.17). The very last step is to integrate out the $\Phi_{i}$, as we did above, the result being equation (7.5.18).

Once we have the result for the field theory action, we must ask whether any symmetry enhancement occurred in the low-energy limit, as happened in the ABJM theory without flavor.

Inspecting the superpotential above, we can see that the theory retains the $U(1)_{b}$ baryon number symmetry under which $A_{a} \rightarrow e^{i \alpha} A_{a}$ and $B_{a} \rightarrow e^{-i \alpha} B_{a}$. The theory additionally has a global $U\left(N_{f}\right) \times U\left(N_{f}\right)$ flavor symmetry, of which the overall, diagonal $U(1)$ (usually also called baryon number) acts as $Q_{i} \rightarrow e^{-i \beta} Q_{i}$ and $\tilde{Q}_{i} \rightarrow e^{i \beta} \tilde{Q}_{i}$.

From the superpotential in equation (7.5.18) we can also see that codimension-zero flavor breaks the $S U(2)_{A} \times S U(2)_{B}$ symmetry to the diagonal subgroup that leaves invariant the product of fields $A_{a} B_{a}$. If we perform $S U(2)_{A}$ and $S U(2)_{B}$ transformations,

$$
\left(\begin{array}{l}
A_{1} \\
A_{2}
\end{array}\right) \rightarrow e^{i m_{j} \sigma_{j}}\left(\begin{array}{l}
A_{1} \\
A_{2}
\end{array}\right), \quad\left(\begin{array}{l}
B_{1} \\
B_{2}
\end{array}\right) \rightarrow e^{i n_{j} \sigma_{j}}\left(\begin{array}{l}
B_{1} \\
B_{2}
\end{array}\right)
$$

where $m_{j}$ and $n_{j}$ are the parameters of the transformation, and the $\sigma_{j}$ are the Pauli matrices $(j=1,2,3)$, then we have

$$
\begin{aligned}
A_{a} B_{a} & =\left(A_{1}, A_{2}\right)\left(\begin{array}{l}
B_{1} \\
B_{2}
\end{array}\right) \rightarrow\left(A_{1}, A_{2}\right) e^{i m_{j} \sigma_{j}^{T}} e^{i n_{j} \sigma_{j}}\left(\begin{array}{l}
B_{1} \\
B_{2}
\end{array}\right), \\
e^{i m_{j} \sigma_{j}^{T}} e^{i n_{j} \sigma_{j}} & =\mathbb{1}_{2}+i m_{j} \sigma_{j}^{T}+i n_{j} \sigma_{j}+\ldots \\
& \left.=\mathbb{1}_{2}+i\left(m_{1}+n_{1}\right) \sigma_{1}+i\left(-m_{2}+n_{2}\right) \sigma_{2}+i\left(m_{3}+n_{3}\right) \sigma_{3}\right)+\ldots,
\end{aligned}
$$

where we have expanded the exponentials, $\mathbb{1}_{2}$ stands for the $2 \times 2$ identity matrix, and $\ldots$ stands for terms of higher order in $m_{j}$ and $n_{j}$. By demanding that the terms linear in 
$m_{j}$ and $n_{j}$ vanish, we find that only the subspace of the $S U(2)_{A} \times S U(2)_{B}$ algebra where $n_{1}=-m_{1}, n_{2}=m_{2}$ and $n_{3}=-m_{3}$ leaves $A_{a} B_{a}$, and hence the superpotential, invariant. We will denote this diagonal subgroup $S U(2)_{D}$.

The theory also has $\mathcal{N}=3$ supersymmetry and hence retains the $S U(2)_{R}$ symmetry, under which $\left(A_{1}, B_{1}^{*}\right),\left(A_{2}, B_{2}^{*}\right)$, and $\left(Q_{i}, \overline{\tilde{Q}}_{i}\right)$ transform as doublets. In the ABJM theory without flavor, the superpotential exhibited the symmetry $S U(2)_{A} \times S U(2)_{B}$, which does not commute with $S U(2)_{R}$. The conclusion was that in fact the full $\mathcal{R}$-symmetry was $S U(4)$, and hence the theory had $\mathcal{N}=6$ supersymmetry, as we reviewed in section 7.3.1.

The crucial question is thus whether or not $S U(2)_{R}$ and the $S U(2)_{D}$ subgroup of $S U(2)_{A} \times$ $S U(2)_{B}$ commute. As mentioned in $[206,220,221]$, they do commute, as we will now show explicitly 10 . Let the $4 \times 4$ matrices $\delta_{j}^{R}=i \sigma_{j} \otimes 1_{2}$ and

$$
\delta_{j}^{A}=\left(\begin{array}{cc}
i \sigma_{j} & 0_{2} \\
0_{2} & 0_{2}
\end{array}\right), \quad \delta_{j}^{B}=\left(\begin{array}{cc}
0_{2} & 0_{2} \\
0_{2} & -i \sigma_{j}^{*}
\end{array}\right),
$$

represent the generators of $S U(2)_{R}, S U(2)_{A}$ and $S U(2)_{B}$, respectively that act on the vector $\left(A_{1}, A_{2}, B_{1}^{*}, B_{2}^{*}\right)$. Here $0_{2}$ represents the $2 \times 2$ null matrix. We then find

$$
\begin{aligned}
& {\left[\delta_{1}^{R}, \delta_{j}^{A}\right]=i \sigma_{2} \otimes \sigma_{j}, \quad\left[\delta_{2}^{R}, \delta_{j}^{A}\right]=-i \sigma_{1} \otimes \sigma_{j}, \quad\left[\delta_{3}^{R}, \delta_{j}^{A}\right]=0,} \\
& {\left[\delta_{1}^{R}, \delta_{j}^{B}\right]=i \sigma_{2} \otimes \sigma_{j}^{*}, \quad\left[\delta_{2}^{R}, \delta_{j}^{B}\right]=-i \sigma_{1} \otimes \sigma_{j}^{*}, \quad\left[\delta_{3}^{R}, \delta_{j}^{B}\right]=0 .}
\end{aligned}
$$

and hence we immediately find that the subgroup $S U(2)_{D}$ commutes with $S U(2)_{R}$ :

$$
\left[\delta_{j}^{R}, \delta_{1}^{A}-\delta_{1}^{B}\right]=\left[\delta_{j}^{R}, \delta_{2}^{A}+\delta_{2}^{B}\right]=\left[\delta_{j}^{R}, \delta_{3}^{A}-\delta_{3}^{B}\right]=0 .
$$

The $S U(2)_{R}$ is therefore not enhanced, so the system has only $\mathcal{N}=3$ supersymmetry.

To summarize: classically the theory has $\mathcal{N}=3$ superconformal symmetry, with bosonic subgroup $S O(3,2)$, and global symmetry $S U(2)_{R} \times S U(2)_{D} \times U(1)_{b} \times U\left(N_{f}\right) \times U\left(N_{f}\right)$ which matches perfectly with the symmetries in the supergravity description above.

As this case was a rather trivial example of our recipe, we now turn to a slightly more involved example, in particular, an example that exhibit supersymmetry enhancement.

\subsection{Codimension One $\mathcal{N}=(0,6)$ Supersymmetric Flavor}

In this section we study an example of a probe brane that introduce codimension-one flavor fields, that is, flavor fields propagating in a $(1+1)$-dimensional subspace of the $(2+1)$-dimensional ABJM theory. The brane we study, a D7/D8/M9-brane, were first studied in type IIA on $A d S_{4} \times \mathbb{C P}^{3}$ in [222]. We review and extend the gravity results of [222], and write the dual field theory Lagrangian explicitly for the D7/D8/M9-brane.

In $(1+1)$ dimensions the supersymmetries divide into left- and right-handed sectors. We begin in this section with a chiral codimension-one theory, which preserves $\mathcal{N}=(0,6)$ supersymmetry. In the next section we study non-chiral flavor.

\footnotetext{
${ }^{10}$ The fact that $S U(2)_{R}$ and $S U(2)_{D}$ commute is a familiar feature of $S U(4)$. The $S U(4)$ algebra has two obvious $S U(2) \times S U(2)$ sub-algebras, whose diagonal $S U(2)$ 's commute with one another. In the ABJM theory these are $S U(2)_{A} \times S U(2)_{B}$, with diagonal $S U(2)_{D}$, and $S U(2)_{1} \times S U(2)_{2}$, with diagonal $S U(2)_{R}$. Here $S U(1)_{1}$ acts on $\left(A_{1}, B_{1}^{*}\right)$ as a doublet and leaves $\left(B_{2}^{*}, A_{2}\right)$ invariant, while for $S U(2)_{2}\left(A_{1}, B_{1}^{*}\right)$ is invariant and $\left(B_{2}^{*}, A_{2}\right)$ is a doublet.
} 


\subsubsection{Supergravity with M9/D8-brane probes}

We begin by adding D7-branes extended along (01345789) in the type IIB setup. We are free to choose their position on the $x^{6}$ circle. We will add two stacks of D7-branes, each with $N_{f}$ coincident D7-branes, on opposite sides of the circle, away from the NS5-brane and $(1, k) 5$-brane. The strings from the D7-branes to the two stacks of D3-branes introduce massless flavor in both gauge groups. (In contrast, the authors of [222] considered matter fields that coupled only to a single gauge group.) Notice also that the D7-branes and D3-branes have 8 ND directions, hence the flavor fields will be chiral, as we mentioned in section 7.4.2. As shown in table 7.1 in section 7.4.1, these D7-branes preserve 3 real supercharges in the type IIB setup.

After T-duality in $x^{6}$ the D7-branes become D8-branes. The $2 N_{f}$ D8-branes are coincident, and have a $U\left(2 N_{f}\right)$ symmetry broken to $U\left(N_{f}\right) \times U\left(N_{f}\right)$ by a $\mathbb{Z}_{2}$-valued Wilson line (similar to what happened in section 7.5.1). After uplift to M-theory and the "near-horizon" limit, the D8-branes become M9-branes extended along (01) and along all of $\mathbb{C}^{4} / \mathbb{Z}_{k}$. Obviously the branes preserve the full $S U(4) \times U(1)_{b}$ symmetry of $\mathbb{C}^{4} / \mathbb{Z}_{k}$. In appendix B.3.1 (see also [222]) we find that the M9-branes preserve 6 real supercharges.

If we take $N_{c} \rightarrow \infty$, we can replace the M2-branes by their near-horizon geometry, which is $A d S_{4} \times S^{7} / \mathbb{Z}_{k}$. The M9-branes are extended along $A d S_{3}$ inside $A d S_{4}$ and wrap all of $S^{7} / \mathbb{Z}_{k}$. In appendix B.3.2 we analyze the $\kappa$-symmetry condition for these branes and find that after the near-horizon limit the number of preserved supercharges has doubled to 12 .

For large $k$, such that $k^{5} \gg N_{c}$, the M9-branes reduce to D8-branes in type IIA that wrap $A d S_{3} \times \mathbb{C P}^{3}$. These probe D8-branes were first studied in [222].

To summarize: in the type IIB setup we can add D7-branes that produce fundamental matter for both gauge group factors. They have $8 \mathrm{ND}$ directions (with respect to the D3-branes), so the flavor fields will be chiral. These D7-branes become M9-branes in Mtheory on $\mathbb{C}^{4} / \mathbb{Z}_{k}$. These M9-branes preserve 6 real supercharges, so we expect a dual field theory with (in $(1+1)$-dimensional notation) $\mathcal{N}=(0,6)$ superconformal symmetry. The corresponding $\mathcal{R}$-symmetry group must be $S U(4) \cong S O(6)$, which fits into the symmetry of the brane construction. We now proceed to construct the dual field theory and check that is has the right symmetries and amount of supersymmetry.

\subsubsection{The Field Theory}

Let us apply our recipe.

\section{Step 1: Construct the D3/D7 Theory}

Once again we consider a single stack of D3-branes alone in flat space (so again let $x^{6}$ be non-compact and remove the NS5-brane and $(1, k) 5$-brane), and add a codimension-two D7-brane. Such a D3/D7 intersection has 8 ND directions and preserves 8 real supercharges. What is the field theory for such a D3/D7 intersection? This question was answered ${ }^{11}$ in [96-98]. With 8 ND directions, the NS sector zero-point energy is $1 / 2$, so

\footnotetext{
${ }^{11}$ Much of the analysis of [96-98] focused on what we would call back-reaction, that is, effects that result from leaving the probe limit. Strictly speaking, all of our statements apply only in the probe limit.
} 
the ground state is in the Ramond sector. What survives the GSO projection is a single Weyl spinor confined to the (1+1)-dimensional intersection, transforming in the $\left(N_{c}, \bar{N}_{f}\right)$. We thus obtain chiral flavor. Our Weyl fermion will be left-handed.

The immediate question is: with only fermions in the ground state, how can the theory be supersymmetric? The answer is that all of the preserved supercharges are right-handed. The theory has $(1+1)$-dimensional $\mathcal{N}=(0,8)$ supersymmetry. The flavor fermions are completely inert under both supersymmetry and the $\mathcal{R}$-symmetry.

The action is then remarkably simple. From the D3-branes we of course have the $(3+1)$ dimensional $\mathcal{N}=4 U\left(N_{c}\right)$ SYM theory action. For the defect flavor fields, the claim of [96-98] is that the only marginal and gauge-invariant terms that respect all of the symmetries are

$$
S_{\text {fund }}=\int d x^{+} d x^{-} \psi_{q}^{\dagger}\left(i \partial_{-}-A_{-}\right) \psi_{q}
$$

where we have used (1+1)-dimensional coordinates $x^{ \pm}=x^{0} \pm x^{1}, \psi_{q}$ is our left-handed Weyl fermion, and $A_{-}$is the restriction of the ambient $U\left(N_{c}\right)$ gauge field to the defect. Of crucial importance is the fact that $A_{-}$is inert under $\mathcal{N}=(0,8)$ supersymmetry transformations [96-98].

\section{Step 2: Add the NS5-branes}

We add the NS5-brane and NS5'-brane along (012345) and separated in $x^{6}$ (which for now is still non-compact), and let the D3-branes end on them in $x^{6}$. The NS5-brane boundary conditions set to zero the $(2+1)$-dimensional $\mathcal{N}=4$ hypermultiplet. The flavor fields only couple to the gauge field, however, so adding the NS5-branes does not alter the action in the flavor sector. The supersymmetry is reduced to $\mathcal{N}=(0,4)$, however.

\section{Step 3: Compactify $x^{6}$, form the $(1, k) 5$-brane, and lift to M-theory}

We compactify $x^{6}$ and add another stack of D3-branes, so that the gauge group of the D3branes' worldvolumes is $U\left(N_{c}\right) \times U\left(N_{c}\right)$, and another stack of $N_{f}$ D7-branes. We obtain two sets of flavor fields, which we will denote as $\psi_{q}^{i}$ with $i=1,2$. We obtain two copies of the action above, one for each $\psi_{q}^{i}$. The rest of the construction (forming the $(1, k) 5$-brane, T-duality, etc.) also leaves the action in the flavor sector untouched. The Chern-Simons terms break the supersymmetry to $\mathcal{N}=(0,3)$.

\section{Step 4: Take the low-energy limit}

Lastly, we must take the low-energy limit, which means writing all terms consistent with the symmetries of the field theory. Let us review the symmetries of the theory at the end of Step 3. Our theory has $\mathcal{N}=(0,3)$ supersymmetry and the corresponding $S U(2)_{R}$ $\mathcal{R}$-symmetry. (The $S U(2)_{R}$ is easy to see in the type IIB setup, being exactly the same $S U(2)_{R}$, which rotates (345) and (789) simultaneously, that appears in the theory without flavor.) The theory also has a baryon number symmetry that shifts the phase of $\psi_{q}^{i}$ and leaves all other fields invariant. Recall also that the theory of course has $(1+1)$-dimensional Lorentz invariance and gauge invariance. We will now argue that in fact these symmetries forbid any new (relevant or marginal) terms. 
First let us do some dimension counting. The fields $\psi_{q}$ are $(1+1)$-dimensional fermions, hence they are dimension $1 / 2$. (We will drop the $i$ index on $\psi_{q}^{i}$ for now.) We must also consider the restriction of the $(2+1)$-dimensional fields to $(1+1)$ dimensions. We will use $\phi$ to denote a generic $(2+1)$-dimensional scalar restricted to the defect, and $\Psi$ to denote a $(2+1)$-dimensional fermion restricted to the defect. $\phi$ is dimension $1 / 2$ and $\Psi$ is dimension 1.

Terms with an odd number of $\psi_{q}$ and $\psi_{q}^{\dagger}$, whether relevant or marginal, are forbidden by gauge invariance and by the $U(1)$ baryon number that shifts the phase of $\psi_{q}$. Terms with two $\psi_{q}$ that are relevant include couplings to scalars, of the form $\phi \psi_{q}^{\dagger} \psi_{q}$, which is dimension $3 / 2$. These are forbidden by Lorentz invariance. $\psi_{q}$ is a $(1+1)$-dimensional left-handed fermion. Its conjugate $\psi_{q}^{\dagger}$ is also left-handed, hence $\psi_{q}^{\dagger} \psi_{q}$ is not a Lorentz singlet. Marginal couplings of the form $\phi^{2} \psi_{q}^{\dagger} \psi_{q}$ and $\Psi \psi_{q}^{\dagger} \psi_{q}$, and the marginal quartic term $\left(\psi_{q}^{\dagger} \psi_{q}\right)^{2}$, are forbidden for the same reason. (We can also eliminate many such terms, and/or linear combinations of them, using the $\mathcal{R}$-symmetry and/or supersymmetry.) The only term involving derivatives and/or the gauge field that is allowed by the symmetries is the gauge-covariant kinetic term itself. The overall normalization of that term can change, but of course such an overall constant can be removed by a rescaling of $\psi_{q}$.

Our conclusion is that the form of the defect action does not change in Step 4. We can thus write the defect action easily. We have two Weyl fermions, $\psi_{q}^{i}$, where again $i=1,2$ labels the gauge group, that is, under $U\left(N_{c}\right)_{k} \times U\left(N_{c}\right)_{-k} \times U\left(N_{f}\right) \times U\left(N_{f}\right)$ the $\psi_{q}^{1}$ fermion transforms as $\left(N_{c}, \mathbf{1}, \bar{N}_{f}, \mathbf{1}\right)$ and the $\psi_{q}^{2}$ fermion transforms as $\left(\mathbf{1}, N_{c}, \mathbf{1}, \bar{N}_{f}\right)$. We add to the ABJM action the terms

$$
S_{\text {fund }}=\int d x^{+} d x^{-} \psi_{q}^{i \dagger}\left(i \partial_{-}-A_{-}^{i}\right) \psi_{q}^{i},
$$

where here again $A_{-}^{i}$ are the defect values of the bulk gauge fields, and summation over $i$ is implicit.

We show in appendix B.4 that $A_{-}$is invariant under $\mathcal{N}=(0,6)$ supersymmetry transformations, hence the flavor action preserves $\mathcal{N}=(0,6)$ supersymmetry. The action is also trivially invariant under the full $S U(4)_{R} \times U(1)_{b}$ symmetry. These symmetries perfectly match those of the brane construction.

\section{7 $\quad S U(4)$ Equivalence of Probe Flavor}

Although the ABJM construction starts with a fairly complicated brane setup in type IIB, we have seen in section 7.3 that after the "near-horizon" limit we end up with M2-branes probing $\mathbb{C}^{4} / \mathbb{Z}_{k}$. The "near-horizon" limit, that is, zooming in on the $\mathbb{C}^{4} / \mathbb{Z}_{k}$ singularity of the space $X_{8}$ mentioned in section 7.3 .3 , discards much of the complicated information of the type IIB setup. After taking $N_{c} \rightarrow \infty$ we reach M-theory on $A d S_{4} \times S^{7} / \mathbb{Z}_{k}$.

For $k=1$ the addition of flavor branes in M-theory, namely codimension-two M2-branes and codimension-one M5-branes, was studied in [113]. There the authors had to consider only one embedding for each probe brane since the $S O(8)$ isometry group of $\mathbb{C}^{4}$ or $S^{7}$ can map any two supersymmetric embeddings into each other. If two brane embeddings are related by such an $S O(8)$ symmetry transformation, then they are physically equivalent. 
In other words, when $k=1$ all supersymmetric codimension-two M2-branes are physically equivalent, and similarly for supersymmetric codimension-one M5-branes.

For general $k$, the $\mathbb{Z}_{k}$ orbifold of $\mathbb{C}^{4}$ breaks the $S O(8)$ isometry group to $S U(4) \times U(1)_{b}$. Two supersymmetric brane embeddings may be related by an $S O(8)$ element that is not contained in $S U(4) \times U(1)_{b}$. In that case, we have two physically distinct ways of adding flavor. An interesting question is whether we can fully classify the supersymmetric embeddings of flavor branes in the ABJM theory, but that is beyond the scope of this paper. Here, we will discuss how to use the unbroken $S U(4) \times U(1)_{b}$ symmetry to show that certain probe branes are physically equivalent although they look very different in the type IIB setup. When that occurs, we will call the two type IIB D-branes $S U(4)$-equivalent.

On the gravity side, we will present an explicit example of $S U(4)$-equivalent pairs. In the field theory, $S U(4)$ equivalence occurs when two different theories flow to the same lowenergy fixed point. In the language of our recipe, the two different theories are the theories we obtain at the end of Step 3, which flow to the same theory at low energy in Step 4. We will present one explicit example of such flow in what follows, for the codimension-zero case.

Two necessary conditions for two D-branes to be $S U(4)$-equivalent are 1.) they become the same object in M-theory and 2.) they have the same codimension. More precisely, as we do a T-duality along $x^{6}$ to go from type IIB to type IIA, two $S U(4)$-equivalent D-branes must have the same codimension in the directions (012). Furthermore, if both D-branes wrap $x^{6}$ or both do not wrap $x^{6}$, then they have to be both Dp-branes. Another possibility is that a type IIB $\mathrm{D}(\mathrm{p}+1)$-brane is equivalent to a type IIB $\mathrm{D}(\mathrm{p}-1)$-brane, if the $\mathrm{D}(\mathrm{p}+1)$ brane wraps $x^{6}$ and the $\mathrm{D}(\mathrm{p}-1)$-brane does not. Notice also that, in M-theory on $\mathbb{C}^{4} / \mathbb{Z}_{k}$, the orientation of the object does not affect the symmetries it preserves. (That is obvious in the $k=1$ case.) That means that, in addition to an $S U(4) \times U(1)_{b}$ transformation, we can also reverse the orientation of an object, so that, in type IIB, Dp-branes and anti-Dpbranes may be $S U(4)$ equivalent. Finally, an especially important point is that, due to the "near-horizon" limit in which the $\mathcal{R}$-symmetry $S O(3)_{R}$ is enhanced to $S U(4)_{R}$, two branes in type IIB that preserve different amounts of supersymmetry and different subgroups of the $S O(3)_{R}$ may still be $S U(4) \times U(1)_{b}$ equivalent.

\subsubsection{Codimension-zero KK monopoles}

We start with the codimension-zero D5-branes along (012789) from section 7.5. As shown in [206], these D5-branes become KK-monopoles on $\mathbb{C}^{4} / \mathbb{Z}_{k}$ with the embedding equations $z^{1}=\bar{z}^{3}, z^{2}=\bar{z}^{4}$. As we argued above, we can perform an $S U(4)$ transformation from the old coordinates $z^{i}$ to new coordinates $z_{\text {new }}^{i}$, such that the embedding becomes $\operatorname{Im}\left(z_{\text {new }}^{i}\right)=0$ for $i \in\{1,2,3,4\}$. Explicitly, the $S U(4)$ transformation is

$z_{\text {new }}^{1}=\frac{1}{\sqrt{2}}\left(z^{1}+z^{3}\right), \quad z_{\text {new }}^{2}=\frac{-i}{\sqrt{2}}\left(z^{1}-z^{3}\right), \quad z_{\text {new }}^{3}=\frac{1}{\sqrt{2}}\left(z^{2}+z^{4}\right), \quad z_{\text {new }}^{4}=\frac{-i}{\sqrt{2}}\left(z^{2}-z^{4}\right)$.

What happens if we start with a KK monopole described by $\operatorname{Im}\left(z^{i}\right)=0$ for $i \in\{1,2,3,4\}$ and return to type IIB (using the results of appendix B.2)? Up to an $S U(4) \times U(1)_{b}$ transformation that only changes the constant value of $x^{6}$, we find D7-branes along (01235679) (If we reverse the orientation of the KK monopole, we can obtain anti-D7-branes, as explained above). These D7-branes are $S O(3)_{R}$ equivalent to the D7-branes along (01234678) 
listed in table 7.1. We summarize the $S U(4)$ equivalence in the following table.

\begin{tabular}{|c|cc|cc|}
\hline Type IIB & D5 & $(012789)$ & D7 & $(01235679)$ \\
\hline M-theory & KK & $z^{1}=\bar{z}^{3}, z^{2}=\bar{z}^{4}$ & KK & $\operatorname{Im}\left(z^{i}\right)=0$ \\
\hline
\end{tabular}

We have found two different types of D-branes in type IIB that lead to the same configuration in M-theory, and are therefore physically identical in M-theory. That might be surprising since the D-branes preserve different amounts of supersymmetry and different subgroups of the $S O(3)_{R}$ symmetry in the type IIB setup. We will, therefore, now show on the field theory side that both D-branes lead to the same theory upon taking the low-energy limit.

For the flavor D5-branes we reviewed the field theory in section 7.5.2, following [206, $220,221]$. The action of the $\mathcal{N}=3$ supersymmetric $(2+1)$-dimensional flavor appears in equations 7.5 .16 and 7.5.18. Here we will begin instead with anti-D7-branes along (01234678). We will apply our recipe once again.

\section{Step 1: Construct the D3/D7 Theory}

We begin with D3-branes along (0126) and anti-D7-branes along (01234678). Such an intersection preserves 8 real supercharges. The intersection has 4 ND directions, hence we obtain non-chiral flavor propagating in $(3+1)$ dimensions (along (0126)). The field theory of the 4 ND D3/D7 intersection is well known: it is (3+1)-dimensional $S U\left(N_{c}\right) \mathcal{N}=4$ supersymmetric Yang-Mills theory coupled to $\mathcal{N}=2$ supersymmetric hypermultiplets in the fundamental representation of $S U\left(N_{c}\right)$. The action is usually written in $\mathcal{N}=1$ superspace, and includes the usual kinetic terms for the flavor fields as well as a superpotential coupling for the flavor fields whose form is dictated by $\mathcal{N}=2$ supersymmetry. If we decompose the $\mathcal{N}=4$ vector multiplet into an $\mathcal{N}=1$ vector multiplet and three $\mathcal{N}=1$ chiral multiplets, then the superpotential includes a coupling of the flavor fields to the $\mathcal{N}=1$ chiral multiplet whose scalars represent fluctuations of the branes in the overall transverse directions, which here are (59). For more details about the D3/D7 theory, see section 2.5.1 as well as [92] and references therein.

\section{Step 2: Add the NS5-branes}

We now add the NS5-brane and NS5'-brane along (012345), and let the D3-branes end on them. As mentioned in appendix B.1, the system then preserves 4 real supercharges. Technically, we should perform a dimensional reduction from $(3+1)$ dimensions to $(2+1)$ dimensions (since the anti-D7-branes are extended along $x^{6}$ ) and then impose the NS5brane boundary conditions. We know what the result has to be, however. The NS5brane boundary conditions set to zero the scalars (789). That means that after imposing those boundary conditions, the flavor fields couple only to the single scalar 5 . We also know that the theory has 4 real supercharges, or in $(2+1)$-dimensional language, $\mathcal{N}=2$ supersymmetry. The flavor fields must of course have kinetic terms, with the usual coupling to the $\mathcal{N}=2$ vector multiplet. The key observation is that the $\mathcal{N}=2$ vector multiplet includes a single real scalar (which, recalling the type IIB construction of the ABJM theory, must indeed be 5). We can conclude that the $(2+1)$-dimensional flavors have no superpotential: any superpotential coupling must preserve $\mathcal{N}=2$ supersymmetry, 
and hence must be a coupling to an $\mathcal{N}=2$ chiral superfield, but that would introduce couplings to additional scalars that are obviously absent here. In short, the flavor fields only couple to enough scalars for an $\mathcal{N}=2$ vector multiplet! The $(2+1)$-dimensional action in the flavor sector is then simply the $\mathcal{N}=2$ kinetic term, whose explicit form appears in equation (7.5.16).

\section{Step 3: Compactify $x^{6}$, form the $(1, k) 5$-brane, and lift to M-theory}

As usual, these steps leave the form of the flavor action untouched. Notice also that in this case the supersymmetry remains $\mathcal{N}=2$ throughout. For example, table 7.1 in section 7.4.1 shows that after we form the $(1, k) 5$-brane the system still preserves 4 real supercharges. Notice also that the symmetries of the field theory and the brane construction agree. $\mathcal{N}=2$ supersymmetry has a $U(1) \mathcal{R}$-symmetry, and the anti-D7-brane along (01234678) clearly preserves the $U(1)$ subgroup of $S O(3)_{R}$ that rotates (34) and (78) simultaneously.

\section{Step 4: Take the low-energy limit}

Now we come to the crucial step. We must write all terms consistent with $\mathcal{N}=2$ supersymmetry, the $U(1) \mathcal{R}$-symmetry, and the $U(1)_{b}$ symmetry. Here we will borrow some arguments from [212]. Only one such term exists, a coupling to the $\mathcal{N}=2$ chiral fields $\Phi_{i}$, of the form written in equation (7.5.17). We must therefore add such a term to the superpotential, with some coefficient. Arguments similar to those in [212], based on the sign of the two-loop beta function, then suggest that the coefficient flows to precisely the right value to produce the enhancement to $\mathcal{N}=3$ supersymmetry. The coupling is then identical to the term in equation (7.5.17), and we thus recover exactly the same theory as in section 7.5 .

We have thus seen how two different field theories flow to the same low-energy fixed point, and hence how $S U(4)$-equivalence appears on the field theory side. Notice that these two theories preserved different symmetries: the D5-brane along (012789) preserved the whole $S O(3)_{R}$ while the anti-D7-brane along (01234678) preserved only a $U(1)$ subgroup.

\subsection{Other Flavor branes in $A d S_{4} / C F T_{3}$}

In this chapter we discussed a general recipe how to add flavor degrees of freedom to the ABJM theory, i.e. to $\mathcal{N}=62+1$-dimensional supersymmetric Chern-Simons-matter theory with gauge group $U\left(N_{c}\right)_{k} \times U\left(N_{c}\right)_{-k}$. We applied this general procedure to two examples: a D6-brane in type IIA (a KK-monopole in M-theory) describing codimensionzero $\mathcal{N}=3$ supersymmetric flavor fields and a D8-brane in type IIA giving rise to chiral, $\mathcal{N}=(0,6)$ supersymmetric flavor fields. In $[6]$ we considered also two other examples:

- A D3-brane that becomes a D4-brane in type IIA and an M5-brane in M-theory, and which introduces codimension-one, non-chiral, $\mathcal{N}=(3,3)$ supersymmetric flavor fields.

- A D3-brane that becomes a D2-brane in type IIA and an M2-brane in M-theory, and which introduces codimension-two, $\mathcal{N}=4$ supersymmetric flavor fields. 
To summarize, in this chapter we have added flavor degrees of freedom to $\mathcal{N}=6 U\left(N_{c}\right) \times$ $U\left(N_{c}\right)$ Chern-Simons-Matter theory, which is the low-energy description of $N_{c}$ coincident M2-branes. The flavor degrees of freedom have been realized on the gravity side by membranes or by other non-perturbative objects, such as KK-monopoles. In particular, we have explicitly written down the couplings of the flavor fields to the Chern-SimonsMatter theory on the gauge theory side and have determined the preserved symmetries.

The analysis presented in this chapter is one of the basic ingredients to study $(2+1)$ dimensional Quantum Hall liquids in a holographic setup. Therefore, in the future we should investigate the thermodynamics of the models discussed here and determine the spectral functions of mesons and mesinos. 


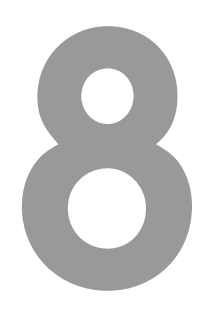

\section{Conclusions and Outlook}

In this thesis we have presented new methods which are necessary to generalize the AdS/CFT correspondence towards applications to condensed matter systems. In particular, we have extend the calculation of direct-current conductivities to arbitrary constant electric and magnetic fields. Moreover we have determined the conductivity for alternating and direct currents for a non-relativistic system with Schrödinger symmetry. Whereas the $\mathrm{AC}$ conductivity is calculated in linear response, the direct-current conductivity capture effects in all orders of the electric and magnetic fields considered.

Moreover we have investigated holographic superconductors in string theory embeddings. We find a second order phase transition between a state spontaneously breaking an $U(1)$ symmetry and a symmetry-preserving state. The phase transition have mean-field critical exponents. Finally we have also studied holographic fermions and non-Fermi liquids in string theory embeddings. In particular, we identify an emerging Fermi surface for low temperatures. Finally we also determine the fermionic response in the superconducting phase.

Before I review how to add charge carriers to Chern-Simons theories, let me recall our string theory setup in more detail. As discussed in section 1.3 of the introduction, we use strongly-coupled $\mathcal{N}=4$ supersymmetric Yang-Mills theory in the large- $N_{c}$ limit. Note that this field theory is conformal. The dual gravity side is given by type IIB supergravity on $A d S_{5} \times S^{5}$. In order to add $N_{f}$ mass-degenerate flavor fields we consider $N_{f}$ Dpbranes in this geometry. The bound state of flavor fields, i.e. mesons and their fermionic superpartners, mesinos, are described by fluctuations of scalar, spinor and gauge fields living on the worldvolume of the Dp-brane.

Note that we assume that the branes do not backreact on the geometry, but rather probe only the geometry. This so-called probe-approximation is valid for $N_{f} \ll N_{c}$ and corresponds to the quenched approximation on the field theory side. This limit has one immediate consequence. The field theory including the matter fields is also conformal, which is convenient for the description of quantum critical theories with dynamical scaling 
exponent $z=1$, as discussed in section 1.2 .

The $N_{f}$ mass-degenerate flavor fields give rise to a $U\left(N_{f}\right)$ global symmetry corresponding to the local $U\left(N_{f}\right)$ gauge symmetry of Dp-branes' scalar, spinor and gauge fields. In particular, we investigate the effect of two subgroups in more details: the overall $U(1)$ symmetry, corresponding to the $U(1)$-baryon symmetry on the field theory side and, in the case of $N_{f}=2$, the non-Abelian subgroup $S U(2)$, which is identified as the isospin symmetry on the field theory. A non-trivial gauge field $A_{t}(u)$, where $u$ is the radial direction of Anti-de Sitter spacetime, corresponds to a state of finite baryon number density and baryon chemical potential on the field theory side. Therefore we can consider a finite density of charge carriers, which are charged under the flavor symmetry subgroup $U(1)$. In the following, we embed holographic superconductors and holographic (non-)Fermi liquids into this or a similar setup. Moreover, we can calculate the conductivities associated with the baryon number density.

The last project presented in the thesis is more technical. We have considered a purely $(2+1)$-dimensional Chern-Simons-Matter theory arising from the low-energy limit of M2branes. This theory may also serve as a candidate for the quantum critical theory. In chapter 7 we have investigated how to add flavor fields, giving rise to charge carriers, to that theory. In particular, we have determined the effective couplings of the flavor fields to the Chern-Simons-Matter theory on the field theory side. Moreover, we have also constructed the dual gravitational configuration consisting of probe D-branes, KKmonopoles or M-branes.

In the following, the main projects are summarized in detail. Furthermore we also suggest directions for future research.

\section{Conductivities in relativistic and non-relativistic theories}

First let us consider the direct-current conductivity tensor for a relativistic system. In particular, in section 3.3 we have computed the conductivity tensor of $\mathcal{N}=2$ supersymmetric flavor fields propagating through a strongly-coupled $\mathcal{N}=4$ SYM theory plasma at finite temperature $T$. We have included a finite $U(1)$ baryon number density $\left\langle J^{t}\right\rangle$ and have considered the most general configuration of constant external fields, namely an electric field $E$ and a magnetic field with a component $B_{z}$ perpendicular to $E$ and a component $B_{x}$ parallel to $E$.

The direct-current conductivity components $\sigma_{x x}$ and $\sigma_{x z}$ consists of two terms adding in quadrature. These two terms can be traced back to two types of charge carriers. First we have the density of charge carriers we introduced explicitly by $\left\langle J^{t}\right\rangle \neq 0$. Even when $\left\langle J^{t}\right\rangle=0$ we find a non-zero conductivity components $\sigma_{x x}$ and $\sigma_{x z}$ and hence a non-zero current $\left\langle J^{x}\right\rangle$ and $\left\langle J^{z}\right\rangle$. We have given evidence that the other type of charge carriers come from pair production in the electric field. In contrast, note that $\sigma_{x y}$ depends only on the density of charge carriers.

Note that in the analysis presented in section 3.3 and reviewed above, we have applied a constant electric fields, i.e. the charge carriers have to be accelerated forever and our quasi-static ansatz for the holographic setup would be inconsistent. However, due to the probe-approximation $N_{f} \ll N_{c}$ we build in a mechanism for dissipation. Since the charge 
carriers are of order $N_{f} N_{c}$ but the quantum critical theory, i.e. $\mathcal{N}=4$ super Yang-Mills theory, is of order $N_{c}^{2}$, the charge carriers can transfer energy and momentum to the quantum critical theory, but the rates at which they do so are of order $N_{c}$. That means that only at times of order $N_{c}$ the charge carriers have transferred order $N_{c}^{2}$ amounts of energy and momentum to the plasma, and hence the motion of the $\mathrm{N}=4 \mathrm{SYM}$ plasma is no longer negligible. Therefore, the probe limit thus provides the charge carriers with an apparent mechanism for dissipation.

For the non-relativistic theories, we have computed in chapter 6 both DC and AC conductivities associated with a finite baryon number density of charge carriers in a stronglycoupled theory with non-relativistic symmetry. The quantum critical theory is $\mathcal{N}=4$ SYM theory deformed by a dimension-five operator that breaks the relativistic conformal group down to the Schrödinger group, with dynamical scaling exponent $z=2$. On the gravity side we have to perform a solution-generating method, the Null-Melvin-Twist (NMT).

The charge carriers are comprised of a finite baryon density of massive $\mathcal{N}=2$ supersymmetric hypermultiplets. We find that, generally speaking, both the DC and AC conductivities exhibit relativistic scaling, with temperature or frequency, respectively in the IR and non-relativistic scaling, with $z=2$, in the UV. These results are in accord with our expectations, given the origin of the non-relativistic symmetry via an irrelevant deformation of the theory.

For the future, we can think of many questions that deserve further research:

First, we could introduce a thermal gradient into both holographic setups and compute the thermal conductivity and the thermo-electric transport coefficients (called $\alpha_{i j}$ in the introduction) associated with the flavor fields. A further extension would be to work with two coincident D7-branes, and hence two flavors in the SYM theory, and to compute the thermal conductivity and thermo-electric transport coefficients associated with isospin charge. As demonstrated in chapter 4, a sufficiently large isospin chemical potential triggers a phase transition to a superconducting, or more accurately a superfluid, phase.

Notice that also a more detailed analysis of probe D-branes in Schrödinger spacetime would be extremely useful. Given background $S c h_{5}$ geometries that preserve some supersymmetry $[187,188]$, can we find supersymmetric embeddings of probe D-branes? What happens to the many phase transitions that occur in the relativistic setting when the probe D-branes are instead in $\mathrm{Sch}_{5}$ ?

Introducing a magnetic field on the worldvolume of a probe D-brane is straightforward, and allows us to compute not only the Hall conductivity but indeed the entire conductivity tensor, in the DC limit $[5,106]$. What scaling does the Hall conductivity have in the Schrödinger case?

Moreover, with probe D-branes that do not wrap all coordinates of $A d S_{5}$, we have more options about how to perform the NMT. Consider for example a D5-brane extended along $A d S_{4} \times S^{2}$ inside $A d S_{5} \times S^{5}$. Here we must make a choice: the D5-brane may be extended along the T-duality direction $y$ or not. If the D5-brane is along $y$, we expect the results to be similar those of section 6.2 .3 for the D7-brane. What happens when the D5-brane is transverse to $y$, however?

Perhaps the most exciting direction for future research would be model-building of the kind 
advocated in [185]. Our results indicate that a straightforward way to engineer scaling exponents would be to start in the relativistic case, i.e. probe D-branes in $A d S_{5} \times S^{5}$, introduce a scalar to produce the desired IR exponents, and then perform the NMT. We generically expect that the result will be DC and AC conductivities that in the UV have non-relativistic scalings, with $z=2$, and in the IR have whatever scalings we initially gave them.

\section{Holographic superconductors}

In chapter 4 we have embedded holographic p-wave superconductors in a string theory setup. In particular, we have considered on the field theory side two different kinds of massdegenerate charge carriers, giving rise to a $S U(2)$ global symmetry. Moreover, we have turned on finite temperature and an $S U(2)$ isospin chemical potential, breaking $S U(2)$ to the subgroup $U(1)_{3}$. The two kinds of charge carriers have opposite chemical potentials.

In the holographic setup we had to embed two coincident Dp-branes into (4+1)-dimensional Schwarzschild Anti-de Sitter spacetime. Since the effective action for two coincident Dpbranes is only known up to order $F^{4}$ in the field strength tensor $F$, we use three different prescriptions for the effective non-Abelian Dp-brane action and compare the results. In all three cases, the physics agrees qualitatively.

Below a critical temperature $T_{c}$, or equivalently above a critical isospin chemical potential, we have found a new phase with non-vanishing meson condensate $\left\langle J_{1}^{x}\right\rangle$. We have explicitly shown that this new phase is favored thermodynamically. The phase transition is second order and has a mean-field critical exponent. Note that $\left\langle J_{1}^{x}\right\rangle$ breaks the remaining $U(1)_{3^{-}}$ symmetry spontaneously. Therefore we have interpreted the meson condensate $\left\langle J_{1}^{x}\right\rangle$ as a meson superfluid. If we would gauge the global $U(1)_{3}$-symmetry on the field theory side, we may view the new state as a superconductor with p-wave symmetry. We have shown that the superconducting phase persists at finite quark mass and have determined the phase diagram as function of the isospin chemical potential, the temperature and the mass of the flavor fields.

In addition, we have considered the linear response of the superconductor to electrical fields. In particular, we have computed the frequency-dependent conductivity for alternating currents. We have found that there is a gap in the real part of the conductivity for small frequencies. The gap increases as we lower the temperature. Moreover, using Kramers-Kronig relations, we have realized that there is a delta peak in the real part of the $\mathrm{AC}$ conductivity for zero frequency. We have also determined the response to a magnetic field. As expected, the critical temperature of the superconductor is lowered if we switch on the finite magnetic field. Note that we do not expect that the system expels magnetic fields since the U(1), which is spontaneously broken, is a global symmetry. But our calculation have shown that if we gauge this symmetry weakly, i.e. by promoting it to a local symmetry and introducing the corresponding gauge fields, we can generate currents which will induce magnetic fields opposite to the external fields [3].

Let me suggest directions for future research.

An interesting question is whether we can only realize p-wave superconductors by embedding probe branes, or if we also find other symmetry breaking patterns. Of course, we can 
first try to find an s-wave superconductor in this framework. For condensed matter applications towards High- $T_{c}$ superconductors, it is interesting to find the dual holographic description of d-wave superconductors. So far first steps in this direction have been taken in the bottom-up approach, but it is not clear whether there is a consistent string theory embedding. To obtain a condensate with d-wave symmetry, a spin-two operator has to condense. An obvious candidate is the graviton. In the holographic setup presented here, we have other fields with the correct symmetry: higher spin mesons. This observation can be the starting point for the construction of d-wave superconductors in the top-down approach.

Moreover, for the application towards High- $T_{c}$ superconductors it is instructive to calculate the thermo-electric transport as mentioned in the previous paragraph. This may be relevant for high- $T_{c}$ superconductors, which exhibit unusually large thermo-electric response even outside the superconducting phase.

\section{Holographic fermions and (non-)Fermi liquids}

In chapter 5 we have embedded fermions and holographic (non-)Fermi liquids in our topdown approach using probe branes. We have considered the fermionic part of the action of Dp-branes, as proposed in [167-169], and have specialized the action to the background of $N_{c}$ D3-branes, which we considered so far. In particular, we have computed the effective mass of the worldvolume fermions for Dp-branes in $A d S_{5} \times S^{5}$, which allowed us to determine the conformal dimensions of the dual field theory operators, i.e. of the mesinos. For a particular example, the codimension one D3/D5 intersection which wraps $A d S_{4} \times S^{2}$ in the dual gravity setup, we have written down in section 2.5.1.2 the explicit form of the mesinos. Moreover, we have explicitly mapped the mesinos to the fermionic fluctuations in section 2.5.2.3, borrowing the results of chapter 5 .

In addition, in section 2.3 we have performed the holographic renormalization for fermions in AdS in order to get a finite partition function and finite renormalized one-point functions. We have explicitly determined the counterterms for massive fermions and compute the renormalized one- and two-point functions in Euclidean signature. Finally, we describe in section 3.2 .4 a new efficient algorithm for computing retarded Green's functions from bulk fermions that couple to one another.

Using the above ingredients, we have numerically computed the fermionic spectral functions in the background used for p-wave superconductors in chapter 4. We have been able to determine the spectral measure, i.e. the trace of the retarded Green's function, but also single components of it. For simplicity, we have considered only the spectral measure in chapter 5 and have left a full-detailed study of the components of the retarded Green's function for the future.

For low temperatures, we have observed an emerging Fermi surface as we cool down the system in the normal phase. However, we know that the normal phase is not thermodynamically preferred. The thermodynamically preferred state is a holographic p-wave superfluid. If we cool the system through the p-wave superfluid transition, the Fermi surface collapses to isolated points.

Using the machinery that we have developed, we can think of a number of directions for 
future research.

Introducing a magnetic field on the worldvolume of a probe Dp-brane is straightforward to do, and has many interesting consequences [3,120,122-126, 130,131, 133, 223]. Our embedding of p-wave superfluids, and of charged fermions, into string theory may be a useful arena in which to study the effects of magnetic fields both on holographic pwave superfluids, along the lines of [3], and on holographic Fermi surfaces, along the lines of [224-227].

Typically the mesinos carry some R-charge, so another avenue to study holographic Fermi surfaces would be to embed probe Dp-branes into background geometries corresponding to field theory states with finite R-charge density. In those cases the $T=0$ limit is accessible within the probe approximation. These geometries are generically charged dilatonic black hole solutions. The most attractive of such solutions, for condensed matter applications, may be the solution discussed in [228], which produced in the field theory specific heats linear in temperature.

We have focused in chapter 5 on probe D5-branes extended along $A d S_{4} \times S^{2}$ inside $A d S_{5} \times$ $S^{5}$, and have worked only with the massless worldvolume fermion. With the methods presented in the thesis, the Fermi surfaces of other types of charge carriers, given by other Dp-branes, and worldvolume fermions of other masses, deserve further study.

\section{Charge carriers in $(2+1)$-dimensional Chern-Simons Theory}

In chapter 7 we have studied a large class of supersymmetric flavor branes in the brane construction of the ABJM theory, and have provided a general method to derive the corresponding field theories. We have applied our method to two different examples. We first have studied codimension-zero $\mathcal{N}=3$ supersymmetric flavor, which appeared in the supergravity description as KK-monopoles in M-Theory or as D6-branes in the type IIA limit. We then have studied codimension-one chiral $\mathcal{N}=(0,6)$ supersymmetric flavor, which appeared in supergravity as a D7/D8/M9-brane.

For both cases, on the field theory side we have written the kinetic terms and couplings of the flavor fields explicitly and have matched the symmetries to the supergravity description. Finally, we have argued how in general different probe branes in type IIB can become physically equivalent in M-theory and therefore give rise to the same field theory.

Many extensions and generalizations are possible. We have not explored the matching of supergravity fields to field theory operators. Many deformations are also possible, for example, we can give the flavor fields a supersymmetric or non-supersymmetric mass in various ways. A nonzero mass, which breaks scale invariance, would allow us to compute meson spectra along the lines of [89]. We can also deform the background, for example by replacing $\mathbb{C}^{4}$ with a cone over some non-trivial seven-dimensional manifold, such that the theory to which we add flavor would have reduced supersymmetry. We can also ask what role flavor fields play in various dualities, such as mirror symmetry [229]. More general types of probe branes are also possible [230], for example the author of [231] studied the addition of co-isotropic codimension zero probe D8-branes on the gravity side, and the authors of [232] studied more generally the addition of codimension-one domain walls. Many applications are also possible, especially in the context of hydrodynamics and/or 
condensed matter physics, for example along the lines of $[127,174,233]$.

Another particularly interesting extension of our work would be to study backreaction effects. The effect of the KK monopoles on the metric of eleven-dimensional supergravity has already been studied in $[220,221,234]$. As stated in $[222,235,236]$, when we include backreaction the D8-brane will source the Romans mass (Ramond-Ramond zero-form field strength), which means that the sum of the Chern-Simons levels of the two gauge groups will no longer be zero.

Our work is only the tip of the iceberg. We can now use the results of chapter 7 to study condensed matter systems near quantum critical points which are effectively described by Chern-Simons theories. The first step towards it is to investigate the phase diagram of Chern-Simons matter theories with a finite baryon or isospin density. Then, we can study fractional Quantum Hall liquids or Luttinger liquids using these models.

\section{Outlook}

In this thesis we have studied strongly coupled theories with a finite density of charge carriers. We have extended the AdS/CFT correspondence towards condensed matter applications by embedding holographic superconductors and (non-)Fermi liquids in string theory. In particular, we have used a top-down approach in which the field theory is explicitly known, as opposed to the bottom-up approach which we discussed in section 1.3 .

A main advantage of the approach used here is that we can compare the results of this thesis, which were obtained at strong coupling, to a perturbative analysis. Therefore we should investigate for example the phase diagram of the field theory for finite chemical potential, temperature and mass of the flavor fields perturbatively. As we discussed in section 4.2, we expect that a meson condense below a critical temperature. But does the critical temperature at weak coupling agree with the critical temperature at strong coupling? Do we also observe a vector meson condensate. What happens to the Fermi surface in the superconducting phase at weak coupling? Does it also collapse to points? What about the non-Fermi liquid behavior we observed?

As emphasized in the introduction, we do not claim to make quantitative predictions in this thesis. However, gauge/gravity dualities are a very convenient tool for discovering universal features. The prime example is, of course, the ratio of the shear viscosity, $\eta$, to the entropy density, $s$. For any strongly-coupled, large- $N_{c}$ gauge theory $\eta / s$ is given by $\eta / s=1 /(4 \pi)[237]$. The smoking gun for High- $T_{c}$ superconductors is Homes' law, which we discussed in section 1.2. Is this really a universal law? Do we find violations of this law in holographic models or, even better, can we find evidence in favor of it? These are natural questions we can ask within the framework of the AdS/CFT correspondence.

Phase transitions can easily be studied in holographic systems. The partition function is given as the exponent of the on-shell action of the corresponding gravity action. In the string theory embedding studied in this thesis, the action is given by the sum of type IIB supergravity and the effective action of the Dp-branes. In the most cases, the phase transition can even be interpreted in a geometric way. Note that most holographic phase transitions are either first order or second order with mean field critical exponents. 
Although higher order phase transitions are not forbidden by any limit we take, but they are not very common. An example of a third order phase transition was already mentioned in the thesis. The meson-melting phase transition above a critical value for the chemical potential is third order.

The mean-field behavior of second order phase transitions can be traced back to the large$N_{c}$ limit of the quantum critical theory. Therefore an obvious question is whether it is possible to study non-mean field phase transitions in holographic systems using probe Dp-branes?

So far we have also considered second order phase transitions in effectively (2+1)-dimensional systems at finite temperature, although, according to the Coleman-Mermin-WagnerHohenberg theorem, there should not be such a phase transition since quantum fluctuations may destroy it. But since we work in the large- $N_{c}$ limit, such quantum fluctuations are suppressed in $1 / N_{c}$. Therefore an interesting question is whether we can do calculations beyond the large- $N_{c}$ limit.

Another question which deserves future study is if it is possible to go beyond Landau's description of a phase transition in holographic systems. According to Landau's theory, an order parameter is associated with each phase transition. However, since the 1980's phase transitions are known which cannot be described by local order parameters or long-ranged correlations, since the order of the phase is of topological nature.

To conclude, this $\mathrm{PhD}$ thesis is only the starting point of applying the AdS/CFT correspondence to condensed matter systems, in particular using probe Dp-branes in the top-down approach. There are further interesting questions which await to be answered using holographic techniques. 


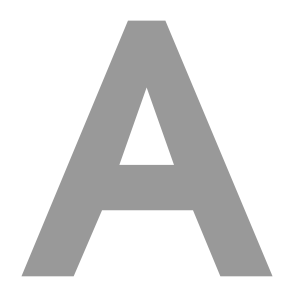

Glossary

Here we explain the most important technical terms of string theory such that the reader who is not familiar with this theory can follow and understand the main points of the thesis. 1 In section A.1 we review the basics of bosonic strings. In particular we discuss the Polyakov action of a fundamental string, the equations of motions as well as the different possible boundary conditions for fundamental strings. After quantizing the string we discuss string perturbation theory and strings in curved backgrounds. Thereafter the generalization to superstring theory is discussed in section A.2. In particular, type IIA and type IIB superstring theories are defined. Moreover, the low-energy theory of type IIB superstring theory, which is called type IIB supergravity, is mentioned. Subsequently, a few examples of dualities are discussed which connect the five consistent string theories: T-duality and S-duality. Finally, in section A.3, D-branes are introduced and their double life - being soliton-like solutions of supergravity and hyperplanes where open strings can end - is discussed in detail.

\section{A.1 Bosonic String Theory}

Whereas in conventional quantum field theory the elementary particles are pointlike objects, the fundamental objects in perturbative string theory are one-dimensional strings. As a string evolves in time, it sweeps out a two-dimensional surface in spacetime, the worldsheet of the string, which is the string counterpart of the world-line for a point particle. To parameterize the worldsheet of the string, two parameters are needed: the worldsheet time coordinate $\tau=\sigma^{0}$, which parameterizes the world-line in the case of a pointlike particle, and $\sigma=\sigma^{1}$ parameterizing the spatial extent of the string. The embedding of the worldsheet of the fundamental string into (target) spacetime is given by the functions $X^{\mu}(\tau, \sigma)$, which are also referred to as the embedding functions or target spacetime string coordinates. Since the action of a pointlike particle is given by the length

\footnotetext{
${ }^{1}$ Parts of the glossary was published in [4].
} 
of the world-line, the natural generalization to the action of a string propagating through flat spacetime is given by the area of the worldsheet,

$$
S=-\frac{1}{2 \pi \alpha^{\prime}} \int d^{2} \sigma \sqrt{-\operatorname{det} \partial_{\alpha} \mathrm{X}^{\mu} \partial_{\beta} \mathrm{X}_{\mu}}
$$

where $d^{2} \sigma=d \sigma^{0} d \sigma^{1}=d \tau d \sigma$. This is the Nambu-Goto action of a fundamental string. The determinant is taken with respect to $\alpha, \beta=0,1$, where $\alpha$ and $\beta$ label the worldsheet coordinates. Moreover we use the short hand notation $\partial_{\alpha}=\frac{\partial}{\partial \sigma^{\alpha}}$. The only free parameter appearing in this action is $\alpha^{\prime}$, which is related to the length of the string, $\alpha^{\prime}=l_{s}^{2}$. The dimensionful prefactor $T=\left(2 \pi \alpha^{\prime}\right)^{-1}$ can be interpreted as the string tension or the energy per length. To get rid of the square root in the action of the fundamental string in view of quantization, an auxiliary field $h_{\alpha \beta}\left(\sigma^{0}, \sigma^{1}\right)$ is introduced, which has to satisfy constraints given below. This gives rise to the Polyakov action,

$$
S=-\frac{1}{4 \pi \alpha^{\prime}} \int d^{2} \sigma \sqrt{-h} h^{\alpha \beta} \partial_{\alpha} X^{\mu} \partial_{\beta} X_{\mu},
$$

which is classically equivalent to (A.1.1) using the equations of motion of $h_{\alpha \beta}$. In (A.1.2), $h$ is the determinant of the matrix $h_{\alpha \beta}$ and $h^{\alpha \beta}$ is the inverse matrix of $h_{\alpha \beta}$, i.e. $h^{\alpha \beta} h_{\beta \gamma}=\delta_{\gamma}^{\alpha}$. The auxiliary field $h_{\alpha \beta}$ is called the worldsheet metric. The Polyakov action is invariant under the following symmetries:

\section{- Poincaré transformations}

These transformations are global symmetries of the worldsheet fields $X^{\mu}$ of the form

$$
\delta X^{\mu}=\Lambda_{\nu}^{\mu} X^{\nu}+a^{\mu} \quad \text { and } \quad \delta h^{\alpha \beta}=0,
$$

where $\Lambda_{\nu}^{\mu}$ and $a^{\mu}$ are Lorentz transformations and space-time translations respectively.

\section{- Reparametrizations}

The Polyakov action is invariant under reparametrizations since a change in the worldsheet parameterization of the form $\sigma^{\alpha} \rightarrow f^{\alpha}(\sigma)=\sigma^{\prime \alpha}$ with

$$
h_{\alpha \beta}(\sigma)=\frac{\partial f^{\gamma}}{\partial \sigma^{\alpha}} \frac{\partial f^{\delta}}{\partial \sigma^{\beta}} h_{\gamma \delta}\left(\sigma^{\prime}\right) \quad \text { and } \quad X^{\prime \mu}\left(\tau^{\prime}, \sigma^{\prime}\right)=X^{\mu}(\tau, \sigma)
$$

does not change the action.

- Weyl transformations

The action is also invariant under rescaling of the worldsheet metric $h_{\alpha \beta}$

$$
h_{\alpha \beta} \rightarrow e^{\omega(\sigma, \tau)} h_{\alpha \beta} \quad \text { and } \quad \delta X^{\mu}=0 .
$$

Since this transformation is a local symmetry of the action, the energy-momentum tensor of the field theory defined on the worldsheet is traceless, i.e. $T_{a}^{a}=0$. After quantization, Weyl Symmetry is potentially broken by a conformal anomaly. In string theory, this anomaly has to be absent, which is only the case if the spacetime dimension of the target space is $D=26$ for bosonic string theory. Moreover there are restrictions on the form of the background fields allowed (see section A.1.4). 
The local symmetries may be used to choose a gauge which brings the components of the worldsheet metric into a simple form. In particular, the equations of motion of the action can be simplified by choosing the gauge

$$
h_{\alpha \beta}=\eta_{\alpha \beta}=\left(\begin{array}{cc}
-1 & 0 \\
0 & 1
\end{array}\right) \text {. }
$$

In this and other conformal gauges, the equation of motion for $X^{\mu}(\tau, \sigma)$ is a relativistic wave equation,

$$
\left(\partial_{\tau}^{2}-\partial_{\sigma}^{2}\right) X^{\mu}=0
$$

supplemented by the Virasoro constraints

$$
\begin{aligned}
\partial_{\tau} X^{\mu} \partial_{\sigma} X_{\mu} & =0, \\
\partial_{\tau} X^{\mu} \partial_{\tau} X_{\mu}+\partial_{\sigma} X^{\mu} \partial_{\sigma} X_{\mu} & =0 .
\end{aligned}
$$

These constraints are derived from the equations of motion of the auxiliary field $h_{\alpha \beta}$ in the Polyakov action and have to be satisfied to ensure the equivalence of the two actions (A.1.1) and (A.1.2) at the classical level.

\section{A.1.1 Open and closed strings}

By applying variational methods, it is possible to derive not only the equations of motion but also the possible boundary conditions for the string. There are two different types of strings: open and closed strings.

\section{A.1.1.1 Closed strings}

Closed strings are topologically equivalent to a circle, i.e. they do not have endpoints. If we parameterize these strings by the parameter $\sigma \in[0,2 \pi[$, the boundary conditions read

$$
X^{\mu}(\tau, 0)=X^{\mu}(\tau, 2 \pi), \quad \partial_{\sigma} X^{\mu}(\tau, 0)=\partial_{\sigma} X^{\mu}(\tau, 2 \pi), \quad h^{\alpha \beta}(\tau, 0)=h^{\alpha \beta}(\tau, 2 \pi) .
$$

This means that the string coordinates $X^{\mu}$ are periodic, i.e. the endpoints are joined to form a closed loop. The mode expansion for the closed string is simply given by a pair of left and right-moving waves, which travel around the string in opposite directions,

$$
X^{\mu}(\tau, \sigma)=X_{R}^{\mu}(\tau-\sigma)+X_{L}^{\mu}(\tau+\sigma)
$$

$X_{R}\left(X_{L}\right)$ are the right (left) moving parts, respectively. The mode decompositions of the left and right-moving parts are given by

$$
X_{R}^{\mu}(\tau-\sigma)=\frac{1}{2} x_{0}^{\mu}+\alpha^{\prime} p_{R}^{\mu}(\tau-\sigma)+i \sqrt{\frac{\alpha^{\prime}}{2}} \sum_{n \neq 0} \frac{1}{n} \alpha_{n}^{\mu} e^{-2 i n(\tau-\sigma)}
$$

and

$$
X_{L}^{\mu}(\tau+\sigma)=\frac{1}{2} x_{0}^{\mu}+\alpha^{\prime} p_{L}^{\mu}(\tau+\sigma)+i \sqrt{\frac{\alpha^{\prime}}{2}} \sum_{n \neq 0} \frac{1}{n} \tilde{\alpha}_{n}^{\mu} e^{-2 i n(\tau+\sigma)} .
$$


$x_{0}^{\mu}$ and $p^{\mu}$ are the center of mass position and momentum of the string, respectively. The periodicity condition requires that $p_{R}^{\mu}=p_{L}^{\mu}$, and reality of $X^{\mu}$ requires the conditions $\alpha_{-n}^{\mu}=\left(\alpha_{n}^{\mu}\right)^{\star}$ and $\tilde{\alpha}_{-n}^{\mu}=\left(\tilde{\alpha}_{n}^{\mu}\right)^{\star}$. Moreover the center of mass momentum $p^{\mu}$ can be identified with the zero mode of the expansion by

$$
\alpha_{0}^{\mu}=\tilde{\alpha}_{0}^{\mu}=\sqrt{\frac{\alpha^{\prime}}{2}} p^{\mu} .
$$

\section{A.1.1.2 Open strings}

For open strings, two different boundary conditions in each direction $\mu$ of the spacetime are possible, Neumann or Dirichlet boundary conditions. In the case of Neumann boundary conditions, the component of the momentum normal to the boundary of the worldsheet vanishes, i.e.

$$
\partial_{\sigma} X_{\mu}(\tau, 0)=\partial_{\sigma} X_{\mu}(\tau, \pi)=0 .
$$

Note that the open string is now parametrized by $\sigma \in[0, \pi]$. The boundary condition implies that there is no momentum flowing through the ends of the string. The mode decomposition of the embedding function $X^{\mu}(\tau, \sigma)$ is given by

$$
X^{\mu}(\tau, \sigma)=x_{0}^{\mu}+2 \alpha^{\prime} p^{\mu} \tau+i \sqrt{2 \alpha^{\prime}} \sum_{n \neq 0} \frac{1}{n} \alpha_{n}^{\mu} e^{-i n \tau} \cos (n \sigma) .
$$

Due to the Neumann boundary condition, the left and right-moving waves of an open string are reflected into each other. As in the case of the closed string, the center of mass momentum $p^{\mu}$ of the string can be identified with the zero mode $\alpha_{0}^{\mu}$ of the expansion,

$$
\alpha_{0}^{\mu}=\sqrt{2 \alpha^{\prime}} p^{\mu} .
$$

If we choose Dirichlet boundary conditions along the $\mu$ direction of spacetime, the endpoints of the string are fixed, i.e.

$$
X^{\mu}(\tau, 0)=X^{\mu}(\tau, \pi)=x_{0}^{\mu}
$$

where $x_{0}^{\mu}$ is a constant. The mode decomposition is then given by

$$
X^{\mu}(\tau, \sigma)=x_{0}^{\mu}+\sqrt{2 \alpha^{\prime}} \sum_{n \neq 0} \frac{1}{n} \alpha_{n}^{\mu} e^{-i n \tau} \sin (n \sigma) .
$$

The string coordinate $X^{\mu}$ is real if the usual property $\left(\alpha_{n}^{\mu}\right)^{\star}=\alpha_{-n}^{\mu}$ holds. Note that the zero mode $\alpha_{0}^{\mu}$ is not present in directions where Dirichlet boundary conditions are imposed, since the center of mass momentum of the string vanishes.

The modern interpretation of open string boundary conditions is that they correspond to hyperplanes, so-called $D p$-branes, on which open strings can end. In $p$ spatial dimensions and in the time direction, Neumann boundary conditions are implemented, whereas in the remaining $26-(p+1)$ dimensions Dirichlet boundary conditions are used. We will have a closer look at D-branes in section A.2 when we discuss T-duality and in section A.3. 


\section{A.1.2 Quantization of bosonic string theory}

The theory can be quantized by using the standard commutation relations for the fields $X^{\mu}$ and the momentum $P^{\mu}$, which is conjugate to $X^{\mu}$. These commutation relations imply commutation relations for creation and annihilation operators, $\alpha_{n}^{\mu}$ and $\tilde{\alpha}_{n}^{\mu}$, acting on the ground state of the fundamental string: 2

The masses squared $M^{2}$ of the excited states are

$$
M^{2}=\frac{1}{\alpha^{\prime}}(N-1)
$$

for open strings and

$$
M^{2}=\frac{2}{\alpha^{\prime}}(N+\bar{N}-2)
$$

for closed strings. $N$ and $\bar{N}$ are the mass levels and are given by

$$
N=\sum_{n=1}^{\infty} \alpha_{-n}^{\mu} \alpha_{n \mu}, \quad \bar{N}=\sum_{n=1}^{\infty} \tilde{\alpha}_{-n}^{\mu} \tilde{\alpha}_{n \mu} .
$$

The mass levels $N$ and $\bar{N}$ have integer eigenvalues, which are also called $N$ and $\bar{N}$, respectively.

Physical string states of the closed string have to obey the level-matching condition $N=\bar{N}$ for the mass levels. Due to this condition, $\alpha_{n}^{\mu}$ and $\tilde{\alpha}_{n}^{\mu}$ are not independent. The spectrum of the closed string at the first two mass levels consists of

- $N=\bar{N}=0:$ tachyon with mass $M^{2}=-\frac{4}{\alpha^{\prime}}$.

- $N=\bar{N}=1$ : a rank two massless tensor field, which can be decomposed into an antisymmetric part $B_{\mu \nu}$ (the Kalb-Ramond field), a symmetric traceless part $g_{\mu \nu}$ (the graviton) and the trace of the symmetric part $\phi$ (the dilaton).

Since every string theory involves closed strings, a rank two symmetric tensor field, which will be identified with the graviton in section A.1.4, is necessarily incorporated in string theory. Moreover we will see that the vacuum expectation value of $\phi$ is related to the string coupling constant. The tachyon in the closed string spectrum is much more severe since it may indicate an instability of the theory. Such a tachyon will not appear in the spectrum of closed superstrings if we demand that supersymmetry is not explicitly broken in the embedding space.

The physical states of the open string ending on a Dp-brane at the first two mass levels are

- $N=0$ : A tachyon with $M^{2}=-\frac{1}{\alpha^{\prime}}$ appears in the spectrum. According to Sen's conjectures [238], this is related to the fact that in bosonic string theories, D-branes are unstable and will decay to radiation of closed strings.

- $N=1$ : A massless vector boson. This will give rise to a $U(1)$ gauge theory on the Dp-brane. Furthermore if $p<25$ massless scalars for each direction normal to the Dp-brane are found in the open string spectrum.

\footnotetext{
${ }^{2}$ We consider only non-interacting strings. The creation and annihilation operators can be considered as exciting internal degrees of freedom of the string.
} 


\section{A.1.3 String Perturbation Theory: Interactions and scattering ampli- tudes}

The Feynman path integral is a very natural method for describing interactions in string theory. In this approach amplitudes are given by summing over all worldsheets, weighted by the factor $\exp \left(i \frac{S}{\hbar}\right)$, which connect initial and final string configurations, as shown in figure A.1 for the closed string.

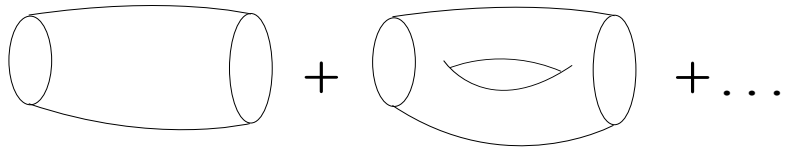

Figure A.1: Examples of worldsheets connecting initial and final string configurations.

For closed oriented strings, to which we restrict ourselves in this section, the sum is taken over all oriented two-dimensional worldsheets without boundaries. To take open strings into account, worldsheets with boundaries have to be included. Interactions of the strings are already implicit in the sum over worldsheets. The worldsheet of a decay of one closed string into two is given in figure A.2. Thus the worldsheet is similar to a Feynman diagram in which propagator lines are replaced by cylinders. A loop now corresponds to a handle of the worldsheet. An example is shown in figure A.3. The partition function $\mathcal{Z}$, i.e. the integral over all (Euclidean) worldsheet metrics $h_{\alpha \beta}$ and over all embeddings $X^{\mu}(\tau, \sigma)$ is given by, with $\hbar=1$,

$$
\mathcal{Z}=\int\left[d X^{\mu}\right]\left[d h_{\alpha \beta}\right] \exp (-S) .
$$

The Euclidean action $S$ contains the usual Polyakov action $S_{p}$, supplemented by a topological term weighting the different topologies of the string worldsheet $\Sigma$,

$$
S=S_{p}+\lambda \chi \quad \text { with } \quad \chi=\frac{1}{4 \pi} \int_{\Sigma} d^{2} \sigma \sqrt{h} R_{(h)},
$$

where $R_{(h)}$ is the Ricci scalar of the worldsheet metric $h_{\alpha \beta}$. Since $\chi$ is a topological term measuring the Euler number of the worldsheet, it does not contribute to the equations of motion. The factor $\exp (-\lambda \chi)$ in the path integral only affects the relative weighting of different topologies. Adding a handle to any worldsheet reduces the Euler number by two and therefore adds a factor of $\exp (2 \lambda)$. Since the process which is described by adding a handle corresponds to emitting and reabsorbing a closed string, the coupling constant of a closed string is given by $g_{\text {closed }}=\exp (\lambda)$. By analogous arguments, a string coupling constant $g_{\text {open }}$ of open strings can be introduced, which is related to $g_{\text {closed }}$ by

$$
g_{s} \equiv g_{\text {closed }}=g_{\text {open }}^{2}=e^{\lambda} .
$$

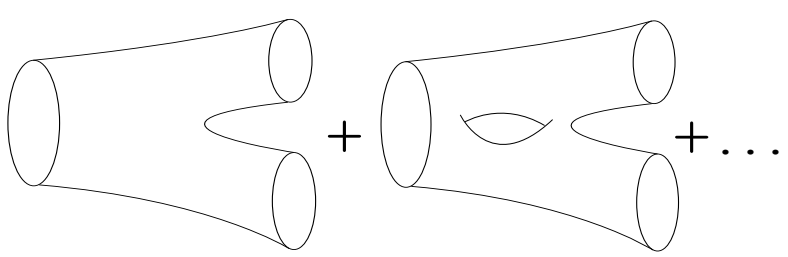

Figure A.2: Joining and splitting of strings. 

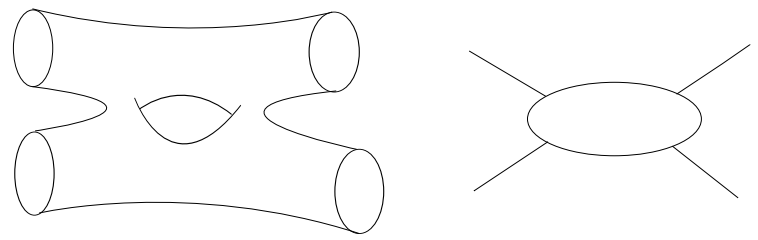

Figure A.3: Comparison between Feynman diagrams of quantum field theory and interacting string diagrams.

In section A.1.4, we will see that the string coupling constant is fixed by the vacuum expectation value of the dilaton field.

\section{A.1.4 Bosonic string theory in background fields}

Up to now we considered the propagation of open and closed strings in Minkowski spacetime. By coupling the fundamental string to the massless closed string excitations (see section A.1.2), strings propagating through curved spacetimes can be described. In particular we will see that the massless closed string excitation $g_{\mu \nu}-$ which is traceless and symmetric - can be identified with the metric of the target spacetime. Since the quantized string theory in curved spacetime should be Weyl invariant, we obtain restrictions on the target spacetime allowed: The spacetime has to satisfy the vacuum Einstein equations (at least in lowest order of $\alpha^{\prime}$ ). This is the goal of this section.

Now we will generalize the (Euclidean) Polyakov action in a simple manner to take into account couplings to the massless closed string excitations: the antisymmetric tensor field $B_{(2)}$ (the components are called $\left.B_{\mu \nu}\right) \cdot 3$ the symmetric traceless component $g_{\mu \nu}$ as well as the trace $\phi$. Since $g_{\mu \nu}$ is symmetric and traceless, the only possibility to couple it to the string (given by $X^{\mu}$ ) is

$$
S_{P}=-\frac{1}{4 \pi \alpha^{\prime}} \int d^{2} \sigma \sqrt{h} h^{\alpha \beta} \partial_{\alpha} X^{\mu} \partial_{\beta} X^{\nu} g_{\mu \nu}\left(X^{\rho}\right) .
$$

We see that this equation is a generalization of the Polyakov action (A.1.2) to curved target spacetimes. Furthermore we can couple the Kalb-Ramond field $B_{\mu \nu}\left(X^{\rho}\right)$ and the dilaton field $\phi\left(X^{\rho}\right)$ to the fundamental string by adding

$$
S_{B, \phi}=\frac{1}{4 \pi \alpha^{\prime}} \int d^{2} \sigma \sqrt{h}\left(i \epsilon^{\alpha \beta} \partial_{\alpha} X^{\mu} \partial_{\beta} X^{\nu} B_{\mu \nu}\left(X^{\rho}\right)+\alpha^{\prime} R_{(h)} \phi\left(X^{\rho}\right)\right)
$$

to the Polykov action (A.1.26), where $R_{(h)}$ is the Ricci scalar with respect to the worldsheet metric $h_{\alpha \beta}$. Comparing the dilaton dependent part of $S_{B, \phi}$ to (A.1.24), we see that the dilaton sets the string coupling constant. Using (A.1.25) the string coupling constant $g_{s}$ is given by

$$
g_{s}=e^{\phi} .
$$

Moreover, for ensuring Weyl invariance of the quantum theory (see remark after (A.1.5)), we impose the tracelessness of the energy momentum tensor of the worldsheet theory in

\footnotetext{
${ }^{3}$ Throughout the thesis we denote the number of indices of an antisymmetric tensor field by numbers in brackets. Therefore $B_{(2)}$ has two indices. If we exchange the indices, we obtain a minus sign, i.e. $B_{\mu \nu}=-B_{\nu \mu}$. Since the number of indices are apparent in the component notation, this number is omitted.
} 
$D=26$ dimensions,

$$
T_{a}^{a}=-\frac{1}{2 \alpha^{\prime}} \beta_{\mu \nu}^{g} h^{a b} \partial_{a} X^{\mu} \partial_{b} X^{\nu}-\frac{i}{2 \alpha^{\prime}} \beta_{\mu \nu}^{B} \epsilon^{a b} \partial_{a} X^{\mu} \partial_{b} X^{\nu}-\frac{1}{2} \beta^{\phi} R
$$

with

$$
\begin{aligned}
& \beta_{\mu \nu}^{g}=-\alpha^{\prime}\left(R_{\mu \nu}+2 \nabla_{\mu} \nabla_{\nu} \phi-\frac{1}{4} H_{\mu \rho \sigma} H_{\nu}{ }^{\rho \sigma}\right)+\mathcal{O}\left(\alpha^{\prime 2}\right), \\
& \beta_{\mu \nu}^{B}=\alpha^{\prime}\left(-\frac{1}{2} \nabla^{\rho} H_{\rho \mu \nu}+\nabla^{\rho} \phi H_{\rho \mu \nu}\right)+\mathcal{O}\left(\alpha^{\prime 2}\right), \\
& \beta^{\phi}=\alpha^{\prime}\left(-\frac{1}{2} \nabla^{2} \phi+\nabla_{\rho} \phi \nabla^{\rho} \phi-\frac{1}{24} H_{\rho \mu \nu} H^{\rho \mu \nu}\right)+\mathcal{O}\left(\alpha^{\prime 2}\right)
\end{aligned}
$$

to lowest order of $\alpha^{\prime} . H_{\rho \mu \nu}$ are the components of the field strength $H_{(3)}$ of the KalbRamond field $B_{(2)}$, i.e.

$$
H_{\rho \mu \nu}=\partial_{\rho} B_{\mu \nu}+\partial_{\mu} B_{\nu \rho}+\partial_{\nu} B_{\rho \mu} .
$$

The Polyakov action leads to a Weyl-invariant quantum theory if all three functions $\beta_{\mu \nu}^{g}, \beta_{\mu \nu}^{B}$ and $\beta^{\phi}$ vanish. Remarkably, the consistency equations $\beta_{\mu \nu}^{g}=\beta_{\mu \nu}^{B}=\beta^{\phi}=0$ can be derived as equations of motion from the target spacetime action

$$
S=\frac{1}{2 \kappa_{0}} \int d^{26} X \sqrt{-g} e^{-2 \phi}\left[R+4 \nabla_{\mu} \phi \nabla^{\mu} \phi-\frac{1}{12} H_{\mu \nu \rho} H^{\mu \nu \rho}+\mathcal{O}\left(\alpha^{\prime}\right)\right] .
$$

This is the effective action for the massless string states $B_{\mu \nu}, g_{\mu \nu}$ and $\phi$ of the closed string sector, where the effects due to the tachyon are omitted. Here $R$ is the Ricci scalar of the symmetric tensor field $g_{\mu \nu}$ and $\nabla_{\mu}$ are the covariant derivatives.

As discussed above, the string coupling constant is given by the expectation value of the dilaton $g_{s}=e^{\phi}$. Moreover the massless rank two symmetric tensor field $g_{\mu \nu}$ can be identified with the graviton since $g_{\mu \nu}$ has to satisfy the equations of motion $\beta_{\mu \nu}^{g}=0$, which also follow immediately from the effective action. The first term in (A.1.34) is an Einstein-Hilbert term coupled to a dilaton. Therefore $g_{\mu \nu}$ is identified with the target spacetime metric (see also (A.1.26) $)$.

Moreover we can canonically normalize the Einstein-Hilbert term of the action (A.1.34). Rescaling the metric 4

$$
\tilde{g}_{\mu \nu}=e^{\frac{1}{6}\left(\phi_{0}-\phi\right)} g_{\mu \nu}
$$

the action (A.1.34) can be rewritten in the form

$$
S=\frac{1}{2 \kappa^{2}} \int d^{26} X \sqrt{-\tilde{g}}\left[\tilde{R}-\frac{1}{6} \nabla_{\mu} \tilde{\phi} \nabla^{\mu} \tilde{\phi}-\frac{1}{12} e^{-\frac{1}{3} \tilde{\phi}} H_{\mu \nu \rho} H^{\mu \nu \rho}+\mathcal{O}\left(\alpha^{\prime}\right)\right]
$$

with $\tilde{\phi}=\phi-\phi_{0}$ and $\kappa=\kappa_{0} e^{\phi_{0}}=\sqrt{8 \pi G_{N}}$. Looking at the part involving the Ricci scalar $\tilde{R}$, which is determined by the rescaled metric $\tilde{g}_{\mu \nu}$, we see that we have removed the factor involving the dilaton $\phi$ in the Einstein-Hilbert part of the action (A.1.36). Whereas the action written in terms of the original fields is called the string-frame action, the latter, canonically normalized action is referred to as the Einstein-frame action.

\footnotetext{
${ }^{4}$ The rescale of the metric depends on the dimension $D$ of the target spacetime. For simplicity we used here $D=26$.
} 
In view of coupling the open string to the Abelian gauge field $A_{\mu}$ living on a D-brane, we have to include a term of the form

$$
S_{A}=\int_{\partial \Sigma} d \tau A_{\mu}\left(X^{\rho}\right) \partial_{\tau} X^{\mu},
$$

where $\partial \Sigma$ denotes the boundary of the worldsheet $\Sigma$. The effective action of the open string sector, summarizing the leading order (in $\alpha^{\prime}$ ) open string physics at tree level, is given by 5

$$
S=-C \int d^{26} X e^{-\phi} \operatorname{Tr} F_{\mu \nu} F^{\mu \nu},
$$

where $C$ is a dimensionful constant. Therefore the physics of the open string sector at tree level is described by Yang-Mills theories. In the case of one D-brane the gauge group is $U(1)$, but can be generalized to non-Abelian gauge groups. In the section A.3.1 we will discuss the effective action of D-branes, which determines the open string physics.

\section{A.1.5 Chan-Paton factors}

So far we have seen that open strings on one Dp-brane are described by a $U(1)$ gauge theory. In order to generalize this to non-Abelian gauge theories, Chan-Paton factors are introduced on a stack of coincident $N$ Dp-branes. Chan-Paton factors are non-dynamical degrees of freedom from the worldsheet point of view, which are assigned to the endpoints of the string. These factors label the open strings that connect the various coincident D-branes. For example, the Chan-Paton factor $\lambda_{i j}$ labels strings stretching from brane $i$ to brane $j$, with $i, j \in\{1, \ldots, N\}$. The resulting matrix $\lambda$ is an element of a Lie algebra. It turns out that the only Lie algebra consistent with open string scattering amplitudes is $U(N)$ in the case of oriented strings, where $N$ is the number of coincident D-branes. Therefore $\lambda$ can be chosen as a hermitean matrix and $\lambda_{i j}$ are the corresponding entries of the matrix.

Although the Chan-Paton factors are global symmetries of the worldsheet action, the symmetry turns out to be local in the target spacetime. The theory of open strings ending on coincident D-branes can effectively be described by a non-Abelian gauge theory.

\section{A.2 Superstring Theory}

The bosonic string theory is unsatisfactory in two respects. Since we observe fermions in nature, these particles should not be excluded in string theory. Moreover the bosonic string theory is inconsistent because tachyons occur in the closed string spectrum. This indicates an severe instability of the theory.

Remarkably, both problems can be solved by incorporating supersymmetry into string theory. There are two different approaches to superstring theory: 6

\footnotetext{
${ }^{5}$ For details how to compute the effective D-Brane action see [239].

${ }^{6}$ Recently, various approaches using spinor formalism were suggested by Berkovits. For a review see [240].
} 
- The Green-Schwarz (GS) formalism is supersymmetric in ten-dimensional Minkowski spacetime, and can be generalized to curved background geometries with fluxes.

- The Ramond-Neveu-Schwarz (RNS) formalism is supersymmetric on the worldsheet of the fundamental string.

These approaches are equivalent at least in ten-dimensional Minkowski spacetime.

In this section the RNS approach to superstring theory is explained.

\section{A.2.1 The RNS formalism of superstring theory}

The Polyakov action of the bosonic string in $D$-dimensional Minkowski spacetime reads, in the conformal gauge $h_{\alpha \beta}=e^{\omega(\tau, \sigma)} \eta_{\alpha \beta}$,

$$
S=-\frac{1}{4 \pi \alpha^{\prime}} \int d^{2} \sigma \partial_{\alpha} X_{\mu} \partial^{\alpha} X^{\mu} .
$$

This action is supplemented by Virasoro constraints (A.1.8) and (A.1.9). For a supersymmetric worldsheet action, we have to introduce $D$ Majorana fermions $\psi^{\mu}$ transforming in the vector representation of the Lorentz group $S O(D-1,1)$. We therefore consider the Polyakov action supplemented by the usual Dirac action for $D$ free massless fermions,

$$
S=-\frac{1}{4 \pi \alpha^{\prime}} \int d^{2} \sigma\left(\partial_{\alpha} X_{\mu} \partial^{\alpha} X^{\mu}+\bar{\psi}^{\mu} \gamma^{\alpha} \partial_{\alpha} \psi_{\mu}\right) .
$$

Here, $\gamma^{\alpha}$ are two-dimensional Dirac matrices satisfying the anticommutation relations $\left\{\gamma^{\alpha}, \gamma^{\beta}\right\}=2 \eta^{\alpha \beta} \mathbb{1}$. A convenient basis is

$$
\gamma^{0}=\left(\begin{array}{cc}
0 & -1 \\
1 & 0
\end{array}\right) \quad \text { and } \quad \gamma^{1}=\left(\begin{array}{ll}
0 & 1 \\
1 & 0
\end{array}\right) .
$$

The worldsheet fields $\psi^{\mu}$ are Grassmann numbers consisting of two components

$$
\psi^{\mu}=\left(\begin{array}{c}
\psi_{-}^{\mu} \\
\psi_{+}^{\mu}
\end{array}\right),
$$

where $\psi_{-}^{\mu}$ and $\psi_{+}^{\mu}$ are real. In this notation, the fermionic part of the action takes the form

$$
S_{f}=\frac{i}{2 \pi \alpha^{\prime}} \int d^{2} \sigma\left(\psi_{-}^{\mu} \partial_{+} \psi_{-\mu}+\psi_{+}^{\mu} \partial_{-} \psi_{+\mu}\right),
$$

with $\partial_{-}=\frac{\partial}{\partial \sigma^{-}}, \partial_{+}=\frac{\partial}{\partial \sigma^{+}}$and $\sigma^{ \pm}=\tau \pm \sigma$. The equations of motion are $\partial_{+} \psi_{-}^{\mu}=\partial_{-} \psi_{+}^{\mu}=0$, which describe left- and right-moving waves. The action is invariant under the infinitesimal transformations

$$
\begin{aligned}
\delta_{\epsilon} X^{\mu} & =\bar{\epsilon} \psi^{\mu}, \\
\delta_{\epsilon} \psi^{\mu} & =\gamma^{\alpha} \partial_{\alpha} X^{\mu} \epsilon,
\end{aligned}
$$

where $\epsilon$ is a constant infinitesimal Majorana spinor. This transformation mixes bosonic and fermionic worldsheet fields and is therefore a global supersymmetry transformation. Unfortunately, the supersymmetry algebra closes only on-shell, i.e. when the equations 
of motion are imposed. 7 Note that in the case of superstrings, the critical dimension is $D=10$.

\section{A.2.2 Boundary conditions for fermions}

Next we consider the boundary conditions that arise from the superstring action. The possible boundary conditions of the bosonic fields $X^{\mu}$ are discussed in A.1.1. The boundary condition for the fermionic part reads

$$
\delta S_{f}=\int d \tau\left[\psi_{+}^{\mu} \delta \psi_{+\mu}-\psi_{-}^{\mu} \delta \psi_{-\mu}\right]_{\sigma=0}^{\sigma=\pi} .
$$

\section{Open strings}

For open strings we have to demand that the two terms for $\sigma=0$ and $\sigma=\pi$ vanish independently, i.e.

$$
\psi_{+}^{\mu} \delta \psi_{+\mu}-\psi_{-}^{\mu} \delta \psi_{-\mu}=0 \quad \text { for } \quad \sigma=0, \pi .
$$

Note that this is equivalent to

$$
\delta\left(\psi_{+\mu}\right)^{2}=\delta\left(\psi_{-\mu}\right)^{2} \quad \text { for } \quad \sigma=0, \pi .
$$

Since the overall sign of the components can be chosen arbitrarily, we demand $\psi_{+}^{\mu}(\tau, 0)=$ $\psi_{-}^{\mu}(\tau, 0)$. If we want to impose the boundary conditions at $\sigma=\pi$ we have two options corresponding to the Ramond (R) sector and the Neveu-Schwarz (NS) sector of the theory,

$$
\begin{aligned}
R: \psi_{+}^{\mu}(\tau, \pi) & =+\psi_{-}^{\mu}(\tau, \pi), \\
N S: \psi_{+}^{\mu}(\tau, \pi) & =-\psi_{-}^{\mu}(\tau, \pi) .
\end{aligned}
$$

The mode decomposition in the R and NS sector is given by

$$
\begin{aligned}
R: \psi_{\mp}^{\mu}(\tau, \sigma) & =\frac{1}{\sqrt{2}} \sum_{n \in \mathbb{Z}} d_{n}^{\mu} e^{-i n \sigma_{\mp}}, \\
N S: \psi_{\mp}^{\mu}(\tau, \sigma) & =\frac{1}{\sqrt{2}} \sum_{r \in \mathbb{Z}+\frac{1}{2}} b_{r}^{\mu} e^{-i r \sigma_{\mp}},
\end{aligned}
$$

where $d_{n}^{\mu}$ and $b_{r}^{\mu}$ are Grassmann numbers.

The string states are constructed by acting on the ground state of the NS and R sector with creation operators. The ground state in the NS sector $|0\rangle_{N S}$ is a spacetime boson and therefore all string states in this sector are bosonic in spacetime since the oscillators act as vectors in spacetime. Furthermore the ground state of the NS sector is tachyonic and will be removed. The first excited string state, which is generated by applying a creation operator to the ground state of the NS sector, we obtain a massless vector boson.

By contrast, the ground states in the R sector, which are massless spacetime fermions, are degenerate and differ by chirality in spacetime. By applying creation operators to the

\footnotetext{
${ }^{7}$ However, closure of the algebra can be achieved by introducing auxiliary fields. Moreover we used the worldsheet theory in conformal gauge. There is a more fundamental formulation in which the worldsheet supersymmetry is a local symmetry.
} 
ground state of the NS sector, massive string states are obtained. Moreover all states of the $\mathrm{R}$ sector are spacetime fermions.

The spectrum of the NS and R sector can be truncated in a specific way which eliminates the tachyon. This truncation is called GSO projection, named after Gliozzi, Scherk and Olive [241]. The GSO projection also ensures that the partition function on the twotorus is modular invariant. In the NS sector only states with an odd number of creation operators $b_{-r}^{\mu}, r>0$ applied to the ground state $|0\rangle_{N S}$ are kept in the spectrum. The GSO projection leaves an equal number of bosons and fermions at each mass level, as required by spacetime supersymmetry. At the massless level the states of the NS sector are massless gauge bosons, whereas the $\mathrm{R}$ sector includes the supersymmetric partner of the gauge boson, the gaugino.

\section{Closed string}

As we saw in bosonic string theory, a closed string consists essentially of left- and rightmoving copies of an open string. Since an open superstring has two different sectors (NS and $\mathrm{R}$ ), the closed string sector can be constructed in four ways by combining the leftmoving sector (NS and R) and the right-moving one (NS and R). The NS-NS and R-R states are spacetime bosons, whereas the NS-R and R-NS states are spacetime fermions. Applying a GSO projection as in the open superstring case lead to a supersymmetric theory in spacetime.

The NS-NS sector of oriented strings includes at the massless level exactly the same states as the closed sector of the oriented bosonic string theory: the graviton $g_{\mu \nu}$, the KalbRamond field $B_{\mu \nu}$ and the dilaton $\phi$. The NS-R and R-NS states, which are fermionic in spacetime, contain the gravitino, the supersymmetric partner of the graviton, and the dilatino, the supersymmetric version of the dilaton. The story for the $\mathrm{R}-\mathrm{R}$ sector is a little more subtle due to the degeneracy of ground states of the R-sector. We will see that two different superstring theories are obtained: type IIA and type IIB.

\section{A.2.3 Type IIA and type IIB superstring}

Since the R-sector has two possible inequivalent ground states, which differ by chirality, we can choose ground states with the same chirality for the left- and right-moving sector. This corresponds to type IIB superstring theory. The R-R sector consists of a scalar field $C_{(0)}$, an antisymmetric field $C_{(2)}$ and a totally antisymmetric rank four tensor field $C_{(4)}$ at the massless level ${ }^{8}$ If the $\mathrm{R}$ sector ground states for the left- and right-moving modes have different chiralities, we are lead to type IIA superstring theory. In the type IIA theory the massless R-R bosons are given by a gauge field $C_{(1)}$ and a totally antisymmetric rank three tensor field $C_{(3)} \cdot 9$

Although type IIA and type IIB superstring theories are inequivalent, there exist dualities between both theories. For example, T-duality relates type IIA and type IIB string theories

\footnotetext{
${ }^{8}$ The components of $C_{(0)}, C_{(2)}$ and $C_{(4)}$ are denoted by $C, C_{\mu \nu}$ and $C_{\mu \nu \rho \sigma}$, i.e. the number of indices is not explicitly specified for the components.

${ }^{9}$ The components of $C_{(1)}$ and $C_{(3)}$ are called $C_{\mu}$ and $C_{\mu \nu \rho}$ respectively.
} 


\begin{tabular}{|l|l|l|l|}
\hline Type & $\begin{array}{l}\text { number of } \\
\text { supercharges }\end{array}$ & bosonic massless fields & non-Abelian gauge group \\
\hline heterotic $S O(32)$ & 16 & $g_{\mu \nu}, \phi, B_{(2)}, A_{\mu}^{a}$ & $S O(32)$ \\
heterotic $E_{8} \times E_{8}$ & 16 & $g_{\mu \nu}, \phi, B_{(2)}, A_{\mu}^{a}$ & $E_{8} \times E_{8}$ \\
IIA & $16+\overline{16}$ & $g_{\mu \nu}, \phi, B_{(2)}, C_{(1)}, C_{(3)}$ & - \\
IIB & $16+16$ & $g_{\mu \nu}, \phi, B_{(2)}, C_{(0)}, C_{(2)}, C_{(4)}$ & - \\
I & 16 & $g_{\mu \nu}, \phi, A_{\mu}^{a}, C_{(2)}$ & $S O(32)$ \\
\hline
\end{tabular}

Table A.1: Bosonic massless fields of the five consistent superstring theories in ten spacetime dimensions. $g_{\mu \nu}$ and $\phi$ are the metric and the dilaton, respectively. The Kalb-Ramond field is denoted by $B_{(2)}$. Moreover in superstring theories there exist $p$-form gauge potentials $C_{(p)}$ in the $\mathrm{R}-\mathrm{R}$ sector of the closed string. In heterotic and type I superstring theories there are also nonAbelian gauge degrees of freedom $A_{\mu}^{a}$ present. The corresponding gauge groups are listed in the last column. By adding D-branes to string theories other gauge groups are possible.

on different target spacetimes. Moreover, in type IIB string theory there is strong-weak duality build in, known as S-duality.

Altogether, there are five consistent superstring theories in ten spacetime dimensions: type I, type IIA and type IIB as well as $S O(32)$ heterotic and $E_{8} \times E_{8}$ heterotic string theory. Although all these theories describe vibrating strings, the details are quite different. We will not discuss type I and the two heterotic string theory in this glossary. A few details of these theories may be found in table A.1.

\section{A.2.4 Low-energy effective action of superstring theory}

As in the bosonic case (see section A.1.4) we can write down a spacetime action taking into account the effects of the massless superstring excitations. These excitations are listed in table A.1. Since the effective supersymmetric action necessarily incorporates gravity, 10 the theory is called supergravity.

The low-energy effective action for type IIA and type IIB superstring theories are called type IIA and type IIB supergravity, respectively. As an example, we consider the action of type IIB supergravity. Type IIB superstring theory consists of the following closed string states at the massless level: the metric $g_{\mu \nu}$, the NS-NS Kalb-Ramond field $B_{\mu \nu}$, the dilaton $\phi$ as well as the $p$-form R-R potentials $C_{(0)}, C_{(2)}$ and $C_{(4)}$. Moreover we define as linear combinations of these fields the axion-dilaton scalar $\tau$ as well as the complex three-form $G_{(3)}$ by

$$
\tau=C_{(0)}+i e^{-\phi}, \quad G_{(3)}=F_{(3)}-\tau H_{(3)} .
$$

Here, $F_{(3)}$ and $H_{(3)}$ are the field strength of $C_{(2)}$ and $B_{(2)}$, i.e. $F_{(3)}=d C_{(2)}$ and $H_{(3)}=$ $d B_{(2)}$. The field strength of $C_{(4)}$ is given by $F_{(5)}=d C_{(4)}$. More important is the self-dual combination

$$
\tilde{F}_{(5)} \equiv F_{(5)}+\frac{1}{2} B_{(2)} \wedge F_{(3)}-\frac{1}{2} C_{(2)} \wedge H_{(3)}
$$

\footnotetext{
${ }^{10}$ These effective spacetime theories are not only invariant under global supersymmetry transformations, but also under local ones. Since the commutator of two supersymmetry transformations is a translation, the theory is also invariant under local diffeomorphisms and therefore contains gravity.
} 
The type IIB supergravity action in the Einstein frame then reads

$$
\begin{aligned}
S_{I I B} & =\frac{1}{2 \kappa_{10}^{2}} \int d^{10} x \sqrt{-g}\left[R-\frac{\left|\partial_{\mu} \tau\right|^{2}}{2(\operatorname{Im} \tau)^{2}}-\frac{\left|G_{(3)}\right|^{2}}{12 \operatorname{Im} \tau}-\frac{\left|\tilde{F}_{(5)}\right|^{2}}{4 \cdot 5 !}\right\} \\
& +\frac{1}{8 i \kappa_{10}^{2}} \int \frac{C_{(4)} \wedge G_{(3)} \wedge \bar{G}_{(3)}}{\operatorname{Im} \tau},
\end{aligned}
$$

where the ten-dimensional gravitational coupling is $2 \kappa_{10}^{2}=16 \pi G_{10}=\frac{1}{2 \pi}\left(2 \pi l_{s}\right)^{8} g_{s}^{2}$ and $l_{s}=\sqrt{\alpha^{\prime}}$ is the string length. Moreover we have to impose the self-duality constraint of $\tilde{F}_{(5)}$ at the level of the equations of motion by hand, i.e. $\star \tilde{F}_{(5)}=\tilde{F}_{(5)}$, where $\star$ denotes the ten-dimensional Hodge star operator.

\section{A.2.5 T-duality}

T-Duality (or target space duality) denotes the equivalence between two superstring theories compactified on different background spacetimes. For simplicity, let us consider only bosonic string theory compactified on a circle, i.e. the coordinate $X^{25}$ is periodically identified in the following way,

$$
X^{25} \sim X^{25}+2 \pi R
$$

\section{T-duality of closed strings}

Now let us restrict ourselves to closed strings. The embedding function $X^{25}(\tau, \sigma)$ has to satisfy the periodicity condition

$$
X^{25}(\tau, \sigma+2 \pi)=X^{25}(\tau, \sigma)+2 m \pi R
$$

where $R$ is the radius of the circle and $m$ is an arbitrary integer. The number $m$ counts how often the closed string winds around the compactified direction $X^{25}$ and is therefore called winding number. In the non-compactified directions, the mode decomposition (A.1.12) and (A.1.13) for the right and left-moving modes can be used subject to $p_{R}^{\mu}=p_{L}^{\mu}$. In the compactified direction the same mode decomposition can be applied, however now with $p_{R}^{25} \neq p_{L}^{25}$. Omitting the oscillatory terms, we have the decomposition

$$
\begin{aligned}
& X_{R}^{25}(\tau-\sigma)=\frac{1}{2} x_{0}^{\mu}+\alpha^{\prime} p_{R}^{\mu}(\tau-\sigma)+\ldots \\
& X_{L}^{25}(\tau+\sigma)=\frac{1}{2} x_{0}^{\mu}+\alpha^{\prime} p_{L}^{\mu}(\tau+\sigma)+\ldots
\end{aligned}
$$

Since $X^{25}=X_{L}^{25}+X_{R}^{25}$, the periodicity condition reads

$$
\alpha^{\prime}\left(p_{L}^{25}-p_{R}^{25}\right)=m R
$$

Since the $X^{25}$ direction is compactified, the center of mass momentum $p_{R}^{25}+p_{L}^{25}$ is quantized in units of $1 / R$, i.e.

$$
p_{L}^{25}+p_{R}^{25}=\frac{n}{R}
$$


Thus $p_{R}^{25}$ and $p_{L}^{25}$ are given by

$$
\begin{aligned}
p_{L}^{25} & =\frac{1}{2}\left(\frac{n}{R}+\frac{m R}{\alpha^{\prime}}\right), \\
p_{R}^{25} & =\frac{1}{2}\left(\frac{n}{R}-\frac{m R}{\alpha^{\prime}}\right) .
\end{aligned}
$$

We are now interested in the spectrum of the closed string states. First of all, the levelmatching condition (see section A.1.2) for the closed string is modified,

$$
\bar{N}-N=n m,
$$

and the mass formula for string states reads

$$
M^{2}=\left(\frac{m R}{\alpha^{\prime}}\right)^{2}+\left(\frac{n}{R}\right)^{2}+\frac{2}{\alpha^{\prime}}(N+\bar{N}-2) .
$$

However this is not the whole story. The closed string sector has an remarkable symmetry. Considering the mass formula, it turns out that the closed string spectrum for a compactification with radius $R$ is identical to the closed string spectrum for a compactification with radius $\tilde{R}=\alpha^{\prime} / R$ if we interchange the winding number $m$ and momentum number $n$,

$$
\begin{aligned}
R & \leftrightarrow \tilde{R}=\frac{\alpha^{\prime}}{R}, \\
(n, m) & \leftrightarrow(m, n) .
\end{aligned}
$$

Although here we have described the proof for T-duality only for free strings, it can be shown that T-duality of closed strings is an exact symmetry at the quantum level also if interactions are included.

In fact it is not possible to distinguish between both compactifications. Note that if $R$ is large, then the dual radius $\tilde{R}$ is small. This is a remarkable feature, which is not present in usual field theories of pointlike particles. Since T-duality exchanges the winding number on the circle with the quantum number of the corresponding (discrete) momentum, it is clear that this symmetry has no counterpart in ordinary point particle field theory, as the ability of closed strings to wind around the compact dimension is essential.

\section{T-duality of open strings}

At first sight, it seems that T-duality does not apply to theories with open strings, since open strings do not have a winding sector. However, this is only apparent [242]. T-duality can be restored in the open string sector with the help of D-branes which are hyperplanes where open strings end. By applying T-duality, not only the radius of the compactified dimension changes, but also the dimension of the D-brane.

To see this, let us consider the propagation of open bosonic strings in a spacetime which is compactified in the $X^{25}$ direction. Furthermore we assume for simplicity that we have a space-filling D25-brane, i.e. the endpoints of the string can move freely. As it was in the case of closed strings, the center of mass momentum in the compactified direction is quantized, i.e. $p^{25}=n / R$ and contributes terms of the form $n^{2} / R^{2}$ to the mass formula of 
string states. However, this contribution changes if we apply the T-duality rules of closed strings only. Since the dual radius is $\tilde{R}=\alpha^{\prime} / R$, the contribution to the mass formula changes to $n^{2} \tilde{R}^{2} / \alpha^{\prime 2}$.

T-duality can be restored in the open string sector by considering D-branes. Instead of the D25-brane described above, consider now a D24-brane in the dual theory, which does not wrap the $X^{25}$-direction. Due to the Dirichlet boundary conditions, we have no momentum states in the compact direction. In addition, the endpoints of the open string must remain attached to points with $x^{25}=x_{0}^{25}+2 \pi n \tilde{R}$, where $x_{0}^{25}$ is the position of the D24-brane in the compactified direction. Therefore we get winding states in the dual theory which contribute to the mass formula by

$$
\left(\frac{n \tilde{R}}{\alpha^{\prime}}\right)^{2}=\left(\frac{n}{R}\right)^{2}
$$

This is precisely the contribution of the momentum states in the original theory with a space filling D25-brane.

Therefore T-duality is an exact symmetry of the open string sector, if the dimension of the D-brane is also changed. This means that the type of boundary conditions of open strings (Neumann or Dirichlet) has to be exchanged in the direction in which T-duality is performed.

As an example consider a D24-brane stretched along the coordinates $X^{0}, X^{1}, \ldots, X^{24}$. In these directions Neumann boundary conditions for open strings are imposed. Moreover in the $X^{25}$ direction open strings will satisfy Dirichlet boundary conditions. Assuming that the $X^{24}$ and $X^{25}$ directions are compactified on circles with radius $R_{24}$ and $R_{25}$ respectively we can apply T-duality to both compact directions. If we perform a T-duality along $X^{25}$, the open strings in the dual theory in the $X^{25}$-direction obey also Neumann boundary conditions. Therefore in the dual theory a D25-brane exists and the radii of the two compactified directions are given by $R_{24}$ and $\alpha^{\prime} / R_{25}$, respectively. If we apply a T-duality along $X^{24}$ instead, the open strings no longer satisfy Neumann boundary conditions in the $X^{24}$-coordinate. Therefore we are left with a D23-brane in the dual theory, which is compactified on circles with radii $\alpha^{\prime} / R_{24}$ and $R_{25}$.

\section{A.2.5.1 T-duality in superstring theory}

Up to now we have only discussed the rules of T-duality in bosonic string theory. However, T-duality is also an exact symmetry of superstring theories. In fact T-duality relates the following superstring theories:

- heterotic $S O(32)$ superstring theory on a circle with radius $R$ and heterotic $E_{8} \times E_{8}$ superstring theory on a circle with radius $\alpha^{\prime} / R$.

- type IIA superstring theory on a circle with radius $R$ and type IIB superstring theory on a circle with radius $\alpha^{\prime} / R$. 


\section{A.2.6 S-duality}

S-duality is a strong-weak coupling duality, in the sense that a superstring theory in the weak coupling regime is mapped to another strongly coupled superstring theory. S-duality relates the string coupling constant $g_{s}$ to $1 / g_{s}$ in the same way that T-duality maps the radii of the compactified dimension $R$ to $\alpha^{\prime} / R$.

The most prominent example where S-duality is present is type IIB superstring theory. This theory is mapped to itself under S-duality. This is due to the fact that S-duality is a special case of the $S L(2, \mathbb{Z})$ symmetry of type IIB superstring theory: In the massless spectrum of type IIB superstring theory, the scalars $\phi$ and $C_{(0)}$ and the 2-form potentials $B_{(2)}$ and $C_{(2)}$ are present in pairs. Arranging the R-R scalar $C_{(0)}$ and the dilaton $\phi$ in a complex scalar $\tau=C_{(0)}+i \exp (-\phi)$, the $S L(2, \mathbb{R})$ symmetry of the equations of motion of type IIB supergravity (see $(\overline{A .2 .55})$ ) acts as

$$
\tau \rightarrow \frac{a \tau+b}{c \tau+d},
$$

with the real parameter $a, b, c, d$ satisfying $a d-b c=1$. Moreover, the R-R 2-form potential $C_{(2)}$ and the NS-NS $B_{(2)}$ transform according to

$$
\left(\begin{array}{l}
B_{(2)} \\
C_{(2)}
\end{array}\right) \rightarrow\left(\begin{array}{cc}
d & -c \\
-b & a
\end{array}\right)\left(\begin{array}{l}
B_{(2)} \\
C_{(2)}
\end{array}\right)
$$

Due to charge quantization, this symmetry group breaks down to $S L(2, \mathbb{Z})$ of the full superstring theory. A particular case of the above symmetry is S-duality. If the R-R scalar $C_{(0)}$ vanishes, the coupling constant $g_{s}=\exp (\phi)$ of type IIB superstring theory can be mapped to $1 / g_{s}$ by the $S L(2, \mathbb{Z})$ transformation with $a=d=0$, and $b=-c=1$, i.e.

$$
\phi \rightarrow-\phi, \quad B_{(2)} \rightarrow C_{(2)}, \quad C_{(2)} \rightarrow-B_{(2)} .
$$

Note that the $S L(2, \mathbb{Z})$ duality of type IIB superstring theory is a strong-weak coupling duality relating different regimes of the same theory.

Since the NS-NS field $B_{(2)}$ couples to the fundamental string, the fundamental string carries one unit of $B_{(2)}$ charge, but is not charged under the NS-NS 2-form field $C_{(2)}$. However, there are also solitonic strings which are charged under the NS-NS 2-form field $C_{(2)}$, but not under the Kalb-Ramond field $B_{(2)}$. This objects are D1-branes (see also section A.3). Under S-duality, a fundamental string is transformed into a D1-brane and vice versa. Moreover a general $S L(2, \mathbb{Z})$ transformation maps the fundamental string into a bound state $(p, q)$, carrying $p$ units of NS-NS charge and $q$ units of R-R charge.

\section{A.3 D-branes}

D-branes are non-perturbative objects in string theory which have an interesting double life. So far we considered D-branes as hyperplanes where fundamental strings can end. This is an microscopic point of view, since D-branes are defined as boundaries of worldsheets of open strings. We will see that this interpretation of D-branes is appropriate if the string coupling constant $g_{s}$ is small, i.e. $g_{s} \ll 1$. In this case the tension (or equivalently the energy density) of the D-brane is much bigger in comparison to the tension of 
a fundamental string. Therefore the deformation of the D-brane in the transverse space may be described by scalar fields. Moreover, the open strings give rise to gauge fields living on the brane. To be precise, the massless string excitations of open strings ending on D-branes can be described in general by a supersymmetric (non-)Abelian gauge theory.

In addition, D-branes are also soliton-like solutions to the macroscopic equations of motion for the low-energy theory of superstring theory, governed effectively by supergravity. In that macroscopic point of view D-branes are objects such as black holes, cosmic strings, monopoles, which curve the surrounding spacetime (and which are charged as we will see below). This description is reliable if the surrounding spacetime is weakly curved. As we will see later in this section this is the case if $g_{s} N \gg 1$, where $N$ is the number of coincident D-branes.You should view these D-branes as further ingredient to superstring theory besides fundamental strings. So called BPS D-branes carry charges of the R-R $p$-form gauge fields $C_{(p)}$. Due to charge conservation BPS D-branes are stable. In type IIA/IIB superstring theory Dp-branes with p even/odd are BPS since in this superstring theories RR gauge potentials $C_{(p+1)}$ are present to which Dp-branes couple [243].

Both interpretations of D-branes will be discussed in the subsequent sections.

\section{A.3.1 Effective action of D-branes}

In this section we consider D-branes as hyperplanes where open strings can end. The massless excitations of open strings give rise to gauge fields and scalar fields (as well as their fermionic superpartners, e.g. gauginos). For simplicity we consider in the following only the effective action for one Dp-brane. Later in this section we generalize the effective action to describe coincident Dp-branes.

The dynamics of the massless bosonic open string modes ending on one Dp-brane (or on one anti-Dp-brane) is described by an effective action

$$
S_{D p}=S_{D B I} \pm S_{C S}
$$

consisting of a Dirac-Born-Infeld (DBI) and a Chern-Simons (CS) action. The relative sign between the DBI and CS part of the action depends whether it is a Dp or anti-Dp-brane (also denoted by $\overline{D p}$-brane). The plus sign corresponds to a Dp-brane.

The interaction of the NS-NS massless fields $g_{\mu \nu}, B_{\mu \nu}$ and $\phi$ as well as the NS field strength $F_{a b}$ on the Dp-brane are given by the DBI action

$$
S_{D B I}=-\tau_{p} \int_{D p} d^{p+1} \zeta e^{-\tilde{\phi}} \sqrt{-\operatorname{det}\left(\mathcal{P}[\mathrm{g}]_{\mathrm{ab}}+\mathcal{F}_{\mathrm{ab}}\right)},
$$

where $\mathcal{F}_{a b}=(2 \pi \alpha)^{\prime 2} F_{a b}+\mathcal{P}[B]_{a b}$ and $\mathcal{P}[B]_{a b}, \mathcal{P}[g]_{a b}$ are the pullback of $B_{\mu \nu}$ and $g_{\mu \nu}$, i.e.

$$
\mathcal{P}[B]_{a b}=\partial_{a} X^{\mu} \partial_{b} X^{\nu} B_{\mu \nu}, \quad \mathcal{P}[g]_{a b}=\partial_{a} X^{\mu} \partial_{b} X^{\nu} g_{\mu \nu},
$$

with $\partial_{a}=\frac{\partial}{\partial \zeta^{a}}$ and $a, b,=0, \ldots, p$ are the worldvolume indices. The Dp-brane is embedded into the target spacetime by the functions $X^{\mu}\left(\zeta^{a}\right)$. Implicitly, equation A.3.72 employs static gauge. We identify the worldvolume coordinates of the Dp-brane with the first $p$ coordinates of the target spacetime, i.e. $X^{a}=\zeta^{a}$. Therefore we are left with $9-p$ dynamical scalar fields, which we sometimes call $\Phi^{i}\left(\zeta^{a}\right)$. 
Finally, $\tilde{\phi}$ is related to the dilaton field $\phi$ by

$$
\tilde{\phi}=\phi-\phi_{\infty},
$$

where $\phi_{\infty}$ is an asymptotic value setting the string coupling constant $g_{s}=\exp \left(\phi_{\infty}\right)$.

The tension of the Dp-brane $\tau_{p}$ is given by $\tau_{p}=(2 \pi)^{-p} \alpha^{\prime-(p+1) / 2} g_{s}^{-1}$ for type II theories. Note that we get the right dilaton dependence $g_{s}^{-1}$ for the effective open string tree level action. Throughout the thesis we use $\tilde{\phi}$ instead of the Dilaton field $\phi$. To simplify the notation we will drop the tilde of $\tilde{\phi}$.

Expanding the DBI action by using $\operatorname{det}(\mathbb{1}+\mathrm{M})=1-\frac{1}{4} \operatorname{tr}\left(\mathrm{M}^{2}\right)$ for antisymmetric matrices $M$ we see that the DBI action is a generalization of a Yang-Mills action

$$
S_{D B I} \simeq \frac{\left(2 \pi \alpha^{\prime}\right)^{2}}{4} \tau_{p} \int_{D p} d^{p+1} \zeta \mathcal{F}_{a b} \mathcal{F}^{a b}
$$

if consider only a constant dilaton field $\tilde{\phi}$. The prefactor of the action $\left(2 \pi \alpha^{\prime}\right)^{2} \tau_{p} / 4$ may be identified with the gauge coupling constant $1 /\left(g_{Y M}^{2}\right)$. Therefore we obtain for $g_{Y M}$

$$
g_{Y M}^{2}=(2 \pi)^{p-2} \alpha^{\prime(p-3) / 2} g_{s} .
$$

For D3-branes, i.e. for $p=3$, this specializes to

$$
g_{Y M}^{2}=2 \pi g_{s} .
$$

The Chern-Simons part of the action describes the interaction of the R-R fields as well as the interaction with the NS-NS fields

$$
S_{C S}=\mu_{p} \int_{D p} \sum_{q} \mathcal{P}\left[C_{(q+1)}\right] \wedge \operatorname{tr} e^{\mathcal{F}}
$$

The integral is restricted only to $p+1$-forms. Moreover, $\mu_{p}$ is related to $\tau_{p}$ by $\mu_{p}=\tau_{p} g_{s}$.

The DBI and CS part together form the relevant action for a single Dp-brane at leading classical order in the string coupling $g_{s}$, i.e. at disk level, and at leading order in the derivatives. Note that it is also possible to write down an effective action of a Dp-brane describing the fermionic massless excitations of open strings. The fermionic part of the Dpbrane action (truncated to order $\alpha^{\prime 2}$ will be discussed in chapter 5. Note that the fermionic part of the effective action for a single D-brane in arbitrary backgrounds (including RR fields) is known to all orders in $\alpha^{\prime}$ and quadratic order in the fermionic fields [167-169]. We will see in section 5.2 that the form of the action is determined by supersymmetry and T-duality [169],

So far we only discussed the effective action for a single Dp-brane. Let us now discuss the generalization of the effective action to multiple coincident Dp-branes. As review in section A.1, we have to introduce Chan-Paton factors to keep track of all different massless open string excitations by numbering the Dp-branes on which the two endpoints of the open string end. That means we have to promote the scalar fields and Abelian gauge fields to non-Abelian scalar and gauge fields, respectively. In the case of $N$ Dp-branes in type IIA/IIB string theory, the gauge group is $U(N)$. The full non-Abelian action for such a stack of Dp-branes is not completely known 11

\footnotetext{
${ }^{11}$ However, note that if we are only interested in the overall $U(1)$ subgroup of the $U(N)$ gauge group the (unknown) non-Abelian action reduces to the Abelian action discussed above if we multiply the whole action by $N$.
} 
Although the action is not completely known, it is possible to write down an effective action [193] which captures the right terms up to order $F^{4}[244], 12$ but fails to be correct at order $F^{5}$. The non-Abelian DBI action in static gauge reads

$$
S_{\mathrm{DBI}}=-\tau_{p} \operatorname{Str} \int_{D p} \mathrm{~d}^{p+1} \xi \sqrt{\operatorname{det} \mathrm{Q}}\left[\operatorname{det}\left(\mathcal{P}_{\mathrm{ab}}\left[\mathrm{E}_{\mu \nu}+\mathrm{E}_{\mu \mathrm{i}}\left(\mathrm{Q}^{-1}-\delta\right)^{\mathrm{ij}} \mathrm{E}_{\mathrm{j} \nu}\right]+2 \pi \alpha^{\prime} \mathrm{F}_{\mathrm{ab}}\right)\right]^{\frac{1}{2}}
$$

with

$$
Q^{i}{ }_{j}=\delta^{i}{ }_{j}+\mathrm{i} 2 \pi \alpha^{\prime}\left[\Phi^{i}, \Phi^{k}\right] E_{k j} .
$$

Here we also used the short-hand notation $E_{\mu \nu}=g_{\mu \nu}+B_{\mu \nu}$. Besides the term $\left(Q^{-1}-\delta\right)$ the other new ingredient in the non-Abelian DBI action is the prescription (denoted by Str) how to order the generators of the gauge group. In this action we have to use the symmetrized trace prescription invented by Tseytlin [245]: first symmetrize the product of the generators of the gauge group and then take the usual trace.

\section{A.3.2 D-branes as charged BPS objects}

In superstring theories Dp-branes carry conserved charges of topological nature. In particular $N$ coincident Dp-branes are described at low energies by solitonic supersymmetric solutions of the effective supergravity equations of motion that carry R-R charge, which is given by (A.3.88). Splitting the ten-dimensional spacetime into the worldvolume directions $a, b=0, \ldots, p$ (with coordinates $x^{a}$ ) and the transverse directions $i, j=p+1, \ldots, 9$ (with coordinates $y^{i}$ ), the solution for the metric, dilaton and the $\mathrm{R}-\mathrm{R}$ p-form potentials are given by

$$
\begin{aligned}
d s^{2} & =H_{p}(r)^{-1 / 2} \eta_{a b} d x^{a} d x^{b}+H_{p}(r)^{1 / 2} d y^{i} d y^{i} \\
e^{\phi} & =g_{s} H_{p}(r)^{(3-p) / 4} \\
C_{(p+1)} & =\left(H_{p}(r)^{-1}-1\right) g_{s}^{-1} d x^{0} \wedge d x^{1} \wedge \cdots \wedge d x^{p} \\
B_{\mu \nu} & =0,
\end{aligned}
$$

where the harmonic function $H_{p}(r)$ with $r^{2}=\sum_{i=p+1}^{9} y_{i}^{2}$ is

$$
H_{p}(r)=1+\left(\frac{L_{p}}{r}\right)^{7-p}
$$

The characteristic length $L_{p}$ is given by

$$
L_{p}^{7-p}=(4 \pi)^{(5-p) / 2} \Gamma\left(\frac{7-p}{2}\right) g_{s} N \alpha^{\prime(7-p) / 2} .
$$

For $N$ D3-branes we get the well-known relation

$$
L_{3}^{4}=4 \pi g_{s} N \alpha^{2} \text {. }
$$

Whereas $C_{(q+1)}$ vanishes for a Dp-brane $(q \neq p)$, the Dp-brane induces a non-trivial $p+2$ form field strength $13 F_{(p+2)}=d C_{(p+1)}$ as well as a non-trivial dilaton for $p \neq 3$. The charge

\footnotetext{
${ }^{12}$ To be precise, the derivative corrections are known up to order $D^{4} F^{4}$ and $D^{8} F^{2}$ where $D$ represents the gauge-covariant derivative.

${ }^{13}$ Note that for type IIB string theory and supergravity $F_{(5)}$ has to be self-dual, i.e. $F_{(5)}=\star F_{(5)}$. Therefore in the case of D3-branes, the five-form field strength can be calculated by $F_{(5)}=\frac{1}{2}\left(d C_{(4)}+\star d C_{4}\right)$.
} 
of Dp-branes can be calculated by integrating the R-R flux through the $(8-p)$-dimensional sphere at infinity (surrounding the pointlike charge in the $(9-p)$-dimensional transversal space)

$$
Q=\int_{S_{\infty}^{8-p}} \star F_{(p+2)},
$$

where $\star$ is the ten-dimensional Hodge operator. For the solution A.3.81 the charge $Q$ is given by $Q=N$, i.e. the charge $Q$ encodes the total number of Dp-branes. 



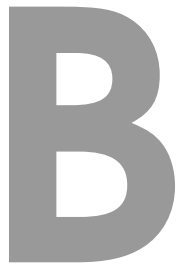

\section{Appendix to Chapter 7}

We present the main calculations for chapter 7 in this appendix. In chapter 7 we have studied a $(2+1)$-dimensional Chern-Simons-Matter theory and added flavor degrees of freedom, transforming in the fundamental representation of the gauge group.

Here, we consider probe branes in the type IIB configuration of the ABJM setup in section B.1. Moreover we show how to lift the type IIB setup to M-theory in section B.2. In particular we relate the coordinates of type IIB setup to the coordinates of $\mathbb{C}^{4} / \mathbb{Z}_{k}$ on the M-theory side. Then we calculate in B.3 the preserved supersymmetry of certain flavor configurations in M-theory. Finally we work out explicitly the $\mathcal{N}=(0,6)$ supersymmetry transformations of the codimension one field theory in section B.4.

\section{B.1 Supersymmetry of Type IIB Probes}

In this section we add supersymmetry-preserving D-branes to the type IIB setup of the ABJM theory. Our starting point is the list of probes in [102], since these are known to be mutually supersymmetric with respect to the D3-branes, but now we have two new ingredients: the NS5-branes and the $(1, \mathrm{k}) 5$-brane 1 These new ingredients break the usual $S O(6)$ symmetry, which rotates the (345789) directions into one another, down to the $S O(3)$ that rotates (345) and (789) simultaneously.

As mentioned in section 7.4.1, we limit our search for supersymmetric probes by imposing four constraints. First, we consider only D1-, D3-, D5- and D7-brane probes (the list from [102]). Second, we do not separate any probes from the D3-branes in overall transverse directions. Third, when we consider multiple probes, i.e. $N_{f}>1$, we do not separate them from each other, so that they retain a $U\left(N_{f}\right)$ symmetry. Fourth, we consider only probes

\footnotetext{
${ }^{1}$ Another new ingredient is that the $x^{6}$ direction is compact. While that is crucially important for deriving the low-energy $(2+1)$-dimensional worldvolume field theory, it does not affect the counting of supersymmetries.
} 
aligned along the coordinate axes. More generally the probe brane could be at an angle with respect to these axes. We studied a few special cases of probes at angles (see below) and found that such probes never appear to exhibit enhanced supersymmetry.

Our results are summarized in table 7.1 in section 7.4.1, reproduced here as table B.1. The details of the notation (such as the column headings) appear in section 7.4.1.

\begin{tabular}{|c|c|c|c|c|c|c|}
\hline Type IIB & Type IIA & M theory & codim & wrapping & SUSY & SUSY (anti) \\
\hline \hline D1 & D2 & M2 & 2 & $0(7)$ & 2 & 2 \\
\hline D3 & D2 & M2 & 0 & 0126 & 6 & 0 \\
\hline D3 & D4 & M5 & 1 & $01(37)$ & 3 & 3 \\
\hline D3 & D4 & M5 & 1 & $01(38)$ & 2 & 2 \\
\hline D3 & D2 & M2 & 2 & $0(34) 6$ & 2 & 2 \\
\hline D3 & D2 & M2 & 2 & $06(78)$ & 2 & 2 \\
\hline D5 & D6 & KK & 0 & $012(347)$ & 2 & 2 \\
\hline D5 & D6 & KK & 0 & $012(349)$ & 4 & 2 \\
\hline D5 & D6 & KK & 0 & 012789 & 6 & 0 \\
\hline D5 & D4 & M5 & 1 & 013456 & 3 & 3 \\
\hline D5 & D4 & M5 & 1 & $01(378) 6$ & 2 & 2 \\
\hline D5 & D4 & M5 & 1 & $01(389) 6$ & 3 & 3 \\
\hline D5 & D6 & KK & 2 & $0(34) 789$ & 2 & 2 \\
\hline D7 & D6 & KK & 0 & $0126(3478)$ & 2 & 4 \\
\hline D7 & D6 & KK & 0 & $0126(3479)$ & 2 & 2 \\
\hline D7 & D8 & M9 & 1 & 01345789 & 3 & 3 \\
\hline
\end{tabular}

Table B.1: List of D-branes (extended along the coordinate axes) that we can add to the type IIB construction while still preserving some supersymmetry.

To count unbroken supercharges we follow [215] very closely. In particular, we perform a different T-duality from the one that leads to the ABJM theory: we T-dualize in $x^{2}$, not $x^{6}$, and then lift to M-theory. The type IIB construction reviewed in section 7.3.2 then has a very simple interpretation in terms of M-branes alone (rather than M-branes in some nontrivial geometry). The D3-branes become M2-branes along (016), the NS5-brane becomes an M5-brane along (012345), and the $(1, k) 5$-brane becomes an M5-brane tilted at an angle $\theta$ in the (37), (48), and (59) directions and an angle $-\theta$ in the (2\#) directions, relative to the other $\mathrm{M} 5$-brane, where $\tan \theta=k$.

Let $\epsilon$ denote the 32-component Majorana spinor and $\Gamma_{A}$ the $32 \times 32 \Gamma$-matrices of 11 dimensional supergravity. The $\Gamma$ matrices obey the flat space Clifford algebra $\left\{\Gamma_{A}, \Gamma_{B}\right\}=$ $2 \eta_{A B}$, where we use a mostly-plus metric. Let $\Gamma_{A B C \ldots}$ denote the totally antisymmetric product of $\Gamma$-matrices, equivalent to the usual product due to the Clifford algebra. The product $\Gamma_{0123456789 \#}=\mathbb{1}_{32}$, where $\mathbb{1}_{32}$ is the $32 \times 32$ identity matrix.

The M2- and M5-branes give rise to projection conditions on $\epsilon$,

$$
\Gamma_{016} \epsilon=\epsilon, \quad \Gamma_{012345} \epsilon=\epsilon, \quad R \Gamma_{012345} R^{-1} \epsilon=\epsilon,
$$

where the last condition, for the rotated M5-brane, involves the rotation matrix

$$
R(\theta)=\exp \left(-\frac{\theta}{2} \Gamma_{2 \#}+\frac{\theta}{2} \Gamma_{37}+\frac{\theta}{2} \Gamma_{48}+\frac{\theta}{2} \Gamma_{59}\right) .
$$


Notice that $R^{-1}(\theta)=R(-\theta)$. Making use of the fact that all of the $\Gamma$-matrices in $R$ anti-commute with $\Gamma_{012345}$, we can simplify the condition for the rotated M5-brane,

$$
R \Gamma_{012345} R^{-1} \epsilon=R^{2} \Gamma_{012345} \epsilon=R^{2} \epsilon=\epsilon,
$$

where we used the projection condition for the un-rotated M5-brane. We can then write the rotated M5-brane's projection condition as $\left(R^{2}-\mathbb{1}_{32}\right) \epsilon=0$. At this point we need to write the matrices appearing in the projection conditions explicitly, in order to count the number of preserved supercharges. Following [215], we use a basis in which the following set of mutually-commuting matrices are diagonal:

$$
\begin{aligned}
\Gamma_{012345} & =\left(\mathbb{1}_{16},-\mathbb{1}_{16}\right) \\
\Gamma_{016} & =\left(\mathbb{1}_{2},-\mathbb{1}_{2},-\mathbb{1}_{2}, \mathbb{1}_{2},-\mathbb{1}_{2}, \mathbb{1}_{2}, \mathbb{1}_{2},-\mathbb{1}_{2}, \ldots\right) \\
\Gamma_{2 \# 37} & =\left(\mathbb{1}_{8},-\mathbb{1}_{8}, \ldots\right) \\
\Gamma_{2 \# 48} & =\left(\mathbb{1}_{4},-\mathbb{1}_{4}, \mathbb{1}_{4},-\mathbb{1}_{4}, \ldots\right) \\
\Gamma_{2 \# 59} & =\left(\mathbb{1}_{2},-\mathbb{1}_{2}, \mathbb{1}_{2},-\mathbb{1}_{2}, \mathbb{1}_{2},-\mathbb{1}_{2}, \mathbb{1}_{2},-\mathbb{1}_{2}, \ldots\right) .
\end{aligned}
$$

In this basis, the matrix in the projection condition for the rotated M5-brane becomes

$$
R^{2}-\mathbb{1}_{32}=2 R \Gamma_{2 \#}\left(\sin (-2 \theta) \mathbb{1}_{2}, \sin (-\theta) \mathbb{1}_{2}, \sin (-\theta) \mathbb{1}_{2}, 0_{2}, \sin (-\theta) \mathbb{1}_{2}, 0_{2}, 0_{2}, \sin (\theta) \mathbb{1}_{2}, \ldots\right) .
$$

The $0_{2}$ 's in this equation that overlap with the $\mathbb{1}_{2}$ 's in $\Gamma_{016}$ indicate which components of $\epsilon$ are preserved, hence the full system of M2-brane, M5-brane, and rotated M5-brane preserves 6 real supercharges.

To study probe branes we first need to translate all the type IIB D-branes to the M-theory description, which produces various M2- and M5-branes, as well as KK monopoles. We do not present every case in detail, rather, we just show a few representative examples, with decreasing amounts of supersymmetry.

First we note that when $k=0$, such that the rotation matrix $R$ is simply the identity (and in the type IIB description we have just NS5-branes), all of the objects we study preserve 4 real supercharges, with two exceptions: the D3-branes along (0126) and the D5-branes along (012789). These two D-branes preserve 8 real supercharges when $k=0$.

For nonzero $k$, the easiest example is in fact the D5-brane along (012789) (see also [203,215]), which upon T-duality in $x^{2}$ and lift to M-theory becomes an M5-brane along $(01789 \#)$. The projection condition is $\Gamma_{01789 \#} \epsilon=\epsilon$. We can use $\Gamma_{0123456789 \#}=\mathbb{1}_{32}$ to write $\Gamma_{01789 \#}=\Gamma_{016} \Gamma_{012345}$, hence this M5-brane does not impose any additional constraint on $\epsilon$, and preserves 6 real supercharges.

An example preserving 4 real supercharges is a D5-brane along (012349), which upon Tduality in $x^{2}$ and uplift to M-theory becomes an M5-brane along (01349\#). In this case we use $\mathbb{1}_{32}=-\Gamma_{25} \Gamma_{25}$ to write $\Gamma_{01349 \#}=-\Gamma_{012345} \Gamma_{2 \# 59}$. In the upper-left $16 \times 16$ block, $\Gamma_{012345}$ is simply the identity, so in this subspace $\Gamma_{01349 \#}=-\Gamma_{2 \# 59}$. Using the $\Gamma$-matrices written explicitly above, we can count that this probe preserves 4 real supercharges. The same steps obviously apply for cases related to this one by $S O(3)$ rotations. An anti-D5brane has $-\Gamma_{01349 \#}=\Gamma_{2 \# 59}$, and hence preserve 2 real supercharges.

An example preserving 3 real supercharges is a D3-brane along (0137). Here we first rotate the D3-brane so that it is extended along (0237), which then becomes an M2-brane along 
(037). We insert $\mathbb{1}_{32}=-\Gamma_{2 \#} \Gamma_{2 \#}$ to write $\Gamma_{037}=-\Gamma_{02 \#} \Gamma_{2 \# 37}$. We need to know the additional $\Gamma$ matrix,

$$
\Gamma_{02 \#}=\left(\sigma_{1}, \sigma_{1},-\sigma_{1},-\sigma_{1}, \sigma_{1}, \sigma_{1},-\sigma_{1},-\sigma_{1}, \ldots\right),
$$

where $\sigma_{1}$ is the first Pauli matrix, $\sigma_{1}=((0,1),(1,0))$. We thus have

$$
\Gamma_{037} \epsilon=-\Gamma_{02 \#} \Gamma_{2 \# 37} \epsilon=\left(-\sigma_{1},-\sigma_{1}, \sigma_{1}, \sigma_{1}, \sigma_{1}, \sigma_{1},-\sigma_{1},-\sigma_{1}, \ldots\right) \epsilon=\epsilon .
$$

Each $\sigma_{1}$ imposes an additional constraint on the two components of $\epsilon$ in that $2 \times 2$ block, hence this brane kills half of the supercharges, i.e it preserves 3 real supercharges.

The cases preserving 2 real supercharges are slightly more involved. For example, consider the D1-brane along (07), which becomes an M2-brane along (027). Inserting $\mathbb{1}_{32}=\Gamma_{3} \Gamma_{3}$ we have $\Gamma_{027} \epsilon=-\Gamma_{23} \Gamma_{037} \epsilon=\epsilon$. We know that $\Gamma_{037}$ is $2 \times 2$ block-diagonal in this basis, but $\Gamma_{23}$ is not (it can be written as $\Gamma_{23}=-i \mathbb{1}_{2} \otimes \mathbb{1}_{2} \otimes \sigma_{1} \otimes \sigma_{1} \otimes \mathbb{1}_{2}$ ). Nevertheless, these cases are straightforward to check explicitly, although we do not present the details.

As mentioned above, we could also consider probe branes rotated with respect to the coordinate axes. In principle, such branes could have enhanced supersymmetry. We have not analyzed all possible rotations. For many of the branes in our table we considered the special case in which the brane is rotated by one angle in the (37), (48) and (59) planes simultaneously and by an independent angle in the (2\#) plane. In all cases the rotated brane never exhibits enhanced supersymmetry, and in most cases preserves fewer supersymmetries.

\section{B.2 Type IIB to M-theory}

When we add flavor branes to the brane construction of the ABJM theory, many aspects of the field theory are best understood from the type IIB description, while the symmetries and the amount of supersymmetry preserved become manifest after the "near-horizon" limit in M-theory. To determine where the probe D-branes of the type IIB setup end up in M-theory on $\mathbb{R}^{2,1} \times \mathbb{C}^{4} / \mathbb{Z}_{k}$, we need to find the coordinate transformations from the type IIB coordinates, $x^{m}$ with $m=0, \ldots, 9$, to the M-theory coordinates $z^{i}, i=1,2,3,4$ on $\mathbb{C}^{4}$. Our objective in this appendix is to write the $z^{i}$ in terms of the $x^{m}$ (and vice versa) explicitly, so that we can translate directly between the two coordinate systems.

As reviewed in section 7.3.3, when we T-dualize along $x^{6}$ and then lift to M-theory, the NS5-brane and $(1, k) 5$-brane both become KK monopoles in M-theory extended along (012), so that we only need to consider the eight other directions, which we denote by

$$
\varphi_{1}=x^{6}, \quad \varphi_{2}=x^{\sharp}, \quad \vec{x}^{1}=\left(\begin{array}{c}
x^{7} \\
x^{8} \\
x^{9}
\end{array}\right), \quad \vec{x}^{2}=\left(\begin{array}{c}
x^{3} \\
x^{4} \\
x^{5}
\end{array}\right) .
$$

Both $x^{6}$ and $x^{\sharp}$ have $2 \pi$ periodicity. The metric describing the intersection of the two KK monopoles is [246]

$$
\begin{aligned}
d s^{2} & =U_{i j} d \vec{x}^{i} \cdot d \vec{x}^{j}+U^{i j}\left(d \varphi_{i}+A_{i}\right)\left(d \varphi_{j}+A_{j}\right), \\
A_{i} & =d \vec{x}^{j} \cdot \vec{\omega}_{j i}=d x_{a}^{j} \omega_{i j}^{a}, \quad \partial_{x_{a}^{j}} \omega_{k i}^{b}-\partial_{x_{b}^{k}} \omega_{j i}^{a}=\epsilon^{a b c} \partial_{x_{c}^{j}} U_{k i},
\end{aligned}
$$


where $U^{i j}$ is the transposed inverse of $U_{i j}$. Notice in particular that the metric is uniquely determined by the matrix $U$ and that the equations are linear in $U$ so that we can obtain the configuration for two monopoles simply by linear superposition.

The NS5-brane becomes a KK monopole associated with the circle $\varphi_{1}=x^{6}$ and transverse directions $\vec{x}^{1}$. The corresponding $U$ matrix reads

$$
U=\left(\begin{array}{ll}
1 & 0 \\
0 & 1
\end{array}\right)+\left(\begin{array}{cc}
h_{1} & 0 \\
0 & 0
\end{array}\right), \quad h_{1}=\frac{1}{2\left|\vec{x}^{1}\right|} .
$$

The identity matrix in $U$ indicates that asymptotically the space is $\mathbb{R}^{6} \times T^{2}$. The $(1, k) 5$ brane is rotated in the $\left(\vec{x}^{1}, \vec{x}^{2}\right)$ - and $\left(\varphi_{1}, \varphi_{2}\right)$-plane. The $U$ matrix of such a KK monopole is

$$
U=\left(\begin{array}{ll}
1 & 0 \\
0 & 1
\end{array}\right)+\left(\begin{array}{cc}
h_{2} & k h_{2} \\
k h_{2} & k^{2} h_{2}
\end{array}\right), \quad h_{2}=\frac{1}{2\left|\vec{x}^{1}+k \vec{x}^{2}\right|} .
$$

The metric describing the two intersecting KK monopoles is then

$$
U=\left(\begin{array}{ll}
1 & 0 \\
0 & 1
\end{array}\right)+\left(\begin{array}{cc}
h_{1} & 0 \\
0 & 0
\end{array}\right)+\left(\begin{array}{cc}
h_{2} & k h_{2} \\
k h_{2} & k^{2} h_{2}
\end{array}\right) .
$$

The metric in equation (B.2.8), with the $U$ matrix in equation (B.2.12), is the metric of the space $X_{8}$ mentioned in section 7.3 .3 .

To relate the type IIB coordinates $\varphi_{1}, \varphi_{2}, \vec{x}^{1}$ and $\vec{x}^{2}$ to the $\mathbb{C}^{4}$ coordinates $z^{i}$, we proceed in five steps. The first step is to take the "near-horizon" limit [137] that we described in section 7.3.3. The four subsequent steps are simply changes of coordinates.

The "near-horizon" limit consists of taking $\vec{x}^{1} \sim \vec{x}^{2} \sim 0$, which in simple terms means we drop the identity matrix from the $U$ in equation (B.2.12).

Now we change coordinates four times. The first change of coordinates diagonalizes the new $U$, producing the "near-horizon" metric of strictly perpendicular KK monopoles [137]:

$$
\begin{aligned}
\vec{x}^{\prime}=\vec{x}^{1}, & \vec{x}^{\prime}=\vec{x}^{1}+k \vec{x}^{2}, \\
\varphi_{1}^{\prime}=x^{6}-\frac{1}{k} x^{\sharp}, & \varphi_{2}^{\prime}=\frac{1}{k} x^{\sharp},
\end{aligned}
$$

with new $U$ matrix $U^{\prime}$,

$$
U^{\prime}=\left(\begin{array}{cc}
\frac{1}{2\left|\vec{x}^{\prime} 1\right|} & 0 \\
0 & \frac{1}{2\left|\vec{x}^{2}\right|}
\end{array}\right) .
$$

The new circle coordinates, $\varphi_{i}^{\prime}, i=1,2$, are $2 \pi$ periodic, but the $2 \pi$ periodicity of $x^{\sharp}$ leads to an extra identification

$$
\left(\varphi_{1}^{\prime}, \varphi_{2}^{\prime}\right) \sim\left(\varphi_{1}^{\prime}, \varphi_{2}^{\prime}\right)+\frac{2 \pi}{k}(-1,1)
$$

that is, if we shift $\varphi_{1}^{\prime}$ by a multiple of $\frac{2 \pi}{k}$ and simultaneously shift $\varphi_{2}^{\prime}$ by the opposite amount, we end up at the same point. The above periodicity leads to the $\mathbb{Z}_{k}$ action on $\mathbb{C}^{4}$, as we see below. In the new coordinates we have two perpendicular KK monopoles so we treat them simultaneously. The Taub-NUT metric of a single KK monopole in the "near-horizon" limit is

$$
d s_{i}^{2}=\frac{1}{2\left|\vec{x}^{\prime} i\right|}\left(d \vec{x}^{\prime}\right)^{2}+2\left|\vec{x}^{i}\right|\left(d \varphi_{i}^{\prime}+A_{i}\right)^{2},
$$


where $i=1,2$ labels the KK monopoles.

Now we do the second change of coordinates, which is simply a change from Euclidean to spherical coordinates, $\left(d \vec{x}^{\prime}\right)^{2}=d r_{i}^{2}+r_{i}^{2}\left(d \theta_{i}^{2}+\sin ^{2} \theta_{i} d \phi_{i}^{2}\right)$, so that we obtain

$$
d s_{i}^{2}=\frac{1}{2 r_{i}} d r_{i}^{2}+\frac{r_{i}}{2}\left(d \theta_{i}^{2}+\sin ^{2} \theta_{i} d \phi_{i}^{2}\right)+2 r_{i}\left(d \varphi_{i}^{\prime}+\frac{1}{2} \cos \theta_{i} d \phi_{i}\right)^{2}
$$

where we have used $A_{i}=\frac{1}{2} \cos \theta_{i} d \phi_{i}$.

In the third change of coordinates we define a new radial coordinate $r_{i}=\rho_{i}^{2} / 2$ and new angles $\varphi_{i}^{\prime}=\psi_{i} / 2$. The metric then becomes that of flat space, with an extra $\mathbb{Z}_{k}$ identification,

$$
d s_{i}^{2}=d \rho_{i}^{2}+\frac{\rho_{i}^{2}}{4}\left(d \theta_{i}^{2}+d \phi_{i}^{2}+d \psi_{i}^{2}+2 \cos \theta_{i} d \phi_{i} d \psi_{i}\right) .
$$

The three angles have ranges $0 \leq \theta_{i}<\pi, 0 \leq \phi_{i}<2 \pi$ and $0 \leq \psi_{i}<4 \pi$, and the $\psi_{i}$ have the extra identification

$$
\left(\psi_{1}, \psi_{2}\right) \sim\left(\psi_{1}, \psi_{2}\right)+\frac{4 \pi}{k}(-1,1)
$$

following from equation (B.2.16).

In the fourth and final change of coordinates, we introduce complex coordinates for the first KK monopole,

$$
z^{1}=\rho_{1} \cos \left(\frac{\theta_{1}}{2}\right) e^{-i\left(\psi_{1}+\phi_{1}\right) / 2}, \quad z^{2}=\rho_{1} \sin \left(\frac{\theta_{1}}{2}\right) e^{-i\left(\psi_{1}-\phi_{1}\right) / 2}
$$

while for the second KK monopole we choose something similar, but with $i \rightarrow-i$,

$$
z^{3}=\rho_{2} \cos \left(\frac{\theta_{2}}{2}\right) e^{i\left(\psi_{2}+\phi_{2}\right) / 2}, \quad z^{4}=\rho_{2} \sin \left(\frac{\theta_{2}}{2}\right) e^{i\left(\psi_{2}-\phi_{2}\right) / 2}
$$

In these coordinates, the Taub-NUT metrics in the "near-horizon" limit have become

$$
d s_{1}^{2}=d z^{1} d \bar{z}^{1}+d z^{2} d \bar{z}^{2}, \quad d s_{2}^{2}=d z^{3} d \bar{z}^{3}+d z^{4} d \bar{z}^{4},
$$

and the $\mathbb{Z}_{k}$ transformation of equation $(\overline{\mathrm{B} .2 .20})$ acts as $z^{j} \rightarrow e^{\frac{2 \pi i}{k}} z^{j}$.

Tracing back through our coordinate transformations, we can write the original type IIB coordinates (plus $x^{\sharp}$ ) in terms of the $z^{i}$ :

$$
\begin{aligned}
x^{6} & =\frac{1}{2} \arg \left(\bar{z}^{1} \bar{z}^{2} z^{3} z^{4}\right), \\
x^{\sharp} & =\frac{k}{2} \arg \left(z^{3} z^{4}\right), \\
\left(\begin{array}{c}
x^{7} \\
x^{8} \\
x^{9}
\end{array}\right) & =\left(\begin{array}{c}
\operatorname{Re}\left(z^{1} \bar{z}^{2}\right) \\
-\operatorname{Im}\left(z^{1} \bar{z}^{2}\right) \\
\frac{1}{2}\left(\left|z^{1}\right|^{2}-\left|z^{2}\right|^{2}\right)
\end{array}\right), \\
\left(\begin{array}{c}
x^{3} \\
x^{4} \\
x^{5}
\end{array}\right) & =\frac{1}{k}\left(\left(\begin{array}{c}
\operatorname{Re}\left(z^{3} \bar{z}^{4}\right) \\
\operatorname{Im}\left(z^{3} \bar{z}^{4}\right) \\
\frac{1}{2}\left(\left|z^{3}\right|^{2}-\left|z^{4}\right|^{2}\right)
\end{array}\right)-\left(\begin{array}{c}
\operatorname{Re}\left(z^{1} \bar{z}^{2}\right) \\
-\operatorname{Im}\left(z^{1} \bar{z}^{2}\right) \\
\frac{1}{2}\left(\left|z^{1}\right|^{2}-\left|z^{2}\right|^{2}\right)
\end{array}\right)\right) .
\end{aligned}
$$


Inverting the above expressions, we can write the $z^{i}$ in terms of the type IIB coordinates (plus $x^{\sharp}$ ):

$$
\begin{aligned}
\left|z^{1}\right|^{2} & =x^{9}+\sqrt{\left(x^{7}\right)^{2}+\left(x^{8}\right)^{2}+\left(x^{9}\right)^{2}}, \\
\left|z^{2}\right|^{2} & =-x^{9}+\sqrt{\left(x^{7}\right)^{2}+\left(x^{8}\right)^{2}+\left(x^{9}\right)^{2}}, \\
\left|z^{3}\right|^{2} & =\left(x^{9}+k x^{5}\right)+\sqrt{\left(x^{7}+k x^{3}\right)^{2}+\left(x^{8}+k x^{4}\right)^{2}+\left(x^{9}+k x^{5}\right)^{2}}, \\
\left|z^{4}\right|^{2} & =-\left(x^{9}+k x^{5}\right)+\sqrt{\left(x^{7}+k x^{3}\right)^{2}+\left(x^{8}+k x^{4}\right)^{2}+\left(x^{9}+k x^{5}\right)^{2}}, \\
\arg z^{1} & =\frac{x^{\sharp}}{k}-x^{6}-\frac{1}{2} \arctan \frac{x^{8}}{x^{7}}, \\
\arg z^{2} & =\frac{x^{\sharp}}{k}-x^{6}+\frac{1}{2} \arctan \frac{x^{8}}{x^{7}}, \\
\arg z^{3} & =\frac{x^{\sharp}}{k}+\frac{1}{2} \arctan \frac{x^{8}+k x^{4}}{x^{7}+k x^{3}}, \\
\arg z^{4} & =\frac{x^{\sharp}}{k}-\frac{1}{2} \arctan \frac{x^{8}+k x^{4}}{x^{7}+k x^{3}} .
\end{aligned}
$$

Recall that the $z^{i}$ transform as a 4 of $S U(4)$, which clearly acts non-trivially on the $x^{m}$. The $U(1)_{b}$ is just a common phase shift $z^{j} \rightarrow e^{i \alpha} z^{j}$. The only coordinate that changes under the $U(1)_{b}$ is $x^{\sharp}$, which shifts as $x^{\sharp} \rightarrow x^{\sharp}+k \alpha$.

As explained in section 7.3.4, we can take a large- $N_{c}$ limit in M-theory, so that the geometry becomes $A d S_{4} \times S^{7} / \mathbb{Z}_{k}$, and then take also large $k$, so that a circle in M-theory shrinks and we obtain type IIA in $A d S_{4} \times \mathbb{C P}^{3}$. Where in the geometry is the circle that shrinks when $k \rightarrow \infty$ ? To answer this question, notice that the circle direction

$$
x^{6}=\frac{1}{2} \arg \left(\bar{z}^{1} \bar{z}^{2} z^{3} z^{4}\right)
$$

is invariant under the $\mathbb{Z}_{k}$ action, hence the circle that shrinks when $k \rightarrow \infty$ must be part of $x^{\sharp}$. To show this explicitly, we return to our third change of coordinates, which involved the angles $\psi_{1}$ and $\psi_{2}$, with $\mathbb{Z}_{k}$ acting as in equation (B.2.20). Tracing back through the changes of coordinates, we find $x^{6}=\frac{1}{2}\left(\psi_{1}+\psi_{2}\right)$, which is of course invariant, and $x^{\sharp}=\frac{k}{2} \psi_{2}$, on which the $\mathbb{Z}_{k}$ acts as a $2 \pi$ shift. We can then define a coordinate $y$,

$$
y=\frac{1}{4}\left(\psi_{2}-\psi_{1}\right)=-\frac{1}{2} x^{6}+\frac{1}{k} x^{\sharp} .
$$

such that the $\mathbb{Z}_{k}$ acts as $y \sim y+\frac{2 \pi}{k}$ but leaves all other coordinates invariant. The direction $y$ is thus the circle that shrinks when $k \rightarrow \infty$. From a type IIB perspective we are decomposing $x^{\sharp}$ as $x^{\sharp}=k y+\frac{k x^{6}}{2}$. In terms of the $z^{i}$,

$$
\begin{aligned}
& \arg z^{1}=y-\frac{x^{6}}{2}-\frac{\phi_{1}}{2}, \\
& \arg z^{3}=y+\frac{x^{6}}{2}+\frac{\phi_{2}}{2}, \quad \arg z^{2}=y-\frac{x^{6}}{2}+\frac{\phi_{1}}{2},
\end{aligned}
$$

which shows that $y$ is simply the sum of the phases of the $z^{i}$.

A crucial question is whether a D-brane in type IIB remains a D-brane in type IIA on $A d S_{4} \times \mathbb{C P}^{3}$. After T-duality from type IIB, when we first lift to M-theory, the circle 


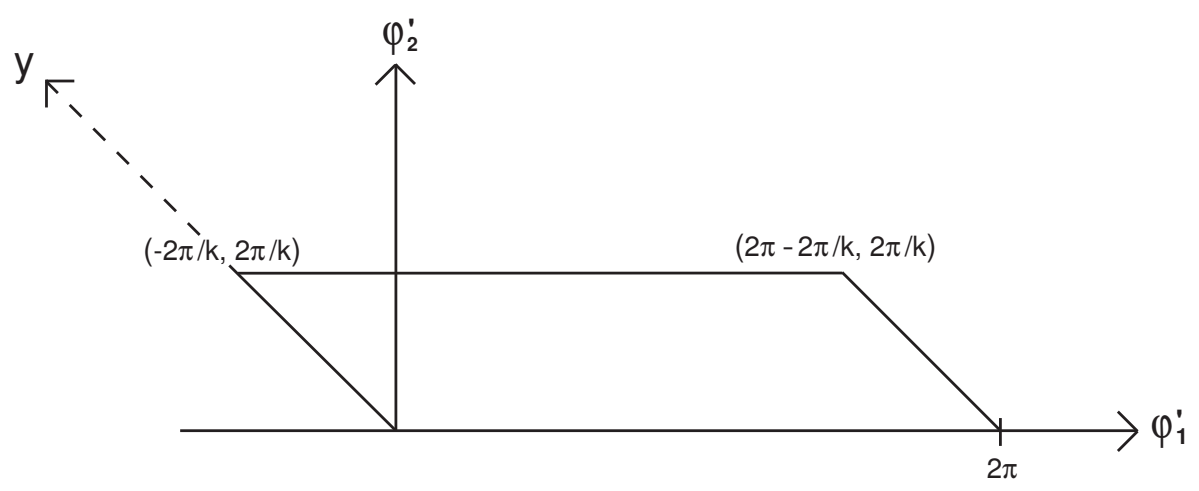

Figure B.1: The torus spanned by $\left(\varphi_{1}^{\prime}, \varphi_{2}^{\prime}\right)$. We have indicated the fundamental domain (the parallelogram) and the $y$ direction (the dotted line). A basis of one cycles is a curve in the $y$ direction and a curve in the $\varphi_{1}^{\prime}$ direction. When $k \rightarrow \infty$, the $y$ circle shrinks, and the parallelogram collapses onto the $\varphi_{1}^{\prime}$ axis.

$x^{\sharp}$ opens up. We then take the "near-horizon" limit and $N_{c} \rightarrow \infty$ to obtain M-theory on $A d S_{4} \times S^{7} / \mathbb{Z}_{k}$, and then we take large $k$, so that the $y$ circle shrinks, and the theory reduces to type IIA on $A d S_{4} \times \mathbb{C P}^{3}$. In short, $x^{\sharp}$ opens up but $y$ shrinks. A D4-brane in type IIA becomes an M5-brane when $x^{\sharp}$ opens up, but what happens when $y$ closes? Does the M5-brane reduce to a D4-brane again, or an NS5-brane with D4-brane flux?

The easiest way to see what happens is to return to our first change of coordinates, and in particular to consider the torus spanned by the coordinates $\varphi_{1}^{\prime}=x^{6}-\frac{1}{k} x^{\sharp}$ and $\varphi_{2}^{\prime}=\frac{1}{k} x^{\sharp}$. These two coordinates are orthogonal (as opposed to, say, $x^{6}$ and $x^{\sharp}$ ). The $\mathbb{Z}_{k}$ action on these coordinates appears in equation (B.2.16). We draw the fundamental domain of the $\left(\varphi_{1}^{\prime}, \varphi_{2}^{\prime}\right)$ torus in the figure. We also indicate the $y$ direction in the figure, where in these coordinates $y=\frac{1}{2}\left(\varphi_{2}^{\prime}-\varphi_{1}^{\prime}\right)$. The generators of homology are the $y$ and $\varphi_{1}^{\prime}$ axes as drawn, i.e. these form a basis of one-cycles. When $k \rightarrow \infty$, the upper bound of the fundamental domain moves down, so that the parallelogram collapses (in the $y$ direction) onto the $\varphi_{1}^{\prime}$ axis. The cycles that shrink in this process are all the ones that have net winding around $y$ and zero winding around $\varphi_{1}^{\prime}$. The shortest cycles that shrink are parallel to the $y$-axis, so here again we identify $y$ as the M-theory circle (when descending to type IIA on $\left.A d S_{4} \times \mathbb{C P}^{3}\right)$.

Let's consider what happens to our flavor branes when $x^{\sharp}$ opens up and then $y$ collapses. We have four options: a brane can wrap $x^{\sharp}$ but not $x^{6}, x^{6}$ but not $x^{\sharp}$, or a brane can wrap both, or a brane can wrap neither.

Consider a brane that wraps $x^{\sharp}$ and sits at fixed $x^{6}$. The key point is that, from the definition of $\varphi_{1}^{\prime}$ and $\varphi_{2}^{\prime}$, we immediately see that such a brane is parallel to the $y$-axis, so such a brane returns to whatever it was in IIA. For example, a D4-brane localized in $x^{6}$, which lifts to an M5-brane wrapping $x^{\sharp}$, would descend back to a D4-brane localized in $x^{6}$ when $y$ closes.

Now consider a brane that wraps $x^{6}$ and sits at fixed $x^{\sharp}$. Such a brane extends along $\varphi_{1}^{\prime}$ at fixed $\varphi_{2}^{\prime}$ (a horizontal line in the figure). Since $\varphi_{1}^{\prime}$ is the direction that remains when $k \rightarrow \infty$ we see that such a brane again returns to whatever it was (now in IIA on $\left.A d S_{4} \times \mathbb{C P}^{3}\right)$. 
The last two cases are basically trivial. A brane that wraps both directions or neither returns to whatever it was. For example, a D2-brane localized in $x^{6}$ lifts to an M2-brane that wraps neither $x^{6}$ nor $x^{\sharp}$, and returns to a D2-brane localized in $x^{6}$ when $y$ collapses. A D4-brane wrapping $x^{6}$ lifts to an M5-brane wrapping both $x^{6}$ and $x^{\sharp}$, and hence wraps the entire $\left(\varphi_{1}^{\prime}, \varphi_{2}^{\prime}\right)$ torus, and becomes a D4-brane wrapping $x^{6}$ when $y$ collapses.

To summarize: $x^{\sharp}$ opens up and $y$ shrinks, and all D-branes remain the same D-branes when we go to IIA on $A d S_{4} \times \mathbb{C P}^{3}$.

\section{B.3 Supersymmetry of M-theory Objects}

In this appendix we calculate the supersymmetry preserved by probes added to the $N_{c}$ M2-branes along $\mathbb{R}^{2,1}$ sitting at the origin of $\mathbb{C}^{4} / \mathbb{Z}_{k}$ and also for probes added to the near-horizon geometry of $N_{c} \rightarrow \infty$ M2-branes, $A d S_{4} \times S^{7} / \mathbb{Z}_{k}$ (see $[247,248]$ for similar calculations).

The number of supersymmetries preserved by our probes is the number of solutions of the $\kappa$-symmetry condition

$$
\Gamma_{\kappa} \epsilon=\epsilon, \quad \Gamma_{\kappa}=\frac{1}{n !} \frac{1}{\sqrt{-g}} \epsilon^{12 \ldots n} \gamma_{12 \ldots n}
$$

where $\epsilon$ is the 32-component spinor of the background, $n$ is the dimensionality of the object (KK monopole or M-brane), and $g_{m n}=\partial_{m} X^{I} \partial_{n} X^{J} g_{I J}$ and $\gamma_{m}=\partial_{m} X^{I} e_{I}^{A} \Gamma_{A}$ are the pullbacks of the background metric and the $\Gamma$-matrices to the worldvolume of the object. Here $X^{I}$ represent the scalars on the worldvolume of the object, $e_{I}^{A}$ are vielbeins, and $A, B \ldots$ are tangent space indices. The $\Gamma_{A}$ satisfy the tangent space Clifford algebra $\left\{\Gamma_{A}, \Gamma_{B}\right\}=2 \eta_{A B}$, where we use a mostly-plus metric. We calculate the spinor $\epsilon$ by demanding that the supersymmetry transformation of the gravitino, $\Psi$, vanishes

$$
\delta \Psi_{I}=\left(\partial_{I}+\frac{1}{4} \omega_{I}^{A B} \Gamma_{A B}\right) \epsilon-\frac{1}{288}\left(\Gamma_{I}^{A B C D} F_{A B C D}^{(4)}-8 \Gamma^{B C D} F_{I B C D}^{(4)}\right) \epsilon=0 .
$$

Here $\omega_{I}^{A B}$ is the spin connection and $F^{(4)}$ is the four-form field strength of M-theory, while $I$ is a general coordinate index $(A, B, C, D$ are still tangent space indices).

\section{B.3.1 Objects in $\mathbb{R}^{2,1} \times \mathbb{C}^{4} / \mathbb{Z}_{k}$}

In this subsection we consider M-theory on $\mathbb{R}^{2,1} \times \mathbb{C}^{4} / \mathbb{Z}_{k}$, without flux $\left(F^{(4)}=0\right)$. We add M2-branes along $\mathbb{R}^{2,1}$ and a variety of KK monopoles and M-branes. We use polar coordinates on $\mathbb{C}^{4}$ such that $z^{i}=r_{i} e^{i \varphi_{i}}$. The metric is

$$
d s^{2}=-\left(d x^{0}\right)^{2}+\left(d x^{1}\right)^{2}+\left(d x^{2}\right)^{2}+\sum_{i=1}^{4}\left(d r_{i}^{2}+r_{i}^{2} d \varphi_{i}^{2}\right) .
$$

In these coordinates the spinor on $\mathbb{R}^{2,1} \times \mathbb{C}^{4}$ is

$$
\epsilon=e^{i \frac{\varphi_{1}}{2} \Gamma_{r_{1} \varphi_{1}}} e^{i \frac{\varphi_{2}}{2} \Gamma_{r_{2} \varphi_{2}}} e^{i \frac{\varphi_{3}}{2} \Gamma_{r_{3} \varphi_{3}}} e^{i \frac{\varphi_{4}}{2} \Gamma_{r_{4} \varphi_{4}}} \epsilon_{0} \equiv M \epsilon_{0},
$$


where $\epsilon_{0}$ is a constant 32-component spinor. The $\mathbb{Z}_{k}$ acts as $\varphi_{i} \rightarrow \varphi_{i}+\frac{2 \pi}{k}, \forall i$. We write $\epsilon_{0}$ as a sum of eigenspinors $\epsilon_{s_{1} s_{2} s_{3} s_{4}}$ that satisfy $\Gamma_{r_{i} \varphi_{i}} \epsilon_{s_{1} s_{2} s_{3} s_{4}}=i s_{i} \epsilon_{s_{1} s_{2} s_{3} s_{4}}$ for $i=1, \ldots, 4$, where $s_{i}= \pm 1$. For the spinor to be invariant we demand that $\sum_{i} s_{i}=0$ for $k>2$. This means that of the 16 combinations of $\left(s_{1}, s_{2}, s_{3}, s_{4}\right)=( \pm 1, \pm 1, \pm 1, \pm 1)$, we project out 10 combinations and preserve 6 . A spinor in $\mathbb{R}^{2,1}$ has two real components so that the 6 preserved combinations correspond to a total of 12 real preserved supercharges. From $\sum_{i} s_{i}=0$ we find that $\prod_{i} s_{i}=1$ and therefore that $\epsilon=\Gamma_{01 \ldots r_{4} \varphi_{4}} \epsilon=\Gamma_{012}(i)^{4} s_{1} s_{2} s_{3} s_{4} \epsilon=$ $\Gamma_{012} \epsilon$, so the projection condition for the color M2-branes is automatically satisfied, i.e. the M2-branes do not break any additional supersymmetry.

Now we can calculate $\Gamma_{\kappa}$ for any given embedding using equation (B.3.31), check how many supercharges are preserved by the condition ${ }^{2} \Gamma_{\kappa} \epsilon=\epsilon \Leftrightarrow M^{-1} \Gamma_{\kappa} M \epsilon_{0}=\epsilon_{0}$, where $\epsilon_{0}$ is the 12-component spinor from above. The calculation is fairly easy. We summarize our results in table B.2, and work out explicit examples in the more complicated background of $A d S_{4} \times S^{7} / \mathbb{Z}_{k}$ in the next subsection. In the table we restrict ourselves to objects that sit at the origin of $\mathbb{C}^{4} / \mathbb{Z}_{k}$. We can use the $S U(4) \times U(1)$ symmetry to set any constant phase factor to zero so that configurations that differ from those in the table by constant shifts in any of the $\varphi_{i}$ preserve the same amount of supersymmetry. The table contains the four examples studied in this paper and also some other easy configurations. The second column indicates what the resulting object is in type IIA for $k \rightarrow \infty$ and the third column gives the codimension of the probe in $\mathbb{R}^{2,1}$.

\begin{tabular}{|c|c|c|c|c|}
\hline M-theory & Type IIA & codim & real supercharges & worldvolume coordinates \\
\hline \hline M2 & D2 & 0 & 12 & $x^{0}, x^{1}, x^{2}$ \\
\hline \hline KK & D6 & 0 & 6 & $x^{0}, x^{1}, x^{2}, r_{1}, r_{2}, r_{3}, r_{4}$ \\
\hline KK & KK & 0 & 8 & $x^{0}, x^{1}, x^{2}, z^{1}=z^{2}, z^{3}=z^{4}$ \\
\hline M5 & D4 & 1 & 6 & $x^{0}, x^{1}, z^{1}=z^{2}, z^{3}=z^{4}$ \\
\hline M5 & NS5 & 1 & 6 & $x^{0}, x^{1}, r_{1}, r_{2}, r_{3}, r_{4}$ \\
\hline M9 & D8 & 1 & 6 & $x^{0}, x^{1}, r_{i}, \varphi_{i}, i=1,2,3,4$ \\
\hline M2 & D2 & 2 & 4 & $x^{0}, r_{3}=r_{4}, \varphi_{3}=-\varphi_{4}$ \\
\hline M2 & F1 & 2 & 6 & $x^{0}, z^{1}=z^{2}=z^{3}=z^{4}$ \\
\hline
\end{tabular}

Table B.2: List of supersymmetry-preserving objects of given codimension and given worldvolume directions in $\mathbb{C}^{4} / \mathbb{Z}_{k}$. The second column indicates what the probes become in type IIA (large $k$ ). For details see the accompanying paragraph.

\section{B.3.2 Objects in $A d S_{4} \times S^{7} / \mathbb{Z}_{k}$}

In this section we study objects in the geometry obtained as the near-horizon limit $\left(N_{c} \rightarrow\right.$ $\infty)$ of the $N_{c}$ M2-branes. First we introduce new coordinates

$$
z^{1}=r \cos \alpha \sin \beta e^{\zeta_{1}}, \quad z^{2}=r \cos \alpha \cos \beta e^{\zeta_{2}} \quad z^{3}=r \sin \alpha \sin \gamma e^{\zeta_{3}}, \quad z^{4}=r \sin \alpha \cos \gamma e^{\zeta_{4}}
$$

\footnotetext{
${ }^{2}$ The cautious reader might worry whether this procedure is applicable to KK monopoles and the mysterious M9-branes. We do not give a direct proof, rather we think of this one condition as a combination of the two projection conditions for the left- and right-handed spinors for D6- and D8-branes in type IIA.
} 
With these coordinates, the metric of $A d S_{4} \times S^{7}$ becomes

$$
\begin{aligned}
d s^{2}= & \frac{R^{2}}{4}\left(d r^{2}+e^{2 r}\left(-d t^{2}+d x_{1}^{2}+d x_{2}^{2}\right)\right)+R^{2}\left(d \alpha^{2}+\cos ^{2} \alpha d \beta^{2}+\sin ^{2} \alpha d \gamma^{2}(\mathrm{~B} .3 .35)\right. \\
& \left.+\cos ^{2} \alpha \sin ^{2} \beta d \zeta_{1}^{2}+\cos ^{2} \alpha \cos ^{2} \beta d \zeta_{2}^{2}+\sin ^{2} \alpha \sin ^{2} \gamma d \zeta_{3}^{2}+\sin ^{2} \alpha \cos ^{2} \gamma d \zeta_{4}^{2}\right)
\end{aligned}
$$

where $0 \leq \alpha, \beta, \gamma \leq \frac{\pi}{2}$ and $0 \leq \zeta_{i}<2 \pi$. We also have the flux $F^{(4)}=\frac{3}{8} R^{3} \Omega_{A d S_{4}}$ with $\Omega_{A d S_{4}}$ being the volume form of $A d S_{4}$.

From the supersymmetry variation of the gravitino we find that the spinor preserved by this background is

$$
\begin{aligned}
& \epsilon=e^{-\frac{r}{2} \Gamma_{r} \hat{\Gamma}}\left(\mathbb{1}_{32}+\frac{1}{2} x^{\mu} \Gamma_{\mu} \hat{\Gamma}\left(\mathbb{1}_{32}+\Gamma_{r} \hat{\Gamma}\right)\right) e^{\frac{\alpha}{2} \Gamma_{\alpha} \hat{\Gamma}} e^{\frac{\beta}{2} \Gamma_{\beta} \hat{\Gamma}} e^{\frac{\gamma}{2} \Gamma_{\alpha \gamma}} e^{\frac{\zeta_{1}}{2} \Gamma_{\beta \zeta_{1}}} e^{\frac{\zeta_{2}}{2} \Gamma_{\zeta_{2}} \hat{\Gamma}} e^{\frac{\zeta_{3}}{2} \Gamma_{\gamma \zeta_{3}}} e^{\frac{\zeta_{4}}{2} \Gamma_{\alpha \zeta_{4}}} \epsilon_{0} \\
& \equiv M_{A d S} M_{\alpha \beta \gamma} M_{\zeta} \epsilon_{0} \equiv M \epsilon_{0},
\end{aligned}
$$

where $\epsilon_{0}$ is a constant 32 -component spinor, $\hat{\Gamma}=\Gamma^{012 r}=-\Gamma_{012 r}, M_{A d S}$ is the part of $M$ that depends on $A d S_{4}$ coordinates, and similarly for $M_{\alpha \beta \gamma}$ and $M_{\zeta}$. The $\mathbb{Z}_{k}$ quotient acts as $\zeta_{i} \rightarrow \zeta_{i}+\frac{2 \pi}{k}$. We write the spinor $\epsilon_{0}$ as a sum of eigenspinors of $\left(\Gamma_{\beta \zeta_{1}}, \Gamma_{\zeta_{2}} \hat{\Gamma}, \Gamma_{\gamma \zeta_{3}}, \Gamma_{\alpha \zeta_{4}}\right)$, that is, $\Gamma_{\beta \zeta_{1}} \epsilon_{s_{1} s_{2} s_{3} s_{4}}=i s_{1} \epsilon_{s_{1} s_{2} s_{3} s_{4}}$, etc.; since only the eigenspinors that satisfy $\sum_{i=1}^{4} s_{i}=0$ are invariant under $\mathbb{Z}_{k}$ for $k>2$, the background preserves 24 real supercharges for $k>2$. This can be seen from $\mathbb{1}_{32}=\Gamma_{01 \ldots \zeta_{3} \zeta_{4}}=s_{1} s_{2} s_{3} s_{4} \mathbb{1}_{32}$ which implies an even number of positive and negative $s_{i}$. The condition $\sum_{i=1}^{4} s_{i}=0$ therefore projects out the two cases where all $s_{i}$ are the same, and is satisfied for the other six cases, so $\frac{6}{8}$ of the 32 supercharges (hence 24) are preserved.

Now we explicitly solve the $\kappa$-symmetry equation for the two objects discussed in sections 7.5 and 7.6 as well as two further examples summarized in section 7.8. As mentioned in the previous subsection, we think of this one condition in M-theory as a combination of the two projection conditions for the left- and right-handed spinors for D-branes in type IIA.

We start with the codimension-zero KK monopole of section 7.5. Although we can use the $S U(4) \times U(1)$ symmetry of the background to set constant phases to zero, we keep them explicitly in our calculation. This is useful if we consider multiple stacks of probes that sit at different constant phases. Instead of choosing the embedding such that $\operatorname{Im}\left(z^{i}\right)=0, \forall i$, we are more general and take the worldvolume coordinates to be $x^{0}, x^{1}, x^{2}, r, \alpha, \beta, \gamma$ and set the phase to constant values $\zeta_{i}^{0}$. For this embedding we find $\Gamma_{\kappa}=\Gamma_{012 r \alpha \beta \gamma}$ and

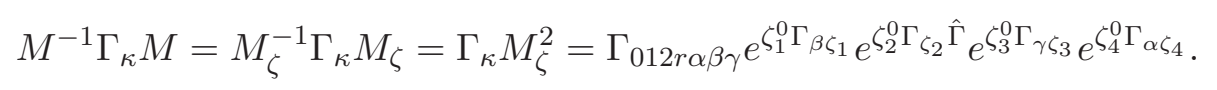

Solving $\Gamma_{012 r \alpha \beta \gamma} e^{\zeta_{1}^{0} \Gamma_{\beta \zeta_{1}}} e^{\zeta_{2}^{0} \Gamma_{\zeta_{2}} \hat{\Gamma}} e^{\zeta_{3}^{0} \Gamma_{\gamma \zeta_{3}}} e^{\zeta_{4}^{0} \Gamma_{\alpha \zeta_{4}} \epsilon_{0}}=\epsilon_{0}$ we find that 12 real components are preserved. Comparing with the second row in table B.2, we see that after the near-horizon limit the amount of supersymmetry has doubled, as expected.

Now we look at the codimension-one case of section [7.6, that is, the lift of D8-branes to M-theory. We choose the ten worldvolume coordinates to be $\left(x^{0}, x^{1}, r, \alpha, \beta, \gamma, \zeta_{1}, \zeta_{2}, \zeta_{3}, \zeta_{4}\right)$, and find that $\Gamma_{\kappa}=\Gamma_{01 r \alpha \beta \gamma \zeta_{1} \zeta_{2} \zeta_{3} \zeta_{4}}=\Gamma_{2}$, where we have used $\Gamma_{012 r \alpha \beta \gamma \zeta_{1} \zeta_{2} \zeta_{3} \zeta_{4}}=\mathbb{1}_{32} . \Gamma_{\kappa}$ commutes with $M_{\alpha \beta \gamma}$ and $M_{\zeta}$, so we find

$$
M^{-1} \Gamma_{\kappa} M=M_{A d S}^{-1} \Gamma_{\kappa} M_{A d S}=\left(\mathbb{1}_{32}-x^{2} \Gamma_{2} \hat{\Gamma}\left(\mathbb{1}_{32}+\Gamma_{r} \hat{\Gamma}\right)\right) \Gamma_{\kappa}=\left(\mathbb{1}_{32}-x^{2} \Gamma_{2} \hat{\Gamma}\left(\mathbb{1}_{32}+\Gamma_{r} \hat{\Gamma}\right)\right) \Gamma_{2} .
$$


Demanding that $M^{-1} \Gamma_{\kappa} M \epsilon_{0}=\epsilon_{0}$ again reduces the components of $\epsilon_{0}$ by a factor of $\frac{1}{2}$, so that we find 12 preserved supercharges.

The next codimension-one case, discussed shortly in section 7.8, are M5-branes extended along $A d S_{3}$ inside $A d S_{4}$ and embedded such that $z^{1}=z^{2}=0$. This embedding is in fact $S U(4)$-equivalent to the one used in table B.2 (see section 8.2 of [6]). The worldvolume coordinates are $\left(x^{0}, x^{1}, r, \gamma, \zeta_{3}, \zeta_{4}\right)$ and we have to set $\alpha=\frac{\pi}{2}$, which leads to $\Gamma_{\kappa}=\Gamma_{01 r \gamma \zeta_{3} \zeta_{4}}$. Since $M_{\alpha \beta \gamma}^{-1} \Gamma_{\kappa} M_{\alpha \beta \gamma}=-\hat{\Gamma} \Gamma_{\alpha} \Gamma_{\kappa}$ and $M_{\zeta}$ commutes with that, we find

$$
\begin{aligned}
M^{-1} \Gamma_{\kappa} M=-M_{A d S}^{-1} \hat{\Gamma} \Gamma_{\alpha} \Gamma_{\kappa} M_{A d S} & =-\left(\mathbb{1}_{32}-x^{2} \Gamma_{2} \hat{\Gamma}\left(\mathbb{1}_{32}+\Gamma_{r} \hat{\Gamma}\right)\right) \hat{\Gamma} \Gamma_{\alpha} \Gamma_{\kappa} \\
& =-\left(\mathbb{1}_{32}-x^{2} \Gamma_{2} \hat{\Gamma}\left(\mathbb{1}_{32}+\Gamma_{r} \hat{\Gamma}\right)\right) \Gamma_{2 \alpha \gamma \zeta_{3} \zeta_{4}}(
\end{aligned}
$$

We then find that 12 real supercharges are preserved. Again we see that the probes reduce the amount of supersymmetry of the background by a factor of $\frac{1}{2}$, and that the near-horizon limit leads to a doubling of the preserved supercharges (see table B.2).

Finally we look at the example mentioned in section 7.8, codimension-two M2-branes embedded such that $z^{1}=z^{2}=0, z^{3}=\bar{z}^{4}$. Again we are slightly more general and allow for constant phases. We take the worldvolume coordinates to be $x^{0}, r, \zeta_{3}$ and set $\alpha=\frac{\pi}{2}, \gamma=\frac{\pi}{4}, \zeta_{1}=\zeta_{1}^{0}, \zeta_{2}=\zeta_{2}^{0}, \zeta_{4}=-\zeta_{3}+\zeta^{0}$, where $\zeta_{1}^{0}, \zeta_{2}^{0}, \zeta^{0}$ are constants. We then find

$$
\Gamma_{\kappa}=\frac{1}{\sqrt{2}} \Gamma_{0 r}\left(\Gamma_{\zeta_{3}}-\Gamma_{\zeta_{4}}\right) \text {. }
$$

Next we write $M=M_{A d S} M_{\alpha \beta \gamma} M_{\zeta}$ and note that the AdS part commutes with the rest, so we first calculate

$$
M_{A d S}^{-1} \Gamma_{\kappa} M_{A d S}=\left(\mathbb{1}_{32}-\left(x^{1} \Gamma_{1}+x^{2} \Gamma_{2}\right) \hat{\Gamma}\left(\mathbb{1}_{32}+\Gamma_{r} \hat{\Gamma}\right)\right) \Gamma_{\kappa} .
$$

We then use $\alpha=\frac{\pi}{2}$ to find

$$
\begin{aligned}
& M_{\alpha \beta \gamma}^{-1} M_{A d S}^{-1} \Gamma_{\kappa} M_{A d S} M_{\alpha \beta \gamma} \\
& =\left(\mathbb{1}_{32}-\left(x^{1} \Gamma_{1}+x^{2} \Gamma_{2}\right) \hat{\Gamma}\left(\mathbb{1}_{32}+\Gamma_{r} \hat{\Gamma}\right)\right) e^{-\frac{\gamma}{2} \Gamma_{\alpha \gamma}} e^{-\frac{\beta}{2} \Gamma_{\beta} \hat{\Gamma}} e^{-\frac{\alpha}{2} \Gamma_{\alpha} \hat{\Gamma}} \Gamma_{\kappa} e^{\frac{\alpha}{2} \Gamma_{\alpha} \hat{\Gamma}} e^{\frac{\beta}{2} \Gamma_{\beta} \hat{\Gamma}} e^{\frac{\gamma}{2} \Gamma_{\alpha \gamma}} \\
& =\left(\mathbb{1}_{32}-\left(x^{1} \Gamma_{1}+x^{2} \Gamma_{2}\right) \hat{\Gamma}\left(\mathbb{1}_{32}+\Gamma_{r} \hat{\Gamma}\right)\right) e^{-\frac{\gamma}{2} \Gamma_{\alpha \gamma}} e^{-\frac{\beta}{2} \Gamma_{\beta} \hat{\Gamma}} \Gamma_{\kappa} e^{\alpha \Gamma_{\alpha} \hat{\Gamma}} e^{\frac{\beta}{2} \Gamma_{\beta} \hat{\Gamma}} e^{\frac{\gamma}{2} \Gamma_{\alpha \gamma}} \\
& =\left(\mathbb{1}_{32}-\left(x^{1} \Gamma_{1}+x^{2} \Gamma_{2}\right) \hat{\Gamma}\left(\mathbb{1}_{32}+\Gamma_{r} \hat{\Gamma}\right)\right) e^{-\frac{\gamma}{2} \Gamma_{\alpha \gamma}} \Gamma_{\kappa} \Gamma_{\alpha} \hat{\Gamma} e^{\frac{\gamma}{2} \Gamma_{\alpha \gamma}} \\
& =\left(\mathbb{1}_{32}-\left(x^{1} \Gamma_{1}+x^{2} \Gamma_{2}\right) \hat{\Gamma}\left(\mathbb{1}_{32}+\Gamma_{r} \hat{\Gamma}\right)\right) \Gamma_{\kappa} \frac{1}{\sqrt{2}}\left(\Gamma_{\alpha}+\Gamma_{\gamma}\right) \hat{\Gamma} \\
& =\left(\hat{\Gamma}+\left(x^{1} \Gamma_{1}+x^{2} \Gamma_{2}\right)\left(\mathbb{1}_{32}-\Gamma_{r} \hat{\Gamma}\right)\right) \Gamma_{\kappa} \frac{1}{\sqrt{2}}\left(\Gamma_{\alpha}+\Gamma_{\gamma}\right) \\
& =\frac{1}{2}\left(\hat{\Gamma}+\left(x^{1} \Gamma_{1}+x^{2} \Gamma_{2}\right)\left(\mathbb{1}_{32}-\Gamma_{r} \hat{\Gamma}\right)\right) \Gamma_{0 r}\left(-\Gamma_{\alpha \zeta_{3}}+\Gamma_{\alpha \zeta_{4}}-\Gamma_{\gamma \zeta_{3}}+\Gamma_{\gamma \zeta_{4}}\right) .
\end{aligned}
$$

Since equation $(\overline{B .3 .42})$ commutes with $\Gamma_{\beta \zeta_{1}}$ and $\Gamma_{\zeta_{2}} \hat{\Gamma}$ we have

$$
\begin{aligned}
& M^{-1} \Gamma_{\kappa} M=e^{-\frac{\zeta_{4}}{2} \Gamma_{\alpha \zeta_{4}}} e^{-\frac{\zeta_{3}}{2} \Gamma_{\gamma \zeta_{3}}} \frac{1}{2}\left(\hat{\Gamma}+\left(x^{1} \Gamma_{1}+x^{2} \Gamma_{2}\right)\left(\mathbb{1}_{32}-\Gamma_{r} \hat{\Gamma}\right)\right) \Gamma_{0 r}
\end{aligned}
$$

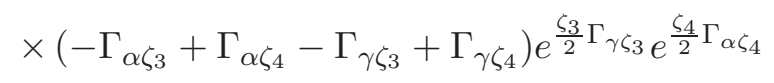

$$
\begin{aligned}
& =\frac{1}{2}\left(\hat{\Gamma}+\left(x^{1} \Gamma_{1}+x^{2} \Gamma_{2}\right)\left(\mathbb{1}_{32}-\Gamma_{r} \hat{\Gamma}\right)\right) \Gamma_{0 r} \\
& \times\left(\Gamma_{\alpha \zeta_{4}}-\Gamma_{\gamma \zeta_{3}}+\cos \zeta^{0}\left(\Gamma_{\gamma \zeta_{4}}-\Gamma_{\alpha \zeta_{3}}\right)+\sin \zeta^{0}\left(\Gamma_{\alpha \gamma}+\Gamma_{\zeta_{3} \zeta_{4}}\right)\right) .
\end{aligned}
$$


Acting with this on the 24-component constant spinor $\epsilon_{0}$, we find that such branes preserve 8 real supercharges. Note that although the projector depends on $\zeta^{0}$, we find that the preserved supercharges depend only on the position in $x^{1}, x^{2}$ and not on the constant phases $\zeta_{1}^{0}, \zeta_{2}^{0}, \zeta^{0}$.

\section{B.4 $\mathcal{N}=(0,6)$ Supersymmetry Transformations}

In this appendix we discuss the supersymmetry transformations of the codimension-one flavor field theories of sections 7.6 as well as the one discussed in section [7.8. In particular, we show that the gauge field component $A_{-}$, appearing in the Lagrangian of the codimension-one chiral field theory of section 7.6 , equation $(7.6 .20)$, is invariant under $\mathcal{N}=(0,6)$ supersymmetry.

The supersymmetry algebra of the ABJM theory is

$$
\left\{\mathcal{Q}_{\alpha}^{(I)}, \mathcal{Q}_{\beta}^{(J)}\right\}=-2 \delta^{I J}\left(\gamma^{\mu}\right)_{\alpha \beta} P_{\mu},
$$

where $\left(\gamma^{\mu}\right)_{\alpha \beta}$ is given by $\gamma^{\mu}=\left(-\mathbb{1},-\sigma^{3}, \sigma^{1}\right)$ and $\mu=0,1,2$. The index $\alpha=1,2$ labels the components of the real two-component spinor $\mathcal{Q}$. (Notice that we are using different conventions from those in section 7.3.1.)

Let us place the defect at $x^{2}=0$. Since the translational invariance in $x_{2}$ direction and therefore the momentum $P_{2}$ is broken, some of the supersymmetry charges are also broken. Let us discuss the broken supersymmetry generators for the $\mathcal{N}=(0,6)$ and $\mathcal{N}=(3,3)$ supersymmetric flavor theories.

The broken supersymmetry generators for the $\mathcal{N}=(0,6)$ supersymmetric flavor theory are $\mathcal{Q}_{1}^{(I)}$. An explicit check shows that, upon setting $\mathcal{Q}_{1}^{(I)}=0$, the algebra reduces to a supersymmetry algebra for a $(1+1)$-dimensional theory, i.e. $P_{2}$ drops out.

The broken supersymmetry generators for the $\mathcal{N}=(3,3)$ supersymmetric flavor theory are more complicated since the obvious guess, setting half of the supersymmetry generators $\mathcal{Q}^{(I)}$ to zero, is wrong. For simplicity let us consider the algebra just for two supersymmetry generators, say $I=1$ and $I=2$,

$$
\left\{\mathcal{Q}_{\alpha}^{(1)}, \mathcal{Q}_{\beta}^{(1)}\right\}=\left\{\mathcal{Q}_{\alpha}^{(2)}, \mathcal{Q}_{\beta}^{(2)}\right\}=-2\left(\gamma^{\mu}\right)_{\alpha \beta} P_{\mu}, \quad\left\{\mathcal{Q}_{\alpha}^{(1)}, \mathcal{Q}_{\beta}^{(2)}\right\}=0
$$

Since we aim to eliminate $P_{2}$ and $\sigma_{2}$ is off-diagonal, we have to define the new supersymmetry charges,

$$
\tilde{\mathcal{Q}}_{1} \equiv \mathcal{Q}_{1}^{(1)}, \quad \overline{\tilde{\mathcal{Q}}}_{2} \equiv \mathcal{Q}_{2}^{(2)}, \quad \tilde{\mathscr{Q}}_{1} \equiv \mathcal{Q}_{1}^{(2)}, \quad \tilde{\mathscr{Q}}_{2} \equiv \mathcal{Q}_{2}^{(1)},
$$

and set $\tilde{\mathscr{Q}}_{1}=\tilde{\mathscr{Q}}_{2}=0$. The remaining supersymmetry generators $\tilde{\mathcal{Q}}_{\alpha}, \quad \alpha=1,2$, satisfy the $(1+1)$-dimensional supersymmetry algebra. This procedure can be straightforward generalized to six supersymmetry generators. The unbroken supercharges generate a $\mathcal{N}=$ $(3,3)$ supersymmetric algebra in $(1+1)$ dimensions.

In order to determine the supersymmetry transformation for $A_{-}$in the $\mathcal{N}=(0,6)$ supersymmetric theory, we use the conventions and supersymmetry transformations of [249]. 
Let us quote the supersymmetry transformation of the gauge fields $A_{\mu}$ and $\hat{A}_{\mu}$ (the gauge fields of the two gauge groups),

$$
\begin{aligned}
& \delta A_{\mu}=\Gamma_{A B}^{I} \bar{\epsilon}^{I} \gamma_{\mu} \Psi^{A} X^{B}-\tilde{\Gamma}^{I A B} X_{B} \bar{\Psi}_{A} \gamma_{\mu} \epsilon^{I}, \\
& \delta \hat{A}_{\mu}=\Gamma_{A B}^{I} X^{B} \bar{\epsilon}^{I} \gamma_{\mu} \Psi^{A}-\tilde{\Gamma}^{I A B} \bar{\Psi}_{A} \gamma_{\mu} \epsilon^{I} X_{B},
\end{aligned}
$$

where $X_{A}, A=1, \ldots, 4$ are the four complex scalars and $\Psi_{A}$ are the spinor fields of the ABJM theory (in the notation of [249]). The spinor field $\Psi_{A \alpha}$ has a lower spinor index, whereas the conjugated field $\bar{\Psi}_{A}^{\alpha}$ carries an upper spinor index. Note that $\left(\gamma^{\mu}\right)_{\alpha}^{\beta}=$ $\left(i \sigma^{2}, \sigma^{1}, \sigma^{3}\right)$ for $\mu=0,1,2$. The conjugate fields $\Psi^{A}$ and $X^{B}$ are denoted by upper indices. Moreover, $\epsilon^{I}$ are real two-component spinors for $I=1, \ldots, 6$, and the $4 \times 4$ matrices $\Gamma^{I}, I=1, \ldots, 6$ satisfy the commutation relation

$$
\Gamma^{I} \tilde{\Gamma}^{J}+\Gamma^{J} \tilde{\Gamma}^{I}=2 \delta^{I J},
$$

where $\tilde{\Gamma}^{I}=\left(\Gamma^{I}\right)^{\dagger}$. Let us decompose $\epsilon^{I}$ into

$$
\epsilon^{I}=\left(\begin{array}{c}
\epsilon_{R}^{I} \\
\epsilon_{L}^{I}
\end{array}\right)
$$

and set $\epsilon_{L}^{I}$ to zero since the $\tilde{\mathcal{Q}}_{2}^{(I)}$ are broken in the $\mathcal{N}=(0,6)$ algebra. Finally, introducing lightcone coordinates $x^{ \pm}=x^{0} \pm x^{1}$, the unbroken right-handed supersymmetry transformations $\delta_{R, I}$ with respect to $\epsilon_{R}^{I}$ of the gauge field components read

$$
\begin{aligned}
& \delta_{R, I} A_{+}=\Gamma_{A B}^{I} \epsilon_{R}^{I} \Psi_{R}^{A} X^{B}-\tilde{\Gamma}^{I A B} X_{B} \Psi_{R A} \epsilon_{R}^{I}, \\
& \delta_{R, I} A_{-}=0, \\
& \delta_{R, I} \hat{A}_{+}=\Gamma_{A B}^{I} X^{B} \epsilon_{R}^{I} \Psi_{R}^{A}-\tilde{\Gamma}^{I A B} \Psi_{R A} \epsilon_{R}^{I} X_{B}, \\
& \delta_{R, I} \hat{A}_{-}=0 .
\end{aligned}
$$

In particular we see that $A_{-}$and $\hat{A}_{-}$do not transform under $\mathcal{N}=(0,6)$ supersymmetry. 


\section{Bibliography}

[1] M. Ammon, J. Erdmenger, S. Höhne, D. Lüst, and R. Meyer, Fayet-Iliopoulos Terms in AdS/CFT with Flavour, JHEP 07 (2008) 068, arXiv:0805.1917.

[2] M. Ammon, J. Erdmenger, M. Kaminski, and P. Kerner, Superconductivity from gauge/gravity duality with flavor, Phys. Lett. B680 (2009) 516-520, arXiv:0810.2316.

[3] M. Ammon, J. Erdmenger, M. Kaminski, and P. Kerner, Flavor Superconductivity from Gauge/Gravity Duality, JHEP 10 (2009) 067, [arXiv:0903.1864.

[4] J. Erdmenger and M. Ammon, Introduction to cosmology and string theory, . In *Erdmenger, J. (ed.): String cosmology* 1-46.

[5] M. Ammon, T. H. Ngo, and A. O'Bannon, Holographic Flavor Transport in Arbitrary Constant Background Fields, JHEP 10 (2009) 027, arXiv:0908.2625.

[6] M. Ammon, J. Erdmenger, R. Meyer, A. O'Bannon, and T. Wrase, Adding Flavor to AdS4/CFT3, JHEP 11 (2009) 125, arXiv:0909.3845.

[7] M. Ammon, J. Erdmenger, V. Grass, P. Kerner, and A. O'Bannon, On Holographic p-wave Superfluids with Back-reaction, Phys. Lett. B686 (2010) 192-198, arXiv:0912.3515].

[8] M. Ammon, J. Erdmenger, M. Kaminski, and A. O'Bannon, Fermionic Operator Mixing in Holographic p-wave Superfluids, JHEP 05 (2010) 053, arXiv:1003.1134.

[9] M. Ammon, C. Hoyos, A. O'Bannon, and J. M. S. Wu, Holographic Flavor Transport in Schroedinger Spacetime, JHEP 06 (2010) 012, [arXiv:1003.5913].

[10] J. M. Maldacena, The large $N$ limit of superconformal field theories and supergravity, Adv. Theor. Math. Phys. 2 (1998) 231-252, hep-th/9711200.

[11] E. Witten, Anti-de Sitter space and holography, Adv. Theor. Math. Phys. 2 (1998) 253-291, hep-th/9802150.

[12] S. S. Gubser, I. R. Klebanov, and A. M. Polyakov, Gauge theory correlators from non-critical string theory, Phys. Lett. B428 (1998) 105-114, hep-th/9802109.

[13] H. J. Schulz, Fermi liquids and non-Fermi liquids, cond-mat/9503150.

[14] J. Bednorz and K. A. Müller, Possible High-Tc superconductivity in the Ba-La-Cu-O system, Zeitschrift fur Physik B condensed matter 64 (1986), no. 2 189-193. 
[15] Z.-A. Ren, G.-C. Che, X.-L. Dong, J. Yang, W. Lu, W. Yi, X.-L. Shen, Z.-C. Li, L.-L. Sun, F. Zhou, and Z.-X. Zhao, Superconductivity and phase diagram in iron-based arsenic-oxides refeaso, EPL (Europhysics Letters) 83 (2008), no. 1 17002 .

[16] F. Steglich, J. Aarts, C. D. Bredl, W. Lieke, D. Meschede, W. Franz, and H. Schäfer, Superconductivity in the presence of strong pauli paramagnetism: Cecu si $_{2}$, Phys. Rev. Lett. 43 (Dec, 1979) 1892-1896.

[17] H. R. Ott, H. Rudigier, Z. Fisk, and J. L. Smith, Ube 13 : An unconventional actinide superconductor, Phys. Rev. Lett. 50 (May, 1983) 1595-1598.

[18] D. C. Tsui, H. L. Stormer, and A. C. Gossard, Two-dimensional magnetotransport in the extreme quantum limit, Phys. Rev. Lett. 48 (May, 1982) 1559-1562.

[19] R. B. Laughlin, Quantized hall conductivity in two dimensions, Phys. Rev. B 23 (May, 1981) 5632-5633.

[20] B. I. Halperin, Quantized hall conductance, current-carrying edge states, and the existence of extended states in a two-dimensional disordered potential, Phys. Rev. B 25 (Feb, 1982) 2185-2190.

[21] S.-I. Tomonaga, Remarks on Bloch's Method of Sound Waves applied to Many-Fermion Problems, Progress of Theoretical Physics 5 (1950), no. 4 544-569.

[22] J. M. Luttinger, An Exactly Soluble Model of a Many-Fermion System, J. Math. Phys. 4 (1963) 1154.

[23] A. Luther and V. J. Emery, Backward scattering in the one-dimensional electron gas, Phys. Rev. Lett. 33 (Sep, 1974) 589-592.

[24] P. Anderson, The resonating valence bond state in la2cuo4 and superconductivity, Science 235 (1987), no. $47931196-1198$.

[25] P. Anderson, c-axis electrodynamics as evidence for the interlayer theory of high-temperature superconductivity, Science 279 (1987), no. 53541196 -1198.

[26] G. Kotliar, Resonating valence bonds and d-wave superconductivity, Phys. Rev. B 37 (Mar, 1988) 3664-3666.

[27] P. Gegenwart, Q. Si, and F. Steglich, Quantum criticality in heavy fermion metals, Nature Physics 4 (2008) 186.

[28] P. Monthoux, D. Pines, and G. Lonzarich, Superconductivity without phonons, Nature 450 (2007) 1177.

[29] N. D. Mathur, F. M. Grosche, S. R. Julian, I. R. Walker, D. M. Freye, R. K. W. Haselwimmer, and G. G. Lonzarich, Magnetically mediated superconductivity in heavy fermion compounds, Nature 394 (1998) 39-43.

[30] C. P. Herzog, Lectures on Holographic Superfluidity and Superconductivity, J. Phys. A42 (2009) 343001, [arXiv:0904.1975]. 
[31] C. C. Homes, S. V. Dordevic, D. A. Bonn, R. Liang, W. N. Hardy, and T. Timusk, Coherence, incoherence, and scaling along the $c$ axis of yba2cu3o6 $+x$, Phys. Rev. B 71 (May, 2005) 184515.

[32] C. C. Homes, S. V. Dordevic, T. Valla, and M. Strongin, Scaling of the superfluid density in high-temperature superconductors, Phys. Rev. B 72 (Oct, 2005) 134517.

[33] C. e. a. Homes, A universal scaling relation in high-temperature superconductors, Nature 430 (July, 2004) 539-541.

[34] J. Zaanen, Superconductivity: Why the temperature is high, Nature 430 (July, 2004) 512-513.

[35] S. Sachdev, Quantum Phase transitions. Cambridge University Press, 1999.

[36] S. A. Hartnoll, Lectures on holographic methods for condensed matter physics, Class. Quant. Grav. 26 (2009) 224002, arXiv:0903.3246.

[37] J. McGreevy, Holographic duality with a view toward many-body physics, arXiv:0909.0518.

[38] S. Sachdev and M. Müller, Quantum criticality and black holes, arXiv:0810.3005.

[39] K. Damle and S. Sachdev, Nonzero-temperature transport near quantum critical points, Phys. Rev. B $\mathbf{5 6}$ (Oct, 1997) 8714-8733.

[40] A. G. Green and S. L. Sondhi, Nonlinear quantum critical transport and the schwinger mechanism for a superfluid-mott-insulator transition of bosons, Phys. Rev. Lett. 95 (Dec, 2005) 267001.

[41] M. J. Bhaseen, A. G. Green, and S. L. Sondhi, Magnetothermoelectric response at a superfluid-mott-insulator transition, Phys. Rev. Lett. 98 (Apr, 2007) 166801.

[42] C. P. Herzog, P. Kovtun, S. Sachdev, and D. T. Son, Quantum critical transport, duality, and M-theory, Phys. Rev. D75 (2007) 085020, hep-th/0701036.

[43] S. A. Hartnoll, P. K. Kovtun, M. Muller, and S. Sachdev, Theory of the Nernst effect near quantum phase transitions in condensed matter, and in dyonic black holes, Phys. Rev. B76 (2007) 144502, arXiv:0706.3215.

[44] S. S. Gubser, Breaking an Abelian gauge symmetry near a black hole horizon, Phys. Rev. D78 (2008) 065034, [arXiv:0801.2977].

[45] S. A. Hartnoll, C. P. Herzog, and G. T. Horowitz, Building a Holographic Superconductor, Phys. Rev. Lett. 101 (2008) 031601, arXiv:0803.3295].

[46] S. A. Hartnoll, C. P. Herzog, and G. T. Horowitz, Holographic Superconductors, JHEP 12 (2008) 015, arXiv:0810.1563.

[47] S. S. Gubser and S. S. Pufu, The gravity dual of a p-wave superconductor, JHEP 11 (2008) 033, arXiv:0805.2960.

[48] S.-J. Rey, String theory on thin semiconductors: Holographic realization of Fermi points and surfaces, Prog. Theor. Phys. Suppl. 177 (2009) 128-142, [arXiv:0911.5295]. 
[49] H. Liu, J. McGreevy, and D. Vegh, Non-Fermi liquids from holography, arXiv:0903.2477.

[50] M. Cubrovic, J. Zaanen, and K. Schalm, String Theory, Quantum Phase Transitions and the Emergent Fermi-Liquid, Science 325 (2009) 439-444, [arXiv:0904.1993].

[51] T. Faulkner, H. Liu, J. McGreevy, and D. Vegh, Emergent quantum criticality, Fermi surfaces, and AdS2, arXiv:0907.2694.

[52] S.-S. Lee, A Non-Fermi Liquid from a Charged Black Hole: A Critical Fermi Ball, Phys. Rev. D79 (2009) 086006, [arXiv:0809.3402].

[53] J.-W. Chen, Y.-J. Kao, and W.-Y. Wen, Peak-Dip-Hump from Holographic Superconductivity, arXiv:0911.2821.

[54] A. Damascelli, Z. Hussain, and Z.-X. Shen, Angle-resolved photoemission studies of the cuprate superconductors, Rev. Mod. Phys. 75 (Apr, 2003) 473-541.

[55] S. S. Gubser, F. D. Rocha, and P. Talavera, Normalizable fermion modes in a holographic superconductor, arXiv:0911.3632.

[56] T. Faulkner, G. T. Horowitz, J. McGreevy, M. M. Roberts, and D. Vegh, Photoemission 'experiments' on holographic superconductors, JHEP 03 (2010) 121, arXiv:0911.3402].

[57] O. Aharony, S. S. Gubser, J. M. Maldacena, H. Ooguri, and Y. Oz, Large $N$ field theories, string theory and gravity, Phys. Rept. 323 (2000) 183-386, hep-th/9905111.

[58] E. D'Hoker and D. Z. Freedman, Supersymmetric gauge theories and the AdS/CFT correspondence, hep-th/0201253.

[59] B. Zwiebach, A first course in string theory, . Cambridge, UK: Univ. Pr. (2004) $558 \mathrm{p}$.

[60] J. Polchinski, String Theory I: An Introduction to the Bosonic String. Cambridge University Press, 1998.

[61] J. Polchinski, String Theory II: Superstring Theory and Beyond. Cambridge University Press, 1998.

[62] C. V. Johnson, D-branes. Univ. Pr., 2003. Cambridge, USA.

[63] K. Becker, M. Becker, and J. H. Schwarz, String theory and M-theory: A modern introduction, . Cambridge, UK: Cambridge Univ. Pr. (2007) 739 p.

[64] M. B. Green, J. H. Schwarz, and E. Witten, Superstring theory. Vol. 2: Loop amplitudes, anomalies and phenomenology, . Cambridge, Uk: Univ. Pr. (1987) 596 P. ( Cambridge Monographs On Mathematical Physics).

[65] M. B. Green, J. H. Schwarz, and E. Witten, Superstring theory. Vol. 1: Introduction, . Cambridge, Uk: Univ. Pr. (1987) 469 P. ( Cambridge Monographs On Mathematical Physics). 
[66] E. Kiritsis, String theory in a nutshell, . Princeton, USA: Univ. Pr. (2007) 588 p.

[67] D. Lüst and S. Theisen, Lectures on string theory, Lect. Notes Phys. 346 (1989) $1-346$.

[68] J. Wess and J. Bagger, Supersymmetry and supergravity, . Princeton, USA: Univ. Pr. (1992) 259 p.

[69] C. Montonen and D. I. Olive, Magnetic Monopoles as Gauge Particles?, Phys. Lett. B72 (1977) 117.

[70] P. Goddard, J. Nuyts, and D. I. Olive, Gauge Theories and Magnetic Charge, Nucl. Phys. B125 (1977) 1.

[71] E. Witten and D. I. Olive, Supersymmetry Algebras That Include Topological Charges, Phys. Lett. B78 (1978) 97.

[72] M. Dohse, Configuration Space Methods and Time Ordering for Scalar Propagators in (Anti and) de Sitter Spacetimes, arXiv:0706.1887.

[73] A. O'Bannon, Holographic Thermodynamics and Transport of Flavor Fields, arXiv:0808.1115.

[74] G. 't Hooft, a planar diagram theory for strong interactions, Nucl. Phys. B72 (1974) 461.

[75] P. Breitenlohner and D. Z. Freedman, Positive Energy in anti-De Sitter Backgrounds and Gauged Extended Supergravity, Phys. Lett. B115 (1982) 197.

[76] I. R. Klebanov and E. Witten, AdS/CFT correspondence and symmetry breaking, Nucl. Phys. B556 (1999) 89-114, hep-th/9905104.

[77] S. de Haro, S. N. Solodukhin, and K. Skenderis, Holographic reconstruction of spacetime and renormalization in the AdS/CFT correspondence, Commun. Math. Phys. 217 (2001) 595-622, hep-th/0002230.

[78] K. Skenderis, Lecture notes on holographic renormalization, Class. Quant. Grav. 19 (2002) 5849-5876, hep-th/0209067.

[79] M. Bianchi, D. Z. Freedman, and K. Skenderis, Holographic Renormalization, Nucl. Phys. B631 (2002) 159-194, hep-th/0112119.

[80] R. G. Leigh and N. N. Hoang, Fermions and the Sch/nrCFT Correspondence, JHEP 03 (2010) 027, arXiv:0909.1883.

[81] N. Iqbal and H. Liu, Real-time response in AdS/CFT with application to spinors, Fortsch. Phys. 57 (2009) 367-384, arXiv:0903.2596.

[82] R. Contino and A. Pomarol, Holography for fermions, JHEP 11 (2004) 058, hep-th/0406257.

[83] M. Henneaux, Boundary terms in the AdS/CFT correspondence for spinor fields, hep-th/9902137. 
[84] M. Henningson and K. Sfetsos, Spinors and the AdS/CFT correspondence, Phys. Lett. B431 (1998) 63-68, hep-th/9803251.

[85] W. Mück and K. S. Viswanathan, Conformal field theory correlators from classical field theory on anti-de Sitter space. II: Vector and spinor fields, Phys. Rev. D58 (1998) 106006, hep-th/9805145].

[86] E. Witten, Anti-de Sitter space, thermal phase transition, and confinement in gauge theories, Adv. Theor. Math. Phys. 2 (1998) 505-532, hep-th/9803131].

[87] S. S. Gubser, I. R. Klebanov, and A. W. Peet, Entropy and Temperature of Black 3-Branes, Phys. Rev. D54 (1996) 3915-3919, hep-th/9602135.

[88] A. Karch and E. Katz, Adding flavor to AdS/CFT, JHEP 06 (2002) 043, hep-th/0205236.

[89] M. Kruczenski, D. Mateos, R. C. Myers, and D. J. Winters, Meson spectroscopy in AdS/CFT with flavour, JHEP 07 (2003) 049, hep-th/0304032.

[90] T. Sakai and J. Sonnenschein, Probing flavored mesons of confining gauge theories by supergravity, JHEP 09 (2003) 047, hep-th/0305049.

[91] J. Babington, J. Erdmenger, N. J. Evans, Z. Guralnik, and I. Kirsch, Chiral symmetry breaking and pions in non-supersymmetric gauge / gravity duals, Phys. Rev. D69 (2004) 066007, hep-th/0306018.

[92] J. Erdmenger, N. Evans, I. Kirsch, and E. Threlfall, Mesons in Gauge/Gravity Duals - A Review, Eur. Phys. J. A35 (2008) 81-133, [arXiv:0711.4467].

[93] A. Karch and L. Randall, Open and closed string interpretation of SUSY CFT's on branes with boundaries, JHEP 06 (2001) 063, hep-th/0105132.

[94] O. DeWolfe, D. Z. Freedman, and H. Ooguri, Holography and defect conformal field theories, Phys. Rev. D66 (2002) 025009, hep-th/0111135.

[95] J. Erdmenger, Z. Guralnik, and I. Kirsch, Four-Dimensional Superconformal Theories with Interacting Boundaries or Defects, Phys. Rev. D66 (2002) 025020, hep-th/0203020.

[96] J. A. Harvey and A. B. Royston, Localized Modes at a D-brane-O-plane Intersection and Heterotic Alice Strings, JHEP 04 (2008) 018, [arXiv:0709.1482].

[97] E. I. Buchbinder, J. Gomis, and F. Passerini, Holographic Gauge Theories in Background Fields and Surface Operators, JHEP 12 (2007) 101, arXiv:0710.5170.

[98] J. A. Harvey and A. B. Royston, Gauge/Gravity duality with a chiral $N=(0,8)$ string defect, JHEP 08 (2008) 006, [arXiv:0804.2854].

[99] J. Gomis and F. Passerini, Holographic Wilson loops, JHEP 08 (2006) 074, hep-th/0604007.

[100] N. R. Constable, J. Erdmenger, Z. Guralnik, and I. Kirsch, Intersecting D3-branes and holography, Phys. Rev. D68 (2003) 106007, hep-th/0211222. 
[101] I. Kirsch, Spectroscopy of fermionic operators in AdS/CFT, JHEP 09 (2006) 052, hep-th/0607205.

[102] K. Skenderis and M. Taylor, Branes in AdS and pp-wave spacetimes, JHEP 06 (2002) 025, hep-th/0204054.

[103] A. Karch, A. O'Bannon, and K. Skenderis, Holographic renormalization of probe D-branes in AdS/CFT, JHEP 04 (2006) 015, hep-th/0512125.

[104] A. Karch and A. O'Bannon, Chiral transition of $N=4$ super Yang-Mills with flavor on a 3-sphere, Phys. Rev. D74 (2006) 085033, hep-th/0605120.

[105] A. Karch and A. O'Bannon, Metallic AdS/CFT, JHEP 09 (2007) 024, [arXiv:0705.3870.

[106] A. O'Bannon, Hall Conductivity of Flavor Fields from AdS/CFT, Phys. Rev. D76 (2007) 086007, arXiv:0708.1994.

[107] I. Kirsch, Generalizations of the AdS/CFT correspondence, Fortsch. Phys. 52 (2004) 727-826, hep-th/0406274.

[108] K. Ghoroku, T. Sakaguchi, N. Uekusa, and M. Yahiro, Flavor quark at high temperature from a holographic model, Phys. Rev. D71 (2005) 106002, hep-th/0502088.

[109] D. Mateos, R. C. Myers, and R. M. Thomson, Holographic phase transitions with fundamental matter, Phys. Rev. Lett. 97 (2006) 091601, hep-th/0605046.

[110] T. Albash, V. G. Filev, C. V. Johnson, and A. Kundu, A topology-changing phase transition and the dynamics of flavour, Phys. Rev. D77 (2008) 066004, hep-th/0605088.

[111] C. Hoyos-Badajoz, K. Landsteiner, and S. Montero, Holographic Meson Melting, JHEP 04 (2007) 031, hep-th/0612169].

[112] D. Mateos, R. C. Myers, and R. M. Thomson, Thermodynamics of the brane, JHEP 05 (2007) 067, hep-th/0701132.

[113] R. C. Myers and R. M. Thomson, Holographic mesons in various dimensions, JHEP 09 (2006) 066, hep-th/0605017].

[114] D. Arean, Holographic flavor in the gauge/gravity duality, arXiv:0906.5183.

[115] S. Kobayashi, D. Mateos, S. Matsuura, R. C. Myers, and R. M. Thomson, Holographic phase transitions at finite baryon density, JHEP 02 (2007) 016, hep-th/0611099.

[116] A. Karch, A. O'Bannon, and E. Thompson, The Stress-Energy Tensor of Flavor Fields from AdS/CFT, JHEP 04 (2009) 021, arXiv:0812.3629.

[117] D. Mateos, S. Matsuura, R. C. Myers, and R. M. Thomson, Holographic phase transitions at finite chemical potential, JHEP 11 (2007) 085, [arXiv:0709.1225].

[118] T. Faulkner and H. Liu, Condensed matter physics of a strongly coupled gauge theory with quarks: some novel features of the phase diagram, arXiv:0812.4278. 
[119] A. Karch and A. O'Bannon, Holographic Thermodynamics at Finite Baryon Density: Some Exact Results, JHEP 11 (2007) 074, [arXiv:0709.0570].

[120] V. G. Filev, C. V. Johnson, R. C. Rashkov, and K. S. Viswanathan, Flavoured large $N$ gauge theory in an external magnetic field, JHEP 10 (2007) 019, hep-th/0701001.

[121] V. G. Filev, Criticality, Scaling and Chiral Symmetry Breaking in External Magnetic Field, JHEP 04 (2008) 088, [arXiv:0706.3811].

[122] T. Albash, V. G. Filev, C. V. Johnson, and A. Kundu, Finite Temperature Large N Gauge Theory with Quarks in an External Magnetic Field, JHEP 07 (2008) 080, [arXiv:0709.1547].

[123] J. Erdmenger, R. Meyer, and J. P. Shock, AdS/CFT with Flavour in Electric and Magnetic Kalb-Ramond Fields, JHEP 12 (2007) 091, arXiv:0709.1551.

[124] K. Jensen, A. Karch, and E. G. Thompson, A Holographic Quantum Critical Point at Finite Magnetic Field and Finite Density, JHEP 05 (2010) 015, arXiv: 1002.2447.

[125] N. Evans, A. Gebauer, K.-Y. Kim, and M. Magou, Holographic Description of the Phase Diagram of a Chiral Symmetry Breaking Gauge Theory, JHEP 03 (2010) 132, [arXiv:1002.1885].

[126] M. C. Wapler, Thermodynamics of Holographic Defects, JHEP 01 (2010) 056, arXiv:0911.2943.

[127] R. C. Myers and M. C. Wapler, Transport Properties of Holographic Defects, JHEP 12 (2008) 115, arXiv:0811.0480.

[128] N. Evans and E. Threlfall, Chemical Potential in the Gravity Dual of a $2+1$ Dimensional System, Phys. Rev. D79 (2009) 066008, [arXiv:0812.3273].

[129] V. G. Filev, C. V. Johnson, and J. P. Shock, Universal Holographic Chiral Dynamics in an External Magnetic Field, JHEP 08 (2009) 013, arXiv:0903.5345.

[130] M. C. Wapler, Holographic Experiments on Defects, arXiv:0909.1698.

[131] V. G. Filev, Hot Defect Superconformal Field Theory in an External Magnetic Field, JHEP 11 (2009) 123, arXiv:0910.0554.

[132] M. C. Wapler, Massive Quantum Liquids from Holographic Angel's Trumpets, JHEP 05 (2010) 019, arXiv:1002.0336].

[133] K. Jensen, A. Karch, D. T. Son, and E. G. Thompson, Holographic Berezinskii-Kosterlitz-Thouless Transitions, arXiv:1002.3159.

[134] N. Evans, A. Gebauer, K.-Y. Kim, and M. Magou, Phase diagram of the D3/D5 system in a magnetic field and a BKT transition, arXiv:1003.2694.

[135] N. Itzhaki, J. M. Maldacena, J. Sonnenschein, and S. Yankielowicz, Supergravity and the large $N$ limit of theories with sixteen supercharges, Phys. Rev. D58 (1998) 046004, hep-th/9802042. 
[136] T. Sakai and S. Sugimoto, Low energy hadron physics in holographic QCD, Prog. Theor. Phys. 113 (2005) 843-882, hep-th/0412141.

[137] O. Aharony, O. Bergman, D. L. Jafferis, and J. Maldacena, $N=6$ superconformal Chern-Simons-matter theories, M2-branes and their gravity duals, JHEP 10 (2008) 091, arXiv:0806.1218.

[138] D. T. Son and A. O. Starinets, Minkowski-space correlators in AdS/CFT correspondence: Recipe and applications, JHEP 09 (2002) 042, hep-th/0205051].

[139] C. P. Herzog and D. T. Son, Schwinger-Keldysh propagators from AdS/CFT correspondence, JHEP 03 (2003) 046, hep-th/0212072.

[140] I. Amado, M. Kaminski, and K. Landsteiner, Hydrodynamics of Holographic Superconductors, JHEP 05 (2009) 021, [arXiv:0903.2209].

[141] M. Kaminski, K. Landsteiner, J. Mas, J. P. Shock, and J. Tarrio, Holographic Operator Mixing and Quasinormal Modes on the Brane, JHEP 02 (2010) 021, [arXiv:0911.3610].

[142] S. A. Hartnoll and P. Kovtun, Hall conductivity from dyonic black holes, Phys. Rev. D76 (2007) 066001, [arXiv:0704.1160].

[143] S. A. Hartnoll and C. P. Herzog, Ohm's Law at strong coupling: S duality and the cyclotron resonance, Phys. Rev. D76 (2007) 106012, arXiv:0706.3228.

[144] K. Ghoroku, M. Ishihara, and A. Nakamura, D3/D7 holographic Gauge theory and Chemical potential, Phys. Rev. D76 (2007) 124006, [arXiv:0708.3706].

[145] T. Albash, V. G. Filev, C. V. Johnson, and A. Kundu, Quarks in an External Electric Field in Finite Temperature Large N Gauge Theory, JHEP 08 (2008) 092, arXiv:0709.1554.

[146] V. G. Filev and C. V. Johnson, Universality in the Large $N_{c}$ Dynamics of Flavour: Thermal Vs. Quantum Induced Phase Transitions, JHEP 10 (2008) 058, [arXiv:0805.1950].

[147] J. Erdmenger, M. Kaminski, P. Kerner, and F. Rust, Finite baryon and isospin chemical potential in AdS/CFT with flavor, JHEP 11 (2008) 031, arXiv:0807.2663.

[148] J. Mas, J. P. Shock, and J. Tarrio, Holographic Spectral Functions in Metallic AdS/CFT, JHEP 09 (2009) 032, [arXiv:0904.3905].

[149] C. P. Burgess, Open string instability in background electric fields, Nucl. Phys. B294 (1987) 427.

[150] C. Bachas and M. Porrati, Pair creation of open strings in an electric field, Phys. Lett. B296 (1992) 77-84, hep-th/9209032.

[151] N. Seiberg, A note on background independence in noncommutative gauge theories, matrix model and tachyon condensation, JHEP 09 (2000) 003, hep-th/0008013. 
[152] C. P. Herzog, A. Karch, P. Kovtun, C. Kozcaz, and L. G. Yaffe, Energy loss of a heavy quark moving through $N=4$ supersymmetric Yang-Mills plasma, JHEP 07 (2006) 013, hep-th/0605158.

[153] S. S. Gubser, Drag force in AdS/CFT, Phys. Rev. D74 (2006) 126005, hep-th/0605182.

[154] S. S. Gubser, S. S. Pufu, and F. D. Rocha, Quantum critical superconductors in string theory and M- theory, Phys. Lett. B683 (2010) 201-204, [arXiv:0908.0011].

[155] S. S. Gubser, C. P. Herzog, S. S. Pufu, and T. Tesileanu, Superconductors from Superstrings, Phys. Rev. Lett. 103 (2009) 141601, arXiv:0907.3510.

[156] J. P. Gauntlett, J. Sonner, and T. Wiseman, Holographic superconductivity in M-Theory, Phys. Rev. Lett. 103 (2009) 151601, arXiv:0907.3796].

[157] D. T. Son and M. A. Stephanov, QCD at finite isospin density: From pion to quark antiquark condensation, Phys. Atom. Nucl. 64 (2001) 834-842, hep-ph/0011365.

[158] F. Sannino, Review of vector condensation at high chemical potential, AIP Conf. Proc. 688 (2004) 121-127, hep-ph/0307053.

[159] O. Aharony, K. Peeters, J. Sonnenschein, and M. Zamaklar, Rho meson condensation at finite isospin chemical potential in a holographic model for QCD, JHEP 02 (2008) 071, [arXiv:0709.3948].

[160] A. Rebhan, A. Schmitt, and S. A. Stricker, Meson supercurrents and the Meissner effect in the Sakai- Sugimoto model, JHEP 05 (2009) 084, arXiv:0811.3533].

[161] S. S. Gubser, F. D. Rocha, and A. Yarom, Fermion correlators in non-abelian holographic superconductors, arXiv:1002.4416.

[162] P. Basu, J. He, A. Mukherjee, and H.-H. Shieh, Hard-gapped Holographic Superconductors, Phys. Lett. B689 (2010) 45-50, [arXiv:0911.4999.

[163] S. S. Gubser and F. D. Rocha, The gravity dual to a quantum critical point with spontaneous symmetry breaking, Phys. Rev. Lett. 102 (2009) 061601, arXiv:0807.1737.

[164] S. S. Gubser and A. Nellore, Ground states of holographic superconductors, Phys. Rev. D80 (2009) 105007, arXiv:0908.1972.

[165] G. T. Horowitz and M. M. Roberts, Zero Temperature Limit of Holographic Superconductors, JHEP 11 (2009) 015, arXiv:0908.3677.

[166] M. M. Roberts and S. A. Hartnoll, Pseudogap and time reversal breaking in a holographic superconductor, JHEP 08 (2008) 035, arXiv:0805.3898.

[167] D. Marolf, L. Martucci, and P. J. Silva, Fermions, T-duality and effective actions for D-branes in bosonic backgrounds, JHEP 04 (2003) 051, hep-th/0303209].

[168] D. Marolf, L. Martucci, and P. J. Silva, Actions and fermionic symmetries for D-branes in bosonic backgrounds, JHEP 07 (2003) 019, hep-th/0306066. 
[169] L. Martucci, J. Rosseel, D. Van den Bleeken, and A. Van Proeyen, Dirac actions for D-branes on backgrounds with fluxes, Class. Quant. Grav. 22 (2005) 2745-2764, hep-th/0504041.

[170] M. Kaminski, Flavor Superconductivity and Superfluidity, arXiv:1002.4886.

[171] Y. Maeno, T. M. Rice, and M. Sigrist, The intriguing superconductivity of strontium ruthenate, Physics Today 54 (Jan., 2001) 010000-47.

[172] R. Heise and H. G. Svendsen, A note on fermions in holographic QCD, JHEP 08 (2007) 065, arXiv:0706.2253.

[173] L.-Y. Hung and A. Sinha, Holographic quantum liquids in $1+1$ dimensions, JHEP 01 (2010) 114, arXiv:0909.3526].

[174] S. Kachru, A. Karch, and S. Yaida, Holographic Lattices, Dimers, and Glasses, Phys. Rev. D81 (2010) 026007, arXiv:0909.2639.

[175] D. Birmingham, I. Sachs, and S. N. Solodukhin, Conformal field theory interpretation of black hole quasi- normal modes, Phys. Rev. Lett. 88 (2002) 151301, hep-th/0112055.

[176] P. K. Kovtun and A. O. Starinets, Quasinormal modes and holography, Phys. Rev. D72 (2005) 086009, hep-th/0506184.

[177] T. Mehen, I. W. Stewart, and M. B. Wise, Conformal invariance for non-relativistic field theory, Phys. Lett. B474 (2000) 145-152, hep-th/9910025.

[178] Y. Nishida and D. T. Son, Nonrelativistic conformal field theories, Phys. Rev. D76 (2007) 086004, [arXiv:0706.3746].

[179] D. T. Son, Toward an AdS/cold atoms correspondence: a geometric realization of the Schroedinger symmetry, Phys. Rev. D78 (2008) 046003, [arXiv:0804.3972].

[180] K. Balasubramanian and J. McGreevy, Gravity duals for non-relativistic CFTs, Phys. Rev. Lett. 101 (2008) 061601, arXiv:0804.4053.

[181] C. P. Herzog, M. Rangamani, and S. F. Ross, Heating up Galilean holography, JHEP 11 (2008) 080, arXiv:0807.1099.

[182] J. Maldacena, D. Martelli, and Y. Tachikawa, Comments on string theory backgrounds with non- relativistic conformal symmetry, JHEP 10 (2008) 072, arXiv:0807.1100.

[183] A. Adams, K. Balasubramanian, and J. McGreevy, Hot Spacetimes for Cold Atoms, JHEP 11 (2008) 059, arXiv:0807.1111].

[184] A. Adams, C. M. Brown, O. DeWolfe, and C. Rosen, Charged Schrodinger Black Holes, Phys. Rev. D80 (2009) 125018, arXiv:0907.1920.

[185] S. A. Hartnoll, J. Polchinski, E. Silverstein, and D. Tong, Towards strange metallic holography, JHEP 04 (2010) 120, arXiv:0912.1061].

[186] M. C. N. Cheng, S. A. Hartnoll, and C. A. Keeler, Deformations of Lifshitz holography, JHEP 03 (2010) 062, [arXiv:0912.2784]. 
[187] N. Bobev, A. Kundu, and K. Pilch, Supersymmetric IIB Solutions with Schródinger Symmetry, JHEP 07 (2009) 107, arXiv:0905.0673.

[188] A. Donos and J. P. Gauntlett, Solutions of type IIB and D=11 supergravity with Schrodinger(z) symmetry, JHEP 07 (2009) 042, arXiv:0905.1098.

[189] E. G. Gimon, A. Hashimoto, V. E. Hubeny, O. Lunin, and M. Rangamani, Black strings in asymptotically plane wave geometries, JHEP 08 (2003) 035, hep-th/0306131.

[190] J. L. F. Barbon and C. A. Fuertes, Ideal gas matching for thermal Galilean holography, Phys. Rev. D80 (2009) 026006, [arXiv:0903.4452].

[191] C. A. Fuertes and S. Moroz, Correlation functions in the non-relativistic AdS/CFT correspondence, Phys. Rev. D79 (2009) 106004, [arXiv:0903.1844].

[192] A. Volovich and C. Wen, Correlation Functions in Non-Relativistic Holography, JHEP 05 (2009) 087, arXiv:0903.2455].

[193] R. C. Myers, Dielectric-branes, JHEP 12 (1999) 022, hep-th/9910053.

[194] S. S. Pal, Null Melvin Twist to Sakai-Sugimoto model, arXiv:0808.3042.

[195] J. Erdmenger, M. Kaminski, and F. Rust, Holographic vector mesons from spectral functions at finite baryon or isospin density, Phys. Rev. D77 (2008) 046005, [arXiv:0710.0334].

[196] J. Bagger and N. Lambert, Modeling multiple M2's, Phys. Rev. D75 (2007) 045020, hep-th/0611108.

[197] A. Gustavsson, Algebraic structures on parallel M2-branes, Nucl. Phys. B811 (2009) 66-76, arXiv:0709.1260].

[198] J. Bagger and N. Lambert, Gauge Symmetry and Supersymmetry of Multiple M2-Branes, Phys. Rev. D77 (2008) 065008, [arXiv:0711.0955].

[199] J. Bagger and N. Lambert, Comments On Multiple M2-branes, JHEP 02 (2008) 105, [arXiv:0712.3738].

[200] M. Van Raamsdonk, Comments on the Bagger-Lambert theory and multiple M2branes, JHEP 05 (2008) 105, arXiv:0803.3803.

[201] N. Lambert and D. Tong, Membranes on an Orbifold, Phys. Rev. Lett. 101 (2008) 041602, arXiv:0804.1114.

[202] J. Distler, S. Mukhi, C. Papageorgakis, and M. Van Raamsdonk, M2-branes on M-folds, JHEP 05 (2008) 038, arXiv:0804.1256.

[203] A. Hanany and E. Witten, Type IIB superstrings, BPS monopoles, and threedimensional gauge dynamics, Nucl. Phys. B492 (1997) 152-190, hep-th/9611230].

[204] D. Gaiotto and E. Witten, Supersymmetric Boundary Conditions in N=4 Super Yang-Mills Theory, arXiv:0804.2902. 
[205] E. Bergshoeff and J. P. van der Schaar, On M-9-branes, Class. Quant. Grav. 16 (1999) 23-39, hep-th/9806069.

[206] Y. Hikida, W. Li, and T. Takayanagi, ABJM with Flavors and FQHE, JHEP 07 (2009) 065, arXiv:0903.2194.

[207] C. M. Hull and P. K. Townsend, Unity of superstring dualities, Nucl. Phys. B438 (1995) 109-137, hep-th/9410167.

[208] E. Witten, String theory dynamics in various dimensions, Nucl. Phys. B443 (1995) 85-126, hep-th/9503124.

[209] N. A. Obers and B. Pioline, U-duality and M-theory, Phys. Rept. 318 (1999) 113-225, hep-th/9809039.

[210] P. K. Townsend, The eleven-dimensional supermembrane revisited, Phys. Lett. B350 (1995) 184-187, hep-th/9501068.

[211] E. A. Ivanov, Chern-Simons matter systems with manifest $N=2$ supersymmetry, Phys. Lett. B268 (1991) 203-208.

[212] D. Gaiotto and X. Yin, Notes on superconformal Chern-Simons-matter theories, JHEP 08 (2007) 056, arXiv:0704.3740].

[213] H.-C. Kao, K.-M. Lee, and T. Lee, The Chern-Simons coefficient in supersymmetric Yang-Mills Chern-Simons theories, Phys. Lett. B373 (1996) 94-99, hep-th/9506170.

[214] O. Bergman, A. Hanany, A. Karch, and B. Kol, Branes and supersymmetry breaking in 3D gauge theories, JHEP 10 (1999) 036, hep-th/9908075.

[215] T. Kitao, K. Ohta, and N. Ohta, Three-dimensional gauge dynamics from brane configurations with (p,q)-fivebrane, Nucl. Phys. B539 (1999) 79-106, hep-th/9808111.

[216] A. Karch, D. Lüst, and D. J. Smith, Equivalence of geometric engineering and Hanany-Witten via fractional branes, Nucl. Phys. B533 (1998) 348-372, hep-th/9803232.

[217] A. Karch, Field theory dynamics from branes in string theory, hep-th/9812072.

[218] J. Park, R. Rabadan, and A. M. Uranga, $N=1$ type IIA brane configurations, chirality and T- duality, Nucl. Phys. B570 (2000) 3-37, hep-th/9907074.

[219] E. G. Gimon and J. Polchinski, Consistency Conditions for Orientifolds and D-Manifolds, Phys. Rev. D54 (1996) 1667-1676, hep-th/9601038.

[220] S. Hohenegger and I. Kirsch, A note on the holography of Chern-Simons matter theories with flavour, JHEP 04 (2009) 129, [arXiv:0903.1730].

[221] D. Gaiotto and D. L. Jafferis, Notes on adding D6 branes wrapping $R P^{3}$ in $A d S_{4} \times C P^{3}$, arXiv:0903.2175.

[222] M. Fujita, W. Li, S. Ryu, and T. Takayanagi, Fractional Quantum Hall Effect via Holography: Chern-Simons, Edge States, and Hierarchy, arXiv:0901.0924. 
[223] A. O'Bannon, Toward a Holographic Model of Superconducting Fermions, JHEP 01 (2009) 074, [arXiv:0811.0198.

[224] T. Albash and C. V. Johnson, Holographic Aspects of Fermi Liquids in a Background Magnetic Field, arXiv:0907.5406.

[225] P. Basu, J. He, A. Mukherjee, and H.-H. Shieh, Holographic Non-Fermi Liquid in a Background Magnetic Field, arXiv:0908.1436.

[226] F. Denef, S. A. Hartnoll, and S. Sachdev, Quantum oscillations and black hole ringing, Phys. Rev. D80 (2009) 126016, [arXiv:0908.1788].

[227] T. Albash and C. V. Johnson, Landau Levels, Magnetic Fields and Holographic Fermi Liquids, arXiv:1001.3700.

[228] S. S. Gubser and F. D. Rocha, Peculiar properties of a charged dilatonic black hole in AdS5, Phys. Rev. D81 (2010) 046001, arXiv:0911.2898.

[229] K. Jensen and A. Karch, ABJM Mirrors and a Duality of Dualities, JHEP 09 (2009) 004, arXiv:0906.3013].

[230] P. Koerber and L. Martucci, D-branes on AdS flux compactifications, JHEP 01 (2008) 047, arXiv:0710.5530.

[231] P. Koerber, Coisotropic D-branes on AdS4 x CP3 and massive deformations, JHEP 09 (2009) 008, [arXiv:0904.0012].

[232] M. Haack, D. Lüst, L. Martucci, and A. Tomasiello, Domain walls from ten dimensions, JHEP 10 (2009) 089, [arXiv:0905.1582].

[233] J. Alanen, E. Keski-Vakkuri, P. Kraus, and V. Suur-Uski, AC Transport at Holographic Quantum Hall Transitions, JHEP 11 (2009) 014, arXiv:0905.4538.

[234] M. Fujita and T.-S. Tai, Eschenburg space as gravity dual of flavored $N=4$ ChernSimons-matter theory, JHEP 09 (2009) 062, arXiv:0906.0253.

[235] D. Gaiotto and A. Tomasiello, The gauge dual of Romans mass, JHEP 01 (2010) 015, arXiv:0901.0969.

[236] D. Gaiotto and A. Tomasiello, Perturbing gauge/gravity duals by a Romans mass, J. Phys. A42 (2009) 465205, [arXiv:0904.3959].

[237] P. Kovtun, D. T. Son, and A. O. Starinets, Viscosity in strongly interacting quantum field theories from black hole physics, Phys. Rev. Lett. 94 (2005) 111601, hep-th/0405231.

[238] A. Sen and B. Zwiebach, Tachyon condensation in string field theory, JHEP 03 (2000) 002, hep-th/9912249.

[239] R. G. Leigh, Dirac-Born-Infeld Action from Dirichlet Sigma Model, Mod. Phys. Lett. A4 (1989) 2767.

[240] N. Berkovits, ICTP lectures on covariant quantization of the superstring, hep-th/0209059. 
[241] F. Gliozzi, J. Scherk, and D. I. Olive, Supersymmetry, Supergravity Theories and the Dual Spinor Model, Nucl. Phys. B122 (1977) 253-290.

[242] J. Dai, R. G. Leigh, and J. Polchinski, New Connections Between String Theories, Mod. Phys. Lett. A4 (1989) 2073-2083.

[243] J. Polchinski, Dirichlet-Branes and Ramond-Ramond Charges, Phys. Rev. Lett. 75 (1995) 4724-4727, hep-th/9510017.

[244] P. Koerber and A. Sevrin, The non-abelian D-brane effective action through order alpha**4, JHEP 10 (2002) 046, hep-th/0208044].

[245] A. A. Tseytlin, On non-abelian generalisation of the Born-Infeld action in string theory, Nucl. Phys. B501 (1997) 41-52, hep-th/9701125.

[246] J. P. Gauntlett, G. W. Gibbons, G. Papadopoulos, and P. K. Townsend, Hyper-Kaehler manifolds and multiply intersecting branes, Nucl. Phys. B500 (1997) 133-162, hep-th/9702202.

[247] T. Nishioka and T. Takayanagi, Fuzzy Ring from M2-brane Giant Torus, JHEP 10 (2008) 082, arXiv:0808.2691.

[248] N. Drukker, J. Plefka, and D. Young, Wilson loops in 3-dimensional N=6 supersymmetric Chern-Simons Theory and their string theory duals, JHEP 11 (2008) 019, arXiv:0809.2787.

[249] M. A. Bandres, A. E. Lipstein, and J. H. Schwarz, Studies of the ABJM Theory in a Formulation with Manifest SU(4) R-Symmetry, JHEP 09 (2008) 027, arXiv:0807.0880. 



\section{Acknowledgements}

There are many people to whom I am very much indebted and it is a pleasure to express my gratitude towards them here.

In the first place, I would like to express my gratitude to my advisor Johanna Erdmenger for her continuous support during the last years. The constant interest in my work and the willingness to discuss problems and achievements at any time, lead to an extremely enjoyable and stimulating working atmosphere.

Furthermore, I would like to thank Dieter Lüst not only for working through this thesis as an interested second referee and for extremely inspiring discussion about string theory, but also for providing brilliant working conditions in his groups at the Max-Planck-Institut for Physik (Werner-Heisenberg-Institut) and at Ludwig-Maximilians-Universität in Munich.

I would like to thank Viviane Grass, Constantin Greubel, Sebastian Halter, Stephan Höhne, Matthias Kaminski, Patrick Kerner, René Meyer, Hai Ngo, Andy O’Bannon, Felix Rust, Timm Wrase as well as Carlos Hoyos and Jackson Wu for being good collaborators, colleagues and friends.

I thank - in alphabetical order - Nikolas Akerblom, Murad Alim, Saeid Aminiam, Ralph Blumenhagen, Alex Buchel, Claudio Caviezel, Michael Haack, Daniel Härtl, Sean Hartnoll, Christopher Herzog, Jegors Korovins, Simon Körs, Benjamin Jurke, Felix Karbstein, Paul Koerber, Luca Martucci, John McGreevy, Sebastian Moster, Enrico Pajer, Erik Plauschinn, Thorsten Rahn, Christian Römelsberger, Ivo Sachs, Oliver Schlotterer, Maximilian Schmidt-Sommerfeld, Stephan Stieberger, Marc Wagner, Amos Yarom, Marco Zagermann and Andrey Zayakin for many hours of interesting discussions and pleasant time together.

I also thank the Max-Planck Institute for Physics and the IMPRS program of the MaxPlanck-Society for providing various useful seminars and promoting interchange among the Ph.D. students. Also, thanks are due to the coordinator of the IMPRS for Particle Physics, Frank Steffen, as well as to the secretaries Monika Goldammer, Roswita Jurgeleit and Carola Reinke. I am also very grateful to Thomas Hahn for IT support. I am grateful to Sebastian Halter, Patrick Kerner, Steffen Müller, Hai Ngo, Andy O'Bannon and HansJörg Zeller for careful proofreading several chapters of this thesis.

I thank the Studienstiftung des Deutschen Volkes for financial support during my studies. Part of this work was also funded by the Cluster of Excellence for Fundamental Physics - Origin and Structure of the Universe and by the International Max Planck Research School.

Finally, I would like to thank my parents, my brother and my friends for their support, love and understanding as well as their patience when I was talking about physics. 